CORSO DI DOTTORATO DI RICERCA IN SCIENZE BIBLIOGRAFICHE, ARCHIVISTICHE, DOCUMENTARIE E PER LA CONSERVAZIONE E IL RESTAURO DEI BENI LIBRARI E ARCHIVISTICI - CICLO XXIV

TESI DI DOTTORATO DI RICERCA

\title{
UN APPROCCIO CONCETTUALE E LINGUISTICO ALL'INDICIZZAZIONE RIFLESSIONI E PROPOSTE SUGLI STRUMENTI INDICALI
}

RELATORE

CHIAR.MO PROF. MAURO GUERRINI 
Tesi di dottorato di Giuliano Genetasio, discussa presso l'Università degli Studi di Udine

\begin{abstract}
La ricerca mira a fornire una serie di proposte teoriche per la revisione degli strumenti indicali, secondo una visione unitaria delle diverse branche dell'indicizzazione, e seguendo due approcci distinti ma correlati: concettuale e linguistico.

La prima parte della ricerca, dedicata all'approccio "concettuale" - nel senso con cui il termine è inteso nell'ambito della progettazione di database -, è rivolta a elaborare un modello dell'universo bibliografico e dei suoi elementi (entità, attributi, relazioni, funzioni dell'utente) e a suggerire le possibilità di una sua realizzazione in termini di strutture catalografiche. Base di partenza del modello concettuale proposto è FRBR, Functional requirements for bibliographic records, pubblicato dall'IFLA nel 1998 e rivolto a stabilire i requisiti funzionali delle registrazioni bibliografiche; FRBR è tuttavia assunto criticamente e il modello proposto se ne differenzia in più punti.

La seconda parte della ricerca, dedicata all'approccio linguistico all'indicizzazione, analizza gli strumenti indicali alla luce di nozioni tratte dalla linguistica; li considera cioè in quanto lingue (documentarie), con il duplice obiettivo di fornire una particolare prospettiva d'analisi dell'indicizzazione e di elaborare un modello di lingua documentaria utile come base per la costruzione di nuove lingue o per il miglioramento di quelle esistenti.

Gli approcci concettuale e linguistico, benché autonomi, concorrono a costituire un unico quadro teorico dell'indicizzazione e non mancano riferimenti reciproci tra i due approcci.

Tra gli elementi peculiari dell'approccio concettuale vi sono: l'introduzione di entità nuove rispetto a FRBR (Tempo, Contenuto editoriale, Tipo di persona, Tipo di testo, Tipo di ente e Tipo di risorsa); una trattazione analitica della nozione di soggetto e dei suoi diversi aspetti (circalità estensionale e intensionale, punti di vista, tema e rema); una trattazione dettagliata delle relazioni semantiche tipiche dell'indicizzazione per soggetto, le quali vengono estese agli elementi della catalogazione per autore; un'articolata tassonomia degli elementi indicali che dovrebbero comparire nelle registrazioni bibliografiche. Tra gli elementi peculiari dell'approccio linguistico vi sono: una visione unificata e integralmente semantica delle diverse branche dell'indicizzazione, ovvero un modello di lingua documentaria che si pone come base per la catalogazione sia per autore sia per soggetto; un'ampia trattazione delle relazioni semantiche delle lingue documentarie, con la proposta di varie tipologie di relazioni tradizionalmente non prese in considerazione dalla teoria dell'indicizzazione (relazioni gerarchiche generiche specificate sulla base delle relazioni sintagmatiche; relazioni semiche e sememiche; relazioni tra nominalizzazioni; relazioni di omotassia; un'articolata tassonomia delle relazioni sintagmatiche); la possibilità di esprimere la totalità delle relazioni bibliografiche (anche quelle relative alla responsabilità, al soggetto) come parte della semantica, o rete sindetica, delle lingue documentarie.
\end{abstract}


Tesi di dottorato di Giuliano Genetasio, discussa presso l'Università degli Studi di Udine

\section{Introduzione}

\subsection{L'attuale contesto dell'accesso bibliografico e il dibattito suscitato dal Rapporto Calhoun}

Il quadro dell'accesso bibliografico - il processo di interazione con le registrazioni bibliografiche da parte di indicizzatori e utenti finali, comprensivo di indicizzazione (catalogazione $)^{1}$ e reperimento dell'informazione - e delle sue modalità esplicative ha subito cambiamenti fondamentali nel corso degli ultimi trent'anni: prima con il passaggio dall'ambiente cartaceo a quello elettronico; poi, grazie alla telematica, con il passaggio dal catalogo locale al catalogo globale; poi ancora con il web sociale (web 2.0) e infine - ma qui siamo ancora agli inizi e l'esito del processo non è scontato - con il web semantico (web 3.0).

In anni recenti, il catalogo e gli strumenti di indicizzazione tradizionali sono stati soggetti ad attacchi da più parti. Karen Calhoun ${ }^{2}$ ha evidenziato un processo parallelo di declino e disintegrazione: secondo l'autrice il catalogo è in declino perché usato sempre meno dagli utenti; perché rappresenta una fetta sempre minore dell'universo informativo; perché alle registrazioni bibliografiche tradizionali, esterne e aggiunte alle risorse, si sostituiscono sempre più metadati inseriti all'interno delle risorse stesse; il catalogo è al contempo soggetto a un processo di disintegrazione, per il quale le registrazioni bibliografiche sono sempre meno unità informative a sé stanti e sempre più prodotti dell'assemblaggio di singoli dati - questi ultimi diverrebbero invece il fulcro dei processi di accesso e scambio bibliografico, utilizzabili da una molteplicità di attori per una molteplicità di scopi anche non precipuamente catalografici. Tanto il rapporto di Calhoun, quanto un altro importante rapporto pubblicato dall'UCLA, University of California at Los Angeles, ${ }^{3}$ hanno attaccato l'accesso per soggetto in generale, ritenuto costoso o non essenziale, e gli strumenti tradizionali di indicizzazione per soggetto in particolare, primo tra tutti le Library of Conress Subject Headings (LCSH), il soggettario della Library of Congress diffuso in tutto il mondo e sviluppato a partire dalla fine del diciannovesimo secolo. ${ }^{4}$

1 Considereremo i due termini come sinonimi.

2 KAREN CALHOUN, The changing nature of the catalog and its integration with other discovery tools. Final report, prepared for the Library of Congress, 2006, disponibile all'indirizzo

$\langle$ http://www.loc.gov/catdir/calhoun-report-final.pdf $>$.

3 UNIVERSITY OF CALIFORNIA LIBRARIES. BIBLIOGRAPHIC SERVICES TASK FORCE,

Rethinking how we provide bibliographic services for the University of California. Final report, 2005, disponibile all'indirizzo <http://libraries.universityofcalifornia.edu/sopag/BSTF/Final.pdf>.

4 AMERICAN LIBRARY ASSOCIATION, List of subject headings for use in dictionary catalogs, Boston, Library Bureau, 1895, disponibile all'indirizzo

<http://ia600304.us.archive.org/4/items/ed2listofsubject00ameruoft/ed2listofsubject00ameruoft.pdf>; LIBRARY OF CONGRESS. POLICY AND STANDARDS DIVISION, Library of Congress subject headings, 32nd ed., Washington, D.C., Library of Congress, Cataloging Distribution Service, 2010. Cfr. NORMAN ODER, The end of LC subject headings?, "Library journal", 13, 9 (2006), p. 14: "Should the Library of Congress (LC) jettison Library of Congress Subject Headings (LCSH), the longstanding professional taxonomy? That's one of the provocative suggestions in a new report released last month by LC "The Changing Nature of the Catalog and Its Integration with Other Discovery Tools," commissioned by LC and written by associate university librarian Karen Calhoun". L'attacco di Calhoun e dell'UCLA alle LCSH è citato anche da ADAM L. SCHIFF, The future of LCSH, 2008, disponibile all'indirizzo 
La maggior parte degli attacchi dei rapporti di Calhoun e dell'UCLA si sono presto rivelati per ciò che erano: goffi tentativi di tagliare i costi della catalogazione poco o per nulla fondati su realtà di fatto. Discutere dell'attuale rilevanza del catalogo rispetto ad altri strumenti di ricerca esula dagli scopi della presente ricerca; facciamo però presenti alcuni punti. Il processo di disintegrazione del catalogo, con l'assurgere dei singoli dati a unità informative di base rispetto ai quali le registrazioni bibliografiche costituiscono un prodotto, è un processo in corso ormai da diversi anni e di cui non si può che prendere atto, ma di cui occorre evidenziare alcune criticità: i dati che troviamo nelle registrazioni bibliografiche nascono e sono concepiti per un ambiente cartaceo, in cui la registrazione andava letta nel suo insieme e in cui l'interpretazione del singolo dato non era sempre autonoma dagli altri; è inoltre vero che oggi i dati bibliografici sono utilizzati da una molteplicità di attori per una molteplicità di fini diversi, ma anche questo dato di fatto va assunto criticamente: cosa succede se i diversi attori utilizzano uno stesso dato con significati o persino scopi differenti? Le biblioteche hanno scopi diversi da quelle delle librerie, degli editori, dei distributori, o di singoli utenti; i significati attributi ai diversi elementi delle registrazioni bibliografiche mutano a partire dai presupposti e dai fini degli utenti di dati bibliografici; il modello distribuito di accesso e controllo bibliografico va senz'altro accettato, ma con alcune riserve. Per quanto riguarda l'accesso per soggetto, va del tutto scartata l'idea secondo cui sia possibile farne a meno: che esso sia un'esigenza fondamentale e indispensabile dell'accesso bibliografico è un dato che emerge da numerose ricerche; la ricerca per soggetto, pur profondamente problematica, è una delle modalità di ricerca principali negli OPAC e, anzi, in forte aumento a partire dall'introduzione dei cataloghi in rete; ${ }^{5}$ in contesti documentalistici essa è poi la modalità di ricerca principale. Va anche smentito il luogo comune secondo cui la ricerca per soggetto sarebbe utilizzata solo da chi ignora specifici autori o titoli: la ricerca per soggetto può essere utilizzata sia come mezzo (per ritrovare testi noti ma di cui non si ricordi l'autore o il titolo) sia come fine (per avere una conoscenza esaustiva dei testi su un soggetto, per navigare registrazioni bibliografiche di testi su argomento identico o correlato, etc.), e in quest'ultimo caso anche a partire da ricerche per autore o tramite altri elementi informativi; la ricerca per soggetto è inoltre, anche in ricerche per autore o di diverso tipo, uno degli elementi fondamentali (forse il più importante) per operare una scelta tra diverse risorse bibliografiche correlate. Per quanto riguarda l'attacco agli strumenti tradizionali dell'accesso per soggetto, la loro superiorità complessiva è stata, in risposta agli attacchi di Calhoun, dimostrata efficacemente dai diversi saggi di Thomas Mann e da un rapporto del CPSO, Cataloging Policy and Standards Office (oggi PSD, Policy \& Standards Division) della Library of Congress; ${ }^{6}$ (la superiorità delle modalità

$\langle$ http://depts.washington.edu/aluw/activities/0708/The_Future_of_LCSH.ppt $>$.

5 Cfr. ELAINE SVENONIUS, The intellectual foundation of information organization, Cambridge, Mass., MIT Press, 2000, p. 46; cfr. anche RAY R. LARSON, The decline of subject searching. Long-term trends and patterns of index use in an online catalog, "Journal of the American Society for Information Science", 42, 3 (1991), disponibile all'indirizzo <http://polaris.gseis.ucla.edu/gleazer/462_readings/Larson_1991.pdf>, p. 197-198; cfr. anche JENS-ERIK MAI, Semiotics and indexing. An analysis of the subject indexing process, "Journal of documentation", 57, 5 (2001), p. 620.

6 LIBRARY OF CONGRESS. CATALOGING POLICY AND SUPPORT OFFICE, Library of Congress subject headings. Pre- vs. Post-coordination and related issues, 2007, disponibile all'indirizzo <http://www.loc.gov/ catdir/cpso/pre_vs_post.pdf $>$; THOMAS MANN, The changing nature of the catalog and its integration with other discovery tools. Final report. March 17, 2006. Prepared for the Library of Congress by Karen Calhoun. A critical 
tradizionali di indicizzazione per soggetto era peraltro già stata dimostrata ampiamente da studi precedenti).

Non intendiamo approfondire qui la questione, che riprenderemo, in riferimento a punti specifici, nei capitoli successivi, ma elenchiamo alcuni dei punti di forza degli strumenti tradizionali di indicizzazione per soggetto e del controllo linguistico che essi comportano; essi offrono all'utente finale la possibilità di: 1) accedere a un elemento di significato a prescindere dalla sua forma o terminologia, anche aldilà delle barriere linguistiche, grazie al raggruppamento di sinonimi e diverse forme del nome, del titolo, etc. di un elemento; 2) suggerirgli sinonimi ed espressioni correlate a quelle ricercate (Larson evidenzia il nesso tra bisogno informativo dell'utente e ignoranza della terminologia specifica); 3) navigare le registrazioni bibliografiche tramite termini correlati in esse registrate; 4) esprimere significati complessi grazie alla coordinazione di più elementi di significato; 5) facilitare l'interpretazione semantica dei termini utilizzati per l'indicizzazione tramite la creazione di contesti artificiali (relazioni tra i diversi elementi componenti di un elemento lessicale in forma composta, rete sindetica, etc.) che disambiguano omonimie e omotassie (sintagmi sintatticamente identici ma semanticamente differenti, ne parleremo in 3.2.5.3.3); 6) offrendogli una rappresentazione sintetica e mediata del soggetto di un testo ${ }^{8} 7$ ) offrendogli la possibilità di uno scorrimento (browsing) facile e ordinato dei risultati di ricerca, in modo da avere una visione complessiva di un soggetto e delle sue articolazioni. Viceversa, nel corso di più di trent'anni numerose sono state le critiche a modalità di accesso per soggetto derivate o non controllate come le parole chiave e l'indicizzazione automatizzata; tra queste, una delle principali è l'impossibilità dell'indicizzazione automatizzata di un'analisi autenticamente semantica dei testi; ${ }^{9}$ tra gli altri, Julian Warner ha nel 2006 fornito una critica radicale della possibilità di una completa sostituzione (mentre ne sostiene l'utilità come strumento integrativo) del computer all'uomo nell'indicizzazione. ${ }^{10}$

\subsection{Verità del dibattito e ragioni della ricerca: carenze teoriche e inadeguatezza tecnologica dell'indicizzazione}

review, 2006, disponibile all'indirizzo <http://www.guild2910.org/AFSCMECalhounReviewREV.pdf>. 7 RAY R. LARSON, The decline of subject searching, cit.

8 Cfr., in riferimento al full text, KAREN SPARCK-JONES - MARTIN KAY, Linguistics and information science, New York, Academic Press, 1973, p. 48; il full text "Has the advantage that it avoids the omissions and distortions inevitably associated with index descriptions. But it ignores the positive role of the summary index description [...] there will always be circumstances in which summaries are used for reasons of convenience as well as economy, and we can therefore continue to assume that document retrieval systems typically involve the substitution of a shorter text for a longer one".

9 Cfr. B. LONG, Linguistique et indexation, "Documentaliste", 17, 3 (1980), p. 105; cfr. anche MAGDELEINE MOUREAU, Les aspects linguistiques des stratégies d'interrogation dans la rcherche bibliographique sur ordinateur, "Documentaliste", 13, 5/6 (1976), p. 190; cfr. anche KAREN COYLE, Machine indexing, "Journal of academic librarianship", 34, 6 (2008), p. 530.

10 Cfr. JULIAN WARNER, Linguistics and information theory. Analytic advantages, "Journal of the American Society for Information Science and Technology”, 58, 2 (2007), p. 283-284, il quale evidenzia

l'impossibilità per i computer: 1) di tenere conto in modo autentico della semantica dei testi; 2) di operare scelte; 3) della loro assenza di intenzionalità; Warner confuta la possibilità di un IR di tipo deterministico ed evidenzia l'influenza di Cartesio (razionalismo) e della filosofia cognitiva riduzionista (equivalenza di mente e macchina) su questo tipo di posizioni. 
Tesi di dottorato di Giuliano Genetasio, discussa presso l'Università degli Studi di Udine

Vi sono alcune verità di fondo nel dibattito suscitato dalla pubblicazione del Rapporto Calhoun. Tanto gli attacchi di Calhoun e dell'UCLA, quanto i testi (Mann, CPSO, etc.) che hanno teso a rispondergli, hanno direttamente o indirettamente contribuito a evidenziare due problemi storici dell'indicizzazione e dei relativi strumenti: 1) la parziale inadeguatezza degli strumenti di indicizzazione tradizionali - ancora per certi aspetti basati sulla logica lineare e fissa dell'ambiente cartaceo - rispetto ai progressi tecnologici degli ultimi trent'anni; da questo punto di vista è emblematico che alcuni argomenti portati da Mann in difesa alle LCSH siano basati su una visione forzosamente lineare dell'accesso per soggetto; 2) l'assenza di una visione solida, unitaria e soddisfacente della natura, delle finalità e degli strumenti dell'indicizzazione, ovvero anche degli elementi dell'universo bibliografico e delle registrazioni che dovrebbero rifletterlo.

Tali carenze teoriche hanno influito enormemente sull'accesso bibliografico nel corso dei decenni e si sono a loro volta tradotte in strumenti indicali insoddisfacenti e tra loro integrati in modo non sufficiente, soprattutto per quanto riguarda l'indicizzazione per soggetto.

\subsection{Contenuti e obiettivi della ricerca: approccio concettuale e linguistico all'indicizzazione}

Questa ricerca mira ad affrontare alcuni aspetti dei suddetti problemi. Essa cerca di offrire: 1) una visione complessiva e unitaria dell'accesso bibliografico e dell'indicizzazione in particolare; 2) un approfondimento teorico di alcuni tra questi elementi; 3) una serie di proposte per la revisione degli strumenti e delle strutture (registrazioni e loro elementi) dell'indicizzazione; 4) una visione dell'accesso bibliografico e dell'indicizzazione adeguata ai cambiamenti tecnologici dell'ambiente catalografico odierno.

Ciò secondo due approcci distinti ma correlati: 1) un approccio concettuale all'indicizzazione ("concettuale" nell'accezione con cui il termine è inteso all'interno della progettazione di database e guardando soprattutto al modello concettuale entità-relazioni pubblicato dall'IFLA: FRBR, Functional Requirements for Bibliographic Records), ${ }^{11}$ che delinei uno schema ideale degli elementi dell'universo bibliografico (in termini di entità, relazioni, attributi, funzioni, etc.) e delle strutture catalografiche che dovrebbero rappresentarli, sulla base di una discussione di tali elementi e strutture e in modo particolare di quelli relativi all'indicizzazione per soggetto; 2) un approccio linguistico all'indicizzazione, che analizzi i rapporti tra linguaggio e indicizzazione ovvero che affronti lo studio degli strumenti di indicizzazione in quanto lingue (documentarie), con il duplice obiettivo di fornire una particolare prospettiva d'analisi dell'indicizzazione e di avanzare alcune proposte volte al miglioramento linguistico degli strumenti di indicizzazione.

Per quanto riguarda l'approccio concettuale, l'utilizzo di modelli concettuali per la catalogazione non è in sé una novità, risalendo almeno agli anni Ottanta e affermandosi

11 IFLA STUDY GROUP ON THE FUNCTIONAL REQUIREMENTS FOR BIBLIOGRAPHIC RECORDS, Functional requirements for bibliographic records. Final report, approved by the Standing Committee of the IFLA Section on Cataloguing, München, Saur, 1998, disponibile all'indirizzo <http://www.ifla.org/files/cataloguing/frbr/frbr.pdf >. 
alla fine degli anni Novanta con FRBR. Tra gli elementi peculiari del nostro approccio concettuale vi sono: un modello concettuale basato su FRBR ma che differisce rispetto a esso in più punti (entità, attributi, relazioni e funzioni); una trattazione esplicita delle "relazioni semantiche" tipiche dell'indicizzazione per soggetto, ${ }^{12}$ estese però anche agli elementi della catalogazione per autore; un'approfondita discussione della nozione di soggetto e dei suoi diversi aspetti; aldilà del modello concettuale vero e proprio, una articolata tassonomia degli elementi che dovrebbero comparire nelle registrazioni bibliografiche. A differenza di FRBR, inoltre, il modello concettuale da noi elaborato non si limita a un'analisi dell'universo bibliografico così come rappresentato dalle attuali registrazioni bibliografiche, ma mira a elaborare un quadro ideale di rappresentazione indicale dell'universo bibliografico, proponendo. laddove opportuno, elementi indicali differenti rispetto a quelli presenti nelle attuali registrazioni bibliografiche.

Con le eccezioni che diremo, non abbiamo generalmente preso in considerazione i modelli dell'IFLA che hanno esteso FRBR all'analisi delle registrazioni di autorità: FRAD, Functional requirements for authority data, e FRSAD, Functional requirements for subject authority data; ${ }^{13}$ ciò sia per ragioni cronologiche (FRAD essendo stato pubblicato nel corso del 2009, quando il nostro modello concettuale era già abbozzato, e FRSAD soltanto nel 2010), sia perché abbiamo ritenuto che FRBR, rivolto alla catalogazione in generale e non a una particolare tipologia di registrazione di autorità, costituisse una migliore base di partenza per sviluppare il nostro modello, sia ancora perché, come diremo nel prossimo capitolo, abbiamo ritenuto alcuni punti di FRAD e FRSAD incompatibili con FRBR o comunque insoddisfacenti.

Per quanto riguarda l'approccio linguistico all'indicizzazione, l'idea per uno suo sviluppo è nata originariamente proprio dal lavoro al modello concettuale, e in particolare dall'idea di Tom Delsey secondo cui sarebbe stato opportuno modellizzare concettualmente le relazioni semantiche tipiche dei thesauri e le relazioni "sintattiche" tra i diversi elementi delle stringhe di soggetto, per poi svilupparsi autonomamente attraverso uno studio della linguistica generale e applicata all'indicizzazione. ${ }^{14}$ Nemmeno l'applicazione della linguistica agli strumenti indicali è, in sé, una novità: vi sono molti autori nell'ambito della teoria dell'indicizzazione e della linguistica che, soprattutto a partire dalla seconda metà degli anni Sessanta, hanno applicato analisi e metodologie linguistiche all'indicizzazione (vedi 3.1.2), nonché un intero filone della linguistica dedicata

12 Spiegheremo più avanti che parlare semplicemente di "relazioni semantiche" non è esatto (vedi 2.14). 13 IFLA UBCIM WORKING GROUP ON FUNCTIONAL REQUIREMENTS AND NUMBERING OF AUTHORITY RECORDS (FRANAR), Functional requirements for authority records. A conceptual model, draft 2005-06-15, 2005, <http://www.cidoc crm.org/docs/frbr_oo/frbr_docs/FRANARConceptual-M-Drafte.pdf>; FLA WORKING GROUP ON THE FUNCTIONAL REQUIREMENTS FOR SUBJECT AUTHORITY RECORDS, Functional requirements for subject authority data (FRSAD). A conceptual model, editors: Marcia Lei Zeng, Maja Žumer, Athena Salaba, approved by the Standing Committee of the IFLA Section on Classification and Indexing, June 2010, <http://www.ifla.org/files/classification-andindexing/functional-requirements-for-subject-authority-data/frsad-finalreport.pdf $>$. 14 Vedremo più avanti $(3.2 .6,3.3 .5)$ che parlare di "relazioni sintattiche" tout court non è del tutto corretto. Cfr. TOM DELSEY, Modeling subject access. Extending the FRBR and FRANAR conceptual models, "Cataloging \& classification quarterly", 39, 3/4 (2005), p. 56-58: "There are, however, a number of additional types of relationships that [...] will also need to be represented in the models. The most prominent of such relationships are the semantic relationships that are commonly reflected in the syndetic structure of thesauri and subject heading lists-in the references between equivalent terms, broader and narrower terms, related terms, etc.". 
all'applicazione automatizzata dell'indicizzazione. Tra gli elementi peculiari del nostro approccio linguistico vi sono: 1 ) una visione unificata e integralmente semantica dell'indicizzazione, con la formulazione di un modello di lingua documentaria che potrebbe costituire la base per l'indicizzazione nella sua totalità (per autore e per soggetto); non soltanto le relazioni semantiche tipiche della linguistica, ma anche le relazioni tipicamente bibliografiche potrebbero essere rappresentate come parte della medesima rete sindetica; 2) un'articolata tassonomia delle relazioni semantiche, basata sul parallelismo tra semantica implicita (paradigmatica) e semantica dei sintagmi (sintagmatica), con la proposta di relazioni semantiche tradizionalmente non prese in considerazione dall'indicizzazione; 3) una visione degli elementi del lessico della lingua documentaria come costituiti idealmente da una serie di componenti che è possibile distinguere e registrare separatamente, a cui si accompagna uno studio dettagliato delle relazioni sintagmatiche tra queste componenti.

Gli approcci concettuale e linguistico costituiscono due diverse prospettive con cui guardare all'indicizzazione; benché autonomi, essi concorrono a costituire un unico quadro teorico dell'indicizzazione e non mancano riferimenti reciproci tra i due approcci.

\subsection{Limiti della ricerca}

\subsubsection{Ambito di analisi}

Via via che la ricerca prendeva forma, è stato inevitabile circoscrivere la ricerca ad alcuni aspetti dell'indicizzazione, escludendone altri, pure molto importanti; ciò principalmente per ragioni di tempo, essendo la presente ricerca circoscritta a un arco temporale di circa tre anni; segnaliamo qui gli aspetti esclusi o trattati solo parzialmente. La ricerca non si occupa, se non tramite sporadici riferimenti, del reperimento dell'informazione; benché indicizzazione e reperimento dell'informazione costituiscano le due facce dell'accesso bibliografico, e benché una loro analisi congiunta fosse auspicabile nell'ottica di una visione unitaria dell'accesso bibliografico, li abbiamo ritenuti aspetti relativamente autonomi, almeno a livello di analisi; data la precedenza logica dell'indicizzazione rispetto al reperimento dell'informazione, abbiamo preferito indagare la prima, riservando l'analisi del reperimento dell'informazione - che per sua natura costituisce il banco di prova migliore delle nostre proposte sull'indicizzazione - a una futura occasione. La presente ricerca non indaga inoltre, se non indirettamente, l'analisi dei processi dell'indicizzazione: analisi concettuale, traduzione, modalità di modifica ed espansione degli strumenti di indicizzazione, etc. Inoltre, gli strumenti di indicizzazione sono indagati pressoché esclusivamente dalla prospettiva di indicizzatori e utenti finali, non da quella di altri utenti di dati bibliografici: bibliotecari di reference, responsabili delle acquisizioni, autori, editori, distributori, etc. L'analisi dell'indicizzazione per soggetto (alfabetica, in particolare) e dei suoi problemi è preponderante rispetto all'analisi della catalogazione per autore, di cui vengono soprattutto indagati gli elementi che più sono legati all'indicizzazione per soggetto. Altro limite della ricerca è che l'ambiente analizzato, del quale si evidenzieranno le carenze e rispetto al quale si avanzeranno alcune proposte di miglioramento, è quello dei cataloghi (OPAC) tradizionali; benché la ricerca abbia tenuto 
conto almeno in parte delle funzioni e di alcuni elementi tipici del web sociale, non è invece stato possibile estendere l'analisi all'universo del web semantico e agli argomenti a esso correlati (SKOS, Linked Data, etc.). Infine, ulteriori limiti della ricerca verranno esposti via via nel corso del lavoro.

\subsubsection{Approccio scelto}

Vi sono inoltre dei limiti intrinseci negli approcci proposti; li approfondiremo in dettaglio nei relativi capitoli, ma anticipiamo già i limiti principali. La modellizzazione concettuale è, per definizione, una schematizzazione dell'universo bibliografico, con tutti i limiti che ciò comporta: rischio di riduzionismo, arbitrarietà, etc. L'approccio linguistico all'indicizzazione, invece, ha il limite di prendere in considerazione soltanto un aspetto dell'indicizzazione: il linguaggio, aspetto fondamentale e che pure non esaurisce la totalità delle problematiche inerenti l'indicizzazione.

\subsubsection{Letteratura analizzata}

Per lo sviluppo dell'approccio concettuale abbiamo preso in considerazione la letteratura sull'indicizzazione, e in particolar modo sull'indicizzazione per soggetto; per lo sviluppo dell'approccio linguistico abbiamo preso in considerazione sia testi di linguistica generale sia di linguistica applicata all'indicizzazione. Per le ricerche bibliografiche sono state utilizzate le banche dati LISA (Library and Information Science Abstracts) e LLIS (Library Literature and Information Science); la ricerca si è servita prevalentemente del posseduto della Biblioteca "Luigi Crocetti" della Regione Toscana, della Biblioteca della Scuola Normale Superiore di Pisa e, in misura minore, della Biblioteca Umanistica dell'Università di Firenze, della Biblioteca Nazionale Centrale di Firenze e della Biblioteca dell'European University Institute presso Fiesole. Non è stato possibile prendere in considerazione la totalità del materiale pertinente, o perché (in pochissimi casi) irreperibile o perché - per incompetenza linguistica di chi scrive - in lingue diverse dall'inglese (che tuttavia costituisce la lingua prevalente dei testi pertinenti la ricerca), italiano, francese, portoghese o spagnolo. 
Tesi di dottorato di Giuliano Genetasio, discussa presso l'Università degli Studi di Udine

\section{Un approccio concettuale all'indicizzazione}

\subsection{L'approccio concettuale all'indicizzazione}

Il primo approccio che seguiremo nella nostra analisi dell'indicizzazione è di tipo concettuale: "concettuale" sia nel senso di teorico, sia nell'accezione propria dei modelli concettuali tipici del database design, e in particolare di tipo entità-relazioni, in cui un dato dominio di interesse viene rappresentato schematicamente in oggetti (entità), caratteristiche degli oggetti (attributi) e relazioni tra questi oggetti.

In ambito bibliografico, l'esempio più noto di modello concettuale è certamente FRBR, Functional requirements for bibliographic records, testo seminale pubblicato nel 1998 che informa i più recenti testi di principi (ICP, Principi internazionali di catalogazione) e codici di catalogazione (RDA, Resource Description and Access; REICAT, Regole italiane di catalogazione). ${ }^{15}$ FRBR ha rivoluzionato la teoria catalografica: 1) offrendo un modello concettuale dell'universo delle registrazioni bibliografiche in termini di entità - gli oggetti della catalogazione -, attributi (caratteristiche o elementi informativi delle entità) relazioni tra le entità e tra gli attributi; 2) rapportando ogni attributo e ogni relazione alle esigenze dell'utente finale; 3) offrendo una visione unitaria dell'indicizzazione, per autore e per soggetto.

FRBR è il modello concettuale da cui prenderemo le mosse, confermandone le scelte di modellizzazione, specificandole o discostandocene a seconda dei casi. Indagheremo in modo particolare l'indicizzazione (alfabetica) per soggetto, poco approfondita dal modello dell'IFLA $;{ }^{16}$ rispetto a FRBR, dedicheremo dunque un minore grado di dettaglio agli oggetti ed elementi indicali dell'indicizzazione per autore - includeremo tutti gli elementi più importanti, più tutti quelli che abbiamo ritenuto rilevanti per l'indicizzazione per soggetto stessa. Cercheremo di discutere ogni elemento nuovo o differente rispetto a

\section{INTERNATIONAL FEDERATION OF LIBRARY ASSOCIATIONS AND INSTITUTIONS,}

Dichiarazione di principi internazionali di catalogazione, traduzione a cura di Carlo Bianchini, Maria De Panicis, Mauro Guerrini, Cristina Magliano, Paola Manoni, in INTERNATIONAL FEDERATION OF LIBRARY ASSOCIATIONS AND INSTITUTIONS, IFLA cataloguing principles. The Statement of international cataloguing principles (ICP) and its Glossary. In 20 languages, edited by Barbara B. Tillett and Ana Lupe Cristán, München, Saur, 2009, p. 126-139, disponibile all'indirizzo

<http://www.ifla.org/files/cataloguing/icp/icp_2009-it.pdf>; JOINT STEERING COMMITTEE FOR DEVELOPMENT OF RDA, Resource description $\mathcal{E}$ access. RDA, developed in a collaborative process led by the Joint Steering Committee for Development of RDA (JSC), Chicago, American Library Association, 2010-; Regole italiane di catalogazione. REICAT, a cura della Commissione permanente per la revisione delle regole italiane di catalogazione, Roma, ICCU, 2009.

16 L'indicizzazione per soggetto viene tradizionalmente distinta in indicizzazione alfabetica o verbale (soggettazione) e in indicizzazione sistematica o classificata (classificazione). Nel testo che segue faremo implicitamente sempre riferimento alla sola indicizzazione alfabetica. Vedi anche la discussione sul rapporto tra soggettazione e classificazione in 2.26. Le registrazioni di autorità "per soggetto" sono state approfondite da FRSAD: IFLA WORKING GROUP ON THE FUNCTIONAL REQUIREMENTS FOR SUBJECT AUTHORITY RECORDS, Functional requirements for subject authority data (FRSAD), cit.; tuttavia, poiché come diremo meglio più avanti (vedi 2.27), non riteniamo soddisfacente né il concetto di registrazione di autorità "per soggetto" né la concezione di soggetto come entità tipica di FRSAD, abbiamo scelto di non prendere in considerazione FRSAD e di utilizzare FRBR come base di partenza per il nostro approccio. 
FRBR, spiegando i motivi della sua inclusione e le modalità della sua modellizzazione concettuale. L'attenzione rivolta all'indicizzazione per soggetto richiederà, in particolare, una lunga digressione sulla nozione di soggetto.

Anticipiamo le maggiori differenze tra FRBR e il nostro modello; noi includeremo: 1) un maggior numero di funzioni dell'utente, le quali a differenza di FRBR saranno parte integrante e anzi prioritaria del modello; 2) una tassonomia delle entità differente da FRBR sia nei criteri sia nel numero e nell'identità delle entità; 3) una modellizzazione differente degli attributi e relazioni che, nel nostro modello, non sono mutualmente esclusivi; 4) una modellizzazione esplicita delle cosiddette "relazioni semantiche", estesa anche alla catalogazione per autore; 5) una modellizzazione articolata della nozione di soggetto, sulla base di un approfondimento e di una revisione di tali nozione; 6) aldilà del modello concettuale vero e proprio, una articolata tassonomia degli indici, che costituiscono la traduzione degli elementi del modello concettuale in strutture fisiche. Inoltre, a differenza di FRBR, che nella sua analisi delle entità, degli attributi, delle relazioni e degli elementi indicali resta entro i limiti di ciò che si trova attualmente nelle registrazioni bibliografiche, il modello concettuale proposto partirà dall'universo bibliografico in quanto tale, considerato prima e a prescindere dalle registrazioni bibliografiche attuali; queste verranno sì prese in considerazione, ma in modo critico e discostandosene laddove opportuno: anche in ciò sta il perseguimento di migliorare gli strumenti dell'indicizzazione. Ciò comporterà una discussione approfondita e una revisione concettuale di alcuni elementi dell'universo bibliografico e della loro rappresentazione indicale, in particolare degli elementi dell'indicizzazione per soggetto: le elaborazioni teoriche che ne scaturiranno costituiscono la parte centrale della nostra proposta di miglioramento dell'indicizzazione tramite un approccio concettuale. Prima di passare alla modellizzazione concettuale proposta, desideriamo evidenziare i limiti intrinseci della modellizzazione concettuale in generale. Ogni modellizzazione concettuale, in quanto schematizzazione, richiede necessariamente una preventiva riduzione e selezione degli elementi di un dato universo di discorso. Essa è dunque una visione sempre parziale di una data realtà. La natura della modellizzazione è sempre convenzionale e pragmatica: suo obiettivo è suggerire rappresentazioni efficaci per determinati scopi. Per esempio, nella modellizzazione e-r la classificazione di un oggetto come entità o attributo può variare a seconda delle esigenze per cui nasce la modellizzazione stessa. ${ }^{17}$ Infine, quanto alla nostra modellizzazione, esplicitiamo sin da adesso due punti importanti: 1) essa non mira a essere esaustiva, nemmeno entro negli aspetti per ciò che riguarda l'indicizzazione per soggetto; il modello offerto è soprattutto un'ipotesi di sviluppo, che può essere modificata o estesa a seconda delle esigenze, e che certamente potrà essere migliorata; 2) essa non richiede l'applicazione integrale di ogni suo aspetto: il suo uso per l'analisi e costruzione degli strumenti indicali può essere modulare e flessibile.

17 Cfr. BIRGER HJØRLAND - KARSTEN NISSEN PEDERSEN, A substantive theory of classification for information retrieval, "Journal of documentation", 61, 5 (2005), p. 588, 593: "The way we conceptualise an object depends on our pre-understanding of our social-cultural background, of our domain-specific knowledge, and of our theoretical outlook [...] a "neutral" system tends to be based on a dominant view and is usually not seen as neutral from outside perspectives". 
Tesi di dottorato di Giuliano Genetasio, discussa presso l'Università degli Studi di Udine

\subsection{L'accesso bibliografico}

Primo passo per uno studio teorico dell'indicizzazione è inquadrarla nel più ampio ambito dell'accesso bibliografico, in modo da mostrare la sostanziale continuità tra questi livelli e la complementarietà dei diversi elementi in essi coinvolti.

Per "accesso bibliografico" intendiamo il processo comunicativo che si instaura tra utenti (finali, cioè lettori, o fruitori andando oltre il mondo del libro; ma anche indicizzatori) e risorse documentarie tramite una mediazione indicale. In altri termini, l'accesso bibliografico può essere visto come processo comunicativo tra utenti finali e indicizzatori circa determinate risorse. Oltre alle risorse vere e proprie, l'utente finale può essere interessato ad altri oggetti bibliografici realizzati nelle o implicati dalle risorse: autori, opere, soggetti, etc.; le risorse in quanto tali sono soltanto uno tra i molteplici oggetti di interesse dell'utente finale. Inoltre, l'utente finale si accosta alle risorse esigenze conoscitive potenzialmente differenti a seconda degli scopi della ricerca. Specifichiamo allora meglio la precedente definizione: l'accesso bibliografico è il processo comunicativo tra utenti finali e indicizzatori circa determinati oggetti di interesse, secondo determinati obiettivi e tramite determinati indici. Tre gli elementi fondamentali di tale processo: attori (utenti finali e indicizzatori) e loro obiettivi, oggetti, indici. Di questi elementi gli attori e i loro obiettivi sono quello centrale e prioritario.

Il processo di accesso bibliografico si svolge in due sensi, o sottoprocessi: come indicizzazione, ovvero interazione tra indicizzatore e risorse, e come recupero dell'informazione, ovvero interazione tra utente finale e risorse per mezzo degli indici; indicizzazione e recupero dell'informazione sono processi speculari e costituiscono le due facce di una stessa medaglia. La relazione tra gli attori non è però paritetica: l'indicizzatore esiste in tale processo soltanto in funzione dell'utente finale e del recupero dell'informazione. Occorre inoltre specificare che gli attori non rappresentano figure necessariamente diverse, ma piuttosto ruoli assunti in modo contingente: l'indicizzatore è prima di tutto un utente di dati bibliografici, poiché la consultazione degli indici, a partire dalle ricerche bibliografiche, è essenziale per l'attività indicale; con il web sociale (web 2.0), si ha anche il rapporto di tipo inverso: l'utente finale può farsi indicizzatore delle risorse di cui usufruisce.

Una specificazione sui rapporti tra obiettivi e oggetti. Elaine Svenonius sostiene che in una modellizzazione concettuale gli obiettivi dovrebbero sempre precedere e determinare gli oggetti di interesse. Sempre secondo Svenonius, l'inclusione di oggetti, indici e altri elementi nel modello concettuale è giustificata solo se essi contribuiscono al soddisfacimento di uno o più obiettivi. ${ }^{18}$ È una critica a FRBR, il quale pone gli obiettivi dell'utente come elemento finale del modello, anzi propriamente come elemento esterno al modello vero e proprio, composto di entità, attributi e relazioni. Anche noi daremo la precedenza agli obiettivi rispetto agli oggetti di interesse, perché riteniamo che la determinazione degli oggetti sia funzionale agli obiettivi dell'utente finale. Includeremo allora gli obiettivi, o funzioni, dell'utente finale come parte integrante e prioritaria del nostro modello. Tuttavia, allo stesso tempo escludiamo l'idea secondo cui sarebbe possibile porre una corrispondenza biunivoca tra obiettivi e oggetti, sia la possibilità di desumere gli oggetti direttamente ed esclusivamente dagli obiettivi. Gli oggetti di interesse, infatti, discendono anche dalle caratteristiche intrinseche dell'universo

18 ELAINE SVENONIUS, The intellectual foundation of information organization, cit., p. 14, 18. 
Tesi di dottorato di Giuliano Genetasio, discussa presso l'Università degli Studi di Udine

bibliografico. Più precisamente, possiamo dire che essi discendono dalle caratteristiche dell'universo bibliografico tramite la mediazione degli obiettivi dell'utente. Porremo dunque l'utente finale a cardine del nostro modello, e parleremo in questo senso di "interesse dell'utente", avvertendo che esso si specifica in obiettivi (elemento prioritario), oggetti e indici.

Una nota su questi ultimi. Gli indici e le loro caratteristiche discendono direttamente dall'incrocio di obiettivi e oggetti di interesse (entità, attributi e relazioni) dell'utente. Se volessimo vedere il processo di accesso bibliografico secondo la prospettiva degli indici, tralasciando gli oggetti di interesse ad essi soggiacenti, dovremmo rilevare che l'accesso bibliografico ha luogo come interazione tra strumenti (gli indici stessi) e processi/obiettivi seguiti dagli attori: utenti finali nel recupero dell'informazione, indicizzatori nell'indicizzazione.

\subsection{L'interesse dell'utente}

Utilizzeremo l'espressione "interesse dell'utente" per indicare l'insieme delle esigenze dell'utente (vedi 2.4), degli oggetti di interesse (vedi 2.5 e ss.), e degli indici (2.19 e ss.) in quanto subordinati all'utente finale. Il principio dell'interesse dell'utente, o del primato o convenienza dell'utente, stabilisce la centralità dell'utente finale nell'accesso bibliografico ed è largamente condiviso e costantemente presente nella letteratura catalografica, fino a diventarne un luogo comune. ${ }^{19}$ Esso è stato declinato in più sensi, e ha ricevuto molteplici interpretazioni. La Dichiarazione di principi internazionali di catalogazione (ICP) del 2009 ne ribadisce in più punti la centralità; anche FRBR, peraltro, poneva l'utente al centro della sua analisi. ${ }^{20}$ Occorrono allora alcune chiarificazioni. La nozione di "interesse dell'utente" implica necessariamente un'astrazione: sarebbe opportuno quantomeno porre l'espressione al plurale, poiché gli "interessi" sono molteplici e vari quanto gli utenti stessi. A diversi utenti corrispondono esigenze diverse: così, le esigenze del pubblico generale differiscono da quelle dei ricercatori, le esigenze dell'utente locale possono differire rispetto a quelle dell'utente remoto, etc. ${ }^{21}$ Per la nostra analisi, ci riferiremo alle esigenze dell'utente generale, e solo in alcune occasioni (ma senza pretesa di sistematicità) prenderemo in considerazione anche le esigenze di un'utenza più specialistica.

Seconda riserva al principio della centralità dell'interesse dell'utente: la nozione di interesse dell'utente può essere interpretata secondo modalità diverse e persino opposte. Uno degli esempi più eclatanti è l'interpretazione opposta che del principio diedero

19 CHARLES A. CUTTER, Rules for a dictionary catalog, 4th edition rewritten, Washington, D.C., Government Printing Office, 1904, p. 6. Cfr. anche DIEGO MALTESE, Principi di catalogazione e regole italiane, Firenze, Olschki, 1965, p. 3.

20 Cfr. per esempio INTERNATIONAL FEDERATION OF LIBRARY ASSOCIATIONS AND INSTITUTIONS, Dichiarazione di principi internazionali di catalogazione, cit., p. 1-2; cfr. anche IFLA STUDY GROUP ON THE FUNCTIONAL REQUIREMENTS FOR BIBLIOGRAPHIC RECORDS, Functional requirements for bibliographic records. Final report, cit., p. 3.

21 Cfr. ELAINE SVENONIUS, The intellectual foundation of information organization, cit., p. 69: "Cutter himself recognized this [...] There is no single public, no user writ large; rather there are many users, and the way they verbalize their search requests represents many different language usages"; cfr. anche RAFAEL CAPURRO, Hermeneutics facing the 'information framing', "Ubiquity", 9, 8 (2008), disponibile all'indirizzo <http://ubiquity.acm.org/article.cfm?id=1361368>. 
Tesi di dottorato di Giuliano Genetasio, discussa presso l'Università degli Studi di Udine

Charles A. Cutter e David J. Haykin, i due studiosi il cui pensiero ha influenzato maggiormente le Library of Congress Subject Headings (LCSH). Benché la centralità dell'utente fosse condivisa da entrambi, ${ }^{22}$ per Cutter essa avrebbe dovuto tradursi in una scelta e forma delle intestazioni che rispecchiassero i termini ricercati con più probabilità dagli utenti, anche qualora ciò comportasse una certa loro incoerenza e illogicità; per Haykin, viceversa, era la rigida coerenza nella scelta e nella forma delle intestazioni a garantire la predittività del catalogo e, di conseguenza, a favorire l'interesse dell'utente. (Proprio queste opposte visioni, tra l'altro, sono alla base di una parte significativa delle contraddizioni nella forma delle LCSH). L'interesse dell'utente non è allora, in sé, un criterio catalografico, quanto piuttosto un ideale che può poi informare criteri catalografici differenti; una ragione necessaria, ma non sufficiente, per determinate scelte catalografiche.

Infine, un'ultima specificazione importante: "interesse dell'utente" non è sinonimo di gratificazione immediata dell'utente, né coincide automaticamente e necessariamente con i termini della sua richiesta. ${ }^{23}$ Gli attori dell'accesso bibliografico si muovono, come in ogni contesto cognitivo, all'interno di un paradigma di razionalità limitata.

Razionalità limitata ("bounded rationality") è una nozione ideata da Herbert A. Simon ${ }^{24}$ per indicare che in una situazione cognitiva vige sì la razionalità, ovvero la presenza di catene mezzi-fini, ma che questa è condizionata: la scelta dei mezzi scelti per perseguire determinati fini, e già la scelta degli stessi fini, è limitata dalle conoscenze disponibili all'attore in un dato momento, ed evolve al mutare delle condizioni conoscitive. Nella ricerca dell'utente vi è dunque un nesso diretto tra l'ignoranza conoscitiva che si cerca di colmare e l'ignoranza dei mezzi (i termini di ricerca) utilizzati. ${ }^{25}$ Una delle spiacevoli conseguenze di questa condizione è che, come evidenzia Diana Brooking, "gli utenti non avvertono la mancanza di ciò che non trovano", ${ }^{26}$ e che i desideri ("wants") di un utente non sempre corrispondono ai suoi bisogni ("needs"). ${ }^{27}$

Come essere certi di perseguire l'interesse dell'utente? La risposta è inevitabilmente politica ed etica. La nostra scelta, che resta un'opzione ma che proprio perciò riteniamo

22 Cfr. CHARLES A. CUTTER, Rules for a dictionary catalog, cit., p. 49; cfr. anche DAVID JUDSON HAYKIN, Subject headings. A practical guide, New York, Gordon Press, 1978, p. 7.

23 Cfr. JOHN TOFANELLI, Written testimony for Working Group, in Testimony submitted to the Library of Congress Working Group on the Future of Bibliographic Control. Part II, 2007, p. 98. Cfr. anche FABIO METITIERI, L'OPAC collaborativo, tra folksonomia e socialità, "Biblioteche oggi”, 27, 2 (2009), p. 7-12, disponibile all'indirizzo <http://www.bibliotecheoggi.it/2009/20090200701.pdf>, p. 8.

24 HERBERT A. SIMON, Il comportamento amministrativo, Bologna, Il mulino, 2001, traduzione di Administrative behavior. A study of decision-making processes in administrative organization, New York, MacMilian, 1957. Alla nozione di razionalità limitata fa riferimento anche SAMANTHA SCHMEHL HINES,

Librarians at the bounds of rationality. How bounded rationality can help us help others, "Behavioral \& social sciences librarian”, 28, 3 (2009), p. 80-86.

25 Cfr. RAY R. LARSON, Classification clustering, probabilistic information retrieval, and the online catalog, "Library quarterly", 61, 2 (1991), p. 147.

26 DIANE BROOKING, Comments for Working Group, in Testimony submitted to the Library of Congress Working Group on the Future of Bibliographic Control. Part II, 2007, p. 14-15. Cfr. anche MARCIA J. BATES, Subject access in online catalogs. A design model, "Journal of the American Society for Information Science", 37, 6 (1986), p. 359.

27 Cfr. JAMES D. ANDERSON - JOSÉ PÉREZ-CARBALLO, The nature of indexing. How humans and machines analyze messages and texts for retrieval. Part I, Research, and the nature of human indexing, "Information processing \& management", 37, 2 (2001), p. 231-254, disponibile all'indirizzo

<http://www.mariapinto.es/ciberabstracts/Articulos/artAnderson2001a.pdf>, p. 250. 
Tesi di dottorato di Giuliano Genetasio, discussa presso l'Università degli Studi di Udine

corretto esplicitare, è che servire l'utente significa essenzialmente servirne l'interesse ultimo, non necessariamente quello immediato; significa servire gli interessi possibili, non soltanto quelli attuali.

\subsection{Le funzioni dell'utente}

Come accennato, l'accesso bibliografico si svolge in due grandi fasi o sottoprocessi: indicizzazione e recupero dell'informazione. A ognuna di queste fasi corrispondono diversi attori (indicizzatore e utente finale). I processi di indicizzazione e recupero dell'informazione sono correlati e speculari: ${ }^{28}$ il recupero dell'informazione parte dall'utente finale, e, attraverso gli indici, arriva agli oggetti di interesse; l'indicizzazione parte dalle risorse e dagli oggetti di interesse in esse realizzati per arrivare, tramite gli indici, agli utenti finali. In entrambi i processi si succedono simili tipi di attività: analisi e interpretazione soprattutto, ma anche ricerche e scelte. Anche gli obiettivi

dell'indicizzazione e del recupero dell'informazione hanno una corrispondenza; tuttavia, essendo l'utente finale il cardine dell'accesso bibliografico, i processi e gli obiettivi dell'indicizzazione sono subordinati e funzionali a quelli del recupero dell'informazione: sarà allora su questi che ci concentreremo.

Gli obiettivi dell'utente, o esigenze dell'utente, o funzioni dell'utente, o ancora funzioni catalografiche (useremo indistintamente tutte queste espressioni) sono i diversi tipi di obiettivi informativi che un utente finale può esprimere nei confronti degli oggetti di interesse, ovvero che il catalogo è teso a soddisfare. ${ }^{29}$ Così come gli oggetti di interesse corrispondono ad aspetti dell'interesse utenziale, anche le funzioni dell'utente non sono altro che una specificazione dell'interesse dell'utente.

La riflessione sulle funzioni è presente già con Thomas Hyde (1674) ${ }^{30}$ ma viene formalizzata forse per la prima volta nella teorizzazione di Charles A. Cutter (1876), secondo cui il catalogo ha la funzione di: 1) mettere in grado una persona di trovare un libro di cui sia noto: (a) l'autore; (b) o il titolo; (c) o il soggetto; 2) mostrare che cosa la biblioteca possiede: (d) di un determinato autore; (e) su un determinato soggetto; (f) di un determinato genere di scritti; 3) facilitare la scelta di un libro: (g) relativamente alla sua edizione (in senso bibliografico); (h) relativamente al suo carattere (in senso letterario o tematico). Le tre funzioni sono anche note come funzione di localizzazione (1), di raggruppamento (2), e di scelta (3). La tassonomia cutteriana verrà ripresa, con modifiche, dai Principi di Parigi del 1961. ${ }^{31}$ Durante la Conferenza di Parigi, da cui emersero i Principi,

28 Molti autori evidenziano, anche tramite schemi, la specularità e reciprocità del processo di accesso bibliografico di indicizzazione e recupero dell'informazione, e delle relative operazioni, vedi p.e. LOURDES Y. COLLANTES, Degree of agreement in naming objects and concepts for information retrieval, "Journal of the American Society for Information Science", 46, 2 (1995), p. 116-132.

29 Esulano completamente da questa modellizzazione delle funzioni, basata sull'acquisizione delle informazioni, le funzioni ed esigenze espresse tipicamente con il web sociale, a cui accenneremo soltanto in alcuni punti del presente lavoro (vedi 2.23, 3.3.3).

30 Cfr. JULIA PETTEE, The development of authorship entry and the formulation of authorship rules as found in the Anglo-American code, in Foundations of cataloging. A sourcebook, edited by Michael Carpenter and Elaine Svenonius, Littleton, Colo., Libraries Unlimited, 1985, p. 79-80.

31 CHARLES A. CUTTER, Rules for a dictionary catalog, cit., p. 12. Cfr. anche INTERNATIONAL CONFERENCE ON CATALOGUING PRINCIPLES, International Conference on Cataloguing Principles. 
vi fu un'ampia e accesa discussione delle funzioni del catalogo tra Seymour Lubetzky ed Eva Verona, in particolare sulla priorità da accordare alla funzione di localizzazione, ritenuta più importante da Verona e dalla maggior parte dei partecipanti, e che certamente godeva di una tradizione più forte, ${ }^{32}$ o raggruppamento, ritenuta da Lubetzky almeno altrettanto importante di quella di localizzazione. Né la discussione si spegnerà con la pubblicazione dei Principi; essa continuerà anche negli anni successivi; ${ }^{33}$ secondo Michael M. Malinconico la funzione di raggruppamento acquisterebbe una maggiore importanza a partire dall'avvento del catalogo elettronico, permettendo di ricostruire la fisionomia culturale delle raccolte. ${ }^{34}$ La differente valutazione dell'importanza delle funzioni di localizzazione e raggruppamento, per esempio, si ritrova nelle differenti branche della letteratura sull'indicizzazione per soggetto: la funzione di raggruppamento è stata enfatizzata dalla teoria della classificazione e dell'organizzazione della conoscenza, le quali assumono spesso che scopo principale dell'indicizzazione sia la classificazione e strutturazione dello scibile umano; viceversa, la documentazione e la letteratura sul recupero dell'informazione sembrano sottovalutare o misconoscere l'esigenza di raggruppamento a favore di quella di localizzazione. ${ }^{35}$

Le funzioni di FRBR non abbandonano quelle di Cutter, ma piuttosto le trasformano, in alcuni casi specificandole, in altre rendendole più generiche, e le adattano all'ambiente del catalogo in rete; esse: 1) non sono più funzioni del catalogo ma dell'utente; più precisamente, e qui sta la maggiore differenza, quelle di FRBR sono operazioni dell'utente ("user-tasks"): gli obiettivi dell'utente vengono messi in relazione con le operazioni compiute dall'utente; 2) comportano una fusione della funzione di raggruppamento e localizzazione nella funzione Trovare - che, però, comprende gli obiettivi di "localizzare un'unica entità o un insieme di entità" -; 3) attuano una distinzione della funzione di localizzazione nelle due funzioni Trovare e Identificare; 4) includono la funzione di scelta come funzione Selezionare; 5) esplicitano la funzione di accesso fisico, Ottenere; 5) non parlano più di titoli, autori, soggetti, né di libri, ma semplicemente di entità e relazioni. Accanto a queste quattro funzioni, FRBR includeva, come sorta di quinta funzione non ufficiale, "Correlare [...] assistere l'utente a mettere in relazione un'entità con un'altra o a 'navigare' il catalogo" ${ }^{36}$ Elaine Svenonius elaborò una propria teorizzazione in cui

Paris, 9th-18th October, 1961. Report, edited by A. H. Chaplin and Dorothy Anderson, London, Organizing Committee of the International Conference on Cataloguing Principles, 1963, disponibile all'indirizzo <http://www.d-nb.de/standardisierung/pdf/paris_principles_1961.pdf>.

32 Secondo ALBERTO PETRUCCIANI, Funzione e struttura del catalogo per autore, Firenze, Giunta regionale toscana, La nuova Italia, 1984, p. 48, la funzione di localizzazione non è tuttavia mai stata definita in modo soddisfacente.

33 Cfr. per esempio SALLY JO REYNOLDS, In theory there is no solution, The impediments to a subject cataloging code, "Library quarterly", 59, 3 (1989), p. 227.

34 S. MICHAEL MALINCONICO, The library catalog in a computerized environment, in The nature and future of the catalog. Proceedings of the ALA's Information Science and Automation Division's 1975 and 1977 Institutes on the Catalog, edited by Maurice J. Freedman and S. Michael Malinconico, Phoenix, Oryx, 1979, p. 48. 35 Con alcune eccezioni, p.e. VANNEVAR BUSH, As we may think, "The Atlantic monthly", 176, 1 (1945), p. 101-108.

36 IFLA STUDY GROUP ON THE FUNCTIONAL REQUIREMENTS FOR BIBLIOGRAPHIC

RECORDS, Functional requirements for bibliographic records, cit., p. 82-83. Cfr. anche MARIA BRUNA BALDACCI, Rappresentazione e ricerca delle informazioni. Come comunicare attraverso i sistemi informativi automatizzati, Roma, NIS, 1988, p. 93: "Il linguaggio per interrogare le basi di dati [...] deve permettere infatti: 1) di fare ricerche negli archivi della base dei dati; b) di passare (o navigare, come si dice) da un 
riprendeva la quinta funzione come Navigare e cercava di fondere le funzioni di Cutter e di FRBR, distinguendo nuovamente le funzioni di localizzazione (Trovare singole entità) e raggruppamento (Trovare gruppi di entità). ${ }^{37}$

La nostra modellizzazione riprenderà pienamente la corrispondenza di FRBR tra funzioni del catalogo e operazioni dell'utente; questa corrispondenza pone implicitamente le basi per una successione cronologica ${ }^{38}$ tra le varie operazioni, che noi cercheremo di esplicitare.

All'inizio del paragrafo, abbiamo detto come sia possibile concepire le funzioni quali specificazioni dell'interesse dell'utente. Cerchiamo allora di specificare progressivamente l'interesse dell'utente fino ad arrivare ai vari obiettivi e operazioni. ${ }^{39}$ Ciò che abbiamo posto come interesse dell'utente è essenzialmente equivalente all'intenzione di acquisire informazioni. "Acquisire informazioni" può essere dunque posta come prima e fondamentale funzione dell'utente. Le informazioni che l'utente intende acquisire possono essere classificate in informazioni primarie (le risorse documentarie) e secondarie (informazioni sulle informazioni primarie, ovvero informazioni su altri oggetti di interesse). Articoliamo così la funzione Acquisire informazioni in due funzioni: Funzione di accesso, rivolta alle risorse documentarie e Funzione di informazione, rivolta agli altri oggetti di interesse. La funzione di informazione potrebbe a sua volta essere ulteriormente articolata. Un utente può essere interessato a ottenere informazioni su specifiche entità (più o meno note, ma di cui l'utente finale si ricordi almeno un'informazione utile alla ricerca),${ }^{40}$ oppure su due o più entità (non necessariamente note), forse anche tutte, che condividono una medesima caratteristica o relazione. Una volta trovata un'entità, l'utente cercherà di identificarla, di sincerarsi, in base a conoscenze pregresse, che sia effettivamente l'oggetto voluto. La ricerca dell'utente potrà arrestarsi a questo punto: così, per esempio, se l'utente è interessato esclusivamente a identificare o citare una data opera o se vuole stilare una prima bibliografia su una determinata persona. Oppure, partendo dalla singola entità l'utente potrebbe essere interessato ad acquisire informazioni su altre entità correlate; per esempio, una volta trovata un'opera di cui conosceva il titolo ma non il nome dell'autore, un utente potrebbe essere interessato a venire a conoscenza delle altre opere dell'autore; l'utente inizierebbe così la sua navigazione del catalogo, acquisendo informazioni sulle relazioni tra le entità rappresentate in esso. L'utente potrebbe così continuare indefinitamente la sua navigazione dell'universo bibliografico, godendo magari di sorprese e scoperte fortuite (serendipità), oppure stancarsi ed abbandonare la ricerca,

archivio all'altro attraverso le associazioni che li connettono".

37 ELAINE SVENONIUS, The intellectual foundation of information organization, cit., p. 20.

38 Cfr. MAURO GUERRINI, I Principi internazionali di catalogazione (ICP). Universo bibliografico e teoria catalografica all'inizio del XXI secolo, con Giuliano Genetasio, postfazione di Attilio Mauro Caproni, Milano, Editrice Bibliografica, 2012, p. 132. La scelta di esplicitare il rapporto tra funzioni del catalogo e le operazioni compiute dall'utente, lungi dal costituire una "cosmesi di tipo operativistico", (ALFREDO SERRAI, Critica dei Functional requirements for bibliographic records (FRBR), "Bibliotheca", 1, 2 (2002), p. 215) comporta il radicamento delle funzioni del catalogo nella dimensione temporale.

39 Escluse dalla modellizzazione delle funzioni rimarranno alcune operazioni preliminari che citiamo qui: 1) l'identificazione del bisogno informativo; 2) la scelta delle fonti e dei repertori per la ricerca (cataloghi, google, contatti personali, etc.). Cfr. GIANFRANCO CRUPI, 010.72. Strumenti e strategie di ricerca bibliografica, in Biblioteconomia. Guida classificata, diretta da Mauro Guerrini, condirettore Gianfranco Crupi, a cura di Stefano Gambari, collaborazione di Vincenzo Fugaldi, presentazione di Luigi Crocetti, Milano, Editrice Bibliografica, 2007, p. 129.

40 Cfr. ALBERTO PETRUCCIANI, Funzione e struttura del catalogo per autore, cit., p. 1-2. 
Tesi di dottorato di Giuliano Genetasio, discussa presso l'Università degli Studi di Udine

oppure ancora potrebbe fissare il suo interesse su una singola entità o su un gruppo di entità. A quel punto, $\mathrm{o}$ anche semplicemente dopo aver trovato uno o più entità senza essere necessariamente essere passato dalla navigazione, l'utente sarà probabilmente interessato ad accedere fisicamente a una risorsa documentaria specifica; prima di farlo, però, potrebbe essere interessato a ottenere ulteriori informazioni per valutare la pertinenza dell'entità rispetto alle proprie esigenze, eventualmente a confronto con altre entità, per esempio quando sia necessario scegliere una tra le diverse edizioni di un'opera, o una tra le diverse opere su un soggetto. Soltanto quando ciò sarà avvenuto, l'utente cercherà di acquisire la risorsa documentaria.

Secondo questo schema, potremmo così articolare sei operazioni dell'utente: 1) cercare specifiche entità: localizzazione; 2) cercare due o più entità, o insiemi di entità, che condividono una medesima caratteristica o relazione: raggruppamento; 3) identificare specifiche entità: Identificare o funzione di identificazione; 4) cercare entità correlate a quelle trovate: Navigare o funzione di navigazione; 5) acquisire ulteriori informazioni per scegliere una o più tra le entità trovate senza dovervi necessariamente accedere fisicamente: Selezionare o funzione di selezione o funzione di scelta; ${ }^{41} 6$ ) accedere fisicamente a una risorsa documentaria: Ottenere o funzione di accesso fisico.

Se ci fermassimo qui avremmo una tassonomia delle funzioni sostanzialmente identica a quella di Elaine Svenonius. Noi, tuttavia, non considereremo la differenza tra la funzione di localizzazione e raggruppamento. Nella tassonomia cutteriana, e nella logica dei Principi di Parigi, localizzazione e raggruppamento sono effettivamente differenti perché rivolte a oggetti diversi. Nella tassonomia di FRBR e nella nostra, invece, la differenza tende sostanzialmente a scomparire, perché entrambe localizzazione e raggruppamento sono rivolte alle entità. La differenza viene meno anche perché la presunta differenza tra localizzazione e raggruppamento, per cui la prima sarebbe rivolta a specifici elementi noti e la seconda a gruppi di elementi non noti - differenza che avevamo sopra menzionata per esigenze di esposizione, ma che qui confutiamo -, è insoddisfacente. Non è infatti vero presumere che possano esistere elementi totalmente non noti: anche per il raggruppamento è infatti indispensabile conoscere almeno un elemento per ricercare l'entità desiderata; poiché, però, nell'ottica entità-relazioni questo elemento non è a sua volta riferito che a un'entità (il nome di una persona, un termine di soggetto, etc.), si intende come ogni raggruppamento richieda una preventiva localizzazione di specifiche entità: la differenza tra localizzazione e raggruppamento viene allora meno. Con FRBR, parleremo dunque semplicemente di un'unica funzione Trovare (che nel nostro modello include le funzioni tradizionali di localizzazione e raggruppamento), con un'unica nota: ovviamente, non tutti gli elementi che servono a localizzare servono con ciò anche a raggruppare, bensì soltanto quegli elementi che si applichino a più entità; identificatori per definizione -, ma anche altri elementi che occorrano un'unica volta in un archivio bibliografico, serviranno esclusivamente a localizzare.

Rivedendo su queste basi la precedente tassonomia, abbiamo le seguenti cinque funzioni:1) Trovare; 2) Identificare; 3) Navigare; 4) Selezionare; 5) Ottenere; sarà questa tassonomia delle funzioni, a metà strada tra FRBR e la sua rielaborazione da parte di Svenonius, che adotteremo per il nostro modello. Sopra, abbiamo accennato che

41 Cfr. KAREN CALHOUN - JOANNE CANTRELL - PEGGY GALLAGHER - JANET HAWK, Online catalogs. What users and librarians want. An OCLC report, Dublin, Ohio, OCLC, 2009, disponibile all'indirizzo <http://www.oclc.org/reports/onlinecatalogs/fullreport.pdf>, p. 11. 
l'equazione posta da FRBR tra funzioni e operazioni lascia intravedere un possibile scarto cronologico tra le varie funzioni-operazioni. Dal suddetto quadro delle funzioni, emerge che esse si svolgono secondo una determinata successione - quella sopra delineata era del tutto indicativa: in realtà accanto ad alcuni passaggi temporali costanti ve ne sono altri variabili.

Sono caratteristiche della ricerca dell'utente che: 1) la funzione Trovare sia sempre presente e sempre come passaggio iniziale, benché possa rientrare in gioco anche in momenti successivi della ricerca; 2) la funzione di identificazione sia sempre presente e sempre successiva a Trovare ma precedente la funzione di selezione; 3) la funzione di navigazione sia opzionale e possa svolgersi, anche a più riprese, tra la funzione di identificazione e quella di accesso fisico; 5) la funzione di selezione sia opzionale e possa svolgersi, anche a più riprese, tra la funzione di identificazione e quella di accesso fisico; 6) la funzione di accesso fisico sia opzionale e, se presente, sia sempre successiva alla funzione di identificazione e sempre finale. Inoltre, non vi è una corrispondenza biunivoca tra funzioni da un lato e attributi o relazioni dall'altro. Piuttosto, almeno un certo numero di attributi e relazioni (spiegheremo dopo quali) è relativamente e reciprocamente funzionale: nell'ambito di una ricerca che utilizzi un certo attributo per Trovare, gli altri serviranno a Identificare, Selezionare, etc., e viceversa.

\subsection{Gli oggetti di interesse}

Già sopra abbiamo fatto più volte riferimento agli oggetti di interesse: entità, attributi e relazioni. Gli oggetti di interesse rappresentano i diversi aspetti bibliografici dell'interesse dell'utente e formano nel loro complesso, come spiegheremo in 3.3.4.2 e ss., la base della semantica degli strumenti di indicizzazione. Possiamo suddividere gli oggetti di interesse in entità, ovvero le diverse componenti di ciò che tradizionalmente è chiamato l'oggetto della catalogazione (per esempio opere, risorse documentarie, persone, etc.), in attributi, ovvero caratteristiche di tali entità (per esempio le caratteristiche fisiche di una pubblicazione, il titolo di un'opera, etc.), e in relazioni tra entità differenti (per esempio: relazione di responsabilità, di adattamento, di omaggio, etc.). Entità, attributi e relazioni costituiscono il nucleo del modello concettuale di FRBR. Utilizzeremo allora le entità, gli attributi e le relazioni di FRBR come base di partenza per il nostro modello, discostandocene, modificandoli o specificandoli ove opportuno.

\subsection{Le entità}

La questione dell'oggetto della catalogazione (ovvero quale o quali fossero gli oggetti della catalogazione) emerge, in ambito catalografico e in riferimento allo specifico mondo del libro, soprattutto a partire dagli anni Cinquanta dello scorso secolo. Fino ad allora i termini "libro", "opera" e "pubblicazione" venivano, benché vi fosse consapevolezza di una loro differenza, impiegati in modo piuttosto flessibile e interscambiabile. Sono Lubetzky, che distingue nettamente l'opera dal libro, ed Eva Verona, che distingue unità letteraria (opera) e unità bibliografica (libro, pubblicazione), a chiarire più precisamente la distinzione tra due entità, tra due oggetti della catalogazione. "Nell'approccio di Lubetzky 
l'interesse per il libro è funzionale all'interesse per l'opera, che è dunque il primo e principale oggetto della catalogazione. Per Verona il lettore è interessato innanzitutto ai libri e solo successivamente alle opere” ${ }^{42}$ FRBR ha avuto il grande merito di riprendere la discussione sull'oggetto della catalogazione, e di mostrare come la questione non fosse quella della scelta o priorità di un oggetto della catalogazione rispetto a un altro, essendo l'oggetto della catalogazione intrinsecamente molteplice, ma semmai quella di decidere quanti e quali aspetti (le entità e le relazioni) dell'oggetto della catalogazione includere. Accanto agli oggetti tradizionali della catalogazione (Opera e Manifestazione, corrispondente alla pubblicazione), FRBR ne ha aggiunti altri: sempre relativi alle risorse documentarie, come l'Espressione (edizione) e l'Item (copia), che insieme a Opera e Manifestazione costituiscono le entità del Gruppo 1; più i responsabili delle entità bibliografiche stesse (entità del Gruppo 2: Persone ed Enti), e le entità che fungono da soggetti (entità del Gruppo 3: Concetti, Oggetti, Eventi e Luoghi).

Nel modello concettuale proposto accettiamo solo parzialmente questa tassonomia delle entità. Discuteremo le singole entità e i singoli gruppi di entità nei paragrafi successivi, ma fin da adesso anticipiamo che mentre riteniamo i Gruppi 1 e 2 sostanzialmente accettabili, con le modifiche che diremo, riteniamo inaccettabile il Gruppo 3, inteso come insieme delle entità che fungono da soggetti. Inoltre, in aggiunta alle entità dei Gruppi 1, 2 e 3 di FRBR, per la nostra modellizzazione concettuale abbiamo stabilito come entità un certo numero di elementi che abbiamo ritenuto potessero interessare l'utente secondo più aspetti, ovvero poter essere l'oggetto di due o più relazioni (e attributi) di tipo differente; ciò perché abbiamo ritenuto che in questi casi un utente potesse essere interessato non soltanto alle relazioni contingenti che l'elemento intrattiene di volta in volta rispetto a un'entità, ma anche all'elemento in sé (a prescindere dagli specifici ruoli assunti rispetto a singole entità). In queste scelte il nostro modello si differenzia da FRBR, che separa nettamente attributi e relazioni e trascura dunque di modellare le entità e relazioni alla base di alcuni attributi (vedi 2.12$).{ }^{43}$ Infine, come diremo meglio dopo, introdurremo una tassonomia trasversale delle entità sulla base della loro natura linguistica.

\subsection{Entità del Gruppo 1 di FRBR: Testi e Risorse ${ }^{44}$}

Le entità del Gruppo 1 di FRBR "rappresentano i differenti aspetti dell'interesse dell'utente nei prodotti di un'attività artistica o intellettuale". ${ }^{45}$ A esse ci si è

42 Cfr. MAURO GUERRINI, I Principi internazionali di catalogazione (ICP). Universo bibliografico e teoria catalografica all'inizio del XXI secolo, con Giuliano Genetasio, postfazione di Attilio Mauro Caproni, Milano, Editrice Bibliografica, 2012, p. 44-45.

43 Cfr. per esempio il caso del Luogo, IFLA STUDY GROUP ON THE FUNCTIONAL REQUIREMENTS FOR BIBLIOGRAPHIC RECORDS, Functional requirements for bibliographic records, cit., p. 31.

44 Per il presente lavoro prenderemo in considerazione soltanto entità bibliografiche (Testi e Risorse) finite; non prenderemo invece in considerazione le entità bibliografiche continuative (periodici, serie, risorse integrative, etc.), il cui approfondimento esula dagli scopi del presente lavoro e avrebbe reso notevolmente più complesso il modello concettuale.

45 IFLA STUDY GROUP ON THE FUNCTIONAL REQUIREMENTS FOR BIBLIOGRAPHIC RECORDS, Functional requirements for bibliographic records, cit., p. 12. 
Tesi di dottorato di Giuliano Genetasio, discussa presso l'Università degli Studi di Udine

successivamente riferiti con l'espressione "entità bibliografiche", un po' generica ma accettabile, che utilizzeremo anche noi. ${ }^{46}$ FRBR include tra le entità bibliografiche l'Opera, l'Espressione, la Manifestazione e l'Item; per una loro definizione, rimandiamo al modello dell'IFLA.

Due aspetti insoddisfacenti e collegati della modellizzazione delle entità bibliografiche di FRBR sono la Manifestazione e gli aggregati. La Manifestazione è insoddisfacente perché sembra a tratti configurarsi come un'entità materiale, a tratti come un'entità intellettuale: alla Manifestazione appartengono gli elementi di tipo editoriale, i quali non possono tuttavia essere considerati elementi esclusivamente materiali. Per quanto riguarda la modellizzazione degli aggregati, in FRBR vige un rapporto uno-a-molti tra le varie entità bibliografiche (l'Opera può realizzarsi in una o più Espressioni, ma l'Espressione può essere la realizzazione di una e soltanto un'Opera), tranne nel caso del rapporto tra Espressione e Manifestazione, per cui vige un rapporto di tipo molti-a-molti: un'Espressione può realizzarsi in una o più Manifestazioni, una Manifestazione può essere la realizzazione di una o più Espressioni. In altri termini, una Manifestazione può raccogliere una o più Espressioni: è il caso delle raccolte (aggregati). Tuttavia, nella discussione degli aggregati e delle relazioni tutto/parte (o relazioni partitive, di cui discuteremo in 2.14), FRBR lascia libertà nella considerazione dell'aggregato come Opera costituita da parti di opere, o viceversa come Opera (complessiva) costituita da più Opere (componenti). Sembrerebbe cioè che la relazione di aggregazione sia posta in questo caso a livello di Opera e non più di Manifestazione.

Per risolvere questi problemi di modellizzazione, Patrick Le Bœuf ha proposto l'introduzione di una quinta entità, il "Contenuto editoriale", posta tra Espressione e Manifestazione. ${ }^{47}$ Questa nuova entità permetterebbe: 1) di scindere gli aspetti intellettuali e materiali presenti nella Manifestazione: il Contenuto editoriale si farebbe carico dei primi, e la Manifestazione resterebbe un'entità puramente materiale - anche se resterebbe, a differenza dell'Item, pur sempre un'astrazione -; 2) di gestire in modo soddisfacente la modellizzazione degli aggregati: in questi casi Opera (ed Espressione) sarebbe sempre la parte, e l'intero sarebbe invece costituito dal Contenuto editoriale; in altri termini, una singola Espressione potrebbe realizzarsi in uno o più Contenuti editoriali, e un Contenuto editoriale potrebbe comprendere una o più Espressioni; a sua volta, il Contenuto editoriale potrebbe realizzarsi in una o più Manifestazioni, ma non viceversa. Accogliamo dunque il Contenuto editoriale come entità del nostro modello: una particolare aggregazione di Opere (e relative Espressioni) che può trovare realizzazione in una o più Manifestazioni, come atti di convegno, miscellanee in onore, etc.

Nell'ambito delle entità bibliografiche, FRBR separa Opera ed Espressione - a cui dobbiamo aggiungere il Contenuto editoriale - da Manifestazione e Item: il primo sottogruppo include le entità bibliografiche di natura artistica e intellettuale, il secondo

46 MAJA ŽUMER - GERHARD J. A. RIESTHUIS, Consequences of implementing FRBR. Are we ready to open Pandora's box?, “Knowledge organization”, 29, 2 (2002), p. 79.

47 Così traduciamo l'espressione "Package content" utilizzata PATRICK LE BCEUF, Brave new FRBR world, in IFLA MEETING OF EXPERTS ON AN INTERNATIONAL CATALOGUING CODE, 1, IFLA cataloguing principles. Steps towards an international cataloguing code. Report from the 1st IFLA Meeting of Experts on an International Cataloguing Code, Frankfurt, 2003, edited by Barbara B. Tillett, Renate Gömpel and Susanne Oehlschläger,München, Saur, 2004, disponibile all'indirizzo <http://www.dnb.de/standardisierung/pdf/papers_leboeuf.pdf>, p. 46-47. 
sottogruppo quelle di natura fisica e materiale. Tale suddivisione ci è particolarmente utile, perché ai fini dell'indicizzazione per soggetto la distinzione interna ai due sottogruppi è generalmente poco rilevante, con le eccezioni che diremo, e viceversa è fondamentale separare le entità bibliografiche intellettuali da quelle materiali.

Per quanto riguarda queste ultime, nella parte iniziale della nostra modellizzazione e nella discussione delle funzioni dell'utente avevamo fatto riferimento alle risorse documentarie, e detto che queste a loro volta sono uno dei molteplici oggetti di interesse dell'utente. Chiameremo Risorse l'insieme delle entità bibliografiche materiali: Manifestazioni e Item; poiché ai fini dell'indicizzazione per soggetto la distinzione tra Manifestazione e Item è il più delle volte irrilevante, li distingueremo nei soli casi in cui l'Item abbia una rilevanza autonoma (studio dei manoscritti, etc.).

Per quanto riguarda le entità artistico-intellettuali, nel presente lavoro approfondiremo esclusivamente quelle entità artistico-intellettuali (Opere, Espressioni e Contenuti editoriali) di tipo testuale, ovvero che presentano un testo di qualche tipo: non necessariamente un testo in forma grafica, ma comunque in forma segnica, scritta o orale che sia; ciò implicherà l'inclusione di discorsi registrati in forma sonora; non, tuttavia, di risorse sonore o video di tipo non segnico (solo musica, solo immagini, etc.). Su queste basi, chiameremo Testi le entità artistico-intellettuali nel loro complesso. Ogni Testo è costituito da una serie di elementi - attributi, di cui parleremo più sotto (vedi 2.12 e ss.) -: un creatore (sia noto o meno), un messaggio o discorso, dei destinatari; più altri elementi come la lingua del testo, la data e il luogo della sua creazione, il tipo testuale a cui appartiene (forma bibliografica, etc.), etc. Il messaggio di un Testo, a sua volta formato da più elementi, di cui parleremo successivamente (vedi 2.16 e ss.), coincide in sostanza con l'ambito del soggetto; soltanto i Testi, lo anticipiamo sin da adesso, sono dunque suscettibili di avere un soggetto.

La suddivisione in Testi e Risorse richiama direttamente quella di Lubetzky tra "opera" e "libro"; nel nostro modello, Testi e Risorse costituiscono però super-entità, ovvero classi o raggruppamenti di entità, le quali possono poi essere ulteriormente specificate nelle entità Opera, Espressione e Contenuto editoriale da un lato, e Manifestazione e Item dall'altro.

\subsection{Entità del Gruppo 2 di FRBR}

Le entità del Gruppo 2 di FRBR rappresentano "i responsabili del contenuto artistico o intellettuale, della produzione, disseminazione, o custodia delle entità" bibliografiche. Esse sono, in FRBR, la Persona e l'Ente; per una loro definizione rimandiamo al modello dell'IFLA. Si tratta evidentemente di una modellizzazione che distingue determinate entità a partire da un determinato tipo di relazione (responsabilità di vario genere), ed è in questo rispetto insoddisfacente. Le entità del Gruppo 2, infatti, possono essere intrattenere anche relazioni di tipo diverso dalla responsabilità con le entità bibliografiche (relazione di soggetto, omaggio, etc.); è dunque più corretto affermare che le entità del Gruppo 2 sono - è così che le accogliamo nella nostra modellizzazione - persone e gruppi di persone (Enti) che intrattengono relazioni di vario tipo con le entità bibliografiche. Alle due entità del Gruppo 2 di FRBR ne aggiungiamo una terza, Famiglia, tratta dal secondo modello dell'IFLA, FRAD, a cui rimandiamo per una sua definizione; ${ }^{48}$ 
Tesi di dottorato di Giuliano Genetasio, discussa presso l'Università degli Studi di Udine

pur non prendendo in considerazione le altre entità di FRAD, alcune delle quali (Nome, Punto di accesso) sono incompatibili con il nostro modello, riteniamo che l'entità Famiglia sia importante e perfettamente compatibile con il nostro modello; essa è prevista, tra le altre cose, dalle Library of Congress subject headings ed è inoltre un'entità fondamentale dell'universo archivistico.

\subsection{Entità del Gruppo 3 di FRBR}

Le entità del Gruppo 3 di FRBR rappresentano "un insieme aggiuntivo di entità che fungono da soggetti delle opere”. Esse sono: Concetto, Oggetto, Evento, Luogo; per una loro definizione rimandiamo al modello dell'IFLA. Anche in questo caso FRBR modellizza un gruppo di entità sulla base di una specifica relazione (relazione di soggetto). Tuttavia la definizione di FRBR trascura: 1) che anche gli altri gruppi di entità (Gruppo 1 e 2) possono intrattenere relazioni di soggetto, cosa che del resto riconosce lo stesso FRBR; 2) che le relazione di soggetto non coinvolge il rapporto tra entità di un certo tipo e opere, ma tra entità di un certo tipo e Testi, cioè Opere, ma anche Espressioni e Contenuti editoriali: il soggetto di un'Opera può infatti cambiare lievemente da Espressione a Espressione; nel caso di aggregati, è possibile avere un soggetto non solo per le singole Espressioni contenute, ma anche per il Contenuto editoriale che le raccoglie; approfondiremo più avanti la discussione dell'entità oggetto dell'indicizzazione per soggetto (vedi 2.16.8); 3) che le entità del Gruppo 3, o almeno alcune tra esse, possono teoricamente fungere non soltanto da soggetti, ma anche da elementi di tipo diverso: luogo di pubblicazione, nel caso dei Luoghi; termini generici o iperonimi (vedi 2.14), nel caso dei Concetti; FRBR, che parte da quanto si trova attualmente nelle registrazioni bibliografiche e che non analizza in profondità l'indicizzazione per soggetto (in cui, tipicamente, i Concetti intrattengono relazioni semantiche di vario tipo con le altre entità), trascura questo aspetto e dichiara esplicitamente di considerare le entità del Gruppo 3 esclusivamente in quanto potenziali soggetti, non elementi d'altro tipo; per quanto ci riguarda, invece, accetteremo pienamente la possibilità per le entità del Gruppo 3 di svolgere ruoli diversi da quelli di soggetti dei Testi.

Per i motivi suddetti, pur accettando pienamente Concetti, Oggetti, Eventi e Luoghi come entità del nostro modello, non li considereremo come parte di un gruppo a sé stante di entità; non parleremo dunque, d'ora in poi, di Gruppo 3, ma semplicemente di Concetti, Oggetti, Eventi e Luoghi. Con alcune differenze rispetto alle entità di FRBR. Per quanto riguarda l'entità Luogo, nella nostra modellizzazione essa funge non soltanto da soggetto di Testi, ma anche come possibile oggetto dei luoghi di pubblicazione, distribuzione, stampa, etc. e, inoltre, come parte del nome di alcuni Eventi (vedi 2. 14). Questa modellizzazione, come già accennato, è funzionale all'idea che un utente interessato in un Luogo può essere interessato sia a tutti i Testi che hanno come argomento quel Luogo, sia a tutte le risorse pubblicate e stampate in quel Luogo (nel caso delle città), sia ancora a tutti gli Eventi occorsi in determinati Luoghi. Inoltre, è bene evidenziare che nell'ambito del nostro modello, e sulla scorta delle osservazioni del GRIS, i Luoghi includono non soltanto località geografiche, ma anche le relative comunità e, se intesi in senso generico, i relativi enti territoriali (viceversa, se intesi in senso specifico

AUTHORITY RECORDS (FRANAR), Functional requirements for authority records, cit., p. 11. 
Tesi di dottorato di Giuliano Genetasio, discussa presso l'Università degli Studi di Udine

consideriamo gli enti territoriali come Enti a tutti gli effetti); ciò perché località geografiche, comunità ed enti territoriali costituiscono accezioni distinte ma spesso inseparabili dei nomi di luogo. ${ }^{49}$

Per quanto riguarda gli Oggetti, vogliamo semplicemente notare che, almeno da un punto di vista linguistico e puramente teorico, Testi e Risorse ricadrebbero tra gli Oggetti: essi sono oggetti bibliografici che però, proprio per la loro natura, FRBR ritiene - del tutto correttamente, visto che l'individuazione delle entità è subordinata alle esigenze dell'accesso bibliografico - utile distinguere. Un'altra entità da approfondire è Concetto: "una nozione astratta o un'idea". Rifacendoci alle categorizzazioni tipiche della linguistica (vedi 2.11 e 3.3.6.4.2.3) notiamo che mentre tutte le entità di FRBR menzionate precedentemente (Opere, Persone, Luoghi, etc.) sono entità individuali, le entità incluse nella definizione di Concetto sono entità collettive, ovvero insiemi di entità individuali; si tratta di una differenza importante. Infine, per quanto riguarda l'entità Evento, e sempre riferendoci alle categorizzazioni della linguistica, esso raccoglie sia entità di tipo linguistico sia attività come discipline, processi, etc.

\subsection{Entità nuove rispetto a FRBR}

Rispetto a FRBR, la nostra modellizzazione presenta diverse entità nuove: individuali (Lingua, Tempo) e collettive (Tipo di testo, Tipo di risorsa, Tipo di persona, Tipo di ente). La ragione per modellizzare questi elementi come entità è che, come spiegato, abbiamo ritenuto che tali elementi costituissero oggetti di interesse per l'utente sotto più rispetti, ovvero che essi potessero svolgere relazioni (ovvero fungere da attributi, vedi 2.12) di tipo diverso.

Iniziamo con le entità individuali. La prima entità nuova del nostro modello è la Lingua: un sistema linguistico; esempi di Lingua sono "italiano", "inglese", etc. Modellizzare la Lingua come entità a sé stante è utile perché un utente interessato a una particolare lingua può essere interessato a tutti i Testi scritti o tradotti in quella Lingua, così come a tutti i Testi di cui quella Lingua è il soggetto, etc.

Seconda entità nuova del nostro modello, ma già proposta da Tom Delsey proprio in riferimento a FRBR, è il Tempo: $:^{50}$ una data o un arco temporale. Esempi di tempo sono “2011”, "Ottocento” (ovvero “Diciannovesimo secolo"), "Medioevo”, etc. Modellizzare il Tempo come entità a sé stante è utile perché un utente interessato a un particolare Tempo può essere interessato a tutti i Testi composti in quel Tempo, a tutte le Risorse pubblicate in quel Tempo, ma anche a tutti i Testi di cui quel Tempo è il soggetto, ai Tempi associati a un particolare Evento, etc.

Oltre a queste entità individuali, il nostro modello concettuale comprende anche una serie di entità collettive: Tipi di testo, Tipi di risorsa, Tipi di persona, Tipi di ente; si tratta in tutti i casi di tipologie generali di appartenenza di entità già discusse: ogni Testo appartiene a un Tipo di testo, ogni Risorsa a un Tipo di risorsa, etc. Si tratta di entità che,

49 ASSOCIAZIONE ITALIANA BIBLIOTECHE. GRUPPO DI RICERCA SULL'INDICIZZAZIONE PER SOGGETTO, Guida all'indicizzazione per soggetto, Roma, Associazione italiana biblioteche, 2001, p. 51, chiarisce che, oltre l'ambiguità con l'ente territoriale, vi è anche quella con la comunità di un luogo: "Italia" può indicare tanto la penisola, quanto la Repubblica italiana, quanto ancora la comunità degli italiani. 50 Cfr. TOM DELSEY, Modeling subject access, cit., p. 52. 
almeno teoricamente, e benché FRBR non faccia esempi espliciti a riguardo, ricadrebbero forse - in quanto entità collettive - nell'entità Concetto di FRBR. Per quanto ci riguarda, riteniamo tuttavia utile distinguerle dai Concetti, sulla base del criterio sopra menzionato. Non abbiamo invece ritenuto opportuno modellizzare come entità a sé stanti le categorie generali di appartenenza di altre entità individuali (Famiglie, Luoghi, Eventi, etc.), che tratteremo nel loro complesso come parte dell'entità residuale Altre entità (vedi sotto). La terza entità nuova del nostro modello è il Tipo di testo: una tipologia formale o concettuale di testo. In questa definizione ricadono dunque tanto forme bibliografiche (saggi, bibliografie, carteggi, cataloghi, diari, letteratura, etc.), quanto forme specificamente letterarie (teatro, narrativa, poesia, etc.), tanto generi letterari e ambientazioni letterarie (commedia, tragedia, etc.; giallo, thriller, romanzo storico, etc.), quanto infine tipologie concettuali di testo (testi sacri, etc.). La distinzione tra forme letterarie e generi letterari è volutamente approssimativa, in quanto non sempre è possibile porre una distinzione netta tra i due: a determinate forme letterarie corrispondono a volte determinati generi o ambientazioni. Il Tipo di testo, come suggerisce il nome, è un'entità prettamente testuale, ovvero legata ai Testi; un utente interessato a un Tipo di testo può essere interessato a tutti i Testi che appartengono a quel Tipo di testo, così come a tutti i Testi di cui quel Tipo di testo è l'argomento, etc. Il Tipo di testo, e in particolare il genere letterario, è stato considerato un oggetto di interesse importante già nella formulazione delle funzioni di Cutter. Il Tipo di testo ha inoltre da sempre rivestito una notevole importanza come base per gli elementi indicali (intestazioni formali o qualificazioni di intestazioni d'altro tipo). Proprio in riferimento a FRBR, l'opportunità di introdurre forma e genere (che ricadono nel nostro Tipo di testo) come nuove entità fu suggerita da Tom Delsey. ${ }^{51}$

La quarta entità nuova del nostro modello è il Tipo di risorsa: tipologie di supporto su cui sono registrate e codificate le Risorse, sia di carattere generale (supporto audio, supporto video, supporto elettronico, supporto cartaceo, etc.; manoscritto, a stampa, etc.) sia specifico (cd, dvd, vinile, libro, ebook, etc.). Un utente interessato a un Tipo di risorsa può essere interessato a tutte le Risorse registrate su quel supporto, ma anche a tutti i Testi che parlano di un determinato Tipo di risorsa, etc.

La quinta entità nuova del nostro modello è il Tipo di persona: una categoria di appartenenza di una persona; la categoria può riguardare la professione, l'età, il sesso, l'etnia, la nazionalità, la religione, la condizione socio-economica, etc. Riteniamo utile modellizzare il Tipo di persona come entità a sé stante perché un utente interessato a un Tipo di persona può essere interessato a tutti i Testi che hanno quel Tipo di persona come soggetto, oppure a tutti i Testi che si rivolgono a quel Tipo di persona (ovvero di cui quel Tipo di persona è il destinatario, vedi 2.14), o ancora a tutti i Testi scritti da un certo Tipo di persona, etc.

La sesta entità nuova del nostro modello è il Tipo di ente: la categoria generale di appartenenza di un ente. Riteniamo utile modellizzare il Tipo di ente come entità a sé stante perché un utente interessato a un Tipo di ente può essere interessato a tutti i Testi che hanno quel Tipo di ente come soggetto, oppure a tutti i Testi che si rivolgono a quel Tipo di ente (ovvero di cui quel Tipo di ente è il destinatario), o ancora a tutti i Testi di cui è responsabile un certo Tipo di ente, etc.

Avevamo sopra accennato a un'ulteriore entità residuale del nostro modello: Altre entità.

51 TOM DELSEY, Modeling subject access, cit, p. 55. 
Oltre a non trattare esplicitamente il caso delle tipologie generali di appartenenza delle entità, FRBR lascia in ombra anche un'altra serie di elementi come gli animali (e i tipi di animale), i personaggi fittizi e leggendari, etc. Per questi casi, così come per altre tipologie generali di appartenenza di entità individuali (tipi di oggetto, tipi di luogo, tipi di evento, etc.), nemmeno noi offriremo una modellizzazione distinta. Piuttosto, includeremo questi diversi elementi nelle Altre entità, super-entità in cui raccoglieremo tutto ciò che non abbiamo ritenuto utile distinguere come tipologia di entità a sé stante - ma che, almeno in linea di principio, sarebbe possibile distinguere se ritenuto opportuno; le Altre entità comprendono sia entità di tipo collettivo che di tipo individuale.

\subsection{Entità concettuali e tipologie lessicali}

Chiudiamo la trattazione delle entità del nostro modello con una lista riassuntiva delle entità e alcune considerazioni di carattere linguistico, che riprenderemo in 3.3.6.4.2.3. Le entità del nostro modello sono: Opere, Edizioni e Contenuti editoriali (Testi); Manifestazioni e Item (Risorse); Persone, Enti e Famiglie; Luoghi, Oggetti, Eventi e Concetti; Tempi, Tipi di persona, Tipi di ente, Tipi di testo, Tipi di risorsa, Altre entità. A livello linguistico, e in particolare di tipologie lessicali, la totalità delle entità del modello concettuale può essere un'entità di tipo linguistico, ma soltanto Eventi e Altre entità (tipi di evento) possono essere attività. Per quanto riguarda le entità di tipo linguistico, sono entità individuali: Opere, Edizioni, Contenuti editoriali, Manifestazioni, Item, Persone, Enti, Famiglie, Luoghi, Oggetti, Eventi, Tempi e Luoghi. Sono invece entità collettive: Concetti, Tipi di persona, Tipi di ente, Tipi di testo e Tipi di risorsa. Un'altra importante differenza di origine linguistica tra le entità è quella tra entità animate e inanimate. Sono entità animate: Persone, Enti e Famiglie; sono entità inanimate tutte le altre. Le Altre entità raccolgono sia attività (tipi di evento), sia entità di tipo linguistico (tutte le altre) e, tra queste, sia entità collettive (tipi di oggetto, tipi di evento, tipi di luogo, tipi di animale, etc.) sia individuali (animali, personaggi fittizi, etc.), sia entità animate (animali, personaggi fittizi, tipi di animale) sia inanimate (tutte le altre).

\subsection{Attributi e relazioni}

Gli altri elementi fondamentali di FRBR e del nostro modello concettuale sono gli attributi e le relazioni. Le relazioni sono rapporti tra le entità e tra attributi delle entità. FRBR afferma al loro riguardo che esse "fungono da espediente per rappresentare il legame tra un'entità e un'altra, e dunque come mezzo per aiutare l'utente a 'navigare' l'universo rappresentato in una bibliografia, un catalogo, o un database bibliografico"; 52 questa affermazione fa esplicito riferimento alla funzione Navigare di cui abbiamo parlato, e che FRBR considera come una sorta di quinta funzione non ufficiale. Benché l'affermazione sia accettabile, essa mostra una certa confusione tra le relazioni in quanto tali e loro rappresentazione indicale. Per una discussione più esaustiva di tutti gli aspetti delle relazioni qui non trattati rimandiamo comunque a FRBR, che fornisce una

52 IFLA STUDY GROUP ON THE FUNCTIONAL REQUIREMENTS FOR BIBLIOGRAPHIC RECORDS, Functional requirements for bibliographic records, cit., p. 56. 
trattazione piuttosto ampia delle relazioni. Altri studi fondamentali sulle relazioni bibliografiche, che hanno tutti più o meno influenzato FRBR, sono quelli di Barbara B. Tillett, Sherry L. Vellucci, Edward T. O'Neill e Diane Vizine-Goetz. In Italia, le relazioni bibliografiche sono recentemente state approfondite da Carlo Bianchini. ${ }^{53}$

Gli attributi di FRBR sono le caratteristiche delle entità, e sono quegli elementi che servono da base per le ricerche degli utenti finali: ${ }^{54}$ se per esempio ci riferiamo a una Manifestazione, sono suoi attributi il titolo della manifestazione, il nome dell'editore, le caratteristiche fisiche, l'ISBN (International Standard Book Number), etc.

Nel modello dell'IFLA vi è una separazione netta tra attributi e relazioni: elementi informativi modellizzati come attributi non lo sono come relazioni, e viceversa; il criterio per stabilire se un determinato elemento informativo sia da considerarsi una relazione o un attributo è legato al fatto che esso faccia o meno riferimento a ulteriori entità: gli attributi sono elementi informativi che non fanno riferimento ad ulteriori entità; le relazioni quelli che fanno riferimento a ulteriori entità, ovvero che legano tra loro due entità. Questa impostazione ha il pregio di offrire un quadro in cui attributi e relazioni sono nettamente distinti, senza sovrapposizioni. Essa ha però anche diversi svantaggi: 1) con essa, i criteri di cosa debba essere stabilito come attributo o relazione rimangono strettamente ancorati agli attuali standard e regole di catalogazione; 2) essa non è coerente con la definizione fornita di "attributo": infatti, se gli attributi sono "quegli elementi che servono da base per le ricerche degli utenti finali", essi dovrebbero comprendere anche ciò che FRBR modellizza come relazione: autori di opere, soggetti, etc.; 3) essa non riesce a giustificare l'esistenza di elementi informativi che in alcuni casi possono implicare relazioni con altre entità, in altri no - quali, nel nostro modello, i nomi, titoli e termini delle entità.

Per il nostro modello concettuale adotteremo perciò un'impostazione dei rapporti tra attributi e relazioni lievemente differente. Nel nostro modello attributi e relazioni non saranno nettamente distinti, ma piuttosto sovrapposti: ogni entità ha un certo numero di attributi; alcuni tra questi, poi, implicano anche relazioni di vario tipo. Secondo questa impostazione, per esempio, il responsabile di un'Opera è al contempo un attributo dell'Opera ma anche una relazione tra l'Opera e una o più ulteriori entità (Persone $\mathrm{o}$ Enti). Data questa impostazione, e al fine di non appesantire troppo la discussione, nei paragrafi successivi ci concentreremo sugli attributi, facendo solo riferimenti espliciti alle relazioni soggiacenti solo in pochi casi.

FRBR non prevede una modellizzazione delle cosiddette "relazioni semantiche" (relazioni gerarchiche, associative, di equivalenza, etc.). Esse sono invece, come avremo modo di dire, previste nel nostro modello. A ciò è legata un'altra differenza tra FRBR e il modello proposto: nell'ambito del nostro modello le relazioni possono essere sia relazioni tra

53 BARBARA B. TILLETT, Bibliographic relationships. Toward a conceptual structure of bibliographic information used in cataloging, Ann Arbor, Mi., University Microfilms International, 1987; SHERRY L. VELLUCCI, Bibliographic relationships in music catalogs, with a foreword by D. W. Krummel, Lanham, Md., Scarecrow, 1997; EDWARD T. O'NEILL - DIANE VIZINE-GOETZ, Bibliographic relationships. Implications for the function of the catalog, in The conceptual foundations of descriptive cataloging, edited by Elaine Svenonius, San Diego, Academic Press, 1989, p. 167-179; CARLO BIANCHINI, Riflessioni sull'universo bibliografico. Funzioni, oggetti e modelli della catalogazione per autore e titolo, prefazione di Mauro Guerrini, Milano, Sylvestre Bonnard, 2005, p. 155-162.

54 IFLA STUDY GROUP ON THE FUNCTIONAL REQUIREMENTS FOR BIBLIOGRAPHIC RECORDS, Functional requirements for bibliographic records, cit., p. 30. 
entità, sia, in alcuni casi (relazioni di equivalenza, relazioni di subordinazione e coordinazione tra temi del soggetto), relazioni tra attributi. Sulle basi suddette, suddivideremo innanzitutto gli attributi in nomi - che comprendono anche titoli e termini -, o attributi nominali, e in attributi diversi da nomi, o attributi non nominali. Gli attributi nominali possono riguardare tanto il nome o i nomi di un'entità, quanto nomi di altre entità in relazione con l'entità stessa, ovvero nomi delle entità che fungono da attributi rispetto all'entità stessa. Nel primo caso si ha che fare con attributi nominali "interni" (il nome o i nomi dell'entità), nel secondo con attributi nominali "esterni" che corrispondono all'incirca alle relazioni di FRBR -; gli attributi non nominali sono tutti indistintamente interni; viceversa, gli attributi nominali possono essere attributi interni o esterni. Ovviamente, la differenza tra attributi non nominali (interni) e attributi (nominali) esterni - ma già tra gli attributi e le relazioni di FRBR, è del tutto convenzionale: essendovi alla base la differenza tra elementi informativi che fanno riferimento o meno a ulteriori entità, la determinazione di cosa debba costituire attributo non nominale o attributo esterno è strettamente legata alla scelta di cosa, tra gli elementi dell'universo bibliografico, è considerato entità e cosa no. Nel nostro modello, tutti i nomi implicano potenzialmente relazioni perché tutti possono avere relazioni di equivalenza con gli altri nomi di un'entità. Inoltre, i nomi si dividono in complessi (attributi nominali complessi) e semplici (attributi nominali semplici), a seconda che esplicitino o meno relazioni dell'entità con altre entità (vedi 2.13); anche la differenza tra nomi semplici e complessi è convenzionale e basata sui criteri di individuazione delle entità e delle relazioni.

Un'altra differenza importante con FRBR è che nel modello dell'IFLA vi è a volte confusione tra attributi e relazioni da un lato e loro rappresentazione dall'altro; nel nostro modello, in cui vi è una trattazione esplicita degli elementi indicali che rappresentano attributi e relazioni, vi è invece uno scarto netto tra entità, attributi e relazioni da un lato e loro rappresentazione catalografica dall'altro: la rappresentazione di entità, attributi e relazioni può restare del tutto implicita oppure può avere una rappresentazione più $\mathrm{o}$ meno esplicita, ovvero diversi gradi di controllo formale (vedi 2.17 e 3.3.4). Adesso che abbiamo esposto l'impostazione generale degli attributi e delle relazioni del nostro modello, passiamo alla trattazione degli specifici tipi di attributi (e, indirettamente, di relazioni) del nostro modello. Non li tratteremo in modo esaustivo, ma parleremo soltanto di quegli attributi e relazioni che riteniamo più importanti o in cui il nostro modello si differenzia esplicitamente da FRBR; in alcuni casi accenneremo soltanto agli attributi e alle relazioni; in altri, e in particolare nel caso del soggetto, offriremo una trattazione molto più approfondita. Inizieremo con la discussione degli elementi nominali, prima considerati in quanto tipologia generale di attributo, e poi discutendo le specifiche tipologie di attributi; quindi continueremo con la discussione degli elementi non nominali. Faremo infine una lunga e approfondita digressione sulla nozione di soggetto e sulla sua modellizzazione: mostreremo come nel soggetto siano fusi elementi diversi che è opportuno distinguere.

\subsection{Attributi nominali}

I nomi, o attributi nominali, costituiscono una tipologia generalissima di attributo del 
nostro modello. Essi comprendono non solo nomi veri e propri, ma anche termini, titoli e altri appellativi di un'entità - a esclusione degli identificatori, concettualmente affini ma distinti e che considereremo attributi non nominali.

Ogni entità può avere uno o più nomi: essi costituiscono il primo e principale attributo di ogni entità; in presenza di più nomi, questi sono tra loro legati da una relazione di equivalenza (vedi 2.14). Accanto ai nomi che sono propri di un'entità, e che ne costituiscono attributi (nominali) interni, vi sono poi i nomi di tutte le entità in relazione con l'entità stessa: questi nomi costituiscono invece attributi (nominali) esterni ovviamente, se una prima entità ha come attributo esterno il nome di una seconda entità, quello stesso nome sarà un attributo interno della seconda entità.

Nell'ambito del nostro modello, i nomi hanno la peculiarità di essere attributi che, a seconda dei casi, possono esplicitare relazioni tra l'entità nominata e altre entità che ne costituiscono i componenti concettuali. Quando ciò avvenga, parleremo di nomi "complessi", o attributi nominali complessi, in contrapposizione a nomi "semplici", o attributi nominali semplici, che non esprimono relazioni tra l'entità nominata e altre entità. L'individuazione di nomi complessi è subordinata a tre condizioni: 1) la presenza, nel nome di una data entità, di elementi indicativi (nomi) di ulteriori entità; 2) l'ulteriore presenza di almeno un altro elemento, che può essere a sua volta il nome di un'altra entità o meno (vedi sotto) - è dunque anche condizione necessaria e non sufficiente che un nome complesso presenti due o più parole; 3) le ulteriori entità menzionate nel nome di una data entità devono costituirne gli effettivi componenti concettuali, ovvero devono essere legati alla data entità da una relazione gerarchica; in tutti gli altri casi, quando cioè la relazione espressa dal nome sia di tipo diverso, non si avrà un nome complesso. ${ }^{55}$ Non costituiscono dunque nomi complessi né titoli (che facciano riferimento a ulteriori entità che siano i soggetti di un Testo), né nomi che contengano elementi sincategorematici ("occhi di vetro", che non sono un tipo di "occhi") ${ }^{56}$ né ancora nomi che non esprimano relazioni autentiche, come per esempio "Biblioteca Alberto Bombace", dove non vi è un'autentica relazione tra l'Ente "Biblioteca Alberto Bombace" e la Persona "Alberto Bombace”. All'interno del nostro modello sono escluse dalla possibilità di nomi complessi alcune tipologie di entità: Persone, Famiglie, Tempi e Lingue; ogni altro tipo di entità può avere nomi semplici, complessi o anche di entrambi i tipi.

Casi di entità con nomi complessi possono essere quelli del nome di un Evento o di un Oggetto che si presenti come combinazione di un nome comune (che non rimanda a ulteriori entità) e di un nome proprio di una o più ulteriori entità legate con l'Evento o l'Oggetto; per esempio il nome dell'Oggetto "Cattedrale di Palermo" esplicita una relazione con il Luogo "Palermo"; il nome dell'Evento "Alluvione di Firenze del '66" esplicita una relazione con il Luogo "Firenze" e con il Tempo "1966". Sono altri esempi di nomi complessi nomi di Luogo come "Golfo di Napoli", che fa a sua volta riferimento al Luogo "Napoli", o nomi di Ente come "Università di Catania”, che fa riferimento al

55 In teoria, sarebbe possibile prendere in considerazione anche altri tipi di relazione tra quelli che determinano nomi complessi: se si prendessero in considerazione relazioni di omonimia e di soggetto, per esempio, una parte considerevole dei titoli costituirebbero nomi complessi. Volendo prendere in considerazione le relazioni retoriche o di senso figurato, cosa che non faremo nel presente lavoro (vedi 3.2.5.3.3), sarebbe possibile considerare nomi complessi anche tutti quelli che esprimano questo tipo di relazioni.

56 Cfr. ASSOCIAZIONE ITALIANA BIBLIOTECHE. GRUPPO DI RICERCA

SULL'INDICIZZAZIONE PER SOGGETTO, Guida all'indicizzazione per soggetto, cit., p. 71-73. 
Luogo "Catania". Un caso tipico di entità individuali il cui nome includa quello di ulteriori entità è poi quello degli enti gerarchici: la "Biblioteca della Regione Sicilia" (ovvero "Biblioteca Alberto Bombace") fa riferimento al nome dell'ente sovraordinato "Regione Sicilia".

Finora abbiamo parlato di nomi complessi di entità individuali; leggermente diverso è il caso dei nomi complessi delle entità collettive. Il nome del Concetto "Etica della vita" (ovvero "Bioetica") esplicita una relazione con i Concetti "Etica" e "Vita". Anche Tipi di persona, Tipi di ente, Tipi di testo e Tipi di risorsa possono presentare nomi complessi. Così, il Tipo di persona "Bibliotecari italiani" esplicita una relazione sia con il Tipo di persona "Bibliotecari" sia con il Luogo "Italia". Il Tipo di testo "Letteratura italiana" esplicita una relazione sia con il Tipo di testo "Letteratura" sia con la Lingua "Italiano"; etc. Nel caso delle entità collettive non si ha a che fare con nomi non distintivi abbinati a nomi propri, ma piuttosto con nomi che fanno riferimento ai componenti concettuali delle entità stesse; a differenza del caso dei nomi complessi delle entità individuali, generalmente nei nomi complessi delle entità collettive ogni elemento esprime potenzialmente una relazione con ulteriori entità.

Riprenderemo e approfondiremo queste considerazioni nel prossimo capitolo, dedicando ampio spazio anche alla trattazione delle relazioni sintagmatiche tra i diversi elementi componenti dei nomi complessi (vedi 3.2.5.3.3, 3.2.7, 3.3.6.2.4 3.3.6.2.5, 3.3.6.4.2.2.2 e ss., 3.3.6.4.3 e ss.).

\subsection{Attributi nominali specifici}

Parliamo adesso degli attributi nominali specifici: sia interni (nel caso delle relazioni di equivalenza) sia esterni (tutti gli altri); ricordiamo anche che tutti gli attributi esterni sono attributi nominali.

Ogni entità (Opere, Manifestazioni, ma anche Eventi, Persone, Enti, etc.) può avere una o più date, dichiarate dalla risorsa oppure congetturate, integrate o corrette

dall'indicizzatore. In questi casi vi è un attributo (esterno) di data - e una corrispondente relazione di data con un Tempo. Le date possono essere di tipo differente sulla base dell'entità di riferimento: vi sono date dell'Opera (data di composizione), dell'Espressione (data di copyright, etc.), del Contenuto editoriale, della Manifestazione (data di pubblicazione, data di stampa, etc.), dell'Item, dell'Evento (data dell'evento), delle Persone ed Enti (date di nascita, di morte, di attività). La data può essere chiusa o aperta, in base all'esistenza o meno di un termine previsto della data.

Le entità possono avere uno o più luoghi di creazione (luogo di composizione dell'Opera), modifica (Espressione), produzione, pubblicazione, distribuzione, stampa, etc.

(Manifestazione), siano o meno dichiarati all'interno di una risorsa; anche Enti, Eventi, Concetti, Tipi di persona, Tipi di ente, Tipi di testo, Tipi di risorsa, etc. possono avere un luogo associato. In tutti questi casi si ha a che fare con attributi (esterni) di luogo di vario tipo - e con corrispondenti relazioni tra l'entità e un Luogo).

A differenza di FRBR, il nostro modello prevede una trattazione esplicita delle cosiddette "relazioni semantiche" ${ }^{57}$ e dei relativi attributi; approfondiremo tali relazioni soprattutto

57 L'espressione "relazione semantica”, nel senso con cui è utilizzata nell'indicizzazione, è del tutto scorretta perché come diremo nel terzo capitolo non soltanto le "relazioni semantiche", ma la totalità di relazioni, 
nel prossimo capitolo (vedi 3.2.5.3.3, 3.3.6.4.2 e ss.), ma ne forniamo qui una breve trattazione funzionale alle esigenze della nostra modellizzazione concettuale degli attributi e delle relazioni. Gli attributi relativi alle "relazioni semantiche" (termine specifico, termine generico, etc.) sono attributi nominali comuni a tutte le entità del nostro modello; a essi corrispondono precise "relazioni semantiche" (iponimia, iperonimia, etc., vedi sotto). L'espressione "relazioni semantiche" è tradizionalmente utilizzata nell'ambito dell'indicizzazione per soggetto (per questo le "relazioni semantiche" sono assenti da FRBR), mentre nell'indicizzazione per autore si utilizzano generalmente termini diversi. Si tratta di una difformità del tutto infondata, poiché come mostreremo in entrambi i casi (indicizzazione per soggetto e per autore) si ha a che fare con il medesimo tipo di relazioni. Nell'ambito dell'indicizzazione per soggetto, e dei thesauri in particolare, l'espressione "relazioni semantiche" è utilizzata per indicare relazioni di significato di vario tipo tra le entità utilizzate come soggetti, tradizionalmente suddivise in tre categorie diverse: relazioni gerarchiche (a loro volta suddivise in relazioni gerarchiche generiche, partitive e d'altro tipo, vedi sotto), relazioni di equivalenza, relazioni associative.

Prima di discuterle, notiamo che le "relazioni semantiche" differiscono in un elemento fondamentale rispetto agli altri attributi nominali del nostro modello concettuale: mentre questi riguardano il rapporto tra una prima entità e una seconda entità che svolge, rispetto alla prima, un ruolo specifico, ovvero ne costituisce una caratteristica specifica (l'autore, il soggetto, il luogo di pubblicazione, il proprietario, etc.), le "relazioni semantiche" riguardano: 1) nel caso delle relazioni gerarchiche (e associative): rapporti tra una prima entità e una seconda entità nella sua interezza; 2) nel caso delle relazioni di equivalenza: rapporti tra i diversi nomi (attributi interni) di un'entità.

Le relazioni gerarchiche sono quelle in cui è presente un concetto di gerarchia tra i termini; ne esistono di diversi tipi. Le relazioni gerarchiche generiche (o di eponimia) sono le relazioni tra un genere o classe e le sue specie o individui. Un esempio di relazione generica è quella che lega "Felini" a "Gatti": "Felini" è in relazione generica o iperonimica con "Gatti", "Gatti" è in relazione specifica o iponimica con "Felini"; riferendoci agli attributi, "Felini" è termine generico o iperonimo di "Gatti", "Gatti" è termine specifico o iponimo di "Felini". Altri esempi di relazione generica sono quelli che legano "Biblioteche" (termine generico) a "Biblioteche ecclesiastiche" (termine specifico), o "Letteratura" (termine generico) a "Letteratura italiana" (termine specifico); sono possibili anche relazioni generiche tra tipi diversi di entità, e in particolare tra entità collettive ed entità individuali, per esempio tra tipi di luogo (Altre entità, nel nostro modello) e Luoghi, come tra "Vulcani" (o più precisamente "Vulcani italiani") ed "Etna"; l'eventuale rappresentazione indicale di quest'ultimo tipo di relazioni gerarchiche è importante perché un utente interessato alla classe ("Vulcani") potrebbe essere interessato anche all'individuo ("Etna"), e viceversa.

Come avremo modo di dire nel prossimo capitolo (vedi 3.2.5.3.3, 3.3.6.4.2.2.2.4), gli elementi dei termini che non indicano il genere o la classe, ma piuttosto la specificano ("ecclesiastiche" in "Biblioteche ecclesiastiche", "italiana" in "Letteratura italiana"), sono a loro volta legati da relazioni semantiche reciproche rispetto a quelle generiche: le relazioni semiche e sememiche, normalmente non trattate dall'indicizzazione per soggetto

attributi ed entità dei modelli concettuali sono da ritenersi di natura semantica (vedi 3.3.4.6); in 2.14 proporremo un'espressione più appropriata, ma almeno per il momento continueremo a utilizzare l'espressione "relazioni semantiche". 
Tesi di dottorato di Giuliano Genetasio, discussa presso l'Università degli Studi di Udine

tradizionale.

Un'altra tipologia di relazione gerarchica sono le relazioni partitive (o partonomiche), che indicano la relazione tra un intero e una sua parte. Un esempio di relazione partitiva è quella che lega "Italia" a "Calabria": "Italia" è in relazione olonimica rispetto a "Calabria", "Calabria" è in relazione meronimica rispetto a "Italia"; referendoci agli attributi, "Italia" è un termine olonimo di "Calabria", "Calabria è un termine meronimo di "Italia"; altri esempi di relazione partitiva sono quelli che legano "Mano" (olonimo) a "Pollice" (meronimo), oppure "Fisica" (olonimo) a "Termodinamica" (meronimo). Le relazioni di equivalenza sono relazioni che indicano una sinonimia o più in generale un rapporto tra elementi (nomi, termini, titoli) che abbiano una forma diversa ma un significato identico o molto simile. Esempi di relazione di equivalenza sono quelli tra "Prima guerra mondiale" e "Grande guerra" oppure tra "Gatto" e "Felis silvestris catus"; sono inoltre esempi di relazione di equivalenza i già citati "Biblioteca della Regione Sicilia" e "Biblioteca Alberto Bombace", o ancora "Bioetica" e "Etica della vita".

Vi sono poi anche le relazioni semantiche tra i diversi elementi di termini composti come "Etica della vita" o "Biblioteca della Regione Sicilia"; nel termine composto "Etica della vita", per esempio, vi è una precisa relazione tra i termini "Etica" e "Vita". Si tratta delle relazioni sintagmatiche che toccheremo più sotto ma che approfondiremo soprattutto nel prossimo capitolo (vedi 3.2.7, 3.3.6.4.3).

Un'altra tipologia di "relazione semantica", spesso non considerata come tale dall'indicizzazione per soggetto, è quella delle relazioni di omonimia e polisemia. Le relazioni di omonimia sono quelle tra termini identici nell'aspetto ma differenti nel significato, come per esempio "Gru" (l'attrezzatura) e "Gru" (l'uccello); simili alle relazioni di omonimia sono le relazioni di polisemia, che esistono tra le diverse accezioni di senso di uno stesso termine, per esempio tra i diversi sensi con cui può essere inteso il termine "Radice" (radice di pianta, radice matematica, etc.).

Le relazioni associative, infine, sono relazioni residuali (super-relazioni) dell'indicizzazione per soggetto che raccolgono tutte le relazioni di significato tra le entità che non ricadono nelle relazioni gerarchiche, di equivalenza, etc.: antonimia (relazioni tra termini di significato contrario), relazioni tra un agente a un'attività o tra un'attività e un oggetto, relazioni causa-effetto, etc. A causa di questa natura eterogenea e poco definita, non prenderemo in considerazione le relazioni associative all'interno del nostro approccio, preferendo parlare piuttosto di antonimi e di relazioni d'altro tipo; spiegheremo motivi e dettagli di queste scelte nel prossimo capitolo (vedi 3.3.6.4.21, 3.3.6.4.2.2.3).

All'inizio del paragrafo, avevamo accennato al fatto che nonostante alcune difformità terminologiche, le "relazioni semantiche" riguardano la catalogazione per autore allo stesso modo della catalogazione per soggetto. Così, anche se nella catalogazione per autore non si parla generalmente di relazioni di equivalenza, le relazioni tra diversi nomi o forme del nome di una Persona o di un Ente, oppure le relazioni tra i diversi titoli di un'Opera, sono in tutto e per tutto identiche alle relazioni di equivalenza che vigono tra Concetti, Oggetti etc.; benché nel caso di nomi personali e di enti, e nel caso dei titoli, non si possa parlare di vera e propria sinonimia, si tratta sempre di relazioni tra elementi che hanno una forma diversa ma uno stesso significato; così, sono altri esempi di relazioni di equivalenza quelli tra le diverse forme del nome di un Ente ("IFLA", "International Federation of Library Associations and Institutions"), o di una Persona ("John Hutchins", "W. John Hutchins" e "William John Hutchins", etc.), o ancora tra titoli differenti di 
Tesi di dottorato di Giuliano Genetasio, discussa presso l'Università degli Studi di Udine

un'Opera ("Pinocchio", "Le avventure di Pinocchio").

Le stesse considerazioni valgono per le relazioni partitive, spesso definite relazioni tutto/parte nell'ambito della catalogazione per autore: sono relazioni partitive quelle tra enti gerarchici, e cioè tra un ente sovraordinato ("Regione Sicilia") e uno subordinato ("Biblioteca della Regione Sicilia"); ma anche tra un'Opera ("Divina commedia") e le sue parti ("Inferno", "Purgatorio", etc.).

Se le relazioni di polisemia e di antonimia riguardano a rigor di termini soltanto Concetti ed altre entità collettive, l'omonimia è invece una "relazione semantica" che ritroviamo spesso nella catalogazione per autore: vi possono essere due Persone omonime ("Thomas Mann" autore de La morte a Venezia, "Thomas Mann" autore di Oxford guide to library research), due Opere dallo stesso titolo, etc.

Infine, attualmente la catalogazione per autore non prevede generalmente la possibilità di rappresentare relazioni gerarchiche generiche per entità come Persone, Enti, Testi, Risorse, etc. Ma si tratta di una mancanza ingiustificata: anche Persone, Enti, Testi, Risorse, etc., come tutte le altre entità, intrattengono relazioni generiche con altre entità. Ogni persona appartiene a una o più categorie: etnia o nazionalità, professione, religione, sesso, etc.; ogni Ente appartiene a una specifica categoria di enti, o in alcuni casi anche a più d'una; e lo stesso può dirsi per Testi e Risorse, ciascuno dei quali può appartenere a più categorie testuali o fisiche. Si tratta, in tutti questi casi, di relazioni generiche (e relativi attributi) tra Persone (iponimo) e Tipi di persona (iperonimo), Enti (iponimo) e Tipi di ente (iperonimo), Testi (iponimo) e Tipi di testo (iperonimo), Risorse (iponimo) e Tipi di risorsa (iperonimo). Così, per esempio, "Luigi Pirandello" appartiene alla categoria degli "Autori” (o più precisamente degli "Autori italiani”); "Leonardo da Vinci” è iponimo di "Scienziati italiani" ma anche di "Artisti italiani"; "Biblioteca della Regione Sicilia" è iponimo di "Biblioteche" (o più precisamente delle "Biblioteche regionali siciliane"), "Associazione italiana biblioteche" è iponimo di "Associazioni bibliotecarie italiane". La classe di appartenenza di un'Opera può coincidere semplicemente con la sua forma testuale (vedi sotto); a volte, invece, alcune Opere appartengono anche a tipologie testuali d'altro tipo, non riconducibili a forme testuali. Per esempio, la Bibbia appartiene ai "Testi sacri del Cristianesimo", ovvero ne è un iponimo.

Riferendoci al livello fisico dell'indicizzazione, potremmo chiederci: la possibilità di una rappresentazione indicale delle relazioni generiche tra entità tipiche della catalogazione per autore è senz'altro possibile; ma essa è utile? Lo è doppiamente. La registrazione delle relazioni generiche tra Opere, Persone, Enti, etc. è utile nelle ricerche per soggetto perché, così come per Luoghi, Eventi, Oggetti, etc., un utente interessato a una certa entità come soggetto (una particolare Persona, un particolare Ente, etc.) potrebbe essere interessato alla relativa categoria di appartenenza (Tipo di persona, Tipo di ente etc.). Ma è anche utile nelle ricerche per autore e titolo: un utente finale, infatti, potrebbe essere interessato a tutte le opere scritte da immigrati, o da artisti, o da altri Tipi di persona; potrebbe essere interessato a tutte le manifestazioni emanate da banche, da enti governativi, da biblioteche, o da altri Tipi di ente; e così via.

Le "relazioni semantiche", dunque, valgono - fatta qualche eccezione come l'antonimia, la polisemia, etc. - per tutte le tipologie di entità. Esse restano identiche a prescindere dal ruolo contingente (attributo o relazione) svolto da un'entità: l'appartenenza di una Persona a una professione vale ed è identica sia nel caso essa sia soggetto di un'Opera, sia nel caso ne sia il responsabile; l'appartenenza di un Luogo a un altro Luogo più ampio 
non varia se esso è luogo di pubblicazione piuttosto che il luogo di cui parla un'Opera. Nel caso in cui un'entità sia il tema di un Testo, ovvero ne rappresenti la circalità estensionale, vi è semmai una potenziale sovrapposizione tra le "relazioni semantiche" dell'entità che costituisce la circalità estensionale del Testo e la circalità intensionale del Testo (per le nozioni di circalità estensionale e intensionale, vedi 2.16.4).

Finora abbiamo utilizzato l'espressione "relazioni semantiche" sempre tra virgolette; prima di passare alla trattazione degli altri attributi e relazioni del nostro modello, ne chiariamo il motivo. L'espressione "relazioni semantiche" non è corretta, perché, come avevamo peraltro anticipato, tutte le relazioni del modello concettuale sono semantiche.

L'individuazione di tutti i vari elementi del modello concettuale (relazioni, ma anche attributi ed entità) scaturisce in ultima analisi da una selezione di elementi di significato del linguaggio naturale, operata sulla base delle specifiche caratteristiche ed esigenze dell'accesso bibliografico: dunque la totalità degli elementi del modello concettuale è di natura semantica. Semmai, è vero che le "relazioni semantiche" sopra discusse sono le uniche relazioni semantiche del modello concettuale che abbiano una corrispondenza diretta (tranne nel caso delle relazioni associative) con le relazioni semantiche trattate esplicitamente dalla linguistica, e cioè quelle indipendenti dal particolare contesto dell'accesso bibliografico. Date queste condizioni, da qui in poi non parleremo - in riferimento alle relazioni gerarchiche, di equivalenza, di omonimia, polisemia, etc. - di "relazioni semantiche", ma piuttosto di "relazioni semantiche paradigmatiche" e, in rapporto alle relazioni tra gli elementi componenti un nome complesso, di "relazioni sintagmatiche"; spiegheremo queste nozioni nel prossimo capitolo (vedi 3.2.3, 3.2.6, 3.3.5, 3.3.6.1, etc.).

Continuiamo con la trattazione di altri attributi nominali del nostro modello. Tra gli attributi esterni specifici a Testi, Risorse, Persone, Enti (e Famiglie, nel nostro modello), vi è quello di provenienza: Persone o Enti che hanno precedentemente posseduto Item o Oggetti.

Altro attributo esterno che riguarda Testi (Contenuti editoriali) da un lato e Persone dall'altro è quello di omaggio; si tratta del caso delle miscellanee in onore o Festschrift; l'omaggio è stato tradizionalmente assimilato a una responsabilità o a un soggetto, ma non ricade in nessuna delle due, e dunque abbiamo preferito distinguerlo nel nostro modello.

La nozione di responsabilità e quelle correlate (autore, attribuzione, etc.) è, come il soggetto, una nozione al contempo centrale e complessa dell'accesso bibliografico. Non discuteremo in profondità la nozione di responsabilità, perché ciò esula dagli scopi della nostra modellizzazione. La responsabilità lega un'entità bibliografica (Testi e Risorse) con Persone ed Enti; non prevediamo la possibilità di una responsabilità da parte di Famiglie. All'attributo "responsabile" dell'entità bibliografica corrisponde un reciproco attributo "responsabile di” della Persona o dell'Ente.

Fin qui abbiamo parlato di responsabilità in modo generico. In effetti, esistono responsabilità di tipo assai diverso, sia sulla base dell'entità di riferimento, sia sulla base del tipo di operazione sui cui è esercitata la responsabilità: così, accanto a responsabilità testuali (intellettuali, artistiche, di curatela) vi sono anche responsabilità non testuali relative alla pubblicazione e alla produzione materiale (stampa, etc.). Le responsabilità possono inoltre essere di gradi di importanza differenti: in presenza di due o più responsabili che svolgono lo stesso tipo di responsabilità, il loro ruolo può essere di pari 
importanza, oppure uno dei responsabili può avere un ruolo principale rispetto agli altri. È teoricamente possibile distinguere le differenti responsabilità sulla base di diversi criteri; per esempio, FRBR suddivide le diverse tipologie di responsabilità sulla base di entità bibliografica di riferimento (responsabilità di creazione per l'Opera, di realizzazione per l'Espressione, di produzione per la Manifestazione); e lo stesso vale per i gradi di responsabilità.

Tra gli attributi esterni specifici di Persone, Enti e Famiglie dobbiamo parlare almeno degli attributi di possesso, ${ }^{58} \mathrm{di}$ affiliazione istituzionale e di componente familiare. L'attributo di possesso riguarda Enti posseduti da Persone o Famiglie che ne sono i possessori. Simile all'attributo di possesso è quello di affiliazione istituzionale; una persona ha un'affiliazione istituzionale rispetto a uno o più Enti, e viceversa un Ente ha come dipendenti o affiliati una o più Persone. Infine, ogni Persona può avere come attributo di famiglia d'appartenenza una Famiglia, e viceversa una Famiglia può avere come attributo di componenti familiari una o più Persone.

Per quanto riguarda gli attributi esterni (e le relazioni) specifici di Testi e Risorse, vanno segnalati innanzitutto quelli (che potremmo chiamare "attributi primari") corrispondenti alle cosiddette "relazioni primarie" di FRBR, che riguardano le entità del Gruppo 1: ${ }^{59}$ un'Opera ha come realizzazioni una o più Espressioni; un'Espressione è la realizzazione di un'Opera e ha a sua volta come realizzazioni uno o più Contenuti editoriali (nel nostro modello, non in FRBR) oppure una o più Manifestazioni - a seconda che l'Espressione sia inserita in una raccolta o meno. Il Contenuto editoriale è la realizzazione di due o più Espressioni, tra loro combinate, e può a sua volta avere come realizzazioni una o più Manifestazioni. La Manifestazione è la realizzazione di un'Espressione o di un Contenuto editoriale e può a sua volta avere come esemplari o copie uno o più Item. L'Item è la copia o l'esemplare di una Manifestazione. Come abbiamo già spiegato, tra le entità bibliografiche del Gruppo 1 vi è un tipo di rapporto uno-a-molti, tranne nel caso del rapporto tra Espressioni e Contenuti editoriali.

Vi sono poi attributi esterni (e relazioni) delle entità bibliografiche che riguardano entità dello stesso tipo (Opere e Opere, Manifestazioni e Manifestazioni, etc.). Di esse FRBR fornisce una modellizzazione piuttosto dettagliata. Tra quelli relativi alle Risorse, il modello dell'IFLA elenca: 1) per quanto riguarda le Manifestazioni: riproduzione, versione alternativa (formati alternativi, edizioni pubblicate contemporaneamente); 2) per quanto riguarda gli Item: riproduzione, riconfigurazione. Ogni Risorsa appartiene a uno o più tipi di supporto. L'attributo delle Risorse "tipo di supporto" fa riferimento a un Tipo di risorsa. Tra gli attributi (e le relazioni) tra entità dello stesso tipo relativi i Testi ed elencate da FRBR vi sono invece: 1) per quanto riguarda le Opere: successione, complemento, riassunto, adattamento, derivazione, imitazione; 2) per quanto riguarda le Espressioni; riduzione, revisione, traduzione, arrangiamento.

Tra gli attributi esterni che riguardano Testi e altre entità, dobbiamo poi menzionare la lingua, la forma testuale, i destinatari e il soggetto. Ogni Testo può presentarsi in una o più lingue. L'attributo esterno di un Testo "lingua" fa riferimento a una Lingua. La lingua può essere quella originale (lingua dell'Opera), oppure, nel caso di traduzioni (Espressioni di un'Opera), una lingua diversa. Opere, Espressioni e Contenuti editoriali

58 Da non confondere con la relazione sintagmatica di possesso, di cui parleremo in 3.2.7. 59 IFLA STUDY GROUP ON THE FUNCTIONAL REQUIREMENTS FOR BIBLIOGRAPHIC RECORDS, Functional requirements for bibliographic records, cit., p. 58-60. 
possono anche presentarsi in più lingue (per esempio opere tradotte con testo a fronte in lingua originale, tête-bêche, bibbie poliglotte, etc.).

Ogni Testo può presentarsi o appartenere a uno o più tipi di forme testuali. L'attributo esterno di un Testo "forma testuale" fa riferimento a un Tipo di testo (forma bibliografica, genere letterario, etc.).

Un attributo esterno importante è quello dei destinatari di un Testo. Ogni Testo è rivolto, esplicitamente o implicitamente, a dei destinatari. I destinatari possono essere rappresentati da una o più categorie di persone o di enti. Alcuni autori e alcuni testi importanti dell'indicizzazione per soggetto hanno considerato i destinatari tra gli elementi del soggetto di un testo: per esempio Derek W. Langridge e Bianca Castañon Moreno, ma anche lo standard NISO Z39.19 per i thesauri multilingue del 2005; ${ }^{60}$ tuttavia, benché concettualmente legati al messaggio di un testo, al suo soggetto, i destinatari restano distinti rispetto a esso; noi considereremo allora i destinatari come un attributo esterno connesso al soggetto ma distinto rispetto a esso.

Infine, l'ultimo attributo esterno che caratterizza i Testi e che occorre menzionare è il soggetto. Qualsiasi tipo di entità può fungere da soggetto di un Testo. Per il nostro modello concettuale, tuttavia, parleremo di soggetto come attributo solo in senso generico. Riteniamo infatti che parlare semplicemente di un unico attributo "soggetto" sia riduttivo, e che sia necessario approfondire e specificare la nozione di soggetto in un certo numero di elementi diversi (vedi 2.16 e ss.). Prima di approfondire la nozione di soggetto, però, parleremo brevemente degli attributi non nominali del nostro modello.

\subsection{Attributi non nominali}

Gli attributi non nominali sono quelli non riconducibili a nomi di entità; secondo la nostra modellizzazione, inoltre, tutti gli attributi non nominali sono anche attributi interni a un'entità: essi descrivono infatti caratteristiche proprie di un'entità senza riferimenti a ulteriori elementi individuati come entità. Presentiamo qui soltanto i più importanti. Tra gli attributi non nominali delle Opere vanno segnalati gli eventuali identificatori dell'Opera: per esempio, gli identificatori di Opere musicali. Molteplici sono invece gli attributi non nominali importanti delle Risorse. Per quanto riguarda gli attributi non nominali delle Manifestazioni, vanno segnalati: l'identificatore della Manifestazione (ISBN, EAN - European Article Number, etc.) e le caratteristiche fisiche (dimensioni, presenza di illustrazioni, etc.). Tra gli attributi non nominali degli Item segnaliamo: lo stato di conservazione, le condizioni di disponibilità (restrizioni all'accesso, disponibilità per il prestito, condizioni per l'acquisto, etc.) e la collocazione fisica.

60 Cfr. DEREK W. LANGRIDGE, Subject analysis. Principles and procedures, London, Bowker-Saur, 1989, p. 9-10; BIANCA CASTAÑÓN MORENO, Análisis temático documental, "Investigación bibliotecológica”, 6,12 (1992), p. 8 elenca diversi elementi del Soggetto: "tópicos o conceptos temáticos", "forma de conocimiento", “destinatario", "nivel”, "idioma”, "tipo de documento” e "forma estructural”. Cfr. inoltre NATIONAL INFORMATION STANDARDS ORGANIZATION, Guidelines for the construction, format, and management of monolingual controlled thesauri. ANSI/NISO Z39.19-2005, Bethesda (Md.), NISO Press, 2005, p. 15: "Attributes that might be selected as facets for content objects are: Topic - the subject of the content object; Format - the format of material (e.g., text, image, sound, etc.); Target audience - the appropriate reader for the content (e.g., Children, Adults); Intellectual level - the type of the content (e.g., K-12, news, scholarly journal)". 
Tesi di dottorato di Giuliano Genetasio, discussa presso l'Università degli Studi di Udine

\subsection{Il soggetto}

\subsubsection{Introduzione}

Sul soggetto FRBR dice soltanto che "le entità di tutti e tre i gruppi sono connesse all'Opera tramite una relazione di soggetto". ${ }^{61}$ Cosa sia il soggetto non viene spiegato in nessun punto del testo. Diversi studiosi hanno evidenziato la carenza della nozione di soggetto e dell'indicizzazione per soggetto di FRBR; FRBR prende in considerazione esclusivamente soggetti costituiti da entità caratterizzate da nomi non relazionali, e non da entità caratterizzate da nomi relazionali. ${ }^{62}$ La carenza dell'analisi delle entità e relazioni dell'indicizzazione per soggetto è peraltro riconosciuta dallo stesso FRBR, che auspicava ulteriori analisi in questo ambito. ${ }^{63}$ Per una modellizzazione soddisfacente del soggetto, dovremo allora mettere da parte FRBR e intraprendere un'analisi autonoma della nozione di soggetto. Ripercorreremo allora il dibattito attorno a tale nozione, verificando e valutando le diverse posizioni per poi articolare una nostra concezione e definizione del soggetto e dell'indicizzazione per soggetto.

\subsubsection{Le diverse concezioni del soggetto}

Quella di soggetto è una nozione sfuggente e difficile da determinare: di essa esistono numerose concezioni e definizioni; esistono inoltre una serie di nozioni il cui significato sembra confondersi o sovrapporsi parzialmente a quella di soggetto. Secondo Patrick Wilson, il soggetto è una nozione intrinsecamente oscura, imperscrutabile, dai contorni

61 IFLA STUDY GROUP ON THE FUNCTIONAL REQUIREMENTS FOR BIBLIOGRAPHIC RECORDS, Functional requirements for bibliographic records, cit., p. 62.

62 VICTORIA FRÂNCU, Subjects in FRBR and poly-hierarchical thesauri as possible knowledge organizing tools, in Knowledge organization for a global learning society. Proceedings of the ninth International ISKO Conference, 4-7 July 2006, Vienna, Austria, edited by Gerhard Budin, Christian Swertz, Konstantin Mitgutsch, Würzburg, Ergon, 2006, p. 375; cfr. anche TOM DELSEY, Modeling subject access, cit, p. 55. Secondo PINO BUIZZA MAURO GUERRINI, Un modello concettuale per il Nuovo soggettario. L'indicizzazione per soggetto alla luce di FRBR, "Bollettino AIB", 41, 3 (2001), p. 330, il soggetto di FRBR è carente perché "I soggetti vengono presentati come unità atomistiche, senza l'articolazione di più concetti che si trova nella maggioranza dei soggetti reali".

63 IFLA STUDY GROUP ON THE FUNCTIONAL REQUIREMENTS FOR BIBLIOGRAPHIC

RECORDS, Functional requirements for bibliographic records, cit., p. 5. L'analisi dei dati di autorità per soggetto è poi stata intrapresa da IFLA WORKING GROUP ON THE FUNCTIONAL REQUIREMENTS FOR SUBJECT AUTHORITY RECORDS, Functional requirements for subject authority data (FRSAD), cit. In FRSAD il soggetto è modellato come entità, non più come relazione, o meglio come super-entità (classe di entità) che comprende tutte le altre entità utilizzate come soggetti. Ma già anni prima di FRSAD l'idea del tema come entità fu anticipata da ALBERTO CHETI, Il punto di vista del GRIS sulla 'relazione di soggetto' in FRBR, in Principi di catalogazione internazionali. Una piattaforma europea? Considerazioni sull'IME ICC di Francoforte e Buenos Aires. Atti del convegno internazionale, Roma, Bibliocom, 51. Congresso AIB, 27 ottobre 2004, a cura di Mauro Guerrini, Roma, Associazione italiana biblioteche, 2008, p. 94-95. 
indefiniti. ${ }^{64}$ Ross J. Todd rileva come nella letteratura sull'indicizzazione esista una certa confusione terminologica sulla nozione di soggetto. ${ }^{65}$

Il soggetto è, afferma Jacques Maniez, una nozione al contempo concettualmente inafferrabile ma praticamente indispensabile. "Soggetto" è un termine che storicamente è stato impiegato per intendere cose assai diverse, a volte consciamente, più spesso inconsapevolmente; esso è a volte stato impiegato in modo residuale come "nozione ombrello" per tutto ciò che non ricadeva o non ricadeva nettamente nell'ambito della catalogazione per autore. ${ }^{67}$

Il soggetto è un concetto che ha ricevuto molteplici definizioni, tese a identificarlo con una o più tra le seguenti nozioni: tema, circalità, concetto, significato, rilevanza, contenuto, informazione, argomento, etc. Ai diversi termini e alle diverse definizioni di soggetto fanno capo diverse e anche opposte visioni della natura del soggetto; Birger Hjørland ha parlato in proposito di quattro diverse filosofie del soggetto, ${ }^{68}$ ma si tratta di

64 PATRICK WILSON, Two kinds of power. An essay on bibliographic control, Berkeley, University of California Press, 1978, p. 70-71: "If there is any obscurity in the notion of "the subject" of writings, it will infect any instrument whose rules of assignment resemble that of The Catalog. Now it seems to me that that notion is in fact a deeply obscure one, that the instruments in whose making that notion is employed are in important respects necessarily inscrutable, that in fact it is almost impossible, in such instruments, to arrive at an adequate sense of the position as to which writings are assigned [Jolley] [...] Behind the use of the definite article, "the subject," lies an apparently innocent assumption that there will be just one thing to mention in answer to the question "What is it about?" And with that assumption goes another, that though there may be many ways of describing roughly or imprecisely what the writing is about, there will be just one perfectly precise description of what it is about"; p. 89-90: "The notion of the subject of a writing is indeterminate, in the following respect: there may be cases in which it is impossible in principle to decide which of two different and equally precise descriptions is a description of the subject of a writing, or if the writing has two subjects rather than one [...] of course we can always formulate descriptions which are obviously and definitely not descriptions of what a writing is about, but we cannot expect to find the subject [...] The uniqueness implied in our constant talk of the subject is non-existent".

65 ROSS J. TODD, Academic indexing. What's it all about?, "The indexer", 18, 2 (1992), p. 101: "First, what is actually meant by the subject of a document? Rather than clarifying this concept, key writers in the field present considerable terminological confusion. Cutter defines the subject as the 'theme or topic of the resource, whether stated in its title or not'. Kaiser clarifies subjects as the 'things in general, real or imaginary, and the conditions attached to them. We shall call them concretes and processes respectively'. Ranganathan talks about the 'thought content of a document'-an assumed term; an 'isolate'. Coates identifies subject as the abstraction of the overall idea embodied in the subject content of a given literary unit. Vickery speaks of 'the theme on which books, parts of books, articles or parts of articles are written; a complex aggregate of specific aspects; a com pound of elementary terms. Borko and Bernier define subjects as the $[. .$.$] central themes toward which the attention and efforts of the author have been directed. They are$ those aspects of a work that contain novel ideas, explanations, or interpretations. And they should all be indexed.' And more recent literature appears to use the term aboutness synonymously with the term subject of a document".

66 JACQUES MANIEZ, Actualité des langages documentaires. Les fondements théoriques de la recherche d'information, Paris, ADBS, 2002, p. 140.

67 Cfr. DORALYN J. HICKEY, Subject analysis. An interpretive survey, "Library trends”, 25, 1 (1976), p. 275. 68 BIRGER HJØRLAND, The concept of 'subject' in information science, "Journal of documentation", 48, 2 (1992), p. 172-200. Cfr. anche BIRGER HJØRLAND, Information retrieval, text composition, and semantics, “Knowledge organization”, 25, 1/2 (1998), p. 20, in cui l'autore individua quattro possibili accezioni di significato: significati come costrutti individuali (idealista/individuale), significati come referenti del mondo (realista/individuale), significati come costrutti sociali (idealista/sociale), significati come scoperte umane stabilizzate nella lingua e nella cultura (realista/sociale). Cfr. anche TERESA GRIMALDI, 025.47.

Catalogazione per soggetto (Teoria dell'indicizzazione semantica), in Biblioteconomia. Guida classificata, diretta da Mauro Guerrini, condirettore Gianfranco Crupi, a cura di Stefano Gambari, collaborazione di Vincenzo 
Tesi di dottorato di Giuliano Genetasio, discussa presso l'Università degli Studi di Udine

una semplificazione.

Il soggetto è stato concepito come qualcosa rilevabile in modo più o meno oggettivo, ${ }^{69}$ oppure come qualcosa di ineludibilmente soggettivo (ogni individuo si approccia a un testo con differenti conoscenze pregresse, punti di vista, aspettative e obiettivi), ${ }^{70}$ relativo (per esempio, ciò che è considerabile un soggetto per una data disciplina può non esserlo per un'altra $)^{71}$ e interpretativo. ${ }^{72}$ Il soggetto è stato concepito ora come tema, come insieme delle "cose" di cui parla il testo; ${ }^{73}$ ora come insieme dei concetti suscitati nella mente del lettore, come significato che il testo assume di volta in volta per l'utente finale ${ }^{74}$ ovvero come uso potenziale del testo in un documento in un determinato contesto. ${ }^{75}$ Il soggetto è stato pensato sia come entità presente nel testo, preesistente l'utente finale e che starebbe all'indicizzatore scoprire o estrarre, sia come relazione che si instaura e vive solo nel momento della mediazione indicale. ${ }^{76}$ Il soggetto è stato visto

Fugaldi, presentazione di Luigi Crocetti, Milano, Editrice Bibliografica, 2007, p. 625, che riguardo all'indicizzazione per soggetto afferma: "Se manca una teoria generale di questa complessa attività intellettuale, essa è stata oggetto di numerose interpretazioni, spesso conflittuali, in relazione alle diverse concezioni filosofiche della conoscenza assunte implicitamente come base per la determinazione del concetto di significato".

69 SAMUEL D. NEILL, The dilemma of the subjective in information organisation and retrieval, "Journal of documentation", 43, 3 (1987), p. 195.

70 Cfr. TERESA GRIMALDI, L'indicizzazione dal punto di vista cognitivo, "Il bibliotecario", 12, 1 (1995), p. 292-293; BRENDA DERVIN, Useful theory of librarianship. Communication, not information, "Drexel library quarterly", 13, 3 (1977), p. 25: "To cope with individuality one must also deal with situationality. It is also suggested that the only way to deal with situationality is through the eyes of a given individual [...] Meanings are in people. No message means the same thing to different people. No situation is seen the same by different people. The same message read on two different days will not mean the same thing to the same person"; SAMUEL D. NEILL, The dilemma of the subjective in information organisation and retrieval, cit., p. 197; ROSS J. TODD, Academic indexing, cit., p. 102.

71 JAMES D. ANDERSON - JOSÉ PÉREZ-CARBALLO, The nature of indexing. How humans and machines analyze messages and texts for retrieval. Part I, Research, and the nature of human indexing, cit., p. 243.

72 JENS-ERIK MAI, Semiotics and indexing. An analysis of the subject indexing process, "Journal of documentation", 57, 5 (2001), p. 619-620.

73 Cfr. CHARLES A. CUTTER, Rules for a dictionary catalog, cit., p. 23; cfr. anche INTERNATIONAL ORGANIZATION FOR STANDARDIZATION, International standard ISO 5963. Documentation. Methods for examining documents, determining their subjects, and selecting indexing terms, Géneve, ISO, 1985, p. 1, che concepisce il soggetto come concetto o combinazione di concetti che rappresetino un tema all'interno di un documento.

74 ALFREDO SERRAI, Del catalogo alfabetico per soggetti. Semantica del rapporto indicale, Roma, Bulzoni, 1979, definisce il soggetto come "il concetto che il discorso suscita nella mente dell'interprete" (p. 61), e come "il significato che un documento possiede per chi lo utilizza o utilizzerebbe" (p. 11). BIRGER HJØRLAND, Information seeking and subject representation. An activity-theoretical approach to information science, Wetsport, Conn., London, Greenwood, 1997, p. 37, riprende, a proposito del soggetto, la nozione gadameriana del significato di un testo come insieme delle interpretazioni passate, presenti e future (e cioè effettive e possibili) all'interno di un orizzonte ermeneutico. Cfr. anche PETER INGWERSEN - IRENE WORMELL, Means to improved subject access and representation in modern information retrieval, "Libri", 38, 2 (1988), p. 108: "The purpose of indexing should not be to provide summarisation of text [...] but rather ought to provide readers with points of contact, leading users from what they know to what they whish to learn".

75 Cfr. CLARE THORNLEY - FORBES GIBB, Meaning in philosophy and meaning in information retrieval (IR), "Journal of documentation", 65, 1 (2009), p. 139-140.

76 Secondo PINO BUIZZA - MAURO GUERRINI, Un modello concettuale per il Nuovo soggettario, cit., p. 330, "Il soggetto appartiene al mondo reale in quanto elaborazione concettuale dell'indicizzatore, il quale rappresenta il contenuto dell'opera in modo sommario e formalizzato. Il soggetto non è un'entità presente 
come valutabile in termini di rispecchiamento della realtà del testo (soggetto come insieme delle affermazioni vere riguardo a un testo), oppure come valutabile in termini di pregnanza, di utilità per l'utente finale; parimenti, la stessa attività di indicizzazione per soggetto è stata concepita dai primi come essenzialmente rappresentativa e descrittiva, dai secondi come essenzialmente decisoria e strumentale. ${ }^{77}$ Il soggetto è stato pensato come argomento di un testo così come dichiarato dai suoi contrassegni formali e innanzitutto dal titolo, in quanto espressione del pensiero dell'autore, ${ }^{78}$ oppure come l'argomento di un testo anche a prescindere dai suoi contrassegni formali e dall'intenzione dell'autore stesso di evidenziarne un aspetto specifico. Il soggetto è stato concepito come elemento

nell'opera e da essa estratta, né un'entità precostituita che esiste di per sé. Esiste come nucleo concettuale informativo creato in funzione di mediazione fra il pensiero svolto nell'opera e l'universo dei discorsi culturali e informativi che danno luogo alle richieste delle interrogazioni bibliografiche"; cfr. anche TERESA GRIMALDI, 025.47. Catalogazione per soggetto (Teoria dell'indicizzazione semantica), cit., p. 627: "Il concetto di significato non va inteso come un'entità oggettiva esistente nei documenti ed estraibile dall'indicizzatore, ma come qualcosa che si realizza nell'atto dell'interpretazione svolta in vista di precise finalità d'uso"; cfr. anche PINO BUIZZA, 025.4 Analisi e controllo per soggetto, in Biblioteconomia. Guida classificata, diretta da Mauro Guerrini, condirettore Gianfranco Crupi, a cura di Stefano Gambari, collaborazione di Vincenzo Fugaldi, presentazione di Luigi Crocetti, Milano, Editrice Bibliografica, 2007, p. 568: "Il significato non si può intendere come un dato oggettivo, ipostatizzato nel testo, da scovare ed estrarre, ma è, dinamicamente e secondo il contesto, ciò che viene ricevuto e recepito e provoca reazioni nella persona che accosta il testo: appartiene alla sua realtà esistenziale ed è quindi potenzialmente diverso da utente a utente [...] la lingua, altro che semplice combinazione di forme lessicali unitarie con cui contrassegniamo oggetti, è un sistema semantico precostituito al parlante, dotato di categorizzazioni logiche, presupposizioni metafisiche, etiche, assiologiche [...] Nella comprensione di un testo stanno dunque in tensione dialettica la soggettività dell'utente e la mentalità condivisa”. Cfr. anche JAMES D. ANDERSON - JOSÉ PÉREZ-CARBALLO, The nature of indexing. How humans and machines analyze messages and texts for retrieval. Part I, Research, and the nature of human indexing, cit., p. 240. Cfr. anche W. JOHN HUTCHINS, Languages of indexing and classification. A linguistic study of structures and functions, Stevenage, Peregrinus, 1975, p. 107.

77 YVES GILBERT COURRIER, Document analysis, verbs and case grammar, Pittsburg, University of Pittsburg, 1976, p. 59: "No written document is absolutely univocal and any condensed representation of it is one choice - among other possible choices - which has been made for communication in a specific context"; cfr. anche DAVID C. BLAIR, Language and representation in information retrieval, Amsterdam, New York, Elsevier Science Publishers, 1990, p. 136-138; cfr. anche JAMES D. ANDERSON - JOSÉ PÉREZCARBALLO, The nature of indexing. How humans and machines analyze messages and texts for retrieval. Part I, Research, and the nature of human indexing, cit., p. 251: "The assignment of a term to a document is justified if the average utility associated with that assignment is positive, and unjustified if it is negative". Cfr. anche TERESA GRIMALDI, 025.47. Catalogazione per soggetto (Teoria dell'indicizzazione semantica), cit., p. 626: "L'indicizzazione per soggetto implica la decisione su ciò intorno a cui verte l'opera, e quindi sulle ragioni per cui essa possa essere di interesse per una determinata categoria di utenti. La stessa opera potrebbe essere indicizzata in modi piuttosto differenti in centri di informazione diversi, e dovrebbe essere indicizzata in modi differenti se i gruppi di utenti sono interessati all'opera per ragioni diverse [...] Ė necessario tener conto anche delle considerazioni extra-testuali e pragmatiche: chi userà il testo e per quale fine; a quale esigenza di ricerca l'autore ha inteso finalizzare il suo messaggio; per quale utente la biblioteca ha acquisito l'opera. Per creare o assegnare dei topici a un testo dobbiamo considerarlo nel più ampio contesto dei presumibili utenti che lo useranno, degli altri testi di cui gli utenti si servono, del modo in cui i testi dipendono l'uno dall'altro". Cfr. anche MARIA BRUNA BALDACCI, Rappresentazione e ricerca delle informazioni, cit., p. 18: "Non si può dunque parlare della rispondenza della rappresentazione all'oggetto rappresentato. Ha senso invece indagare se la rappresentazione risponde alla finalità per la quale è stata creata: comunicare dell'oggetto informazioni utili a scopi definiti”. Cfr. anche GARY STEELE, The wisdom of the cataloguers. Using collective intelligence to assign Library of Congress subject headings, 2010, disponibile 
permanente e atemporale ${ }^{79}$ oppure come elemento storico, esposto ai cambiamenti. ${ }^{80}$ Infine, ma la lista di queste contrapposizioni potrebbe continuare pressoché infinitamente, il soggetto è stato pensato come qualcosa di tendenzialmente definibile in termini certi e unici ("il" soggetto di un testo) - siamo nell'ambito di questa posizione unicista anche quando ammettiamo che "il" soggetto possa di volta in volta essere di natura e origini diverse, ovvero appartenere a una tra le diverse contrapposizioni suddette ,$-{ }^{81}$ oppure come qualcosa di tendenzialmente indefinito e plurale ("i" soggetti di un testo). Come comportarsi di fronte alla molteplicità di queste concezioni del soggetto, di fronte alla profondità delle contrapposizioni da esse implicate? È possibile scegliere una tra le alternative in gioco, o magari anche solo un gruppo di alternative? O piuttosto le contrapposizioni in gioco sono tali da costituire uno scacco per qualsivoglia tentativo di costruire una teoria soddisfacente del soggetto? Per Thornley e Gibb la nozione di soggetto sarebbe intrinsecamente aporetica e le opposizioni in essa implicate di carattere dilemmatico, cioè irresolubili. ${ }^{82}$

\subsubsection{Antinomie del soggetto: pragmatismo e oggettivismo del soggetto}

Se accettassimo la posizione di Thornley e Gibb, la nostra discussione sul soggetto finirebbe qui. Noi proveremo invece a scegliere tra le varie alternative concettuali in

all'indirizzo <http://www.iskouk.org/conf2011/papers/steele.pdf>, p. 6: "When discussing LCSH most commentators take the objective epistemological position that there is an absolute, definable reality that exists independently of any cataloguer [...] For me, the problem lies not in judging the truth or falsehood of a heading, but rather in judging if the heading is the "right" one for the situation. The art of subject heading assignment lies in finding the "fittest" heading for a particular item [...] the correct heading is the one that fits the criteria of appropriate application. Thus it is more useful to discuss LCSH in degrees of appropriateness rather than the dichotomy true or false".

78 Una nota sui titoli. Il titolo può essere un'espressione della volontà dell'autore, più spesso è invece concordato tra autore ed editore. Il soggetto enunciato dal titolo spesso non coincide per estensione o per argomenti con l'effettivo contenuto del testo: si danno spesso casi di titoli che indicano un argomento più generale di quello effettivamente trattato nel testo, con lo scopo di dare un respiro più ampio al testo, di estenderne l'interesse. Viceversa, la condizione di un titolo il cui soggetto corrisponda effettivamente a una sola parte del testo può avere principalmente due origini: nel caso di raccolte, di un singolo autore o di più autori (e rientrano pienamente in questa casistica i saggi raccolti sulla base di un progetto di ricerca, o gli atti di convegni), il titolo tende a evidenziare una singola tematica (nel caso di convegni) o un singolo saggio incluso nella raccolta (quello che l'autore ha ritenuto più importante); per le raccolte di più autori, la scelta della tematica o del saggio da evidenziare è spesso del curatore. Nel caso di saggi e monografie scientifiche o tecniche, capita spesso che il titolo voglia evidenziare la tesi sostenuta dall'autore o i capitoli più importanti del testo (il rema a cui abbiamo fatto riferimento), tralasciando di rappresentare il resto. Per opere di carattere generale, il titolo può rappresentare il tema, il rema o entrambi; capita anche che il titolo rappresenti il rema, e il sottotitolo il tema, o viceversa. In una raccolta, capita anche che il titolo rappresenti un saggio, e il sottotitolo un altro saggio. Il sottotitolo si configura in alcuni casi cioè come un vero e proprio secondo titolo del testo.

79 Cfr. ROSS J. TODD, Academic indexing., cit., p. 102.

80 BIRGER HJØRLAND, Information seeking and subject representation, cit., p. 74.

81 Cfr. per esempio la posizione di JOHN WALLACE METCALFE, Tentative code of rules for alphabeticospecific entry, in Subject classifying and indexing, by John Wallace Metcalfe, New York, Scarecrow, 1959, p. 278, che rifiuta un'unica concezione del soggetto ma continua apparentemente a pensare a un unico soggetto. 82 CLARE THORNLEY, Dilemmas in information science (IS) and information retrieval (IR). Recurring challenges or new solutions?, "Aslib proceedings", 61, 3 (2009), p. 323-330; cfr. anche CLARE THORNLEY - FORBES GIBB, A dialectical approach to information retrieval, "Journal of documentation", 63, 5 (2007), p. 755-764. 
gioco, o almeno una parte di esse.

Sintetizziamo e precisiamo le varie antinomie del soggetto a cui abbiamo sopra fatto riferimento: 1) tesi: oggettività del soggetto; antitesi: soggettività e relatività del soggetto;

2) tesi: soggetto come insieme degli argomenti trattati esplicitamente da un testo; antitesi: soggetto come significato che il testo assume per l'utente; 3) tesi: indicizzazione per soggetto come rappresentazione fedele del contenuto di un testo; antitesi: indicizzazione per soggetto come creazione di strumenti utili per l'utente; 4) tesi: soggetto come derivato dai contrassegni formali del testo, innanzitutto dal titolo; antitesi: soggetto come dipendente dal contesto d'uso del testo, e relativamente indipendente dai contrassegni formali del testo; 5) tesi: esiste "il" soggetto di un testo, e il problema è trovarlo; antitesi: un testo ha potenzialmente più soggetti, ovvero può interessare gli utenti sotto più aspetti; 6) tesi: soggetto come entità presente nel testo; antitesi: soggetto come relazione tra testo e argomenti che esso implica, stabilita dall'indicizzatore in funzione dell'interesse dell'utente; 7) tesi: soggetto come elemento permanente e atemporale; antitesi: soggetto come elemento dinamico e storico.

Se guardiamo ad esse, notiamo che sono tutte collegate tra loro più o meno fortemente. In alcuni casi il legame tra le contrapposizioni è molto forte, per esempio tra la prima e la seconda contrapposizione, che potrebbero essere considerate perfino un'unica contrapposizione; in altri il legame è più debole. Le varie tesi da una parte, e le varie antitesi dall'altra, concorrono a formare due diverse visioni o filosofie del soggetto. Intuitivamente, e senza aspirare a un'esattezza assoluta, i tipi di problemi implicati dalle tesi sono di natura conoscitiva; quelli implicati dalle antitesi sono relativi ad azioni e decisioni. Le tesi delineano una visione fondamentalmente oggettivista e, nel caso delle antinomie 5, 6 e 7 (che non riguardano tanto la questione della conoscenza del soggetto quanto piuttosto quella della sua realtà), realista ed essenzialista, del soggetto; parleremo in questo caso, e solo ai fini di una semplificazione terminologica, di oggettivismo (del soggetto). Le antitesi delineano una visione pragmatista, relativista e, nel caso della settima antinomia, storicista del soggetto; parleremo in questo caso, solo ai fini di una semplificazione terminologica, di pragmatismo (del soggetto). L'oggettivismo del soggetto è forse la posizione più antica - possiamo far risalire i suoi riferimenti filosofici al positivismo ottocentesco, oltre che al neopositivismo novecentesco - e per lungo tempo è stata anche la più accettata nella teoria dell'indicizzazione per soggetto. Secondo l'oggettivismo il soggetto coincide essenzialmente con l'argomento di cui parla esplicitamente un testo, così come rilevabile con un ragionevole certezza dai suoi contrassegni formali (titolo, etc.). L'indicizzazione è qui concepita essenzialmente come processo di sommarizzazione del contenuto concettuale del testo e, per attuarlo, l'indicizzatore non dovrebbe fare altro che "estrarre", cioè scegliere, alcuni termini tratti dai contrassegni formali. La fedeltà di rispecchiamento del testo è posta dall'oggettivismo a garanzia dell'universalità e assolutezza del soggetto così individuato. L'indicizzazione che ne conseguirebbe sarebbe perciò valutabile tramite semplici confronti intertestuali: 1) il testo primario (il soggetto del Testo) confrontato con il testo secondario (indici), al fine di verificarne la fedeltà di rispecchiamento; 2) i testi secondari confrontati tra loro (indicizzazione di uno stesso testo o di testi simili) al fine di verificarne la coerenza quella della coerenza tra gli indicizzatori, o "inter-indexer consistency", è stata per decenni una delle tematiche più dibattute nella letteratura di taglio oggettivista.

Il pragmatismo del soggetto è una posizione più recente, i cui riferimenti filosofici 
possiamo far risalire al pragmatismo filosofico di fine Ottocento, e per molto tempo minoritaria nella teoria dell'indicizzazione per soggetto; essa ha negli ultimi decenni acquisito una maggiore forza; per esempio, è la posizione oggi dominante nell'ambito del movimento dell'organizzazione della conoscenza (“Knowledge organization”). Secondo il pragmatismo il soggetto di un testo coincide essenzialmente con il significato o i significati (il plurale è importante) che un testo può rivestire per l'utente finale; esso non sarebbe semplicemente rilevato o estratto, ma sarebbe frutto di processi analitici e decisionali che partono sì dai contrassegni formali di un testo, ma che poi se ne discostano per guardare alle intenzioni del testo e all'interesse che i vari concetti trattati o implicati dal testo possono rivestire per i diversi utenti finali. Il pragmatismo parte infatti dall'assunto che le esigenze degli utenti differiscono e che un testo possa essere usato in diversi momenti, in diversi contesti e per diversi scopi. Un testo non avrebbe un unico soggetto ("il" soggetto dell'oggettivismo), ma tanti soggetti quante sono le prospettive sotto cui esso può essere utile: teoricamente infinite - Blair ha su questa base ripreso la nozione peirciana di semiosi infinita,$-{ }^{83}$ praticamente selezionate sulla base di una valutazione (soggettiva e intuitiva) delle probabilità che un testo sia utile per una determinata prospettiva - Hjørland ha parlato a questo proposito di potenziale epistemologico di un documento o messaggio; ${ }^{84}$ valutazione ipotetica che, proprio perché nata in determinati contesti e guardando alle esigenze di determinati tipi di utenti, non potrebbe in ogni caso aspirare né all'universalità, né all'esaustività, né a una validità assoluta nel tempo. ${ }^{85}$

È possibile prendere posizione a favore dell'oggettivismo o del pragmatismo del soggetto? Secondo noi sì, fare una scelta fondamentale tra queste due visioni del soggetto è possibile e anzi necessario. La nostra scelta è a favore del pragmatismo, ma è una scelta l'esposizione delle due diverse posizioni era un artificio necessario per spiegare la nostra posizione - che avevamo già preso quando abbiamo posto a cardine del nostro modello l'interesse dell'utente e le sue esigenze, quando abbiamo detto che gli obiettivi dell'indicizzazione sono funzionali a quelli del recupero dell'informazione e quando

83 DAVID C. BLAIR, Language and representation in information retrieval, cit., p. 136-138.

84 BIRGER HJØRLAND, Theory and metatheory of information science. A new interpretation, "Journal of documentation", 54, 5 (1998), p. 610: "The subject of a book (or any other document, or message) is closely related to what kind of answers people can find from reading the book. Different people put different questions in different situations and one single document can in principle answer an infinite number of questions. I define the subject of a document (or of any message or sign for that matter) as the epistemological potentialities of that document".

85 JENS-ERIK MAI, Semiotics and indexing, cit., p. 606: "It would be nearly impossible, of course, for any single person or, in this case, any single indexer, to determine all of the ideas and meanings which might be associated with any particular document, since there might always be potential ideas and meanings which different people at different times and places might find in the document. Furthermore, it would be well nigh impossible to predict precisely which of the many possible ideas and meanings that could be associated with the document would be specifically valuable to the users or would have some sort of lasting value for the document. To recognise and accept this fundamental openness is of utmost importance. The indexer must realise from the start that he or she will never discover all the ideas and meanings that could be associated with the document and that, therefore, it is not possible to describe all these ideas and meanings”. Cfr. anche TERESA GRIMALDI, 025.47 Catalogazione per soggetto (Teoria dell'indicizzazione semantica), cit., p. 627: "Il compito dell'indicizzatore è di rappresentare la conoscenza registrata in vista dei motivi, dei progetti e delle circostanze che determinano le esigenze informative degli utenti [...] l'individuazione del soggetto è un'attività intellettuale che ha il carattere dell'ipotesi sulla valenza informativa attuale e futura dei discorsi documentari”. 
abbiamo posto che tutti gli altri aspetti del nostro modello sono stati concepiti su questa base: nell'utente e nelle sue esigenze, oltre e più ancora che nei Testi, va secondo noi cercata la ragione ultima del soggetto e della sua formulazione. Accettiamo pienamente la prospettiva pragmatista assunta da Robert A. Fairthorne, quando afferma incisivamente che il soggetto di un testo "dipende dagli usi che ne farà il suo lettore, oltre che da ciò che l'autore dice. Qualsiasi persona può scrivere qualsiasi cosa su ogni cosa, e qualsiasi lettore nel futuro potrà usarla per qualsiasi altra cosa" ${ }^{86}$

La scelta a favore del pragmatismo non è tuttavia di natura assoluta: riteniamo cioè che, pur privilegiando la prospettiva pragmatista, sia in via di principio auspicabile l'assunzione di almeno alcuni aspetti o elementi della visione oggettivista. Le tesi più estreme dell'oggettivismo, o meglio quelle che costituiscono una degenerazione dell'oggettivismo, vanno senz'altro scartate. L'unicismo del soggetto - l'idea ingenua o peggio consapevole che sia possibile trovare o formulare "il" soggetto di un testo - va senz'altro rifiutato, così come va rifiutata la concezione essenzialista e innatista del soggetto come entità da estrarre. ${ }^{87}$ Il giudizio di Patrick Wilson sull'unicismo del soggetto è netto, e lo condividiamo appieno: "l'unicità implicata costantemente nella nostra discussione del soggetto è inesistente". Secondo W. John Hutchins: "non dovremmo mai parlare 'del' soggetto di un documento [...] i giudizi sul soggetto (da parte di autori, lettori e indicizzatori) sono influenzati da una tale quantità di fattori che nessuna formulazione particolare del soggetto di un documento non dovrebbe essere considerata altro che soltanto una tra le molteplici formulazioni possibili" ${ }^{88}$ Rifiutare l'unicismo del soggetto in un'ottica pragmatista comporta da un lato il riconoscimento della pluralità, e forse anche infinità del soggetto: riallacciandoci a Blair potremmo parlare di "soggettualità infinita" di un Testo - con l'avvertimento che il riconoscimento che un testo possa avere infiniti soggetti non significa che possa averli tutti $-;^{89}$ dall'altro la consapevolezza della necessaria finitudine della rappresentazione del soggetto. L'indicizzazione viene a delinearsi come ricerca perenne dell'equilibrio tra la realtà trascendente e infinita del soggetto e sua rappresentazione indicale, necessariamente finita e dunque imperfetta. ${ }^{90}$ Secondo Rafael Capurro, che riprende il rapporto tra Essere e linguaggio posto da Martin Heidegger, il soggetto si nasconderebbe nelle sue rappresentazioni, piuttosto che rivelarsi in esse. ${ }^{91}$

86 ROBERT A. FAIRTHORNE, Content analysis, specification, and control, "Annual review of information science and technology", 4 (1969), p. 84.

87 Cfr. ALBERTO PETRUCCIANI, I fondamenti semantici della catalogazione per soggetto, "Accademie e biblioteche d'Italia" 50, 3 (1982), p. 219: "La natura dell'indice catalografico è oscura. Per molto tempo lo si è creduto un'essenza nascosta nel documento che qualche alchimia appresa con lungo tirocinio avrebbe dovuto permettere di estrarre".

88 PATRICK WILSON, Two kinds of power. An essay on bibliographic control, Berkeley, University of California Press, 1978, p. 90; W. JOHN HUTCHINS, Languages of indexing and classification. A linguistic study of structures and functions, Stevenage, Peregrinus, 1975, p. 116.

89 Del Fedone di Platone si può dire che è un testo che parla dell'immortalità dell'anima, o di Socrate, o semplicemente di filosofia e altre cose ancora; non si può però dire, per fare alcuni esempi, che esso parli di industrie o di nazismo. Cfr. UMBERTO ECO, I limiti dell'interpretazione, 2. ed., Milano, Bompiani, 1995, p. 23-24.

90 BIRGER HJØRLAND, Theory and metatheory of information science, cit., p. 610: "Because a document does have an infinite number of subjects, the process of subject analysis is a process of giving priority to those subjects which best serve the needs of the users of the information system in question. This view of subject matter differs basically from mainstream approaches in IS”.

91 RAFAEL CAPURRO, Hermeneutics facing the 'information framing', "Ubiquity", 9, 8 (2008), disponibile all'indirizzo <http://ubiquity.acm.org/article.cfm?id=1361368>: "Written as well as spoken logos never 
Quanto alla settima antinomia, va accantonata definitivamente l'idea che il soggetto possa essere qualcosa di permanente, statico e indipendente dai cambiamenti storici e culturali. Il soggetto è intrinsecamente storico, perché storiche sono le realtà documentarie e già gli stessi saperi e culture in quelle realtà riflesse. Soggetti e indici utilizzati per esprimerli riflettono lo stato della conoscenza di una data collettività in un dato momento storico. La terminologia utilizzata per esprimere i soggetti può divenire obsoleta nel tempo, oppure subire slittamenti di significato, o ancora divenire pregiudiziale ("negro" diventa solo da un certo momento in poi un termine razzista). Ciò che "è" soggetto in una data epoca può non esserlo per un'altra, perché cambiamenti paradigmatici nella visione del mondo hanno reso irrilevanti determinati temi, problemi, etc. o viceversa i testi possono assumere nuovi soggetti se analizzati nell'ottica di temi e problemi nati di recente. Per fare degli esempi estremi: ciò che nella Germania degli anni Trenta era un libro sugli ebrei è oggi una fonte per lo studio dell'ideologia nazista; ciò che ieri era un libro di viaggio è oggi letteratura (Il milione). Se va senz'altro accettata l'idea che l'indicizzazione, oltre a essere un'attività strumentale e decisoria, sia anche un'attività descrittiva - le due cose non si escludono necessariamente a vicenda -, va però rifiutata l'idea che tale descrizione possa essere oggettiva o del tutto neutra. Innanzitutto: neutrali rispetto a cosa e a chi? La neutralità rispetto a ciò che dice un autore, per esempio, non coincide necessariamente con la neutralità rispetto alla realtà. La menzogna, l'errore e l'ironia sono tutti elementi che possono essere presenti in un testo e creare un conflitto tra affermazioni dell'autore e realtà. In questi casi, perseguire la neutralità nei confronti delle asserzioni dell'autore significherebbe formulare soggetti falsi e ostacolare l'utente finale, piuttosto che aiutarlo. Anche a voler seguire sempre la realtà, in caso di conflitti, il problema è che non esistono punti di vista assoluti da cui guardare la realtà: pensare il contrario significa soltanto esporsi al rischio di assumere un particolare punto di vista senza esserne consapevoli, spesso quello dominante in un dato contesto. E ciò si ricollega alla storicità del soggetto: una formulazione di soggetto neutrale rispetto a ciò che è accettabile oggi può essere parziale rispetto a ciò che era accettabile ieri o che sarà accettabile domani. Scartando dunque la possibilità di una descrizione obiettiva e del tutto neutra, dobbiamo riconoscere che la formulazione del soggetto di un testo è piuttosto una descrizione strumentale (secondo determinati fini) e relativa (secondo determinati punti di vista). Abbiamo finora fatto riferimento alla maggior parte delle "antinomie del soggetto", tranne alla prima e alla seconda, tra loro strettamente connesse. Esse sono senza dubbio le più critiche, le uniche rispetto alle quali non prenderemo una posizione netta. Per quanto riguarda la seconda antinomia: l'indicizzazione è un processo oggettivo o soggettivo? Pur privilegiando la posizione pragmatista, e riconoscendo dunque la natura essenzialmente relativa ed ermeneutica dell'indicizzazione, riteniamo che sia se non altro possibile distinguere gli elementi totalmente soggettivi dell'indicizzazione, frutto di intuizioni, congetture e scelte, da quelli solo parzialmente soggettivi, ovvero maggiormente accertabili. Per quanto riguarda la prima antinomia: il soggetto di un testo è l'insieme degli oggetti di cui parla, a cui fa riferimento esplicito (oggettivismo), oppure l'insieme dei concetti suscitati nel lettore (pragmatismo)? Anche in questo caso, pur privilegiando la posizione pragmatista, occorre riconoscere le ragioni della posizione oggettivista e, piuttosto che operare una scelta netta tra le due opzioni tra loro incompatibili, accettarle entrambe come parte del soggetto. Ripartiremo da qui per

comes to an end, can never be definitively fixed once and for all. It conceals itself in its re-presentations”. 
Tesi di dottorato di Giuliano Genetasio, discussa presso l'Università degli Studi di Udine

(de)costruire la nostra nozione di soggetto.

\subsubsection{Verso una visione pragmatista e complessa del soggetto: circalità estensionale e intensionale}

Il soggetto è inestricabilmente costituito di significati posti esplicitamente da un testo e di significati che quel testo assume per l'utente finale. A differenza di Thornley e Gibb, che parlano di due concezioni dilemmatiche del soggetto, noi suggeriamo piuttosto di vedere significati espliciti ed assunti come due aspetti compresenti e anzi essenzialmente complementari del soggetto. La distinzione è tra una concezione estensionale e intensionale del soggetto. ${ }^{92}$

Apriamo una breve parentesi. Nozione strettamente correlata, anzi sostanzialmente sinonimica, a quella di soggetto, è la nozione di circalità. "Circalità" (“aboutness") è un termine che compare a partire dalla fine degli anni sessanta ma si diffonde soprattutto a partire dalla metà degli anni Settanta per indicare l'insieme dei significati di un Testo; tra i primi proponenti del concetto possiamo annoverare Fairthorne, M. E. Maron, Hutchins e D. F. Swift-V. Winn-D. Bramer. ${ }^{93}$

Partendo da un lato dalla contrapposizione tra piano estensionale ed intensionale del soggetto, e dall'altro dalla nozione di circalità, Fairthorne ha sviluppato le nozioni di circalità estensionale ("extensional aboutness") e intensionale ("intensional aboutness"). ${ }^{94}$ La circalità estensionale è l'insieme degli argomenti a cui fa espresso riferimento un dato

92 L'estensione di un termine è l'insieme degli oggetti a esso riferibili o che esso comprende (denotati). L'intensione di un concetto l'insieme delle caratteristiche, degli elementi concettuali (connotati) che costituiscono il concetto stesso. I termini assumono una particolare accezione nell'indicizzazione, cfr. ALBERTO PETRUCCIANI, I fondamenti semantici della catalogazione per soggetto, cit., p. 221-222: "nel campo della catalogazione semantica l'alternativa è tra una garanzia estensionale, data dall'enumerazione delle cose di cui si parla, e una garanzia intensionale, ricercata nell'aderenza ai significati che il discorso convoglia [...] La tesi estensionale prospetta l'elusione della complessità delle reti di significati tramite un inventario di cose o una mappa che permetta di ridurre i discorsi ai loro agganci al reale [...] Il linguaggio d'indicizzazione avrebbe uno status semantico inferiore a quello del linguaggio dei documenti, ed è quindi presupposta la possibilità di una riduzione [...] dei discorsi alla loro componente referenziale o fattuale. Possiamo parlare di una riduzione empiristica perché essa esibisce i tratti caratteristici dei programmi del Circolo di Vienna e dell'empirismo logico [...] Dal punto di vista semantico la tesi estensionale consiste nella scelta di un linguaggio che enumeri le cose di cui si parla, ciò su cui l'autore fornisce e il lettore ricerca informazione, in termini il più possibile neutri, che non sfaccettino l'oggetto secondo una prospettiva di interesse e non lo inquadrino in un corpo di conoscenze o in una particolare concezione del mondo. Sul piano terminologico ciò significa ricorrere in primo luogo al vocabolario dei nomi propri, e successivamente a termini che abbiano una estensione saldamente delimitata e su cui non pesino eccessive presupposizioni intensionali [...] La tesi intensionale richiede invece un ampliamento dei livelli semantici del sistema d'indicizzazione: il linguaggio d'indicizzazione deve essere un metalinguaggio semantico [...] che assuma a proprio oggetto i discorsi contenuti nei documenti e renda conto di ciò che con essi e in essi si vuol dire". 93 ROBERT A. FAIRTHORNE, Content analysis, specification, and control, cit.; W. JOHN HUTCHINS, The concept of 'aboutness' in subject indexing, "Aslib proceedings", 30, 5, p. 172-181; D. F. SWIFT - V. WINN D. BRAMER, 'Aboutness' as a strategy for retrieval in the social sciences, "Aslib proceedings", 30, 5 1978, p. 182-187.

94 Cfr. ALFREDO SERRAI, Del catalogo alfabetico per soggetti, cit., p. 71; cfr. anche JAMES D.

ANDERSON - JOSÉ PÉREZ-CARBALLO, The nature of indexing. How humans and machines analyze messages and texts for retrieval. Part I, Research, and the nature of human indexing, cit., p. 240. 
testo, ciò che è menzionato dal testo; la circalità intensionale l'insieme dei significati implicati da quegli argomenti e dalla loro trattazione, ovvero le circostanze del possibile impiego del testo in riferimento: a) a un particolare compito o problema; b) un particolare utente o agente; c) un requisito espresso dal lettore. ${ }^{95}$ A volte circalità estensionale e intensionale possono coincidere (per esempio nelle introduzioni, nei manuali, etc.), più spesso differiscono perché il significato complessivo di un testo non equivale necessariamente alla somma dei significati delle sue parti. Moby Dick, ricorda Fairthorne, è un libro su una balena, ma anche su altre cose. ${ }^{96}$ La circalità estensionale è spesso insufficiente nella definizione di un soggetto, perché una "formulazione che elenchi tutte le cose menzionate da una domanda non è necessariamente una risposta soddisfacente alla domanda" e, viceversa, la circalità intensionale non può essere desunta dal solo testo in sé, ma è necessario guardare anche all'utente finale perché "non è possibile dedurre una domanda dalla sua risposta". ${ }^{97}$

La distinzione di Fairthorne è stata ripresa, tra gli altri, da Clare Beghtol (come differenza tra circalità e significato $)^{98} \mathrm{e}$, in Italia, da Alfredo Serrai, che contrappone oggetto e soggetto. ${ }^{99}$ Corrispondente alla distinzione tra circalità estensionale ed intensionale è quella, posta da Dagobert Soergel e ripresa da Hjørland e Raya Fidel, tra indicizzazione orientata al contenuto ("content-oriented indexing", "entity-oriented indexing", "document-oriented indexing") e indicizzazione orientata alle richieste ("request-oriented indexing" o "problem-oriented indexing”). ${ }^{100}$ L'indicizzazione orientata alle richieste

95 ROBERT A. FAIRTHORNE, Content analysis, specification, and control, cit., p. 76, 80: "The distinction between intensional and extensional aboutness must be considered not only when considering the document as such, as opposed to the aggregate of sentences it contains, but also when considering a particular collection as such, as opposed to to the separate documents that compose it, and a corpus of recorded discourse as opposed to the partitular collections that form it. At each level, one depends upon the environment defined by the other, and content analysis must be conducted in this light".

96 ROBERT A. FAIRTHORNE, Content analysis, specification, and control, cit., p. 79: "Moby Dick is about a whale; Othello about a handkerchief, and about other things [...] The difficulties are to identify which of the things mentioned refer to relevant topics, and how to deal with topics of the document that are not mentioned explicitly [...] parts of a document are not always about what the entire document is about, nor is a document usually about the sum of the things it mentions. A document is a unit of discourse [...] This may be called its "intensional" aboutness, while the aboutness of its components is its "extensional" aboutness".

97 ROBERT A. FAIRTHORNE, Content analysis, specification, and control, cit., p. 79-80.

98 CLARE BEGHTOL, Bibliographic classification theory and text linguistics. Aboutness analysis, intertextuality and the cognitive act of classifying documents, "Journal of documentation", 42, 2 (1986), p. 84-85.

99 ALFREDO SERRAI, Del catalogo alfabetico per soggetti, cit., p. 61: "L'oggetto è il denotato del discorso, ossia le cose reali o immaginarie, concrete od astratte, fisiche o concettuale, delle quali il discorso tratta e alle quali si riferisce. Il soggetto è, invece, il significato del discorso, ossia il concetto che il discorso suscita nella mente dell'interprete; come tale il soggetto implica da una parte le intenzioni di chi produce il messaggio, dall'altra le reazioni di chi lo riceve".

100 DAGOBERT SOERGEL, Organizing information. Principles of data base and retrieval systems, Orlando, Academic Press, 1985, p. 227: "Request-oriented indexing (problem-oriented indexing) is a logical development o the method of anticipated queries. It contrast with entity-oriented indexing, where the focus is on the entity and its faithful description. Entity-oriented indexing is the approach most commonly used [...] However, the preceding examination of the retrieval problem shows that request-oriented indexing promises improved performance, possibly at higher cost"; cfr. inoltre BIRGER HJØRLAND, Information seeking and subject representation, cit., p. 5, BIRGER HJØRLAND, Towards a theory of aboutness, subject, topicality, theme, domain, field, content...and relevance, "Journal of the American Society for Information Science and Technology", 52, 9 (2001), p. 776; cfr. anche RAYA FIDEL, User-centered indexing, "Journal 
comporta sia la predizione alle domande che possono essere poste a un testo, sia la valutazione della possibile rilevanza del testo rispetto a ciascuna domanda. ${ }^{101}$ Maron, uno degli studiosi che ha offerto il maggior contributo al concetto di circalità, ha invece distinto tra circalità oggettiva ("objective about", corrisponde all'incirca alla circalità estensionale di Fairthorne), soggettiva e di recupero ("subjective about" e "retrieval about", insieme corrispondono approssimativamente alla circalità intensionale di Fairthorne). ${ }^{102}$ Alla distinzione elaborata da Fairthorne corrisponde invece solo parzialmente la differenza elaborata da Alberto Cheti e dal GRIS tra tema di base e tema particolare, poiché quest'ultimo comprende sia aspetti della circalità intensionale (soggetto del testo in riferimento a usi ed esigenze degli utenti) sia della circalità estensionale (soggetto di parti testuali del testo). ${ }^{103}$ Nell'elaborazione originaria di Fairthorne, la differenza tra circalità estensionale ed intensionale non è quella tra due elementi totalmente distinti e opposti, quanto piuttosto quella tra i due poli di un continuum, che va dai significati delle parti di un testo, e già dai termini a cui esso fa riferimento, al significato complessivo che il testo assume per l'utente; essa è cioè soprattutto una differenza di grado, relativa, come evidenzia Alberto Petrucciani. ${ }^{104}$ Nella nostra modellizzazione accetteremo la distinzione tra circalità estensionale e intensionale in termini di due diversi attributi esterni di un Testo, ovvero di due diverse relazioni tra un Testo e una o più entità di qualsiasi tipo. Tuttavia, e discostandoci da Fairthorne, nel nostro modello la distinzione tra circalità estensionale ed intensionale non è soltanto una differenza di grado. Noi concepiremo infatti la circalità intensionale non soltanto come l'insieme dei significati assunti da ciò di cui parla un Testo, ovvero a partire dalla circalità estensionale di un Testo, bensì come l'insieme dei significati assunti dal Testo nella sua totalità: ciò di cui parla più tutti gli altri attributi testuali.

Concepiremo cioè la circalità intensionale come insieme dei significati assunti a partire: 1) dalla circalità estensionale dell'entità testuale; 2) dagli altri attributi testuali: autore, data, destinatari, forma testuale, etc.; 3) da una loro combinazione. Un inventario dei beni di una determinata chiesa compilato nel tredicesimo secolo è, sia a causa di ciò a cui fa riferimento (i beni mobili e immobili della chiesa), sia a causa della sua data e della sua forma testuale, rilevante per lo studio della storia della Chiesa locale. L'enciclica Deus caritas est parla dell'amore cristiano e di Dio (circalità estensionale) ma, per il fatto stesso di essere scritta dal papa e di essere un'enciclica, è anche una fonte della dottrina cattolica (circalità intensionale). Nel nostro modello, dunque, la circalità intensionale indicherà non soltanto gli argomenti implicati dalla circalità estensionale (argomenti espressamente trattati) del Testo, ma più in generale l'insieme degli argomenti implicati da un Testo a partire dalla sua circalità estensionale o da altri attributi testuali. La circalità intensionale

of the American Society of Information Science", 45, 8 (1994), p. 572-574.

101 DAGOBERT SOERGEL, Organizing information, cit., p. 230-232.

102 M. E. MARON, On indexing, retrieval and the meaning of about, "Journal of the American Society for Information Science", 28, 1 (1977), p. 40-41.

103 ALBERTO CHETI, Il punto di vista del GRIS sulla 'relazione di soggetto' in FRBR, cit., p. 92-93.

104 ALBERTO PETRUCCIANI, I fondamenti semantici della catalogazione per soggetto, cit., p. 226: "In molte discipline soprattutto storiche la rete dei nomi propri particolari costituisce il principale punto di riferimento: quando vogliamo abbandonare questo livello enumerativo, ai fini di generalizzazione o anche di economia nell'indicizzazione, siamo costretti a indicare gli oggetti di cui si parla in maniera indiretta, tramite una somiglianza o un principio di riunione, un concetto sotto cui cadono, che ha necessariamente carattere intensionale". 
è allora, lo si deduce da quanto detto, strettamente correlata alle relazioni semantiche paradigmatiche (quelle gerarchiche in particolare) delle entità coinvolte a vario titolo in un Testo: la circalità intensionale è correlata tanto all'insieme delle entità che intrattengono relazioni semantiche paradigmatiche con le entità che costituiscono la circalità estensionale di un testo, quanto all'insieme delle entità che intrattengono relazioni paradigmatiche con entità che costituiscono altri attributi esterni (autori, date, luoghi, destinatari, forme testuali, etc.) dei Testi. In tal senso la circalità intensionale può essere concepita come un'amplificazione, un'accentuazione delle implicazioni di significato che scaturiscono dai diversi attributi testuali, con le rispettive relazioni semantiche paradigmatiche, e dalla loro combinazione. Dunque, ritornando alla differenza tra circalità estensionale ed intensionale, possiamo schematizzarne la differente natura dicendo che nel nostro modello la circalità estensionale è una circalità esplicita o "in partenza", quella intensionale una circalità implicita o "in arrivo". La differenza tra i due tipi di circalità non è naturalmente di una differenza assoluta, ma relativa. La differenza tra ciò che è esplicito e ciò che è implicito in un testo, infatti, non sempre è netta. Inoltre, aver assunto una posizione fondamentalmente pragmatista, come noi facciamo, comporta una concezione della circalità estensionale in cui anche questa (e non solo la circalità intensionale) è determinata a partire da una considerazione delle esigenze degli utenti e del contesto d'uso del testo, ovvero è sempre subordinata a una valutazione della rilevanza degli argomenti esplicitamente trattati nel testi. Nonostante queste necessarie riserve alla possibilità di distinguere in modo assoluto circalità estensionale ed intensionale, riteniamo fondamentalmente valida la distinzione: essa individua aspetti potenzialmente differenti del significato di un testo, e dunque dell'interesse che esso può rivestire per l'utente finale.

La differenza tra circalità estensionale e intensionale e il diverso grado di oggettività che le caratterizza ha tre importanti conseguenze a livello di indicizzazione: 1) l'indicizzazione della circalità estensionale è regolabile e valutabile in modo più oggettivo e verificabile; viceversa, l'indicizzazione della circalità intensionale resiste a ogni sistematizzazione e rimane nella sua essenza un'arte; 2) la circalità estensionale coinvolge in misura minore le soggettività di indicizzatori e utenti finali; essa è dunque anche maggiormente valida come base per un'indicizzazione utilizzabile in più contesti, mentre l'indicizzazione della circalità intensionale rimane strettamente legata alle esigenze e caratteristiche di un'utenza particolare; il perseguimento dell'uniformità dell'indicizzazione per soggetto è dunque possibile soltanto per gli aspetti estensionali della circalità, non per quelli intensionali; 3) poiché l'individuazione della circalità estensionale è basata sui termini che compaiono esplicitamente in un testo, non è azzardato pensare che la sua indicizzazione possa consistere tanto di termini assegnati dal catalogatore sulla base di una riflessione sul significato del testo, quanto di una trascrizione di parti più o meno ampie (abstract, sommari, etc. che potrebbero essere trascritti manualmente o automaticamente) del testo stesso, quanto ancora di una loro combinazione, in questo caso prevedendo magari un loro impiego differenziato, per esempio un'indicizzazione della circalità estensionale di livello generale di tipo assegnato, affiancata da un'indicizzazione della circalità estensionale di livello analitico (indicizzazione degli argomenti di capitoli o parti di un testo) di tipo derivato, cioè frutto di trascrizione.

Una nota finale sulla soggettività dell'indicizzazione. Eliminarla totalmente dall'indicizzazione non è possibile. È semmai possibile esercitare un controllo sulla 
Tesi di dottorato di Giuliano Genetasio, discussa presso l'Università degli Studi di Udine

soggettività dell'indicizzazione attraverso: a) una salda conoscenza da parte dell'indicizzatore delle esigenze e caratteristiche delle tipologie di utenti finali servite dall'indicizzazione; b) l'esplicitazione dell'indicizzatore o dell'agenzia bibliografica responsabile dell'indicizzazione, il cui nome, così come avviene per gli autori di opere letterarie o scientifiche, servirà all'utente per valutare l'autorevolezza dell'indicizzazione o per preferire uno stile di indicizzazione rispetto a un altro. ${ }^{105}$

\subsubsection{Gli elementi della circalità estensionale}

Abbiamo scomposto il soggetto negli attributi di circalità estensionale e di circalità intensionale. Dobbiamo tuttavia parlare dell'articolazione interna alla circalità estensionale.

Una delle contrapposizioni tra diverse concezioni del soggetto che non abbiamo sinora menzionato, perché non collegata all'opposizione tra oggettivismo e pragmatismo, è quella tra tema e rema. Il soggetto è stato concepito da alcuni come argomento svolto o oggetto di studio scelto dall'autore (tema o argomento in senso proprio), da altri come ciò che l'autore afferma relativamente a quel tema: tesi sostenute, osservazioni, novità apportate, etc. (rema o commento). ${ }^{106}$ Come già per la coppia circalità estensionale e intensionale, è possibile vedere tema e rema in termini di complementarietà piuttosto che di opposizione, ovvero come aspetti distinti ma entrambi importanti del soggetto, e in particolare della circalità estensionale; anche il rema è infatti, quando presente, un elemento esplicito del messaggio di un testo (non necessariamente dei suoi contrassegni formali: oltre ai titoli, che possono avere una valenza rematica, anche introduzioni, conclusioni, quarta di copertina, etc. sono importanti per l'individuazione del rema di un testo).

Tema e rema contribuiscono insieme alla circalità estensionale di un testo, ma non sono elementi di pari importanza: il rema è infatti concettualmente dipendente dal tema allo stesso modo in cui un predicato dipende da un soggetto e non ha una rilevanza autonoma per l'utente. Inoltre, mentre il tema punta direttamente a una o più entità, il rema non è concepibile semplicemente come rapporto tra due entità diverse; per questo motivo, all'interno del nostro modello il tema è un attributo esterno, il rema un attributo

105 Cfr. JAMES D. ANDERSON - JOSÉ PÉREZ-CARBALLO, The nature of indexing. How humans and machines analyze messages and texts for retrieval. Part II, Machine indexing, and the allocation of human versus machine effort, "Information processing \& management", 37, 2 (2001), p. 274.

106 W. JOHN HUTCHINS, The concept of 'aboutness' in subject indexing, cit., p. 173-174: "In any sentence [...] there are some elements which the speaker [...] assumes his hearer or reader knows of already and which he takes as "given", and there are other elements which he introduces as "new" elements conveying information not previously known [...] we may regard the "given" elements of a sentence as constituting its "theme"; and those elements expressing anything new or otherwise unpredictable (from the text or environment) as constituting its "rheme" [...] In the normal case, the "theme" precedes the "rheme" [...] It is natural to begin by saying which "given" elements are going to be talked "about" ”. Cfr. anche PINO BUIZZA, 025.4 Analisi e controllo per soggetto, cit., p. 570. Cfr. anche ELAINE SVENONIUS, The intellectual foundation of information organization, cit., p. 47. Cfr. anche PETER INGWERSEN - IRENE WORMELL, Means to improved subject access and representation in modern information retrieval, cit., p. 108: "The reader is primarily interested in the new information of documents, in the additions and changes that are made to his knowledge structures as a result of reading the text. But for the indexer, 'aboutness' must be formulated in terms of the familiar, the given framework of recorded knowledge". 
non nominale interno, implicitamente dipendente dal tema.

Vi è poi un'ulteriore articolazione interna al tema. Finora abbiamo schematizzato parlando di tema e rema, ma in effetti un testo può avere anche più temi. Questi temi possono essere tra loro in diversi rapporti; spesso vi è una relazione di subordinazione tra i diversi temi: un'Opera può presentare un tema generale, e poi articolarlo attraverso più sottotemi, oppure presentare una certa problematica, e poi trattarne anche un'applicazione particolare (casi studio, etc.).

In altri casi, invece, la relazione tra i diversi temi non è riconducibile alla subordinazione a un tema comune, ma vi possono essere due o più temi di pari importanza, anche non direttamente collegati tra loro: così, un Contenuto editoriale (per esempio una miscellanea in onore) può contenere Opere (saggi) su temi disparati; in questo secondo caso non abbiamo una relazione tra un tema e uno o più sottotemi, ma tra temi diversi di pari importanza e tra loro in relazione di coordinazione. ${ }^{107}$

\subsubsection{Punti di vista}

Un ultimo elemento (attributo) importante del soggetto di cui occorre parlare sono i punti di vista assunti da un Testo, ovvero da un autore, ${ }^{108}$ nella trattazione di un tema o argomento. I punti di vista comprendono sia le prospettive ideologiche assunte sia le particolari metodologie utilizzate per lo studio di un argomento. I punti di vista sono concettualmente dipendenti da un tema ma rispetto a esso rivestono un interesse autonomo, a differenza di quanto avviene per il rema; per l'utente finale i punti di vista possono in alcuni casi essere addirittura più importanti del tema stesso: un libro sul comunismo scritto da un neoliberista sarà rilevante per chi cerca materiale sul comunismo, ma soprattutto sarà rilevante per chi cerca materiale sul liberismo; nelle scienze sociali, il fenomeno studiato può essere spesso di secondaria importanza rispetto alla metodologia impiegata per il suo studio.

Proprio in riferimento alle scienze sociali, Swift, Winn e Bramer arrivarono a distinguere tre diversi tipi di punti di vista. ${ }^{109}$ I punti di vista sono stati considerati un importante aspetto del soggetto anche da Langridge, da Hutchins e da Hjørland, il quale ne ha

107 La relazione di coordinazione tra temi non va confusa con la relazione sintagmatica tra gli elementi componenti gli elementi lessicali in forma composta, di cui parleremo nel prossimo capitolo (vedi 3.2.8, 3.3.6.4.3.4).

108 Ovviamente, lo stesso autore può essere considerato parte del punto di vista di trattazione del soggetto, ma per la nostra modellizzazione lo considereremo un elemento distinto.

109 D. F. SWIFT - V. WINN - D. BRAMER, 'Aboutness' as a strategy for retrieval in the social sciences, cit., p. 186; cfr. anche D. F. SWIFT - V. WINN - D. BRAMER, Information systems for subject specialist. A multimodal approach to indexing and classification, Washington, D.C., distributed by ERIC Clearinghouse, 1977, disponibile all'indirizzo <http://www.eric.ed.gov/contentdelivery/servlet/ERICServlet?accno=ED139396>, p. 8: "The dimensions we have selected allow us to describe and group documents in terms of [1] theoretical orientation, [2] method of research, [3] empirical situation understudy (concern may be either description, prescription), [4] data collected and [5] form (e.g. bibliography, reader). The indexer systematically takes these as viewpoints and generates a set of title-like phrases for each document, each phrase taking a different aspect of document content as its focus but referring to the whole. The phrases associated with each dimension are presented as separate series of data in effect as separate indexes, each organised according to its own set of headings". 
Tesi di dottorato di Giuliano Genetasio, discussa presso l'Università degli Studi di Udine

sottolineato l'importanza per valutare la rilevanza di un testo; ${ }^{110}$ più recentemente, il Léon manifesto dell'ISKO - International Society of Knowledge Organization - ha sottolineato l'importanza di distinguere punti di vista ideologici e metodologici rispetto ai fenomeni studiati. ${ }^{111}$ Poiché tuttavia a determinate metodologie sono legati precisi assunti teorici, distinguere ideologie e metodologie non è sempre possibile; per la nostra modellizzazione parleremo allora semplicemente di punti di vista, senza ulteriori distinzioni.

I punti di vista sono spesso stati considerati come parte del significato assunto da un testo, ovvero della sua circalità intensionale. Ma poiché nel nostro modello la differenza tra circalità estensionale ed intensionale è legata al carattere esplicito o implicito di un certo argomento, e i punti di vista possono essere sia espliciti che impliciti, e poiché inoltre i punti di vista rivestono un'importanza autonoma rispetto alla circalità estensionale ed intensionale, per la nostra modellizzazione considereremo i punti di vista come un attributo esterno a sé stante.

\subsubsection{Una definizione di soggetto e di indicizzazione per soggetto}

Riepilogando, abbiamo considerato il soggetto di un Testo come formato dai seguenti elementi (attributi): circalità estensionale, circalità intensionale, punti di vista; la circalità estensionale è a sua volta formata da tema (uno o più) e rema; in caso di più temi, questi possono essere tra loro in relazione di subordinazione o coordinazione. Tali elementi sono tutti attributi esterni, tranne nel caso del rema.

Proviamo adesso, sulle basi della precedente discussione, a dare una nostra definizione di soggetto e di indicizzazione per soggetto. Le seguenti definizioni non aspirano a una verità assoluta: più semplicemente esse, in accordo con la posizione pragmatista da noi scelta, sono soltanto strumenti utili per gli scopi della nostra modellizzazione. Definiamo il soggetto come "l'insieme degli argomenti per cui un testo è potenzialmente utile perché in esso trattati o altrimenti implicati", ovvero, con una formulazione lievemente diversa, "l'utilità potenziale di un testo in rapporto a determinati argomenti in esso trattati o da esso implicati"; la circalità estensionale è parte degli argomenti trattati, la circalità intensionale è parte degli argomenti implicati, mentre i punti di vista possono ricadere, a seconda che siano espliciti o meno, negli argomenti trattati o implicati. Se volessimo specificare l'elemento soggettivo costituito dal giudizio dell'indicizzatore dovremmo dire che il soggetto è "l'insieme degli argomenti per cui un testo è ritenuto potenzialmente utile perché in esso trattati o altrimenti implicati”. La definizione riprende quella di

110 DEREK W. LANGRIDGE, Subject analysis, cit., p. 45; cfr. anche BIRGER HJØRLAND - LYKKE KYLLESBECH NIELSEN, Subject access points in electronic retrieval, "Annual review of information science and technology", 35 (2001), p. 259; cfr. anche W. JOHN HUTCHINS, Languages of indexing and classification, cit., p. 104; cfr. anche BIRGER HJØRLAND, Nine principles of knowledge organization, in Knowledge organization and quality management. Proceedings of the third International ISKO Conference, 20-24 June 1994, Copenhagen, Denmark, organized by the Royal School of Librarianship, Copenhagen, Denmark in cooperation with the International Society for Knowledge Organization, Germany, with support from Dan Fink's Foundation, Denmark, edited by Hanne Albrechtsen and Susanne Oernager, Frankfurt/Main, Indeks, 1994, p. 98.

111 INTERNATIONAL SOCIETY FOR KNOWLEDGE ORGANIZATION. SEZIONE ITALIA. INTEGRATIVE LEVELS CLASSIFICATION, León manifesto, 2007, disponibile all'indirizzo <http://www.iskoi.org/ilc/leon.php〉. 
Hjørland, in cui il soggetto è concepito come potenziale epistemologico di un documento rispetto a determinate domande; su questa base l'indicizzazione per soggetto, oltre che essere un'attività descrittiva, è innanzitutto una valutazione che l'indicizzatore fa sulla possibile utilità di un documento, una "prognosi" sui possibili usi futuri del documento. ${ }^{112}$ Un'attività, dunque, di natura essenzialmente predittiva. Riprendendo e completando la precedente definizione di soggetto, possiamo allora definire l'indicizzazione per soggetto come "l'attività di predizione dell'insieme degli argomenti per cui un testo è potenzialmente utile perché in esso trattati o altrimenti implicati”, ovvero come "l'attività di predizione dell'insieme delle domande che un testo potrebbe soddisfare efficacemente per via degli argomenti in esso trattati o da esso implicati”. Perché la predizione non abbia carattere aleatorio, è fondamentale che essa sia ancorata a una salda conoscenza degli utenti finali dell'indicizzazione e delle loro esigenze. ${ }^{113}$

\subsubsection{L'oggetto dell'indicizzazione per soggetto}

Riteniamo a questo punto importante approfondire la questione dell'entità oggetto dell'indicizzazione per soggetto, a cui avevamo già accennato nella discussione delle entità del Gruppo 3 di FRBR.

Per FRBR il soggetto riguarda il rapporto tra un'Opera e le altre entità che costituiscono la base del soggetto. Tuttavia, il soggetto di un'Opera può cambiare lievemente da Espressione a Espressione: per esempio quanto a copertura cronologica, ${ }^{114}$ ma in alcuni casi anche ad argomenti affrontati o tesi sostenute. Sarebbe allora più corretto dire che il soggetto è un insieme di attributi che può riguardare sia Opere sia Espressioni.

Vi è poi la questione del soggetto degli aggregati (raccolte, atti, etc.) che, nel nostro modello, abbiamo modellato come Contenuti editoriali, i quali contengono un certo numero di Opere (i saggi raccolti): in questi casi la relazione di soggetto riguarda l'Opera (e la sua Espressione), ovvero le parti, oppure il Contenuto editoriale, ovvero l'intero? Secondo Pino Buizza e Mauro Guerrini dovremmo seguire FRBR nella scelta di porre la relazione di soggetto esclusivamente a livello di Opera, al contrario di quanto avviene oggi, in cui il soggetto è attribuito spesso alla Manifestazione (al Contenuto editoriale, nel nostro modello) e a tutto ciò che contiene. ${ }^{115}$ Tuttavia, secondo noi anche in questo caso

112 BIRGER HJØRLAND, Towards a theory of aboutness, subject, topicality, theme, domain, field, content... and relevance, cit., p. 776.

113 ROBERT A. FAIRTHORNE, Content analysis, specification, and control, cit., p. 77: "An indexer does not and cannot index all the ways in which a document will interest all kinds of readers, present and future [...] a document can be of interest for reasons that appear only when it is judged against the background of its environment of use".

114 Cfr. PINO BUIZZA, Indicizzazione per soggetto e FRBR, "Bibliotime”, 6, 1 (2003), disponibile all'indirizzo <http://didattica.spbo.unibo.it/bibliotime/num-vi-1/buizza.htm>: "La relazione [di soggetto] permane attraverso le diverse espressioni e manifestazioni in cui l'opera si realizza e materializza, salvo leggere variazioni che sono possibili (ad esempio, un'opera aggiornata avrà una maggiore copertura temporale del soggetto)".

115 PINO BUIZZA - MAURO GUERRINI, Un modello concettuale per il Nuovo soggettario, cit., p. 335-336; cfr. anche PINO BUIZZA, Indicizzazione per soggetto e FRBR, cit.; anche HERBERT H. HOFFMAN, Making sense of serials. Subject access to works in online catalogs, “Technicalities”, 21, 5 (2001), p. 9-13, difende la posizione per cui oggetto della soggettazione è l'Opera, non la Risorsa: ciò implicherebbe una soggettazione analitica per raccolte, atti, etc. 
siamo in cospetto di una contrapposizione che è possibile trasformare in complementarietà: soggetto del Contenuto editoriale e delle Opere in esso contenute possono essere visti come aspetti o livelli diversi e complementari del soggetto. $O$, in altri termini, accanto al soggetto delle singole Opere (livello analitico) vi è un soggetto del Contenuto editoriale (livello generale). Il tipo di relazione tra i due soggetti (del Contenuto editoriale e dell'Opera) è una relazione di subordinazione tra temi della circalità estensionale. A livello fisico (elementi indicali), la scelta di porre l'indicizzazione per soggetto a livello del Contenuto editoriale, o delle Opere, o di entrambi, sarebbe poi una questione relativa alle politiche di indicizzazione.

Riepilogando, riteniamo che il soggetto debba essere posto come insieme di attributi che può riguardare non soltanto l'Opera, ma tutti i Testi in generale (Opere, Espressioni e Contenuti editoriali).

\subsection{Dal modello concettuale alla sua realizzazione fisica}

Gli oggetti di interesse (entità, attributi e relazioni) del modello concettuale possono riflettersi in una varietà di elementi a livello fisico, e cioè di strutture catalografiche. FRBR non fornisce indicazioni esplicite sulle strutture catalografiche che dovrebbero realizzare il modello concettuale, se non nella sua parte finale, Basic requirements for national bibliographic records, ${ }^{116}$ esterna al modello concettuale vero e proprio e dedicata a rapportare il modello e le funzioni dell'utente con le attuali strutture catalografiche, con l'obiettivo di raccomandare gli elementi indicali che dovrebbero essere inclusi nelle registrazioni bibliografiche delle biblioteche nazionali. In questa seconda parte del capitolo cercheremo di fare qualcosa di simile a Basic requirements for national bibliographic records: parleremo cioè delle strutture catalografiche (elementi indicali, collegamenti e registrazioni bibliografiche) che dovrebbero corrispondere al modello concettuale sopra esposto, ovvero di raccomandare gli elementi indicali che dovrebbero essere inclusi nelle registrazioni bibliografiche (di biblioteche nazionali e non); con la differenza fondamentale che nel nostro approccio il riferimento alle attuali strutture catalografiche sarà molto più libero: ci discosteremo da ciò che si trova nelle attuali strutture catalografiche ogni qual volta ciò sia richiesto da un'adeguata rappresentazione degli elementi del nostro modello concettuale.

Nel nostro approccio assumeremo che le entità si riflettano generalmente in registrazioni; in ciò possiamo vedere un primo grado di controllo dell'universo bibliografico.

Assumeremo inoltre che gli attributi si riflettano in indici o elementi indicali delle registrazioni; l'esplicitazione dei diversi attributi di un'entità tramite diversi elementi indicali costituisce un secondo grado di controllo dell'universo bibliografico. Assumeremo infine che le relazioni, se segnalate in modo esplicito, si riflettano in collegamenti tra le registrazioni relative alle entità - qui con alcune eccezioni che diremo -; in ciò possiamo vedere un terzo grado di controllo dell'universo bibliografico. Approfondiremo il rapporto tra elementi del modello concettuale, loro traduzione fisica e gradi di controllo dell'universo bibliografico nel prossimo capitolo (3.3.4, con una

116 IFLA STUDY GROUP ON THE FUNCTIONAL REQUIREMENTS FOR BIBLIOGRAPHIC RECORDS, Functional requirements for bibliographic records, cit., p. 97-112. 
Tesi di dottorato di Giuliano Genetasio, discussa presso l'Università degli Studi di Udine

terminologia un po' diversa: parleremo di "controllo linguistico").

Per quanto riguarda il rapporto tra entità e registrazioni, non ne riteniamo necessaria una discussione approfondita, ma ci limitiamo a segnalare che nel nostro approccio concettuale non è prevista la differenziazione in registrazioni bibliografiche e di autorità. Dedicheremo invece gran parte di ciò che resta del capitolo all'esposizione e discussione degli elementi (indici e collegamenti) relativi ad attributi e relazioni del modello concettuale; così come per gli attributi e le relazioni, ci concentreremo soprattutto sulla trattazione degli indici, facendo occasionali riferimenti ai collegamenti. Dopo una premessa sull'impatto che i cambiamenti tecnologici hanno avuto e hanno sulle strutture catalografiche, esporremo una tassonomia generale degli indici e, dunque, ne discuteremo quelli che riteniamo più importanti o peculiari del nostro approccio.

\subsection{Indici e collegamenti: premessa: l'impatto dei cambiamenti tecnologici}

\subsubsection{Introduzione}

Con la discussione degli indici e dei collegamenti passiamo dal modello concettuale vero e proprio alle possibilità della sua implementazione fisica. Prima di trattare i singoli tipi di indici proposti e di stabilirne una tassonomia, è indispensabile una premessa sull'impatto che i cambiamenti tecnologici degli ultimi cinquant'anni circa hanno avuto sulle strutture della mediazione indicale. È essenziale parlarne perché proprio la sottovalutazione del ruolo della tecnologia nell'indicizzazione è alla base di molti fraintendimenti, preconcetti e luoghi comuni che caratterizzano la stessa teoria dell'indicizzazione.

La mediazione indicale è un'operazione di natura eminentemente tecnica. E dunque storica, perché fortemente legata ai cambiamenti delle tecnologie. Due i cambiamenti cruciali nelle tecnologie di mediazione indicale: 1) il passaggio dall'ambiente cartaceo a quello elettronico; 2) l'affermazione del web, che ha comportato il passaggio da un ambiente locale a uno di tipo globale.

Esiste una letteratura sterminata dedicata al tema delle conseguenze catalografiche del passaggio dal catalogo cartaceo a schede mobili a quello elettronico e in rete. Tuttavia standard, formati di dati e strutture catalografiche arrancano dietro le conseguenze di tale passaggio. $^{117}$

Il catalogo elettronico nasce storicamente come catalogo 'automatizzato', cioè come replica potenziata di quello cartaceo; esempio di tale catalogo 'automatizzato' sono le registrazioni MARC, che ricalcano nella struttura quelle cartacee; ciò che è problematico è il fatto che

117 ELAINE SVENONIUS, The intellectual foundation of information organization, cit., p. 63-64: "The transition from card to online catalogs, though ongoing for over thirty years now, is still in its initial stages. This slow transition is due in part to difficulty in understanding the function of the bibliographic record in an electronic environment". Cfr. anche MARCIA J. BATES, Subject access in online catalogs. A design model, "Journal of the American Society for Information Science", 37, 6 (1986), p. 357: "With the advent of online catalogs, the nature of catalogs and access to library materials has changed more in a handful of years than it has in all the rest of the twentieth century. But the changes have been more technological than conceptual. Online catalogs to date have added powerful capabilities to the traditional catalog, yet system designs, generally, have still not gone beyond implementing the card catalog in online form". 
il catalogo elettronico sia rimasto a tutt'oggi, nonostante sia passato circa mezzo secolo dalla sua introduzione, una replica di quello cartaceo ${ }^{118}$ per quanto riguarda molte sue caratteristiche strutturali; e ciò a dispetto delle sue enormi potenzialità, delle sue caratteristiche fondamentalmente altre rispetto a quello cartaceo.

Esaminiamo le conseguenze maggiori - effettive o potenziali - di questo passaggio a livello di mediazione indicale.

\subsubsection{Ordine lineare e uniformità ${ }^{19}$}

Nel catalogo cartaceo, forma e sequenza delle informazioni bibliografiche in immissione (indicizzazione) ed emissione (visualizzazione) coincidevano. Le informazioni erano disposte in un ordine lineare, a lettura sequenziale. ${ }^{120}$ In un contesto di sequenza fissa, la scelta di un ordine fisso tra gli elementi garantiva la coerenza necessaria allo scambio e alla comprensione delle registrazioni bibliografiche; esso richiedeva tuttavia la scelta di un unico elemento (intestazione principale) sugli altri, che stabilisse il posto che la scheda dovesse avere all'interno del catalogo; la possibilità di avere le stesse informazioni in più punti del catalogo tramite l'utilizzo di più elementi (intestazioni aggiunte) per l'ordinamento era prevista, ma ridotta al minimo per ragioni di economia. La scelta di un ordine fisso tra gli elementi costituiva inoltre una lingua internazionale artificiale con cui identificare gli elementi descrittivi della registrazione; su tale è idea è fondato lo standard ISBD - International Standard Bibliographic Description. ${ }^{121}$ In un contesto di forma fissa, era necessario scegliere un'unica forma degli elementi in fase di immissione, e soprattutto di quelli atti a determinare l'ordinamento (intestazione uniforme). A differenza del catalogo cartaceo, le informazioni nelle registrazioni del catalogo elettronico non richiedono una sequenza e una forma fissa: la sequenza (ordine) e la forma delle informazioni in immissione non sono necessariamente le stesse che quelle in emissione. La ricerca avviene, almeno in principio, per "accesso casuale": le informazioni possono essere estrapolate dalla registrazione a prescindere dalla posizione in cui sono state disposte all'interno della registrazione. Non soltanto ogni intestazione (ma venendo meno la struttura che caratterizzava il catalogo cartaceo è più corretto parlare di "punti di accesso") permette di trovare la registrazione in modo identico, che sia principale o aggiunta, ma inoltre ogni altro elemento della registrazione costituisce un punto di

118 MAJA ŽUMER, FRBR. The end of the road or a new beginning?, "Bulletin of the American Society for Information Science and Technology", 33, 6 (2007), p. 27.

119 Per questi paragrafi cfr. MAURO GUERRINI, I Principi internazionali di catalogazione (ICP). Universo bibliografico e teoria catalografica all'inizio del XXI secolo, con Giuliano Genetasio, postfazione di Attilio Mauro Caproni, Milano, Editrice Bibliografica, 2012, p. 151-159.

120 Come ci ricorda ROSSELLA DINI, Principi e standard, in Il futuro della descrizione bibliografica. Atti della giornata di studio, Firenze, 13 novembre 1987, a cura di Mauro Guerrini, Roma, Associazione italiana biblioteche, 1988, p. 13, riferendosi a ISBD: "gli standard attuali sono concettualmente legati a una cultura catalografica di tipo preinformatico. Dietro alla loro elaborazione stanno infatti la tecnologia della scheda e la lettura sequenziale dei dati - con la conseguente presentazione monodimensionale delle informazioni". 121 INTERNATIONAL FEDERATION OF LIBRARY ASSOCIATIONS AND INSTITUTIONS, International standard bibliographic description (ISBD). Preliminary consolidated edition, recommended by the ISBD Review Group, approved by the Standing Committee of the IFLA Cataloguing Section, München, Saur, 2007. 
accesso alla registrazione, almeno potenzialmente; è comunque possibile escludere alcuni elementi della registrazione per la ricerca, rendendoli non ricercabili (ed è proprio ciò che noi proponiamo, vedi 2.19). Accanto a questa condizione naturale del catalogo elettronico che è rappresentata dalla ricerca per parole chiave, è auspicabile prevedere la possibilità di ricerche differenziate per tipo di elemento indicale. Per fare un esempio a livello di indicizzazione per soggetto, la fine di un ordine fisso significa, tra le altre cose, che la questione dell'utilizzo della forma diretta o inversa nelle intestazioni di soggetto divenga obsoleta. ${ }^{122}$

Inoltre, oggi lo scambio delle registrazioni bibliografiche prevede la possibilità di includere o escludere i vari elementi indicali della registrazione bibliografica a seconda delle esigenze; l'ordine fisso non è più necessario nemmeno per lo scambio delle registrazioni. La possibilità di predisporre gli elementi indicali secondo un ordine lineare in emissione continua a essere un elemento essenziale per lo scorrimento dei risultati, ma ciò non richiede che l'ordine sia fisso: l'ordine degli elementi può variare sulla base delle ricerche dell'utente, in modo che in prima posizione sia posto l'elemento che di volta in volta è ricercato dall'utente. La fine di una forma necessariamente fissa in immissione ed emissione riduce l'utilità dell'idea di una lingua internazionale artificiale fondata su elementi convenzionali come l'ordine fisso. A divenire controversi sono tanto l'ideale di una lingua basata su elementi simbolici, piuttosto che verbali, quanto l'idea che tale lingua debba essere necessariamente internazionale. A livello di emissione, una lingua simbolica internazionale può infatti oggi essere sostituita dal linguaggio naturale in forma verbale; la lingua potrebbe essere una lingua nazionale di valore internazionale (inglese, spagnolo, etc.) oppure la stessa lingua del catalogo o dell'utente. Dalla fine di una forma fissa consegue che l'uniformità delle intestazioni (intestazione uniforme) non è più indispensabile, almeno a livello di indicizzazione - secondo Tillett, rimarrebbe sempre necessaria la scelta di una forma predefinita per la visualizzazione. ${ }^{123}$ Ayres e Weston si sono spinti fino a proporre di abbandonare anche a livello locale il principio di uniformità e di indicizzare un elemento nella forma in cui di volta in volta compare su una risorsa documentaria. ${ }^{124}$

Approfondiremo le possibilità e i limiti di una realizzazione tecnica del superamento dell'uniformità nel prossimo capitolo (vedi 3.3.6.4.2.2.5).

\subsubsection{Oltre la separazione tra indicizzazione per autore e per soggetto, verso una visione unitaria dell'accesso bibliografico: prima parte}

122 KAREN MARKEY - DIANE VIZINE-GOETZ, Using subject headings for online retrieval. Theory, practice, and potential, San Diego, Academic Press, 1994, p. 333.

123 BARBARA B. TILLETT, AACR and authority control, in The future of the descriptive cataloging rules. Papers from the ALCTS Preconference. AACR2000, American Library Association Annual Conference, Chicago, June 22, 1995, edited by Brian E. C. Schottlaender, Chicago, American Library Association, 1998, p. 31.

124 PAUL GABRIELE WESTON, Il catalogo elettronico. Dalla biblioteca cartacea alla biblioteca digitale, postfazione di Giovanni Solimine, Roma, Carocci, 2005, p. 138. F. H. AYRES, Time for change. A new approach to cataloguing concepts, "Cataloging \& classification quarterly", 28, 2/3 (2000), p. 9: "The cataloguer must not dictate the preferred heading. To the user the preferred heading is the heading that he searches by and when he searches he wants the headings that he has thought about to be linked to the heading that he has not thought about. This means that the place for authority control is not at the cataloguing stage but at the searching stage". 
Tesi di dottorato di Giuliano Genetasio, discussa presso l'Università degli Studi di Udine

Con il passaggio dal catalogo a schede a quello elettronico cade la separazione fisica tra catalogo per soggetto e per autore. L'OPAC è tipicamente unico (benché alla sua base vi siano spesso ancora oggi archivi di autorità separati). La fine di questa separazione dei cataloghi riduce anche la portata della tradizionale divisione tra indicizzazione per autore e per soggetto. Continueremo ad approfondire la questione in 2.27.

\subsubsection{Oltre la precoordinazione?}

La coordinazione è l'operazione di combinazione di due o più parole all'interno di una stessa struttura indicale; ${ }^{125}$ essa può svolgersi in una o più fasi dell'accesso bibliografico: durante l'indicizzazione, durante il recupero dell'informazione, o entrambe. Nel primo caso è detta precoordinazione, nel secondo postcoordinazione; è possibile anche un terzo caso: una ricerca postcoordinata su elementi precoordinati. ${ }^{126}$

La coordinazione ha lo scopo di sopperire all'assenza di contestualità che caratterizza l'accesso bibliografico rispetto a una situazione comunicativa ordinaria (discussione tra due persone, etc.) fornendo un contesto artificiale: specificando una parola tramite ulteriori termini, l'indicizzatore (precoordinazione) o l'utente finale (postcoordinazione) fornisce un contesto artificiale alla parola ricercata, permettendo una maggiore precisione della ricerca. ${ }^{127}$ Il tipo di contestualità fornita dalla precoordinazione tradizionale è molto più espressiva di quella che caratterizza la postcoordinazione: mentre quest'ultima si limita generalmente a esprimere la sola relazione di congiunzione, la prima permette di esprimere (a prezzo di una complessità e dunque costi maggiori) una più ampia gamma di significati.

Tradizionalmente, elemento fondamentale della precoordinazione è l'uso di un ordine fisso tra le parole che vengono combinate. L'ordine fisso, cioè, viene utilizzato come espediente per fornire un significato alle relazioni tra le diverse parole inserite in una struttura indicale, e ciò secondo uno o più principi di costruzione e interpretazione: per esempio, secondo il principio d'inversione, per cui l'ordine delle parole inserite in una struttura indicale sarebbe invertito rispetto a quello vigente nel linguaggio naturale (p.e. "Education-Philosophy-History" andrebbe interpretato non come "educazione alla filosofia della storia" ma come "storia della filosofia dell'educazione").

Abbiamo tuttavia detto sopra (vedi 2.18.2) che con il passaggio al catalogo elettronico l'ordine fisso tra gli elementi diviene obsoleto. Ciò comporta anche l'obsolescenza della precoordinazione? Fin dall'avvento del catalogo elettronico, una parte significativa degli studiosi di indicizzazione e recupero dell'informazione ha sostenuto l'obsolescenza o comunque l'inferiorità della precoordinazione rispetto alla postcoordinazione nel nuovo ambiente, ${ }^{128}$ sulla base dell'argomento dell'obsolescenza dell'ordine fisso. L'ordine fisso è

125 L'operazione di coordinazione, di cui parliamo in questo paragrafo, non va confusa con la relazione sintagmatica di coordinazione, di cui parleremo in 3.2.8.

126 PINO BUIZZA, 025.4 Analisi e controllo per soggetto, cit., p. 567.

127 DANIEL N. JOUDREY - ARLENE G. TAYLOR, RE: LCSH Strings - some thoughts, in Library of

Congress subject headings. Pre- vs. Post-coordination and related issues, 2007, disponibile all'indirizzo

<http://www.loc.gov/catdir/cpso/pre_vs_post.pdf>, p. 22.

128 A favore della precoordinazione si schiera LIBRARY OF CONGRESS. CATALOGING POLICY

AND SUPPORT OFFICE, Library of Congress subject headings. Pre-vs. Post-coordination and related issues, 2007, 
però soltanto uno strumento della precoordinazione, non coincide con essa: ciò che è e continua ad essere essenziale è la creazione di contestualità, non i mezzi per realizzarla. Metodo alternativo all'utilizzo di un ordine fisso (e a principi impliciti o espliciti per interpretarlo) per realizzare la precoordinazione, e già utilizzato da alcuni strumenti di indicizzazione come il PRECIS, ${ }^{129}$ è l'assegnazione di ruoli di significato alle singole parole o espressioni componenti la struttura indicale (stringa); per realizzare una contestualità soddisfacente dal punto di vista della precisione, dell'espressività e della comprensibilità, sarà dunque necessario uno studio delle relazioni sintagmatiche (o ruoli semantici) che possono essere svolte dalle singole parole o espressioni componenti la struttura indicale; cercheremo di realizzare questo studio nel prossimo capitolo (vedi 3.2.7, 3.3.6.4.3). Con un'assegnazione dei ruoli semantici a parole e espressioni delle strutture indicali l'ordine fisso non è più necessario: i diversi ordini possibili tra gli elementi non diventano altro che diverse forme (sintagmatiche) varianti, che può essere utile fornire all'utente; ciò potrebbe avvenire anche in maniera automatica, per esempio tramite un programma che generi automaticamente tutti i possibili ordini sintatticamente corretti tra le singole parole o espressioni componenti a partire dai rispettivi ruoli semantici. Rinunciare alla precoordinazione sulla base dell'obsolescenza dell'ordine fisso significherebbe rinunciare alla contestualità in indicizzazione. La contestualità ottenuta tramite postcoordinazione si aggiunge ma non sostituisce quella creata in fase di indicizzazione. Del resto, una postcoordinazione che sfrutti i ruoli semantici delle parole ed espressioni è molto più espressiva di una che utilizzi la semplice combinazione di parole ed espressioni e costituisce il complemento naturale della precoordinazione tramite assegnazione di ruoli semantici. La precoordinazione in quanto tale, dunque, non è affatto superata. Piuttosto, il tipo di coordinazione che proponiamo: 1) è basata sull'assegnazione di ruoli semantici degli elementi componenti la struttura indicale, non sull'ordine fisso; 2) non si basa su una contrapposizione, ma piuttosto su una complementarietà, di precoordinazione e postcoordinazione: la coordinazione si svolge in fase di indicizzazione e possibilmente anche in fase di recupero dell'informazione.

\subsubsection{Dal catalogo locale al catalogo in rete: ancora sull'uniformità}

Conseguenze altrettanto rilevanti ha avuto, a partire dagli anni Novanta, la diffusione di internet. Il web ha nel giro di pochi anni scavalcato il ruolo tradizionale del catalogo come ambiente entro cui si realizzano le ricerche bibliografiche. $O$ più precisamente, il catalogo (OPAC) è oggi parte stessa della rete, ne è una sua terminazione nervosa. La rete ha facilitato enormemente lo scambio dei dati bibliografici. Essa ha soprattutto comportato una globalizzazione della realtà del catalogo: prima dell'era internet, il catalogo era soprattutto uno strumento locale per un'utenza locale. A partire da internet, il catalogo è diventato un portale rivolto a un'utenza potenzialmente globale. La globalizzazione del catalogo ha a sua volta comportato la revisione di alcuni principi fondanti la teoria catalografica, per esempio quello di uniformità.

disponibile all'indirizzo <http://www.loc.gov/catdir/cpso/pre_vs_post.pdf>.

129 DEREK AUSTIN, PRECIS. A manual of concept analysis and subject indexing, London, Council of the British National Bibliography, 1974; cfr. anche JUTTA SØRENSEN - DEREK AUSTIN, PRECIS in a multilingual context. Part 2, A linguistic and logical explanation of the syntax, "Libri", 26, 3 (1976), p. 108-139. 
Tesi di dottorato di Giuliano Genetasio, discussa presso l'Università degli Studi di Udine

Avevamo detto come nel catalogo elettronico divenisse non più indispensabile la scelta di una forma preferita di un elemento indicale (intestazione uniforme) rispetto ad altre non preferite, almeno a livello di indicizzazione. Scelte alternative a quella proposta da Ayres e Weston di usare per l'indicizzazione la forma dell'elemento che compare sulla risorsa, sono sia la possibilità, aperta da VIAF, Virtual International Authority File, di mantenere l'uniformità a livello locale ma non globale, collegando tra loro le diverse forme preferite appartenenti a contesti (archivi di autorità) differenti, sia la possibilità, preconizzata da Michael Gorman negli anni Settanta, di codificare in fase di indicizzazione ogni forma dell'attributo (nome, titolo, termine, etc.) sulla base della lingua, dell'archivio di autorità di riferimento, etc., in modo di permettere poi al singolo sistema bibliotecario o persino al singolo utente finale la possibilità di scegliere, in fase di recupero dell'informazione, le forme dei nomi degli indici che preferisce sulla base del criterio specificato. ${ }^{130}$

\subsection{Indici e collegamenti: introduzione}

Gli indici o elementi indicali costituiscono la traduzione degli attributi del modello concettuale in strutture catalografiche fisiche; più precisamente, gli indici sono il prodotto dell'incrocio di attributi da un lato e funzioni catalografiche dall'altro - ciò è vero per la maggior parte degli indici, ma non per tutti: come diremo, alcuni tipi di indici non nominali non sono necessariamente legati ad attributi specifici dell'entità -; allo stesso modo, i collegamenti sono la traduzione delle relazioni del modello concettuale in strutture catalografiche fisiche.

Nelle discussioni che seguiranno, ci concentreremo soprattutto sugli indici, facendo riferimento ai collegamenti più che altro in rapporto a specifiche tipologie di indici. Suddivideremo la trattazione degli specifici indici sulla base dei criteri già adottati per la trattazione degli attributi e delle relazioni: prima, dunque, gli indici che corrispondono agli attributi nominali; poi gli indici che corrispondono agli attributi non nominali, più alcuni indici che non corrispondono necessariamente a particolari attributi e che non fanno comunque riferimento a ulteriori entità: sono indici di questo tipo i metaindici e gli indici valutativi; infine, discuteremo a parte gli indici relativi alla rappresentazione del soggetto.

Prima di passare alla discussione dei vari indici, però, è necessario suddividere gli indici in

130 VIAF. Virtual international authority file, disponibile all'indirizzo <http://www.viaf.org/>. Cfr. anche MICHAEL GORMAN, Cataloging and the new technologies, in The nature and future of the catalog. Proceedings of the ALA's Information Science and Automation Division's 1975 and 1977 Institutes on the Catalog, edited by Maurice J. Freedman and S. Michael Malinconico, Phoenix, Oryx, 1979, p. 133-134. Cfr. anche PAUL GABRIELE WESTON, FRBR e utente. Considerazioni sulla ricerca, in SEMINARIO FRBR, Seminario FRBR. Functional requirements for bibliographic records, Requisiti funzionali per record bibliografici, Firenze, 27-28 gennaio 2000, atti a cura di Mauro Guerrini, Roma, Associazione italiana biblioteche, 2000, disponibile all'indirizzo <http://www.aib.it/aib/sezioni/toscana/conf/frbr/weston.htm>, p. 127: "Rinunciare ad una forma autorevole non significa rinunciare al controllo o alla struttura sindetica che collega forme alternative o correlate, ma nasce dall'esigenza di rispettare, anche nei fatti, la filosofia user-oriented che informa il modello strutturale di FRBR [...] pare più opportuno lasciare ad ogni biblioteca [...] di stabilire la forma preferita per l'accesso". Cfr. anche LIBRARY OF CONGRESS. WORKING GROUP ON THE FUTURE OF BIBLIOGRAPHIC CONTROL, On the Record. Report of the Library of Congress Working Group on the Future of Bibliographic Control, Washington, D.C., Library of Congress, 2008, disponibile all'indirizzo <http://www.loc.gov/bibliographic-future/news/lcwg-ontherecord-jan08-final.pdf>, p. 19. 
diverse tipologie sulla base delle loro specifiche caratteristiche, ovvero abbozzare una tassonomia generale degli indici. La prima classificazione degli elementi indicali è legata alla natura fondamentale degli indici: questi possono essere suddivisi in indici (senza ulteriori specificazioni), che registrano attributi di un'entità, e in metaindici, che non riflettono direttamente nessun attributo di un'entità ma piuttosto forniscono informazioni sugli indici stessi; approfondiremo i metaindici in 2.24.

Una seconda classificazione degli indici è quella, già menzionata indirettamente, tra indici nominali e indici non nominali: gli indici nominali sono quelli che corrispondono agli attributi nominali; gli indici non nominali sono quelli che corrispondono agli attributi non nominali, più indici non necessariamente riferiti a specifici attributi come metaindici e indici valutativi.

Una terza classificazione degli indici è quella, basata sulle corrispondenti tipologie di attributi, in indici interni ed indici esterni; tutti gli indici esterni sono indici nominali. Una quarta classificazione degli indici è possibile sulla base delle funzioni dell'utente: gli indici (e metaindici) possono così essere suddivisi in punti di accesso (funzionali a Trovare e Navigare) ed elementi che definiamo "non ricercabili"; questi ultimi possono essere ulteriormente suddivisi in elementi "descrittivi" (funzionali a Identificare e Selezionare) ed "inventariali" (funzionali a Ottenere) - la terminologia è di nostra elaborazione. La scelta di quali indici costituiscano punti di accesso e quali elementi non ricercabili deriva in parte dalla natura degli attributi stessi cui fanno riferimento gli indici, in parte da nostre scelte. Abbiamo sopra spiegato come la natura del catalogo elettronico faccia sì che ogni elemento indicale costituisca potenzialmente un punto di accesso. D'altro canto, occorre riconoscere che non tutti gli attributi sembrano idonei a costituire chiavi di ricerca utili per la prima fase di recupero dell'informazione, quella costituita dall'interrogazione del catalogo: non tutti gli attributi sembrano funzionali a Trovare. I punti di accesso del nostro modello sono dunque gli elementi indicali che corrispondono ad attributi che abbiamo ritenuto essere funzionali a Trovare, a seconda dei casi, anche a Navigare, oltre che a Identificare, Selezionare e Ottenere: dunque tutti gli attributi nominali (interni o esterni), più gli identificatori delle entità (ISBN, EAN, etc.); ai punti di accesso corrispondenti a questi attributi si aggiungono gli identificatori delle registrazioni, elementi metaindicali che individuano le entità oggetto di registrazione. In 2.4 avevamo detto che almeno un certo numero di attributi svolge due o più funzioni, in modo relativo e complementare; tali attributi, lo esplicitiamo adesso, sono quelli che abbiamo individuato come attributi funzionali a Trovare e che a livello fisico, dunque, corrispondono ai punti di accesso.

Qui è necessaria una nota sulla questione dell'autonomia dei punti di accesso ai fini della ricerca (funzioni Trovare e Identificare): un singolo punto di accesso non è necessariamente autonomo in una ricerca, cioè tale da Identificare un'entità sempre e comunque: soltanto gli identificatori delle entità e gli identificatori delle registrazioni, che mirano a Identificare un'entità in modo assoluto e preventivo, possono aspirare a tale capacità; tutti gli altri punti di accesso permettono di Trovare un'entità, ma non garantiscono di Identificarla; gli identificatori hanno dunque un ruolo fondamentale nella disambiguazione delle relazioni di omonimia tra le entità; riprenderemo la discussione della funzione di disambiguazione degli identificatori nel prossimo capitolo (vedi 3.3.6.4.2.2.6).

Accanto ai punti di accesso, gli elementi non ricercabili sono tutti quegli indici funzionali 
esclusivamente a descrivere un'entità (funzioni Identificare e Selezionare) come descrizioni fisiche o d'altro tipo, note, etc. ("elementi descrittivi"), oppure a Ottenere una risorsa come la segnalazione delle condizioni di disponibilità, della collocazione fisica, etc. ("elementi inventariali").

A questo punto è necessario parlare della rappresentazione indicale delle relazioni. Le relazioni del modello possono essere rappresentate, a livello indicale, in modo implicito o esplicito: sono rappresentate in modo implicito quando le entità i cui nomi fungono da attributi dell'entità descritta sono registrate in forma discorsiva, senza collegamenti tra le registrazioni delle diverse entità. Viceversa, quando le relazioni sono rappresentate in modo esplicito si ha a che fare con un terzo e duplice grado di controllo formale della relazione; esso implica: 1) la registrazione autonoma dell'entità il cui nome funge da attributo dell'entità descritta; 2) la creazione di collegamenti fisici tra le registrazioni delle due entità.

Ciò vale, lo precisiamo, per tutte le relazioni che riguardano una prima entità e una seconda entità il cui nome sia un attributo nominale esterno della prima entità, e cioè per la maggior parte delle relazioni. Diversa è la questione per le relazioni di equivalenza tra i diversi attributi nominali interni di un'entità, per le relazioni di subordinazione e coordinazione tra i diversi temi estensionali (attributi nominali esterni) di un Testo, e per le relazioni di omonimia e polisemia. In presenza di più nomi (attributi nominali interni) di un'entità, essi sono legati da una relazione di equivalenza. Però, a differenza delle altre relazioni che intercorrono tra gli attributi nominali esterni, la rappresentazione necessariamente esplicita - di una relazione di equivalenza avviene già con la sola registrazione dei vari (due o più) nomi di un'entità: la relazione di equivalenza non comporta dunque collegamenti fisici ma soltanto la registrazione di attributi dell'entità. Una condizione simile vale anche per i diversi temi estensionali di un Testo, le cui relazioni, se controllate formalmente, possono essere registrate non tramite reciproci collegamenti, ma semplicemente tramite elementi indicali differenziati: semplicemente più indici di tema estensionale, nel caso delle relazioni di coordinazione; indice di tema estensionale e indici di sottotema estensionale, nel caso delle relazioni di subordinazione. Infine, per quanto riguarda le relazioni di omonimia e polisemia, nemmeno esse necessitano di particolari collegamenti fisici; tradizionalmente, la disambiguazione di omonimie e polisemie avviene tramite l'utilizzo di "qualificazioni", elementi indicali non previsti nel nostro approccio, e note; per quanto ci riguarda, riteniamo che la rappresentazione delle relazioni di omonimia e polisemia possa restare nella maggior parte dei casi implicita, essendo la disambiguazione già svolta indirettamente dalle altre relazioni semantiche paradigmatiche (gerarchiche, di equivalenza, di antonimia, etc.) e dagli identificatori; in via del tutto residuale, può comunque essere prevista la possibilità di elementi descrittivi (note) che chiariscano la relazione di omonimia. Approfondiremo nel prossimo capitolo la discussione delle relazioni di omonimia e polisemia, e della loro trattazione indicale (vedi 3.2.5.3.3, 3.3.6.4.2.2.6).

Questo terzo grado di controllo formale, sia nel caso delle relazioni di equivalenza tra nomi dell'entità, sia nel caso delle relazioni tra temi estensionali, sia ancora nel caso delle relazioni tra attributi (nominali) esterni e delle relazioni di omonimia e polisemia, corrisponde a ciò che tradizionalmente viene definito authority control e gli indici che ne risultano vengono tradizionalmente detti "controllati". Pur precisando che, come abbiamo detto, il processo di controllo formale delle relazioni è soltanto il terzo grado di 
controllo dell'universo bibliografico, adotteremo anche noi questa terminologia e parleremo, per attributi nominali di cui siano state controllate le relazioni, di indici controllati. Parleremo invece di indici non controllati sia per gli attributi non soggetti a questo terzo grado di controllo formale.

Fin qui non abbiamo parlato in modo esplicito della rappresentazione indicale delle relazioni sintagmatiche. Nella tassonomia degli attributi, abbiamo mostrato come gli attributi nominali si suddividano in "semplici" e "complessi". La rappresentazione indicale dei nomi complessi e delle relazioni tra i diversi elementi (entità o elementi componenti non significativi, vedi sotto) a cui essi fanno riferimento può anche in questo caso essere implicita o esplicita (verosimilmente soltanto per gli attributi nominali complessi stabiliti come indici controllati). In questo secondo caso il controllo formale comporta: 1) la registrazione autonoma dell'ulteriore entità o delle ulteriori entità menzionate esplicitamente nel nome complesso di un'entità; 2) la creazione di collegamenti fisici tra le registrazioni tra le diverse entità coinvolte, per esempio un collegamento dalla registrazione della "Biblioteca della Regione Sicilia" alla registrazione della "Regione Sicilia"; 3) la creazione di collegamenti fisici (o espedienti simili, come operatori di ruolo, etc.) tra i diversi elementi - a esclusione di articoli, preposizioni, etc. che fanno parte nel nome complesso, per esempio "Biblioteca - [parte/intero] - Regione Sicilia” o "[parte:] Biblioteca - [intero:] Regione Sicilia”.

Parleremo in questo caso di indici (nominali controllati) collegati; parleremo invece di indici non collegati per indici nominali relativi a nomi complessi le cui relazioni sintagmatiche non siano state controllate formalmente. Nessuno strumento di indicizzazione attuale prevede indici collegati o espedienti simili; essi costituiscono una possibilità teorica, peculiare del nostro approccio. Il controllo formale dei nomi complessi e delle relative relazioni sintagmatiche costituisce un vero e proprio ulteriore grado di controllo formale dell'universo bibliografico.

Quando tutti gli elementi (non relativi a preposizioni, articoli, etc.) di un nome complesso individuino entità e siano pertanto collegati a proprie registrazioni, parleremo di indici totalmente collegati (per esempio in presenza di collegamenti da "Etica della vita" alle registrazioni di "Etica" e "Vita"); quando invece non tutti gli elementi di un nome complesso individuino entità, perché nel nome è presente un elemento che non è ritenuto significativo in modo autonomo, parleremo di indici parzialmente collegati (vedi l'esempio suddetto di "Biblioteca della Regione Sicilia"); nomi complessi di entità individuali avranno generalmente indici parzialmente collegati; nomi complessi di entità entità collettive avranno generalmente indici totalmente collegati.

Approfondiremo gli indici (parzialmente e totalmente) collegati e non collegati nel prossimo capitolo, con una terminologia un po' diversa (vedi 3.3.6.2.5). Gli indici controllati e collegati (e i relativi collegamenti) sono le strutture catalografiche principali per il soddisfacimento della funzione Trovare e - tranne nel caso del controllo delle relazioni di equivalenza, di subordinazione e coordinazione tra temi estensionali, e di omonimia - Navigare.

Il processo di formalizzazione delle relazioni tra le differenti entità e tra i nomi delle entità comporta la creazione di una rete, detta rete sindetica, che rispecchia in modo formalizzato e sintetico la semantica dell'universo bibliografico nel suo complesso e aiuta l'utente a muoversi tra le entità e a chiarire i loro rapporti reciproci. Le varie classificazioni delle tipologie di elementi indicali sopra delineate gettano nuove luci sui 
rapporti tra funzioni del catalogo e indici.

Se guardiamo alle suddette classificazioni nella prospettiva delle funzioni ci accorgiamo che: 1) la funzione Trovare è soddisfatta dai punti di accesso in generale (indici nominali o non nominali, controllati o non controllati, ed eventualmente collegati o non collegati); 2) la funzione Navigare è soddisfatta soltanto dai punti di accesso controllati (e da eventuali punti di accesso collegati); 3) le funzioni Identificare e Selezionare sono soddisfatte sia dai punti di accesso sia dagli elementi descrittivi, i quali possono servire a seconda dei casi sia a individuare elementi già noti all'utente finale (Identificare), sia a fornire ulteriori informazioni su elementi noti o non noti (Selezionare); 4) la funzione Ottenere è soddisfatta sia dai punti di accesso sia dagli elementi inventariali - i quali riguardano però soltanto le Risorse.

Queste osservazioni ci portano a stabilire la seguente gerarchia tra gli elementi indicali volti a soddisfare le funzioni: 1) gli elementi indicali più importanti sono i punti di accesso controllati (e gli eventuali punti di accesso collegati), funzionali a tutte e cinque le funzioni (Trovare, Navigare, Identificare, Selezionare, Ottenere); 2) seguono i punti di accesso non controllati (nominali o non nominali) che svolgono le funzioni Trovare, Identificare, Selezionare e Ottenere; 3) seguono infine gli elementi descrittivi, funzionali a Identificare e Selezionare, da un lato, e gli elementi inventariali, funzionali a Ottenere, dall'altro; elementi descrittivi e inventariali sono funzionalmente esclusivi (i primi servono a Identificare e Selezionare; i secondi a Ottenere) e dunque incommensurabili in termini di importanza funzionale, ma sono entrambi meno importanti dei punti di accesso; se volessimo stabilire una gerarchia tra tali elementi, dovremmo porre gli elementi inventariali all'ultimo posto perché relativi soltanto a Ottenere (mentre gli elementi descrittivi servono a Identificare e Selezionare) e perché Ottenere è, come già detto, cronologicamente l'ultima delle cinque funzioni. Un'ultima classificazione delle tipologie indicali è quella, sulla base della fonte dell'indicizzazione, e relativa alle sole entità bibliografiche, tra indici derivati e assegnati. Una parte degli elementi informativi di un'entità bibliografica può essere derivata direttamente da essa o da entità bibliografiche associate (per esempio le edizioni di un'Opera, oppure, nel caso del nome di un Ente, dal sito ufficiale dell'Ente); gli elementi così indicizzati sono detti indici derivati.

Una parte degli elementi informativi di un'entità bibliografica può essere invece costituita da: 1) elementi segnici presenti nell'entità bibliografica ma totalmente rielaborati dall'indicizzatore; 2) elementi non segnici dell'entità bibliografica, la cui registrazione è necessariamente frutto di un esame dell'entità bibliografica da parte dell'indicizzatore; sono elementi di questo tipo il tipo di supporto, le dimensioni, etc.; 3) elementi assenti in quanto tali dall'entità bibliografica ma escogitati dall'indicizzatore sulla base di un'analisi dell'entità bibliografica. In tutti questi tre casi parleremo di indici assegnati.

Parlando del soggetto, avevamo spiegato che esso è costituito in parte da elementi espliciti (circalità estensionale), in parte da elementi impliciti (circalità intensionale), in parte da elementi che possono essere espliciti o impliciti (punto di vista). Gli elementi impliciti non possono che essere frutto della riflessione dell'indicizzatore e dunque essere registrati come indici assegnati. Diversa è la questione per la circalità estensionale: questa può essere o individuata dall'indicizzatore sulla base di una preventiva analisi dei termini e discorsi di un Testo, e dunque tradotta successivamente in un'espressione sintetica scelta dall'indicizzatore - sulla base di un controllo formale, cioè dell'utilizzo di uno strumento di indicizzazione, o meno -, o semplicemente essere determinata tramite una trascrizione 
(manuale o automatizzata) di parti attinenti la circalità di un Testo come titoli, sommari, abstract, quarte di copertina, etc. Vi sono pro e contro in entrambi i casi: l'indicizzazione assegnata è frutto di una mediazione dell'indicizzatore e dunque soltanto essa permette di mettere in rapporto la circalità estensionale con le esigenze dell'utente finale; mentre l'indicizzazione derivata si ferma all'aspetto esteriore dei segni delle parole (significanti, come spiegheremo nel prossimo capitolo, vedi 3.2.1), l'indicizzazione assegnata permette di risalire ai significati delle parole; le formulazioni (termini o frasi) tipiche dell'indicizzazione assegnata sono generalmente maggiormente sintetiche di quelle ottenute tramite l'indicizzazione derivata; l'indicizzazione assegnata permette di differenziare lingua del testo e lingua dell'indicizzazione, ovvero permette di raggruppare tutti i testi su un determinato argomento a prescindere dalla loro lingua; l'indicizzazione assegnata è però molto più costosa di quella derivata. Così come abbiamo fatto nella discussione del soggetto, e pur nella consapevolezza di una complessiva superiorità qualitativa dell'indicizzazione assegnata su quella derivata, anche in questo caso suggeriamo di vedere indici assegnati e derivati non come alternativi ma piuttosto come complementari tipi di indice: li considereremo entrambi come indici del nostro approccio, con l'indicazione che uno strumento di indicizzazione dovrebbe prevedere l'impiego di entrambi i tipi di indice, magari differenziandone l'utilizzo; per esempio, come già accennato, prevedendo un'indicizzazione per soggetto di livello complessivo di carattere assegnato, e un'indicizzazione per soggetto di livello analitico di carattere derivato. In questo caso, gli indici di soggetto derivati potrebbero integrare o sostituire l'indicizzazione assegnata dei sottotemi subordinati al tema principale di un'entità testuale. L'opportunità dell'integrazione di indici derivati e assegnati è naturalmente estendibile anche agli attributi non relativi al soggetto: frontespizio, copertina, illustrazioni, etc. possono infatti essere oggi facilmente registrate o collegate all'interno di una registrazione tramite digitalizzazione.

Passiamo adesso alla discussione delle singole tipologie di indici; discuteremo soltanto quelle più importanti o su cui è opportuno fare delle precisazioni.

\subsection{Indici nominali interni}

Attributi nominali (nomi, titoli, termini, etc.) interni dell'entità possono essere rappresentati a livello indicale come indici nominali interni; di essi abbiamo già parlato più sopra nel testo (vedi $2.13,2.14$ ). Gli indici nominali interni sono punti di accesso. Se un'entità presenta più nomi, questi possono essere registrati come altrettanti indici nominali interni; la registrazione dei diversi nomi di un'entità equivale al controllo della relazione di equivalenza tra questi nomi. Porre sotto controllo formale i diversi nomi di un'entità favorisce enormemente la possibilità di Trovare un'entità. Quando gli indici nominali interni traducono nomi complessi, è possibile controllare esplicitamente le entità e relazioni coinvolte dal nome complesso: in questo caso, come detto, si avranno indici (nominali interni) collegati. I diversi tipi di nome, termine o titolo dell'entità possono essere modellati anche tramite indici nominali differenziati: nel caso dei nomi, sulla base della lingua, della completezza del nome, delle regole di indicizzazione di riferimento, etc.; nel caso di titoli, sulla base del tipo di titolo: titolo proprio, alternativo, sottotitolo, etc.; nel caso dei termini, sulla base del carattere scientifico o popolare del 
Tesi di dottorato di Giuliano Genetasio, discussa presso l'Università degli Studi di Udine

termine, della forma sciolta o contratta, della diversa grafia, etc.

\subsection{Indici nominali esterni}

Parliamo adesso della rappresentazione indicale degli attributi esterni di un'entità, ovvero degli indici nominali esterni; tutti gli indici nominali esterni sono punti di accesso, e possono essere controllati o non controllati; soltanto alcuni di essi possono, sulla base della natura semplice o complessa dei relativi nomi, essere collegati o non collegati. Le date di un'entità possono essere registrate come indici di data. Gli indici di data collegano un'entità a un Tempo; nel caso delle entità bibliografiche, possono essere derivati (se desunti dall'entità bibliografica o da fonte esterna) o assegnati (se frutto di una valutazione o congettura dell'indicizzatore). Ai differenti tipi di data possono corrispondere differenti tipi di indice di data.

I luoghi relativi a un'entità possono essere registrati come indici di luogo. Gli indici di luogo collegano l'entità a un Luogo; nel caso delle entità bibliografiche, possono essere derivati (se desunti dall'entità bibliografica o da fonte esterna) o assegnati (se frutto di una valutazione o congettura dell'indicizzatore). I differenti tipi di luogo possono essere registrati come differenti tipi di indice di luogo.

Abbiamo già detto della rappresentazione indicale delle relazioni di equivalenza e delle relazioni di omonimia e polisemia; parliamo adesso brevemente della rappresentazione indicale delle altre relazioni semantiche paradigmatiche, che approfondiremo però soprattutto nel prossimo capitolo (vedi 3.3.6.4.2.2 e ss.).

Gli attributi relativi alle relazioni gerarchiche generiche che un'entità intrattiene con altre entità possono essere registrati come indici gerarchici generici (iperonimici e iponimici) e, se controllati formalmente, con relativi collegamenti gerarchici generici, che collegano l'entità registrata a qualsiasi tipo di entità collettiva (Concetti, Tipi di persona, Tipi di ente, Tipi di testo, Tipi di risorsa, Altre entità, etc.). Nella trattazione delle relazioni gerarchiche generiche, abbiamo spiegato come nel nostro approccio concettuale esse siano previste anche per entità del Gruppo 1 e del Gruppo 2; anticipando la discussione della loro trattazione indicale, abbiamo anche detto che nonostante il controllo formale delle relazioni gerarchiche generiche tra questi tipi di entità non sia, attualmente, previsto dalla maggior parte degli strumenti di indicizzazione, esso sarebbe invece opportuno, perché importante per le funzioni catalografiche (Trovare e Navigare in particolare). Gli attributi relativi alle relazioni semiche e sememiche, che approfondiremo nel prossimo capitolo (vedi 3.2.5.3.3 3.3.6.4.2.2.2.4), possono essere registrati come indici gerarchici sememici e semici. Gli attributi relativi agli antonimi, che approfondiremo prossimo capitolo (vedi 3.2.5.3.3 3.3.6.4.2.2.4), possono essere registrati come indici antonimici.

Gli attrbuti relativi alle relazioni partitive che un'entità intrattiene con ulteriori entità possono essere registrati come indici gerarchici olonimici (se l'ulteriore entità rappresenta la parte) o meronimici (se l'ulteriore entità rappresenta l'intero), e, se controllati formalmente, con relativi collegamenti partitivi.

Non approfondiremo qui la rappresentazione indicale delle relazioni sintagmatiche e delle relazioni di antonimia; lo faremo direttamente nel prossimo capitolo (vedi 3.2.5.3.3, 3.2.7, 3.3.6.4.2.2.4, 3.3.6.4.3 e ss.). 
Per quanto riguarda gli indici che coinvolgono entità del Gruppo 1 in rapporto a entità del Gruppo 2, ad attributi di provenienza e omaggio corrispondono indici (nominali esterni) di provenienza e omaggio e, se controllati formalmente, relativi collegamenti. Se la responsabilità di un'entità bibliografica sono note, perché dichiarate all'interno di una risorsa o ricavate in altro modo (ricerche bibliografiche, etc.), possono essere registrate come indici di responsabilità che, se controllati formalmente, collegano l'entità bibliografica con i nomi di Persone o Enti che esercitano un qualche tipo di responsabilità sull'entità bibliografica. Le diverse tipologie di responsabilità possono essere modellate tramite differenti tipi di indici di responsabilità. È teoricamente possibile anche la registrazione degli indici di responsabilità che una Persona o un Ente esercita su entità bibliografiche; gli attuali strumenti di indicizzazione generalmente non prevedono questo tipo di indice, ma si tratta di una carenza non giustificata, e che invece faciliterebbe le funzioni catalografiche Trovare e Navigare.

Per quanto riguarda gli indici che coinvolgono tra loro entità del Gruppo 2, attributi di possesso, di affiliazione istituzionale e di componente familiare possono essere registrati tramite corrispondenti indici.

Gli "attributi primari” delle entità del Gruppo 1 possono essere registrati tramite corrispondenti indici primari; gli indici primari si differenziano in un punto fondamentale rispetto a tutti gli altri: essi devono essere necessariamente controllati. Gli attributi relativi alle varie relazioni che possono esistere tra un medesimo tipo di entità bibliografica (successione, complemento, riassunto, adattamento, derivazione, imitazione, riduzione, revisione, traduzione, arrangiamento, riproduzione, versione alternativa, riproduzione, riconfigurazione) possono essere registrati tramite corrispondenti indici.

L'attributo del tipo di supporto, tipico delle Risorse, può essere registrato tramite un corrispondente indice di tipo di supporto che, se controllato formalmente, collega la Risorsa con un Tipo di risorsa. Veniamo infine alla discussione degli indici esterni tipici dei Testi in rapporto ad altre entità. La lingua di un Testo può essere registrata tramite un corrispondente indice di lingua che, se controllato formalmente, collega il Testo a una Lingua. La forma testuale di un Testo può essere registrata tramite un corrispondente indice di forma testuale che, se controllato formalmente, collega il Testo a un Tipo di testo. I destinatari di un Testo possono essere registrati tramite un corrispondente indice di destinatari che, se controllato formalmente, collega il Testo a un Tipo di persona o un Tipo di ente.

\subsection{Indici non nominali corrispondenti ad attributi}

Gli indici non nominali, come accennato, possono essere suddivisi in quelli che corrispondono agli attributi non nominali delle entità, e in quelli che non corrispondono necessariamente a precisi attributi ma che comunque non si riferiscono a ulteriori entità: indici valutativi e metaindici.

Iniziamo parlando degli indici non nominali che corrispondono agli attributi non nominali. Gli identificatori di Opere, Manifestazioni, etc. possono essere rappresentati a livello indicale tramite corrispondenti indici di identificatore; gli indici di identificatore sono punti di accesso non controllati. 
Le caratteristiche fisiche di una Manifestazione (dimensioni, estensione, presenza di illustrazioni, altre caratteristiche fisiche) possono essere registrate tramite indici di descrizione fisica. Gli indici di descrizione fisica sono elementi descrittivi assegnati, perché ricavati da elementi non segnici della risorsa.

I differenti tipi di caratteristiche fisiche di una risorsa possono essere registrati tramite differenti tipi di indice di descrizione fisica.

Lo stato di conservazione di un Item può essere registrato tramite un corrispondente indice; l'indice di stato di conservazione è un elemento descrittivo assegnato.

Le condizioni di disponibilità fisica di un Item possono essere registrate tramite un corrispondente indice di disponibilità; l'indice di disponibilità è un elemento inventariale assegnato; a differenti tipi di condizioni di disponibilità (informazioni sull'accesso, sul prestito, sul prezzo, etc.) possono corrispondere indici di disponibilità differenziati. La collocazione fisica di un Item all'interno di una raccolta può essere registrata tramite la segnatura di collocazione; la segnatura di collocazione consiste di una formula numerica, alfabetica o simbolica o di una combinazione tra queste, la cui formulazione può avere più origini e può seguire più criteri - che non approfondiremo nel presente lavoro -; la segnatura di collocazione è un elemento inventariale assegnato.

\subsection{Indici valutativi}

Avevamo detto come nella visione dell'oggettivismo del soggetto, a tutt'oggi quella più diffusa, la funzione dell'indicizzazione sia intesa come eminentemente descrittiva e il più possibile neutrale. Abbiamo anche detto come quella della neutralità assoluta della descrizione sia un ideale nei fatti impossibile da perseguire. Al contrario, ogni descrizione ha una natura innanzitutto valutativa. Inoltre, e contro l'ideale della funzione esclusivamente descrittiva dell'indicizzazione, gli utenti finali hanno in anni recenti espresso sempre più fortemente l'esigenza di informazioni più marcatamente valutative, che, cioè, esprimano opinioni, giudizi o anche voti sui diversi oggetti di interesse; e hanno chiesto in modo sempre più forte di partecipare a questo processo valutativo esprimendo le proprie opinioni. Con Amazon (e siti simili) e con la diffusione del web sociale, l'impiego di indici assegnati non controllati (tag) di natura descrittiva ma anche valutativa da parte degli utenti finali è divenuta sempre più diffusa, fino a riguardare anche i cataloghi (cosiddetti Social OPAC o SOPAC).

Noi riteniamo che questo tipo di valutazioni, da parte dell'utente finale ma già dell'indicizzatore, non soltanto siano utili, ma che anzi possano contribuire in modo essenziale alla funzione Selezionare. ${ }^{131}$ Per questo motivo nel nostro approccio

131 L'importanza degli indici "sociali" (tipo web 2.0) e valutativi è evidenziata da KAREN CALHOUN JOANNE CANTRELL - PEGGY GALLAGHER - JANET HAWK, Online catalogs, cit., p. 17; cfr. anche LIBRARY OF CONGRESS. WORKING GROUP ON THE FUTURE OF BIBLIOGRAPHIC

CONTROL, On the Record, cit., p. 31: "Library users [...] value features and data that help them make sense of results by ranking, organizing, and clustering. Library catalogs have consciously presented a neutral and authoritative view of the bibliographic universe. Evaluative information, such as reviews and reading lists, has not traditionally been part of the library catalog"; cfr. anche JAMES D. ANDERSON - JOSÉ PÉREZCARBALLO, The nature of indexing. How humans and machines analyze messages and texts for retrieval. Part I, Research, and the nature of human indexing, cit., p. 250; cfr. anche TANJA MERČUN - MAJA ŽUMER, New generation of catalogues for the new generation of users. A comparison of six library catalogues, "Program", 42, 3 
prevediamo la possibilità di indici valutativi o di valutazione: essi esprimono valutazioni che possono a seconda dei casi riguardare o specifici attributi dell'entità (per esempio il soggetto: il tema o il rema), o l'entità nel suo complesso. I differenti oggetti delle valutazioni possono essere rappresentati anche tramite indici valutativi differenziati. Gli indici valutativi sono elementi descrittivi; nel caso degli indici valutativi, è opportuna la presenza di un relativo metaindice che segnali il nome della persona o agenzia responsabile dell'indicizzazione (vedi prossimo paragrafo): è importante sapere almeno se l'indicizzazione valutativa sia di origine professionale (indicizzatore) o amatoriale (utente finale).

\subsection{Metaindici}

I metaindici, o elementi metaindicali, non riflettono direttamente nessun attributo di un'entità ma piuttosto forniscono informazioni sugli indici stessi che registrano i vari attributi. I metaindici, inoltre, nascono principalmente per le funzioni gestionali del catalogo - quelle relative all'uso e alla manutenzione del catalogo da parte di indicizzatori e altri utenti professionali, che non tratteremo nel presente lavoro - e solo indirettamente sono utili alle funzioni dell'utente discusse; in particolare, la maggior parte dei metaindici è utile alla funzione Selezionare. Nel nostro modello prenderemo in considerazione solo una piccola selezione dei metaindici che si trovano in una registrazione: una discussione approfondita dei metaindici non rientra negli scopi del presente lavoro.

Un elemento metaindicale importante è il nome dell'agenzia bibliografica o dell'indicizzatore (professionale o amatoriale) che ha effettuato l'indicizzazione; esso può riferirsi all'indicizzazione dell'entità nella sua interezza, oppure gruppi di elementi indicali oppure ancora a singoli elementi indicali. I nomi dell'agenzia bibliografica e dell'indicizzatore sono importanti per l'utente finale (e già per gli indicizzatori) al fine di valutare la qualità e l'autorevolezza dell'indicizzazione e dunque, indirettamente, per la funzione di selezione.

Secondo elemento metaindicale rilevante è la data di indicizzazione: anch'essa può riferirsi a uno o più elementi indicali o all'indicizzazione nel suo complesso; la data di indicizzazione è utile a valutare la novità dell'indicizzazione e dunque, indirettamente, alla funzione di selezione; tra gli studiosi che hanno evidenziato l'importanza di segnalare la data di indicizzazione (in particolare riferendosi al soggetto) vi è Julius O. Kaiser. ${ }^{132}$ Altro elemento metaindicale importante è lo status dell'indicizzazione: se essa sia cioè completa o incompleta, rivista o non ancora rivista, etc. Lo status dell'indicizzazione può riferirsi a uno o più elementi indicali o all'indicizzazione nel suo complesso; esso è utile a valutare la qualità e l'autorevolezza dell'indicizzazione, dunque, indirettamente, anche alla funzione di selezione.

Vi sono poi le regole, strumenti, standard, etc. di indicizzazione; anch'esse sono un

(2008), p. 243-261, disponibile all'indirizzo <http://www.ff.uni-

lj.si/oddelki/biblio/oddelek/osebje/dokumenti/MercunZumer08_New_generation_of_catalogues_for_the _new_generation_of_users.pdf>.

132 Kaiser propone vari elementi aggiuntivi per la descrizione di soggetto, tra cui la "date of information" (data di indicizzazione), cfr. ELAINE SVENONIUS, Facet definition. A case study, "International classification" 5, 3 (1978), p. 138. 
elemento metaindicale che serve a valutare le modalità di indicizzazione e dunque utile, indirettamente, alla funzione di selezione.

La lingua di indicizzazione è un elemento metaindicale che serve a valutare le modalità di indicizzazione e dunque utile, indirettamente, alla funzione di selezione.

Un ulteriore elemento metaindicale importante è quello delle informazioni relative alle fonti consultate (con successo o meno) nell'indicizzazione, in riferimento a un particolare attributo o all'entità nel suo complesso; anch'esso fornisce informazioni sull'autorevolezza di una registrazione ed è, dunque, indirettamente utile alla funzione di selezione. Infine, un ultimo elemento metaindicale importante è l'identificatore della registrazione dell'entità. L'identificatore della registrazione si presenta generalmente in forma numerica o alfanumerica; esso serve soprattutto per scopi gestionali ma può servire anche all'utente finale per Trovare e Identificare con certezza l'entità oggetto della registrazione, e dunque costituisce un potenziale punto di accesso; come abbiamo detto sopra (vedi 2.19), l'identificatore della registrazione costituisce inoltre un espediente fondamentale per la disambiguazione di entità omonime.

\subsection{Indici di soggetto}

Risultato della confusione che esiste attualmente sulla nozione di soggetto è che il prodotto dell'analisi documentaria, l'indice di soggetto, è stato ed è una struttura impiegata con scopi e significati differenti; i nomi, termini, etc. utilizzati negli indici di soggetto possono anche essere identici, ma il loro senso può nondimeno differire sulla base del significato che l'indicizzatore ha di volta in volta teso a esprimere. Punto essenziale della nostra proposta di miglioramento dell'indicizzazione per soggetto è allora l'idea che sia necessario articolare l'indice di soggetto unico in più indici di soggetto corrispondenti ai diversi elementi componenti il soggetto, più sopra discussi: circalità estensionale (tema e rema), circalità intensionale, punti di vista.

L'eventuale differenziazione dell'indice di soggetto in più indici di soggetto è, lo ribadiamo, di tipo modulare e flessibile: ogni strumento di indicizzazione particolare può impiegare un numero diverso di indici. Un'articolazione completa dei diversi aspetti del soggetto in altrettanti indici è naturalmente la scelta più complessa ed impegnativa ma anche quella che, idealmente, permette di ottimizzare il soddisfacimento dell'interesse dell'utente. Con una fondamentale riserva: la distinzione dei diversi aspetti del soggetto e, di conseguenza, dei diversi indici di soggetto è condizione necessaria ma non sufficiente per un controllo del significato degli indici di soggetto; questi ultimi restano sempre etichette il cui significato d'uso è però stabilito sempre dagli indicizzatori al momento dell'indicizzazione; la definizione dei diversi tipi di relazioni e indici di soggetto mitiga, ma non annulla, la soggettività intrinseca all'indicizzazione.

La circalità estensionale di un'entità testuale può essere indicizzata tramite indici derivati e/o assegnati. Gli indici assegnati di circalità estensionale si dividono in indici di tema estensionale e indici di rema. Il tema o i temi di un Testo possono essere indicizzati tramite uno o più indici di tema estensionale; gli indici di tema estensionale sono punti di accesso e sono indici nominali esterni. Come abbiamo detto più sopra, in caso di più temi estensionali, l'eventuale rappresentazione formale delle relative relazioni di coordinazione o subordinazione può avvenire tramite tipologie differenti di indici; la 
relazione di coordinazione non necessita di tipologie particolari di indici; il controllo formale della relazione di subordinazione tra un tema generale e uno o più sottotemi può invece avvenire tramite l'utilizzo di specifici indici di sottotema estensionale per i sottotemi. Gli indici di tema estensionale possono essere controllati o non controllati; se controllati, e relativi a nomi complessi, possono essere collegati o non collegati. Il rema di un Testo può essere indicizzato tramite un indice di rema; l'indice di rema è un elemento descrittivo, concettualmente dipendente dall'indice o dagli indici di tema estensionale. La circalità estensionale di un testo può essere indicizzata anche tramite uno o più indici derivati; gli indici derivati di circalità estensionale sono prodotti da una trascrizione automatizzata o manuale di una o più parti pertinenti la circalità estensionale di un testo: titoli, sommari, abstract, indici, etc. ${ }^{133}$ Ogni parte può esser trascritta in un diverso indice derivato di circalità estensionale. Gli indici derivati di circalità estensionale non permettono di distinguere tema e rema: essi possono, a seconda dei casi, riguardare il tema, il rema o entrambi, anche all'interno di uno stesso indice. Gli indici derivati di circalità estensionale sono indici nominali esterni non controllati.

Una nota sul rapporto tra indici derivati e assegnati relativi alla circalità estensionale. L'uso di indici assegnati o derivati di circalità estensionale è una scelta pertinente ai singoli strumenti di indicizzazione. Tuttavia, e come abbiamo già detto, gli indici derivati di circalità estensionale dovrebbero idealmente integrare (piuttosto che sostituire) gli indici assegnati di circalità estensionale. Nel caso di una compresenza di indici assegnati e derivati di circalità estensionale, è opportuno che i diversi tipi di indice riguardino diversi livelli di analiticità dell'indicizzazione: agli indici assegnati di tema estensionale e rema spetterebbe il compito di rappresentare sinteticamente e a un livello generale la circalità estensionale dell'entità; agli indici derivati di circalità estensionale spetterebbe il compito di rappresentare la circalità estensionale delle diverse parti dell'entità. L'indice di rema può essere invece sostituito senza problemi da un abstract, se presente, perché gli abstract esprimono generalmente sia il tema che il rema di un testo.

La circalità intensionale di un Testo può essere rappresentata tramite uno o più indici di circalità intensionale; gli indici di circalità intensionale sono punti di accesso e sono indici nominali esterni di natura assegnata (la circalità intensionale esclude per definizione una possibilità di derivazione). Gli indici di circalità intensionale sono concettualmente dipendenti da uno o più attributi testuali, ma sono tuttavia autonomi ai fini dell'interesse dell'utente e delle sue ricerche. Abbiamo visto sopra (vedi 2.16.4) come la circalità intensionale di un'entità testuale sia una nozione complessa, che coinvolge $\mathrm{i}$ significati impliciti del messaggio di un Testo così come dei suoi altri attributi; abbiamo anche detto come la circalità intensionale sia strettamente correlata alle relazioni semantiche paradigmatiche delle altre entità i cui nomi costituiscono i vari attributi dell'entità testuale. Quando tali relazioni semantiche paradigmatiche siano state registrate formalmente, la rappresentazione indicale della circalità intensionale creerebbe in alcuni casi una ridondanza, seppur indiretta: ciò che fosse registrato come attributo semantico paradigmatico di una prima entità dovrebbe in alcuni casi essere registrato anche come parte della circalità intensionale dell'entità testuale il cui nome della prima entità costituisca uno degli attributi. Per questo motivo, il grado e le modalità della rappresentazione indicale della circalità intensionale di un Testo non possono essere

133 Cfr. KAREN CALHOUN - JOANNE CANTRELL - PEGGY GALLAGHER - JANET HAWK, Online catalogs, cit., p. 17. 
Tesi di dottorato di Giuliano Genetasio, discussa presso l'Università degli Studi di Udine

stabilite in modo assoluto a priori, ma vanno valutate di volta in volta da strumento di indicizzazione a strumento di indicizzazione, e forse addirittura da caso a caso. Per quanto riguarda il nostro approccio, suggeriamo la rappresentazione integrale della circalità intensionale, anche quando ciò comporti una ridondanza indiretta: questa non inficia in nessun caso le funzioni catalografiche, ma piuttosto offre all'utente più percorsi per Trovare, Navigare, etc. le entità dell'universo bibliografico.

I punti di vista (ideologie, metodologie, etc.) possono essere registrati tramite uno o più indici (assegnati) di punto di vista. Gli indici di punto di vista sono punti di accesso e sono indici nominali esterni; essi sono concettualmente dipendenti dagli indici di tema estensionale, ma autonomi ai fini dell'interesse dell'utente e della ricerca.

\subsection{Indicizzazione alfabetica e sistematica per soggetto}

In una nota all'inizio del capitolo avevamo premesso che il nostro discorso sull'indicizzazione per soggetto avrebbe riguardato la sola indicizzazione alfabetica (o indicizzazione verbale o soggettazione), non quella sistematica (o classificata o classificazione bibliografica). Adesso è giunto il momento di parlare anche delle classificazioni, e dei motivi per cui abbiamo deciso di non includerle esplicitamente nel nostro approccio concettuale.

Soggettazione e classificazione sono tradizionalmente state ritenute ambiti affini ma distinti. Sono state accomunate perché entrambe riguardavano l'argomento o il significato, il messaggio di un testo. Più spesso, pur notandone la parentela concettuale, sono però state distinte: perché - si diceva tradizionalmente - funzionali a scopi in parte differenti (trovare opere su determinati argomenti per la soggettazione, trovare opere su determinati argomenti ma anche collocazione dei libri a scaffale, per la classificazione), o relative a diversi oggetti (l'argomento specifico di un'opera per la soggettazione, la disciplina per la classificazione; un'entità individuale per la soggettazione, una classe di entità per la classificazione), o rivolte a diversi utenti (nella concezione biblioteconomica statunitense fino ai primi decenni del Novecento la soggettazione è rivolta a tutti, ma soprattutto all' "uomo della strada" che ignora specifici autori o titoli, la classificazione è invece rivolta alla persona colta, che ha una chiara visione delle discipline e delle loro articolazioni), o ancora caratterizzate da differenti modalità di organizzazione delle informazioni (in forma verbale e diretta, con termini che presentano l'argomento in isolamento rispetto agli altri argomenti; in forma simbolica e sistematica, con notazioni di classificazione che presentano l'argomento in contesto rispetto agli altri argomenti correlati).

Benché la separazione tra soggettazione e classificazione rimanga la posizione ancora oggi dominante, ormai da diversi decenni si sono levate voci tese a ridimensionare, quando non ad annullare, le differenze tra soggettazione e classificazione: esse sono cioè state considerate come differenti modalità di organizzazione, o forse solo di presentazione, dello stesso tipo di oggetto. ${ }^{134}$

134 Cfr. ALFREDO SERRAI, Indici logica e linguaggio. Problemi di catalogazione semantica, Roma, Consiglio nazionale delle ricerche, Laboratorio di studi sulla ricerca e sulla documentazione scientifica, 1974, p. 43-48; cfr. anche TERESA GRIMALDI, L'indicizzazione dal punto di vista cognitivo, cit., p. 287; cfr. anche W. JOHN HUTCHINS, Linguistic processes in the indexing and retrieval of documents, "Linguistics", 61 (1970), p. 
Noi ci schieriamo a favore di questa seconda posizione. Soggettazione e classificazione riguardano un solo e medesimo oggetto: il soggetto. La differenza tra argomento e disciplina è in alcuni casi una differenza tra due entità correlate (disciplina e suo oggetto di studio), in altre una semplice differenza di specificità tra una disciplina e le sue articolazioni, cioè tra entità tra loro in relazione gerarchica generica, in altri ancora una differenza tra un tema e il punto di vista con cui è affrontato. Né è fondato sostenere che la soggettazione riguardi entità individuali e la classificazione classi di entità: nelle classificazioni, la possibilità di scendere fino al livello di entità individuali è stata spesso prevista per entità individuali la cui garanzia bibliografica fosse tale da richiedere una notazione di classificazione specifica. Le modalità di organizzazione delle informazioni sono state, questo sì, realmente differenti fino a quando e laddove la soggettazione consistesse soltanto di una lista di intestazioni di soggetto senza collegamenti tra loro. Con l'introduzione della rete sindetica (la rete di collegamenti semantici tra le entità di cui abbiamo sopra detto), e l'inclusione delle relazioni tipiche dei thesauri, la soggettazione si è però progressivamente avvicinata alla classificazione nelle modalità di organizzazione della conoscenza. A partire da quel momento la differenza tra l'ordinamento alfabetico (ascendente) e quello classificato (discendente) sono venute affievolendosi - la rete sindetica della soggettazione permettendo tanto di risalire quanto di scendere le gerarchie concettuali. Tradizionalmente si è detto che la classificazione tendeva a riunire opere di argomento simile in un unico punto, mentre la soggettazione tendeva a disperdere opere di argomento simile in diversi punti del catalogo sulla base dell'ordinamento alfabetico; ciò è vero, ma la rete sindetica riduce fortemente questa differenza, mitigando l'effetto dispersivo dovuto alle diverse lettere iniziali di argomenti correlati. Uno dei vantaggi della classificazione rispetto alla soggettazione, si diceva tradizionalmente, era la sua maggiore contestualità, ovvero il fatto che essa presentasse ogni argomento in contesto, con le relazioni gerarchiche esplicitamente scandite nei diversi punti della notazione di classificazione, a differenza della soggettazione che presentava i termini di soggetto isolati tra loro. Ma l'esplicitazione delle relazioni semantiche implicata dalla rete sindetica della soggettazione cambia anche questo: $i$ termini di soggetto presentano anch'essi esplicitamente un contesto formato dai relativi termini più generici, specifici, etc.

Riassumendo, soprattutto a partire dall'introduzione della rete sindetica e del catalogo elettronico le differenze tra soggettazione e classificazione si riducano sensibilmente. Non totalmente: alcune differenze rilevanti tra soggettazione e classificazione nell'organizzazione e presentazione del soggetto restano ancora. Le classificazioni rimangono ancora oggi legate alla necessità di scegliere un unico elemento del soggetto sugli altri come elemento valido ai fini dell'ordinamento; ciò avviene perché la notazione di classificazione ha un compito doppio: da un lato quello della rappresentazione di un oggetto per sua natura polidimensionale (il soggetto), dall'altro quella della collocazione fisica di una risorsa; questa doppia finalità diventa un problema nel momento in cui i vincoli che caratterizzano la seconda attività (collocazione fisica) si traducono in limiti della prima attività (rappresentazione del soggetto). ${ }^{135}$ Le classificazioni bibliografiche

31-34.

135 Cfr. IRENE WORMELL, Subject access redefined. How new technology changes the conception of subject representation, in Knowledge organization and quality management. Proceedings of the third International ISKO Conference, 20-24 June 1994, Copenhagen, Denmark, organized by the Royal School of Librarianship, 
tradizionali sono fondate, ai loro più alti livelli, sulla divisione delle discipline.

L'organizzazione basata sulle discipline tipica delle classificazioni tradizionali è tuttavia più fortemente afflitta dalla storicità del soggetto di cui abbiamo parlato; le discipline si determinano storicamente e sono soggette a cambiamenti lenti ma inesorabili: vecchie discipline muoiono o si fondono, nuove discipline nascono, in alcuni casi (per esempio l'informatica) affermandosi prepotentemente sulle altre discipline fino a divenirne base o strumento applicativo indispensabile; altre discipline, prima considerate branche di una materia, acquistano una loro autonomia; negli ultimi decenni, l'interdisciplinarietà, piuttosto che l'appartenenza a una disciplina predefinita, è diventata la norma, e il paradigma di serendipità ha messo in crisi un'organizzazione della conoscenza fondata su rigidi steccati tra le discipline. ${ }^{136}$

Infine, un'ultima differenza evidente tra soggettazione e classificazione è che la prima presenta il soggetto in forma di termini ed espressioni verbali in una delle lingue naturali, mentre la seconda presenta il soggetto in forma di notazione simbolica (numerica, alfabetica o mista; a cui fa da complemento l'utilizzo di forme verbali ausiliarie, dette equivalenti verbali). Si tratta di una differenza formale, ma rilevante. È in virtù di tale differenza che le classificazioni costituiscono una lingua (artificiale) a valore internazionale, mentre gli strumenti di soggettazione rimangono vincolati a una singola lingua naturale. Questa caratteristica costituisce un punto di forza delle classificazioni, pur tuttavia viziato da alcuni problemi: 1) benché la forma delle classificazioni sia internazionale, i loro presupposti semantici e culturali rimangono legati alla lingua e alla cultura d'origine della classificazione: la classificazione decimale Dewey, per fare un esempio, rimane concettualmente legata all'universo culturale degli Stati Uniti di fine Ottocento e alla semantica della lingua inglese; le classificazioni presentano questo e altri problemi tipici delle "lingue universali" bene evidenziati da Umberto Eco nel saggio La ricerca della lingua perfetta nella cultura europea $;{ }^{137}$ ritorneremo sulla problematicità delle lingue universali nel prossimo capitolo (vedi 3.2.2, 3.3.2); 2) è controverso se per l'utente finale sia più utile una lingua internazionale che però deve essere appresa ex novo (la classificazione), oppure una lingua naturale straniera ma magari diffusa. Date le suddette condizioni, riteniamo che gli strumenti di indicizzazione alfabetica siano complessivamente più efficaci per la rappresentazione indicale del soggetto rispetto alle classificazioni bibliografiche. Non riteniamo dunque indispensabile l'uso di notazioni di classificazione per la rappresentazione indicale del soggetto; piuttosto, all'interno del

Copenhagen, Denmark in cooperation with the International Society for Knowledge Organization, Germany, with support from Dan Fink's Foundation, Denmark, edited by Hanne Albrechtsen and Susanne Oernager, Frankfurt/Main, Indeks, 1994, p. 433: "A book [...] By its inherent nature, it is a composite, poly-dimensional intellectual product which can hardly be put into a limited physical and monodimensional system occupying only one linear position”.

136 INTERNATIONAL SOCIETY FOR KNOWLEDGE ORGANIZATION. SEZIONE ITALIA. INTEGRATIVE LEVELS CLASSIFICATION, León manifesto, cit.: "The current trend towards an increasing interdisciplinarity of knowledge calls for essentially new knowledge organization systems (KOS), based on a substantive revision of the principles underlying the traditional discipline-based KOS [...] instead of disciplines, the basic units of the new KOS should be phenomena of the real world as it is represented in human knowledge. The new KOS should allow users to shift from one perspective or viewpoint to another, thus reflecting the multidimensional nature of complex thought. In particular, it should allow them to search independently for particular phenomena, for particular theories about phenomena (and about relations between phenomena), and for particular methods of investigation".

137 UMBERTO ECO, La ricerca della lingua perfetta nella cultura europea, 5. ed., Roma, Laterza, 2006. 
Tesi di dottorato di Giuliano Genetasio, discussa presso l'Università degli Studi di Udine

nostro modello prenderemo in considerazione le notazioni di classificazione come espediente opzionale e complementare all'indicizzazione di tipo alfabetico, in qualità di indici (punti di accesso) esterni, ma non ne approfondiremo la trattazione. Per le stesse ragioni, non approfondiremo lo studio delle classificazioni nemmeno nel prossimo capitolo.

\subsection{Oltre la separazione tra indicizzazione per autore e per soggetto, verso una visione unitaria dell'accesso bibliografico: seconda parte}

Nell'ambito dell'accesso bibliografico vige tradizionalmente una separazione tra indicizzazione per autore e per soggetto. A essere separati sono o sono stati i relativi oggetti, cataloghi e archivi di autorità, oltre che strumenti, figure e gruppi professionali. L'indicizzazione per soggetto riguarda tradizionalmente la rappresentazione indicale del soggetto e delle entità che fungono da soggetti, con i relativi attributi e le relative relazioni; l'indicizzazione per autore, tendenzialmente, tutto il resto: attributi e relazioni dei Testi - tranne il soggetto -, delle Risorse, di Persone ed Enti, etc; vi sono poi alcuni elementi di cui a seconda dei casi si occupa l'indicizzazione per autore, quella per soggetto, o entrambe: forma testuale, destinatari, lingua, sommari, per citarne solo alcuni. Alla differenza di oggetti è stata a volte collegata una differenza della natura fondamentale delle due attività: l'indicizzazione per autore sarebbe un'indicizzazione semiotica, rivolta agli elementi segnici delle risorse documentarie; l'indicizzazione per soggetto sarebbe un'indicizzazione semantica, rivolta ai significati. Nell'ambiente cartaceo all'indicizzazione per soggetto e per autore hanno corrisposto cataloghi differenti: catalogo per soggetto, nel primo caso; catalogo per autore, nel secondo. Quest'ultima differenza, come abbiamo detto più sopra (vedi 2.18.3), è oggi superata: con l'ambiente elettronico la separazione fisica tra catalogo per autore e catalogo per soggetto viene meno. Nell'ambiente odierno rimangono tuttavia spesso separati i relativi archivi di autorità: la separazione tra differenti tipi di registrazioni di autorità, a cui corrispondono differenti archivi di autorità, è quella che esiste attualmente in molti sistemi di accesso bibliografico. È, tra l'altro, anche la condizione che caratterizza l'authority system (il complesso degli archivi di autorità) della Library of Congress, scisso, secondo la denominazione non ufficiale di "Division of the world", in archivio dei "nomi" (in cui rientrano però anche i titoli) e archivio dei "soggetti". 138

Ma quanto è fondata questa separazione tra indicizzazione per autore e per soggetto? È adesso giunto il momento di approfondire la questione dei rapporti tra le diverse branche della catalogazione, per appurare se e in quale grado la loro separazione sia fondata, e quali ne siano le conseguenze.

Per quanto riguarda la supposta differenza tra la natura semiotica dell'indicizzazione per autore e la natura semantica dell'indicizzazione per soggetto, essa è infondata perché, come abbiamo detto nella discussione degli attributi nominali, l'indicizzazione nella sua totalità e i presupposti concettuali che ne sono alla base sono di natura semantica, in quanto presuppongono una selezione di natura semantica di elementi del linguaggio naturale da rappresentare.

138 LIBRARY OF CONGRESS. CATALOGING POLICY AND SUPPORT OFFICE, Subject Headings Manual, Washington, D.C., Library of Congress, Cataloging Policy and Support Office, 2008-, H 405. 
La separazione tra gli archivi di autorità per soggetto e per autore continua ad avere senso nell'ambiente elettronico? E il criterio che ne costituisce la base, la separazione di "nomi" e "soggetti" tipico della Library of Congress, è un criterio sensato? La risposta a entrambe le domande è negativa. Il criterio di creazione del tipo di registrazione di autorità sulla base di una condizione bibliografica (il soggetto) suscettibile di cambiare in modo contingente è profondamente problematico e ha storicamente comportato una forte incoerenza nell'authority system della Library of Congress: in passato, identici tipi di entità (edifici, luoghi, etc.) venivano registrati come "nomi" o "soggetti" sulla base dell'utilizzo bibliografico che ne doveva essere fatto in modo contingente, ovvero sulla base del tipo di attributo o relazione (responsabilità, soggetto, etc.) che l'entità di volta in volta svolgeva in rapporto con un'altra entità, e non sulla base della natura dell'entità in sé stessa. Il problema non è solo terminologico. Le entità registrate come "nomi" (Persone, Opere, etc.), infatti, possono anch'esse essere soggetti di altre entità. Viceversa, non è nemmeno possibile ammettere che i Concetti - per parlare solo delle entità tradizionalmente caratteristiche dell'indicizzazione per soggetto -, registrati come "soggetti", si limitino a fungere esclusivamente da attributi di soggetto; abbiamo infatti già visto come essi possano intrattenere relazioni semantiche paradigmatiche con le altre entità. La differenza tra "nomi" e "soggetti", e quella correlata tra differenti tipi di registrazioni di autorità, va allora abbandonata.

Il passo decisivo in direzione di un superamento di una rigida separazione tra indicizzazione per autore e per soggetto è stato compiuto da FRBR: il modello dell'IFLA ha riportato la differenza tra indicizzazione per soggetto e per autore a una differenza tra tipi differenti di relazioni - e attributi, nel nostro approccio -, entro un quadro di entità e funzioni fondamentalmente unitario. Si tratta di un punto ben evidenziato da Pino Buizza, il quale ha però anche notato come FRBR abbozzasse soltanto questa unificazione della catalogazione per autore e per soggetto, senza svilupparla. ${ }^{139}$ I modelli successivi dell'IFLA, FRAD e FRSAD, hanno invece ridimensionato la portata rivoluzionaria di FRBR, accettando, fin dal loro titolo, la separazione tradizionale tra indicizzazione per autore e per soggetto e relative registrazioni di autorità. FRSAD, il modello che sviluppato l'analisi delle registrazioni di autorità "per soggetto", ha poi posto per la sua analisi il soggetto come (super-)entità, non più come relazione come avveniva in FRBR, ritornando con ciò anche a una visione ingenua del soggetto come qualcosa di esistente in sé. Lasceremo da parte FRAD e FRSAD, e ripartiremo da FRBR per ripensare i rapporti tra

139 PINO BUIZZA, Indicizzazione per soggetto e FRBR, cit., La tradizione ha separato catalogazione descrittiva e indicizzazione per soggetto, perché ciascuna maturasse i propri principi e affinasse le proprie tecniche. Nel Rapporto FRBR, con i limiti anzidetti, autori e soggetti sono portati a incontrarsi intorno alle opere e alle espressioni. In virtù delle relazioni fra soggetto e opera e delle relazioni fra le entità dei gruppi uno e due di FRBR, tipiche della ricerca semiotica, si ricompone l'unità del record e del catalogo, la complessa globalità della rete di relazioni, resa interamente percorribile secondo mappe e percorsi opzionali selezionabili. Per esempio, un'entità persona o ente può relazionarsi distintamente a un'opera come autore o creatore, come concetto componente del soggetto, come responsabile della realizzazione di un'espressione, come tipografo o editore di una manifestazione, come proprietario o restauratore di un item, e senza ingenerare confusione svolgere ciascuna di queste funzioni rispetto a diverse opere, espressioni, manifestazioni, item, perché tutte le entità sono collegabili, ma i tipi di relazioni sono distinti. Una migliore interazione dei cataloghi, che in FRBR non è tema svolto, può essere immaginata come stimolo e direzione del lavoro futuro [...] L'assetto stesso dei cataloghi elettronici attuali, con la possibilità di ricerche su tutto il record (key anywhere) e di ricerche combinate su diversi elementi, scardina l'abitudine all'uso di cataloghi separati e pone con forza il problema di ripensare l'offerta variegata di accessi". 
indicizzazione per autore e per soggetto, concependo la differenza tra indicizzazione per autore e per soggetto come differenza tra attributi e relazioni differenti, all'interno di una visione sostanzialmente unitaria (entità e funzioni) dell'accesso bibliografico. Tale unitarietà è strettamente correlata, nell'ambito del nostro approccio concettuale, alla comune natura semantica dell'indicizzazione per autore e per soggetto. Ogni entità appartiene a o comprende idealmente altre entità, ovvero intrattiene relazioni gerarchiche con altre entità, più una serie di ulteriori relazioni semantiche paradigmatiche; ciò rende possibile la concezione di un'unica rete sindetica: una semantica bibliografica unitaria dell'indicizzazione per autore e per soggetto che, assieme al lessico comune costituito dalle entità, potrebbe persino costituire la base per uno strumento unificato di indicizzazione per autore e per soggetto - svilupperemo questa idea nel prossimo capitolo (vedi 3.3.4.6, 3.3.6.2 e ss., 3.3.6.4.2.2.7).

Al carattere unitario delle relazioni semantiche paradigmatiche delle entità è connesso un altro elemento fondamentale della nostra visione unitaria dell'indicizzazione: la nostra concezione del soggetto, e in particolare della circalità intensionale, poggia sull'idea che il significato implicito di un testo si determini non solo a partire dagli argomenti di cui il testo parla, ma anche dai significati implicati dagli altri elementi testuali tipici dell'indicizzazione per autore. 
Tesi di dottorato di Giuliano Genetasio, discussa presso l'Università degli Studi di Udine

\section{Lista riassuntiva delle entità}

Opera

Edizione

Contenuto editoriale

Manifestazione

Item

Persona

Ente

Famiglia

Oggetto

Concetto

Evento

Luogo

Tempo

Lingua

Tipo di persona

Tipo di ente

Tipo di testo

Tipo di risorsa

Altre entità (tipi di oggetto, tipi di evento, tipi di luogo, tipi di animale, animali, personaggi fittizi, etc.) 


\section{Lista riassuntiva degli attributi e delle relazioni}

\section{Legenda}

Attributi non nominali $\underline{X}$

Attributi nominali

Interni $\underline{T}$

Esterni $\underline{E}$

Nome dell'entità $\underline{T}$

Date (data di composizione, di copyright, di pubblicazione, etc. $\underline{E}$

Luoghi (di creazione, modifica, produzione, pubblicazione, etc.) $\underline{E}$

Iperonimi e iponimi (termine generico/termine specifico) $\underline{E}$

Semi (non generici) e sememi $\underline{E}$

Olonimi e meronimi (tutto/parte) $\underline{E}$

Sinonimi ed elementi equivalenti $\underline{T}$

Omonimia e polisemia $\underline{E}$

Ruoli semantici (relazioni sintagmatiche)*

Antonimi $\underline{E}$

Provenienza $\underline{E}$

Omaggio $\underline{E}$

Responsabilità** $^{*}$

Possesso $\underline{E}$

Affiliazione istituzionale $\underline{\mathrm{E}}$

Componente familiare $\underline{E}$

Attributi primari $\underline{E}$

Attributi relativi a relazioni tra Opere (successione, complemento, riassunto, adattamento, derivazione, imitazione) $\underline{\mathrm{E}}$

Attributi relativi a relazioni tra Espressioni (riduzione, revisione, traduzione, arrangiamento) $\underline{E}$

Attributi relativi a relazioni tra Manifestazioni (riproduzione, versione alternativa) $\underline{E}$ Attributi relativi a relazioni tra Item (riproduzione, riconfigurazione) $\underline{E}$

Tipo di supporto $\underline{\mathrm{E}}$

Lingua $\underline{E}$

Forma testuale $\underline{\mathrm{E}}$

Destinatari $\underline{E}$

Soggetto

Circalità estensionale

Tema $\underline{E}$

Rema $\underline{X}$

Circalità intensionale $\underline{E}$

Punto di vista $\underline{E}$

Identificatore dell'Opera $\underline{X}$

Identificatore della Manifestazione $\underline{X}$

Caratteristiche fisiche $\underline{X}$ 
Tesi di dottorato di Giuliano Genetasio, discussa presso l'Università degli Studi di Udine

Stato di conservazione $\underline{X}$

Condizioni di disponibilità $\underline{X}$

Collocazione fisica $\underline{X}$

* I ruoli semantici, approfonditi nel prossimo capitolo (vedi 3.2.7, 3.3.6.4.3), non rientrano nella classificazione degli attributi sopra esposta (attributi nominali, non nominali; interni, esterni; etc.)

** Opzionalmente, i diversi tipi di responsabilità possono essere considerati attributi di tipo diverso 


\title{
Lista riassuntiva degli indici e dei collegamenti
}

\author{
Legenda \\ Indici I \\ Metaindici $\underline{\mathrm{M}}$ \\ Indici non nominali $\underline{X}$ \\ Indici nominali

$$
\text { interni } \underline{T}
$$$$
\text { esterni } \underline{E}
$$ \\ Punti di accesso $\underline{P}$ \\ Elementi non ricercabili \\ descrittivi $\underline{\mathrm{D}}$ \\ inventariali $\underline{\mathrm{V}}$
}

Indice nominale interno ITP

Indici di data IEP

Indici di luogo IEP

Indici iperonimici e iponimici IEP

Indici semici e sememici IEP

Indici antonimici IEP

Indici olonimici e meronimici IEP

Indici di possesso IEP

Indici di provenienza IEP

Indici di omaggio $\underline{\text { IEP }}$

Indici di responsabilità ${ }^{*}$ IEP

Indici di affiliazione istituzionale IEP

Indici di componente familiare IEP

Indici primari IEP

Attributi relativi a relazioni tra Opere IEP

Attributi relativi a relazioni tra Espressioni IEP

Attributi relativi a relazioni tra Manifestazioni IEP

Attributi relativi a relazioni tra Item IEP

Indice di tipo di supporto IEP

Indice di lingua IEP

Indice di forma testuale IEP

Indice di destinatari IEP

Indici di soggetto

Indici di circalità estensionale IEP

Assegnati

Indice di tema estensionale IEP

Indice di rema $\underline{\mathrm{IX}}$

Derivati IEP

Indice di circalità intensionale IEP 
Tesi di dottorato di Giuliano Genetasio, discussa presso l'Università degli Studi di Udine

Indice di punto di vista IEP

Notazione di classificazione ${ }^{* *} \underline{\text { IEP }}$

Indice di identificatore dell'Opera IXP

Indice di identificatore della Manifestazione IXP

Indice di descrizione fisica IXD

Indice di stato di conservazione IXV

Indice di condizioni di disponibilità IXV

Segnatura di collocazione IXV

Indici valutativi $\underline{\mathrm{IXD}}$

Nome dell'agenzia bibliografica/indicizzatore MXD

Data di indicizzazione MXD

Status dell'indicizzazione MXD

Regole, strumenti e standard di indicizzazione $\underline{\text { MXD }}$

Lingua di indicizzazione $\underline{\mathrm{MXD}}$

Fonti dell'indicizzazione $\underline{\mathrm{MXD}}$

Identificatore della registrazione $\underline{\mathrm{MXP}}$

* Opzionalmente, i diversi tipi di responsabilità possono essere registrati tramite indici di tipo diverso

** Opzionale 


\section{Un approccio linguistico all'indicizzazione}

\subsection{Introduzione}

\subsubsection{Opportunità di un approccio linguistico}

Durante la discussione dei vari elementi del modello concettuale, abbiamo fatto riferimento a nozioni linguistiche come significato, significante, segni, relazioni semantiche, etc. Sarà adesso opportuno svilupparle.

L'approccio concettuale all'indicizzazione, pure fondamentale per la nostra discussione, è in sé insufficiente per uno studio approfondito dell'accesso bibliografico (e dell'indicizzazione). Questo è essenzialmente un processo comunicativo, ovvero linguistico. Linguistica è la natura di una parte rilevante delle entità, le entità testuali (Testi); linguistica è poi la natura della mediazione indicale sia da parte dell'utente finale che interroga il catalogo, sia da parte dell'indicizzatore che ne crea i contenuti. Appare allora evidente l'opportunità di sviluppare un approccio linguistico all'indicizzazione che estenda ed integri alcuni aspetti di quello concettuale.

Nel corso di tale approccio, cercheremo di sviluppare alcune ipotesi: 1) la rilevanza dello studio della linguistica per l'indicizzazione; e in particolare: 2) l'idea che gli strumenti di indicizzazione (regole, codici, standard di indicizzazione, sia per autore sia per soggetto) siano interpretabili linguisticamente, ovvero siano vere e proprie lingue (documentarie o indicali), ${ }^{140}$ il cui studio non può prescindere da quello del linguaggio tout court; 3) l'idea che, nonostante tutti o quasi gli aspetti degli strumenti indicali siano interpretabili linguisticamente, molti di essi necessitino inevitabilmente di una spiegazione che faccia ricorso a fattori extralinguistici, e nella fattispecie prettamente legati all'indicizzazione; ovvero che le lingue documentarie costituiscano lingue ausiliarie delle lingue naturali, in parte dipendenti da esse, in parte dipendenti dal contesto e dalle finalità dell'accesso bibliografico, per cui nascono; 4) l'idea che, sulla base dei punti 2 e 3, e al fine di migliorare gli strumenti di indicizzazione, sia fondamentale riavvicinare le lingue documentarie alle caratteristiche del linguaggio naturale da cui originano, verificando al contempo se e in quali aspetti sia opportuno che le lingue documentarie si discostino, per via delle loro finalità e caratteristiche indicali, dalla lingua naturale (come vedremo, la nostra analisi ha confermato solo parzialmente quest'ultima ipotesi). ${ }^{141}$

140 Sulla base della distinzione vigente in linguistica tra linguaggio, inteso come facoltà umana o come attività, e lingue, intese come sistemi linguistici particolari (ritorneremo più avanti su questa distinzione, vedi 3.2.1), parleremo generalmente di "lingue documentarie" per riferirci agli strumenti di indicizzazione dal punto di vista linguistico, riservando l'uso dell'espressione "linguaggio documentario" per riferirci in modo generico all'uso del linguaggio nell'accesso bibliografico e nell'indicizzazione. Va invece rifiutato, perché scorretto, l'utilizzo assai diffuso (e forse derivato da una traduzione erronea dell'inglese "language", che può indicare sia il linguaggio sia le lingue) delle espressioni "linguaggi documentari", "linguaggi di indicizzazione", etc. per riferirsi agli strumenti di indicizzazione.

141 L'idea del riavvicinamento delle lingue documentarie al linguaggio naturale fu già avanzata da W. JOHN HUTCHINS, Linguistic processes in the indexing and retrieval of documents, cit., p. 62: "We can see the value of improving ILs (whether used in manual or in automated systems) towards a closer proximity with 
Le prime due ipotesi sono in realtà ampiamente (seppure non universalmente) consolidate, nonché implicite nella nostra scelta di un approccio linguistico all'indicizzazione; riteniamo tuttavia proprio per questo indispensabile spiegarle, costituendo esse le premesse fondamentali del nostro discorso. Obiettivo centrale del nostro approccio linguistico sarà, sulla base delle summenzionate ipotesi, l'analisi e il miglioramento delle lingue documentarie, tramite l'elaborazione di un modello di lingua documentaria che possa servire al contempo come termine di confronto per l'analisi di specifiche lingue documentarie e come base per una loro costruzione, ${ }^{142}$ e di cui di volta in volta cercheremo di distinguere gli aspetti di natura prettamente indicale da quelli più marcatamente linguistici.

Dopo un'introduzione sulla letteratura relativa all'applicazione della linguistica e delle discipline correlate all'indicizzazione, dedicheremo una seconda parte del capitolo alla discussione delle caratteristiche del linguaggio naturale in sé (linguistica generale), e una terza parte all'applicazione della linguistica all'indicizzazione, ovvero alla discussione delle lingue documentarie e del nostro modello di lingua documentaria in particolare; non discuteremo comunque se non saltuariamente, poiché esulano dagli scopi del presente lavoro, gli aspetti relativi all'applicazione automatizzata della linguistica all'interpretazione e indicizzazione dei testi (linguistica computazionale).

\subsubsection{L'applicazione della linguistica all'indicizzazione: letteratura favorevole}

Avevamo posto come prima ipotesi la rilevanza dello studio della linguistica per l'indicizzazione; in realtà, si tratta di una "ipotesi" in realtà ampiamente consolidata. Tuttavia la rilevanza della linguistica per l'indicizzazione non è a dire il vero è universalmente accettata; esiste infatti una parte di autori, di certo minoritaria, che escludono o limitano fortemente l'idea dell'utilità della linguistica per l'analisi o il miglioramento dell'indicizzazione; di questa posizione renderemo conto nel prossimo

the structure of NL, so that they may express any concept which can be now, or may be in the future, expressed in NL. The ideal IL would presumably be the SL itself, for the following reasons: (i) Its semantic structure would be based on actual language usage and not upon the conceptual patternings of the designers of the IL [...] the possible conceptual classifications of the world are infinite but that the semantic structure of $\mathrm{NL}$ is relatively constant. (ii) Its syntactic structure would retain as much of the variety of NL syntax as necessary. It would not, unlike many existing ILs, abandon NL syntax altogether or set up a system of logical syntax unrelated to language habits [...] (iii) It would be as flexible and expressive as the best ILs, because, being based on NL structure, it would be able to express any concept which can be expressed in NL. (iv) If incorporated in an automated system, no SL $\rightarrow$ IL algorithm would, of course, be required, Thus, the translation processes would be greatly simplified. (v) Being based on language usage, it would be more easily adaptable to changes in semantic structure: in many fields of learning and research terminology is frequently redefined and new terms are created".

142 Idea già di JESSICA S. MELTON, A use for the techniques of structural linguistics in documentation research, in Classification research. Proceedings of the second International Study Conference held at Hotel Prins Hamlet, Elsinoore, Denmark, 14th to 18th September, 1964, edited by Pauline Atherton, Copenhagen, Munksgaard, 1965, p. 472. Cfr. anche ELAINE SVENONIUS, Directions for research in indexing, classification, and cataloging, "Library resources and technical services", 25, 1 (1981), p. 91: "A stumbling block in the way of developing better means of bibliographical control has been the lack of a general theory of bibliographic description. An instance of this, mentioned earlier, is the lack of a metalanguage suitable for the desription and comparison of various indexing languages". 
Tesi di dottorato di Giuliano Genetasio, discussa presso l'Università degli Studi di Udine

paragrafo.

Per discutere la questione della rilevanza della linguistica per l'indicizzazione, è utile iniziare facendo riferimento a un articolo di Amy J. Warner del 1991, che presenta una lista e una discussione della letteratura pertinente. ${ }^{143}$ Warner evidenzia come alcune aree della linguistica - in particolare la linguistica chomskyana - siano già state ampiamente studiate nell'ambito dell'indicizzazione; ${ }^{144}$ secondo l'autrice rimarrebbero tuttavia alcune aree della linguistica esplorate solo parzialmente dall'indicizzazione. ${ }^{145}$ A partire da un'analisi della letteratura bibliografica pertinente, Warner mostra come le citazioni a lavori di linguistica tendano a essere: 1) relative a un numero ristretto di lavori prodotti tra la fine degli anni Sessanta e l'inizio degli anni Settanta da un numero ristretto di linguisti (Chomsky e la sua scuola, principalmente); 2) relative sempre agli stessi lavori a distanza di decenni, ovvero senza seguire le novità emerse negli anni successivi in ambito linguistico; 3) relative soprattutto a semantica e sintassi, tralasciando invece tendenzialmente pragmatica e fonologia. ${ }^{146}$ Dopo questa panoramica iniziale, sostanzialmente corretta anche a distanza di circa vent'anni, ripercorriamo adesso cronologicamente la letteratura relativa alla questione della rilevanza della linguistica per l'indicizzazione e alle questioni correlate. Pioniere di uno studio linguistico dell'indicizzazione fu, negli anni Dieci del Novecento, Kaiser: la contrapposizione tra "concreti" e "processi" che costituisce uno dei fulcri del suo sistema, infatti, è grammaticale nella sua essenza, ricalcata com'è su quella tra argomenti/sostantivi e predicati/verbi. ${ }^{147}$

Per avere uno studio specificamente dedicato all'utilizzo della linguistica per l'indicizzazione bisogna tuttavia attendere la seconda metà degli anni Sessanta; è a partire da allora, e fino alla metà degli anni Settanta, che un gruppo di linguisti prestati alla documentazione producono i primi lavori importanti (e che tali rimangono ancora oggi): sono gli studi di Maurice Coyaud, Jean-Claud Gardin e W. John Hutchins. ${ }^{148}$

143 AMY J. WARNER, Quantitative and qualitative assessments of the impact of linguistic theory on informations science, "Journal of the American Society for Information Science", 42, 1 (1991), p. 64: "Linguistics has received attention in the literature of information science, indicating that a possible relationship exists or has existed between the two fields. Some authors have explored the interdisciplinary nature of information science and specifically mention linguistics as a relevant part of this discipline. These include discussions of information science as part of the "information sciences" of which linguistics is also a part [...] of the "communication sciences" [...] Others have attempted to outline specific areas where linguistics might benefit information science".

144 AMY J. WARNER, Quantitative and qualitative assessments of the impact of linguistic theory on informations science, cit., p. 70.

145 AMY J. WARNER, Quantitative and qualitative assessments of the impact of linguistic theory on informations science, cit., p. 64: "One might claim from the amount of discussion which has taken place that the relevance of linguistics to information science has been fully explored. However, the research reported on here will suggest that this is not the case and that in fact information scientists may have neglected portions of linguistics which are of potential value to the field".

146AMY J. WARNER, Quantitative and qualitative assessments of the impact of linguistic theory on informations science, cit., p. 65.

147JULIUS OTTO KAISER, Systematic indexing, London, Pitman, 1911.

148 MAURICE COYAUD, Introduction a l'ètude des langages documentaires. Thèse de doctorat du 3e cycle présentée à la Faculté des lettres et sciences humaines de Paris, Paris, Klincksieck, 1966; MAURICE COYAUD, Linguistique et documentation. Les articulations logiques du dicours, Paris, Larousse, 1972; R. C. CROS - JEANCLAUDE GARDIN - F. LÉVY, L'automatisation des recherches documentaires. Un modèle général 'Le SYNTOL', 2e édition revue et augmentée, Paris, Gauthier-Villars, 1968; JEAN-CLAUDE GARDIN, Semantic analysis 
Coyaud è forse il primo studioso nell'ambito dell'indicizzazione a utilizzare esplicitamente la linguistica per lo studio dell'indicizzazione, e in particolare a tentare un confronto sistematico tra linguaggio naturale e lingue documentarie; ${ }^{149}$ qualche anno dopo, Coyaud si spingerà fino a sostenere il rapporto inverso, ovvero la rilevanza dello studio dell'indicizzazione per la linguistica. ${ }^{150}$ Gardin evidenzia fin dalla fine degli anni Sessanta la rilevanza della linguistica, e in particolare della semantica, per l'indicizzazione; uno dei frutti del suo studio linguistico dell'indicizzazione è la lingua documentaria SYNTOL. W. John Hutchins, uno dei primi proponenti del concetto di circalità, ha prodotto nella prima metà degli anni Settanta alcuni lavori importanti, tra cui un monumentale studio delle lingue documentarie, rapportate al linguaggio naturale secondo le categorie tipiche della linguistica; in questo lavoro l'autore lamenta in apertura l'assenza di uno studio linguistico generale dell'indicizzazione (che lui stesso prova a fornire); tra le altre cose, sottolinea la rilevanza della linguistica testuale per l'analisi documentaria; ${ }^{151}$ l'imponente lavoro di Hutchins è tuttavia di taglio soprattutto descrittivo, non volto a un miglioramento delle lingue documentarie; esso non considera inoltre - ciò è per l'epoca inevitabile - alcune delle modificazioni fondamentali del linguaggio dovute al passaggio da un ambiente cartaceo a uno elettronico. La rilevanza della linguistica per l'indicizzazione viene in quegli anni notata, benché solo en passant poiché si tratta di lavori prettamente linguistici, da linguisti del calibro di Julien A. Greimas e dallo stesso Noam Chomsky. ${ }^{152}$ Un altro lavoro importante, forse il primo prodotto da autori che non sono linguisti, e risalente sempre alla prima metà degli anni Settanta, è quello di Karen Sparck-Jones e Martin Kay; si tratta di uno studio dedicato alla tematica dell'applicazione della linguistica all'indicizzazione automatizzata, che dal quel momento in poi sarà anche l'ambito di maggiore applicazione della linguistica all'indicizzazione (linguistica computazionale). ${ }^{153}$

procedures in the sciences of man, "Social science information", 8, 1 (1969), p. 17-42; JEAN-CLAUDE GARDIN, Document analysis and linguistic theory, "Journal of documentation", 29, 2 (1973), p. 137-168; W. JOHN HUTCHINS, Linguistic processes in the indexing and retrieval of documents, cit.; W. JOHN HUTCHINS, Languages of indexing and classification, cit.

149MAURICE COYAUD, Introduction a l'ètude des langages documentaires, cit., p. 5-6, 79-122.

150MAURICE COYAUD, Linguistique et documentation, cit., p. 11.

151 W. JOHN HUTCHINS, Languages of indexing and classification, cit., p. 1, 108.

152 ALGIRDAS JULIEN GREIMAS, Semantica strutturale, Roma, Meltemi, 2000, traduzione di Sémantique structurale. Recherche de méthode, Paris, Larousse, 1966, p. 98: "L'analisi semica del livello semiologico del linguaggio appare il compito primo della semantica strutturale. Tale analisi [...] renderebbe inoltre grandi servigi ai vari procedimenti della linguistica applicata [...] e anche alla costruzione di linguaggi documentari”; cfr. anche NOAM CHOMSKY, La grammatica trasformazionale. Scritti espositivi, Torino, Boringhieri, 1975, traduzione di Logical structures in language, A transformational approach to syntax, On the notion 'Rule of grammar', The formal nature of language, p. 30-31: "Finora ho discusso impostazioni della teoria del significato da un punto di vista abbastanza remoto dagli interessi pratici del campo della documentazione. Penso che ciò sia inevitabile. Lo studio del significato è disgraziatamente in uno stato di arretratezza e penso che non possa ancora pretendere di offrire risultati significativi, che abbiano immediate implicazioni pratiche per problemi come quelli del reperimento dell'informazione [...] Ciò che ho suggerito è che la teoria della struttura linguistica può riuscire a incorporare una parte di quell'enorme e confuso groviglio che è chiamato problema del significato [...] è stato suggerito che il tipo di linguaggio artificiale [...] potrebbe costituire un ponte tra il linguaggio comune e qualche tipo di linguaggio di macchina”. 153 KAREN SPARCK-JONES - MARTIN KAY, Linguistics and information science, New York, Academic Press, 1973. L'opportunità dell'applicazione della linguistica all'indicizzazione (automatizzata) è evidenziata da EUGENE GARFIELD, The relationship between mechanical indexing, structural linguistics and information 
Negli anni successivi diverranno sempre di più i lavori di teorici dell'indicizzazione riguardanti in modo più o meno diretto la tematica dei rapporti tra linguistica e indicizzazione. Risalgono a questo periodo alcuni importanti lavori di Svenonius, Derek Austin, Yves Gilbert Courrier e lo stesso Fairthorne. Austin, creatore del PRECIS, già nella seconda metà degli anni Settanta fa riferimento all'impiego dell'analisi trasformazionale nell'ambito dell'indicizzazione; qualche anno dopo, lo studioso britannico sottolineerà l'importanza del linguaggio naturale come fonte per l'elaborazione delle lingue documentarie. ${ }^{154}$ Courrier evidenzia la natura linguistica dell'indicizzazione e, dunque, il fatto che la teoria dell'indicizzazione non possa prescindere da uno studio del linguaggio e del significato. ${ }^{155}$ Fairthorne, a cui abbiamo fatto più volte riferimento nella discussione della circalità, ha evidenziato come quest'ultima, in quanto proprietà di un discorso, sia strettamente connessa ai problemi generali del linguaggio e del significato. ${ }^{156}$ L'attenzione di Svenonius alle problematiche linguistiche risale alla seconda metà degli anni Settanta, con l'idea di una sistematizzazione delle lingue documentarie attraverso uno studio di tipo linguistico, ed è stata una costante della sua produzione, culminata nel capolavoro The intellectual foundation of information organization (2000). Qui la studiosa americana, oltre a sottolineare in più luoghi la rilevanza della linguistica per l'indicizzazione, compie un importante passo in avanti nell'applicazione della linguistica: propone infatti la nozione di "lingua bibliografica", ovvero documentaria, per analizzare non soltanto gli strumenti dell'indicizzazione per soggetto, ma anche quelli (regole e codici di catalogazione) tipici dell'indicizzazione per autore; accomunando strumenti di indicizzazione per soggetto e per autore sotto l'etichetta dei lingue bibliografiche, Svenonius pone la pietra angolare per la costruzione di una visione unitaria della catalogazione. ${ }^{157}$

\subsubsection{L'applicazione della linguistica all'indicizzazione: discipline correlate e letteratura contraria}

Alcuni autori hanno menzionato altre discipline utili, oltre o più della linguistica, per l'indicizzazione: Julian Warner e Jacques Maniez, per esempio, hanno preferito parlare di una rilevanza della semiotica in generale (la scienza dei segni, comprensiva ma non limitata alla linguistica) per l'indicizzazione. Il già menzionato Gardin, invece, ha proposto

retrieval, "Journal of information science", 18, 5 (1992), p. 344.

154 JUTTA SØRENSEN - DEREK AUSTIN, PRECIS in a multilingual context. Part 2, cit., p. 111; DEREK AUSTIN, Basic concept classes and primitive relations, in Universal classification I. Subject analysis and ordering systems. Proceedings. 4th Internatl. Study Conference on Classification Research, 6th Annual Conference of Gesellschaft für Klassification e.V., Augsburg, 28 June-2 July 1982. Vol. 1, editor Ingetraut Dahlberg, Frankfurt, Indeks, 1982, p. 93.

155 YVES GILBERT COURRIER, Document analysis, verbs and case grammar, cit., p. 8, 10.

156 ROBERT A. FAIRTHORNE, Content analysis, specification, and control, "Annual review of information science and technology", cit., p. 78.

157 ELAINE SVENONIUS - HELEN F. SCHMIERER, Current issues in the subject control of information, "Library quarterly", 47, 3 (1977), p. 343; ELAINE SVENONIUS, The intellectual foundation of information organization, cit. p. xi, 194; cfr. anche RICHARD P. SMIRAGLIA, The progress of theory in knowledge organization, "Library trends", 50, 3 (2002), p. 344, il quale riconosce l'importanza dell'opera di Svenonius e in particolare della sua elaborazione delle "lingue bibliografiche". 
l'utilizzo della logica simbolica come base per entrambe la linguistica e l'indicizzazione. Jens-Erik Mai, infine, ha recentemente notato le affinità tra indicizzazione per soggetto e filosofia del linguaggio. ${ }^{158}$

Avevamo anticipato l'esistenza di una minoranza di autori che escludono o limitano la rilevanza della linguistica per l'indicizzazione. Tra costoro possiamo includere S. E. Robertson, che ha apertamente contestato l'utilità dell'applicazione della linguistica all'indicizzazione, ${ }^{159}$ ma in un certo senso la stessa Amy Warner, la quale sottolinea alcuni limiti intrinseci della linguistica; questa: 1) non sarebbe ancora riuscita a dare una spiegazione esaustiva di alcuni fenomeni linguistici importanti per l'indicizzazione, come la gestione dell'ambiguità e della sinonimia; 2) avrebbe indagato solo parzialmente la semantica e, soprattutto, la pragmatica. ${ }^{160}$

Pur sostenendo l'ipotesi che la linguistica sia effettivamente rilevante per l'indicizzazione, ciò non ci esime dall'evidenziare un limite fondamentale della linguistica e, dunque, del nostro approccio stesso: la linguistica è stata e rimane a tutt'oggi un campo di studi piuttosto frammentato, diviso in scuole diverse che faticano a comunicare tra loro, e che non ha ancora risolto alcuni dei suoi problemi maggiori; questo limite della linguistica incide ovviamente in modo negativo sul tentativo di applicarla all'indicizzazione.

\subsection{Nozioni di linguistica generale}

\subsubsection{Nozioni base della semiotica}

In questa seconda parte del capitolo forniremo un'introduzione ad alcuni dei concetti principali della linguistica (generale) rilevanti per l'indicizzazione; in alcuni casi andremo oltre un livello meramente introduttivo, approfondendo alcuni aspetti più complessi ma essenziali per il nostro approccio linguistico all'indicizzazione. I concetti che qui spiegheremo saranno utilizzati nella terza e ultima parte del capitolo (3.3 e ss.), dedicata allo studio linguistico dell'indicizzazione vero e proprio.

La linguistica studia il linguaggio, inteso come facoltà o sistema di comunicazione tramite segni linguistici, e le lingue, i particolari sistemi (naturali o artificiali, storici o inventati, etc.) attraverso cui si esprime il linguaggio. Parleremo dunque, come spiegato in nota, di

158 JULIAN WARNER, Semiotics, information science, documents and computers, "Journal of documentation", 46, 1 (1990), p. 17; cfr. anche JACQUES MANIEZ, Les langages documentaires et classificatoires. Conception, construction et utilisation dans les systèmes documentaires, Paris, Editions d'organisation, 1987, p. 280-281; JEAN-CLAUDE GARDIN, Document analysis and linguistic theory, p. 158; JENS-ERIK MAI, Semiotics and indexing, cit., p. 592.

159 STEPHEN E. ROBERTSON, Between aboutness and meaning, in Informatics 5. The analysis of meaning. Proceedings of a conference held by the ASLIB Informatics Group and the BCS Information Retrieval Specialist Group, 26-28 march 1979 Oxford, edited by Maxime McCafferty and Kathleen Gray, London, Aslib, 1979, p. 202 : "There is a persistent feeling, on both sides, that those of use who are concerned with documentary information retrieval have something to learn from linguistics [...] The feeling persists despite the evident lack of progress on this front [...] The feeling might e seen as deriving from a long term vision with a science fiction flavour, of an intelligent machine such as the Librarian in James Blish's "Cities in flight", which contains the record of human knowledge in its memory bans, and answers questions thereon". 160 AMY J. WARNER, Quantitative and qualitative assessments of the impact of linguistic theory on informations science, cit., p. 69-70. 
Tesi di dottorato di Giuliano Genetasio, discussa presso l'Università degli Studi di Udine

"lingue documentarie" o "bibliografiche", e non di "linguaggi documentari". Nell'ambito della linguistica, la linguistica generale cerca di studiare il linguaggio in sé, cioè a prescindere dalle particolari lingue; ciò che diremo d'ora in poi sarà prevalentemente riferito alla linguistica generale.

È comunemente accettato considerare la linguistica, intesa come studio dei segni linguistici, quale parte o branca della semiotica, lo studio dei segni in generale. Prima di parlare dei concetti specifici della linguistica, è allora indispensabile introdurre le nozioni più elementari della semiotica, che ritroviamo poi anche nella linguistica: la nozione di segno, che costituisce l'oggetto precipuo della semiotica, e quelle correlate di significato e significante.

Due sono le concezioni più note della nozione di segno; esse corrispondono alle due scuole che hanno posto le basi della semiotica: la prima è quella del padre della linguistica Ferdinand de Saussure (scuola europea della semiotica o semiologia), la seconda quella del filosofo pragmatista Charles Sanders Peirce (scuola americana della semiotica). La differenza tra le due posizioni è stata recentemente riassunta da Jens-Erik Mai. ${ }^{161}$ Saussure concepisce il segno essenzialmente come sinolo di significante (l'espressione fonica o grafica di un segno) ${ }^{162}$ e significato (il contenuto concettuale che scaturisce dalla combinazione dei significanti). La posizione di Saussure è stata centrale per i successivi studi linguistici di area europea; essa è stata accolta, tra gli altri, da Greimas e dal linguista danese Louis T. Hjelmslev, fondatore della glossematica. ${ }^{163}$ La semiotica peirciana, più complessa, si articola invece in tre elementi: il segno stesso ("qualsiasi cosa riferita a qualcosa sotto qualche aspetto o qualità, cioè secondo una certa interpretazione"), l'interpretante (o significato) e il referente (o oggetto). ${ }^{164}$

Le due concezioni si differenziano in due punti fondamentali; la concezione peirciana del segno, rispetto a quella saussuriana: 1) introduce un terzo elemento extralinguistico: il referente o oggetto, ovvero le cose a cui si riferiscono i segni; ${ }^{165} 2$ ) caratterizza esplicitamente il significato in senso interpretativo; il significato è nella concezione peirciana definito in rapporto alla necessaria presenza di qualcuno che operi un'interpretazione: non vi può essere significato senza interpretazione e, dunque, lo stesso significato varia al variare delle interpretazioni.

Per quanto ci riguarda, per il nostro approccio linguistico seguiremo una posizione a metà

161 JENS-ERIK MAI, Semiotics and indexing, cit., p. 596; SAUSSURE, FERDINAND DE, Corso di linguistica generale, traduzione e commento di Tullio De Mauro, 22. ed., Roma, GLF Laterza, 2009, traduzione di Cours de linguistique générale; cfr. anche CHARLES SANDERS PEIRCE, Collected papers of Charles Sanders Peirce. Volume 1, Principles of philosophy, and Volume II, Elements of logic, edited by Charles Hartshorne and Paul Weiss, Belknap Press of Harvard University Press, Cambdridge, Massachusetts, 1960.

162 Nella concezione originaria saussuriana, così come in buona parte della linguistica successiva, la scrittura e i segni grafici sono considerati come derivati dai suoni e dal linguaggio orale; per questo motivo lo studio della fonologia è parte essenziale della linguistica saussuriana; poiché noi ci rivolgeremo esclusivamente alle lingue documentarie, di natura essenzialmente grafica e non fonetica, nella nostra trattazione la fonologia sarà invece irrilevante.

163 ALGIRDAS JULIEN GREIMAS, Semantica strutturale, cit., p. 27; cfr. anche LOUIS T. HJELMSLEV, I fondamenti della teoria del linguaggio, introduzione e traduzione di Giulio C. Lepschy, Torino, Einaudi, 1987, traduzione di Omkring Sprogteoriens Grundlæggelse, p. 52.

164 CHARLES SANDERS PEIRCE, Semiotica, testi scelti e introdotti da Massimo A. Bonfantini, Letizia Grassi, Roberto Grazia, Torino, Einaudi, 1980; cfr. anche AUGUSTO PONZIO, Il linguaggio e le lingue. Introduzione alla linguistica generale, Bari, B.A. graphis, 2004, p. 29-30.

165 Cfr. ALGIRDAS JULIEN GREIMAS, Semantica strutturale, cit., p. 31. 
Tesi di dottorato di Giuliano Genetasio, discussa presso l'Università degli Studi di Udine

tra quella saussuriana e quella peirciana: utilizzeremo la terminologia saussuriana e lasceremo generalmente impliciti i riferimenti extralinguistici del linguaggio (i referenti); adotteremo invece l'idea peirciana del carattere interpretativo del significato (cioè, secondo Augusto Ponzio, come "percorso interprativo"), ovvero l'idea che ogni segno abbia tanti significati possibili quante siano le possibili interpretazioni di esso o i sensi che esso può assumere nei diversi contesti. ${ }^{166}$ Quando, nel precedente capitolo (vedi 2.16.2 e ss.), abbiamo sostenuto il carattere intrinsecamente interpretativo del soggetto, lo abbiamo fatto implicitamente secondo una posizione peirciana.

\subsubsection{Il presupposto fondamentale della linguistica generale: gli universali linguistici}

La linguistica generale mira a studiare il linguaggio come distinto dai particolari sistemi linguistici in cui esso si estrinseca: le lingue. Presupposto essenziale della linguistica generale è, ne consegue, che sia possibile considerare il linguaggio in sé a prescindere dalle lingue, ovvero che sia possibile stabilire delle basi su cui confrontare tra loro le diverse lingue. La linguistica generale postula cioè l'esistenza di un numero più o meno ampio di elementi o caratteristiche presenti in tutte le lingue e costituenti la base del linguaggio: gli universali linguistici.

L'esistenza degli universali linguistici è, tra l'altro, la base della scuola saussuriana di linguistica: lo strutturalismo; lo strutturalismo (linguistico) è fondato sull'idea dell'esistenza di strutture universali del linguaggio: vocabolario, grammatica, semantica, etc. ${ }^{167}$ Questi universali linguistici sono oggi ampiamente accettati al punto da essere parte fondamentale dello studio di ogni lingua. La linguistica generale si è spesso spinta oltre, postulando per esempio che l'opposizione soggetto-nomi e predicato-verbi fosse una struttura che caratterizza la grammatica di tutte le lingue. ${ }^{168}$ Uno dei linguisti che maggiormente hanno creduto nella possibilità degli universali linguistici è stato Chomsky: il lavoro del linguista statunitense - che non a caso nasce

166 GAETANO BERRUTO, Nozioni di linguistica generale, 3. ed., Napoli, Liguori, 2004, p. 77: "Un segno ammette sempre moltissimi significati concreti, cioè una classe di significati specifici di volta in volta realizzati dalla situazione [...] quelli che potremo in certo modo chiamare significati contestuali [...] Questo significato contestuale [...] è il "senso" [...] Il significato è allora l'insieme dei sensi possibili di un segno". 167 Cfr. HOWARD MACLAY, Overview, in Semantics. An interdisciplinary reader in philosophy, linguistics and psychology. Part II. Linguistics, edited by Danny D. Steinberg \& Leon A. Jakobovits, Cambridge, Cambridge University Press, 1971, p. 159. Contro tale presupposto, il poststrutturalismo ha mirato a mostrare la relatività delle strutture linguistiche, ovvero il fatto che queste pertengano il piano dell'analisi linguistica, e non del linguaggio in sé. Sul poststrutturalismo cfr. GARY P. RADFORD - MARIE L. RADFORD, Structuralism, post-structuralism and the library. De Saussure and Foucault, "Journal of documentation", 61, 1 (2005), p. 61, 69: "Post-structuralism [Foucault...] suggests that such descriptions are themselves always high contextual [...] Whereas de Saussure's structuralism was confident that the principles by which language is organized can be fully determined [....] post-structuralism calls into question all such assumptions and suggests that such conclusions are always fragile and open to subversion [...] After all, de Saussure's theory of language is itself a particular parttern of signs created within language [...] Saussure cannot detach himself from the language system he is attempting to make sense of because the language he uses to make sense of it is also a part of and made possible by, that very system".

168 NOAM CHOMSKY, La grammatica trasformazionale, cit., p. 114; cfr. anche MAURICE COYAUD, Linguistique et documentation, cit., p. 10. 
dall'alveo strutturalista - è incentrato sul progetto di creazione di una grammatica universale, costituita da una fonetica, una sintassi e una semantica universali. ${ }^{169}$ Questo ed altri progetti di grammatiche universali hanno subito una critica radicale da parte dei sostenitori della relatività linguistica, ovvero dell'inscindibilità di linguaggio e pensiero (e dunque anche cultura), la quale minerebbe alla base qualsivoglia tentativo di formulare grammatiche universali; tra costoro vi è anche Umberto Eco. ${ }^{170}$ A parte queste critiche radicali, il punto in cui i sostenitori degli universali linguistici incontrano maggiori difficoltà è la semantica: l'idea di fissare a priori e in modo universale i significati delle parole è intrinsecamente problematica; lo stesso Chomsky propose, ma non realizzò mai, il progetto di creazione di una semantica universale. ${ }^{171}$ Coyaud, pur credendo nella possibilità di una sintassi universale (quale base da utilizzare per l'elaborazione dei linguaggi documentari), non credeva che fosse possibile universalizzare in modo analogo la semantica. ${ }^{172}$ L'accusa più frequente che si sono attirati i fautori degli universali linguistici è stata quella di etnocentrismo: poiché il linguaggio è concretamente e invariabilmente incarnato nelle singole lingue, postulare la possibilità di universali linguistici sulla base dell'astrazione di alcuni elementi delle lingue particolari comporta potenzialmente sempre il rischio di trattare come universali elementi che tali non sono, caratterizzanti magari uno o più gruppi linguistici ma non tutti. Alcuni esempi indicativi di etnocentrismo linguistico: nel saggio già citato in cui Chomsky teorizza la possibilità di una grammatica universale e, tra le altre cose, l'universalità del binomio soggetti-predicati, gli esempi portati sono esclusivamente in lingua inglese. Saussure, nell'opera monumentale che gli valse il titolo di fondatore della linguistica, poneva alcune caratteristiche universali delle lingue: linearità, arbitrarietà, storicità, etc. Di queste, solo l'ultima può dirsi effettivamente universale (ma il carattere diacronico delle lingue è un elemento che gioca contro la possibilità di considerare il linguaggio in modo sincronico, cioè universale). La linearità che secondo Saussure (che però partiva dal francese) avrebbe caratterizzato l'organizzazione delle parole nelle frasi e nei periodi (sintagmi) è stata successivamente smentita: l'organizzazione sintagmatica è semmai gerarchica, con uno degli elementi del sintagma che funge da nodo centrale attorno al quale si strutturano e dipendono gli altri, e cioè non necessariamente lineare. ${ }^{173}$ L'arbitrarietà del segno, e cioè

169 NOAM CHOMSKY, La grammatica trasformazionale, cit., p. 113; il progetto chomskyano di una grammatica universale è aspramente criticato in UMBERTO ECO, La ricerca della lingua perfetta nella cultura europea, cit.

170 UMBERTO ECO, La ricerca della lingua perfetta nella cultura europea, cit., p. 311: "La cultura settecentesca [...] sostiene che pensiero e linguaggio si influenzano mutuamente e procedono di pari passo [...] Se è così, non si può più sostenere l'ipotesi razionalista di una grammatica del pensiero, universale e stabile, che i vari linguaggi in qualche modo riflettono. Nessun sistema delle idee [...] può diventare parametro e criterio per la costruzione di una lingua perfetta: la lingua non riflette un universo concettuale platonicamente precostituito, ma concorre a formarlo".

171 NOAM CHOMSKY, La grammatica trasformazionale, cit., p. 116-117.

172 MAURICE COYAUD, Linguistique et documentation, cit., in un paragrafo significativamente intitolato Grammaire générale et ethnocentrisme, cerca di rispondere all'accusa di etnocentrismo rivolta a tutti coloro (tra cui Chomsky) che hanno voluto sostenere la possibilità di universali linguistici; secondo Coyaud, invece, questi esistono, benché limitati alla sola grammatica (Sintassi) e alla struttura profonda: in particolare, Coyaud sostiene l'universalità dell'opposizione verbi-nomi a livello di struttura profonda.

173 Cfr. GIOVANNI GOBBER - MORENO MORANI, Linguistica generale, Milano, McGraw-Hill, 2010, p. 17; cfr. anche REBECCA GREEN, Syntagmatic relationships in index languages. A reassessment, "Library quarterly”, 65, 4 (1995), p. 369. 
il fatto che il legame tra significante e significato sia casuale o convenzionale, ma non intrinseco, è uno dei capisaldi della linguistica (saussuriana e successiva); è l'arbitrarietà del segno, tra le altre cose, che rende possibile l'evoluzione dei significati delle parole; ${ }^{174}$ tuttavia l'arbitrarietà segnica non è un carattere assolutamente universale del linguaggio: essa non si applica a quei sistemi linguistici (come i geroglifici egizi, ma parzialmente anche la scrittura ideogrammatica cinese) che pongono invece un legame intrinseco tra significante e significato, ovvero in cui il significante imita in modo stilizzato l'oggetto a cui si riferisce.

Gli universali linguistici sono allora un fondamento al contempo indispensabile e problematico della linguistica generale: ogni progetto di elaborazione di universali linguistici, ovvero anche di linguaggi o parti di linguaggi universali, deve tenere conto del rischio etnocentristico cui si espone.

Una distinzione utile in riferimento a tale questione, e più in generale alla questione dei rapporti tra universalità e particolarità delle teorie linguistiche, è quella tra universali linguistici formali e sostanziali (e dunque tra piano formale e sostanziale delle lingue). Universali formali sono la già menzionata suddivisione delle lingue in semantica, sintassi e pragmatica; sono universali formali anche l'esistenza di determinate tipologie lessicali e di relazioni semantiche o sintattiche. Universali sostanziali sono principalmente quelli relativi al lessico di una lingua e alle specifiche relazioni semantiche tra gli elementi lessicali. ${ }^{175}$ Postulare l'esistenza di universali sostanziali, come è evidente anche da ciò che abbiamo detto sopra, è certamente più difficile, forse persino impossibile senza incorrere nel rischio di etnocentrismo. In realtà avvertiamo che la differenza tra piano linguistico formale e sostanziale non è così netta; per esempio non è scontato se l'esistenza di determinate tipologie semantiche o sintattiche sia da considerare un universale formale o sostanziale, o ancora a metà tra questi due livelli. Pur con questa riserva, riteniamo comunque utile la distinzione.

Nei paragrafi seguenti, dedicati soprattutto alla discussione degli universali linguistici più importanti, daremo per scontate queste considerazioni.

\subsubsection{Langue e parole, competenza ed esecuzione, sistema e processo, asse paradigmatico e sintagmatico}

In qualche modo correlata alla distinzione tra linguaggio e lingue è la celebre coppia saussuriana "langue" e "parole" (lasciamo i termini francesi perché "parole" è intraducibile in italiano). "Langue" è il linguaggio inteso come sistema a prescindere dalle sue manifestazioni concrete. "Parole" è invece il linguaggio così come utilizzato nei discorsi, con le particolarità che caratterizzano i diversi parlanti e contesti. La linguistica generale, secondo Saussure, studia la "langue" e non la "parole".

Alla coppia "langue-parole" è strettamente connessa quella chomskyana tra competenza

174 GIOVANNI GOBBER - MORENO MORANI, Linguistica generale, cit., p. 21: "Si è osservato che l'arbitrarietà è fondamentale per il cambiamento del potenziale semantico di un'unità linguistica: poiché non vi è ragione per legare un dato suono a un dato significato, è possibile attribuire altri significati a quel suono"; cfr. anche W. JOHN HUTCHINS, Linguistic processes in the indexing and retrieval of documents, cit., p. 31-34, 39.

175W. JOHN HUTCHINS, Languages of indexing and classification, cit., p. 127-130. 
Tesi di dottorato di Giuliano Genetasio, discussa presso l'Università degli Studi di Udine

(“competence") ed esecuzione ("performance"): ogni parlante ha una propria competenza linguistica, un insieme di parole e di regole (per semplificare) che è in grado di utilizzare per comprendere ed esprimersi, la quale differisce dall'esecuzione linguistica, cioè dai diversi modi effettivamente utilizzati per esprimersi e comprendere ciò che gli viene detto. Anche per Chomsky la competenza, e non l'esecuzione, è l'oggetto della linguistica. ${ }^{176}$ Analoga alle coppie "langue-parole" e competenza-esecuzione è quella hjelmsleviana di sistema e processo. Secondo Hjelmslev il linguaggio sarebbe un sistema (o lingua, secondo la terminologia del linguista danese), un insieme di regole a partire dal quale sono possibili un certo numero di processi (o testi, secondo la terminologia del linguista danese) o discorsi. ${ }^{177}$

Alle summenzionate coppie è infine collegata una quarta e fondamentale opposizione: quella tra asse paradigmatico e sintagmatico del linguaggio. ${ }^{178}$ La distinzione tra asse sintagmatico e paradigmatico del linguaggio nasce con Saussure, benché con una terminologia parzialmente differente: le relazioni sintagmatiche sono quelle che valgono "in praesentia", cioè tra le diverse parole che fanno parte di una stessa sequenza o discorso; le relazioni associative (questo il termine usato da Saussure, da non confondere con le relazioni associative delle lingue documentarie), viceversa, sono quelle che valgono "in absentia", cioè che legano le parole a prescindere da specifici discorsi; in esse la singola parola è dunque considerata come parte di una "serie virtuale" cui fanno parte tutte le altre a essa associate; ${ }^{179}$ nella concezione originaria saussuriana le relazioni associative sono tanto di tipo semantico, quanto fonetico o morfologico (stessa radice, stesso suffisso, etc. delle parole).

La coppia sintagmatico e associativo ha avuto un'enorme fortuna nella linguistica successiva (e anche nella teoria dell'indicizzazione, come avremo modo di dire in 3.3.5), subendo però alcuni cambiamenti terminologici e di significato; per ciò che riguarda le relazioni associative: 1) il termine "associative" viene sostituito da "paradigmatiche"; non si parla più di "associazione" ma di "paradigma"; questo viene definito come "la classe degli elementi che possono comparire in una data posizione sull'asse sintagmatico" oppure, più estesamente (sarà questa l'accezione che accoglieremo), come la "rete di relazioni" che una parola ha o può acquisire a prescindere da specifici discorsi; ${ }^{180}$ 2) le relazioni paradigmatiche vengono a essere definite sempre meno in termini fonetici e morfologici e sempre più in termini semantici. ${ }^{181}$ A partire da questa prospettiva, definiremo dunque paradigmatiche le relazioni esistenti al di fuori di specifici discorsi; sintagmatiche le relazioni poste all'interno di specifici discorsi.

Notiamo bene che (a differenza di quanto viene spesso posto nella teoria

176 NOAM CHOMSKY, La grammatica trasformazionale, cit., p. 107-108.

177 Cfr. LOUIS T. HJELMSLEV, I fondamenti della teoria del linguaggio, cit., p. 11-12.

178 YVES GILBERT COURRIER, Document analysis, verbs and case grammar, cit., p. 74: "The distinction between langue and parole leads to the distinction between paradigmatic and syntagmatic relations. The formers are present in the language as a system; the second ones are found in actual statements"; cfr. anche LOUIS T. HJELMSLEV, I fondamenti della teoria del linguaggio, cit., p. 43.

179 SAUSSURE, FERDINAND DE, Corso di linguistica generale, cit., p. 149-153.

180 Cfr. GIOVANNI GOBBER - MORENO MORANI, Linguistica generale, cit., p. 16-17: cfr. anche JULIAN WARNER, Analogies between linguistics and information theory, "Journal of the American Society for Information Science and Technology", 58, 3 (2007), p. 315.

181 Cfr. ALEXIS RIVIER, Construction des langages d'indexation. Aspects théoretiques, "Documentaliste", 27, 6 (1990), p. 268. 
dell'indicizzazione, vedi 3.3.5) la coppia paradigmatico e sintagmatico non implica una differenza tra relazioni di carattere universalmente valido o a priori e relazioni di carattere contingente o a posteriori; né essa è una differenza mutualmente esclusiva: nel linguaggio naturale non esistono relazioni intrinsecamente paradigmatiche o intrinsecamente sintagmatiche. ${ }^{182}$ Piuttosto, paradigmaticità e sintagmaticità sono strettamente correlate: ogni sintagma, in quanto associazione di due o più elementi lessicali, presuppone una qualche paradigmaticità tra di essi; viceversa, sono le associazioni di elementi lessicali nate all'interno di specifici discorsi che concorrono a formare, nel loro complesso, l'insieme delle associazioni valide al di fuori di specifici discorsi. Questa idea è collegata al carattere idiolettico dei testi di cui parla Greimas: ogni sintagma, quale è ogni discorso e dunque anche ogni testo, tende a stabilire rapporti paradigmatici propri. ${ }^{183}$ Continuando secondo questa prospettiva, dobbiamo riconoscere che in ogni contesto linguistico organizzazione paradigmatica e sintagmatica coesistono costantemente; all'interno di ogni discorso sono cioè presenti sia relazioni sintagmatiche che paradigmatiche: nella frase "l'Umbria e il Lazio fanno parte dell'Italia", "Umbria", "Lazio" e "Italia" sono legate tra loro da relazioni sintagmatiche ma già - almeno per chi abbia una qualche nozione sulla geografia della penisola italiana - da relazioni paradigmatiche; nella frase "il cane abbaia" la relazione che lega il sostantivo al verbo è sintagmatica ma, già, paradigmatica (in quanto il verbo "abbaia" è semanticamente appropriato solo per un cane, eccettuati usi retorici del termine). Così come ogni sintagma è più o meno paradigmatico, ogni relazione paradigmatica può essere espressa in modo più o meno sintagmatico, ovvero esplicito: in un discorso in cui si parli soltanto dell'Umbria, il riferimento all'Italia, di cui l'Umbria è parte, resta confinato al piano paradigmatico; in un discorso che riguardi "l'Umbria e l'Italia" i due termini della relazione paradigmatica vengono esplicitati senza che però venga specificato il tipo di relazione che li lega; in un discorso che riguardi "l'Umbria, parte dell'Italia" o ancora più fortemente nella frase "l'Umbria è parte dell'Italia", la relazione paradigmatica tra Umbria e Italia è completamente esplicitata, ovvero espressa sintagmaticamente. ${ }^{184}$

Dobbiamo allora riconoscere la relatività di ciò che è paradigmatico e di ciò che è sintagmatico nel linguaggio naturale, ovvero, ribaltando la questione, dobbiamo riconoscere che una stessa relazione può assumere una forma paradigmatica (in quanto esistente implicitamente) o sintagmatica (in quanto espressa in un discorso); ${ }^{185}$ in questo

182 REBECCA GREEN, Syntagmatic relationships in index languages, cit., p. 374-376: "The strict dichotomy traditionally drawn between paradigmatic and syntagmatic relationships is somewhat artificial, in that all paradigmatic relationships on the lexical level have syntagmatic counterparts on the conceptual level". 183 ALGIRDAS JULIEN GREIMAS, Semantica strutturale, cit., p. 134-135.

184 REBECCA GREEN, Syntagmatic relationships in index languages, cit., p. 368-369: "The two types of relationship overlap [...] The distinction between paradigmatic and syntagmatic relationships is the only relative and not absolute, since a given relationship can be expressd either paradigmatically or syntagmatically or both ways simultaneously"; cfr. anche KAREN SPARCK-JONES - MARTIN KAY, Linguistics and information science, cit., p. 77, secondo cui le relazioni tra termini: "May be expressed paradigmatically or syntagmatically, but [...] this is a formal distinction, since the same predicate may be used either way. A generic relationship, for example, may be indicated paradigmatically or syntagmatically; equally, an associative relation, though usually represented syntagmatically, may, if it is constant, be expressed paradigmatically".

185 W. JOHN HUTCHINS, Languages of indexing and classification, cit., p. 38: "Many linguists have remarked on the high degree of relativity surrounding the notions of "paradigmatic" and "syntagmatic". What may be expressed by a paradigmatic relation in one language may have to be expressed syntagmatically 
Tesi di dottorato di Giuliano Genetasio, discussa presso l'Università degli Studi di Udine

senso, Coyaud ha affermato che la differenza tra sintagmatico e paradigmatico non è una differenza di natura, ma di impiego. ${ }^{186}$

Possiamo andare oltre ed estendere la coppia paradigmatico e sintagmatico a un'altra accezione, includendovi i rapporti espliciti (sintagmatici) o impliciti (paradigmatici) tra gli elementi lessicali: ogni elemento lessicale è formato a sua volta da più elementi di significato, e può essere espresso sintagmaticamente, cioè come sintagma (elemento lessicale in forma composta) formato da più elementi lessicali tra loro in relazione (per esempio "etica della vita" o "cristiano democratico"), oppure "paradigmaticamente", cioè come parola (elemento lessicale in forma semplice), all'interno del quale le relative relazioni concettuali rimangono implicite (per esempio "bioetica" o "democristiano"); nel primo caso le relazioni tra i singoli elementi di significato componenti ("etica" e "vita", "cristiano" e "democratico") vengono esplicitati nel discorso, formando un sintagma; nel secondo esse rimangono impliciti, esistendo cioè a livello paradigmatico ("bioetica" fa riferimento sia alla vita che all'etica; "democristiano" fa riferimento sia alla democrazia che al cristianesimo) all'interno dell'elemento lessicale in forma semplice.

In linguistica, gli elementi lessicali componenti il significato di un altro elemento lessicale sono detti marche semantiche (o semi, vedi 3.2.5.3.3) e l'analisi volta a determinarli analisi componenziale. (In effetti, in linguistica l'analisi componenziale riguarda tratti semantici primari e non elementi di significato già in sé complessi come "vita" o "etica", ma ai fini del nostro discorso possiamo trascurare questo aspetto).

Spingendoci oltre, possiamo anche vedere la differenza tra paradigmaticità e sintagmaticità degli elementi lessicali come una differenza esclusivamente relativa alla forma: ogni singolo elemento lessicale in forma semplice può assumere una forma sintagmatica (parafrasi, di cui un tipico esempio è la definizione) ${ }^{187}$ e viceversa ogni elemento in forma sintagmatica può assumere una forma semplice (così per esempio "etica della vita" che diventa "bioetica"). ${ }^{188}$

Se facciamo riferimento alle tipologie lessicali sottostanti gli elementi lessicali, potremmo dire che ogni elemento lessicale è a sua volta concepibile come combinazione di singoli elementi lessicali; per esempio, l'elemento lessicale "bioetica" è concepibile come interazione concettuale degli elementi lessicali "vita" ed "etica". L'elemento lessicale

in another" [...] The same relativity of the paradigmatic-syntagmatic distinction is observable $\mathrm{n}$ comparisons of DLs. As Gardin puts it "this dichotomy can be shown to have no justification other than practical. A given relationship betweem two or more concepts can be expressed either analytically [i.e. on the paradigmatic axis] ... or syntactically [i.e. syntagmatically]. The choice...depends on practical circumstances that are of the same nature in [DLs] as they are in NL [...] the more straightforward relations, those which "go without saying" by which are nevertheless frequently put to use in a given universe of discouse tend to be transferred to the semantic structure, whereas the more unpredictable relations, bringing forth new knowledge, find their expression in the syntactical structure"”. 186 MAURICE COYAUD, Introduction a l'ètude des langages documentaires, cit., p. 23. 187 GUGLIELMO CINQUE, Introduzione del curatore, in La semantica generativa, a cura di Guglielmo Cinque, Torino, Boringhieri, 1979, p. 17; cfr. anche MAURICE COYAUD, Linguistique et documentation, cit., p. 118.

188 Cfr. ROBERT A. FAIRTHORNE, Content analysis, specification, and control, cit., p. 89: "If the topic of, say, "green-lizards-linking-northwestern-table-legs-on-Fridays-if-it-rains" is of wide concern to saurosociologists, they will give it a name and treat it as a unit in normal discouse with their fellows"; cfr. anche GUGLIELMO CINQUE, Introduzione del curatore, cit., p. 16-17: "Sono ugualmente conseguenze ultime di questi stessi assunti l'ipotesi che le voci lessicali abbiano una struttura per l'essenziale identica a quella delle frasi, essendo tutte e due le forme scomponibili negli stessi elementi atomici”. 
"dantistica" è concepibile come interazione concettuale degli elementi lessicali "studio" e "Dante Alighieri". Cambiando prospettiva, è cioè possibile vedere gli elementi lessicali come articolazioni concettuali di uno dei singoli elementi lessicali (ed entità) componenti; ritornando all'esempio precedente, è allora possibile vedere la "bioetica" sia come un'articolazione concettuale dell'elemento lessicale "etica", sia come articolazione concettuale dell'elemento lessicale "vita" (bioetica come una delle discipline o prospettive di cui la vita è oggetto, assieme alla biologia, biografia, etc.); allo stesso modo, è possibile vedere la "dantistica" come articolazione di studi, oppure come articolazione concettuale di "Dante Alighieri".

Finora abbiamo implicitamente fatto riferimento a elementi lessicali relativi a entità collettive (vedi 3.2.5.3.3 per una categorizzazione delle tipologie lessicali), che sono quelle tipicamente studiate dall'analisi componenziale. Tuttavia, e andando aldilà dell'analisi componenziale propriamente detta, possiamo estendere il concetto di scomposizione semantica degli elementi lessicali a includere non soltanto quelli relativi a entità collettive, ma persino quelli relativi a entità individuali: nomi, titoli ed espressioni come "Dante Alighieri", "Sicilia", etc. Anche gli elementi lessicali relativi a entità individuali, infatti, partecipano di più elementi di significato, che nel loro complesso definiscono l'elemento lessicale; così l'elemento lessicale riferito a un'entità individuale "Dante Alighieri" partecipa dei concetti di "uomo", "autore" (e più precisamente "autore della Divina commedia", che ne è una parafrasi), "fiorentino", etc.

Riassumendo, abbiamo mostrato due diverse accezioni secondo cui è possibile intendere la coppia paradigmatico e sintagmatico: 1) come differenza tra le relazioni esistenti al di fuori di specifici discorsi da un lato e relazioni poste esplicitamente all'interno di specifici discorsi dall'altro: questa è l'accezione più autentica della coppia paradigmatico e sintagmatico, e quella che adotteremo più spesso nel resto della nostra discussione; 2) come differenza tra elementi lessicali in forma semplice (parole) ed elementi lessicali in forma composta (sintagmi): parleremo in questi casi di forme semplici (o paradigmatiche) o composte (o sintagmatiche) degli elementi lessicali. Infine, pur accettando pienamente l'idea della sostanziale relatività di paradigmatico e sintagmatico, anticipiamo fin da qui che alcune relazioni sintagmatiche esulano da tale relatività, perché compaiono esclusivamente nei sintagmi, ovvero sono esclusivamente funzionali a costruire specifici discorsi: sono le relazioni di coordinazione, disgiunzione, subordinazione, etc., che discuteremo più avanti (vedi 3.2.8), e che chiameremo relazioni "sintagmatiche logiche".

\subsubsection{Funzioni linguistiche}

Nelle discussioni precedenti abbiamo accennato a una delle grandi scuole della linguistica: lo strutturalismo. L'altra grande scuola linguistica è quella del funzionalismo. Mentre lo strutturalismo incentra la sua analisi sulle strutture del linguaggio, il funzionalismo pone al centro della sua attenzione le azioni o funzioni svolte dal linguaggio: essenzialmente comunicative, ma che si possono declinare in più sensi. Roman Jakobson è il linguista che ha sviluppato la prima teoria sistematica delle funzioni linguistiche.

Riallacciandosi alla teoria dell'informazione di Shannon-Weaver, Jakobson considerò sei funzioni svolte dalle espressioni linguistiche: 1 ) referenziale (o denotativa, o cognitiva, o 
descrittiva), volta a produrre descrizioni, spiegazioni o informazioni sulla realtà extralinguistica; 2 ) emotiva (o espressiva), volta a esprimere determinate emozioni, sentimenti o opinioni del parlante; 3 ) conativa (o persuasiva), volta a promuovere o evitare un'azione da parte del destinatario. Queste prime tre costituiscono le funzioni principali del linguaggio, a cui si aggiungono le funzioni: 4) fatica (o di contatto), volta a stabilire, prolungare o chiudere la comunicazione stessa; 5) metalinguistica, volta a fornire informazioni e spiegazioni sulla comunicazione stessa; 6) poetica (o estetica), volta a produrre messaggi esteticamente efficaci o divertenti (piuttosto che o oltre che informativi). ${ }^{189}$

Le funzioni non sono mutualmente esclusive: una stessa espressione linguistica svolge anzi quasi sempre più funzioni, di cui una è però prevalente. Lo studio delle funzioni linguistiche è stato successivamente sviluppato da altri linguisti, ma per il nostro approccio linguistico all'indicizzazione le sei funzioni delineate da Jakobson sono già sufficienti.

Una nota sul rapporto tra funzioni linguistiche e branche della linguistica: lo studio delle funzioni del linguaggio può essere considerato parte della pragmatica (vedi 3.2.5.3.1).

\subsubsection{Aspetti e componenti del linguaggio}

\subsubsection{Introduzione}

Il linguaggio può essere visto come un insieme di componenti diverse. Da un lato un insieme di parole e locuzioni, il vocabolario o lessico; dall'altro un insieme di aspetti o rapporti di diverso tipo: rapporti grammaticali tra parole (e locuzioni); rapporti di significato tra parole (e locuzioni); rapporti tra queste parole (e locuzioni) e un contesto extralinguistico. A ciascuna tipologia di rapporti è associata una tipologia di regole d'uso del lessico: regole sintattiche, regole semantiche, regole relative all'uso o contesto linguistico; a ciascuna tipologia di rapporti e di regole, inoltre, corrisponde una delle principali branche della linguistica: rispettivamente morfosintassi, semantica e pragmatica (le discuteremo in 3.2.5.3 e ss.). Vedere il linguaggio come composto di lessico più una serie di aspetti o regole significa schematizzare in modo estremo una situazione molto più complessa: il lessico è in realtà composto di più elementi; i diversi rapporti e regole del linguaggio sono legati tra loro da relazioni complesse, e ciascuno di essi è a sua volta composto da più elementi. Per la nostra trattazione, tuttavia, questa schematizzazione è molto utile, e quindi almeno per il momento la accetteremo.

\subsubsection{Lessico}

Il lessico è l'insieme delle singole parole e locuzioni (elementi lessicali, detti lessemi in linguistica) dotate di significato di una lingua, ovvero l'insieme dei segni linguistici.

189 ROMAN JAKOBSON, Saggi di linguistica generale, cura e introduzione di Luigi Heilmann, traduzione di Luigi Heilmann e Letizia Grassi, 3a ed., Milano, Feltrinelli, 2008, traduzione di Essais de linguistique générale, p. 185-191. Cfr. anche GAETANO BERRUTO, Nozioni di linguistica generale, cit., p. 56-57. 
Ciascun lessema è in realtà composto di più elementi o strati o aspetti.

Quando avevamo parlato dei segni, avevamo detto che ogni segno è un sinolo di significato e significante. Dal punto di vista lessicale ogni lessema, cioè, esiste su due piani distinti: da un lato come significato (semema), oggetto di studio della semantica; dall'altro come significante, oggetto di studio della morfosintassi. Possiamo allora parlare di una biplanarità del lessico del linguaggio naturale, che è al contempo un lessico dei significati e un lessico dei significanti. Ritornando sulla schematizzazione del paragrafo precedente (3.2.5.1), notiamo già che è possibile solo per astrazione separare lessico ed altri aspetti o rapporti del linguaggio; tali rapporti riguardano infatti già il singolo lessema. Mantenere una distinzione tra il lessico in sé e i suoi aspetti è nondimeno utile per il nostro discorso.

Parlando della relatività di paradigmaticità e sintagmaticità, avevamo detto come un ogni lessema riferito a entità collettive conosca virtualmente una forma semplice e una forma composta, ovvero come le forme semplici dei lessemi siano concettualmente scomponibili in sintagmi costituiti da più elementi (marche semantiche o semi), che compongono a loro volta la forma semplice. Ciò che avevamo detto, lo esplicitiamo adesso, era riferito ai lessemi considerati in quanto significati, cioè come sememi.

\subsubsection{Aspetti linguistici e branche della linguistica}

\subsection{Introduzione}

Abbiamo detto che il linguaggio può essere visto secondo diversi aspetti o tipi di rapporti: grammaticali, di significato, relativi all'uso o al contesto; abbiamo detto che a tali aspetti (che chiameremo d'ora in poi aspetti linguistici) corrispondono differenti tipologie di regole e differenti branche della linguistica: morfosintassi, semantica, pragmatica. Tale tripartizione non è assoluta né universalmente accettata in ambito linguistico; benché essa sia una delle più comuni, e certamente quella che ha avuto maggiore fortuna nell'ambito dell'indicizzazione, in ambito linguistico non esiste a tutt'oggi accordo sul numero e sui rapporti tra i diversi aspetti del linguaggio e, dunque, tra le relative branche di studio. ${ }^{190}$

La formulazione originaria di tale tripartizione fu ideata dal filosofo pragmatista Charles Morris che, sulla base della visione peirciana del segno, e riferendosi al più vasto ambito semiotico (non solo linguistico) distinse sintattica, semantica e pragmatica. Nell'originaria tassonomia morrisiana, la sintattica indicava il modo in cui i segni si combinano tra loro; la semantica i significati dei segni; la pragmatica gli effetti dei segni,${ }^{191}$ ovvero il rapporto tra segni e loro utenti.

Nel corso degli anni, molti altri sono stati i termini utilizzati in ambito di linguistica per

190 Cfr. W. JOHN HUTCHINS, Languages of indexing and classification, cit., p. 4-6; cfr. anche SYDNEY M. LAMB, The sememic approach to structural semantics, "American anthropologist", 66, 3 (1964), p. 58. 191 AUGUSTO PONZIO, Il linguaggio e le lingue, cit., p. 50-53; cfr. anche CHARLES W. MORRIS, Lineamenti di una teoria dei segni, nella traduzione di Ferruccio Rossi-Landi, a cura di Susan Petrilli, nuova ed. rivista e aggiornata, Lecce, Pensa multimedia, 2009, traduzione di Foundations of the theory of signs, p. $87-93$. 
indicare aspetti del linguaggio e le relative branche di studio. Per esempio, si è variamente parlato di sintassi, morfologia o morfosintassi. Un'altra branca linguistica, centrale per esempio nella linguistica di Saussure (per il quale il segno grafico è considerato come derivazione del segno fonetico), è la fonologia, ovvero lo studio degli aspetti fonetici del linguaggio. La pragmatica è stata, infine, e aldilà dell'originaria accezione con cui il termine è inteso da Morris, estesa a indicare lo studio dell'uso del linguaggio da parte dei parlanti, ovvero lo studio del linguaggio come azione, oppure lo studio del rapporto tra linguaggio e contesto extralinguistico, o ancora lo studio del significato d'uso dei lessemi in contrapposizione al significato letterale dei lessemi (studiato dalla semantica). ${ }^{192}$ Per quanto ci riguarda, escluderemo la fonologia dalla nostra analisi e dunque dalla nostra tassonomia linguistica, perché poco rilevante ai fini dell'indicizzazione (data la natura scritta delle attuali lingue documentarie). Preferiremo inoltre parlare di morfosintassi, a sua volta costituita di morfologia (studio delle forme grammaticali dei lessemi) e sintassi (studio della combinazione grammaticale tra lessemi), piuttosto che di sintattica. Intenderemo la semantica come studio del significato dei lessemi. Intenderemo infine la pragmatica come studio del rapporto tra linguaggio e contesto (linguistico ed extralinguistico), ovvero anche come studio dell'uso del linguaggio in quanto azione: poiché in tal senso la pragmatica riguarda soprattutto lo stesso studio delle funzioni linguistiche e l'insieme degli espedienti linguistici per perseguirle, non ne daremo una trattazione autonoma. Accetteremo anche la concezione della pragmatica come studio del significato d'uso, ma con le riserve che espliciteremo più sotto (vedi 3.2.5.3.4).

\subsection{Morfosintassi}

Con il termine morfosintassi, o grammatica, ${ }^{193}$ indicheremo quella parte della linguistica che studia la combinazione delle unità linguistiche ai diversi livelli della parola e dei sintagmi (frasi e periodi); tradizionalmente, essa comprende anche la fonologia, che però noi non tratteremo perché poco rilevante ai fini del nostro discorso sull'indicizzazione. Oggetto della morfosintassi nel suo complesso sono i lessemi considerati in quanto significanti (o espressioni, nel senso hjelmsleviano del termine). La morfologia studia le proprietà o forme grammaticali dei lessemi sulla base della combinazione delle unità linguistiche sottostanti; essa comprende categorie grammaticali come il verbo, il sostantivo, l'aggettivo, l'avverbio, la preposizione, l'articolo, etc; ma anche proprietà come il genere (maschile, femminile, neutro, etc.), il numero (singolare, plurale, etc.) e, per i verbi, categorie come il modo, il tempo, la persona.

192 Cfr. ANDRZEJ GRZEGORCZYK, The pragmatic foundations of semantics, "Synthese", 8, 6/7 (1950-1951), p. 300; cfr. anche GAETANO BERRUTO, Nozioni di linguistica generale, cit., p. 73, 93; cfr. anche GIOVANNI GOBBER - MORENO MORANI, Linguistica generale, cit., p. 117-118; cfr. anche ALFREDO SERRAI, Problemi linguistici e implicazioni epistemologiche della catalogazione semantica, "Accademie e biblioteche d'Italia”, 40, 2 (1972), p. 113; cfr. anche CHINGKWEI ADRIENNE LEE - JOHN N. OLSGAARD, Linguistics and information science, in Principles and applications of information science for library professionals, John N. Olsgaard editor, Chicago, American Library Association, 1989, p. 28-29. 193 Nel corso della nostra discussione parleremo di morfosintassi o grammatica in modo indifferenziato: storicamente il termine "grammatica" è stato riferito ora alla morfologia e alla fonologia, ora esteso alla sintassi, etc.; per la nostra discussione, che non prevede una trattazione della fonologia, considereremo comunque morfosintassi e grammatica come sinonimi. 
Tesi di dottorato di Giuliano Genetasio, discussa presso l'Università degli Studi di Udine

La sintassi studia la combinazione dei lessemi in un sintagma, ovvero i ruoli grammaticali svolti dai singoli lessemi all'interno del sintagma. Essa può riguardare diversi livelli: frasi, periodi, etc. Categorie sintattiche sono per esempio quelle di soggetto, oggetto, predicato, etc.

Morfologia e sintassi sono inestricabilmente legate (è anche per questo motivo che preferiamo parlare di darne una trattazione unitaria): la combinazione dei lessemi di un sintagma, infatti, è fondata innanzitutto dalle relative proprietà morfologiche.

Gli aspetti morfosintattici del linguaggio sono certamente quelli che hanno goduto storicamente della maggiore (e più antica) attenzione nell'ambito della linguistica. Fino alla metà del secolo scorso, e oltre, la morfosintassi è stata l'unico aspetto studiato sistematicamente dalla linguistica. La scuola chomskyana di linguistica (grammatica generativo-trasformazionale) ha creduto per diversi anni che la morfosintassi fosse l'aspetto principale di una lingua, da cui gli altri (semantica, perché la pragmatica non era all'epoca una branca di studio autonoma) sarebbero dipesi. ${ }^{194}$ Proprio la grammatica trasformazionale è importante ai fini del nostro discorso per la distinzione che essa ha posto tra struttura profonda e struttura di superficie del linguaggio. Il progetto chomskyano di una grammatica universale è originariamente fondato sull'idea che in ogni lingua esistano due livelli grammaticali: da un lato un livello fenomenico e superficiale della grammatica ("struttura di superficie"), diverso per ogni lingua e corrispondente alle sue manifestazioni fonetiche e grafiche; dall'altro un livello concettuale sottostante ("struttura profonda"), che esprimerebbe i rapporti sintattici originari vigenti all'interno dei sintagmi e che sarebbe comune a tutte le lingue, ovvero e in ciò sta il fondamento psicolinguistico della teoria chomskyana - proprio del linguaggio in quanto sistema e facoltà cognitiva. Secondo la versione originaria del progetto chomskyano, i rapporti tra struttura profonda (dipendente a sua volta da specifiche regole di sintassi, o componente di base o sintattico) e molteplici strutture di superficie sarebbero regolati da una serie di "regole di trasformazione" (o componente trasformazionale, da cui prende il nome la grammatica chomskyana). ${ }^{195}$ Alcuni autori, tra cui Fillmore, hanno successivamente mostrato come categorie grammaticali quali soggetto, predicato, etc. sarebbero proprie esclusivamente del livello superficiale della sintassi, e siano invece irrilevanti a livello di struttura profonda. ${ }^{196}$ Riprenderemo in 3.2.5.3.4 la discussione della struttura profonda e superficiale del linguaggio.

\subsection{Semantica}

194 Cfr. JEAN-CLAUDE GARDIN, Document analysis and linguistic theory, cit., p. 153-156. 195 ARMANDO DE PALMA, Presentazione, in NOAM CHOMSKY, La grammatica trasformazionale. Scritti espositivi, Torino, Boringhieri, 1975, traduzione di Logical structures in language, A transformational approach to syntax, On the notion 'Rule of grammar', The formal nature of language, p. 12-13; NOAM CHOMSKY, La grammatica trasformazionale, cit., p. 136, 153; cfr. anche CHARLES J. FILLMORE, Il caso del caso, traduzione di G. R. Cardona, in Gli universali nella teoria linguistica, Torino, Boringhieri, 1978, p. 27-131, traduzione di The case for case, in Universals in linguistic theory, edited by Emmon Bach, Robert T. Harms, contributing authors Charles J. Fillmore, Paul Kiparsky, James D. McCawley, New York, Holt, Rinehart and Winston, 1968, p. 27-28.

196 Cfr. JUTTA SØRENSEN - DEREK AUSTIN, PRECIS in a multilingual context. Part 2, cit., p. 113; cfr. anche CHARLES J. FILLMORE, Il caso del caso, cit., p. 44-46, 50. 
Tesi di dottorato di Giuliano Genetasio, discussa presso l'Università degli Studi di Udine

La semantica è al contempo un aspetto fondamentale del linguaggio eppure tra i più complessi della linguistica. ${ }^{197}$ Storicamente, la semantica ha iniziato a essere studiata solo molto tempo dopo la fonologia e la sintassi: il termine stesso di semantica nasce solo alla fine dell'Ottocento, e i primi studi sistematici della semantica risalgono agli anni Sessanta; per molti decenni la semantica è stata oggetto di veri e propri pregiudizi da parte della linguistica; almeno inizialmente, il progetto chomskyano di una grammatica universale credette di poter fare a meno della semantica o di derivarla dalla sintassi; per molti anni la semantica ha goduto di uno spazio esclusivamente residuale all'interno della linguistica. ${ }^{198}$ Dati questi elementi, non sorprende che l'insigne linguista Greimas abbia parlato della semantica come del "parente povero" della linguistica. Proprio Greimas è stato uno dei primi studiosi a occuparsi in modo sistematico (da un punto di vista strutturalista) di semantica. ${ }^{199}$ Altri linguisti che hanno dato importanti contributi alla semantica e le cui elaborazioni sono fondamentali per il nostro discorso sono Jerrold J. Katz, Jerry J. Fodor e Charles J. Fillmore.

La semantica è lo studio dei significati dei lessemi; i significati, come detto sopra, vengono generalmente detti sememi in linguistica. ${ }^{200}$ Secondo una concezione ingenua della semantica, a ogni lessema è associato un significato. Ma la situazione è più complessa: non è possibile porre corrispondenze biunivoche tra lessemi e sememi, sia perché ogni significante può avere più significati diversi (p.e. "gru" uccello e "gru" attrezzatura), sia perché come abbiamo visto ogni semema è a sua volta costituito da più componenti semantiche, o marche semantiche, o semi: il significato dei lessemi non ha un carattere atomico, ma complesso. ${ }^{201}$ Ogni lessema ha una valenza semantica distribuita lungo due assi o dimensioni: l'estensione e l'intensione.

Avevamo già fatto riferimento nel precedente capitolo a estensione e intensione parlando del soggetto e, in particolare, delle nozioni di circalità estensionale ed intensionale

197 Cfr. DAVID CRYSTAL, Semantic targeting. Past, present and future, “Aslib proceedings”, 62, 4/5 (2010), p. 356: "The [...] linguistic approach to semantics has taught us [...] that the meaning system of a language is immensely complex. Meanings are notoriously difficult things to pin down, as illustrated by such wellinvestigated notions as connotation, collocation and fuzziness [...] Linguistic semanticists have been working on semantic questions of this kind for over half a century [...] their unanimous conclusion is that this is probably the most complex area of linguistic enquiry, and one which is still in its early stages of development".

198 Cfr. NOAM CHOMSKY, La grammatica trasformazionale, cit., p. 17-18; ARMANDO DE PALMA, Presentazione, cit., p. 14; cfr. anche JERROLD J. KATZ - JERRY ALAN FODOR, The structure of a semantic theory, "Language", 39, 2 (1963), p. 172.

199 ALGIRDAS JULIEN GREIMAS, Semantica strutturale, cit., p. 22.; cfr. anche UMBERTO ECO, Trattato di semiotica generale, 17. ed., Milano, Bompiani, 1999, p. 111.

200 Cfr. ALGIRDAS JULIEN GREIMAS, Semantica strutturale, cit., p. 72; cfr. anche W. JOHN HUTCHINS, Linguistic processes in the indexing and retrieval of documents, cit., p. 31-34.

201 Cfr. W. JOHN HUTCHINS, Linguistic processes in the indexing and retrieval of documents, cit., p. 39; cfr. anche ALGIRDAS JULIEN GREIMAS, Semantica strutturale, cit., p. 80, in cui l'autore evidenzia i limiti di una semantica lessematica (cioè di una semantica che prende come unità di analisi il singolo lessema, cioè la parola, piuttosto che le componenti semantiche a essa interne; cfr. anche JULIAN WARNER, Linguistics and information theory, cit., p. 282; cfr. anche ELAINE SVENONIUS, Indexical contexts, in Universal classification I. Subject analysis and ordering systems. Proceedings. 4th Internatl. Study Conference on Classification Research, 6th Annual Conference of Gesellschaft für Klassification e.V., Augsburg, 28 June-2 July 1982. Vol. 1, editor Ingetraut Dahlberg, Frankfurt, Indeks, 1982, p. 125-138; cfr. anche JERROLD J. KATZ, Semantic theory, New York, Harper and Row, 1972, p. 298. 
sviluppate da Fairthorne (vedi 2.16.4 e ss.). In linguistica, il significato di intensione ed estensione è differente, benché correlato a quello utilizzato da Fairthorne: l'estensione indica l'insieme dei sememi inclusi da un semema: per esempio, l'estensione del semema "quadrupedi" è costituita da tutte le specie di animali che camminano su quattro zampe; l'intensione indica l'insieme delle proprietà che caratterizzano un semema ovvero dei suoi semi. ${ }^{202}$ Estensione e intensione sono inversamente proporzionali: man mano che si passa da elementi lessicali relativi a entità collettive di carattere generale o astratto a elementi lessicali relativi a entità più specifiche, fino ad arrivare a elementi lessicali relativi a entità individuali, l'estensione tende a ridursi e l'intensione ad accrescersi.

Ogni elemento lessicale in quanto significato (semema) è allora composto da più elementi semantici componenti o semi; ciascuno di essi è a sua volta costituisce un altro semema, secondo un processo di significazione virtualmente infinito (in modo simile alla "semiosi infinita" di Peirce); ${ }^{203}$ i semi sono ciò che altrove abbiamo chiamato marche semantiche ("semantic markers"), secondo la terminologia sviluppata da Katz e Fodor. Questi ultimi hanno elaborato una teoria linguistica ${ }^{204}$ in cui le parole (lessemi) sono costituite da una rappresentazione fonologica (o grafica), delle marche sintattiche (pertinenti la sintassi), e un significato (semema); quest'ultimo consiste in una serie di "letture lessicali" o percorsi interpretativi, costituiti a loro volta da un insieme di marche semantiche. ${ }^{205}$ Dobbiamo notare qui un punto importante: nella prospettiva adottata da Katz e Fodor, che sarà anche la nostra, non esiste un significato intrinseco o predefinito dei lessemi: per i due linguisti, che intendono la semantica essenzialmente come studio delle modalità e regole interpretative da parte dei parlanti, ogni significato è costituito a partire da un insieme di elementi semantici (marche semantiche) che si determinano sempre a partire da processi di tipo analitico e interpretativo; ogni semema viene a configurarsi come l'insieme dei semi che è possibile attribuirgli, secondo cui è possibile interpretarlo - la semantica assume un carattere intrinsecamente ermeneutico.

Collegata all'ermeneuticità della semantica è il suo carattere culturale: Umberto Eco definisce il significato come "unità culturale", esprimendo con ciò l'idea che i significati delle parole abbiano radici e confini eminentemente culturali, cioè anche contingenti e storici. ${ }^{206}$ Tutti i semi di un semema sono, in ultima analisi, sempre attribuiti dall'uomo e dunque di carattere soggettivo e contestuale: alcuni sono accettati da tutti in un determinato contesto e tempo - ma non perciò hanno una natura ontologica -, altri lo sono in misura minore o lo sono solo in determinati contesti. Non esiste, né può esistere, qualcosa come una semantica universale o assolutamente a priori del linguaggio (da qui l'erroneità di concepire la dimensione paradigmatica come dimensione relativa ai

202 W. JOHN HUTCHINS, Linguistic processes in the indexing and retrieval of documents, cit., p. 39; cfr. anche WOLFGANG G. STOCK, Concepts and semantic relations in information science, "Journal of the American Society for Information Science and Technology”, 61, 10 (2010), p. 1952; cfr. anche INGETRAUT DAHLBERG, A referent-oriented, analytical concept theory of INTERCONCEPT, "International classification", 5, 3 (1978), p. 148.

203 CHARLES SANDERS PEIRCE, Collected papers of Charles Sanders Peirce. Volume 1, Principles of philosophy, and Volume II, Elements of logic, cit., p. 171.

204 JERROLD J. KATZ - JERRY ALAN FODOR, The structure of a semantic theory, cit., p. 185-186; cfr.

anche ARMANDO DE PALMA, Presentazione, cit., p. 14.

205 Cfr. JERROLD J. KATZ, Semantic theory, cit., p. 298-299.

206 UMBERTO ECO, Trattato di semiotica generale, cit., p. 98. 
Tesi di dottorato di Giuliano Genetasio, discussa presso l'Università degli Studi di Udine

significati a priori degli elementi lessicali). ${ }^{207}$

Terzo carattere fondamentale della semantica è la sua relazionalità: sia nel senso che il significato di un lessema è costituito da un insieme di elementi (semi) tra loro in relazione, sia nel senso che il significato di un lessema si determina sempre a partire da relazioni con altri sememi che ne costituiscono i semi o che ne condividono un certo numero di semi, secondo rapporti di differenza-disgiunzione e identità-congiunzione. ${ }^{208}$ La semantica si costituisce cioè innanzitutto attraverso una serie di relazioni: le relazioni semantiche. Più precisamente, sulla base della differenza tra asse paradigmatico e sintagmatico, possiamo parlare di relazioni semantiche paradigmatiche e sintagmatiche. Di queste ultime parleremo soltanto più avanti (vedi $2.27,2.28$ ); delle prime abbiamo già discusso brevemente nel precedente capitolo (vedi 2.14), ma sarà adesso necessario approfondirle. Non esiste una tassonomia esaustiva o universalmente accettata in linguistica delle relazioni semantiche paradigmatiche. Quelle più comunemente riconosciute sono le relazioni semantiche di sinonimia, antonimia, eponimia, meronimia/olonimia, omonimia (o omografia, nel caso di segni grafici); ma si tratta di una classificazione aperta.

La relazione di eponimia (che comprende le relazioni di iperonimia, iponimia e coiponimia) è quella che caratterizza un semema (iperonimo) la cui estensione includa un altro semema (iponimo; i vari iponimi di un iperonimo sono detti co-iponimi se considerati in relazione l'uno all'altro), ovvero la relazione tra un primo semema e un secondo semema di cui il primo semema costituisce il sema che ne definisce la classe di appartenenza. ${ }^{209}$ Per questo motivo, le relazioni di eponimia sono anche dette relazioni generiche, in quanto legano un genere, o classe, alle sue specie, o individui; esse godono delle proprietà logiche di riflessività, transitività e asimmetria: ciò che è vero per un genere è vero anche per tutte le sue specie, e non viceversa. ${ }^{210}$ Nell'ambito dell'indicizzazione le relazioni eponimiche o generiche sono dette relazioni gerarchiche generiche (così le abbiamo chiamate nel precedente capitolo, vedi 2.14) per sottolineare l'aspetto gerarchico a esse intrinseco.

Sulla base del carattere fondamentalmente culturale della semantica del linguaggio naturale, risulta ovvio che non è possibile stabilire un unico genere o classe per ogni elemento lessicale, ma piuttosto che ogni elemento lessicale ha più generi o classi di appartenenza: teoricamente infiniti, poiché infinite sono gli insiemi concettuali in cui può essere racchiuso un elemento lessicale; praticamente corrispondenti a quelle relazioni di eponimia largamente accettate o accettabili in un dato contesto. La relazione eponimica raccoglie in effetti un insieme di relazioni gerarchiche diverse: essa potrebbe essere ulteriormente specificata sulla base del criterio che di volta in volta serve a differenziare la specie rispetto al genere. Il legame tra una classe e i suoi individui, allora, non è di tipo ontologico o intrinseco ma piuttosto tassonomico, cioè classificatorio. Per

207 Cfr. ALFREDO SERRAI, Del catalogo alfabetico per soggetti, cit., p. 83; cfr. anche TERESA GRIMALDI, L'indicizzazione dal punto di vista cognitivo (II), "Il bibliotecario", 13, 2 (1996), p. 264-265: "nessun sistema può pretendere di valere come una visione globale dell'universo semantico, e nessun modello di analisi componenziale può portare alla luce le strutture universali del significato".

208 Cfr. ALGIRDAS JULIEN GREIMAS, Semantica strutturale, cit., p. 39, 51.

209 Nelle discussioni che seguono tratteremo i vari tipi di relazione semantica (sinonimia, eponimia, olonimia, etc.) non in quanto riferite ai nomi in quanto tali, ma piuttosto ai sememi (significati) soggiacenti.

210ELAINE SVENONIUS, The intellectual foundation of information organization, cit., p. 163. 
fare un esempio: la classe "Biblioteche" comprende individui differenziati sulla base del possessore (biblioteche statali, biblioteche universitarie, biblioteche aziendali, biblioteche private), della funzione o destinazione (biblioteche nazionali, biblioteche pubbliche, biblioteche di ricerca), etc.; ma la stessa cosa può dirsi per esempio di tutte le relazioni eponimiche (genere/specie) tipiche delle scienze biologiche. Così come virtualmente infiniti sono i generi a cui può appartenere un elemento lessicale, virtualmente infiniti sono i criteri in base a cui specificare le relazioni eponimiche.

Per quanto ci riguarda, e sulla base del nesso tra piano paradigmatico e sintagmatico del linguaggio che abbiamo sopra discusso, il criterio di suddivisione delle relazioni eponimiche che adotteremo sarà basato sulla relazione sintagmatica (grammaticale) che differenzia un iperonimo o genere dai suoi iponimi o specie (vedi 3.2.7).

Accanto a relazioni di eponimia, vi sono altri "percorsi lessicali" o semantici di tipo gerarchico tra un semema e i suoi semi: sono quelle che legano un semema agli altri suoi semi che pur costituendone componenti di significato non ne determinano la classe di appartenenza ma piuttosto costituiscono gli elementi di differenziazione rispetto a quella classe. In linguistica manca una specifica definizione di questo tipo di relazione semantica. Le chiameremo relazioni di semia, sememia e co-sememia, ovvero relazioni semiche, sememiche e co-sememiche: ${ }^{211}$ parleremo di relazione semica per indicare la relazione che lega un primo semema a un secondo semema di cui il primo costituisce un sema componente, che non ne definisce la classe ma piuttosto differenzia il semema rispetto alla classe d'appartenenza; parleremo di relazione sememica per la relazione di senso opposto; due elementi lessicali in relazione sememica con un altro elemento lessicale saranno detti co-sememi se considerati l'uno in rapporto all'altro. Per fare un esempio: l'elemento lessicale "Ospedali per bambini" (ovvero "Ospedali pediatrici") è iponimo di "Ospedali", ma è subordinato concettualmente anche al concetto di "Bambini" che ne costituisce uno dei suoi semi, e a cui è legato - secondo il nostro approccio - da una relazione sememica. Sono relazioni sememiche, per esempio, anche quelle tra una disciplina o attività ("bioetica”, ovvero "etica della vita”) e il suo oggetto ("vita"). Così come per le relazioni generiche, anche le relazioni semiche e sememiche possono essere ulteriormente suddivise sulla base del tipo di relazione sintagmatica grammaticale coinvolta ma in senso inverso rispetto alle relazioni eponimiche. Se volessimo espandere ulteriormente questi concetti, tenendo ferma l'idea che tutti gli elementi lessicali siano almeno idealmente composti di più elementi semantici componenti (semi), ci accorgeremmo che un elemento gerarchicamente sovraordinato a un altro sulla base di un determinato criterio di specificazione, è invece gerarchicamente subordinato allo stesso elemento secondo un tipo di criterio di specificazione inverso: partendo dal semema "fumo", potremmo dire che esso è legato da una relazione semica a "conseguenze del fumo" - il fatto che "conseguenze del fumo" sia un sintagma è del tutto irrilevante, poiché come abbiamo spiegato il carattere semplice o composto della forma di un elemento lessicale è del tutto relativo ai diversi contesti linguistici -; "conseguenze del fumo", a sua volta, può essere concepito come iperonimo di "cancro"; quindi, potremmo

211 Relazioni semiche e sememiche sono più propriamente tutte le relazioni che riguardano semi e sememi del linguaggio, dunque anche quelle eponimiche; piuttosto di parlare di relazioni semiche/sememiche eponimiche e non eponimiche, semplificheremo la terminologia utilizzata e parleremo semplicemente di relazioni (semiche/sememiche) eponimiche da un lato, e di relazioni semiche/sememiche (non eponimiche) dall'altro. 
dire che "cancro" è legato da una relazione semica a "cause del cancro", il quale può a sua volta essere concepito come iperonimo di "fumo": abbiamo così un circolo gerarchico, cioè una catena circolare di relazioni gerarchiche di tipo inverso (il cancro come uno degli effetti possibili del fumo, il fumo come uno delle possibili cause del cancro; fig. A). Un'altra relazione semantica spesso menzionata in linguistica è la relazione partitiva o di partonomia, comprensiva delle relazioni di olonimia, meronimia e co-meronimia, che vige tra un semema (olonimo) che costituisce l'intero rispetto a un altro semema (meronimo) che costituisce una sua parte: il corpo umano rispetto ai suoi vari organi, un ente e le sue articolazioni istituzionali, una disciplina e le sue branche di studio, etc. ${ }^{212}$ Due meronimi di uno stesso elemento lessicale sono detti co-meronimi se considerati l'uno in rapporto all'altro. La relazione partitiva non gode delle stesse proprietà logiche della relazione gerarchica generica: ciò che è vero per una classe di tipo partitivo (l'intero) non è sempre vero per tutti i suoi membri (le parti). ${ }^{213}$ La relazione partitiva è detta anche relazione gerarchica partitiva nell'ambito della teoria dell'indicizzazione.

Prima di passare alle altre relazioni semantiche, precisiamo che, a differenza degli elementi lessicali riferiti a entità collettive, gli elementi lessicali riferiti a entità individuali non possono intrattenere né relazioni di iperonimia (non possono avere iponimi) né relazioni semiche (non possono avere sememi di tipo non eponimico); alcuni tipi di entità individuali possono invece intrattenere relazioni di olonimia.

La relazione di sinonimia è quella che vige tra due lessemi morfosintatticamente diversi ma di significato identico o molto simile (per esempio "nave" e "imbarcazione"), ovvero tra due o più lessemi che appartengano a uno stesso genere e la cui estensione e intensione coincidano in larga parte; secondo la terminologia di Katz e Fodor, la sinonimia è la condizione che caratterizza lessemi con un certo numero di "letture lessicali" in comune: più questo è alto, più i lessemi possono pienamente dirsi sinonimi. La sinonimia conosce cioè diversi gradi; nel linguaggio naturale, la sinonimia assoluta è rarissima, se non addirittura assente: ${ }^{214}$ nella logica di economia che caratterizza il linguaggio, essa è un inutile lusso; è invece molto più comune la condizione di lessemi semanticamente affini ma che differiscono quanto a uno o più sensi, ovvero semi o marche semantiche: in questo caso i lessemi sono pienamente sinonimi in contesti linguistici in cui quel particolare senso è irrilevante, ma non in contesti specialistici in cui quel senso assume una rilevanza. "Cane" e "Canis lupus familiaris" sono sinonimi, tuttavia il loro impiego varia in base al contesto linguistico. La relazione di sinonimia riguarda elementi lessicali riferiti a entità collettive, ma anche alcuni tipi di entità individuali (luoghi, etc.).

Relazione strettamente correlata a quella di sinonimia è la relazione di parafrasi, che riguarda l'equivalenza semantica tra elementi lessicali in forma semplice o composta da un lato ed elementi lessicali in forma composta dall'altro: "diversità biologica" è una parafrasi di "biodiversità"; quando la parafrasi è semanticamente esaustiva, si ha una vera e propria definizione; una sottotipologia delle parafrasi sono le allotassie, o sinonimie

212 WOLFGANG G. STOCK, Concepts and semantic relations in information science, cit., p. 1956; cfr. anche CRISTOPHER S. G. KHOO - JIN-CHEON NA, Semantic relations in information science, "Annual review of information science and technology", 40 (2006), p. 176.

213 W. JOHN HUTCHINS, Languages of indexing and classification, cit., p. 45; cfr. anche ELAINE

SVENONIUS, The intellectual foundation of information organization, cit., p. 164.

214 JERROLD J. KATZ - JERRY ALAN FODOR, The structure of a semantic theory, cit., p. 184-185; cfr. anche JACQUES MANIEZ, Les langages documentaires et classificatoires, cit., p. 241. 
sintagmatiche, che riguardano una relazione di sinonimia tra sintagmi i cui elementi lessicali componenti abbiano sequenze diverse: per esempio "Giovanni ama Maria" rispetto a "Maria è amata da Giovanni".

Fin qui abbiamo considerato i sinonimi e le parafrasi come elementi lessicali semanticamente molto simili ma distinti. La semantica generativa si è spinta oltre, fino a formulare, con l'idea delle trasformazioni linguistiche, la possibilità di una piena identità semantica tra sintagmi differenti solo morfosintatticamente (cioè quanto alla loro "struttura di superficie"), ovvero fino a concepire tali sintagmi come forme diverse di uno stesso significato. Hutchins, partendo da un'impostazione concettuale simile, elaborò la nozione di "formula sememica": il semema (significato) in quanto considerato a prescindere dalla forma paradigmatica o sintagmatica dei suoi lessemi. ${ }^{215}$ Relazioni di sinonimia, parafrasi, allotassia, etc. corrispondono nel loro complesso alle relazioni di equivalenza a cui abbiamo fatto riferimento nell'approccio concettuale all'indicizzazione, con l'avvertenza che le relazioni di equivalenza tipiche dell'indicizzazione includono anche rapporti di tipo leggermente diverso dalla vera e propria sinonimia come le relazioni di equivalenza tra differenti nomi o forme del nome di una persona, diversi titoli con cui è

215 W. JOHN HUTCHINS, Linguistic processes in the indexing and retrieval of documents, cit., p. 35-36: "The semantic abstract for any phrase or sentence - its sememic formula (SF) - must, therefore, specify the semons attached to its lexemes, the relationships between them, and the syntactic relationships between the lexemes [...] Different phrases may have the same SF in the following instances: (a) If they have the same lexemes but in different syntactic structures, when these structures are semantically equivalent (e.g. our example of active and passive forms) (b) If they have lexemes of different graphic form but with the same semons (i.e. synonyms) and at the same time they have the same syntactic structure, e.g. oculists sometimes marry shorthand-typists and eye-doctors sometimes marry stenographers (c) If both (a) and (b) occur simultaneously; thus, two phrases can differ in both their constituent lexemes and in their syntactic structures and yet still have the same SF (i.e. one is a paraphrase of the other)"; p. $42-45$ "A common and fruitful method is that of commutation: two units which are able to replace each other with no change in the response of speakers of the language are held to be variants of the same element [...] On the semantic plane, two units (i.e. lexemes) may be held to have the same semantic value (i.e. the same sememe or SF) if they are mutually replaceable [...] K. Sparck Jones (1965) has described a method for finding groups (or 'rows') of synonyms from definitions in dictionaries. (The use of general dictionaries to establish communal language usage is obviously valid.) When a dictionary does not define a word directly, e.g. by describing its referents, but instead indirectly by listing its synonyms, these synonyms and the word itself form a 'row' of lexemes all having the same sememe [...] It must, however, be kept in mind that many lexemes will appear in different rows because they are homonyms. 7.3 James H. White (1964) has attempted to establish the semons of English prepositions [...] Because they occur in so many idiomatic phrases, prepositions are likely to prove extremely difficult to analyse.[...] 7.5 Paraphrases. - In many cases lexemes do not have synonyms but may nevertheless be replaced by a sequence of lexemes with the same meaning (paraphrases: section 3). Dictionaries give many examples of definitions in the form of paraphrases [...] 7.8 Syntax. - Analogous to the synonym relation between lexemes there are, as we mentioned in section 3, certain phrase-structures which are semantically equivalent and hence commutative. We take again our active-passive transformation: the boy loves the girl and the girl is loved by the boy. In transformational grammar the passive construction can be derived from the active by a 'transformational rule' applied to the whole”; p. 46-47, in cui Hutchins, a partire dai grafici di Lamb (SYDNEY M. LAMB, The sememic approach to structural semantics, cit.), in cui dei lessemi componenti una frase vengono evidenziati in orizzontale le relazioni sintagmatiche e in verticale quelle paradigmatiche, sviluppa in forma di grafici l'idea di formula sememica; tramite trasformazioni, è possibile ottenere tutte le altre varianti linguistiche paradigmatiche e sintagmatiche di una determinata formula sememica; da notare che Hutchins segnala che gli equivalenti sintagmatici delle forme paradigmatiche degli elementi lessicali hanno precise relazione sintagmatiche, che vanno rappresentate nel grafico, per esempio "storico" può essere trasformato in "uomo che scrive storia" evidenziando le relative relazioni sintagmatiche. 
Tesi di dottorato di Giuliano Genetasio, discussa presso l'Università degli Studi di Udine

conosciuta un'opera, etc.

La relazione di antonimia o antonimica è quella che lega sememi di significato opposto, ovvero sememi che condividono un certo numero di semi, ma non abbastanza da essere considerati sinonimi, e che per il resto partecipano di semi di valore contrario: per esempio "bianco" e "nero". Vi sono molteplici classificazioni degli antonimi; tra le relazioni di antonimia più spesso citate, e che adotteremo per il nostro discorso, vi sono: l'antonimia scalare, tra antonimi che si situano agli estremi di un unico continuum o scala ("bianco" e "nero", "alto" e "basso") e polare, tra antonimi che non prevedono gradi intermedi ("vivo" e "morto", "falso" e "vero", etc.); vi è poi l'antonimia complementare, tra antonimi che si definiscono reciprocamente ("marito" e "moglie", "compratore" e "venditore", etc.). ${ }^{216}$

Un'altra relazione semantica è la cosiddetta "famiglia semantica": espressione utilizzata in linguistica per indicare la relazione che lega tutti i lessemi che condividono uno stesso sema e anche parte del significante (p.e. biologia, biochimica, biopolitica, bioetica, biopsia, biografia, etc.; ma anche scultori, armatori, scrittori, educatori, etc.): si tratta, secondo la nostra terminologia, di co-sememi. Co-sememi sono anche le relazioni tra quei sostantivi deverbali, cioè derivati da un verbo, o nominalizzazioni (vedi 3.3.6.3) che appartengono a tipologie lessicali differenti (vedi più sotto per una discussione delle tipologie lessicali): per esempio "agricoltura" e "agricoltori", "educazione” ed "educatori", etc.

La linguistica parla solitamente di relazioni di eponimia, meronimia, olonimia, sinonimia, etc. senza spiegarne le reciproche relazioni; per il nostro discorso è invece importante cercare almeno di abbozzare i rapporti tra le diverse relazioni semantiche. Da quello che abbiamo detto sopra, è già chiara la stretta correlazione tra sinonimi e antonimi; la differenza tra antonimia e sinonimia non è dunque una differenza qualitativa, ma fondamentalmente quantitativa: sono antonimi i lessemi che condividono uno o più semi ma che sono opposti per almeno un sema; sono invece sinonimi i lessemi che condividono la maggior parte dei semi (tutti solo nel caso limite della sinonimia assoluta). Inoltre, vi è una stretta correlazione anche tra le relazioni di sinonimia e antonimia da un lato e relazioni di eponimia dall'altro: sinonimi e antonimi sono anche co-iponimi di uno stesso semema.

Passiamo oltre. Il linguaggio naturale è tipicamente caratterizzato da ambiguità. Parole e sintagmi possono essere ambigui dal punto di vista semantico, sintattico, etc. Fonte principale e condizione necessaria (benché non sufficiente) dell'ambiguità semantica del linguaggio sono i fenomeni dell'omonimia, della polisemia e dell'omotassia. ${ }^{217}$ Omonimia e polisemia sono due relazioni semantiche affini, ma lievemente diverse: l'omonimia (e l'omografia, riferendoci a segni grafici) indica la condizione di parole con significanti identici ma differenti significati e, generalmente, etimi; in tal senso essa è la relazione inversa a quella di sinonimia; la polisemia indica la condizione di una parola con più significati tra loro correlati, ovvero con più accezioni di significato; a differenza dell'omonimia, la polisemia riguarda generalmente lessemi con uno stesso etimo; essa, inoltre, è una condizione connaturata al linguaggio naturale: ${ }^{218}$ ogni parola acquisisce nel

216 Cfr. W. JOHN HUTCHINS, Languages of indexing and classification, cit., p. 41-42.

217 Cfr. JERROLD J. KATZ - JERRY ALAN FODOR, The structure of a semantic theory, cit., p. 187-188; cfr. anche JERROLD J. KATZ, Semantic theory, cit., p. 300.

218 Cfr. ELAINE SVENONIUS, The intellectual foundation of information organization, cit., p. 148, 127-128; 
tempo più significati correlati o accezioni di uno stesso significato; ciò è anzi una delle condizioni fondamentali perché una lingua possa evolvere. L'omotassia, invece, è l'ambiguità semantica di origine sintattica che coinvolge elementi lessicali in forma sintagmatica, identici nell'aspetto ma differenti nel significato: "Maria e Giovanni cantano e danzano", che ne è un esempio, può significare tanto che sia "Maria" sia "Giovanni" "cantano e danzano", quanto che "Maria" canti e "Giovanni" danzi, etc.; un altro esempio è l' "Amore di Maria", laddove "di" può introdurre un agente (è Maria che ama) o un oggetto (Maria è amata); Hutchins fa riferimento all'omotassia definendola "ambiguità sistematica". 219

Che si parli di omonimia o di polisemia o di omotassia, l'ambiguità semantica è da un lato una condizione naturale del linguaggio (su di essa è fondata tra l'altro anche la funzione poetica del linguaggio), dall'altro un potenziale ostacolo per una comunicazione efficace; quando ciò avviene, il linguaggio naturale dispone comunque di una serie di elementi e meccanismi che permettono di sciogliere l'ambiguità: 1) nell'ambito di un elemento lessicale in forma sintagmatica, un ruolo di disambiguazione importante è svolto dagli altri elementi del sintagma rispetto a quello semanticamente ambiguo: l'ambiguità semantica può essere cioè spesso sciolta grazie ai rapporti sintattici e semantici tra gli elementi lessicali componenti il sintagma; ciò significa anche che un elemento lessicale in forma semplice, fuori da un sintagma, è più facilmente esposto al rischio di ambiguità semantica; 2) un ruolo di disambiguazione importante è giocato dal contesto linguistico (sintagmi precedenti e seguenti quello preso in considerazione) ed extralinguistico (situazioni a cui fa riferimento il sintagma in oggetto, più o meno note ai parlanti); 3) nei dialoghi, un ruolo di disambiguazione importante è svolto dal feedback (o retroazione), tramite cui il destinatario di un messaggio si rivolge all'autore del messaggio per assicurarsi di avere inteso correttamente il messaggio o chiedergli ulteriori spiegazioni. Vi sono infine le relazioni semantiche tipiche della retorica, legate alla funzione poetica del linguaggio: metonimia, metafora, etc., ma tralasceremo di parlarne perché le riteniamo non essenziali per il nostro discorso (con l'avvertenza che uno studio di queste relazioni è invece fondamentale per l'analisi o l'elaborazione di lingue documentarie di ambito specificamente retorico o poetico).

Più sopra, abbiamo detto che uno dei caratteri fondamentali della semantica è la sua relazionalità. Ma non tutto, nella semantica, è riducibile a relazioni; strettamente connessa alla semantica delle relazioni è una semantica delle tipologie lessicali o "entità"220 linguistiche: tipologie semantiche fondamentali degli elementi lessicali; a esse avevamo già fatto riferimento quando avevamo distinto elementi lessicali riferiti a entità collettive o individuali. Non vi è precedenza della semantica delle tipologie lessicali rispetto alla semantica delle relazioni, o viceversa, ma piuttosto esse si determinano reciprocamente. Non esiste una classificazione universale o totalmente a priori delle tipologie lessicali, tale da valere per ogni lingua; tuttavia in ogni lingua è implicita una simile classificazione: l'uso dei diversi elementi lessicali, ovvero la formazione di sintagmi, la presuppone; così, sappiamo che soltanto un'azione può avere un agente o un oggetto; che soltanto un'entità animata può intraprendere volontariamente azioni; che all'interno

cfr. anche JULIAN WARNER, Analogies between linguistics and information theory, cit., p. 315.

219 Cfr. W. JOHN HUTCHINS, Languages of indexing and classification, cit., p. 64.

220 "Entità" solo in senso generico, perché come diremo tra le tipologie lessicali vi sono anche le attività, che non sono immediatamente riconducibili a entità. 
di una stessa frase non possiamo coordinare, rispetto a un'azione, elementi di natura semantica diversa; etc. Ciò è collegato alla già menzionata relatività dei piani paradigmatico e sintagmatico del linguaggio.

Dicevamo che semantica delle relazioni e delle tipologie lessicali sono strettamente legate; la classificazione delle tipologie semantiche di elementi lessicali è legata in modo particolare alla semantica delle relazioni gerarchiche di ogni lingua, in modo tale che ogni classe di una relazione gerarchica costituisce in sé un naturale raggruppamento semantico lessicale, una naturale tipologia di elemento lessicale: dai livelli più alti delle relazioni gerarchiche fino ai livelli più specifici; la classificazione delle tipologie di elementi lessicali determina a sua volta quali elementi lessicali possano comparire nei sintagmi e in quali posizioni - tanto a livello di relazioni sintagmatiche (vedi 3.2.7) quanto di specifici elementi lessicali - perché si abbiano sintagmi dotati di senso.

Non esiste, lo ripetiamo, una semantica universale delle tipologie lessicali; quella che qui proponiamo, di carattere assolutamente generale e contingente, ha semplicemente i vantaggi di essere relativamente condivisa e utile per il nostro discorso; premettiamo anche che le diverse dicotomie su cui si basa la classificazione non sono, a nessun livello, esenti da problemi e aporie. Con queste doverose precisazioni, possiamo dividere gli elementi lessicali (in quanto sememi) in: entità (cose) e attività (azioni o processi); nell'ambito delle entità, poi, possiamo stabilire ulteriori distinzioni: entità animate (viventi) e inanimate (non viventi); entità collettive (la cui estensione include ulteriori entità) e individuali (entità uniche, la cui estensione non include ulteriori entità); più problematiche sono le distinzioni tra entità astratte ed entità concrete, e tra entità con nomi propri ed entità con nomi comuni. ${ }^{221}$

\subsection{Rapporti tra sintassi, semantica e pragmatica}

Finora, parlando dei diversi aspetti linguistici (morfosintassi, semantica, pragmatica), abbiamo dato per scontato che tali aspetti fossero ben distinti tra loro, che ciascuno di essi avesse una propria autonomia; tra le altre cose, abbiamo visto come la linguistica chomskyana avesse posto, nell'ambito della morfosintassi, una distinzione netta tra "struttura profonda" e "struttura di superficie". In effetti, però, nella realtà linguistica concreta i tre aspetti linguistici si trovano sempre sovrapposti tra loro; essi sono, piuttosto che realtà autonome e discrete, sono realtà profondamente interconnesse e dai confini non definiti in modo netto. ${ }^{222}$ La semantica riguarda il significato dei lessemi, soprattutto

221 Anche ASSOCIAZIONE ITALIANA BIBLIOTECHE. GRUPPO DI RICERCA

SULL'INDICIZZAZIONE PER SOGGETTO, Guida all'indicizzazione per soggetto, cit., 2001, p. 23, parte dalla distinzione fondamentale di entità e attività; p. 77: "In alcuni casi è difficile stabilire se si è in presenza di un nome proprio o di un nome comune, perché non si può tracciare una netta linea di separazione: uno stesso nome può venire considerato in periodi, luoghi, e presso gruppi diversi come proprio o comune".

222 Cfr. AUGUSTO PONZIO, Il linguaggio e le lingue., cit., p. 50-53; cfr. anche CHARLES J. FILLMORE, Pragmatics and the description of discourse, in Radical pragmatics, edited by Peter Cole, New York, Academic Press, 1981, p. 144: "In the extent to which the types of observations one is dealing with are distinc, one can regard these three fields as more or less auonomous; but in the extent to which observations in one of these areas yeld to explanations drawing onanother, they are interdependent"; cfr. anche ALFREDO SERRAI, Problemi linguistici e implicazioni epistemologiche della catalogazione semantica, cit., p. 113. 
in quanto preso in sé; la pragmatica riguarda invece, tra le altre cose, il significato d'uso o contestuale dei lessemi.

Ma fino a che punto è possibile distinguere i significati in sé dei lessemi e i loro significati d'uso? Se accettiamo l'assunto saussuriano dell'arbitrarietà del segno - e lo faremo perché il contrario vorrebbe dire rifugiarsi in insostenibili posizioni innatiste oppure ingenuamente realiste del linguaggio - non possiamo che acconsentire all'idea che il significato nasca a partire dall'uso e qui sempre ritorni. Non possiamo, cioè, che accettare l'idea secondo cui è attraverso l'uso dei lessemi nei diversi contesti linguistici e momenti storici che si producono slittamenti di senso (nuovi significati di un lessema), che si producono nuovi lessemi o che viceversa alcuni lessemi muoiono perché non più utilizzati.

Accettare il primato del significato d'uso, e dunque della pragmatica, può tradursi in due diverse conseguenze a livello di analisi linguistica: 1) rinunciare del tutto all'idea dell'esistenza dei significati a prescindere dal loro uso e, dunque, all'idea di una semantica come distinta dalla pragmatica; esponente di tale posizione è Ludwig Wittgenstein, secondo cui "il significato di una parola è il suo uso nel linguaggio"; ${ }^{223}$ essa è anche, nell'ambito dell'indicizzazione, la posizione di David C. Blair; ${ }^{224}$ 2) continuare a mantenere distinte semantica e pragmatica, pur nella consapevolezza della loro stretta interconnessione e, in particolare, subordinazione concettuale della prima alla seconda, ovvero anche dell'impossibilità di una semantica assolutamente a priori; la semantica stessa verrebbe cioè a configurarsi come una sorta di "pragmatica decontestualizzata", costruita a partire da astrazioni dal significato d'uso dei lessemi, da fotografie del significato di un lessema in un dato punto spazio-temporale, laddove la pragmatica propriamente intesa guarderebbe al significato di un lessema in modo dinamico, nel suo farsi attraverso le diverse situazioni e funzioni linguistiche con cui è utilizzato: referenziali, ma anche retoriche, metalinguistiche, etc.

Sarà quest'ultima la posizione che adotteremo: riteniamo infatti che distinguere aspetti semantici e pragmatici dei lessemi sia, pur con le suddette riserve, possibile e utile ai fini dell'analisi linguistica. Allo stesso tempo, anticipiamo che vi è una costante sovrapposizione di semantica e pragmatica nel linguaggio: poiché tutti gli elementi linguistici assolvono a una qualche funzione linguistica, essi sono con ciò intrinsecamente pragmatici (oltre che semantici, sintattici, etc.); tutte le relazioni semantiche di carattere più contestuale (come per esempio le relazioni gerarchiche atipiche, vedi 3.3.6.4.2.2.2.4), così come tutti gli espedienti utilizzati dal linguaggio naturale per la disambiguazione di omonimia, polisemia e omotassia, appartengono anch'essi sia alla semantica sia alla pragmatica del linguaggio.

Il progetto originario di Chomksy poneva, come abbiamo mostrato, una priorità della morfosintassi rispetto agli altri aspetti linguistici e una sua totale autonomia; nell'ambito della morfosintassi, sarebbe stato possibile distinguere nettamente una struttura "profonda" da una "superficiale". Queste posizioni si rivelarono ben presto insostenibili,

223 LUDWIG WITTGENSTEIN, Ricerche filosofiche, edizione italiana a cura di Mario Trinchero, Torino, Einaudi, 2009, traduzione di Philosophische Untersuchungen, p. 33.

224 DAVID C. BLAIR, Language and representation in information retrieval, Acit., p. 144: "A word (like a tool), regardless of how it is used always looks the same. It is this similarity in appearance that makes use think that there is some essence or meaning that accompanies the word all times. This is the mistake that Semiotics [...] makes when it ties a word/expression to some "content" ". 
per due motivi: 1) come notò Chomsky, la morfosintassi non riusciva a rendere conto delle omotassie; ${ }^{225}$ 2) la morfosintassi non riusciva a rendere conto dell'inaccettabilità di tutti i sintagmi sintatticamente corretti e tuttavia (semanticamente) insensati come il celebre "le idee verdi dormono furiosamente". 226

Passo successivo e inevitabile a tali acquisizioni non poté che essere l'introduzione formale della semantica come elemento della grammatica, cosa che venne realizzata con la teoria semantica di Katz e Fodor. Ma le nozioni di regole di proiezione, marche semantiche, etc., introdotte dai due linguisti, lungi dall'estendere o modificarne singoli aspetti del sistema chomskyano, erano le premesse per una sua trasformazione essenziale: con esse veniva tendenzialmente meno sia l'autonomia della morfosintassi dalla semantica, sia la possibilità di isolare la "struttura profonda" dalla "struttura di superficie" del linguaggio. ${ }^{227}$ L'affermazione della semantica generativa mise definitivamente in crisi una concezione della morfosintassi come aspetto autonomo dalla semantica; la semantica generativa mostrò inoltre, come abbiamo avuto modo di dire, la natura intrinsecamente sintagmatica dei significati dei lessemi e, con ciò, la sostanziale affinità tra strutture sintattiche e semantiche. ${ }^{228}$

La sovrapposizione tra semantica e morfosintassi era stata fatta propria, già prima $\mathrm{e}$ indipendentemente dalla semantica generativa, da due linguisti esterni alla scuola chomskyana: Greimas e Hutchins. Questi autori non si limitarono a constatare la sovrapposizione di semantica e morfosintassi, ma notarono come la seconda fosse essenzialmente subordinata e funzionale alla prima. Essi si spinsero cioè a concepire la morfosintassi come una funzione, una parte o ancora uno strumento della semantica: Greimas ha parlato a proposito di "sintassi semantica"; ${ }^{229}$ Hutchins ha mostrato come il significato di un sintagma derivi non solo dai significati dei singoli lessemi del sintagma, ma anche dai rapporti sintattici tra essi. ${ }^{230}$ In ambito bibliografico, Alfredo Serrai ha posto la sintattica come "primo gradino della semantica", rifacendosi alla tripartizione

225 NOAM CHOMSKY, La grammatica trasformazionale, cit., p. 23-24; cfr. anche W. JOHN HUTCHINS, Languages of indexing and classification, cit., p. 66, il quale nota come la relazione sintagmatica "coordinativa" di congiunzione possa creare sintagmi semanticamente ambigui.

226 Cfr. YVES GILBERT COURRIER, Document analysis, verbs and case grammar, cit., p. 85; cfr. anche UMBERTO ECO, La ricerca della lingua perfetta nella cultura europea, cit., p. 337: "L'idea chomskyana della differenza tra "struttura profonda" e "di superficie" è stata duramente critica anche da Eco, per cui essa rappresenterebbe una forma di universalismo linguistico inaccettabile; riferendosi a essa, l'autore afferma: "Per procedere in tal modo occorre non essere sfiorati neppure per un istante dall'idea che una data lingua rifletta un dato modo di pensare e di vedere il mondo, non un Pensiero Universale; occorre cioè che quello che è stato chiamato il "genio" di una lingua venga relegato tra le modalità di uso superficiale che non affettano la struttura profonda uguale per tutte le lingue. Solo in tal modo è possibile assumere come universali, perché corrispondenti alla sola logica possibile, quelle strutture che identifichiamo nella sola lingua in cui ci si dispone a pensare".

227 Cfr. JEAN-CLAUDE GARDIN, Document analysis and linguistic theory, cit., p. 153-156; cfr. anche YVES GILBERT COURRIER, Document analysis, verbs and case grammar, cit., p. 85-86.

228 W. JOHN HUTCHINS, Languages of indexing and classification, cit., p. 58: "The approach of the generative semanticists is important for [...] it recognises the interdependence of syntax and semantics - i.e. that the syntagmatic organisation of semantic structures, including the internal composition of individual sememes [...] is similar to that of surface syntactic (lexemic) structures"; cfr. anche HOWARD MACLAY, Overview, cit., p. 178-179; cfr. anche GUGLIELMO CINQUE, Introduzione del curatore, cit., p. 17-18. 229 Cfr. ALGIRDAS JULIEN GREIMAS, Semantica strutturale, cit., p. 159-160.

230 W. JOHN HUTCHINS, Linguistic processes in the indexing and retrieval of documents, cit., p. 35. 
Tesi di dottorato di Giuliano Genetasio, discussa presso l'Università degli Studi di Udine

morrisiana degli aspetti linguistici. ${ }^{231}$

Anche noi seguiremo, in generale, questa impostazione dei rapporti tra semantica e morfosintassi, con l'avvertenza che se è possibile affermare che la "struttura di superficie" del linguaggio è interamente pertinente la morfosintassi, più complessa è la situazione della "struttura profonda" del linguaggio, nella quale semantica e morfosintassi coesistono (con la seconda sempre in una certa misura dipendente dalla prima, vedi 3.2.6, 3.2.7).

Riassumendo, gli aspetti linguistici (morfosintassi, semantica, pragmatica) non soltanto non sono autonomi, ma è anzi possibile porre una precisa gerarchia tra di essi. A questa conclusione giunse forse per primo Charles J. Fillmore: a partire da una personale interpretazione della tripartizione morrisiana, Fillmore da un lato affermò la possibilità di distinguere fenomeni semantici, sintattici e pragmatici del linguaggio, dall'altro evidenziò che in alcuni casi i fenomeni sintattici richiedevano una spiegazione semantica e che in alcuni casi i fenomeni semantici richiedevano una spiegazione pragmatica. Secondo il linguista, in altri termini, la sintassi riguarderebbe esclusivamente la forma del linguaggio, la semantica riguarderebbe forma e funzioni comunicative del linguaggio, la pragmatica riguarderebbe forma del linguaggio, funzioni del linguaggio e contesto extralinguistico; l'impostazione di Fillmore fu seguita anche da Hutchins. ${ }^{232}$ Più recentemente, e nell'ambito della teoria dell'indicizzazione, a tale impostazione si è riallacciato anche Blair, che ha affermato la priorità della pragmatica sugli altri aspetti linguistici, derivati per astrazione da quella. ${ }^{233}$

\subsubsection{Ancora su asse paradigmatico e sintagmatico, semantica e sintassi}

Questa impostazione dei rapporti tra gli aspetti linguistici, e in particolare tra semantica e sintassi, getta nuove luci sul rapporto tra asse paradigmatico e sintagmatico del linguaggio. Esiste certamente un nesso tra semantica e asse paradigmatico da un lato, e sintassi e asse sintagmatico dall'altro; molti autori nell'ambito della teoria dell'indicizzazione hanno portato tale nesso fino a una vera e propria identificazione, contrapponendo così le relazioni semantiche o paradigmatiche alle relazioni sintattiche; così la stessa Svenonius e

231 ALFREDO SERRAI, Problemi linguistici e implicazioni epistemologiche della catalogazione semantica, cit., p. 113.

232 W. JOHN HUTCHINS, Languages of indexing and classification, cit., p. 4 ; CHARLES J. FILLMORE, Pragmatics and the description of discourse, cit., p. 1: "I believe that syntactic, semantic and pragmatics facts can be distinguished from each other, but $\mathrm{i}$ also believe that some syntactic facts require semantic and pragmatic explanations and that some semantic facts require pragmatic explanations. Put differently, interpreters sometimes use semantic and pragmatic informatioin making judgments about the syntactic structure of a sentence, and they sometimes use pragmatic facts in making semantic judgments [...] Syntax, in short, characterizes the grammatical forms that occur in a language, while semantics pairs these forms with their potential comunicative functions. Pragmatics is concerned with the three-termed relation which unites (i) linguistic form and (ii) the communicative functions which these forms are capable of serving, with (iii) the contexts or settings in which those linguistic forms can have those communicative functions. [...]

SYNTAX [form]

SEMANTICS [form, function]

PRAGMATICS [form, function, setting]".

233 DAVID C. BLAIR, Language and representation in information retrieval, cit., p. 136. 
Tesi di dottorato di Giuliano Genetasio, discussa presso l'Università degli Studi di Udine

molti altri. ${ }^{234}$

In realtà la situazione è più complessa. L'asse paradigmatico del linguaggio è, in effetti, di esclusiva pertinenza della semantica: ciò è universalmente accettato dalla linguistica postsaussuriana.

Molto più articolata è la situazione dell'asse sintagmatico. Per spiegarla possiamo ripartire dalla distinzione tra "struttura profonda" e "di superficie". La struttura superficiale dell'asse sintagmatico, o asse sintagmatico di superficie, riguarda esclusivamente la morfosintassi così come l'abbiamo intesa sinora, ovvero le caratteristiche morfologiche dei lessemi (sostantivi, aggettivi, verbi, avverbi, etc.) e le relazioni sintattiche dei lessemi (soggetto, predicato, oggetto, etc.) in quanto significanti; non approfondiremo ulteriormente l'analisi della morfosintassi di superficie dell'asse sintagmatico, che ricade in pieno nella morfosintassi così come l'avevamo più sopra trattata (vedi 3.3.6.3). La struttura profonda dell'asse sintagmatico, o asse sintagmatico profondo, riguarda invece gli elementi lessicali considerati in quanto sememi, pertinenti dunque la semantica ma - e qui sta la differenza rispetto alla distinzione chomskyana originale di "struttura profonda" e "di superficie" - anche un certo tipo di sintassi: la sintassi della struttura profonda o semplicemente "sintassi profonda" (vedi 3.2.7).

Sull'asse sintagmatico profondo si sovrappongono in effetti tre diversi sistemi, compresenti all'interno di ciascun sintagma: 1) la presenza implicita della semantica paradigmatica delle relazioni e delle tipologie lessicali, la quale pone dei vincoli alla combinazione dei diversi elementi lessicali (ovvero alla formazione di sintagmi) che ne garantiscono la sensatezza; abbiamo già fatto l'esempio della frase "il cane abbaia": in essa, oltre alle relazioni sintattiche (superficiali) entrano in gioco dei vincoli semantici tali che il sostantivo "cane" possa avere soltanto un certo numero di valori lessicali, tra cui "abbaia" o "guaisce" o "scodinzola", e che viceversa il verbo "abbaia" sia semanticamente appropriato soltanto per il lessema "cane"; Hutchins ha chiamato le relazioni semantiche paradigmatiche presenti nei sintagmi relazioni "fraseologiche"; ${ }^{235}$ 2) la presenza di relazioni sintagmatiche (casi profondi e relazioni sintagmatiche d'altro tipo) che costituiscono i "significati relativi" assunti dagli elementi del sintagma, e che hanno una natura al contempo sintattica e semantica; 3) la presenza di altre relazioni sintagmatiche, di natura sostanzialmente sintattica, che abbiamo definito "logiche", presenti esclusivamente all'interno dei sintagmi e funzionali alla loro costruzione. ${ }^{236}$

234 REBECCA GREEN, Syntagmatic relationships in index languages, cit., p. 374-376: "It has been reasoned that if syntagmatic relationships are syntactic, they cannot then be semantic [...] Symptomatic of this approach is the recognition of two separate relationship system in index languages, one semantic and the other syntactic. Where such a distinction is drawn, it is often the case that the semantic relationships are seen to correspond with paradigmatic relationships, while the syntactic relationships correspond with syntagmatic relationships".

235 W. JOHN HUTCHINS, Languages of indexing and classification, cit., p. 32.

236 Cfr. JACQUES MANIEZ, Actualité des langages documentaires, cit., p. 214-215, 216 (nota): "En linguistique, ce schéma à deux coordonnées perpendiculaires, qu'il soit implite ou explicite, et quels que soient les termes techniques utilisés, est admis par tous les théoriciens [...] On peut y voir une image de la dualité lexique /syntaxe (certains disent malencontrousement sémantique / syntaxe) [...] Sur l'axe syntagmatique, la relation est de type contrastif et connectif, c'est une relation de compatibilité à la fois syntaxique et sémantique [...] Sur l'axe paradigmatique, la relation est de type oppositionnel et comparatif [...] Opposer syntaxe et sémantique, ce que est d'usage courant dans notre discipline, donne à penser que la syntaxe des langues n'est pas de nature sémantique. Il serait préférable de distinguer sémantique lexicale et sémantique syntaxique"; cfr. anche REBECCA GREEN, Syntagmatic relationships in index languages, cit., p. 
Tesi di dottorato di Giuliano Genetasio, discussa presso l'Università degli Studi di Udine

\subsubsection{Asse sintagmatico: semantica sintagmatica o sintassi profonda}

Approfondiamo adesso le relazioni (semantiche) sintagmatiche. Fillmore spiegò che accanto alle relazioni semantiche paradigmatiche, le quali fanno parte di una semantica esterna o della verità o dell'implicitazione, esiste un secondo genere di relazioni semantiche relative alla struttura interna di una proposizione; una semantica interna o relativa ai significati che i lessemi assumono all'interno di un sintagma. ${ }^{237}$ Guardando alle relazioni sintagmatiche, e riallacciandosi alla distinzione chomskyana tra "struttura profonda" e "struttura di superficie", Fillmore ipotizzò che accanto alle relazioni sintagmatiche morfologiche (aggettivo, sostantivo, etc.) e sintattiche (verbo, soggetto, etc.) tradizionali, fisse a livello di "struttura di superficie" ma non necessariamente a livello di "struttura profonda", vi fosse un secondo tipo di relazioni sintagmatiche giocate dai lessemi di un sintagma, come per esempio la relazione tra agente e attività, o tra attività e oggetto, di natura essenzialmente semantica e che non sarebbero mutate nemmeno a livello di "struttura profonda": una sintassi profonda o semantica sintagmatica. Così come a livello di struttura superficiale il sintagma si organizza attorno a un nucleo costituito dal verbo o dal sintagma verbale, rispetto a cui tutti gli altri elementi sono argomenti che completano, specificano o modificano quel verbo, Fillmore teorizzò che nella struttura profonda i sintagmi assumessero una forma in cui l'attività rappresentata dal verbo o da elementi deverbali fosse messa in relazione con i diversi argomenti o ruoli (agente, oggetto, strumento, etc.) rappresentati dagli altri lessemi. Il linguista denominò questo tipo di relazioni semantiche tipiche della struttura profonda "casi profondi" (poi conosciuti anche come "ruoli semantici" o "tematici": useremo indistintamente le due espressioni). ${ }^{238}$

La prima lista dei casi profondi elaborata da Fillmore includeva il caso agentivo (relativo all'agente, ovvero all'entità che compie volontariamente un'azione), strumentale (relativo allo strumento, l'entità che serve involontariamente al compimento di un'azione), ${ }^{239}$ fattitivo (o relativo al prodotto di un'azione), oggettivo (relativo all'oggetto del compimento di un'azione) ${ }^{240}$ e locativo (relativo alla collocazione spaziale dell'azione;

382: "On this conceptual level, the traditional distinction between paradigmatic and syntagmatic relationships is moot, since all semantic relationships can be expressed syntagmatically".

237 CHARLES J. FILLMORE, Si riapre il caso del caso, traduzione di G. R. Cardona, in Gli universali nella teoria linguistica, Torino, Boringhieri, 1978, p. 271-300, traduzione di The case for case reopened, p. 272 :

"Quella che potremmo chiamare semantica "interna" rispetto alla semantica "esterna"; essa interesessa cioè non la semantica della verità o dell'implicitazione o della forza illocutiva, ma piuttosto la natura semantica della struttura interna di una proposizione. Nella semantica interna l'interesse è sintagmatico più che paradigmatico".

238 CHARLES J. FILLMORE, Si riapre il caso del caso, cit., p. 272, 284: "Quel che io sostenevo è che la teoria grammaticale richiede un livello di struttura casuale che io ritenevo identico, o molto vicino, a un livello di rappresentazione semantica”.

239 Comunemente si afferma che il caso strumentale coinvolga esclusivamente entità inanimate; tuttavia in alcuni casi anche alcune entità animate (animali) possono svolgere un caso strumentale se utilizzate da agenti.

240 Successivamente saranno proposte altre denominazioni indicanti ruoli sostanzialmente simili: paziente, tema, etc. 
indicante stato in luogo); ${ }^{241}$ negli anni successivi, questa lista venne estesa progressivamente da Fillmore stesso o da altri autori. Vennero così aggiunti tra gli altri il caso benefattivo (relativo al beneficiario di un'azione), ${ }^{242}$ causativo (relativo alla causa, ovvero all'entità o attività che provoca involontariamente il compimento di un processo; molto simile allo strumento ma non utilizzabile da un agente), temporale (relativo alla circostanza temporale di un'azione), comitativo (relativo a una compagnia indicata dall'azione), di scopo o funzionale, di origine o fonte (relativo alla provenienza spaziale dell'azione; indicante moto da luogo; lo definiremo anche caso originario), di direzione o meta (relativo alla direzione spaziale dell'azione; indicante moto a luogo; lo definiremo anche caso direttivo), per menzionare soltanto quelli più citati.

Non esiste ad oggi un consenso universale sul numero o sull'identità dei casi profondi; piuttosto, l'individuazione di un certo numero di casi profondi è una funzione della granularità di analisi semantica degli elementi dei sintagmi; inoltre, non sempre è possibile stabilire in modo netto a quale caso profondo appartenga un elemento del sintagma, e ciò vale già per i casi profondi elaborati originariamente da Fillmore; per esempio, in alcuni casi è difficile stabilire se un luogo geografico svolga un caso oggettivo o locativo: i "bombardamenti in Sicilia" sono anche i "bombardamenti della Sicilia", o perlomeno i due sintagmi sono così strettamente correlati che una loro distinzione dal punto di vista semantico risulta problematica.

Ciononostante, i casi profondi di Fillmore hanno avuto un'immensa fortuna critica e influenza sulla linguistica e sulla teoria dell'indicizzazione successive: essi hanno, tra le altre cose, influenzato un importante sistema di indicizzazione come il PRECIS. ${ }^{243}$ Hutchins, basandosi sulla rielaborazione dei casi profondi realizzata da Walter A. Cook, sviluppò una lista composta di undici casi profondi, in cui metteva in rapporto "relazioni casuali", "categorie casuali" ed "elementi verbali". ${ }^{244}$ I casi profondi sono stati ripresi anche da Brian C. Vickery e, in anni più recenti, da Christopher S. G. Khoo e Jin-Cheon

241 CHARLES J. FILLMORE, Il caso del caso, cit., p. 52.

242 Successivamente saranno proposte altre denominazioni per ruoli sostanzialmente simili: fine, dativo, recipiente, etc.

243 Cfr. per esempio JUTTA SØRENSEN - DEREK AUSTIN, PRECIS in a multilingual context. Part 2, cit., p. 112.

244 WALTER A. COOK, A case grammar matrix, "Languages and linguistics", 6 (1972), p. 15-47; W. JOHN HUTCHINS, Languages of indexing and classification, cit., p. 65: the cases we have identified in NL may be represented as case categories [...] or as case relations [...] In our discussion we have referredd exlusively to case categories, establishing the following eleven: Patient, Factitive, Agent, Instrument, Experiencer,

Beneficiary, Source, Goal, Location, Time, and Purpose. Each of them has a particular case relation to the "verbal" elements State, Process, Action and Action-process, giving eleven corresponding case relations as follows:

$\begin{array}{lll}\text { CASE RELATION } & \text { CASE CATEGORY } & \text { VERBAL ELEMENT(S) } \\ \text { Affective/Objective } & \text { Patient } & \text { any (except Action? [...]) } \\ \text { Effective } & \text { Factitive } & \text { Action-process } \\ \text { Agentive } & \text { Agent } & \text { Action, Action-process } \\ \text { Instrumental } & \text { Instrument } & \text { Action-process } \\ \text { Experiential } & \text { Experiencer } & \text { State, Process (+ experiential) } \\ \text { Benefactive } & \text { Beneficiary } & \text { State, Process, Action-process } \\ \text { Ablative } & \text { Source } & \text { Process, Action, Action-process } \\ \text { Directional } & \text { Goal } & \text { Process, Action, Action-process } \\ \text { Locative } & \text { Location } & \text { any } \\ \text { Temporal } & \text { Time } & \text { any } \\ \text { Purposive } & \text { Purpose } & \text { Action-process". }\end{array}$


Na. Rebecca Green, pur non riallacciandosi esplicitamente ai casi profondi di Fillmore, elaborò negli anni Novanta la nozione affine di "relazioni sintagmatiche concettuali", definite come relazioni semantiche grammaticalmente rilevanti. ${ }^{245}$

I casi profondi di Fillmore costituiscono una parte rilevante della semantica sintagmatica del nostro approccio linguistico; tuttavia, accanto a essi includeremo altre relazioni sintagmatiche di natura semantica, ai casi profondi non riconducibili, e che spesso non coinvolgono attività: relazioni sintagmatiche partitive ("motori di automobili", "dita delle mani", "biblioteche dell'Università di Firenze", etc.), possessive o di proprietà

("trascendenza divina", "beni della Chiesa”, "qualità dell'educazione”, etc.), e d'altro tipo. Tutte le relazioni semantiche sintagmatiche sopra esposte (casi profondi e relazioni sintagmatiche d'altro tipo) hanno una natura binaria (indicano una relazione tra due elementi del sintagma) e orientata (indicano una dipendenza concettuale di un elemento rispetto a un altro).

Avevamo anticipato una correlazione tra relazioni semantiche sintagmatiche e relazioni semantiche paradigmatiche; più precisamente, secondo l'approccio linguistico da noi adottato le diverse tipologie di relazioni semantiche sintagmatiche (a esclusione di quelle "logiche", di cui parleremo nel prossimo paragrafo) hanno una corrispondenza con i criteri di suddivisione delle diverse tipologie di relazioni gerarchiche eponimiche e semiche/sememiche (vedi 3.3.6.4.2.2.2.4 per una discussione più approfondita).

\subsubsection{Asse sintagmatico: relazioni sintagmatiche logiche}

L'altro sistema di organizzazione sintagmatica è quello delle relazioni che abbiamo definito "sintagmatiche logiche" (termine preso in prestito da Austin, vedi 3.3.6.4.3.1): relazioni esclusivamente funzionali alla costruzione di sintagmi, e che possono comparire solo in essi.

L'unica relazione sintagmatica logica tipica della linguistica che ci interessa per il nostro discorso è la relazione di coordinazione; tralasceremo le relazioni di subordinazione, disgiunzione, etc. La coordinazione è una relazione non orientata che può coinvolgere due o più elementi che svolgono lo stesso tipo di caso (profondo o d'altro tipo); ${ }^{246}$ esempi di relazione di coordinazione sono "Sessismo in Italia e in Spagna" oppure "Scuole e università in Francia”.

Le relazioni sintagmatiche logiche sono relazioni di natura fondamentalmente sintattica, come abbiamo accennato: tuttavia nemmeno esse sono esenti dai vincoli semantici paradigmatici di cui abbiamo detto; poiché le relazioni sintagmatiche (casi profondi e altre relazioni sintagmatiche) sono in una certa misura dipendenti dalla semantica paradigmatica, e poiché le relazioni sintagmatiche logiche coinvolgono elementi che

245 BRIAN C. VICKERY, Knowledge representation. A brief review, "Journal of documentation”, 42, 3 (1986), p. 147; CRISTOPHER S. G. KHOO - JIN-CHEON NA, Semantic relations in information science, cit., p. 169-171; REBECCA GREEN, Syntagmatic relationships in index languages, cit.; REBECCA GREEN, Topical relevance relationships. I, Why topic matching fails, "Journal of the American Society for Information Science", 46, 9 (1995), p. 660.

246 CHARLES J. FILLMORE, Il caso del caso, cit., p. 49, spiega come soltanto i sintagmi nominali che rappresentano uno stesso caso (ovvero uno stesso ruolo sintattico "profondo": Agente, Strumento, etc., in contrapposizione a ruoli sintattici "di superficie” come Soggetto, Oggetto, etc.) possano venire congiunti in una frase. 
svolgono una relazione semantica sintagmatica di qualche tipo, anch'esse dipendono indirettamente dalla semantica paradigmatica; per esempio, due elementi lessicali possono essere coordinati in un sintagma (e formare così un elemento lessicale in forma composta) non soltanto se svolgono lo stesso caso, ma anche se appartengono allo stesso tipo di tipologia lessicale (da qui l'insensatezza di sintagmi come "Giovanni e il martello hanno rotto la finestra").

\subsection{L'uso del linguaggio per l'indicizzazione}

\subsubsection{Indicizzazione e linguaggio}

In 3.1 avevamo posto come seconda e terza ipotesi che, rispettivamente, gli strumenti indicali fossero strumenti di natura linguistica e che dunque fosse opportuno parlarne in termini di lingue documentarie, e che però tale natura linguistica non esaurisse la natura complessiva delle lingue documentarie le quali, in parte, restano determinate da elementi e fini prettamente legati all'indicizzazione; avevamo poi posto come quarta ipotesi quella dell'opportunità di un riavvicinamento delle lingue documentarie al linguaggio naturale. La seconda ipotesi è ampiamente, benché non universalmente, accettata. Gli strumenti di indicizzazione hanno una natura linguistica in quanto, come le lingue naturali, anch'essi: 1) hanno una funzione essenzialmente comunicativa e, nell'ambito di questa, le specifiche funzioni dell'indicizzazione sono inquadrabili negli stessi termini delle funzioni linguistiche più sopra esposte (vedi 3.3.3); 2) presentano - almeno in principio - le stesse caratteristiche fondamentali che caratterizzano le lingue naturali, come per esempio l'arbitrarietà del segno; ${ }^{247} 3$ ) presentano un lessico e aspetti linguistici definiti (morfosintassi, semantica, pragmatica; è invece generalmente assente la fonologia). ${ }^{248}$ Qui è indispensabile una precisazione. La parte preponderante delle tassonomie linguistiche degli strumenti indicali in voga nella teoria dell'indicizzazione ha mutuato la tripartizione morrisiana del linguaggio (sintassi, semantica, pragmatica), in forme più o meno modificate. Caso emblematico, e che ha influenzato molti altri autori, è quello di Svenonius; la studiosa americana ha: a) considerato il lessico come aspetto ulteriore e separato da semantica e sintassi; b) identificato asse sintagmatico del linguaggio e sintassi; c) operato una tripartizione della semantica (categoriale, relazionale e referenziale) di propria elaborazione; ${ }^{249} \mathrm{~d}$ ) inteso la pragmatica, a differenza di quanto avviene in linguistica, come insieme delle regole applicative della lingua documentaria. ${ }^{250}$

247 Cfr. W. JOHN HUTCHINS, Languages of indexing and classification, cit., p. 19.

248 Cfr. MAURICE COYAUD, Introduction a l'ètude des langages documentaires, cit., p. 129-131; cfr. anche W. JOHN HUTCHINS, Linguistic processes in the indexing and retrieval of documents, p. 31-34.

249 ELAINE SVENONIUS, LCSH. Semantics, syntax and specificity, "Cataloging \& classification quarterly", 29, 1/2 (2000), p. 19.

250 ELAINE SVENONIUS, The intellectual foundation of information organization, cit., p. 55; Svenonius sviluppa in realtà la sua tassonomia delle componenti linguistiche delle lingue documentarie almeno dalla metà degli anni Settanta, cfr. ELAINE SVENONIUS - HELEN F. SCHMIERER, Current issues in the subject control of information, cit., p. 338; cfr. anche PINO BUIZZA, 025.4 Analisi e controllo per soggetto, cit., p. 577. 
Ritornando nella suddetta ottica agli aspetti linguistici delle lingue documentarie, dobbiamo riconoscere che lessico e aspetti linguistici delle lingue documentarie coincidono - in via generale - con quelle della lingua naturale stessa, rispetto alla quale sono però generalmente ridotte o semplificate. Gli strumenti di indicizzazione sono allora considerabili a pieno titolo lingue, che diremo documentarie o bibliografiche. ${ }^{251}$ Linguistici, inoltre, sono non soltanto gli strumenti dell'indicizzazione, ma anche una parte significativa dei processi di indicizzazione. Da ciò consegue anche l'inesattezza di tutte quelle teorie che hanno concepito le lingue documentarie come lingue artificiali a sé stanti e indipendenti dalla lingua naturale. ${ }^{252}$

Allo stesso tempo, però, e veniamo alla terza ipotesi, le lingue documentarie presentano caratteristiche proprie, legate al contesto e alle finalità per cui nascono: quelle dell'indicizzazione e dell'accesso bibliografico in generale.

Qui occorre una precisazione. Finora abbiamo parlato di linguaggio naturale tout court; si tratta di un'astrazione, perché il linguaggio naturale in effetti ha luogo sempre in determinati situazioni e secondo determinati codici; possiamo così distinguere un linguaggio scritto da uno parlato, un codice formale e informale dell'uso delle lingue; e vari altri contesti e codici speciali. ${ }^{253}$ Le lingue documentarie costituiscono appunto applicazioni delle lingue naturali a contesti e codici dell'indicizzazione. Tutta l'indicizzazione nel suo complesso potrebbe essere considerata un particolare contesto extralinguistico che informa l'uso della lingua naturale, ovvero parte della sua pragmatica: ma tale categorizzazione, linguisticamente soddisfacente, è "muta" ai fini del miglioramento delle lingue documentarie. Dobbiamo allora riconoscere che l'analisi linguistica degli strumenti indicali non è in sé sufficiente, e che per spiegare alcuni aspetti delle lingue documentarie e del loro uso dobbiamo necessariamente far riferimento a fattori prettamente indicali; esiste cioè una parte delle lingue documentarie che è sì inquadrabile in termini linguistici, ma la cui analisi e il cui miglioramento non può prescindere ed è anzi essenzialmente dipendente da elementi pertinenti la teoria dell'indicizzazione. Per questi aspetti, la lettura linguistica resta comunque utile a livello di analisi, costituendo una prospettiva di lettura non convenzionale.

Veniamo così alla quarta ipotesi che avevamo posto: le condizioni suddette, e cioè il fatto che gli strumenti indicali siano al contempo vere e proprie lingue, dipendenti dal linguaggio naturale ma informate nelle loro caratteristiche anche dalle esigenze dell'indicizzazione, suggeriscono da un lato l'opportunità complessiva del riavvicinamento delle lingue documentarie al linguaggio naturale - che ne costituisce l'origine, ne determina la natura fondamentale, ed è più vicino al linguaggio conosciuto dagli utenti finali -, dall'altro la consapevolezza che non sempre sia possibile o opportuno ricalcare le lingue documentarie sulle caratteristiche del linguaggio naturale quando ciò contrasti con le finalità dell'indicizzazione. Una conferma parziale della quarta ipotesi, dunque, che

251 Lingue bibliografiche ("bibliographic languages") è l'espressione coniata da Elaine Svenonius. 252 Cfr. JACQUES MANIEZ, Actualité des langages documentaires, cit., p. 205-206; cfr. anche MAURICE COYAUD, Introduction a l'ètude des langages documentaires, cit., p. 128: "Malgré le role indéniable que jouent dans la construction des LD la logique et l'épistémologies, les LD sont avant tout, et comme la logique, une émanation des LN. Ces métalangages [...] doivent nécessairement "refléter la structure du premier langage" [...] sur lequel ils sont batis, c'est-à-dire des LN"; per W. JOHN HUTCHINS, Languages of indexing and classification, cit., p. 7, le lingue documentarie sono pienamente lingue artificiali. 253 Cfr. BENEDETTO ASCHERO, Teoria e tecnica dell'indicizzazione per soggetto, nuova ed. riveduta e ampliata, Milano, Editrice Bibliografica, 1993, p. 74. 
Tesi di dottorato di Giuliano Genetasio, discussa presso l'Università degli Studi di Udine

però verificheremo nel corso delle discussioni successive.

\subsubsection{Universali linguistici e lingua documentaria ideale}

Una parte significativa delle riflessioni sulle lingue documentarie mira a stabilire (esplicitamente o implicitamente) una forma di lingua documentaria "ideale": perfetta, universale, neutrale, etc. - a questa condizione non si sottrae, malgrado le riserve che porremo, nemmeno il presente lavoro.

La ricerca di una lingua documentaria ideale è collegata e discendente da quelle, tipiche della linguistica, di una lingua universale e di uno o più universali linguistici (caratteri o elementi universali del linguaggio, vedi 3.2.2): ${ }^{254}$ così, l'idea di stabilire elementi e regole universali delle lingue documentarie non è dissimile al progetto chomskyano di una grammatica universale, con tutti i limiti che abbiamo evidenziato.

Storicamente, i primi tentativi di ideare una lingua (o più precisamente una grammatica) universale artificiale speciale nascono all'inizio degli anni Cinquanta dello scorso secolo nell'ambito della ricerca sulla traduzione automatizzata dei testi ("machine translation" o "mechanical translation"). ${ }^{255}$

La ricerca di una lingua documentaria ideale, ovvero di un insieme di universali linguistici indicali, può trovare fondamento in obiettivi diversi, tra loro collegati ma distinti: la ricerca di una vera e propria lingua documentaria perfetta e definitiva, oppure più modestamente l'ideazione di un modello che serva da base ideale per la creazione o analisi di diverse lingue documentarie, o ancora l'ideazione di una lingua documentaria che serva da ponte o meccanismo di scambio tra le diverse lingue documentarie. ${ }^{256}$ La ricerca di una lingua documentaria ideale (universale, neutrale, perfetta, unica, etc.) è profondamente problematica, per diversi motivi. Hutchins, che è stato il primo studioso a compiere uno studio sistematico degli universali linguistici (in primo luogo in termini di lessico, semantica, sintassi e pragmatica), ha escluso innanzitutto la possibilità di universalizzare la pragmatica delle lingue documentarie (intesa come rapporto tra lingua documentaria e contesto d'uso), perché troppo contingenti e soggettivi sono i fattori contestuali (natura specifica dei documenti indicizzati, tipologie di ricerca, tipologie di utenti, etc.) che informano l'uso delle lingue documentarie. ${ }^{257}$ Tutte le lingue documentarie, anche quelle considerate come universali, sono inoltre caratterizzate da idiosincrasie e peculiarità proprie che le rendono invariabilmente più o meno particolari. Specificamente, la gran parte delle lingue documentarie è basata quanto a semantica e

254 Cfr. W. JOHN HUTCHINS, Languages of indexing and classification, cit., p. 133; cfr. anche BIRGER HJØRLAND - LYKKE KYLLESBECH NIELSEN, Subject access points in electronic retrieval, cit., p. 284. 255 Cfr. ÉRIC DE GROLIER, A study of general categories applicable to classification and coding in documentation, translated from the French text by the National Science Foundation, revised by Anthony Thompson, Paris, UNESCO, 1962, p. 12-13.

256 W. JOHN HUTCHINS, Languages of indexing and classification, cit., p. 127: "we may seek features that are universal for all DLs. Such a discussion thas not only an importance for the theory and description of DL structures and functions, but also a practical value in the formulation of intermediary DLs, i.e. "switching languages" for the conversion of subject descriptions from one DL to another. Furthermore, it is plausible that the results would be significant for NL universals also".

257 W. JOHN HUTCHINS, Languages of indexing and classification, cit., p. 130, 134. 
sintassi sulle lingue (naturali) occidentali e sulla tradizione filosofica occidentale. ${ }^{258}$ All'idea della ricerca di una lingua documentaria universale e neutrale è collegata quella della ricerca di un lingua documentaria unica: secondo Gardin, la molteplicità delle lingue documentarie non fa che riflettere la molteplicità dei punti di vista tipici del mondo reale (e delle lingue naturali); la riconciliazione dei diversi punti di vista in un punto di vista e in una lingua unica e universale è un obiettivo di carattere paradossale, che implicherebbe il compimento, dunque la fine, della ricerca scientifica e, con essa, la stessa necessità di una lingua documentaria. ${ }^{259}$ Infine, va senz'altro abbandonata l'idea di un lingua documentaria perfetta e definitiva sulla base della natura indeterminata, storica e soggettiva dell'universo bibliografico e delle esigenze che l'utente finale ha rispetto a esso. ${ }^{260}$

Date queste condizioni, che ne è della ricerca di un lingua documentaria ideale (ovvero di universali linguistici indicali) e degli obiettivi sopra menzionati? L'ideale di una lingua documentaria universale, assolutamente neutrale, perfetta e unica va senz'altro scartata. All'ideale della neutralità assoluta va opposta, secondo la lezione di Hjørland, una visione consapevolmente conflittuale della conoscenza: le lingue documentarie, piuttosto che pretendere di essere neutrali, dovrebbero invece prevedere la possibilità di una rappresentazione dei diversi punti di vista esplicita e distinta dalla rappresentazione degli argomenti (è ciò che noi abbiamo proposto di fare con l'indice di punto di vista nel nostro modello concettuale, vedi 2.16.6). ${ }^{261}$

Diversa è la questione relativa all'ideazione di un modello linguistico per le lingue documentarie e di una lingua documentaria di scambio: si tratta di obiettivi che, pur con le riserve sopra esposte, riteniamo plausibile perseguire. Nel discutere questi obiettivi, è utile la distinzione tra universali linguistici formali e sostanziali (e tra piano formale e sostanziale di una lingua) a cui abbiamo accennato in 3.2.2. La ricerca o ideazione di universali formali (semantica, sintassi, etc.) è funzionale a quella di un modello di lingua documentaria utile come base per la creazione di future lingue documentarie o per l'analisi di quelle esistenti. ${ }^{262}$ La ricerca o ideazione di universali sostanziali è funzionale a

258 W. JOHN HUTCHINS, Languages of indexing and classification, cit., p. 133-134.

259 JEAN-CLAUDE GARDIN, Semantic analysis procedures in the sciences of man, cit., p. 22-23: "A single documentary language is inconceivable [...] the very diversity of documentary languages is, on the contrary, the reflection of the necessary multiplicity of points of view on the empirical world [...] one has to develop different symbolic quantifications in regard to experimental phenomena of the same order, so as to build up complementary theories about them. That all these theories may ultimately be reconciled [...] but one must always bear in mind the paradoxical characteristic of the temporal success of any such project: it would imply that scientific research has come to an end, having reached its ultimate goal".

260 MARCIA J. BATES, Subject access in online catalogs, cit., p. 260.

261 BIRGER HJØRLAND, Theory of knowledge organization and the feasibility of universal solutions. Presented at the Eighth International ISKO Conference, London, July 13-16, 2004, 2004, disponibile all'indirizzo

<http://arizona.openrepository.com/arizona/bitstream/10150/105303/1/hjorlandisko8.pdf>, p. 8-9, 38. 262 JEAN-CLAUDE GARDIN, Semantic analysis procedures in the sciences of man, cit., p. 23: "In the second category, it is not a matter of the content of information retrieval languages, but of their structural properties, quite apart from the field they cover [...] These properties can be considered on two planes: a) the semantic organization of the lexicon, defined by the nature and number of the "analytic" relationships established a priori between the terms of the documentary language, and by the configuration of the resulting semantic network; b) grammar, as defined by the nature and number of logical indicators [...] so as to express the syntactic or syntagmatic relationships binding them together in a given documentary representation [...] From a structural viewpoint such models play a unifying role, much as the abovementioned "intermediary lexicons" do on the content level". 
quella di una lingua documentaria di scambio o ausiliaria, utile come ponte per la traduzione delle diverse lingue documentarie; questo tipo di lingue sono sempre fortemente esposte al rischio di etnocentrismo, perché è il piano sostanziale di una lingua quello più fortemente caratterizzato dai limiti suddetti di storicità, particolarità, contingenza, etc.

Per il nostro approccio linguistico all'indicizzazione tralasceremo allora l'obiettivo di formulare una vera e propria lingua documentaria di scambio, con un lessico proprio, e universali linguistici di tipo puramente sostanziale. Ci porremo principalmente sul piano formale e proveremo a formulare un modello di lingua documentaria a partire dall'ideazione di un certo numero di elementi formali delle lingue documentarie: funzioni linguistiche svolte; rapporto tra linguaggio e indicizzazione e, dunque, modalità di controllo linguistico e divisione tra parti più e meno controllate delle lingue documentarie; aspetti linguistici e indicali delle lingue documentarie; etc. Alcuni di questi elementi formali sono universali che si trovano già attualmente nelle lingue documentarie. La parte preponderante è tuttavia costituita di universali "ideali" della lingua documentaria, ovvero che le lingue documentarie dovrebbero presentare universalmente ma che attualmente sono spesso assenti le lingue documentarie esistenti.

\subsubsection{Funzioni linguistiche dell'indicizzazione}

Le funzioni linguistiche delle lingue documentarie sono essenzialmente comunicative ed inquadrabili negli stessi termini delle funzioni linguistiche di Jakobson (vedi 3.2.4). Tuttavia, rispetto a queste, le funzioni linguistiche delle lingue documentarie sono di numero ridotto e declinate secondo gli specifici scopi dell'indicizzazione. Le funzioni linguistiche delineate da Jakobson, lo ricordiamo, sono quelle referenziali, emotive, conative, metalinguistiche, fatiche, poetiche. La penultima funzione è trattata implicitamente nelle lingue documentarie; l'ultima può dirsi generalmente assente. ${ }^{263}$ Tradizionalmente, viene affermato che la funzione delle lingue documentarie è essenzialmente quella referenziale o descrittiva; ${ }^{264}$ ciò è senz'altro vero, almeno nel senso che la funzione referenziale resta quella principale delle lingue documentarie. Tuttavia, e contrariamente a quanto ha affermato Hutchins, le lingue documentarie hanno anche una funzione metalinguistica. ${ }^{265}$ Svenonius si è riferita a questa funzione, con particolare riferimento alla disambiguazione delle omonimie, con l'espressione di "semantica referenziale", ma la valenza metalinguistica delle lingue documentarie si estende ben oltre la semplice funzione di disambiguazione. Intendendo la funzione metalinguistica come uso del linguaggio per chiarire e spiegare il linguaggio stesso (formulazioni linguistiche di vario tipo: singoli elementi lessicali, sintagmi, etc.), è

263 Ciò è vero soltanto se ci riferiamo alle lingue documentarie tradizionali o professionali; non è invece vero nel caso del tagging degli utenti (anche le tag possono essere considerate una forma di lingua documentaria per quanto basilare), in cui la funzione poetica ha (insieme a quella emotiva) una parte rilevante, e che però non prenderemo in considerazione nel presente lavoro.

264 Cfr. per esempio MAURICE COYAUD, Introduction a l'ètude des langages documentaires, cit., p. 128. 265 W. JOHN HUTCHINS, Languages of indexing and classification, cit., p. 7: "Whereas NLs can functionas their own metalanguage, DLs cannot [...] We can argue that DLs are metalanguages insofar as they are used to talk about the (NL) texts of documents [...] But even if this is true, it is still not the case that DLs can be their own metalanguage, i.e. "a metalanguage of a metalanguage". 
possibile dire che la totalità degli elementi indicali ha una funzione metalinguistica: ogni elemento indicale, espresso in forma verbale o simbolica, serve tra le altre cose a chiarire il significato contestuale dei diversi elementi delle registrazioni bibliografiche (nei termini delle funzioni dell'utente finale, serve a Identificare un certo elemento bibliografico); così, il fatto che, all'interno di una registrazione bibliografica, venga segnalato che "Luigi Pirandello" sia un "soggetto" e non un "autore" è un fatto pienamente metalinguistico; approfondiremo più sotto quest'aspetto dell'indicizzazione (vedi 3.3.4.7).

L'ideale dell'obiettività dell'indicizzazione esclude che questa possa esprimere opinioni, valutazioni, etc. Tuttavia, nel precedente capitolo abbiamo mostrato sia l'erroneità di un tale ideale inteso in modo assoluto (vedi 2.16.3), sia viceversa l'opportunità di prevedere elementi indicali distinti e di tipo eminentemente valutativo (gli indici valutativi, vedi 2.23), creati dall'indicizzatore o dagli utenti finali stessi (tag) e volti a soddisfare la funzione di selezione del catalogo. ${ }^{266}$ Gli indici valutativi svolgono quella che abbiamo definito come funzione emotiva del linguaggio: anche questa, dunque, è potenzialmente parte delle funzioni linguistiche delle lingue documentarie.

Infine, l'uso delle lingue documentarie nell'interrogazione del catalogo ha sempre una valenza implicitamente conativa: le formule linguistiche immesse nella maschera di ricerca da parte degli utenti valgono come sorta di "ordini" impartiti al catalogo; ${ }^{267}$ ponendo una corrispondenza con le funzioni del catalogo, potremmo dire che l'utente il quale immette una formula linguistica nella maschera di ricerca, chiede di volta in volta al catalogo di Trovare, di Navigare, etc.

Una nota finale sul rapporto tra funzioni e aspetti linguistici: così come per le lingue naturali, le funzioni linguistiche possono essere considerate la parte fondamentale della pragmatica delle lingue documentarie (che non tratteremo autonomamente).

\subsubsection{I diversi gradi di controllo linguistico}

\subsubsection{Introduzione}

Il processo di indicizzazione, come affermato nel precedente capitolo (vedi 2.2), consiste fondamentalmente nell'attività di rappresentazione linguistica di determinati oggetti di interesse (entità, attributi, relazioni, etc.) sulla base di specifiche funzioni o esigenze dell'utente. Le registrazioni bibliografiche, che di tale attività sono il prodotto, sono allora "discorsi bibliografici" attorno a entità e relazioni; il catalogo, l'archivio di autorità e ogni altro repertorio bibliografico che di tali registrazioni è la cumulazione, può a sua volta

266 Le funzioni tipiche del social web possono naturalmente essere anche referenziali o d'altro tipo; inoltre spesso esulano dalle funzioni linguistiche sopra delineate. Per una tassonomia più dettagliata delle funzioni svolte dal social tagging cfr. MARLIESE THOMAS - DANA M. CAUDLE - CECILIA M. SCHMITZ, To tag or not to tag?, "Library hi tech", 27, 3 (2009), p. 412-413; cfr. anche TOM STEELE, The new cooperative cataloging, "Library hi tech", 27, 1 (2009), p. 69; cfr. anche CAIMEI LU - JUNG-RAN PARK - XIAOHUA $\mathrm{HU}$, User tags versus expert-assigned subject terms. A comparison of LibraryThing tags and Library of Congress subject headings, "Journal of Information Science", 36, 6 (2010), p. 763-779, disponibile all'indirizzo <http://jis.sagepub.com/content/36/6/763.full.pdf>, p. 764, 777.

267 Cfr. JACQUES MANIEZ, Les langages documentaires et classificatoires, cit., p. 280-281. 
essere inteso come insieme di discorsi bibliografici. Riallacciandoci alla differenza tra tema e rema posta nel precedente capitolo (vedi 2.16.5), potremmo anche dire che l'entità di cui di volta in volta si parla costituisce il tema di questi discorsi bibliografici, mentre l'insieme degli attributi e delle relazioni che di quell'entità vengono predicate costituisce il rema. I discorsi bibliografici hanno una duplice natura, linguistica e indicale: essi sono il prodotto dell'interazione del linguaggio con le esigenze e caratteristiche dell'indicizzazione; le lingue documentarie, allora, sono una sorta di traduzione delle lingue naturali in una forma controllata sulla base delle esigenze e caratteristiche dell'indicizzazione (vedi 2.17); possiamo allora parlare di un vero e proprio controllo linguistico esercitato dalle lingue documentarie.

\subsubsection{Semantica delle entità concettuali e delle registrazioni}

Il primo e fondamentale elemento caratterizzante le lingue documentarie, nessuna eccettuata, è la distinzione e scelta di un certo numero di elementi dell'universo bibliografico da rappresentare: virtualmente infiniti, ma quelli da noi considerati sono le entità del modello concettuale (vedi 2.6 e ss.). Abbiamo così un primo grado di controllo linguistico delle lingue documentarie; un controllo di natura essenzialmente semantica, con cui un certo numero di elementi di significato del linguaggio naturale (opere, edizioni, persone, etc.) viene selezionato per la sua rilevanza rispetto alle esigenze degli utenti finali e controllato esplicitamente tramite registrazioni bibliografiche. Questi elementi di significato e le registrazioni bibliografiche che li rappresentano costituiscono nel loro insieme la semantica delle entità concettuali e delle registrazioni della lingua documentaria: una semantica "ad hoc", cioè specifica del contesto dell'indicizzazione, che si sovrappone alla semantica delle tipologie lessicali o "entità" linguistiche del linguaggio naturale più sopra discussa (vedi 2.11, 3.2.5.3.3); così, in alcuni casi le entità concettuali raccolgono una molteplicità di tipologie lessicali; in altri, viceversa, le entità concettuali specificano sottotipi di sottotipi di tipologie lessicali (entità inanimate); etc.

Approfondiremo in 3.3.6.4.2.3 i rapporti tra semantica delle entità concettuali e semantica delle tipologie lessicali.

\subsubsection{Semantica degli attributi e degli indici}

Un secondo elemento caratterizzante la gran parte delle lingue documentarie è la distinzione e scelta di un certo numero di caratteristiche dei suddetti elementi di significato (le entità) da rappresentare: quelle particolari da noi considerate sono gli attributi del nostro modello concettuale; abbiamo qui un secondo grado di controllo linguistico delle lingue documentarie. Anche in questo caso si tratta di un controllo natura essenzialmente semantica, con cui un certo numero di elementi di significato del linguaggio naturale relativi alle caratteristiche delle entità (nomi, responsabili, soggetti, possessori, etc.) viene selezionato per la sua rilevanza rispetto alle esigenze degli utenti finali e controllato esplicitamente tramite indici o elementi indicali - e metaindicali, che forniscono informazioni sugli indici stessi. Tale secondo grado di controllo linguistico costituisce nel suo insieme la semantica degli attributi e degli indici della lingua 
Tesi di dottorato di Giuliano Genetasio, discussa presso l'Università degli Studi di Udine

documentaria; anch'esso costituisce parte della semantica "ad hoc" (cioè specificamente bibliografica) della lingua documentaria.

\subsubsection{Modalità di realizzazione del controllo linguistico: linguaggio naturale e lingue documentarie}

Il controllo linguistico può almeno in linea di principio essere realizzato in diversi modi. Il linguaggio naturale conosce diversi espedienti per evidenziare o distinguere gli oggetti a cui si riferisce: può distinguerli o spiegarli esplicitandone verbalmente il significato, oppure utilizzare un codice di natura convenzionale sulla base del quale gli elementi lessicali assumono un preciso significato; avviene così per esempio anche nel linguaggio editoriale usato nei libri, in cui alla posizione degli elementi lessicali sul frontespizio e sulle altre parti formali del libro sono associati dei significati, che poi vanno confermati o smentiti con una successiva analisi. Il linguaggio naturale può evidenziare lo specifico significato degli elementi del discorso tramite l'uso della ridondanza, oppure - nel corso di un'interazione linguistica - tramite retroazione; se orale, tramite il sistema dell'intonazione; in generale, le lingue naturali possono giovarsi dell'uso del contesto (linguistico, cioè sintagmi precedenti e seguenti a quello preso in analisi; ed extralinguistico: fatti, situazioni e valori condivisi dai parlanti) per spiegare o evidenziare gli oggetti di cui parlano. Le lingue documentarie, come ogni linguaggio scritto, non possono contare su molti dei suddetti espedienti. Per permettere il controllo degli oggetti di interesse (entità, attributi, relazioni, etc.), e dunque il soddisfacimento delle diverse funzioni dell'utente, le lingue documentarie devono allora ricorrere a un contesto artificiale con cui segnalare, distinguere e spiegare il significato di ciascun elemento del discorso bibliografico. In alcuni casi tale contesto artificiale può essere del tutto assente, confidando sull'evidenza della natura semantica degli oggetti di discorso; in altri può essere presente in forma implicita, per esempio tramite l'impiego di un ordine fisso convenzionale tra gli elementi del discorso bibliografico.

Entrambi questi criteri sono fortemente problematici: il primo espone la lingua documentaria (ammesso sia possibile parlare a questo livello di lingua documentaria) a tutti i rischi dell'ambiguità del linguaggio naturale senza però i meccanismi di disambiguazione che lo caratterizzano; l'ordine convenzionale è invece un meccanismo che può funzionare bene in un contesto lineare e cartaceo, ma è insufficiente in un ambiente elettronico (quale è l'odierno ambiente dei cataloghi) in cui l'ordine degli elementi può essere manipolato dal computer, ovvero ogni elemento del discorso può essere ricercato autonomamente. Non resta allora che la possibilità di un controllo esplicito di ciascun elemento dei discorsi bibliografici (registrazioni), che può essere verbale (tag o etichette in una delle lingue naturali) o simbolica (per esempio una punteggiatura convenzionale, o ancora l'utilizzo di etichette numeriche come nel caso del MARC); ciò, come diremo in 3.3.4.7, ha alcune importanti conseguenze linguistiche.

\subsubsection{Semantica delle relazioni e dei collegamenti}

Un terzo elemento che caratterizza alcune lingue documentarie (tradizionalmente 
denominate "controllate") è la distinzione e scelta di un certo numero di relazioni dell'universo bibliografico da rappresentare; quelle da noi considerate sono le relazioni esposte nel predente capitolo (vedi 2.12 e ss.), più, in questo caso, le ulteriori relazioni che svilupperemo nel presente capitolo sulla base di un confronto con la linguistica. Abbiamo così un terzo grado di controllo linguistico, di natura fondamentalmente semantica, con cui un certo numero di relazioni di significato del linguaggio naturale viene selezionato per la sua rilevanza rispetto alle esigenze degli utenti finali e controllato esplicitamente - con le eccezioni che abbiamo già detto ${ }^{268}$ - tramite indici controllati e collegamenti fisici tra le registrazioni delle entità.

Il controllo esplicito delle relazioni tramite indici controllati e collegamenti non è soltanto un processo tecnico: fino a quando le entità che fungono da attributi di un'altra entità sono registrate come elementi descrittivi esse fungono sì da elementi semantici e metalinguistici rispetto all'entità registrata, ma questa rimane nel suo complesso isolata linguisticamente dalle altre entità. Soltanto quando il controllo linguistico delle relazioni è esplicito le diverse entità menzionate nelle registrazioni vengono collegate tra loro e con ciò comunicano tra loro; in questo caso, entità e relazioni così collegate vanno a formare parte di una medesima e ulteriore lingua documentaria, con un lessico, una semantica e una morfosintassi distinte: una vera e propria lingua nella lingua, o lingua documentaria interna, che chiameremo "sottolingua" documentaria (vedi 3.3.6).

\subsubsection{Unitarietà e semanticità dell'indicizzazione}

Esplicitiamo a questo punto un importante presupposto del nostro approccio linguistico (peraltro già anticipato nel precedente capitolo, vedi 2.27): nella prospettiva sopra esposta, in cui la totalità di entità, attributi e relazioni, e degli elementi indicali che li rappresentano, è parte della semantica della lingua documentaria, risulta evidente il carattere unitario e integralmente semantico dell'indicizzazione: l'indicizzazione nella sua totalità (non soltanto quella per soggetto) è di natura fondamentalmente semantica, poiché ha sempre a che fare con la scelta e rappresentazione di elementi di significato. Ciò vale tanto per le relazioni semantiche tipiche della linguistica quanto per le relazioni semantiche tipiche dell'indicizzazione, le quali costituiscono nel loro complesso una sorta di semantica a posteriori o "ad hoc" della lingua documentaria. Il carattere unitario e integralmente semantico dell'indicizzazione è ancora più evidente nella sottolingua, nella cui rete sindetica possono almeno in linea di principio coesistere le relazioni dell'indicizzazione per autore e per soggetto.

\subsubsection{Implicazioni per gli aspetti linguistici: lessicalizzazione e metasintagmi}

Il controllo linguistico esplicito operato dalle lingue documentarie ha una conseguenza importante: esso comporta una lessicalizzazione degli aspetti linguistici (semantica, morfosintassi, etc.) del linguaggio naturale. Tali aspetti devono cioè essere nella lingua documentaria espressi tramite ulteriori elementi lessicali con funzione metalinguistica, i

268 Relazioni di equivalenza, di omonimia, relazioni tra temi estensionali. 
quali possono poi (nel caso del terzo grado di controllo) essere parte integrante del lessico della sottolingua, oppure restare esterni ad esso (primo e secondo grado di controllo, rispetto a elementi espressi in forma discorsiva; terzo grado di controllo, rispetto a elementi lessicali della sottolingua); in questo secondo caso essi costituiscono elementi "metalessicali", cioè elementi lessicali che forniscono informazioni su altri elementi lessicali della lingua documentaria (vedi 3.3.6.2.3): per esempio, la semantica paradigmatica delle relazioni della sottolingua può essere esplicitata tramite l'utilizzo di simboli e sigle, quali i vari BT ("broader term", cioè termine generico), NT ("narrower term”, cioè termine specifico), etc. tipici dei thesauri; anche la semantica paradigmatica delle tipologie lessicali della sottolingua può essere esplicitata sotto forma di norme d'uso per la combinazione degli elementi lessicali, esterne al lessico della sottolingua in quanto tale. A causa di tale funzione essenzialmente metalinguistica delle lingue documentarie, non è azzardato denominarle anche "metalingue". ${ }^{269}$

Spingendo oltre queste idee, potremmo arrivare a concepire il rapporto tra elementi lessicali e metalessicali della lingua documentaria come quello tra diversi elementi di un sintagma, in cui il predicato sia presente in forma implicita, in una sorta di sintagmatizzazione ideale delle relazioni paradigmatiche; per esempio, la relazione tra l'elemento lessicale "Etna", subordinato genericamente (tramite l'elemento metalessicale "BT" o suo equivalente) all'elemento lessicale "Vulcani", può essere concepito come sintagma "l'Etna è un vulcano"; la relazione ad hoc tra "Luigi Pirandello" e l'elemento metalessicale "autore" può essere concepito come sintagma "Luigi Pirandello è l'autore" o "l'autore è Luigi Pirandello". Elementi lessicali e relativi elementi metalessicali formano cioè idealmente dei sintagmi impliciti, che potremmo chiamare metasintagmi. In riferimento al livello fisico dell'indicizzazione, non sarebbe almeno in linea di principio impensabile esplicitare tali metasintagmi in fase di visualizzazione, fornendo all'utente una descrizione dell'entità ricercata in forma discorsiva (per esempio, l'utente finale che cercasse "Etna" troverebbe una registrazione dell'entità accompagnata dalla descrizione "l'Etna è un vulcano", o l'utente che cercasse materiale relativo a Pirandello potrebbe trovare un insieme di risorse bibliografiche di cui "Luigi Pirandello è l'autore", o "Luigi Pirandello è il soggetto”, etc.).

\subsubsection{Asse paradigmatico e sintagmatico: prima parte}

La coppia "paradigmatico" e "sintagmatico" ha avuto una grande fortuna nella teoria dell'indicizzazione; essa è utilizzata, per esempio, da Gardin per la sua classificazione delle lingue documentarie; Gardin distingue le lingue documentarie che non fanno uso né di strutture paradigmatiche né sintagmatiche (UNITERM), da quelle che fanno uso soltanto di strutture sintagmatiche (indicizzazione relazionale di Farradane) o paradigmatiche (thesauri), da quelle che fanno uso di entrambe (come per esempio il SYNTOL, linguaggio sviluppato dallo stesso Gardin); tra le altre cose, Gardin notava già la relatività dell'opposizione paradigmatico-sintagmatico. Di relazioni paradigmatiche e sintagmatiche hanno parlato, tra gli altri, anche Magdeleine Moureau e, più recentemente, Rebecca Green. ${ }^{270}$

269 Cfr. MAURICE COYAUD, Introduction a l'ètude des langages documentaires, cit., p. 128.

270 Cfr. R. C. CROS - JEAN-CLAUDE GARDIN - F. LÉVY, L'automatisation des recherches documentaires, 
Tuttavia, nella teoria dell'indicizzazione la coppia paradigmatico e sintagmatico ha subito spesso distorsioni di senso. Un primo esempio di questa distorsione è il collegamento che la teoria dell'indicizzazione tradizionale ha posto tra asse paradigmatico e relazioni universali, valide in modo assoluto (relazioni analitiche o a priori) e tra asse sintagmatico e relazioni di carattere contingente (relazioni sintetiche o a posteriori). Si è così compiuta un'operazione - assente nella coppia originaria di paradigmatico e sintagmatico - in base a cui "paradigmatico" (o meglio a priori: noi continueremo a utilizzare questo termine) e "sintagmatico" (o meglio a posteriori: noi continueremo a utilizzare questo termine) venivano concepiti come ambiti separati e mutualmente esclusivi. In realtà, diversi autori hanno mostrato come la differenza tra ciò che è a priori o universalmente accettato e a posteriori o contingente non è affatto assoluta; ${ }^{271}$ poiché ciò che è comunemente accettato o condiviso cambia nelle diverse epoche, culture e più in generale nei diversi contesti, ciò che è a priori in una data lingua non lo è necessariamente in un'altra, in cui la stessa relazione è a posteriori; ciò che è a priori in un dato momento, può non esserlo in un altro. La differenza tra a priori e a posteriori è una differenza di cui tenere conto, e sui cui ritorneremo più sotto (vedi 3.3.6.4.2.2.2.4), ma è una differenza del tutto relativa. Altro esempio tipico di distorsione della coppia paradigmatico e sintagmatico è la sua identificazione, da parte di una parte significativa della teoria dell'indicizzazione, con la coppia semantica (o lessico) e sintassi, ovvero l'identificazione di relazioni semantiche e paradigmatiche da un lato, e di relazioni sintattiche e sintagmatiche dall'altro. La letteratura successiva ha sempre più spesso teso a ridurre la distinzione tra sintagmatico e paradigmatico a quella tra sintassi e semantica: ritroviamo questo tipo di rapporto, tra gli altri, nel lavoro di Svenonius. ${ }^{272}$

Per il nostro modello di lingua documentaria, manterremo invece la distinzione di paradigmaticità e sintagmaticità nel suo senso originario, con le riserve già esposte, $\mathrm{e}$ in primo luogo nella consapevolezza della loro relatività; ${ }^{273}$ secondo l'impostazione esposta

cit., p. 42, 102-103, in cui gli autori parlano del "Caractère à nos yeux contingent de l'opposition entre le deux axes de mise en relation, la différence entre réseaux syntagmatique et paradigmatique tenant moins à leur structure qu'ax conditions pratiques de leur élaboration et de leur mise en oeuvre"; cfr. anche JEANCLAUDE GARDIN, Document analysis and linguistic theory, cit., p. 146-147; cfr. anche ALEXIS RIVIER, Construction des langages d'indexation, cit., p. 268; cfr. anche MAGDELEINE MOUREAU, Les aspects linguistiques des stratégies d'interrogation dans la rcherche bibliographique sur ordinateur, cit., p. 91; cfr. anche REBECCA GREEN, Syntagmatic relationships in index languages, cit., p. 366.

271 Cfr. per esempio R. C. CROS - JEAN-CLAUDE GARDIN - F. LÉVY, L'automatisation des recherches documentaires, cit., p. 42-43.

272 W. JOHN HUTCHINS, Languages of indexing and classification, cit., p. 6; ELAINE SVENONIUS, The intellectual foundation of information organization, cit., p. 130; ELAINE SVENONIUS, LCSH, cit., p. 23. 273 Cfr. JEAN-CLAUDE GARDIN, Document analysis and linguistic theory, cit., p. 145-148: "The distinction made above between analytical (or semantic) vs. syntactical (or logical) relations. Although it occurs in a large number of MLs, this dichotomy can be shown to have no justification other than practical. A given relationship between two or more concepts can be expressed either analytically, through a semantic device of some sort (e.g. classification, factoring, compounding, etc.) or syntactically through the generation o a grammatical construct in ML (contrast for instance the semantic relation inherent in the compound terms "insecticides", or in the analytical structure "drugs used on insects", irrespective of its codification [...] Obviously, the choice between one or the other relational device depends on practical circumstances that are of the same nature in ML as they are in NL: to be very brief, the more straight-forward relations, whose which "go without saying" but which are nevertheless frequently put to use in a given universe of discourse tend to be trasferred to the semantic structure, whereas the more undpredictable relations, bringing forth new knowledge, find their expression in the syntactical structure [in nota: This functional interpretation of 
già nella sezione precedente del capitolo (vedi 3.2.3, 3.2.6), allora, intenderemo l'asse paradigmatico come esclusivamente semantico, e l'asse sintagmatico come composto di relazioni che sono al contempo semantiche e sintattiche (sintassi profonda). L'asse paradigmatico comprende, nell'ambito della nostra lingua documentaria, sia la semantica delle entità concettuali, sia la semantica degli attributi e delle relazioni, sia ancora la semantica paradigmatica delle tipologie lessicali e delle relazioni della sottolingua. L'asse sintagmatico comprende invece la semantica sintagmatica o sintassi profonda della sottolingua, oltre che la semantica paradigmatica vigente nei sintagmi. Un'altra precisazione. Nel linguaggio naturale, la differenza tra paradigmatico e sintagmatico è quella tra relazioni esistenti implicitamente e relazioni poste esplicitamente in un discorso; nelle lingue documentarie, viceversa, il processo di lessicalizzazione ed esplicitazione degli aspetti linguistici fa sì che la differenza tra paradigmatico e sintagmatico si configuri in modo differente. Nell'ambito della semantica degli attributi e della semantica delle relazioni della lingua documentaria, ciò comporta l'utilizzo di simboli o etichette verbali (elementi metalessicali). Nell'ambito della sottolingua, le relazioni paradigmatiche e sintagmatiche sono dunque non soltanto esplicitate, ${ }^{274}$ ma parte di uno stesso sistema, la rete sindetica della sottolingua, costituendone rispettivamente gli assi verticale e orizzontale (fig. C); ciò, come avremo modo di dire, riduce sensibilmente la portata della relatività della paradigmaticità e della sintagmaticità all'interno delle lingue documentarie. Inoltre, la coppia paradigmatico e sintagmatico è presente anche nei sintagmi della sottolingua come rapporto tra le relazioni sintagmatiche e la semantica paradigmatica vigente nei sintagmi.

\subsubsection{La sottolingua documentaria}

La nozione di sottolingua documentaria corrisponde a ciò che la letteratura dell'indicizzazione tradizionale chiama solitamente "lingua documentaria" (o "linguaggio documentario"), ma noi abbiamo mostrato che vi è una lingua documentaria già nel controllo di entità e registrazioni, attributi e indici delle registrazioni bibliografiche, e perciò preferiamo parlare di sottolingua documentaria per riferirci alla lingua documentaria interna che scaturisce dal terzo grado di controllo linguistico.

\footnotetext{
the contrast between semantic structure and syntactical devices, as used in document analysis, is nothing but a reformulation of the Saussurian distinction between "pensée" \{langue $\}$ and "parole", paradigmatic andy syntagmatic organization, etc., in linguistic analysis] [...] Once it was acknowledged that the distinction between paradigmatic and syntagmatic organization was one of convenience, and that it was desirable to provide "bridgs" from one to the other for purpose of intra-ML as well as inter-ML [cioé tra diversi ling. ind.] conversions [...] it was natural to a dopt a basic relational unit that could be used in both categories of structure [...] our preference went to the minimum syntagm "Ri $(x, y)$ ", where " $x$ " and "y" are terms of the lexicon, and " $\mathrm{R}$ " is a binary relation whose eaning is specified if necessary by the subscript "i". Orientation rules may be added when a given relation is not reciprocal (e.g. cause/effect, etc.) [...] n-ary relations [relazioni tra più di due elementi] can be envisaged as aggregates of binary relations [...] by compouning unrary predicates and by resolving n-ary predicates into binary ones only, a suitable ground is provided for undertaking a genearl inventory of the relational tools of MLs, both on the semantic and on the grammatical plane, irrespective of differences in codification”.

274 Per quanto riguarda le relazioni sintagmatiche ciò non è però generalmente vero per i soggettari tradizionali, che si limitano generalmente a segnalare l'esistenza di una relazione sintagmatica senza specificarne la tipologia.
} 
Inoltre, mentre la letteratura dell'indicizzazione tradizionale utilizza l'espressione "lingua documentaria” solitamente per riferirsi agli elementi dell'indicizzazione per soggetto, la nostra sottolingua riguarda gli elementi dell'indicizzazione nella sua totalità. La semantica delle relazioni della sottolingua (o rete sindetica) costituisce ovviamente la parte centrale della sottolingua e quella a cui a cui dedicheremo maggiore spazio. ${ }^{275}$ Così come noi la concepiamo, la semantica delle relazioni della sottolingua comprende sia relazioni che corrispondono alle relazioni semantiche trattate tipicamente dalla linguistica (omonimia, sinonimia, etc.), sia - ma nel presente lavoro abbozzeremo soltanto quest'aspetto - le relazioni "ad hoc" prettamente tipiche del contesto bibliografico (responsabilità, soggetto, etc.); nulla impedisce infatti che, accanto alla rappresentazione di relazioni linguistiche come sinonimia, eponimia, etc., vengano allo stesso modo rappresentate le relazioni come gli adattamenti, le responsabilità, le relazioni di affiliazione istituzionale tra Persone ed Enti, etc.; è grazie alla rete sindetica che la sottolingua permette un soddisfacimento delle funzioni dell'utente non raggiungibili con la semplice registrazione degli elementi bibliografici in forma di elementi descrittivi.

In questa seconda parte della terza sezione ci concentreremo sulla discussione dei vari aspetti della sottolingua, e in particolare sull'analisi e le possibilità di miglioramento delle relazioni semantiche tratte dalla linguistica; faremo anche riferimento alle relazioni ad hoc (relazioni di responsabilità, di soggetto, etc.), considerate quali possibili elementi della rete sindetica della sottolingua.

\subsubsection{Asse paradigmatico e sintagmatico: seconda parte}

Ribadiamo alcuni caratteri della coppia paradigmatico e sintagmatico nell'ambito della sottolingua da noi proposta: 1) asse paradigmatico della sottolingua: include la semantica paradigmatica delle relazioni e delle tipologie lessicali della sottolingua; asse sintagmatico della sottolingua: include le relazioni sintagmatiche della sottolingua ed è al contempo semantico e sintattico; 2) possibilità, per gli elementi lessicali della sottolingua, di assumere indifferentemente una forma semplice o composta; 3) nesso tra relazioni semantiche sintagmatiche e relazioni semantiche paradigmatiche di eponimia e semiche/ sememiche specificate, in linea con quanto esposto nella precedente sezione (vedi 3.2.5.3.3, 3.2.7); 4) relazioni paradigmatiche e relazioni sintagmatiche sono presenti in forma lessicale o metalessicale nella sottolingua, costituendo rispettivamente gli assi verticale e orizzontale della sua rete sindetica.

Quest'ultimo punto ci porta a un importante elemento di differenza della sottolingua rispetto al linguaggio naturale. Nel linguaggio naturale le relazioni paradigmatiche sono tutte quelle che un elemento lessicale intrattiene con altri a prescindere da specifici discorsi, e viceversa le relazioni sintagmatiche sono le relazioni che un elemento lessicale intrattiene con altri all'interno di specifici discorsi; tutte le relazioni paradigmatiche possono, teoricamente, comparire nei sintagmi, e viceversa - ma qui con la suddetta riserva delle relazioni sintagmatiche logiche - , ovvero ogni relazione semantica può

275 Poiché, come abbiamo spiegato, la sottolingua esiste nel momento in cui le relazioni sono realizzate tramite collegamenti fisici, indici controllati, etc., daremo d'ora in poi per scontati tali aspetti fisici e parleremo semplicemente di semantica delle relazioni e di relazioni semantiche, tranne nei casi in cui sia necessario distinguere tra la rappresentazione implicita ed esplicita (indici e collegamenti) delle relazioni. 
assumere indifferentemente una forma paradigmatica o sintagmatica. Nella sottolingua entra invece in gioco una questione di ottimizzazione della ridondanza del linguaggio naturale: in virtù di quel processo di esplicitazione e lessicalizzazione delle relazioni paradigmatiche per cui esse vanno a formare parte della rete sindetica della sottolingua, la rappresentazione delle relazioni paradigmatiche anche a livello sintagmatico risulterebbe ridondante senza essere funzionale. Per questo motivo la sottolingua proposta - distaccandosi in ciò dalle caratteristiche del linguaggio naturale a causa delle esigenze indicali - non prevede la possibilità di una medesima relazione semantica sia a livello paradigmatico sia a livello sintagmatico: avremo piuttosto da un lato le relazioni paradigmatiche, per la cui trattazione svilupperemo quanto esposto nella precedente sezione circa le relazioni semantiche (eponimia, sinonimia, etc., vedi 3.2.5.3.3); dall'altro le relazioni semantiche sintagmatiche, per la cui trattazione svilupperemo quanto esposto nella precedente sezione circa i casi profondi e le altre relazioni sintagmatiche (vedi 3.2.7, 3.2.8). Anche il nesso tra relazioni semantiche sintagmatiche e relazioni semantiche paradigmatiche gerarchiche specificate determina un certo grado di ridondanza della sottolingua; tuttavia si tratta in questo caso di una ridondanza indiretta, e che riteniamo utile ai fini dell'accesso bibliografico. Per fare un esempio: l'utente che parta dall'elemento lessicale "bambini" per scorrere le sue articolazioni concettuali, si soffermi quindi sull'elemento "ospedali pediatrici" (sinonimo di "ospedali per bambini"), ritroverebbe poi l'elemento lessicale "bambini" a livello di semantica paradigmatica come elemento lessicale in relazione semica benefattiva rispetto a "ospedali per bambini" (vedi 3.3.6.2.5). In questi casi la ridondanza indiretta di un elemento lessicale coinvolto da relazioni sintagmatiche e dalle correlate relazioni paradigmatiche gerarchiche specificate è utile perché offre all'utente finale più percorsi per Trovare, Identificare e Navigare le entità coinvolte nei vari elementi lessicali. Sempre per ragioni legate all'esigenza di ridurre la ridondanza linguistica, la sottolingua proposta esclude la possibilità della rappresentazione di elementi lessicali in forma sintagmatica i cui elementi lessicali componenti siano tra loro legati da relazioni paradigmatiche; con alcune eccezioni: elementi lessicali co-iponimi, co-sememi, co-meronimi, antonimi complementari, alcuni tipi di meronimi e olonimi (vedi 3.3.6.4.2.2.2.4, 3.3.6.4.2.2.2.5 e 3.3.6.4.2.2.4 per la discussione delle tipologie di relazioni menzionate) potranno invece essere rappresentati all'interno dello stesso sintagma.

\subsubsection{Lessico}

\subsection{Controllo della biplanarità del lessico della sottolingua e suoi limiti}

Parlando del lessico del linguaggio naturale (vedi 3.2.5.2), avevamo fatto riferimento alla sua naturale biplanarità (o doppia articolazione: lessemi in quanto composti di significanti e significati). Il processo di lessicalizzazione degli aspetti linguistici implicato dal controllo linguistico esplicito tipico delle lingue documentarie comporta inevitabilmente una scissione del lessico nei due piani semantico e morfosintattico, 
ovvero una separazione dei significati e dei significanti dei lessemi; ${ }^{276}$ ciò vale in linea di principio anche per il primo e secondo grado di controllo linguistico ma è un processo particolarmente evidente nel lessico della sottolingua. Si tratta di un'operazione problematica, perché essenzialmente contraria alla logica del linguaggio, eppure al contempo inevitabile per una lingua in forma scritta (quali le lingue documentarie prese in considerazione) che voglia circoscrivere le ambiguità del linguaggio naturale.

Prima di proseguire la nostra discussione, sono però indispensabili alcune specificazioni relative alla questione del controllo semantico del lessico della sottolingua; una serie di riserve, riguardanti sia il senso complessivo dell'operazione del controllo semantico del lessico, sia la sua reale possibilità ed efficacia.

Uno dei luoghi comuni di tale controllo, un vero e proprio leit motiv della teoria dell'indicizzazione, è quello secondo cui uno dei suoi scopi o il suo scopo principale sia quello di "eliminare" l'ambiguità (omonimia, omotassia, polisemia, etc.) delle lingue naturali. ${ }^{277}$ Innanzitutto ribadiamo che l'ambiguità linguistica non è, in sé, qualcosa di intrinsecamente problematico, ma semmai lo diventa in ambito documentario perché questo è privo espedienti pragmatici (legati al contesto) di disambiguazione. Anzi, l'ambiguità linguistica assolve nelle lingue naturali a funzioni importanti: 1) essa contiene una eccessiva proliferazione lessicale, permettendo di rapportare in modo economico lessico e semantica; 2) essa è la base per la possibilità di evoluzione di una lingua, e in particolare della sua semantica. L'eliminazione totale dell'ambiguità linguistica è dunque un'operazione contraria alla natura del linguaggio e nemmeno auspicabile. Conseguenza del perseguimento dell'ideale dell'eliminazione totale dell'ambiguità linguistica e della biplanarità del lessico, secondo una concezione in cui a ogni significante deve corrispondere uno e un solo significato, è stata storicamente la creazione di lingue documentarie (sottolingue, secondo la nostra terminologia) caratterizzate da lessici e semantiche tendenti all'artificiosità e alla prolissità.

Un controllo totale dei significati dei lessemi è comunque impossibile, per un altro motivo; se è teoricamente possibile cercare di controllare il significato dei lessemi in sé, è molto più difficile controllare il significato d'uso dei lessemi, ovvero la particolare

276 Cfr. JEAN-CLAUDE GARDIN, Semantic analysis procedures in the sciences of man, cit., p. 21: "[in documentary languages] the iregularity of correspondences between signifiants an signifiés, or linguistic form and meaning, is replaced by a normalization of the former grounded on the relative invariance bestowed on the latter in a given reference scheme"; cfr. anche W. JOHN HUTCHINS, Languages of indexing and classification, cit., p. 7. La tendenza delle lingue documentarie a eliminare la doppia articolazione del linguaggio è la stessa che caratterizza le lingue filosofiche e "caratteristiche", a cui fa riferimento UMBERTO ECO, La ricerca della lingua perfetta nella cultura europea, cit., p. 29: "Questo principio della doppia articolazione è da considerare con attenzione perché vedremo come molte lingue filosofiche cercano appunto di eliminarlo. In termini hjelmsleviani una lingua è biplanare ma non conforme $[. .$.$] molte lingue perfette aspirano a questa corrispondenza tra segni e realtà o tra segni e concetti$ corrispondenti"; p. 267: "in una lingua caratteristica si è obbligati a trovare un contenuto per ogni elemento dell'espressione. La lingua caratteristica, non essendo fondata sulla doppia articolazione propria alle lingue naturali (dove suoni privi di significato si combinano per produrre sintagmi dotati di significato), fa sì che una minima variazione di suono o di carattere un cambiamento di senso [...] da cui deriva un totale isomorfismo tra espressione e contenuto".

277 Cfr. per esempio W. JOHN HUTCHINS, Languages of indexing and classification, cit., p. 7, ELAINE SVENONIUS, The intellectual foundation of information organization, cit, p. 14, NATIONAL INFORMATION STANDARDS ORGANIZATION, Guidelines for the construction, format, and management of monolingual controlled thesauri, cit., p. 12. 
accezione semantica con cui essi vengono utilizzati nell'indicizzazione. ${ }^{278}$ Il significato con cui un lessema è utilizzato nell'indicizzazione può infatti variare in base al contesto e al momento storico. Ciò è vero soprattutto per lessemi astratti o ideologici come "arte", "libertà", "democrazia", etc., ovvero quelli che, in termini peirciani, hanno un referente incerto; ciò che oggi è considerato "arte" potrebbe non esserlo stato ieri o non esserlo domani (la fotografia non lo è stata per un certo periodo di tempo). Alcuni termini, come per esempio "sessismo", potrebbero essere utilizzati diversamente sulla base dei presupposti vigenti in un dato contesto storico-culturale.

Date queste condizioni, è innanzitutto d'obbligo rinunciare all'ideale di "eliminare" l'ambiguità linguistica, e semmai puntare su un controllo (sempre parziale) di essa, ovvero una sua registrazione. In secondo luogo è opportuno distinguere l'ambiguità linguistica dovuta a omonimia (e omografia) e omotassia, da un lato, e quella dovuta a polisemia dall'altro: quest'ultima è una condizione tendenzialmente naturale dei lessemi della lingua naturale ed è alla base dell'evoluzione linguistica; inoltre, le diverse accezioni semantiche di un lessema sono strettamente correlate e dunque suscettibili di essere trattate contestualmente dai testi che costituiscono l'oggetto della catalogazione. Pur nella inevitabile riduzione della biplanarità del linguaggio da parte delle lingue documentarie, bisognerebbe pertanto limitare o meglio ancora rinunciare del tutto al controllo della polisemia nelle sottolingue, e limitarsi invece a controllare l'omonimia e l'omotassia.

\subsection{Natura parzialmente chiusa del lessico della sottolingua}

Alla questione dell'ambiguità linguistica è collegata quella dello status aperto o chiuso di una lingua. Le lingue naturali sono per loro natura aperte, cioè suscettibili di un'evoluzione spontanea tanto a livello lessicale (nascita o caduta in disuso di determinati lessemi), quanto semantica (nascita o caduta in disuso di determinati sememi, cambiamento del rapporto tra significanti e significati, ecc.). Viceversa, il controllo semantico del lessico tende a creare lingue parzialmente o totalmente chiuse, in cui l'evoluzione linguistica è possibile, ma solo tramite una regolazione artificiale: lessico e semantica delle sottolingue dovrebbero essere modificate e aggiornate continuamente per non divenire inattuali o incomprensibili; più aumenta la consistenza documentaria indicizzata dalle sottolingue, più aumenta l'estensione del loro lessico e la complessità della loro semantica; inoltre, se è possibile intervenire sul lessico e sulla semantica della sottolingua in sé, è molto difficile, per non dire impossibile, farlo in modo sistematico sugli indici pregressi che utilizzano la sottolingua, ovvero intervenire sui cambiamenti del significato d'uso dei lessemi: ciò crea col tempo uno iato sempre maggiore tra i significati dei medesimi lessemi utilizzati in periodi storici diversi. ${ }^{279}$

Si tratta di un limite intrinseco delle sottolingue, che è importante evidenziare; è a causa di questo limite che alcuni autori nell'ambito della teoria dell'indicizzazione si sono spinti

278 Cfr. ELAINE SVENONIUS, The intellectual foundation of information organization, cit, p. 153: "It would seem that the more closely the meaning of a term is prescribed, the more consistently it would be used by indexers [...] But recent research has challenged this supposition. Michèle Hudon examined the hypothesis that indexing consistency could be improved by augmenting a standard thesaurus with definitions for each of its terms and found that hte hyphotesis could not be supported".

279 Cfr. BIRGER HJØRLAND, Information retrieval, text composition, and semantics, cit., p. 24. 
a difendere l'utilizzo nell'indicizzazione del linguaggio naturale, tale e quale (cioè senza il terzo grado di controllo linguistico). ${ }^{280}$

Il limite della natura chiusa che caratterizza le sottolingue sarebbe eliminabile solo a patto di escogitare un sistema in grado di modificare automaticamente in modo retrospettivo l'indicizzazione pregressa sulla base dei cambiamenti lessicali e degli slittamenti di significato dei lessemi che avvengono col passare del tempo: cosa del tutto utopistica. Più realisticamente, un modo per mitigare tale limite è quello di prevedere specifici indici che segnalino la data in cui è avvenuta l'indicizzazione, e dunque permettano all'utente di rapportare l'utilizzo indicale di un elemento lessicale con la data in cui esso è stato utilizzato: si tratta dei metaindici di data dell'indicizzazione di cui abbiamo parlato nel precedente capitolo (vedi 2. 24).

\subsection{Elementi lessicali e metalessicali}

Gli aspetti linguistici della lingua documentaria, e la semantica in particolare, possono presentarsi in forma lessicale o metalessicale; per quanto riguarda la sottolingua, ciò riguarda in particolar modo le relazioni sintagmatiche: queste possono infatti essere espresse o in forma lessicale (implicita), cioè far parte dello stesso lessico della sottolingua (per esempio: "Educazione in Italia"), o in forma metalessicale (esplicita), cioè presentarsi come elementi esterni al lessico vero e proprio (per esempio: "[delocativo:] Educazione [locativo:] Italia", vedi 3.3.6.4.3 e ss.; il "delocativo" e tutti gli altri elementi metalessicali introdotti dal prefisso "-de" costituiscono le controparti dei vari ruoli semantici della sottolingua) e atti a identificare o spiegare il significato relativo degli elementi lessicali componenti il sintagma; le relazioni paradigmatiche della sottolingua hanno invece sempre una forma metalessicale. L'espressione delle relazioni sintagmatiche in forma lessicale caratterizza gli elementi lessicali in forma non collegata, l'espressione delle relazioni sintagmatiche in forma metalessicale caratterizza invece gli elementi lessicali in forma collegata (vedi 3.3.6.2.5).

\subsection{Elementi lessicali in forma semplice e composta}

Coerentemente con la precedente discussione sulla relatività della forma semplice o composta degli elementi lessicali, così come con la nostra discussione del carattere composito dei sememi, il lessico della sottolingua che proponiamo prevede la presenza sia di elementi lessicali in forma semplice (un'unica parola) sia di elementi lessicali in forma composta (sintagmi); prevede inoltre che di uno stesso elemento lessicale siano registrate sia le le forme semplici sia le forme composte: dunque sia "bioetica" (forma semplice) sia "etica della vita" (forma composta). ${ }^{281}$

\subsection{Elementi lessicali in forma composta non collegata, parzialmente}

$280 \mathrm{~K}$. BHATTACHARYYA, The effectiveness of natural language in science indexing and retrieval, "Journal of documentation”, 30, 3 (1974), p. 251.

281 Cfr. W. JOHN HUTCHINS, Languages of indexing and classification, cit., p. 24. 
Tesi di dottorato di Giuliano Genetasio, discussa presso l'Università degli Studi di Udine

\section{collegata e totalmente collegata ${ }^{282}$}

L'impostazione adottata nella precedente sezione del capitolo (vedi 3.2.3, 3.2.6) esclude la possibilità di porre il "singolo concetto" o "unità di pensiero" a base del lessico della sottolingua, ovvero di stabilire in modo aprioristico i confini concettuali degli elementi di significato; lo standard NISO2005, che lo fa, è poi costretto a riconoscere come la percezione di cosa sia un "singolo concetto" muti da contesto a contesto. ${ }^{283}$ I limiti di scomposizione semantica sintagmatica vanno stabiliti in modo convenzionale sulla base di criteri precisi; come abbiamo spiegato nel precedente capitolo, quando abbiamo parlato dei nomi complessi (vedi 2.13), il criterio da noi scelto è l'individuazione, all'interno di un dato elemento lessicale, di un riferimento ad almeno un ulteriore elemento lessicale che sia ritenuto significativo in sé (ovvero relativo a un'entità, nei termini dell'approccio concettuale) e a cui il dato elemento lessicale sia legato da una relazione gerarchica; come già detto, sono esclusi dalla scomposizione sintagmatica quei nomi che facciano riferimento ad altre entità solo in senso figurato o esclusivamente formale; non è dunque possibile scomporre l'elemento lessicale in forma composta "occhi di vetro" in "occhi" e "vetro", oppure "Biblioteca Alberto Bombace" in "Biblioteca" e "Alberto Bombace", perché il riferimento a "occhi" in "occhi di vetro" è di senso figurato (non vi è una relazione eponimica tra "occhi" e "occhi di vetro"), e perché "Alberto Bombace" è solo parte del nome proprio della "Biblioteca Alberto Bombace". Negli elementi lessicali in forma composta che fanno riferimento a ulteriori entità, gli elementi lessicali componenti che specificano l'elemento lessicale in forma composta rispetto alla classe d'appartenenza sono sempre indicativi di ulteriori entità, mentre l'elemento componente che rappresenta la classe d'appartenenza può o meno essere indicativo di un'ulteriore entità.

La sottolingua proposta prevede due tipologie fondamentali di forme per gli elementi lessicali in forma composta che facciano riferimento a ulteriori elementi lessicali con cui intrattengano relazioni gerarchiche: da un lato forme lessicali composte in linguaggio naturale, cioè in forma discorsiva e non controllata formalmente, con le relazioni

282 L'idea della differenza tra tra forme collegate e non collegate degli elementi lessicali, e più in generale tra forme semplici e composte, è scaturita sia da una riflessione sull'attuale struttura delle LCSH, e dalla relativa incoerenza di utilizzo di termini composti, termini in forma inversa e suddivisioni, sia dalla lettura dell'opera di Hutchins, e in particolare della sua discussione della questione della scomposizione semantica dei termini, cfr. W. JOHN HUTCHINS, Languages of indexing and classification, cit., p. 101-102: "What may be expressed in one DL [...] by a single descriptor may be conveyed in another [...] by a syntagm of descriptors [...] The latter may have fewer and less specific descriptors than the former but it will compensate for this by a richer syntagmatic organisation [...] For example [...] the specific NL word "aniline" might be expressed by the combination of abstract terms (i.e. IL descriptors) "amine, benzene derivative, sixcarbon compund, seven-hydrogen compund, one-nitrogen compound, dye intermediate, ioly substance, toxic substance, combustible base", each representing either a genus or an attribute of "aniline". If each genus and each attribute is then further analysed in the samy way into a set of even more "abstract" terms, and each of these into yet more abstract ones, the result is a "compressed vocabulary" of descriptors - a small set of abstract terms that in combination are capable of expressing higly specific DFs. If these descriptors are not themselves capable of further analysis [...] then is legitimate to treat them as the primitives of a "minimum vocabulary" [ o "compressed vocabularies":] The descriptors of "compressed vocabularies" are often referred to as "semantic factors", and the technique used for discovering them as "semantic factoring" ".

283 Cfr. NATIONAL INFORMATION STANDARDS ORGANIZATION, Guidelines for the construction, format, and management of monolingual controlled thesauri, cit., p. 36, 38. 
sintagmatiche espresse lessicalmente ("etica della vita"); dall'altro forme lessicali composte espresse in forma artificiale, che presentino esclusivamente gli elementi lessicali significativi componenti, in forma sostantivale e con le reciproche relazioni sintagmatiche espresse in forma metalessicale (p.e. "[deoggettivo:] Etica - [oggettivo:] Vita").

Quest'ultimo tipo di forme lessicali composte costituisce a tutti gli effetti un ulteriore grado di controllo linguistico esplicito, tramite cui gli elementi componenti di un elemento lessicale in forma composta vengono messi in relazione grazie alle loro relazioni sintagmatiche interne. Invece che parlare però di forme sintagmatiche non controllate e controllate, ed esclusivamente per non creare confusione con la terminologia utilizzata altrove, parleremo di forme lessicali non collegate per riferirci alle forme composte degli elementi lessicali in linguaggio naturale (forma discorsiva e non controllata; corrispondono agli indici non collegati dell'approccio concettuale); parleremo invece di forme lessicali collegate per riferirci invece alle forme lessicali composte controllate tramite relazioni sintagmatiche espresse in modo metalessicale (corrispondono agli indici collegati dell'approccio concettuale).

Mentre per le forme sintagmatiche non collegate è opportuno prevedere soltanto quelle esistenti anche nella relativa lingua naturale (p.e. "etica della vita" e basta; "diritto canonico amministrativo" e "diritto amministrativo canonico"), per le forme sintagmatiche collegate è opportuno prevedere (in fase di indicizzazione o anche solo di visualizzazione, possibilmente automaticamente) un numero di sequenze possibili corrispondente al numero delle relazioni sintagmatiche coinvolte: due sequenze per una relazione (due elementi lessicali in relazione), sei sequenze per due relazioni (tre elementi lessicali in relazione), ventiquattro sequenze per tre relazioni (quattro elementi lessicali in relazione), etc.

Le forme sintagmatiche collegate, evidentemente, sono delle forme lessicali del tutto estranee alla natura del linguaggio naturale, e legate alle finalità dell'accesso bibliografico; si tratta di uno di quei punti del nostro modello di lingua documentaria in cui linguaggio naturale e finalità dell'indicizzazione divergono.

Le forme sintagmatiche collegate svolgono alcune importanti funzioni catalografiche: permettono, congiuntamente alle relazioni di equivalenza con l'elemento lessicale in forma semplice o composta non collegata, di Trovare un elemento lessicale sia a partire dalla sua forma semplice sia a partire da una delle sue forme composte, per poi Navigare attraverso uno dei suoi elementi lessicali componenti: un utente potrebbe partire da "[deoggettivo:] Etica [oggettivo:] Vita" per spostarsi poi all'elemento lessicale "Etica" o "Vita", etc., senza dover visualizzare la semantica paradigmatica (iperonimi, olonimi, etc.) dell'elemento lessicale; le varie sequenze artificiali che caratterizzano le forme sintagmatiche collegate rendono inoltre possibile Trovare l'elemento lessicale a partire da qualsiasi suo elemento componente che si riferisca a un elemento lessicale registrato autonomamente. Ovviamente, ciò crea una ridondanza indiretta con la semantica paradigmatica, in cui gli elementi lessicali componenti riferiti a ulteriori elementi lessicali sono invariabilmente registrati anche tramite relazioni di iperonimia, sememia, olonimia, etc., tuttavia abbiamo spiegato che si tratta di percorsi semantici alternativi (l'utente non è così obbligato ad accedere alla semantica paradigmatica di un elemento) e dunque entrambi utili.

A questo punto dobbiamo introdurre un'ulteriore differenziazione nell'ambito delle forme sintagmatiche collegate: possiamo suddividerle in parzialmente collegate e 
totalmente collegate a seconda che l'elemento lessicale componente che indichi la classe di appartenenza dell'elemento lessicale nel suo complesso sia o meno un elemento lessicale rilevante in sé, ovvero registrato autonomamente; se lo è, ovvero se tutti gli elementi lessicali componenti il sintagma sono ritenuti significativi e controllati fisicamente tramite proprie registrazioni, parleremo di una forma lessicale totalmente collegata; altrimenti di una forma lessicale parzialmente controllata: quest'ultimo tipo di forma lessicale riguarderà quegli elementi relativi a entità individuali che presentino forme lessicali caratterizzate dalla combinazione di un nome comune e uno o più nomi propri, i quali a loro volta corrispondano agli elementi lessicali sovraordinati gerarchicamente (olonimi e semi non generici). Esempi di forme lessicali parzialmente controllate sono "[meronimico:] Biblioteca - [olonimico:] CGIL", ovvero "Biblioteca della CGIL", "[delocativo + detemporale:] Alluvione - [locativo:] Firenze - [temporale:] 1966", ovvero "Alluvione di Firenze del 1966, "[meronimico:] Cattedrale - [olonimico:] Palermo", ovvero "Cattedrale di Palermo".

Abbiamo così distinto quattro tipologie fondamentali di forme lessicali della sottolingua: forme lessicali semplici, forme lessicali composte non collegate, forme lessicali composte parzialmente collegate e totalmente collegate.

Una nota sulla visualizzazione degli elementi lessicali in forma sintagmatica collegata. Mentre a livello di indicizzazione gli elementi lessicali in forma sintagmatica collegata dovrebbero assumere una forma in cui le relazioni sintagmatiche siano esplicitate tramite elementi metalessicali, del tipo "[dedirettivo:] Deportati [direttivo:] Germania" ("Deportati in Germania"), a livello di visualizzazione è opportuno che l'uso degli elementi metalessicali sia ridotto a quelli indispensabili, per esempio "Deportati [direttivo:] Germania”; inoltre, non è detto che la forma verbale metalessicale debba essere la stessa in fase di indicizzazione e in fase di visualizzazione; la forma metalessicale di visualizzazione potrebbe riprendere direttamente il linguaggio naturale (per esempio "Deportati - [in:] - Germania"). ${ }^{284}$

\subsection{Uso degli elementi lessicali e semantica paradigmatica dei sintagmi}

Una questione di cui non abbiamo parlato sinora è quella della differenziazione del lessico in lessico generale e lessici dipendenti o speciali, uso differenziato del lessico, etc. Alcune lingue documentarie enumerative, per esempio le LCSH, presentano una differenziazione del lessico che non ha una vera giustificazione semantica (elementi lessicali di significato identico ma terminologicamente diversi), ma che è basata su differenze tra strutture indicali (lessico utilizzato nelle "voci principali” e lessico utilizzato nelle "suddivisioni") oppure disciplinari (p.e. uso di "History and criticism" dietro determinati tipi di intestazioni, "Criticism and interpretation" dietro altre, "Criticism, interpretation, etc." dietro altre ancora).

Contro tale impostazione, il nostro modello di sottolingua prevede un lessico fondamentalmente unico, in cui tutte le differenze terminologiche non corrispondenti a differenze di significato siano ricondotte a relazioni di equivalenza tra forme diverse di

284 Questo sistema potrebbe apparire preferibile, tuttavia il problema di questo tipo di forme metalessicali è che per ogni tipo di relazione sintagmatica esistono due o più preposizioni, preposizioni articolate, etc., corrispondenti alle varie forme di superficie di quella relazione sintagmatica. 
uno stesso elemento lessicale. Un lessico unico, ma composto di elementi lessicali il cui uso dovrebbe essere differenziato, così come avviene nel linguaggio naturale, sulla base della semantica paradigmatica delle tipologie lessicali vigente nei sintagmi, la cui discussione riprenderemo più sotto (vedi 3.3.6.4.3.2). Facciamo già qui qualche esempio: l'elemento lessicale "critica" (o "interpretazione" o altro termine simile; tutte le varianti sarebbero considerati come elementi lessicali semanticamente equivalenti) dovrebbe poter essere combinato con tutti gli elementi appartenenti a tipologie lessicali per cui è sensato parlare di critica: Persone, Testi, Tipi di testo, etc. Determinati Tempi, nella forma di archi cronologici, dovrebbero poter essere utilizzati soltanto in combinazione con elementi lessicali per i quali sono significativi: l'arco cronologico "1994-2011”, per esempio, è specificamente significativo per la storia d'Italia, indicando il periodo che va dal primo all'ultimo governo Berlusconi, e dunque potrà essere utilizzato in combinazione con l'elemento lessicale "Italia" più eventuali altri paesi per cui quell'arco cronologico è significativo.

Questa impostazione del lessico della lingua documentaria cerca di cogliere il meglio delle lingue documentarie di tipo sintetico ed enumerativo: su una base lessicale sostanzialmente sintetica, essa intende valorizzare l'espressività tipica delle lingue enumerative, le quali riescono a esprimere significati specifici di determinati raggruppamenti lessicali (e perfino di singoli elementi lessicali) non esprimibili con un lessico puramente sintetico (le LCSH, per esempio, prevedono una serie di elementi lessicali come specificazioni cronologiche, articolazioni disciplinari, etc., utilizzabili soltanto in combinazione con determinati elementi lessicali). Riferendoci alle entità del modello concettuale (vedi 2.6 e ss.), possiamo dire che all'interno della sottolingua proposta soltanto Eventi, Tempi e Concetti possono costituire elementi lessicali specifici di singoli elementi lessicali o di gruppi di elementi lessicali.

\subsubsection{Morfosintassi di superficie}

Il ruolo della morfosintassi (di superficie) della sottolingua, è come già anticipato, fortemente ridotto rispetto a quello delle lingue naturali. ${ }^{285}$ Nella maggior parte delle lingue documentarie, le differenze morfologiche tra diversi elementi lessicali semanticamente equivalenti vengono riportate a forme varianti di uno stesso elemento lessicale (varianti morfologiche); diversi tra gli elementi morfologici tipici del linguaggio naturale sono assenti nelle lingue documentarie. Tra i primi a notarlo vi fu Hutchins, il quale notò come le lingue documentarie ignorino generalmente le differenze semantiche tra le varianti morfologiche di un elemento lessicale, trattandone come sinonimi le diverse forme grammaticali.

La morfologia delle sottolingue consiste allora soprattutto nell'insieme delle regole in base a cui: 1) scegliere quali elementi morfologici (categoria morfologica, genere, numero, etc.) accogliere nella lingua documentaria; 2) stabilire le relazioni semantiche tra le diverse varianti morfologiche di un elemento lessicale. ${ }^{286}$

285 Cfr. ELAINE SVENONIUS, Facet definition, cit., p. 139.

286 NATIONAL INFORMATION STANDARDS ORGANIZATION, Guidelines for the construction, format, and management of monolingual controlled thesauri, cit., p. 25-27, fornisce molte regole, lessicologiche e grammaticali (sintattiche e morfologiche), per operare tali scelte; tra le categorie grammaticali, NISO2005 
Tesi di dottorato di Giuliano Genetasio, discussa presso l'Università degli Studi di Udine

Hutchins notava la generale preferenza delle lingue documentarie per i sostantivi, piuttosto che per aggettivi o verbi, spiegando linguisticamente questa scelta sulla base del fatto che mentre è possibile ottenere facilmente sostantivi da verbi e aggettivi, non sempre è vero il contrario; Maniez ha inoltre notato che il nome è l'unica categoria lessicale a poter esprimere sia oggetti che azioni e stati. ${ }^{287}$ Quanto al numero, Hutchins notava la preferenza generale delle lingue documentarie per il plurale piuttosto che per il singolare; riferendosi alla lingua inglese, Hutchins notava che il singolare è generalmente preferito per tutti i sostantivi numerabili, mentre il plurale per i sostantivi non numerabili. ${ }^{288}$ NISO2005 conferma questo criterio, specificando che il singolare è da preferire però sia per termini che siano generalmente usati al singolare (così come emerge dalla letteratura) sia per concetti ed entità uniche: ma queste eccezioni costituiscono vere e proprie contraddizioni rispetto alla regola generale. Tuttavia, lo stesso Hutchins non mancava di notare che la differenza tra sostantivi numerabili e non numerabili non è un universale linguistico: elementi lessicali che in una lingua sono numerabili in un'altra possono essere non numerabili e viceversa. ${ }^{289}$ Infine, ciò che è accaduto spesso nelle lingue documentarie tradizionali è l'uso del numero come espediente per distinguere elementi lessicali omonimi ("pesca" per indicare l'attività, "pesche" per indicare il frutto); ritroviamo questo criterio anche in NISO2005: ma si tratta evidentemente di un uso improprio del numero, un criterio di differenziazione semantica che non ha rispondenza nelle lingue naturali e che spesso può confondere l'utente finale. ${ }^{290}$ Con queste scelte NISO2005 si adegua all'esistente e alle sue contraddizioni, rinunciando a porre regole coerenti per la costruzione delle lingue documentarie.

Per il nostro modello di lingua documentaria, proponiamo una morfologia con un numero variabile di elementi morfologici presi in considerazione della sottolingua, il più possibile vicina a quella delle lingue naturali. Quanto alle categorie morfologiche: oltre a comprendere i sostantivi, che costituiranno la categoria morfologica principale del lessico di superficie della sottolingua, ed escludendo gli avverbi la cui inclusione nella sottolingua non appare generalmente necessaria, il lessico della sottolingua dovrebbe ovviamente includere aggettivi che formino parte di sintagmi nominali; eventualmente, e soltanto se necessario, gli aggettivi potrebbero essere inclusi anche come elementi a sé stanti, in quanto varianti morfologiche di forme sostantivali. Il lessico dovrebbe includere anche preposizioni che formino parte di sintagmi nominali (non come elementi a sé stanti); in questi casi è opportuno che di un sintagma preposizionale vengano indicizzate tutte le varianti ritenute verosimili, anche quelle in cui la preposizione può essere semanticamente ambigua (per esempio quando la preposizione "di" possa rivestire significati differenti); tali ambiguità andrebbero poi sciolte tramite espedienti

predilige i sostantivi, eventualmente - nel caso di termini composti - anche qualificati da aggettivi (preferibilmente) o preposizioni; prevede anche l'eventuale impiego di aggettivi a sé stanti, ma non di verbi a sé stanti. Cfr. anche W. JOHN HUTCHINS, Languages of indexing and classification, cit., p. 18-19.

287 JACQUES MANIEZ, Actualité des langages documentaires, cit., p. 127.

288 W. JOHN HUTCHINS, Languages of indexing and classification, cit., p. 20; cfr. anche ELAINE

SVENONIUS, The intellectual foundation of information organization, cit, p. 157.

289 NATIONAL INFORMATION STANDARDS ORGANIZATION, Guidelines for the construction, format, and management of monolingual controlled thesauri, cit., p. 28; W. JOHN HUTCHINS, Languages of indexing and classification, cit., p. 22.

290 NATIONAL INFORMATION STANDARDS ORGANIZATION, Guidelines for the construction, format, and management of monolingual controlled thesauri, cit., p. 29. 
metalessicali (esplicitazione delle relazioni sintagmatiche in forma metalessicale, nelle forme sintagmatiche collegate), ma non dovrebbero essere escluse dalla lingua documentaria se hanno una garanzia linguistica. Il lessico della sottolingua dovrebbe parimenti includere articoli indispensabili alla formazione corretta di sintagmi nominali (non come elementi a sé stanti). Per quanto riguarda il numero degli elementi lessicali, è importante che questo non venga utilizzato come espediente metalinguistico per la disambiguazione di omonimi (da realizzarsi tramite altri espedienti, vedi 3.3.6.4.2.2.6), e che invece singolare e plurale di un elemento lessicale vengano considerati legati da una relazione di equivalenza, ovvero come varianti morfologiche, quando si riferiscano a entità effettivamente equivalenti (per esempio "Scienza" e "Scienze"). Viceversa, è importante registrare - almeno in linea di principio - la differenza di numero quando indicativa di differenti entità, tra loro correlate ma distinte, come per esempio un'entità collettiva ("Alluvioni a Firenze nel 1966") e un'entità individuale che ne costituisca una specifica occorrenza (“Alluvione di Firenze del 1966”): la tendenza delle lingue documentarie a utilizzare la classe ("Alluvioni a Firenze nel 1966") per indicare l'individuo (“Alluvione di Firenze del 1966") è, almeno dal punto di vista teorico, inadeguata linguisticamente, oltre che carente dal punto di vista della specificità dell'indicizzazione; nella pratica, un criterio accettabile, anche se non universalizzabile, è quello dell'esistenza di una formulazione ricorrente per riferirsi a una determinata entità. I diversi casi (di superficie; non quelli profondi) di un elemento lessicale andrebbero invece considerati come varianti morfologiche tra loro semanticamente equivalenti. Ancora diversa, almeno potenzialmente, la situazione del genere degli elementi lessicali. In lingue documentarie in cui la differenza di genere non sia rilevante, maschile e femminile di un elemento lessicale dovrebbero essere trattati come varianti morfologiche legate da relazione di equivalenza. Ma in lingue documentarie dove la prospettiva di genere sia importante (femminismo, ma non solo, per esempio quando sia importante distinguere la condizione delle lavoratrici - in quanto donne - da quella dei lavoratori tout court), sarebbe invece opportuno considerare i differenti generi di un elemento lessicale come pertinenti significati ed entità distinte, tra loro legate da una relazione semantica di tipo diverso, per esempio gerarchica. Infine, per quanto riguarda i verbi, noi non li includeremo esplicitamente, ma teoricamente anch'essi potrebbero essere inclusi almeno in alcune forme: per esempio l'inclusione dell'infinito di un verbo quale variante morfologica di un elemento lessicale (per le sottolingue basate su lingue naturali che prevedono l'infinito) potrebbe essere utile, se si ritiene che esso possa essere utilizzato dall'utente finale per la ricerca (per esempio un utente che cerchi informazioni su come "navigare", o su come "costruire", etc.); l'utilizzo di verbi come parte di elementi lessicali è previsto per esempio, seppur raramente, dalle LCSH (vedi le voci del tipo "how to stop smoking", "how to start a business", etc., utilizzate come forme non preferite). Aldilà della sottolingua, sono possibili ulteriori spazi per i verbi nella lingua documentaria: 1) per quanto riguarda elementi indicali che non fanno parte della sottolingua: il rema di un soggetto potrebbe essere espresso tramite l'utilizzo di costruzioni verbali; 2) i metasintagmi della lingua documentaria presentano verbi impliciti, che come già detto sarebbe possibile esplicitare in fase di visualizzazione.

Passiamo adesso a parlare della sintassi (di superficie) della sottolingua. Dalla generale assenza dei verbi nelle sottolingue consegue che le sottolingue sono generalmente composte di nomi e sintagmi nominali - i quali possono però includere 
nominalizzazioni, relative a entità che racchiudono in sé il concetto di un'attività e che sul piano sintagmatico si comportano almeno parzialmente come attività, vedi 3.3.6.4.3.2 - e che la sintassi di superficie delle sottolingue è fortemente ridotta rispetto a quella del linguaggio naturale e riguarda principalmente le relazioni sintattiche tra gli elementi dei sintagmi nominali; Maniez ha giustificato la preferenza delle lingue documentarie per il sintagma nominale sulla base della sua natura di universale linguistico. ${ }^{291}$ In modo simile alla morfologia, il ruolo della sintassi di superficie nella sottolingua si riduce principalmente all'insieme delle regole in base a cui stabilire la corretta formazione (dal punto di vista sintattico) di sintagmi nominali. Si tratta dunque di regole di sintassi in tutto e per tutto discendenti dalla sintassi della lingua naturale. Vedremo più sotto (3.3.6.4.3 e ss.) che un ruolo molto importante ha invece la sintassi profonda, o semantica sintagmatica, della sottolingua.

\subsubsection{Semantica}

\subsection{Introduzione}

Con la discussione della semantica della sottolingua arriviamo alla parte centrale del nostro modello di lingua documentaria. A differenza di quanto avviene per la morfosintassi, la semantica della sottolingua è piuttosto complessa e articolata. Pur nella sua complessità, essa non aspira a essere esaustiva: la semantica della sottolingua che proponiamo è costituita di un insieme di proposte sulle entità e relazioni delle lingue documentarie che potranno o essere attuate solo parzialmente, o specificate in ulteriori tipologie di entità e relazioni.

Ribadiamo che la semantica della sottolingua riguarda il controllo linguistico esplicito di quegli elementi lessicali registrati tramite indici (punti di accesso) controllati del precedente capitolo (vedi 2.19).

Così come per la lingua documentaria in generale, anche la semantica della sottolingua non è e non può essere autoreferenziale, ma dipendente dalla semantica della lingua naturale, di cui è un'esplicitazione e semplificazione: i significati a cui fa riferimento la sottolingua sono fondamentalmente gli stessi del linguaggio naturale; a questo ideale sarà ispirata la nostra modellizzazione linguistica della semantica della sottolingua. ${ }^{292}$ Questa

291 JACQUES MANIEZ, Actualité des langages documentaires. Les fondements théoriques de la recherche d'information, Paris, cit., p. 127.

292 Cfr. MASSIMO ROLLE, Per un nuovo modello di linguaggio documentario, "Bollettino AIB", 41, 3 (2001), p. 324-325: "La formalizzazione del vocabolario e delle relazioni semantiche è un principio irrinunciabile dei linguaggi di indicizzazione [...] Nello stesso tempo va detto che la formalizzazione non deve essere più intesa come arbitrarietà nell'individuazione del rapporto termine-concetto [...] Il rapporto con i significati del linguaggio naturale [...] deve essere considerato il riferimento centrale per tutte le attività di controllo. Per questo motivo va tenuto conto anche del fatto che il termine, in quanto significato formalizzato, individua un significato specifico, che però, in rapporto ai significati del linguaggio naturale [...] va considerato come un fascio di significati coerenti e tra loro confinanti. Questo tipo di impostazione permette di ridurre le dimensioni del vocabolario per mezzo di un controllo più rigoroso del proliferare di termini diversi impiegati in letteratura per concetti simili e con l'eliminazione drastica di "falsi" termini creati per disambiguazione di concetti semanticamente non indipendenti, per esempio, "Arte (Concetto)" ”. 
impostazione ha una conseguenza fondamentale: porre la semantica della sottolingua come discendente da quella della lingua naturale significa accettarne almeno in parte la naturale ambiguità e soggettività; significa anche rinunciare a una semantica del tutto universale e assoluta, che non esiste peraltro nemmeno nel linguaggio naturale. ${ }^{293}$ Ciò nonostante, e come anticipato, sarà in alcuni casi necessario che la semantica della sottolingua si discosti, per esigenze strettamente legate all'indicizzazione, da quella del linguaggio naturale.

La semantica della sottolingua è composta: 1) di una semantica paradigmatica e di una semantica sintagmatica; 2) di una semantica (paradigmatica) delle entità e di una semantica (paradigmatica e sintagmatica) delle relazioni.

Suddivideremo la trattazione della semantica della sottolingua sulla base del primo criterio, ma prima di andare avanti desideriamo fare alcune osservazioni sulla natura generale della semantica delle relazioni (paradigmatiche e sintagmatiche) della sottolingua. La rappresentazione indicale delle relazioni semantiche del linguaggio naturale nella sottolingua può teoricamente conoscere diversi gradi di precisazione sulla base delle particolari esigenze degli utenti finali in un determinato contesto di accesso bibliografico. Così, una sottolingua con finalità di tipo basilare potrebbe limitarsi a segnalare un unico tipo di relazione semantica "di base", senza nemmeno una specificazione della sua natura paradigmatica o sintagmatica. Una sottolingua appena più complessa potrebbe invece limitarsi a distinguere relazioni paradigmatiche "di base" e relazioni sintagmatiche "di base", senza ulteriori specificazioni. Una sottolingua relativamente più complessa potrebbe invece distinguere un numero limitato di tipi di relazione paradigmatica (per esempio relazioni gerarchiche, associative e di equivalenza, così come avviene in una parte significativa dei thesauri) e/o un numero limitato di tipi di relazione sintagmatica (per esempio distinguere relazioni sintagmatiche grammaticali e relazioni sintagmatiche logiche, vedi 3.3.6.4.3 e ss.). E via così fino a distinzioni tra diversi tipi di relazioni gerarchiche e/o di equivalenza e associative, o tra diversi tipi di relazioni sintagmatiche grammaticali o logiche, etc. Il modello di sottolingua proposto rientra in quest'ultima e più articolata tipologia di tassonomie delle relazioni semantiche: essa prevede svariati tipi di relazioni gerarchiche, diversi tipi di relazioni di equivalenza, relazioni di antonimia e d'altro tipo; prevede inoltre diversi tipi e sottotipi di relazioni sintagmatiche.

Inoltre, i diversi gradi di precisazione della semantica delle relazioni della sottolingua che possono caratterizzare le lingue documentarie possono essere visti non soltanto come tipi distinti di semantiche documentarie, ma anche come un'unica semantica documentaria con diversi gradi di specificazione; in altri termini, a partire dalle suddette tassonomie

293 Una critica di taglio pragmatista della possibilità di una semantica "a priori" (cioè decontestualizzata, staccata dall'impiego della terminologia negli specifici ambiti di studio), e rivolta specificamente alla semantica 'a priori' di D. Austin, è fornita da BERND FROHMANN, An investigation of the semantic bases of some theoretical principles of classification proposed by Austin and the CRG, "Cataloging \& classification quarterly", 4, 1 (1983), p. 11-27; cfr. anche ALGIRDAS JULIEN GREIMAS, Semantica strutturale, cit., p. 131: "La constatazione che allo stato attuale delle conoscenze è difficile da immaginare una griglia culturale che risponda alle esigenze dell'analisi meccanica, significa che la descrizione stessa dipende ancora in larga misura dall'apprezzamento soggettivo di chi compie l'analisi [...] Lo stesso problema si incontra nel campo della costruzione dei linguaggi documentari: infatti Coyaud, nella sua recente tesi su I linguaggi documentari, mette chiaramente in evidenza la contraddizione interna, alla quale non sfuggono neppure i migliori linguaggi fino ad ora costruiti”. 
delle relazioni semantiche è possibile concepire una seconda semantica o metasemantica delle relazioni semantiche di una lingua documentaria: l'insieme delle relazioni semantiche tra le diverse tipologie semantiche di una lingua documentaria. Tale metasemantica sarebbe utile sia per gli utenti di una sottolingua, fornendo ulteriori percorsi di navigazione delle relazioni semantiche della sottolingua e chiarendone i reciproci rapporti, sia nell'eventualità di una integrazione o comunicazione tra differenti lingue documentarie le cui semantiche siano più o meno articolate. Metasemantiche di questo tipo sono recentemente state sviluppate - per quanto riguarda le relazioni paradigmatiche in particolare - da Khoo e Na e Wolfgang G. Stock, in entrambi i casi sulla base delle proprietà di riflessività, simmetria e transitività delle varie relazioni. ${ }^{294}$ Per quanto riguarda il nostro modello di lingua documentaria, non approfondiremo la questione, ma ci limitiamo a fornire un abbozzo della metasemantica delle relazioni semantiche (gerarchiche) della sottolingua proposta: 1) a livello paradigmatico, vi è una prima suddivisione (relazione gerarchica) tra relazioni paradigmatiche in generale e macrotipologie di relazioni paradigmatiche: gerarchiche, di equivalenza, di antonimia, di omonimia/omografia, ad hoc (vedi 3.3.6.4.2.2 e ss.); ciascuna macrotipologia è poi suddivisibile (relazione gerarchica) in diverse tipologie di relazioni semantiche: le relazioni gerarchiche sono suddivisibili in relazioni eponimiche (a loro volta suddivisibili in relazioni eponimiche pure e specificate), partonomiche (pure), semiche/sememiche (specificate), e tra nominalizzazioni; le relazioni di antonimia sono suddivisibili (relazione gerarchica) in relazioni di antonimia scalare (se previste esplicitamente), polare e complementare; quelle di equivalenza sono suddivisibili (relazione gerarchica) in relazioni di equivalenza non controllate, monocontrollate e bicontrollate (vedi 3.3.6.4.2.2.5); infine, vi sono le relazioni ad hoc che, se previste esplicitamente, sono suddivisibili (relazione gerarchica) nelle varie tipologie corrispondenti agli elementi indicali del modello concettuale (vedi 3.3.6.4.2.2.7); 2) a livello sintagmatico, le relazioni possono essere suddivise (relazione gerarchica) in relazioni sintagmatiche grammaticali e sintagmatiche logiche (vedi 3.3.6.4.3 e ss.); le prime possono essere ulteriormente suddivise (relazione gerarchica) nelle varie tipologie di casi profondi (relazione di agente, beneficiario, oggetto, strumento, partitive, possessive, etc., vedi 3.3.6.4.3.3) e in relazioni sintagmatiche d'altro tipo; le seconde possono essere suddivise in relazioni di coordinazione e interazione (vedi 3.3.6.4.3.4).

\subsection{Semantica paradigmatica}

\subsection{Introduzione}

Con la trattazione della semantica paradigmatica entriamo nel vivo della discussione della semantica della nostra sottolingua. Prima di discuterla in dettaglio, ribadiamo alcuni concetti. La semantica paradigmatica può essere suddivisa in semantica paradigmatica delle relazioni (equivalente, all'incirca, all'insieme di semantica relazionale e referenziale di Svenonius) e in semantica (paradigmatica) delle tipologie lessicali della sottolingua

294 Cfr. WOLFGANG G. STOCK, Concepts and semantic relations in information science, cit., p. 1964; CRISTOPHER S. G. KHOO - JIN-CHEON NA, Semantic relations in information science, cit., p. 161. 
(equivalente, all'incirca, alla semantica categoriale di Svenonius); semantica paradigmatica delle relazioni e delle tipologie lessicali sono, come avremo modo di spiegare, strettamente correlate tra loro. La suddivisione della semantica (paradigmatica) della sottolingua in semantica relativa alle entità e relazioni ricorda da vicino la terminologia tipica dei modelli concettuali: ed è così infatti che alcuni autori, tra cui Victoria Francu, hanno concepito le lingue documentarie quali i thesauri come forme di modelli concettuali entità-relazioni; ${ }^{295}$ lo stesso può dirsi per il nostro modello di lingua documentaria, che in tal senso costituisce una forma di espressione e precisazione linguistica del modello concettuale proposto nel precedente capitolo.

\subsection{Semantica paradigmatica delle relazioni}

\subsection{1. Introduzione}

Quando, nel precedente capitolo, avevamo parlato di indicizzazione alfabetica e classificazione (vedi 2.26), avevamo anticipato che con l'introduzione e lo sviluppo della rete sindetica gli strumenti di indicizzazione alfabetica si sono avvicinati alle classificazioni bibliografiche: è anzi proprio la semantica paradigmatica delle relazioni a costituire una sorta di "classificazione" interna alla sottolingua. ${ }^{296}$

In termini di funzioni dell'utente finale, la semantica paradigmatica delle relazioni mira a soddisfare le funzioni Trovare, Identificare, Selezionare e Navigare. Più precisamente, e riferendoci alle entità concettuali soggiacenti gli elementi lessicali, le relazioni paradigmatiche: 1) permettono di stabilire raggruppamenti gerarchici delle entità concettuali su base semantica; 2) permettono il reperimento e la localizzazione di entità concettuali su base semantica (paradigmatica), in quanto utilizzate in elementi indicali di vario tipo: indici di soggetto, di termine generico, etc.; 3) permettono di navigare facilmente ed esaustivamente la rete dei significati associati alle entità concettuali; 4) aiutano l'utente a ricordare entità concettuali correlate a quella ricercata e che potrebbero essergli utili; 5) facilitano la disambiguazione di omonimie, polisemie e omotassie; 6) permettono di selezionare le entità concettuali sulla base dei loro significati paradigmatici. $^{297}$

295 VICTORIA FRÂNCU, Subjects in FRBR and poly-hierarchical thesauri as possible knowledge organizing tools, in Knowledge organization for a global learning society. Proceedings of the ninth International ISKO conference, 4-7 July 2006, Vienna, edited by Gerhard Budin, Christian Swertz, Konstantin Mitgutsch, Würzburg, Ergon, 2006, p. 379.

296 Cfr. R. C. CROS - JEAN-CLAUDE GARDIN - F. LÉVY, L'automatisation des recherches documentaires, cit., p. 88-89; cfr. anche JEAN-CLAUDE GARDIN, Semantic analysis procedures in the sciences of man, cit., p. 27: "A second component of a metalanguage [...] is its semantic organization [...] This normally assumes the form of a classification [...] in which analytical relationships are indicated between the lexical items"; cfr. anche ASSOCIAZIONE ITALIANA BIBLIOTECHE. GRUPPO DI RICERCA SULL'INDICIZZAZIONE PER SOGGETTO, Guida all'indicizzazione per soggetto, cit., p. 84: "La struttura delle relazioni svolge alcune funzioni fondamentali: (a) costituisce una vera e propria struttura classificatoria, che raggruppa, in base a criteri omogenei, termini che condividono una o più caratteristiche di significato e mette in relazione termini legati da relazioni di tipo definitorio [...] c) integra, per quel che riguarda le funzioni di ricerca, l'accesso casuale dell'ordine alfabetico con una struttura classificata, individuata dal reticolo delle relazioni”. 297 Cfr. ELAINE SVENONIUS, The intellectual foundation of information organization, cit., p. 88, 127, 134, 
La tassonomia della semantica paradigmatica di gran lunga più utilizzata nell'ambito della teoria dell'indicizzazione è quella tipica dei thesauri, che divide le relazioni semantiche paradigmatiche in relazioni gerarchiche (generiche, partitive, a volte esemplificative), di equivalenza e associative; tale tassonomia è accolta, tra gli altri, anche da Hutchins, Delsey e dalla semantica relazionale di Svenonius. ${ }^{298}$

Per quanto ci riguarda, accoglieremo solo parzialmente questa suddivisione delle relazioni paradigmatiche, e in particolare: 1) mentre nel caso delle relazioni gerarchiche (eponimia, partonomia, semia/sememia) e di equivalenza (sinonimia, etc.) vi è una sostanziale corrispondenza con le relazioni semantiche tipicamente studiate dalla linguistica, ciò non può dirsi per le relazioni associative, le quali raccolgono un insieme di relazioni semantiche diverse; noi non accoglieremo le relazioni associative nella nostra sottolingua, preferendo parlare di relazioni di tipo diverso (antonimia scalare, polare e complementare, etc.); 2) alle relazioni di equivalenza e gerarchiche vanno aggiunte le relazioni di omonimia e omotassia, non previste come vere e proprie relazioni semantiche dai thesauri ma che invece sono relazioni semantiche paradigmatiche a pari titolo delle altre, con l'unica differenza che, come già accennato nel precedente capitolo, esse non devono essere registrate esplicitamente tramite collegamenti fisici distinti (vedi 2.19); e le relazioni co-sememiche tra nominalizzazioni (vedi 3.3.6.4.2.2.2.6); 3) aldilà della linguistica, noi aggiungeremo inoltre, almeno in linea di principio, la possibilità di una rappresentazione esplicita delle relazioni ad hoc, cioè tipiche dell'indicizzazione. Inoltre, all'interno della sottolingua le differenze gerarchiche saranno suddivise in: relazioni eponimiche pure, relazioni partonomiche pure, relazioni eponimiche e semiche/sememiche specificate, relazioni (co-sememiche) tra nominalizzazioni. Sulla base della differenza tra forme lessicali collegate e non collegate le relazioni di equivalenza (sinonimia, parafrasi, etc.) verranno suddivise in relazioni di equivalenza non controllate, monocontrollate e bicontrollate (vedi 3.3.6.4.2.2.5). Ribadiamo inoltre che nella nostra sottolingua le relazioni paradigmatiche non possono comparire come tali anche sul piano sintagmatico, e che le relazioni paradigmatiche assumono necessariamente una forma metalessicale.

\subsection{2. Relazioni gerarchiche}

\subsection{2.1. Introduzione}

Le relazioni gerarchiche sono le prime relazioni semantiche paradigmatiche di cui parleremo. La teoria dell'indicizzazione ha tradizionalmente incluso, tra le relazioni gerarchiche, le relazioni generiche (relazioni di eponimia, tra genere o classe e specie o

156; cfr. anche THOMAS MANN, Will Google's keyword searching eliminate the need of LC cataloging and classification?, 2005, disponibile all'indirizzo <http://www.guild2910.org/searching.htm>; cfr. anche R. C. CROS - JEAN-CLAUDE GARDIN - F. LÉVY, L'automatisation des recherches documentaires, cit., p. 27. 298 W. JOHN HUTCHINS, Languages of indexing and classification, cit., p. 90; TOM DELSEY, Modeling subject access, cit., p. 56-58; ELAINE SVENONIUS, The intellectual foundation of information organization, cit., p. 131. Una tassonomia diversa è quella di PINO BUIZZA - MAURO GUERRINI, Un modello concettuale per il Nuovo soggettario, cit. p. 333. 
individui) e partitive (partonomiche, tra l'intero e le sue parti); alcuni testi escludono le relazioni partitive, altri aggiungono le relazioni esemplificative (relazioni eponimiche tra entità collettive e individuali). ${ }^{299}$

Per il nostro modello di sottolingua suddivideremo le relazioni gerarchiche in relazioni eponimiche pure, partitive pure, e in relazioni eponimiche e semiche/sememiche specificate; parleremo anche della trattazione dei co-iponimi, co-meronimi e co-sememi all'interno della sottolingua, e in particolare delle relazioni co-sememiche tra nominalizzazioni. Non includeremo esplicitamente nel nostro modello di sottolingua le relazioni esemplificative, le quali non hanno una corrispondenza in linguistica e possono comunque essere considerate sottotipologie delle relazioni eponimiche che riguardano relazioni tra entità collettive ed entità individuali.

Prima di entrare nel vivo della discussione delle relazioni gerarchiche della nostra sottolingua, desideriamo esplicitare un importante presupposto della sua struttura: la poligerarchia. Una parte significativa della teoria dell'indicizzazione ha concepito la rete delle relazioni gerarchiche come una sorta di albero, caratterizzato da relazioni di tipo uno-a-molti, secondo cui un elemento lessicale può avere più elementi lessicali subordinati ma soltanto un elemento lessicale sovraordinato: una classe o intero può avere più individui o parti ma ogni individuo o parte uno e un solo genere o intero, etc.; questo tipo di architettura gerarchica è detta monogerarchia. Essa è certamente rigorosa, ma anche artificiosa e riduttiva, non corrispondendo né alla realtà bibliografica (e già extrabibliografica) né alle caratteristiche del linguaggio naturale. Nella realtà si incontrano frequentemente situazioni diverse, in cui un elemento lessicale fa parte semanticamente di due o più elementi lessicali. Abbondano i casi in cui un'entità collettiva può essere ricondotta a due o più entità semanticamente più ampie: per esempio l'elemento lessicale "Sociolinguistica" partecipa, ovvero è subordinato semanticamente, a entrambe la "Linguistica" e la "Sociologia". Più in generale, la monogerarchia non è soddisfacente per molti altri motivi: essa è incompatibile con la concezione comunemente accettata in linguistica secondo cui un semema è composto di più semi, oltre che con la nostra idea di varie tipologie di relazioni eponimiche specificate, che suddividono la relazione generica attraverso un particolare criterio. ${ }^{300}$

Per il nostro modello di sottolingua, adotteremo un tipo di architettura gerarchica reticolare, caratterizzata da rapporti molti-a-molti tra gli elementi lessicali, secondo cui un elemento lessicale può avere più elementi lessicali subordinati ma anche più elementi lessicali sovraordinati; tale architettura gerarchica è detta poligerarchia o struttura poligerarchica (essa è prevista da NISO2005, che però la considera un caso eccentrico). ${ }^{301}$

299 TOM DELSEY, Modeling subject access, cit., p. 56-58; NATIONAL INFORMATION STANDARDS ORGANIZATION, Guidelines for the construction, format, and management of monolingual controlled thesauri, cit., p. 48; NISO considera "mutualmente esclusive" le relazioni gerarchiche generiche ed esemplificative. 300 Cfr. CLASSIFICATION RESEARCH GROUP, The need for a faceted classification as the basis of all methods of information retrieval, "Library Association record", 57, 7 (1955), p. 262-268, poi pubblicato in Theory of subject analysis. A sourcebook, edited by Lois Mai Chan, Phyllis A. Richmond, Elaine Svenonius, Littleton, Colo., Libraries unlimited, 1985, p. 158: "This paper examines whther the generic relations of the field of knowledge can be adequately displayed by a simple enumerative tree-of-knowledge classification. It concludes that they cannot. The terms subsumed under a given genus [...] are not all derived from that genus by differentiation using a single characteristic of division. They can be sorted into groups or facets, each of which is derived from the genus by a different characteristic".

301 NATIONAL INFORMATION STANDARDS ORGANIZATION, Guidelines for the construction, format, and management of monolingual controlled thesauri, cit., p. 49. 
La poligerarchia è più flessibile della monogerarchia perché permette di considerare idealmente tutte le catene gerarchiche cui può appartenere concettualmente un elemento lessicale, tutte le prospettive e i ruoli semantici secondo cui può essere considerato un elemento lessicale; ${ }^{302}$ essa permette di rendere conto in modo più soddisfacente della polidimensionalità semantica degli elementi lessicali, ${ }^{303}$ ovvero del processo di formazione dei significati (sememi) come composti di una serie di elementi semantici (semi). La poligerarchia permette di ridurre eccessive proliferazioni del lessico della sottolingua che caratterizzano spesso le lingue documentarie monogerarchiche (in cui a ogni ambito di un concetto polisemico o con diverse applicazioni dovrebbero corrispondere elementi lessicali diversi, con una conseguente "classificazione nascosta"); la poligerarchia, inoltre, garantisce una maggiore flessibilità e capacità di adattamento agli slittamenti di significato degli elementi lessicali: in una poligerarchia, infatti, i singoli nodi gerarchici possono essere modificati più facilmente senza che ne risenta la rete sindetica nel suo complesso. ${ }^{304}$ Dal punto di vista delle funzioni del catalogo, infine, la poligerarchia permette di Navigare in modo più soddisfacente il catalogo. ${ }^{305}$ Infine, la teoria e gli standard di indicizzazione tradizionali prevedono solitamente la sola rappresentazione di rapporti gerarchici di subordinazione (iponimia, meronimia) e di sovraordinazione (iperonimia, olonimia); così avverrà generalmente anche nella nostra sottolingua, in cui la rappresentazione dei rapporti gerarchici di tipo paritetico tra elementi lessicali che fanno parte di una stessa gerarchia (co-iponimi, co-meronimi e co-sememi) rimarrà nella maggior parte dei casi implicita, con l'unica eccezione delle relazioni co-sememiche tra nominalizzazioni. ${ }^{306}$

\subsection{2. Relazioni eponimiche pure}

Abbiamo già discusso delle relazioni eponimiche pure, o tipiche, nel corso della precedente sezione (vedi 3.2.5.3.3); le relazioni eponimiche pure della sottolingua proposta sono simili alle relazioni eponimiche pure del linguaggio naturale, ma con alcune differenze.

Nell'ambito della sottolingua: 1) le relazioni eponimiche pure potranno comparire soltanto sull'asse paradigmatico; mentre infatti nel linguaggio naturale le relazioni generiche pure (p.e. "felini" rispetto a "gatti") possono comparire come parte di sintagmi

302 Cfr. ELAINE SVENONIUS, Indexical contexts, cit., p. 133-134.

303 ALFREDO SERRAI, Del catalogo alfabetico per soggetti, cit., p. 141-142: "Il multiversum dei significati non è riducibile ad un universum come pretendono gli schemi di classificazione; il rimedio sta nel proporre tanti microuniversi, ognuno dei quali ha la complessità semantica sufficiente a non escludere relazioni importanti e tutti insieme forniscono una mappa adeguata della realtà intellettuale, culturale e tecnologica [...] Il catalogo alfabetico per soggetti può avere un vantaggio importante sui cataloghi per classi; grazie alla accoglibilità illimitata delle voci alfabetiche, esso può accettare non una sola struttura catalografica, di solito monodimensionale, come avviene per il catalogo sistematico ma più di una struttura catalografica; esso può acquisire il pregio della polidimensionalità ossia della contemporanea presenza di molteplici organizzazioni semantiche ognuna delle quali sia impostata su una propria relazione strutturante”.

304 JACQUES MANIEZ, Actualité des langages documentaires, cit. p. 243-244.

305 Cfr. VICTORIA FRÂNCU, Subjects in FRBR and poly-hierarchical thesauri as possible knowledge organizing tools, cit., p. 376.

306 L'importanza della rappresentazione indicale dei rapporti gerarchici paritetici è evidenziata da W. JOHN HUTCHINS, Languages of indexing and classification, cit., p. 51. 
(p.e. "i gatti sono felini") oppure, a livello paradigmatico, possono essere ulteriormente specificate (la relazione tra "felini" e "gatti" potrebbe essere specificata come relazione tra "specie di felini" e "gatti"), nella lingua documentaria entrambi i tipi di specificazione sarebbero superflui (se non lo fossero, esse verrebbero trattate come relazioni gerarchiche specificate, vedi 3.3.6.4.2.2.2.4); 2) per lo stesso motivo, escludiamo la possibilità di elementi lessicali in forma composta che includano elementi lessicali componenti tra loro in relazione di iperonimia e iponimia (ma non co-iponimia) pura; 3 ) mentre nel linguaggio naturale le relazioni eponimiche pure o non specificate, sono tutte quelle i cui criteri di specificazione non siano stati distinti, ma che potrebbero esserlo, nella sottolingua proposta vi è una differenza più netta tra relazioni eponimiche pure da un lato e le relazioni eponimiche (e semiche/sememiche) specificate dall'altro; le relazioni eponimiche pure raccolgono tutte le relazioni eponimiche in cui l'iponimo non si differenzia dall'iperonimo per un numero maggiore di elementi componenti significativi, e quindi: a) relazioni tra elementi che presentino entrambi solo forme semplici ("Felini" e "Gatti"); b) relazioni tra elementi lessicali che presentino forme composte con due o più elementi componenti tutti significativi, ovvero totalmente controllate, per esempio "Cani da caccia" (con "Cani" e "Caccia" entrambi significativi), ed elementi che presentino solo forme semplici, per esempio "Beagle" o "Bassotto"; c) relazioni tra elementi lessicali con forme composte di due o più elementi componenti tutti significativi ed elementi lessicali con forme composte di un identico numero di elementi componenti tutti significativi, per esempio "Sport per bambini" rispetto a "Calcio per bambini"; d) relazioni tra elementi lessicali con forme composte di due o più elementi componenti tutti significativi ed elementi lessicali con forme composte di due o più elementi componenti di cui uno non significativo, per esempio "Cattedrali in Sicilia" rispetto a "Cattedrale di Palermo" (con "Cattedrale" non significativo).

Così, mentre nel linguaggio naturale sarebbero teoricamente possibili anche relazioni semiche e sememiche pure o non specificate, ciò non avverrà nella lingua documentaria, che sulla base dei suddetti presupposti esclude la possibilità di relazioni semiche e sememiche per elementi lessicali in forma composta che non individuino ulteriori elementi lessicali ritenuti significativi.

\subsection{2. Relazioni partonomiche pure}

Khoo e Na e Stock hanno sviluppato interessanti tassonomie delle relazioni partonomiche o partitive: Khoo e Na includono nelle relazioni partitive quelle che esistono tra gli organi di un corpo, tra luoghi geografici, tra articolazioni di un ente o di una disciplina (che invece altri autori fanno rientrare tra le relazioni generiche: questo caso è rappresentativo di come non sia sempre facilissimo distinguere tra relazioni generiche e partitive); le relazioni partitive vengono divise in quattro categorie: componenti funzionali di un intero, segmenti di un intero, membri di un insieme di elementi, sottoinsiemi. Stock definisce una tassonomia delle relazioni partonomiche su due livelli: un primo livello in cui vengono differenziate relazioni partitive tra "oggetti strutturati" e "non strutturati"; le relazioni partitive tra oggetti strutturati vengono a loro volta suddivise in relazioni tra parte di un oggetto strutturato e oggetto strutturato nella sua interezza, tra unità e sub-unità geografica, tra elemento e raccolta, tra unità e 
Tesi di dottorato di Giuliano Genetasio, discussa presso l'Università degli Studi di Udine

organizzazione, tra componente e insieme, tra segmento di un evento ed evento nel suo insieme; le relazioni partonomiche tra oggetti non strutturati vengono a loro volta suddivise in relazioni tra porzione e intero, tra azione e fase, tra oggetto e materiale, tra unità e sub-unità dimensionale. ${ }^{307}$ Poiché le relazioni partonomiche non godono delle stesse proprietà logiche delle relazioni generiche, una parte significativa degli standard di indicizzazione considera le relazioni partitive tra quelle associative, e non gerarchiche. ${ }^{308}$ Per quanto riguarda il nostro modello di lingua documentaria, segnaliamo che all'interno della nostra sottolingua: 1) le relazioni partitive esistono sia come relazioni paradigmatiche (pure e come specificazioni di relazioni eponimiche e semiche/sememiche) sia come relazioni sintagmatiche; ma, a differenza del linguaggio naturale, in cui una stessa relazione può assumere una forma paradigmatica o sintagmatica, nella sottolingua proposta vi è - anche sulla base dell'esigenza di riduzione della ridondanza linguistica - una esclusione reciproca tra i due tipi di relazione: le relazioni (paradigmatiche) partitive pure riguardano elementi lessicali che presentano forme semplici o forme composte che non individuino ulteriori elementi lessicali significativi, per esempio "Sicilia" o a "Reggio Calabria" in relazione a "Italia"; le relazioni sintagmatiche partitive riguardano elementi lessicali che presentino forme composte che facciano riferimento a ulteriori elementi lessicali significativi, cioè parzialmente o totalmente collegate (che a loro volta saranno correlate sul piano paradigmatico con le relazioni eponimiche e semiche/sememiche partitive), per esempio "Golfo di Napoli" (in cui vi è un riferimento all'elemento lessicale significativo "Napoli", mentre "Golfo" è un elemento lessicale non significativo) o "Motori di automobili" (in cui sia "Motori" sia "Automobili" sono elementi lessicali significativi); 2) le relazioni partonomiche sono considerate relazioni gerarchiche, e non associative: il tipo di rapporto tra l'intero e la parte implica un tipo di rapporto intrinsecamente gerarchico; piuttosto, occorre riconoscere che si tratta di un tipo di gerarchia diversa rispetto alle relazioni generiche; 3 ) sulla base della suddetta differenza tra elementi lessicali che fanno o non fanno riferimento a ulteriori elementi lessicali, accanto alle relazioni partonomiche pure (o non specificate), vi sono le relazioni eponimiche e semiche/sememiche di tipo partitivo, di cui parleremo nel prossimo paragrafo. Alcune tipologie di entità concettuali non possono intrattenere relazioni di tipo partitivo (Persone); altre tipologie di entità concettuali possono intrattenere esclusivamente relazioni partitive pure; nella nostra sottolingua: Testi, Risorse, Eventi e Tempi.

\subsection{2.4. Relazioni eponimiche, semiche e sememiche specificate}

Abbiamo già iniziato la discussione delle relazioni gerarchiche eponimiche e semiche/sememiche specificate quando abbiamo parlato della poligerarchia (vedi 3.3.6.4.2.2.2.1) - relazioni gerarchiche specificate e struttura poligerarchica vanno di pari passo.

All'interno della sottolingua la differenza tra relazioni eponimiche pure e relazioni

307 Cfr. CRISTOPHER S. G. KHOO - JIN-CHEON NA, Semantic relations in information science, cit., p. 176-177; cfr. anche WOLFGANG G. STOCK, Concepts and semantic relations in information science, cit., p. 1957.

308 Cfr. ELAINE SVENONIUS, The intellectual foundation of information organization, cit., p. 165. 
eponimiche (e semiche/sememiche) specificate non è semplicemente la differenza tra relazioni eponimiche il cui criterio è o meno stato specificato, ma è una differenza legata all'individuazione o meno, nell'iponimo (o nel semema), di un elemento componente significativo in più rispetto all'iperonimo (o al sema). Le relazioni eponimiche e semiche/ sememiche specificate sono cioè quelle in cui l'iponimo o il semema è riconducibile alla combinazione dell'iperonimo o del sema (che può presentare un unico elemento componente significativo o più elementi componenti significativi) con un ulteriore elemento lessicale significativo. Sono esempi di relazioni eponimiche specificate "Sport" e "Sport in Italia" oppure "Motori" e "Motori di automobili"; sono esempi di relazioni semiche specificate "Italia" e "Sport in Italia" oppure "Automobili" e "Motori di automobili”. Le relazioni eponimiche e semiche/sememiche specificate riguardano dunque le relazioni: 1) tra un primo elemento lessicale che presenti un unico elemento componente significativo e un secondo elemento lessicale che presenti forme composte con due o più elementi componenti significativi e di cui uno corrisponda al primo elemento lessicale, p.e. "Sport" e "Sport per anziani" (se "Anziani" è ritenuto significativo); 2) tra un primo elemento lessicale con forme composte di due o più elementi componenti tutti significativi e un secondo elemento lessicale che specifica il primo tramite l'aggiunta di un ulteriore elemento componente significativo, p.e. "Educazione delle donne" ed "Educazione delle donne in Italia" (se "Italia" è ritenuto significativo). Alle relazioni eponimiche specificate corrispondono relazioni semiche e sememiche speculari e inverse, in modo tale che, per esempio, "Educazione delle donne" sia in relazione sememica specificata rispetto a "Donne", che ne costituisce il sema non generico. Per quanto riguarda le relazioni semiche/sememiche specificate, va aggiunto un terzo tipo di relazioni possibili tra elementi lessicali alle due sopra menzionate: 3) le relazioni tra un primo elemento lessicale con uno o più elementi componenti significativi, e un secondo elemento lessicale che presenti gli stessi elementi componenti significativi più un ulteriore elemento componente non significativo: è il caso delle relazioni semiche/sememiche partitive tra enti sovraordinati (per esempio "ENI") ed enti subordinati il cui nome includa quello dell'ente sovraordinato (per esempio "Biblioteca dell'ENI"; vedi più sotto per una discussione delle relazioni eponimiche e semiche/sememiche partitive), e delle relazioni semiche/sememiche locative o temporali tra entità concettuali di un certo tipo (generalmente Luoghi e Tempi) ed entità individuali di vario tipo (Oggetti, Luoghi, Eventi, Enti il cui nome includa quello dei Luoghi e Tempi) dall'altro, per esempio "Palermo" e "Cattedrale di Palermo" o “2008-2009” e "Crisi del 2008-2009”.

Finora abbiamo parlato semplicemente di relazioni eponimiche e semiche/sememiche specificate, ma senza approfondire il criterio di tale specificazione. Questo può teoricamente essere dei più vari. Quello particolare scelto per la nostra sottolingua è basato su una corrispondenza con le relazioni semantiche sintagmatiche della sottolingua, e in particolare con le relazioni sintagmatiche grammaticali: in tal senso potremmo definire le relazioni gerarchiche (eponimiche e semiche/sememiche) come la "semantica casuale" paradigmatica della sottolingua; ${ }^{309}$ avremo così relazioni eponimiche e semiche/sememiche specificate: agentive, oggettive, strumentali, causative, benefattive, funzionali, locative e temporali, originarie, direttive, partitive, possessive e atipiche (vedi sotto per una lista più dettagliata). Come le altre relazioni paradigmatiche, anche le

309 Cfr. TERESA GRIMALDI, L'indicizzazione dal punto di vista cognitivo (II), cit., p. 267-268. 
relazioni gerarchiche specificate possono comparire, nell'ambito della sottolingua proposta, esclusivamente sull'asse paradigmatico; per lo stesso motivo, escludiamo all'interno della sottolingua la possibilità di elementi componenti di elementi lessicali in forma composta che siano tra loro legati da relazioni di iperonimia/iponimia e semiche/sememiche specificate (ma non co-iponimi e co-sememi specificati, che possono invece essere presenti all'interno di uno stesso elemento lessicale). Non tutte le lingue documentarie attuali prevedono la possibilità di relazioni gerarchiche specificate: spesso le relazioni gerarchiche specificate sono presenti ma non distinte da quelle pure (così nelle LCSH). Un meccanismo simile alle relazioni gerarchiche specificate, se non identico nella sostanza, e già attualmente esistente nelle lingue documentarie, è quello delle etichette di nodo, elementi meta-lessicali che specificano il criterio di suddivisione delle relazioni gerarchiche eponimiche. ${ }^{310}$ Discuteremo soltanto più avanti (3.3.6.4.3) le differenti relazioni sintagmatiche grammaticali che stanno alla base delle differenti tipologie di relazioni gerarchiche specificate. Tuttavia, forniamo già qui una lista dettagliata delle relazioni gerarchiche specificate, sia eponimiche sia semiche/sememiche; come è evidente dalla lista, relazioni eponimiche e semiche/sememiche specificate risultano chiaramente invertite, in modo che, per esempio, a una relazione iperonimica deagentiva, in cui l'iperonimo esprime l'attività svolta dall'agente dell'iponimo, p.e. "Terrorismo", corrisponda una relazione iponimica agentiva, in cui l'iponimo specifica l'iperonimo tramite l'aggiunta di un agente, p.e. "Terrorismo di stato"; e che viceversa a una relazione semica agentiva, in cui il sema (non generico) esprime l'agente del semema, p.e. "Stato", corrisponda una relazione sememica deagentiva, in cui il semema si differenzia dal sema tramite l'aggiunta di un'attività svolta dall'agente, p.e. "Terrorismo di stato" (fig. B1, B2, D).

Le relazioni gerarchiche eponimiche specificate includono:

- relazione iperonimica deagentiva, relazione iponimica agentiva

- relazione iperonimica deoggettiva, relazione iponimica oggettiva

- relazione iperonimica destrumentale, relazione iponimica strumentale

- relazione iperonimica debenefattiva, relazione iponimica benefattiva

- relazione iperonimica decausativa, relazione iponimica causativa

- relazione iperonimica defunzionale, relazione iponimica funzionale

- relazione iperonimica delocativa, relazione iponimica locativa

- relazione iperonimica deoriginaria, relazione iponimica originaria

- relazione iperonimica dedirettiva, relazione iponimica direttiva

- relazione iperonimica detemporale, relazione iponimica temporale

- relazione iperonimica depossessiva, relazione iponimica possessiva

- relazione iperonimica meronimica, relazione iponimica olonimica

- relazione iperonimica atipica, relazione iponimica atipica. ${ }^{311}$

Viceversa, le relazioni gerarchiche semiche/sememiche specificate includono:

310 Cfr. NATIONAL INFORMATION STANDARDS ORGANIZATION, Guidelines for the construction, format, and management of monolingual controlled thesauri, cit., p. 51.

311 Più precisamente dovremmo parlare di relazioni iperonimiche iperonimiche atipiche, di relazioni iponimiche iponimiche atipiche, e, per quanto riguarda le relazioni semiche/sememiche, di relazioni semiche iponimiche atipiche e relazioni sememiche iperonimiche atipiche, ma per semplificare parleremo semplicemente di relazioni iperonimiche atipiche, relazioni iponimiche atipiche, relazioni semiche atipiche e relazioni sememiche atipiche. 
Tesi di dottorato di Giuliano Genetasio, discussa presso l'Università degli Studi di Udine

- relazione semica agentiva, relazione sememica deagentiva

- relazione semica oggettiva, relazione sememica deoggettiva

- relazione semica strumentale, relazione sememica destrumentale

- relazione semica benefattiva, relazione sememica debenefattiva

- relazione semica causativa, relazione sememica decausativa

- relazione semica funzionale, relazione sememica defunzionale

- relazione semica locativa, relazione sememica delocativa

- relazione semica originaria, relazione sememica deoriginaria

- relazione semica direttiva, relazione sememica dedirettiva

- relazione semica temporale, relazione sememica detemporale

- relazione semica possessiva, relazione sememica dedepossessiva

- relazione semica olonimica, relazione sememica meronimica

- relazione semica atipica, relazione sememica atipica.

Riteniamo necessaria una precisazione; l'implementazione delle relazioni gerarchiche specificate in base alle relazioni sintagmatiche è a nostro avviso un'utile specificazione delle relazioni eponimiche e semiche/sememiche; quelle lingue documentarie che vogliano accogliere le relazioni gerarchiche specificate in base alle relazioni sintagmatiche grammaticali potranno, naturalmente, farlo anche soltanto in modo progressivo o parziale. La rappresentazione di alcune tipologie di relazioni gerarchiche specificate in base alle relazioni sintagmatiche è tuttavia particolarmente importante: per esempio le relazioni eponimiche e semiche/sememiche benefattive, funzionali e strumentali, le relazioni eponimiche e semiche/sememiche partitive; un altro importante tipo di relazione semica e sememica è quella - spesso trattata dalle lingue documentarie come relazione associativa - che lega un'attività o una disciplina (caso deoggettivo) al suo oggetto (caso oggettivo) tipico: per esempio la relazione tra "ornitologia" e "uccelli" o tra "biblioteconomia" e "biblioteche".

Un gruppo di relazioni che riteniamo utile approfondire è quello delle relazioni eponimiche e semiche/sememiche partitive. In linguistica si parla semplicemente di relazioni di eponimia e partitive. Tuttavia, già nel linguaggio naturale è possibile specificare le relazioni di eponimia (e le corrispondenti relazioni semiche e sememiche) attraverso vari criteri, tra cui quello partitivo. Facciamo un esempio. Se analizziamo l'elemento lessicale in forma composta "Motori di automobili" dal punto di vista delle relazioni paradigmatiche, ${ }^{312}$ vediamo che esso è meronimo (puro, se volessimo fermarci a questo livello di analisi) di "Automobili"; la relazione tra "Motori di automobili" e "Motori", viceversa, è a livello paradigmatico una relazione iponimica (pura, se volessimo fermarci a questo livello di analisi: i "motori di automobili" come tipi di motori), perché i motori automobilistici sono un tipo di motore. Potremmo fermarci a questo tipo di analisi, e sarebbe sostanzialmente corretta benché asimmetrica - da una parte una relazione partitiva, dall'altra una relazione generica (fig. E1); oppure potremmo spingerci oltre e dire che tra "Motori di automobili" e "Automobili" vi è una relazione sememica meronimica, tra "Automobili" e "Motori di automobili" una relazione semica olonimica; tra "Motori di automobili" e "Motori" una relazione iponimica olonimica e tra "Motori" e "Motori di automobili" una relazione iperonimica meronimica (fig. E2). ${ }^{313}$

312 Dal punto di vista sintagmatico la relazione espressa dal "di” è una relazione sintagmatica partitiva. 313 In effetti, e a differenza delle altre relazioni gerarchiche specificate, le relazioni gerarchiche eponimiche 
Le relazioni eponimiche partitive della nostra sottolingua sono quelle che riguardano la relazione tra una classe che costituisce una parte e un individuo che costituisce l'intero; le relazioni semiche e sememiche partitive della nostra sottolingua sono quelle che riguardano la relazione tra un sema che costituisce l'intero e un semema che costituisca la parte; in questi casi, individui e sememi saranno elementi lessicali in forma composta e totalmente collegata, per gli elementi lessicali relativi a entità collettive; elementi lessicali in forma composta e parzialmente collegata, per elementi lessicali relativi a entità individuali. Per fare un esempio: l'elemento lessicale in forma parzialmente collegata "[meronimico:] Centro storico - [olonimico:] Palermo" (cioè "Centro storico di Palermo"), dove "Centro storico" è un elemento lessicale non controllato, è in relazione sememica meronimica rispetto a "Palermo" e relazione iponimica olonimica rispetto a "Centri storici" (ma in relazione iponimica pura rispetto, per esempio a "[delocativo:] Centri storici - [locativo:] Sicilia", ovvero "Centri storici in Sicilia"), etc. Infine, ricordiamo che le relazioni eponimiche e semiche/sememiche partitive sono correlate alle relazioni sintagmatiche partitive che legano i due elementi lessicali componenti della forma sintagmatica (parzialmente o totalmente) collegata di un elemento lessicale. Un altro gruppo di relazioni di cui occorre parlare è quello delle relazioni gerarchiche (eponimiche e semiche/sememiche) atipiche, chiamate relazioni di "classe quasi generica" da GRIS. ${ }^{314}$ Più sopra, nella discussione della ricezione della coppia paradigmatico e sintagmatico del linguaggio all'interno della teoria dell'indicizzazione (vedi 3.3.5), abbiamo fatto riferimento alle nozioni di a priori (l'insieme delle relazioni semantiche comunemente accettate o analitiche) e a posteriori (l'insieme delle relazioni semantiche diverse da quelle comunemente accettate o sintetiche). A tale differenza corrisponde quella tra relazioni eponimiche a priori, comunemente accettate, e relazioni eponimiche a posteriori, o atipiche, legate al contesto. Come abbiamo accennato più sopra (vedi 3.2.5.3.3), la relazione di eponimia che vige tra una classe e i suoi membri non è una relazione di tipo ontologico o intrinseco, bensì contingente e classificatorio; inoltre la stessa percezione di quali elementi lessicali siano membri di una classe e quali no è un dato eminentemente culturale e contingente. Già nel linguaggio naturale, le relazioni eponimiche a priori comprendono quelle comunemente accettate in una data cultura al punto da essere scontate; altre relazioni eponimiche invece indicano individui non tipici di determinate classi, ovvero considerano un elemento lessicale secondo uno specifica prospettiva, funzione o punto di vista atipici in un determinato contesto culturale: sono relazioni eponimiche che definiremo atipiche (o a posteriori). Esse non godono

e semiche/sememiche partitive (e anche possessive, tra l'altro) non sono autentiche sottotipologie delle relazioni eponimiche e semiche/sememiche, quanto piuttosto ulteriori relazioni paradigmatiche che si sovrappongono a quelle eponimiche e semiche/sememiche: per ritornare all'esempio già fatto, in effetti le "Automobili" non sarebbero, rispetto ai "Motori di automobili", in relazione semica olonimica, ma piuttosto sia in relazione semica sia in relazione olonimica. Ciò perché, come vedremo quando discuteremo la semantica sintagmatica, le relazioni partitive (e possessive) non sono veri casi profondi ma piuttosto relazioni logiche. Pur lasciando aperta la possibilità, per tutti questi casi (molti, almeno tutti quelli che riguardano relazioni partitive tra entità collettive) di prevedere una doppia relazione paradigmatica, cioè di registrare tanto la relazione partitiva quanto quella eponimica o semica/sememica, cosa che sarebbe più corretta ma che appesantirebbe la struttura della lingua documentaria, per il nostro modello di sottolingua considereremo - si tratta di una semplificazione - le relazioni partitive di questo tipo come sottotipologie o criteri di suddivisione delle relazioni eponimiche e semiche/sememiche.

314 ASSOCIAZIONE ITALIANA BIBLIOTECHE. GRUPPO DI RICERCA SULL'INDICIZZAZIONE PER SOGGETTO, Guida all'indicizzazione per soggetto, cit., p. 44. 
ovviamente delle stesse proprietà logiche delle relazioni eponimiche a priori. ${ }^{315}$ Facciamo un esempio. In un contesto linguistico generico, i criceti sono considerati generalmente come roditori (relazione eponimica a priori), ma possono essere considerati anche come animali da laboratorio, ovvero cavie (relazione eponimica a posteriori); in contesti di sperimentazione medica, farmacologica, etc., tuttavia, i criceti sono considerati innanzitutto come cavie, e solo secondariamente, in quanto roditori; quindi in quel tipo di contesto la relazione semantica tra criceti e animali da laboratorio è una relazione eponimica a priori. Nell'ambito della sottolingua, le relazioni che abbiamo definito eponimiche a priori altro non sono che le relazioni eponimiche pure (e in teoria anche tutte le altre relazioni eponimiche specificate, ma trascureremo questo aspetto); le relazioni eponimiche a posteriori sono le relazioni che abbiamo definito come relazioni eponimiche atipiche. Inoltre, accanto a relazioni eponimiche atipiche vi saranno corrispettive relazioni semiche e sememiche atipiche. Così come per gli altri tipi di relazioni gerarchiche specificate, le relazioni eponimiche e semiche/sememiche atipiche hanno una precisa corrispondenza a livello sintagmatico: in particolare, con le relazioni sintagmatiche eponimiche atipiche.

Delle relazioni sintagmatiche eponimiche atipiche parleremo in modo più approfondito soltanto più avanti (vedi 3.3.6.4.3.3), ma facciamo già qui una precisazione. In virtù della necessità di creare un contesto artificiale che caratterizza le lingue documentarie e che determina la lessicalizzazione delle relazioni semantiche, gli elementi lessicali della sottolingua che rappresentano una relazione sintagmatica eponimica atipica: 1) non hanno mai una forma semplice (un'unica parola) ma soltanto forme composte (collegate e non collegate), perché non sarebbe altrimenti possibile evidenziare la relazione gerarchica atipica; 2) le forme non collegate di tali elementi lessicali presentano una forma certamente artificiosa ma inevitabile - del tipo "criceti in quanto animali da laboratorio" o "criceti come animali da laboratorio" etc., esplicitando cioè a livello sintagmatico la relazione paradigmatica; ${ }^{316} 3$ ) non esistono forme parzialmente collegate, ma soltanto totalmente collegate, degli elementi lessicali che rappresentano relazioni sintagmatiche eponimiche atipiche. La differenza tra relazioni gerarchiche (eponimiche e semiche/sememiche) pure e atipiche non è, lo ribadiamo, nemmeno nelle lingue documentarie una differenza assoluta; ciò che è considerato una relazione gerarchica atipica in una determinata lingua documentaria può non esserlo in un altra, e viceversa; così, all'interno di una lingua documentaria di ambito generalista, potremmo avere l'elemento lessicale "granchi" a indicare i granchi in generale o in quanto specie biologica, e l'elemento lessicale "granchi in quanto cibo", legato da una relazione iponimica atipica a "cibo" (fig. F1); viceversa, in un'ipotetica lingua documentaria di ambito gastronomico, non ci sarebbe bisogno di specificare "granchi in quanto cibo", e magari potrebbe esservi semplicemente una relazione eponimica pura tra "granchi" e "cibo" (fig. F2). Nelle lingue documentarie (sottolingue), le relazioni eponimiche (e semiche/sememiche) pure e atipiche costituiscono due architetture gerarchiche parallele, che veicolano significati diversi e che possono tuttavia convivere all'interno della stessa

315 ELAINE SVENONIUS, The intellectual foundation of information organization, cit., 2000, p. 151-152, 164. 316 Non si può, però, parlare nemmeno in questo caso di una stessa relazione semantica che possa presentarsi indifferentemente sul piano paradigmatico e su quello sintagmatico, poiché in effetti, in virtù dell'artificiosità suddetta, sul piano sintagmatico la relazione semantica è quella tra "animali da laboratorio" e "criceti", mentre sul piano sintagmatico la relazione è quella tra "animali da laboratorio" e "criceti in quanto animali da laboratorio". 
Tesi di dottorato di Giuliano Genetasio, discussa presso l'Università degli Studi di Udine

lingua; e ciò non solo per elementi lessicali differenti, ma - almeno in linea di principio - anche in riferimento a uno stesso elemento lessicale; per tornare all'esempio dei granchi, l'elemento lessicale "cibo" potrebbe essere legato da una relazione iperonimica pura a "granchi", e da una relazione iperonimica atipica a "granchi in quanto cibo"; "granchi", a sua volta, sarebbe legato da una relazione semica atipica a "granchi in quanto cibo" (fig. F3).

La possibilità della compresenza di relazioni gerarchiche tipiche e atipiche per uno stesso elemento lessicale, lungi dal costituire un mero esercizio teorico, potrebbe costituire la base per una gestione dinamica dei percorsi gerarchici degli elementi lessicali della sottolingua sulla base del contesto d'uso: l'assegnazione di specifici codici, relativi al contesto d'uso (cultura, disciplina, etc.), alle differenti tipologie di relazioni gerarchiche permetterebbe ai sistemi bibliotecari e agli utenti che utilizzano una determinata lingua documentaria di includere o escludere determinati percorsi gerarchici sulle base delle proprie esigenze.

\subsection{2.5. Relazioni co-iponimiche, co-meronimiche e co-sememiche}

Non abbiamo sinora accennato ai co-iponimi, co-meronimi e co-sememi, solo per esigenze di semplificazione. Così come nel linguaggio naturale, anche nella nostra sottolingua sono contemplati co-iponimi, co-meronimi e co-sememi, sia puri, sia, nel caso di co-iponimi e co-sememi, specificati.

Co-iponimi sono tutti gli antonimi; co-sememi tutti gli elementi lessicali appartenenti alla stessa "famiglia semantica" (biologia, biografia, etc.) e le diverse forme di nominalizzazione di un verbo ("educatori" ed "educazione", etc.).

All'interno del nostro modello di lingua documentaria, tuttavia, la rappresentazione delle relazioni co-iponimiche, co-sememiche e co-meronimiche resterà generalmente implicita; è possibile creare collegamenti fisici tra i co-iponimi, co-meronimi e co-sememi, ma riteniamo che di essi sia generalmente sufficiente una trattazione implicita tramite la comune segnalazione all'interno della registrazione del relativo elemento lessicale iperonimo, olonimo e semico e/o, soprattutto nel caso dei co-sememi, tramite la semplice prossimità nell'ordine alfabetico. La rappresentazione esplicita delle relazioni co-sememiche ritenute importanti può tuttavia essere prevista nel caso di co-sememi morfosintatticamente differenti (per esempio "Germania" e "Tedeschi"), in questo caso o tramite collegamenti (soluzione preferibile) o tramite elementi metalessicali (quali i cosiddetti "rinvii generici"). ${ }^{317}$ Una nota: a differenza della maggior parte degli elementi lessicali legati da altre relazioni paradigmatiche, due o più co-iponimi, co-sememi e co-meronimi possono essere rappresentati all'interno di uno stesso elemento lessicale in forma composta (collegata o non collegata); in questo caso, sono generalmente legati da una relazione sintagmatica di coordinazione (vedi 3.3.6.4.3.4).

\subsection{2.6. Relazioni tra nominalizzazioni}

317 NATIONAL INFORMATION STANDARDS ORGANIZATION, Guidelines for the construction, format, and management of monolingual controlled thesauri, cit., p. 26. 
L'unico tipo di relazioni co-sememiche che sia necessario rappresentare esplicitamente nella sottolingua è quello delle relazioni tra co-sememi che siano nominalizzazioni di uno stesso verbo e che appartengano a tipologie lessicali differenti; le nominalizzazioni, lo ricordiamo, sono elementi lessicali (sostantivi deverbali) relativi a entità che racchiudono in sé il concetto di un'attività, e sul piano sintagmatico si comportano almeno parzialmente come attività. Relazioni tra nominalizzazioni tipiche sono quelle tra un elemento lessicale che rappresenti l'attività ("educazione") e un altro che ne rappresenti l'agente tipico ("educatori"). Poiché in questi casi non sembra però opportuno registrare autonomamente il sema comune ai due elementi lessicali, e poiché dunque la relazione co-sememica in quanto tale rimane implicita anche nelle relazioni tra nominalizzazioni, non parleremo di relazioni co-sememiche tra nominalizzazioni ma semplicemente di relazioni tra nominalizzazioni. Tali relazioni, che nella teoria dell'indicizzazione tradizionale vengono spesso considerate come relazioni associative, ma che in realtà sono relazioni di natura gerarchica, costituiranno all'interno della sottolingua proposta una tipologia di relazioni semantiche gerarchiche a sé stanti. In virtù dell'esigenza di ridurre la ridondanza linguistica, nella nostra sottolingua non è prevista la possibilità di elementi lessicali in forma composta che includano, come elementi lessicali componenti, elementi tra loro in relazione di nominalizzazione.

\subsection{3. Relazioni associative}

Non accoglieremo le relazioni associative nel nostro modello di lingua documentaria. Tuttavia, riteniamo utile parlarne sia per mostrare la loro problematicità di definizione, sia per spiegare a cosa corrispondono le varie tipologie di relazioni associative nell'ambito del nostro modello di lingua documentaria.

Le relazioni definite come associative non hanno un corrispettivo diretto nell'ambito delle relazioni semantiche trattate normalmente in linguistica. La loro definizione come gruppo unitario di relazioni nell'ambito dell'indicizzazione è stata sempre difficile e problematica: la teoria dell'indicizzazione, benché vi abbia dedicato molti sforzi, non è ad oggi riuscita a definire le relazioni associative in modo soddisfacente e universalmente accettato; semmai, l'unico punto universalmente accettato della definizione delle relazioni associative è il riconoscimento della loro natura sfuggente, vaga e di tipo eterogeneo; molti autori riconoscono che sotto l'etichetta di relazioni associative vengono raccolte tipi di relazioni differenti. La maggior parte delle definizioni di relazione associativa vigente sono di tipo residuale, negativo e pragmatista: le relazioni associative vengono definite come quelle relazioni semantiche diverse dalle relazioni gerarchiche o di equivalenza e che si ritiene possa essere utile ricordare o segnalare all'utente. ${ }^{318}$

Tassonomie delle relazioni associative sono state offerte da diversi autori e testi: NISO2005, Delsey, Svenonius, Hutchins, Lancaster, Dahlberg, GRIS, per citarne solo alcuni. Le tipologie di relazioni associative più frequentemente elencate sono, in ordine sparso: relazioni di antonimia di vario tipo, relazioni tra attività o discipline e il loro

318 Cfr. W. JOHN HUTCHINS, Languages of indexing and classification, cit., p. 48; cfr. anche ELAINE SVENONIUS, The intellectual foundation of information organization, cit., p. 161; cfr. anche WOLFGANG G. STOCK, Concepts and semantic relations in information science, cit., p. 1963. 
oggetto tipico, relazioni tra attività o entità e agenti tipici, relazioni tra elementi lessicali appartenenti a catene gerarchiche differenti, relazioni corrispondenti ai vari ruoli semantici (agente, strumento, funzione, etc.), relazioni causa-effetto, relazioni di influenza, relazioni partitive, relazioni tra elementi lessicali di significato fortemente sovrapposto, tra elementi lessicali coordinati all'interno di una stessa gerarchia, relazioni tra elementi lessicali che nel linguaggio naturale sono sinonimi ma che nella lingua documentaria vengono utilizzati per indicare significati diversi, e molte altre. ${ }^{319}$ Guardando con attenzione questa lista vediamo che una parte significativa di "relazioni associative" sono già state trattate in altro modo all'interno della nostra sottolingua: relazioni partitive, relazioni tra elementi lessicali coordinati all'interno di una stessa gerarchia (co-iponimi), relazioni tra attività o discipline e il loro oggetto tipico e tra elementi lessicali appartenenti a catene gerarchiche differenti (relazioni semiche, sememiche e co-sememiche), relazioni tra elementi lessicali di significato fortemente sovrapposto. Altre corrispondono a relazioni semantiche che tratteremo più sotto: così le relazioni di antonimia, i ruoli semantici, tra cui includiamo le relazioni causa-effetto (relazioni sintagmatiche causative, ma intese in senso diverso rispetto a quanto avviene normalmente nelle lingue documentarie, vedi 3.3.6.4.3.3), relazioni tra attività o entità e agenti tipici (particolari relazioni co-sememiche che riguardano nominalizzazioni). Restano invece fuori dal nostro modello altri tipi di relazione associativa come la relazione di influenza, non riconducibile a quelle studiate dalla linguistica e trattate nell'ambito del nostro modello semplicemente come normali elementi lessicali. Nel nostro modello di sottolingua non prevediamo nemmeno la possibilità di relazioni tra elementi lessicali che nella lingua naturale sono sinonimi, ma che nelle lingue documentarie vengono considerate come elementi lessicali di significato differente, e il cui uso è differenziato sulla base di specifiche note. Riteniamo che la pratica di separare nella lingua documentaria elementi lessicali sinonimi per ragioni legate all'indicizzazione, segnalandone il legame tramite note, sia un artificio ingiustificato, che allontana la semantica della lingua documentaria da quella della lingua naturale e che proprio perciò rischia di essere ignorato o frainteso dall'utente finale: essa va abbandonata, trattando le relazioni semantiche tra gli elementi lessicali per quello che sono (sinonimi, antonimi, etc.).

\subsection{4. Relazioni di antonimia}

Abbiamo già introdotto le relazioni di antonimia nella sezione precedente: possiamo distinguere antonimia scalare, polare e complementare (vedi 3.2.5.3.3).

La trattazione degli antonimi all'interno delle lingue documentarie è controversa: alcune lingue documentarie li trattano come relazioni di equivalenza, cioè come relazioni simili

319 Cfr. NATIONAL INFORMATION STANDARDS ORGANIZATION, Guidelines for the construction, format, and management of monolingual controlled thesauri, cit., p. 51-56; TOM DELSEY, Modeling subject access, cit., p. 56-58; FREDERICK WILFRID LANCASTER, Vocabulary control for information retrieval, 2nd ed., Arlington, Va., Information Resources Press, 1986, p. 46-47; ELAINE SVENONIUS, The intellectual foundation of information organization, cit., p. 162; ASSOCIAZIONE ITALIANA BIBLIOTECHE. GRUPPO DI RICERCA SULL'INDICIZZAZIONE PER SOGGETTO, Guida all'indicizzazione per soggetto, cit., p. 89-90; INGETRAUT DAHLBERG, A referent-oriented, analytical concept theory of INTERCONCEPT, cit., p. 148. 
alla sinonimia; altri preferiscono distinguerli e considerarli come relazioni (associative) a sé stanti, come per esempio Svenonius, sulla base del fatto che sono relazioni linguistiche di tipo differente. ${ }^{320}$ Hutchins ha proposto di trattare l'antonimia scalare e polare come relazione di equivalenza, l'antonimia complementare come relazione associativa, sulla base dell'idea secondo cui mentre potrebbero esistere utenti finali interessati a un elemento lessicale complementare senza essere interessati all'altro, è verosimile che un utente interessato a un antonimo scalare sia interessato anche agli altri. ${ }^{321}$

Per la nostra sottolingua accoglieremo le tre relazioni di antonimia suddette (relazione di antonimia scalare o di scalarità, relazione di antonimia polare o polarità, relazione di antonimia complementare o complementarietà); accoglieremo anche, ma solo parzialmente, la proposta di Hutchins: nella sottolingua non solo la relazione di antonimia complementare, ma anche quella di antonimia polare sarà distinta e a sé stante rispetto alle relazioni di equivalenza; lasciamo invece, sulla scorta dell'argomento di Hutchins, aperta l'opzione di trattare gli antonimi scalari tramite una specifica relazione di antonimia scalare oppure come collegati da una relazione di equivalenza (trattati cioè come sinonimi).

Riferendoci alle entità del modello concettuale, dobbiamo dire innanzitutto che le relazioni di antonimia riguardano esclusivamente entità concettuali dello stesso tipo ed esclusivamente entità collettive. L'antonimia scalare e polare può riguardare in linea di massima soltanto Concetti. Più ampio il ventaglio di entità concettuali che possono essere coinvolte da relazioni di antonimia complementare: Tipi di persona ("compratori" e "venditori", etc.), Tipi di ente, Concetti, Altre entità (tipi di evento, tipi di oggetto, etc.). Infine, ribadiamo alcune cose già dette: 1 ) all'interno della sottolingua proposta le relazioni di antonimia (scalare, polare e complementare) sono esclusivamente paradigmatiche; 2) gli antonimi sono anche co-iponimi, benché non sia opportuno registrare esplicitamente anche la relazione di co-iponimia.

Osserviamo anche che la compresenza, all'interno di una forma lessicale composta, di due elementi lessicali componenti che siano antonimi è da escludere, per ragioni legate alla riduzione della ridondanza del linguaggio naturale, tranne nel caso di co-iponimi complementari, che possono essere spesso i due termini di una relazione di interazione (vedi 3.3.6.4.3.4).

\subsection{5. Relazioni di equivalenza}

Le relazioni di equivalenza sono relazioni tipiche dell'indicizzazione, ma che hanno una corrispondenza linguistica con quelle relazioni linguistiche in cui sono in rapporto tra loro elementi lessicali differenti morfosintatticamente ma di significato identico o molto simile: sinonimia, parafrasi, allotassia, etc., a cui si aggiungono altre relazioni non previste esplicitamente dalla linguistica ma concettualmente simili: relazioni tra diversi nomi e forme del nome di una persona o titoli di un testo, varianti ortografiche o morfologiche di uno stesso semema, etc. Le relazioni di equivalenza godono delle proprietà logiche di simmetria, riflessività e transitività. ${ }^{322}$

320 ELAINE SVENONIUS, The intellectual foundation of information organization, cit., p. 159.

321 W. JOHN HUTCHINS, Languages of indexing and classification, cit., p. 42.

322 ELAINE SVENONIUS, The intellectual foundation of information organization, cit., p. 156. 
Tesi di dottorato di Giuliano Genetasio, discussa presso l'Università degli Studi di Udine

Prima di passare alla specifica trattazione delle relazioni di equivalenza all'interno del nostro modello di lingua documentaria, passiamo in rassegna la trattazione delle relazioni di equivalenza all'interno della teoria dell'indicizzazione tradizionale. NISO2005 elenca tra le relazioni di equivalenza sinonimi, varianti lessicali, quasi-sinonimi (e antonimi), "collocazioni generiche" ("generic posting") e rinvii da elementi lessicali in forma composta ai singoli elementi lessicali componenti in forma semplice. ${ }^{323}$ NISO2005 include indirettamente tra le relazioni di equivalenza anche le traduzioni, per quelle lingue documentarie che prevedano la possibilità di includere elementi lessicali in lingue naturali diverse da quella su cui è basata la lingua documentaria, nel caso in cui si ritenga possano essere utili per gli utenti finali. ${ }^{324}$ Tra i sinonimi vengono inclusi e distinti elementi lessicali di origine linguistica differente, versioni popolari e scientifiche di un elemento lessicale, nomi generici e commerciali di un termine, neologismi, termini gergali, varianti dialettali. Tra le varianti lessicali vengono incluse le varianti ortografiche e morfologiche di un elemento lessicale, ma anche abbreviazioni e acronimi. I quasisinonimi, come spiega NISO2005, sono elementi lessicali semanticamente vicini ma non sinonimi, che però vengono considerati tali nella lingua documentaria a causa delle esigenze dell'indicizzazione (funzione Trovare del catalogo, garanzia bibliografica, etc.); ${ }^{325}$ NISO2005 accetta i quasi-sinonimi tra le relazioni di equivalenza, includendovi anche gli antonimi non trattati come relazioni associative, limitandone tuttavia l'applicazione alle "fringe areas", cioè alle aree semanticamente marginali del lessico della lingua documentaria (la considerazione vale ovviamente solo per una sottolingua relativa a un ambito disciplinare o di soggetto specifico).

Oltre a questi tipi di relazioni di equivalenza, NISO2005 ne include altri due, spesso non prese in considerazione ma che caratterizzano una parte significativa delle lingue documentarie per soggetto (thesauri e soggettari). La collocazione generica è una sorta di quasi-sinonimia espansa fino a comprendere la trattazione di una classe e di uno dei suoi individui - spesso quello più rappresentativo ovvero che gode della maggiore garanzia bibliografica (p.e. nelle LCSH la voce di soggetto "Encyclopedias and dictionaries" è trattata come sinonimo di "Encyclopedias and dictionaries, English") - come sinonimi, e non come legati da una relazione gerarchica eponimica; si tratta, in altri termini, di relazioni gerarchiche eponimiche trattate come relazioni di equivalenza. Infine, per quanto riguarda i rinvii da elementi lessicali in forma sintagmatica ai suoi singoli elementi lessicali componenti, si tratta di una relazione di equivalenza tipica di quelle lingue documentarie che preferiscono rinunciare alla coordinazione, cioè alla combinazione di più elementi lessicali all'interno di un unico elemento lessicale in forma composta, e

323 NATIONAL INFORMATION STANDARDS ORGANIZATION, Guidelines for the construction, format, and management of monolingual controlled thesauri, cit., p. 45-46; cfr. anche MARGARET WILLETTS, An investigation of the nature of the relation between terms in thesauri, "Journal of documentation", 31, 3 (1975), p. 160.

324 NATIONAL INFORMATION STANDARDS ORGANIZATION, Guidelines for the construction, format, and management of monolingual controlled thesauri, cit., p. 32-33.

325 NATIONAL INFORMATION STANDARDS ORGANIZATION, Guidelines for the construction, format, and management of monolingual controlled thesauri, cit., p. 7; cfr. anche W. JOHN HUTCHINS, Languages of indexing and classification, cit., p. 41; cfr. anche ELENA FRANCHINI, 025.47. Tesauri, in Biblioteconomia. Guida classificata, diretta da Mauro Guerrini, condirettore Gianfranco Crupi, a cura di Stefano Gambari, collaborazione di Vincenzo Fugaldi, presentazione di Luigi Crocetti, Milano, Editrice Bibliografica, 2007, p. 646. 
utilizzare in suo luogo la coordinazione all'interno di una stessa registrazione bibliografica di un testo di più elementi lessicali in forma semplice.

Il tipo di relazione di equivalenza più tipico è senz'altro quella tra varianti semantiche, o sinonimia: di essa non esiste una tassonomia esaustiva o universalmente condivisa. Oltre a quella di NISO2005 riteniamo utile ricordare quella di Alan Gilchrist, riportata da Hutchins, che divide i sinonimi in nomi commerciali, varianti lessicali dalla differente origine linguistica, varianti lessicali cadute in disuso, varianti locali, varianti popolari, quella di Koo e Na, che elencano sinonimi veri e propri, quasi sinonimi e sinonimi parziali, e quella del GRIS. ${ }^{326}$

Passando al nostro modello di sottolingua, le relazioni di equivalenza costituiscono uno di quei punti in cui la sottolingua si discosta parzialmente dalle caratteristiche del linguaggio naturale e soprattutto dalle relazioni semantiche studiate solitamente in linguistica, almeno per quanto riguarda il piano formale della semantica. All'interno della sottolingua proposta, le relazioni di equivalenza della sottolingua costituscono una sorta di super-relazione che comprende tutte quelle relazioni semantiche tra elementi lessicali di significato identico o molto simile ma morfosintatticamente differenti: quindi sinonimie, parafrasi, allotassie, diverse grafie, etc., così come nel linguaggio naturale; ma anche, e a differenza del linguaggio naturale, e con la teoria dell'indicizzazione tradizionale, relazioni di tipo diverso. Vediamole più in dettaglio. Innanzitutto includiamo tra le relazioni di equivalenza quelle (concettualmente simili ai sinonimi) tra differenti nomi e forme del nome di una persona e titoli di un testo. Discostandoci da quanto avviene nel linguaggio naturale, e rifacendoci invece a una trattazione tipica di molte lingue documentarie, includiamo tra le relazioni di equivalenza quelle tra varianti morfologiche (quanto al caso di superficie, al numero, al genere, etc.) di un elemento lessicale - purché la differenza morfologica non individui anche una differenza di significato rilevante all'interno del particolare contesto di una lingua documentaria: in quel caso (p.e. quando sia importante distinguere il genere: femminismo ma non solo) è invece opportuno non trattare gli elementi lessicali come semanticamente equivalenti. Accettiamo senz'altro tra le relazioni di equivalenza le varianti grafiche di un elemento lessicale, così come abbreviazioni, acronimi, diverse forme del nome, etc. Inoltre, e a differenza del linguaggio naturale, lasciamo aperta l'opzione di trattare gli antonimi scalari come semanticamente equivalenti o meno. Rifiutiamo viceversa la possibilità dei quasi-sinonimi, cioè termini che non siano sinonimi nel linguaggio naturale, perché si tratta di un espediente fortemente contrario alle caratteristiche del linguaggio naturale. Rifiutiamo altresì la possibilità della collocazione generica, sia perché in contrasto con il principio di specificità, sia perché nettamente in contrasto con la semantica della lingua naturale, sia perché essa introduce forti pregiudizi culturali nella lingua documentaria: le LCSH, per esempio, che praticano spesso la collocazione generica, tendono a considerare generalmente sinonimi determinati elementi lessicali e loro corrispettivi americani (o inglesi se linguistici), bianchi, cristiani (e, ovviamente, maschili). Piuttosto, secondo il nostro modello di lingua documentaria le relazioni tra classi e membri, per quanto questi ultimi possano essere rappresentativi,

326 W. JOHN HUTCHINS, Languages of indexing and classification, cit., p. 38; cfr. anche ASSOCIAZIONE ITALIANA BIBLIOTECHE. GRUPPO DI RICERCA SULL'INDICIZZAZIONE PER SOGGETTO, Guida all'indicizzazione per soggetto, cit., p. 85; cfr. anche CRISTOPHER S. G. KHOO - JIN-CHEON NA, Semantic relations in information science, cit., p. 177-178. 
verranno considerate per quello che sono, cioè relazioni eponimiche, e non di equivalenza. Per quanto riguarda i rinvii da elementi lessicali in forma composta ai singoli elementi lessicali componenti, previsti da NISO2005 come possibile tipo di relazione di equivalenza, essi non saranno presi in considerazione nel nostro modello di lingua documentaria: noi escludiamo la possibilità di una post-coordinazione di più elementi lessicali per formare un elemento lessicale in forma composta e proponiamo invece di stabilire gli elementi lessicali in forma composta ogni qualvolta ciò sia possibile. Infine, tipologie fondamentali di relazioni di equivalenza della sottolingua proposta sono quelle tra elementi lessicali in forma semplice e composta (sintagmi), così come avviene nel linguaggio naturale.

Un'altra differenza fondamentale tra le relazioni di sinonimia, parafrasi, etc. tipiche del linguaggio naturale e le relazioni di equivalenza tipiche delle lingue documentarie, finora non menzionata esplicitamente, è che mentre nel linguaggio naturale le relazioni di sinonimia, etc., riguardano sempre relazioni tra differenti elementi lessicali, che abbiamo uno stesso semema o sememi molto simili, il processo di riduzione o eliminazione della biplanarità del lessico che caratterizza le lingue documentarie - che è una conseguenza inevitabile del perseguimento delle funzioni catalografiche - fa sì che il lessico delle lingue documentarie sia soprattutto un lessico dei sememi; ciò ha come conseguenza fondamentale che le relazioni di equivalenza sono relazioni tra diverse forme di un elemento lessicale piuttosto che tra differenti elementi lessicali; ciò si riflette, a livello di registrazione fisica, nel fatto che nelle lingue documentarie le relazioni eponimiche, associative, etc., siano relazioni semantiche tra elementi lessicali registrati separatamente, e che invece le relazioni di equivalenza siano relazioni interne alla registrazione di un unico elemento lessicale (nel precedente capitolo abbiamo espresso questa condizione dicendo che le relazioni di equivalenza sono relazioni che vigono tra gli attributi di una stessa entità, e non tra entità diverse; lo stesso avviene, abbiamo anche detto, per le relazioni tra temi estensionali; vedi 2.14). Così sarà anche per la sottolingua proposta, in cui le relazioni di equivalenza legheranno le diverse forme di un elemento lessicale (un "gruppo di equivalenza" secondo la terminologia GRIS). 327

Altri elementi peculiari delle relazioni di equivalenza della sottolingua in contrasto con il linguaggio naturale sono, come già per le altre relazioni paradigmatiche, quelle legate all'esigenza di ridurre la ridondanza linguistica: 1) le relazioni di equivalenza possono comparire esclusivamente sull'asse sintagmatico della sottolingua; 2) gli elementi lessicali legate (ovvero le diverse forme di uno stesso elemento lessicale legati) da relazioni di equivalenza non possono comparire all'interno di uno stesso elemento lessicale in forma sintagmatica.

Infine, ultima e più importante differenza tra la nostra sottolingua da un lato e il linguaggio naturale, ma anche tutte le altre lingue documentarie, dall'altro, è la possibilità, prevista in modo sistematico all'interno della sottolingua, di relazioni di equivalenza tra elementi lessicali in forma semplice (p.e. "bioetica") o composta non collegata (p.e. "Etica della vita" o "Biblioteca dell'ENI") da un lato ed elementi lessicali in forma composta parzialmente (p.e. "[meronimico:] Biblioteca [olonimico:] ENI") o totalmente collegata (p.e. "[deoggettivo:] Etica - [oggettivo:] Vita") dall'altro, di cui sia possibile navigare i cui singoli elementi lessicali componenti; per questi ultimi è inoltre

327 ASSOCIAZIONE ITALIANA BIBLIOTECHE. GRUPPO DI RICERCA SULL'INDICIZZAZIONE PER SOGGETTO, Guida all'indicizzazione per soggetto, cit., p. 84. 
prevista la creazione sistematica di allotassie (sequenze differenti ma semanticamente equivalenti degli elementi lessicali di un sintagma) artificiali in base al numero delle relazioni sintagmatiche dell'elemento lessicale in forma collegata (due sequenze per una relazione, sei sequenze per due relazioni, etc.), che può essere realizzata a livello di indicizzazione oppure soltanto di visualizzazione; questa creazione sistematica di allotassie, fondata sulle relazioni semantiche sintagmatiche tra gli elementi lessicali componenti il sintagma, ha una somiglianza concettuale con la nozione chomskyana delle trasformazioni linguistiche. ${ }^{328}$ Sulla base della differenza tra forme lessicali semplici e composte non collegate da un lato e collegate dall'altro parleremo allora di: relazioni di equivalenza non controllate (tra forme lessicali semplici o composte non collegate, p.e. tra "Bioetica" ed "Etica della vita"); relazioni di equivalenza monocontrollate (tra una forma lessicale collegata e una forma composta non collegata o semplice, p.e. tra "Etica della vita" e "[deoggettivo:] Etica - [oggettivo:] Vita"); relazioni di equivalenza bicontrollate (tra forme lessicali collegate, p.e. tra "[deoggettivo:] Etica - [oggettivo:] Vita" e "[oggettivo:] Vita [deoggettivo:] Etica”). Questi differenti tipi di relazione di equivalenza potrebbero essere ulteriormente specificati sulla base di altri criteri, per esempio lo specifico tipo di sinonimia (forme scientifiche e popolari di un elemento lessicale, forme sciolte e contratte, forme standard e dialettali, forme correnti o arcaiche, etc.); riteniamo che si tratti di specificazioni utili, benché non indispensabili.

Finora abbiamo lasciato in ombra un aspetto fondamentale: quello delle finalità delle relazioni di equivalenza. Sinonimia, allotassie, parafrasi, differenti nomi e titoli di un'entità, esistenza di forme semplici e composte di un elemento lessicale, e più in generale tutti i casi in cui due elementi differiscano morfosintatticamente ma non semanticamente, costituiscono elementi problematici per il soddisfacimento delle funzioni catalografiche. In questi casi l'uso, da parte della lingua documentaria, di espressioni differenti per indicare significati identici o molto simili tenderebbe a disperdere ciò che è relativo a un'entità in diversi punti del catalogo. Il controllo linguistico della sinonimia e dei fenomeni correlati facilita enormemente le funzioni del catalogo, e la funzione Trovare in particolare. Per quanto riguarda le funzioni svolte dalle relazioni di equivalenza monocontrollate e bicontrollate, vi abbiamo già fatto indirettamente riferimento quando abbiamo discusso le funzioni catalografiche degli elementi lessicali in forma collegata (vedi 3.3.6.2.5): sono le relazioni di equivalenza monocontrollate e bicontrollate a permettere di Trovare un elemento lessicale in forma semplice o composta non collegata per poi visualizzarne e Selezionarne le eventuali forme composte collegate, e dunque Navigarne uno degli elementi lessicali componenti, o viceversa partire da uno dei suoi elementi lessicali componenti per poi arrivare all'elemento lessicale nella sua interezza, in forma semplice o composta. ${ }^{329}$ In riferimento a una visualizzazione lineare degli elementi lessicali in forma composta, l'utente finale potrebbe cioè partire da un elemento lessicale "vita", per poi scorrerne le articolazioni

328 Cfr. NOAM CHOMSKY, La grammatica trasformazionale, cit., p. 49: "Avendo a disposizione strumenti trasformazionali, possiamo semplificare enormemente la grammatica limitando la generazione diretta a struttura sintagmatica a quel piccolo insieme di sequenze per le quali opera agevolmente e derivando tutte le altre frasi mediante trasformazione delle sequenze così derivate".

329 Cfr. ELAINE SVENONIUS, The intellectual foundation of information organization, cit., p. 190-191: "The syntax used in subject languages offers a means to organize large retrievals conceptually [...] The effect of assigning precoordinate subject headings to documents is to pack them, or rather their surrogates, into an orderly sequence of Chinese boxes”. 
concettuali dell'elemento lessicale fino a trovare elementi in forma composta di cui quel primo elemento lessicale è parte (“[oggettivo:] Vita - [deoggettivo:] Etica”), per poi ancora risalire alla forma semplice dell'elemento trovato ("Bioetica") e da lì continuare a navigare il catalogo in un processo di semiosi idealmente infinito.

Per concludere la trattazione delle relazioni di equivalenza, non possiamo non menzionare la questione, a esse tradizionalmente centrale, della scelta della forma preferita di un elemento lessicale. ${ }^{330} \mathrm{Nel}$ precedente capitolo (vedi 2.18 .2 e 2.18.5) abbiamo già spiegato come non sia più necessaria né auspicabile la scelta di una forma preferita (uniformità) a livello internazionale. Diversa è la questione per quanto riguarda la scelta di una forma preferita a livello locale, ovvero di singola lingua documentaria. Una minoranza di autori (Ayres e, in Italia, Paul Gabriele Weston) hanno proposto di abbandonare il principio di uniformità, rinunciando a stabilire una forma preferita sulle altre e scegliendo, come forma di un elemento lessicale da utilizzare quale indice nelle registrazioni di altre entità, la forma lessicale che di volta in volta compare all'interno del testo; ${ }^{331}$ il ruolo di raggruppare tutte le registrazioni relative a un certo elemento lessicale nelle sue varie forme - purché naturalmente si sia provveduto a collegare tra loro le diverse forme (semplici e composte, collegate e non collegate, etc.) dell'elemento lessicale - potrebbe essere secondo questa prospettiva lasciato agli automatismi. Nei sistemi bibliografici odierni, l'identificazione di un oggetto informativo (elemento lessicale, in termini linguistici; entità, in termini concettuali) è affidata innanzitutto a un elemento di natura non linguistica: l'identificatore della relativa registrazione; possiamo concepire tale identificatore come una forma simbolica (numerica o alfanumerica) dell'elemento lessicale, e più precisamente come una forma preferita naturale dell'elemento lessicale, rispetto alla quale tutte le forme verbali non costituiscono che forme varianti.

Spingendo oltre questa idea, e sulla scorta del suggerimento di Ayres, potremmo immaginare un sistema in cui tutte le forme verbali di un elemento lessicale costituiscano forme preferite: a livello di indicizzazione, potrebbe essere utilizzata una qualsiasi tra esse o direttamente l'identificatore; a livello di visualizzazione, l'utente potrebbe accedere direttamente alla registrazione completa dell'elemento lessicale a partire da qualsiasi forma semplice o composta, e ogni forma dell'elemento lessicale presenterebbe la totalità delle relazioni paradigmatiche con altri elementi lessicali e con altre forme dell'elemento lessicale; a quel punto, le relazioni di equivalenza diverrebbero almeno apparentemente identiche alle altre relazioni semantiche, collegando elementi lessicali diversi. La possibilità tecnica di questo sistema si fonderebbe sull'automazione, e in particolare sulla creazione automatica di "alias" o meccanismi simili per la registrazione di ogni elemento lessicale che presenti più forme. Per quanto suggestivo, questo sistema sembra idoneo soprattutto per la rappresentazione di entità che presentino poche (o nessuna) relazioni paradigmatiche oltre a quelle di equivalenza (come, forse, gli elementi lessicali relativi a Persone nell'ambito di una concezione tradizionale dell'indicizzazione, cioè senza relazioni paradigmatiche con Tipi di persona, con Testi, etc.), e non per elementi lessicali inseriti all'interno di una complessa rete sindetica, quale quella da noi proposta; l'applicazione coerente di questo sistema richiederebbe infatti la condizione di segnalare, all'interno della registrazione di un elemento lessicale, la totalità delle forme di tutti gli ulteriori

330 Cfr. NATIONAL INFORMATION STANDARDS ORGANIZATION, Guidelines for the construction, format, and management of monolingual controlled thesauri, cit., p. 45-46. 331 PAUL GABRIELE WESTON, Il catalogo elettronico, cit. p. 138; F. H. AYRES, Time for change, cit., p. 9. 
elementi lessicali in relazione paradigmatica con l'elemento lessicale stesso; condizione che in un'ultima analisi avrebbe l'effetto di ostacolare, invece di facilitare, l'utente finale. Un'alternativa a questo sistema è invece la possibilità, suggerita da Gorman negli anni Settanta, di registrare la differenza tra le diverse tipologie di sinonimi o forme lessicali (per esempio forma sciolta o contratta, forma popolare o scientifica, forma nella lingua originale o nella lingua del catalogo, etc.), in modo da permettere all'utente, in fase di visualizzazione, di scegliere la forma che preferisce; si tratta di una proposta interessante, che pure, essendo per molti elementi lessicali il numero di forme lessicali superiore al numero di tipologie di forme, non eliminerebbe in molti casi la necessità di scegliere una forma preferita per ciascuna tipologia di forma lessicale di un elemento.

Per questo motivo, all'interno della nostra sottolingua accetteremo la necessità pratica della scelta di una forma preferita verbale per la visualizzazione, lasciando aperta l'opzione sulla possibilità di differenziare le diverse tipologie di forma lessicale.

\subsection{6. Relazioni di omonimia, polisemia e omotassia}

Le relazioni semantiche prese in considerazione dalla teoria dell'indicizzazione tradizionale, soprattutto da quella relativa ai thesauri, si fermano qui. Abbiamo invece detto come tra le relazioni semantiche paradigmatiche vadano annoverate anche le relazioni di omonimia (che nelle lingue documentarie è generalmente omografia) e quelle correlate di omotassia e polisemia (Svenonius le tiene in considerazione, ma considerandole a parte dalla semantica paradigmatica, come facenti parte della "semantica referenziale"); esse costituiscono un tipo di relazione semantica inversa rispetto alla sinonimia e alle relazioni di equivalenza in generale: le relazioni di omonimia, omotassia e polisemia sono quelle che legano elementi lessicali dallo stesso significante ma dai differenti significati (o sensi, nel caso della polisemia).

Abbiamo già discusso di omonimia, omotassia e polisemia nella precedente sezione (vedi 3.2.5.3.3): esse sono una delle cause principali dell'ambiguità semantica del linguaggio. Omonimia e polisemia riguardano elementi lessicali in forma semplice. Generalmente gli elementi lessicali omonimi hanno origini linguistiche differenti, a differenza dei polisemi; la polisemia è viceversa una condizione almeno potenzialmente intrinseca agli elementi lessicali, il cui significato può mutare parzialmente in base al contesto. Riferendoci alle entità del modello concettuale, possiamo dire che l'omonimia può riguardare, almeno teoricamente, qualsiasi tipo di entità. Nel linguaggio naturale, l'omotassia è la relazione che lega elementi lessicali in forma sintagmatica i cui significanti siano identici ma i cui significati differiscano ("l'amore di Maria" può indicare tanto l'amore da parte di Maria quanto l'amore per Maria); essa è dunque originata da un'ambiguità semantica di tipo sintagmatico, e riguarda principalmente l'ambiguità semantica delle preposizioni (semplici e articolate). Nell'ambito dell'indicizzazione, a questo tipo di omotassia si aggiunge quella, più grave perché relativa alla struttura profonda dei sintagmi, che caratterizza quelle lingue documentarie che esprimano in modo implicito le relazioni sintagmatiche; per esempio le LCSH, in cui il sintagma "Deportees-Germany" può riferirsi tanto ai deportati in Germania quanto ai deportati dalla Germania, o "Italy-Emigration and immigration" che può riferirsi tanto all'immigrazione in Italia quanto all'emigrazione dall'Italia. Come abbiamo avuto modo di dire, nelle lingue naturali utilizzate in una situazione ordi- 
naria, per esempio un dialogo tra due persone, le ambiguità dovute a omonimia, polisemia e omotassia vengono risolte principalmente tramite il riferimento al contesto linguistico ed extralinguistico (situazioni, presupposti concettuali, emozioni comuni ai parlanti). Nell'accesso bibliografico è tuttavia proprio questo tipo di riferimento che manca: ciò rende problematica la altrimenti accettabile ambiguità delle lingue naturali. Uno degli scopi principali delle lingue documentarie è pertanto quello di controllare l'ambiguità semantica dovuta a omonimia, polisemia e omotassia; in termini di funzioni catalografiche, tale controllo linguistico è essenziale per Trovare e soprattutto Identificare un elemento lessicale (e l'entità concettuale soggiacente).

A differenza delle relazioni semantiche discusse più sopra, la rappresentazione dell'omonimia, della polisemia e dell'omotassia all'interno delle lingue documentarie non avviene in forma di collegamenti tra gli elementi lessicali, quanto piuttosto tramite una serie di espedienti (espliciti o impliciti) di natura metalinguistica, tutti indistintamente rivolti alla creazione di un contesto artificiale per l'elemento lessicale caratterizzato da omonimia, polisemia o omotassia.

Gli espedienti tradizionalmente utilizzati per la disambiguazione di omonimia, polisemia e omotassia sono stati diversi: qualificazioni e note, tra quelli espliciti; a cui va aggiunto l'espediente implicito (quanto all'omonimia, polisemia e omotassia) della rete sindetica stessa, sia a livello delle relazioni sintagmatiche sia, e grazie al processo di lessicalizzazione cui sono soggette, delle relazioni paradigmatiche. ${ }^{332}$ Le qualificazioni degli elementi lessicali, l'espediente di disambiguazione in assoluto più diffuso, creano un vero e proprio contesto artificiale permanente che viene inserito all'interno dello stesso elemento lessicale da disambiguare, generalmente distinto grazie a parentesi o altri espedienti simbolici o stilistici (p.e. "Gru (Attrezzatura)" e "Gru (Uccello)"); si tratta dunque di espedienti metalinguistici ma lessicali a tutti gli effetti, e che come tali sono estranei alle caratteristiche del linguaggio naturale. Inoltre, le qualificazioni vengono spesso utilizzate anche per altri scopi oltre a quello della disambiguazione, come per esempio spiegare il significato di un termine anche in assenza di omonimie, polisemie e omotassie. Il secondo espediente tradizionale delle lingue documentarie per la disambiguazione semantica sono note (elementi metalessicali in forma discorsiva e non controllata) che forniscono una chiarificazione o definizione del significato dell'elemento lessicale stesso; ${ }^{333}$ rispetto alle qualificazioni, le note sono più in linea con la logica del linguaggio naturale, ma anche meno efficaci perché non visualizzate dall'utente immediatamente insieme all'elemento lessicale ricercato. Spesso le note sono, come già le qualificazioni, utilizzate nelle lingue documentarie anche per altri scopi oltre a quello della disambiguazione semantica vera e propria: per definire un termine, per prescriverne l'uso come particolare tipo di indice, etc. ${ }^{334}$ Nell'ambiente catalografico odierno, un importante espediente di disambiguazione è, a livello fisico, l'identificatore della registrazione; naturalmente, si tratta di una disambiguazione che permette di sciogliere soltanto quelle ambiguità linguistiche che siano già state registrate come tali (ovvero che abbiano portato alla registrazione di elementi lessicali omonimi in due differenti registrazioni). Inoltre, per l'utente un identificatore non è in sé significativo, e la di-

332 Cfr. ELAINE SVENONIUS, The intellectual foundation of information organization, cit., p. 149, 151-152, 582.

333 ELAINE SVENONIUS, The intellectual foundation of information organization, cit., p. 150. 334 Cfr. NATIONAL INFORMATION STANDARDS ORGANIZATION, Guidelines for the construction, format, and management of monolingual controlled thesauri, cit., p. 9, 22. 
sambiguazione verbale mantiene ancora un'importanza a livello di visualizzazione. Per il nostro modello di lingua documentaria, allora, accanto all'espediente di disambiguazione fornito dall'identificatore, la disambiguazione dell'omonimia e dell'omotassia è affidata principalmente alla rete sindetica di un elemento lessicale: è sufficiente che l'utente visualizzi le relazioni sintagmatiche in forma metalessicale o le relazioni paradigmatiche (eponimia, antonimia, equivalenza, etc.) di un elemento lessicale per Identificarlo e disambiguare eventuali omonimie e omotassie. È vero che le relazioni paradigmatiche non vengono visualizzate immediatamente dall'utente (a differenza di quelle sintagmatiche), almeno nelle attuali lingue documentarie, ma ciò è tecnicamente superabile escogitando automatismi che permettano di visualizzare immediatamente le relazioni paradigmatiche di un elemento lessicale. Solo in via residuale, la nostra sottolingua prevede anche l'utilizzo di note; non prevede, invece, l'utilizzo di qualificazioni all'interno della sottolingua, giacché si tratta di espedienti non indispensabili e oltretutto contrari alla natura del linguaggio. Non abbiamo parlato della disambiguazione della polisemia all'interno della sottolingua, e non lo faremo: la disambiguazione della polisemia è una condizione né del tutto possibile né auspicabile, visto che sarebbe totalmente contraria alla natura del linguaggio; pertanto, nel nostro modello di sottolingua non prevediamo la possibilità di una disambiguazione delle polisemie.

\subsection{7. Relazioni ad hoc}

Chiudiamo la trattazione delle relazioni paradigmatiche della sottolingua con le cosiddette relazioni ad hoc: tutte quelle relazioni di significato non trattate normalmente in linguistica ma tipiche dei discorsi bibliografici (relazione di responsabilità, di soggetto, di affiliazione istituzionale, di adattamento, etc.). Nella sottolingua proposta, la trattazione esplicita delle relazioni ad hoc rimarrà solo abbozzata. Avevamo anticipato in 3.3.4.5 che le relazioni ad hoc possono teoricamente far parte della sottolingua allo stesso titolo delle altre relazioni paradigmatiche più sopra discusse.

Più precisamente, all'interno della sottolingua le relazioni ad hoc potrebbero assumere fondamentalmente due forme: 1) una forma implicita e lessicale: non venire trattate come relazioni semantiche a sé stanti, ma piuttosto essere trattate come particolari elementi lessicali della sottolingua; 2) una forma esplicita e metalessicale, cioè come relazioni semantiche paradigmatiche a sé stanti.

Facciamo un esempio. La relazione di tra un'opera (Le avventure di Pinocchio di Collodi) e altre opere che ne costituiscono adattamenti per altre forme di espressione (per esempio Le avventure di Pinocchio di Luigi Comencini) potrebbe essere rappresentata paradigmaticamente in due modi: o come relazione semica possessiva che "Le avventure di Pinocchio" di Collodi intrattiene con "Adattamenti delle Avventure di Pinocchio" di Collodi, il quale a sua volta intratterrebbe una relazione iperonimica pura con "Le avventure di Pinocchio" di Comencini; oppure come relazione paradigmatica "di adattamento" che "Le avventure di Pinocchio" di Collodi intrattiene con "Le avventure di Pinocchio" di Comencini. Nel primo caso l'adattamento compare in forma lessicale all'interno di un elemento lessicale in forma sintagmatica (caratterizzato da una relazione sintagmatica possessiva tra "Adattamenti" e "Le avventure di Pinocchio" di Collodi; nel secondo caso l'adattamento compare in forma metalessicale come relazione paradigmatica 
distinta. Dall'esempio fatto risulta chiara una cosa: la distinzione di relazioni ad hoc come vere e proprie relazioni paradigmatiche della sottolingua comporta una complessità maggiore della tassonomia delle relazioni paradigmatiche ma, in compenso, una rete semantica della sottolingua meno complessa. Le relazioni ad hoc, infine, come le relazioni di equivalenza e a differenza di tutte le altre relazioni paradigmatiche sin qui discusse, non riguardano un elemento lessicale nella sua interezza ma piuttosto un suo aspetto o caratteristica.

\subsection{Semantica paradigmatica delle tipologie lessicali}

Nella discussione di diversi gradi di controllo linguistico (3.3.4 e ss.), abbiamo visto come pressoché la totalità delle lingue documentarie operi una partizione semantica del lessico sulla base delle caratteristiche dell'universo bibliografico e delle esigenze dell'utente finale rispetto a esso, per individuare un certo numero di oggetti di interesse o entità concettuale: è la semantica delle entità concettuali della lingua documentaria. Accanto a tale semantica delle entità concettuali, le lingue documentarie (tradizionalmente solo per soggetto) che prevedono una sottolingua presentano spesso una seconda partizione semantica del lessico, più o meno basata sulla semantica delle tipologie lessicali del linguaggio naturale. La chiameremo semantica (paradigmatica) delle tipologie lessicali della sottolingua.

Come la sua omologa semantica del linguaggio naturale, la semantica delle tipologie lessicali della sottolingua: 1) è strettamente legata alla semantica paradigmatica delle relazioni (della sottolingua); 2) ha come finalità principale quella di regolare l'uso del lessico ovvero la formazione di sintagmi (elementi lessicali in forma composta), andando a costituire parte fondamentale della semantica paradigmatica dei sintagmi.

Per quanto riguarda la sottolingua proposta, la semantica delle tipologie lessicali coinciderà in tutto e per tutto con quella già discussa nella precedente sezione (vedi 3.2.5.3.3), nell'ottica di un riavvicinamento delle lingue documentarie al linguaggio naturale; suddivideremo così le tipologie lessicali della sottolingua in attività ed entità (di tipo linguistico); e queste ultime in entità individuali e collettive (insiemi di entità individuali) e in entità animate e inanimate; alcune entità (collettive, sia di tipo animato che inanimato) possono sussumere in sé o fare riferimento a un'attività: è questo il caso di nominalizzazioni come "scultori", "trattori", etc; le entità appartenenti a questa particolare tipologia possono svolgere un ruolo simile alle attività all'interno dei sintagmi, come diremo più avanti (vedi 3.3.6.4.3.2). Ribadiamo che la classificazione delle tipologie lessicali non ha valore ontologico o universale, giacché ogni lingua naturale, e prevedibilmente ogni lingua documentaria, è caratterizzata ad una propria semantica. La semantica delle tipologie lessicali della sottolingua può comparire o in forma metalessicale (etichette verbali, alfanumeriche o numeriche, come per esempio sono quelle del MARC - le quali però mischiano in una sola etichetta semantica delle entità e semantica degli attributi) o come parte dello stesso lessico della sottolingua: per esempio, il fatto che l'educazione sia un'attività può essere segnalato o includendo le "Attività" nel lessico della sottolingua e trattandole come un iperonimo di "Educazione", o tramite elementi metalessicali di vario tipo (etichette di nodo, etc.) che segnalino che "Educazione" è un'attività. 
Parliamo adesso del rapporto tra semantica delle tipologie lessicali e semantica delle entità concettuali, ovvero anche del rapporto tra modello di lingua documentaria e modello concettuale; ne avevamo già parlato nel precedente capitolo (vedi 2.11), ma è adesso necessario riprenderne la discussione. Semantica delle tipologie lessicali e semantica delle entità concettuali coesistono e sono anzi sovrapposte all'interno della sottolingua: la prima costituisce una semantica ad hoc della sottolingua sulla base delle esigenze catalografiche, la seconda una seconda semantica direttamente discendente da quella del linguaggio naturale. In alcuni casi le entità concettuali individuate dalla lingua documentaria sono semanticamente più ampie delle tipologie lessicali, perché riferite a tipologie lessicali che non hanno un valore o un'importanza autonoma a livello bibliografico (è questo il caso degli Eventi, i quali possono riguardare attività o entità di tipo linguistico, e soprattutto delle Altre entità, le quali raggruppano un numero elevato di tipologie lessicali differenti: attività, nel caso dei tipi di evento; entità linguistiche di vario tipo, nel caso dei tipi di oggetto - tranne quelli prettamente bibliografici -, degli animali, dei tipi di animale, dei personaggi fittizi e leggendari, etc.). In altri casi le entità concettuali sono più specifiche, anche di molto, rispetto alle tipologie lessicali: così Opere, Edizioni e Contenuti editoriali sono Testi (una super-entità concettuale), che a loro volta sono una particolare categoria di prodotti bibliografici, che a loro volta sono una particolare categoria di oggetti, che a loro volta sono entità individuali e inanimate. Manifestazioni e Item sono Risorse (un'altra super-entità concettuale), che a loro volta sono una particolare categoria di prodotti bibliografici, che a loro volta sono una particolare categoria di oggetti, che a loro volta sono entità individuali e inanimate. Persone, Famiglie ed Enti sono invece particolari tipi di entità individuali e animate. Tipi di ente e Tipi di persona sono particolari tipi di entità collettive e animate. Tipi di testo e Tipi di risorsa sono particolari tipi di entità collettive e inanimate. Oggetti, Eventi, Tempi e Lingue sono particolari tipi di entità individuali e inanimate; i Concetti sono invece entità collettive e inanimate; e così via.

\subsection{Semantica sintagmatica o sintassi profonda}

\subsection{Introduzione}

Il linguaggio naturale, come ricorda Hutchins, conosce fondamentalmente quattro generi di espedienti di organizzazione sintagmatica: ordine delle parole, concordanza (basata sulle proprietà morfologiche dei lessemi), sistema dei casi e connettivi di vario tipo (preposizioni, congiunzioni, verbi ausiliari, etc.). ${ }^{335}$ Le lingue documentarie che operano un qualche tipo di organizzazione sintagmatica hanno selezionato e semplificato uno o più tra $i$ suddetti espedienti sintagmatici, modificandoli sulla base delle esigenze dell'indicizzazione. ${ }^{336}$

335 W. JOHN HUTCHINS, Languages of indexing and classification, cit., p. 25-26.

336 Cfr. YVES GILBERT COURRIER, Document analysis, verbs and case grammar, cit., p. 54: "Whether few in number or several, whether natural or artificial, they all play the same role: they give a more precise representation of the content of the documents because they reproduce in the document representation the relations among the concepts that were present in the original documents". 
L'espediente sintagmatico in assoluto più utilizzato storicamente dalle lingue documentarie è quello dell'ordine tra le parole (ordine fisso, ordine di citazione, ordine di combinazione, etc.), utilizzato come mezzo per assegnare un significato relativo agli elementi componenti di un elemento lessicale in forma composta. Tuttavia, l'ordine fisso tra le parole è un espediente sintagmatico efficace solo in un ambiente linguistico lineare, quale è il linguaggio naturale (scritto o orale), non in un ambiente elettronico in cui l'ordine delle parole può essere manipolato e in cui è possibile l'accesso a singoli elementi lessicali dei sintagmi a prescindere dalla loro posizione. Continuare a utilizzare l'ordine fisso come espediente sintagmatico, come tuttora viene fatto in molte lingue documentarie, significa vincolare l'accesso bibliografico a una logica del tutto estranea alle potenzialità dell'ambiente elettronico. Una differenza importante di cui abbiamo parlato è la differenza tra livello di superficie e profondo del linguaggio, e in particolare tra (morfo)sintassi di superficie e profonda; la sintassi profonda del linguaggio ha una natura fondamentalmente semantica, e per questo possiamo parlarne anche nei termini di una semantica dell'asse sintagmatico, o semantica sintagmatica. L'ordine delle parole, così come la concordanza, i casi morfosintattici e i connettivi di vario tipo sopra menzionati appartengono alla morfosintassi di superficie; questa ha un ruolo molto limitato all'interno della lingua documentaria. Molto più importante nelle lingue documentarie è invece il livello profondo del linguaggio, quello della sintassi profonda o semantica sintagmatica: le relazioni sintagmatiche, le quali sono relativamente indipendenti dall'ordine, dai casi di superficie, dai connettivi usati, etc. Porre la base dell'organizzazione sintagmatica delle lingue documentarie al livello profondo del linguaggio equivale a dire che è possibile mantenere l'organizzazione sintagmatica del linguaggio, la sua contestualità sintagmatica (quella paradigmatica essendo fornita dalla semantica paradigmatica), ${ }^{337}$ rinunciando a fissare la sua morfosintassi di superficie e dunque, tra le altre, cose, all'ordine fisso tra gli elementi componenti degli elementi lessicali in forma composta. Quando, nel precedente capitolo, avevamo parlato della coordinazione (vedi 2.18.4), avevamo già detto in nuce la maggior parte di queste cose; avevamo detto anche come la chiave per un'efficace coordinazione fosse quella di escogitare un sistema che permettesse di mantenere la contestualità (sintagmatica) rinunciando all'ordine fisso: il sistema di relazioni sintagmatiche di cui abbiamo parlato, e che adesso approfondiremo, vuole rappresentare proprio questo.

La teoria dell'indicizzazione e le lingue documentarie tradizionali hanno rifiutato o accolto le teorie grammaticali della linguistica in modo generalmente acritico (con qualche eccezione, soprattutto autori che erano anche linguisti o che avevano condotto uno studio approfondito della linguistica, vedi sotto); si è spesso pensato o che la sintassi delle lingue naturali non potesse essere una base idonea per quella delle lingue documentarie, o viceversa che le trasformazioni grammaticali elaborate da Chomsky esaurissero le problematiche legate all'organizzazione sintagmatica del linguaggio. ${ }^{338}$ La teoria dell'indicizzazione

337 Sulla contestualità cfr. ELAINE SVENONIUS, The intellectual foundation of information organization, cit., p. 189: "Related to specificity and expressive power, contextuality is the ability of a subject language to recognize distinctions in meaning. Since the task of vocabulary disambiguation is not limited to omonyms and polysemes but extends to all words whose meaning is in part contributed to by context, context is needed for making distinctions in meaning. The contexts shown by Boolean intersections of terms are relationally indiscriminate and therefore cannot disambiguate sufficiently to achieve acceptable precision". 338 Cfr. JEAN-CLAUDE GARDIN, Document analysis and linguistic theory, "Journal of documentation", 29, 2 (1973), p. 142-143: "Our thinking has been somewhat obscured by the stubborness of two extreme views [...] (a) the earlier one, according to which the whole issue was pointless, to the extent that the co- 
tradizionale ha inoltre teso a ignorare o confondere la differenza tra sintassi profonda e di superficie, ${ }^{339}$ tra sintassi e asse sintagmatico del linguaggio - contrapponendo così, erroneamente, sintassi e semantica -, tra relazioni paradigmatiche e sintagmatiche, tra i diversi tipi di relazione sintagmatica.

Nonostante queste imprecisioni, riteniamo utile ripercorrere le categorie, relazioni, e ruoli sintattici elaborati da alcuni autori della teoria dell'indicizzazione. La prima teorizzazione rilevante è quella di Kaiser, già menzionata, che categorizzò gli elementi delle voci di soggetto in concreti e processi (e poi anche località). ${ }^{340} \mathrm{~S}$. R. Ranganathan individuò cinque tipologie di relazioni tra "soggetti", o relazioni di fase: relazione generale, inclinazione, comparazione, differenza, influenza; le relazioni di fase furono poi rielaborate da Antony C. Foskett, che vi aggiunse i destinatari; Hutchins offrì a sua volta una reinterpretazione in termini linguistici delle relazioni di fase di Foskett. ${ }^{341}$ Proprio Hutchins comparò in modo sistematico le modalità e gli espedienti di organizzazione sintagmatica delle lingue naturali e delle lingue documentarie; Hutchins fu forse il primo a riprendere e applicare la nozione dei casi profondi di Fillmore alle lingue documentarie; raccomandò che i particolari espedienti scelti dalla lingua documentaria per rappresentare le relazioni sintagmatiche esplicitassero i casi profondi tra gli elementi del sintagma. ${ }^{342}$ I casi profondi furono proprio in quegli anni (siamo nella metà degli anni Settanta dello scorso secolo) ripresi da Derek Austin, la cui teorizzazione, che sfociò nell'elaborazione del PRECIS, è molto importante per il nostro discorso; Austin: 1) suddivise le relazioni sintagmatiche in relazioni "logiche" (corrispondenti agli operatori booleani) e "grammaticali"; ${ }^{343} 2$ ) approfondì e rielaborò i casi profondi di Fillmore. ${ }^{344}$ In particolare, considerò i seguenti tipi di relazione sintagmatica o "operatori di ruolo": locativa, sistema chiave (oggetto dell'azione, è l'operatore di ruolo centrale nella costruzione del sintagma e corrisponde idealmente al verbo della lingua naturale), azione/effetto, agente, punto di vista, regione/popolazione di studio, destinatari (o beneficiari)/forma, più altri tra cui parte/proprietà, membro di

occurrence of (index) terms in a given document (representation) was enough to indicate their mutual relations; (b) the other, born with generative grammar, for which the kind of syntactical parsers and trees developed in contemporary linguistics provided "the" answer to the relational requirements of document processing [...] although a large number of information systems do take into account syntactical data, none of them seems ever to have contemplated that NL grammars, including the more traditional ones, provided a convenient framework for that purpose. It is not enough to observe that the more "modern" information systems make no use of the more recent grammatical theories, one has also o acknowledge the much more significant fact that none of the many "conventional" systems evolved in the last hundred years for purposes of document analysis hase deemed it convenitent to use the grammatical tools available in the linguistics of their time for the handling of syntactical relations".

339 Cfr. JEAN-CLAUDE GARDIN, Document analysis and linguistic theory, "Journal of documentation”, 29, 2 (1973), p. 142.

340 Cfr. ELAINE SVENONIUS, The intellectual foundation of information organization, cit., p. 129.

341 SHIYALI RAMAMRITA RANGANATHAN, Prolegomena to library classification, assisted by M. A.

Gopinath, 3rd ed., New York, Asia, 1967, disponibile all'indirizzo

<http://arizona.openrepository.com/arizona/handle/10150/106370>, p. 358, 461-462; ANTONY

CHARLES FOSKETT, The subject approach to information, 2nd ed. revised and enlarged, London, Bingley,

1971, p. 125-128; cfr. anche W. JOHN HUTCHINS, Languages of indexing and classification, cit., p. 104-105.

342 W. JOHN HUTCHINS, Languages of indexing and classification, cit., p. 68, 71.

343 DEREK AUSTIN, Basic concept classes and primitive relations, cit., p. 87.

344 JUTTA SØRENSEN - DEREK AUSTIN, PRECIS in a multilingual context. Part 2, cit., p. 108-111; cfr. anche DEREK AUSTIN, Basic concept classes and primitive relations, cit., p. 90; cfr. anche ALBERTO CHETI, Le categorie nell'indicizzazione, "Biblioteche oggi", 8, 1 (1990), p. 48. 
un gruppo quasi-generico, e concetto coordinato. ${ }^{345}$ Il PRECIS prevede la possibilità di coordinare, cioè congiungere, due elementi che svolgano lo stesso caso profondo all'interno del sintagma. ${ }^{346}$ Tra le tassonomie successive, dobbiamo menzionare almeno quella di Coates, simile a quella del PRECIS ma più approfondita, ${ }^{347}$ e le elaborazioni sorte sulla scorta del PRECIS in ambito italiano: GRIS e Nuovo soggettario - che dal lavoro del GRIS ha preso avvio. La "sintassi" del GRIS prevede diverse relazioni sintagmatiche: agenti, attività, strumenti, proprietà, materiali, spazio e tempo; prevede inoltre una suddivisione in ruoli nucleari (azione, oggetto, agente, strumento, beneficiario) e "extranucleari" (locazione: luogo, tempo, etc.), nonché in ruoli primari e secondari (cioè dipendenti: parte, proprietà, etc.). ${ }^{348}$

\subsection{Relazioni sintagmatiche}

Dopo questo breve excursus, possiamo discutere le relazioni sintagmatiche della nostra sottolingua. Prima di entrare nel vivo della discussione, però, vogliamo esplicitare alcune premesse alla base della nostra trattazione delle relazioni sintagmatiche. Così come per molti altri autori e testi della teoria dell'indicizzazione, per la modellizzazione linguistica della semantica sintagmatica della sottolingua siamo partiti dalla nozione di ruolo o caso. In particolare, il nostro sviluppo della semantica sintagmatica ha preso le mosse dall'idea di Tom Delsey secondo cui l'approfondimento delle relazioni sintagmatiche offrirebbe la possibilità di specificare la modellizzazione concettuale degli indici di soggetto (anche se come diremo noi siamo andati oltre e abbiamo esteso tale possibilità anche agli indici della catalogazione per autore). ${ }^{349} \mathrm{Nel}$ fare ciò, e come già Hutchins, PRECIS e altri autori

345 JUTTA SØRENSEN - DEREK AUSTIN, PRECIS in a multilingual context. Part 2, cit., p. 108-111; cfr. anche DEREK AUSTIN, Basic concept classes and primitive relations, cit., p. 115. Cfr. anche TOM DELSEY, Modeling subject access, cit., p. 58-59.

346 JUTTA SØRENSEN - DEREK AUSTIN, PRECIS in a multilingual context. Part 2, cit., p. 118, in cui gli autori spiegano che il PRECIS, pensato per una piena coestensione, prevede la possibilità di coordinare due elementi semantici dello stesso tipo di operatore di ruolo (dunque lo stesso tipo sintattico o sintatticosemantico, cioè che hanno lo stesso caso profondo) in un'unica stringa (per esempio: "Lilies-Growth regulation + Pollination"); cfr. anche DEREK AUSTIN, Basic concept classes and primitive relations, cit., p. 90. 347 ERIC J. COATES, Subject catalogues. Headings and structure, London, Library Association, 1988, p. 55. 348 ASSOCIAZIONE ITALIANA BIBLIOTECHE. GRUPPO DI RICERCA SULL'INDICIZZAZIONE PER SOGGETTO, Guida all'indicizzazione per soggetto, cit., p. 23, che suddivide i ruoli (semantici, ma GRIS li considera relazioni sintattiche) in primari (relazioni sintagmatiche semantiche) e secondari (relazioni sintagmatiche sintattiche: tra questi ultimi include i ruoli di dipendenza e coordinazione. Cfr. anche BIBLIOTECA NAZIONALE CENTRALE DI FIRENZE, Nuovo soggettario, disponibile all'indirizzo <http:// thes.bncf.firenze.sbn.it/>. Cfr. anche MASSIMO ROLLE, Per un nuovo modello di linguaggio documentario, cit., p. 322.

349 TOM DELSEY, Modeling subject access, cit., p. 58-59: "Issues of a different nature will emerge as consideration is given to extending the relationships represented in the FRBR and FRANAR models to cover the syntactic relationships that are reflected within individual indexing strings, as distinct from the semantic relationships between terms, concepts, etc., that are reflected in thesauri and subject heading lists [...] From a modeling perspective, the first issue to be addressed with respect to the representation of syntactic relationships is how to reflect the context-dependent nature of the relationships. The aim would be to delineate the relationships between each of the entities represented in the indexing string and the string as a whole [...] Syntactic relationships could then be defined to operate between specific instances of the entities concept, object, event, place, work, expression, manifestation, item, person, family, and corporate body (as 
prima di noi, abbiamo individuato nei casi profondi (o ruoli semantici) di Fillmore la base di partenza ideale per sviluppare il nostro sistema di relazioni sintagmatiche - è per questo motivo che abbiamo discusso in maniera approfondita i casi profondi di Fillmore nel corso della precedente sezione. ${ }^{350}$

Fatte queste doverose premesse, passiamo a parlare della semantica sintagmatica (o sintassi profonda) della nostra sottolingua e delle sue differenze da un lato rispetto ai casi profondi così come concepiti da Fillmore e dalla linguistica successiva, dall'altro rispetto alla teoria dell'indicizzazione tradizionale. Innanzitutto dobbiamo esplicitare una differenza fondamentale tra i casi profondi così come presenti nelle lingue naturali e la loro applicazione alle lingue documentarie (in generale, non solo la sottolingua da noi proposta): mentre i casi profondi funzionano generalmente bene nelle lingue naturali - con qualche eccezione, vedi la già menzionata ambiguità tra casi oggettivo e locativo per alcuni tipi di evento ("Bombardamenti della Sicilia" e "Bombardamenti in Sicilia") -, la loro applicazione alle lingue documentarie è in alcuni casi problematica; nelle lingue documentarie, infatti, non è auspicabile distinguere significati relativi o sintagmatici che siano molto simili; per questo motivo, inoltre, non è opportuno che la semantica sintagmatica della sottolingua preveda tutti i casi profondi che sono stati proposti nel corso degli anni - sui quali non vi è peraltro accordo nemmeno in linguistica.

Con l'avvertenza che non si tratta di una lista esaustiva o obbligatoria (ogni lingua documentaria che vorrà applicare il nostro sistema di semantica sintagmatica potrà includere un numero maggiore o minore di casi profondi), i casi profondi previsti dalla nostra semantica sintagmatica saranno allora: agentivo, strumentale, oggettivo, locativo, benefattivo, causativo, temporale, funzionale, originario e direttivo; escluderemo invece i casi fattitivo (coperto dal caso oggettivo) e comitativo (che non riteniamo necessario e che in molti casi si sovrappone casi oggettivo e benefattivo); a ognuno di tali casi corrisponde una specifica tipologia di relazione sintagmatica, come diremo sotto.

Abbiamo introdotto tali casi già nella precedente sezione (vedi 3.2.7); discuteremo invece adesso le peculiarità della loro applicazione all'interno del nostro sistema di semantica sintagmatica. I casi profondi di Fillmore corrispondono fondamentalmente agli argomenti della struttura profonda che ruotano attorno al verbo di una frase. Poiché, con le eccezioni effettive o ipotetiche che abbiamo detto più sopra (vedi 3.3.6.3), i verbi sono assenti dalla sottolingua proposta, e dunque tutti i sintagmi della sottolingua saranno sintagmi nominali, il ruolo centrale che nel sistema sintagmatico di Fillmore ha il verbo verrà nella nostra sottolingua assunto da un sostantivo che costituisca una nominalizzazione di verbo. In alcuni casi si tratterà di un sostantivo che rappresenta o faccia riferimento direttamente a una attività o un processo ("educazione", "influenze", "violenza”, etc.); in altri si tratterà di sostantivi che rappresentino entità collettive ma che sussumano in sé o includano il riferimento ad attività: è il caso di alcune tipologie di nominalizzazioni come "scultori”, "scrittori" (entità animate), ma anche "trattori”, "fertilizzanti” (entità inanimate), etc.

well as any relevant additional entities defined in the course of extending the models to cover subject access) and specific instances of the newly defined entity topic or subject matter. The second issue to be addressed is how to define and categorize the various types of syntactic relationships. Essentially the model needs to reflect the role or function of each concept, etc., represented in the indexing string relative to the string as a whole, or more precisely, to the topic or subject matter represented by the string".

350 A differenza del PRECIS, nel nostro approccio il punto di vista è modellato come elemento esterno (ed eventualmente collegato) ai sintagmi utilizzati per rappresentare il tema di un testo; la regione o popolazione di studio non è prevista come elemento autonomo. 
Mentre le attività possono reggere tutti i casi profondi, le entità che facciano riferimento ad attività possono reggere un numero più limitato di casi profondi: non, per esempio, $\mathrm{i}$ casi strumentale e agentivo.

Inoltre, mentre nel sistema di Fillmore tutti i casi profondi sono singoli elementi lessicali che ruotano attorno al ruolo centrale giocato dal verbo, in modo da configurare una organizzazione gerarchica del sintagma di due soli livelli, con un ruolo centrale da un lato e l'insieme dei casi profondi, subordinati al ruolo centrale, dall'altro, nel nostro sistema è prevista la possibilità di un numero maggiore di livelli di organizzazione gerarchica dei sintagmi: ogni caso profondo, infatti, potrà essere costituito da più elementi lessicali componenti i quali intrattengano tra loro ulteriori relazioni sintagmatiche, in modo del tutto identico alla struttura del sintagma generale, cioè con un elemento lessicale che svolge un ruolo sovraordinato rispetto a uno o più elementi lessicali che svolgono casi profondi subordinati (per esempio: in "Valutazione dell'educazione in Italia", "educazione in Italia" è oggettivo di "Valutazione"; "educazione in Italia" è strutturato a sua volta in modo che "Italia" sia locativo di "educazione"). Una struttura, dunque, gerarchicamente complessa dell'organizzazione dei sintagmi, in cui un ruolo sovraordinato regge uno o più ruoli subordinati, ciascuno dei quali può a sua volta svolgere un ruolo sovraordinato rispetto ad altri ruoli subordinati; questo tipo di struttura è prevista anche dal GRIS (che non parla di casi o di elementi sovraordinati e subordinati ma di testa e modificatore). ${ }^{351}$ Proprio per permettere di rappresentare adeguatamente questo tipo di struttura a più livelli gerarchici, oltre che per facilitare la creazione di sequenze artificiali tra gli elementi lessicali del sintagma, nella nostra sottolingua - come si era già accennato indirettamente nella trattazione delle relazioni paradigmatiche gerarchiche specificate - è prevista la rappresentazione obbligatoria non soltanto degli elementi che svolgono un caso profondo, ma anche degli elementi lessicali che svolgono il caso reciproco (per esempio "Educazione" rispetto al caso oggettivo "Donne" in "Educazione delle donne"), secondo una logica binaria; come anticipato, ci riferiremo a tali casi reciproci aggiungendo il prefisso "de-" al nome del rispettivo caso profondo (quindi, ritornando all'esempio precedente, la forma sintagmatica collegata sarebbe "[deoggettivo:] Educazione - [oggettivo:] Donne"); questa terminologia non appartiene alla linguistica ma è di nostra invenzione. Ciò ha come conseguenza che l'elemento lessicale che assume il ruolo sovraordinato del sintagma svolgerà, secondo il nostro sistema, un numero di casi profondi (reciproci) pari a quello dei casi profondi che regge (in "Abusi sui detenuti da parte della polizia", avremmo "[deagentivo + deoggettivo:] Abusi - [agentivo:] Polizia - [oggettivo:] Detenuti”). Precisando quanto detto sopra, possiamo dire che nel nostro sistema sintagmatico ogni elemento lessicale può svolgere più ruoli semantici, secondo una organizzazione sintagmatica al contempo binaria (caso profondo e suo reciproco) e orientata o gerarchizzata (ruolo sovraordinato e ruoli subordinati, che a loro volta possono essere composti da un ruolo sovraordinato con ruoli subordinati). ${ }^{352}$

Fin qui ci siamo limitati deliberatamente a esempi in cui il ruolo sovraordinato è svolto da un elemento lessicale che rappresenta un'attività. Un'altra differenza, ancora più importante della precedente, tra il sistema dei casi di Fillmore e il nostro sistema sintagmati-

351 ASSOCIAZIONE ITALIANA BIBLIOTECHE. GRUPPO DI RICERCA SULL'INDICIZZAZIONE PER SOGGETTO, Guida all'indicizzazione per soggetto, cit., p. 22.

352 Anche JEAN-CLAUDE GARDIN, Document analysis and linguistic theory, cit., si schiera a favore della riduzione di relazioni plurime a relazioni binarie. 
co è che mentre nel primo i casi profondi si applicano esclusivamente a sintagmi verbali, ovvero a sintagmi nominali in cui sia presente una sostantivo deverbale (nominalizzazione del verbo, che rappresenti un'attività o un'entità la quale faccia riferimento a un'attività), nel nostro sistema abbiamo esteso l'applicazione dei casi profondi anche ai sintagmi nominali in cui sono del tutto assenti sostantivi deverbali o riconducibili a un'attività, e che potremmo chiamare sintagmi nominali non deverbali. Per esempio, all'interno del nostro sistema sintagmatico concepiremo la forma sintagmatica collegata di "Edifici in Italia" come "[delocativo:] Edifici - [locativo:] Italia". Si tratta di un'operazione non soltanto estranea alla natura dei casi profondi di Fillmore così come originariamente concepiti, ma persino alla stessa nozione di argomento così come intesa in linguistica; un artificio, che trova però una sua giustificazione teorica nella comune struttura di fondo dei diversi tipi di sintagmi, tutti indistintamente dominati da un nodo rispetto a cui ruotano (secondo due o più livelli gerarchici) gli altri elementi lessicali del sintagma. Inoltre, l'estensione del sistema dei casi profondi ai sintagmi nominali non deverbali conosce un limite: la semantica paradigmatica impone che soltanto un sostantivo deverbale (cioè indicativo di un'attività o processo) possa prevedere casi profondi di agente, oggetto, strumento, causa; viceversa, i sintagmi nominali non deverbali potranno prevedere casi profondi di funzione, beneficiario, stato in luogo, tempo, origine e direzione. Sostantivi deverbali (attività) e sostantivi non deverbali non hanno comunque, nemmeno nel nostro sistema, un'importanza sintagmatica paritetica: quando presenti, i sostantivi deverbali tenderanno ad assumere il ruolo sovraordinato. ${ }^{353}$

I casi profondi di Fillmore, secondo la suddetta rielaborazione, costituiscono la base del nostro sistema di semantica sintagmatica; tuttavia questo include, in linea con la teoria dell'indicizzazione tradizionale, una serie aggiuntiva di relazioni semantiche sintagmatiche non riconducibili ai casi profondi. Vi abbiamo già fatto riferimento in più punti del testo. Si tratta delle relazioni sintagmatiche partonomiche o partitive, delle relazioni sintagmatiche possessive e le relazioni sintagmatiche eponimiche atipiche. Avevamo già introdotto alcune di queste relazioni nella precedente sezione (vedi 3.2.7). Parte di esse (relazioni sintagmatiche partitive e possessive) sono considerate anche dalla linguistica, ma non come casi profondi; le relazioni sintagmatiche eponimiche atipiche, così come le omologhe relazioni paradigmatiche, esprimono invece sì un tipo di relazione presente anche nel linguaggio naturale (l'appartenenza di un elemento lessicale a un genere che gli è atipico), ma in una forma artificiale tipica delle lingue documentarie in quanto tali (vedi il già menzionato "granchi in quanto cibo"). Anticipiamo sin da qui che l'introduzione di tali relazioni sintagmatiche (partitive, possessive ed eponimiche atipiche), esterne al sistema dei casi profondi di Fillmore, acuisce quei problemi di ambiguità e sovrapposizione delle relazioni sintagmatiche a cui abbiamo più sopra fatto riferimento; in particolare, vedremo come ci siano una serie di casi in cui vi è una sovrapposizione di relazioni sintagmatiche partitive e locative.

Oltre a casi profondi e alle altre relazioni sintagmatiche suddette, il nostro modello di sottolingua prevede anche, come già anticipato, quell'insieme di relazioni sintagmatiche che abbiamo denominato relazioni "sintagmatiche logiche": relazioni di natura fondamentalmente sintattica (benché soggette a vincoli semantici), esistenti esclusivamente nei sintag-

353 Cfr. ASSOCIAZIONE ITALIANA BIBLIOTECHE. GRUPPO DI RICERCA

SULL'INDICIZZAZIONE PER SOGGETTO, Guida all'indicizzazione per soggetto, cit., p. 22-23; GRIS

include nelle azioni (attività) anche quelle "entità" che esprimono azione, come p.e. violenza, danni, etc. 
mi e funzionali alla loro costruzione, che non hanno alcuna corrispondenza a livello paradigmatico. L'espressione "relazioni sintagmatiche logiche", lo esplicitiamo qui, è presa in prestito dalla terminologia utilizzata da Austin (anche se a differenza di quelle le nostre relazioni sintagmatiche logiche non corrispondono semplicemente agli operatori booleani); sempre sulla base di tale terminologia, parleremo invece di relazioni "sintagmatiche grammaticali" quando dovremo riferirci all'insieme delle relazioni sintagmatiche (casi profondi e altre) diverse dalle relazioni sintagmatiche logiche. All'interno del nostro modello, oltre alla relazione di coordinazione, a cui abbiamo già fatto riferimento nella precedente sezione (vedi 3.2.8), sarà inclusa anche una relazione sintagmatica logica di nostra elaborazione: la relazione di interazione (vedi 3.3.6.4.3.4).

A relazioni sintagmatiche grammaticali e logiche va aggiunta, come già abbiamo accennato nella sezione precedente (vedi 3.2.6), la semantica paradigmatica vigente nei sintagmi, ovvero l'insieme della semantica delle relazioni paradigmatiche e delle tipologie lessicali della sottolingua, la quale condiziona la combinazione degli elementi lessicali e dunque la formazione di sintagmi (elementi lessicali in forma composta).

Riassumendo, il nostro sistema di semantica sintagmatica o sintassi profonda è costituito da: 1) relazioni sintagmatiche grammaticali, le quali comprendono: a) casi profondi; b) relazioni sintagmatiche d'altro tipo: partitive, possessive, ed eponimiche atipiche; 2) relazioni sintagmatiche logiche: coordinazione e interazione, 3) semantica paradigmatica vigente nei sintagmi - che non approfondiremo essendo questa già stata discussa a sufficienza nei paragrafi precedenti. Queste tre tipologie fondamentali di relazioni esistenti nei sintagmi non sono alternative: piuttosto, esse formano tre sotto-sistemi che sono compresenti all'interno dei sintagmi.

Un'importante differenza, peraltro già anticipata, tra il nostro sistema di semantica sintagmatica e la teoria (e gli standard) dell'indicizzazione tradizionale è che le nostre relazioni sintagmatiche si applicano non soltanto agli elementi dell'indicizzazione per soggetto, ma a tutti gli elementi dell'indicizzazione in generale: ciò è parte del nostro proposito di una visione unificata delle diverse branche della catalogazione.

Infine, ribadiamo già qui alcuni aspetti peculiari del nostro sistema delle relazioni sintagmatiche già discussi più sopra: distingueremo da un lato le relazioni sintagmatiche in forma lessicale, che caratterizzano le forme sintagmatiche non collegate degli elementi lessicali (p.e. "Deportati in Germania"), dall'altro le relazioni sintagmatiche in forma metalessicale, che caratterizzano invece le forme sintagmatiche collegate degli elementi lessicali, e che sono tipiche della sottolingua a differenza del linguaggio naturale (p.e. "[dedirettivo:] Deportati - [direttivo:] Germania"); nel corso delle discussioni seguenti, ci riferiremo soprattutto alle forme metalessicali delle relazioni sintagmatiche della sottolingua. Inoltre, sulla base dell'esigenza di riduzione della ridondanza linguistica non soltanto le relazioni sintagmatiche logiche (così come nel linguaggio naturale), ma anche le relazioni sintagmatiche grammaticali potranno comparire esclusivamente sul piano sintagmatico della sottolingua: queste sarebbero teoricamente rappresentabili sul piano paradigmatico, ma ciò non sarebbe funzionale alle funzioni catalografiche, e dunque ne escluderemo la rappresentazione sul piano paradigmatico. Tuttavia, tra le relazioni sintagmatiche grammaticali e le relazioni paradigmatiche gerarchiche specificate vi è comunque una correlazione: l'elemento lessicale componente che svolge il caso sovraordinato rispetto agli altri elementi corrisponde all'iperonimo dell'elemento lessicale nel suo complesso, laddove viceversa l'elemento lessicale o gli elementi lessicali componenti che svolgono casi subordinati corri- 
spondono ai semi (non generici) dell'elemento lessicale nel suo complesso. Per fare un esempio: il sintagma "[deoggettivo:] Violenza - [oggettivo:] Donne", ovvero "Violenza sulle donne", sarà legato da una relazione paradigmatica iponimica oggettiva a "Violenza" e da una relazione paradigmatica sememica deoggettiva a "Donne". Inoltre, sempre sulla base della stessa esigenza di riduzione della ridondanza, all'interno dei sintagmi (elementi lessicali in forma composta) della sottolingua potranno comparire soltanto elementi lessicali componenti legati da relazioni paradigmatiche co-iponimiche, co-sememiche, co-meronimiche e di antonimia complementare, e non da relazioni paradigmatiche d'altro tipo. Riferendoci al modello concettuale, le relazioni sintagmatiche della sottolingua riguardano tutti gli elementi lessicali utilizzati in quegli indici definiti come indici collegati.

\subsection{Relazioni sintagmatiche grammaticali}

Dopo questa esposizione generale del nostro sistema di relazioni sintagmatiche, passiamo a discutere più in dettaglio le singole tipologie di relazioni sintagmatiche della sottolingua. Iniziamo con la discussione delle relazioni sintagmatiche grammaticali, e innanzitutto dei casi profondi della sottolingua.

Il caso o ruolo di agente o agentivo è quello relativo all'entità che compie volontariamente un'azione, ed è invariabilmente relativo a un'entità (collettiva o individuale) animata, a differenza di quanto avviene nel GRIS; ${ }^{354}$ suo caso reciproco è quello deagentivo (l'attività coinvolta dall'agente). Un esempio di relazione sintagmatica agentiva/deagentiva è "[deagentivo:] Terrorismo - [agentivo:] Stato" ovvero "Terrorismo di Stato". Riferendoci al modello concettuale, possiamo dire che la relazione sintagmatica agentiva/deagentiva può coinvolgere: Persone, Enti, Famiglie, Tipi di persona, Tipi di ente, Altre entità (animali, tipi di animale, personaggi fittizi e leggendari), nel ruolo agentivo, Altre entità (tipi di evento) nel ruolo deagentivo.

Il caso o ruolo oggettivo o di oggetto è quello relativo all'oggetto del compimento di un'azione, e nella nostra semantica sintagmatica copre anche ciò che nel sistema dei casi profondi di Fillmore è il caso fattitivo (relativo al prodotto); suo caso reciproco è quello deoggettivo (l'attività che coinvolge l'oggetto). Un esempio di relazione sintagmatica oggettiva/ deoggettiva è "[deoggettivo:] Coltivazione - [oggettivo:] Grano" ovvero "Coltivazione del grano". A differenza del sistema di Fillmore, inoltre, in cui il caso oggettivo è svolto necessariamente da un'entità, nel nostro sistema di relazioni sintagmatiche il caso oggettivo può essere svolto anche da un'attività, per esempio "[deoggettivo:] Valutazione - [oggettivo:] Educazione", ovvero "Valutazione dell'educazione"; in questi casi, il ruolo oggettivo può a sua volta reggere altri ruoli oggettivi o d'altro tipo, secondo il sistema a più livelli gerarchici sopra esposto (p.e. "[deoggettivo:] Valutazione - [oggettivo: deoggettivo:] Educazione - [oggettivo: oggettivo:] Disabili", ovvero "Valutazione dell'educazione dei disabili"). Avevamo accennato sopra come già nel sistema dei casi di Fillmore vi possa essere, per le attività (Eventi e Altre entità: tipi di evento), un'ambiguità tra caso oggettivo e caso locativo; un esempio relativo ai tipi di evento è quello dei sintagmi "[deoggettivo:] Bombardamenti - [oggettivo:] Sicilia", ovvero "Bombardamenti della Sicilia" e "[delocativo:] Bombardamenti - [locativo:] Sicilia", ovvero "Bombardamenti in Sicilia"; la Sicilia può in questi

354 ASSOCIAZIONE ITALIANA BIBLIOTECHE. GRUPPO DI RICERCA SULL'INDICIZZAZIONE PER SOGGETTO, Guida all'indicizzazione per soggetto, cit., p. 34. 
casi essere cioè vista o come l'oggetto dell'attività di bombardamento, o come il suo luogo; un esempio di sovrapposizione relativo a un Evento è quello di "Alluvione di Firenze" (relazione sintagmatica oggettiva) che è anche "Alluvione a Firenze" (relazione sintagmatica locativa); nel linguaggio naturale, tali significati restano distinti benché strettamente correlati; nella lingua documentaria, l'opportunità di una loro distinzione è invece controversa. Si tratta di un punto profondamente problematico dell'applicazione dei casi profondi alle lingue documentarie. Inoltre, come vedremo l'estensione da noi scelta delle relazioni sintagmatiche grammaticali a relazioni di tipo diverso dai casi profondi avrà come conseguenza il crearsi di più di una situazione di questo tipo. Non c'è una soluzione perfetta a questo tipo di problemi, ma piuttosto una serie di espedienti necessariamente artificiosi e imperfetti; pur non fornendo una risposta definitiva a questo tipo di situazioni in cui vi è una sostanziale sovrapposizione tra relazioni sintagmatiche, abbozziamo alcune possibilità di trattazione di questo tipo di situazione (a valere anche per altre situazioni simili): 1) i due diversi tipi di relazioni sintagmatiche vengono considerate distinte (così come i relativi sintagmi): è la soluzione più corretta sotto il profilo linguistico, ma dal punto di vista delle esigenze dell'indicizzazione comporterebbe una complessità quasi sempre inutile; 2) scelta arbitraria e convenzionale tra le due tipologie di relazioni (e relativi sintagmi); per esempio si può scegliere di rappresentare un determinato tipi di sintagma sempre tramite una relazione sintagmatica oppure sempre secondo l'altra: è una scelta che non ha una corrispondenza nel linguaggio naturale, e che comporta la necessità di stabilire criteri in base a cui operare la scelta tra i due tipi di relazione sintagmatica coinvolta; ha però il vantaggio di mantenere la coerenza e la semplicità della struttura della lingua documentaria; 3) un unico sintagma con una doppia registrazione delle relazioni sintagmatiche (e delle corrispondenti relazioni gerarchiche specificate): scelta che garantisce la coerenza della struttura della lingua documentaria senza soluzioni artificiose ma a prezzo di una maggiore complessità e senza evidenti vantaggi per l'utente finale. Per concludere con il caso oggettivo, e riferendoci alle entità del modello concettuale, possiamo dire che esso può riguardare relazioni tra: Altre entità (tipi di evento) come caso deoggettivo e Concetti, Testi, Risorse, Persone, Enti, Famiglie, Luoghi, Tempi, Lingue, Eventi, Tipi di persona, Tipi di ente, Tipi di testo, Tipi di risorsa, Oggetti e Altre entità (tipi di oggetto, tipi di animale, tipi di evento, etc.) come caso oggettivo.

Il caso o ruolo strumentale o di strumento è quello relativo all'entità che serve involontariamente al compimento di un'azione, e che è generalmente un'entità (collettiva o individuale) inanimata, con l'eccezione degli animali che possono svolgere un caso strumentale; suo caso reciproco è il caso destrumentale (attività coinvolta dallo strumento). A livello morfosintattico di superficie, il caso strumentale può presentarsi con espressioni come "in", "nel”, "tramite", "per mezzo di", etc. Un esempio di relazione sintagmatica strumentale/destrumentale è "[destrumentale:] Formazione - [strumentale:] Internet" ovvero "Formazione tramite internet". Se ci riferiamo alle entità del modello concettuale, la relazione sintagmatica strumentale/destrumentale può riguardare: Altre entità (tipi di evento) per il caso destrumentale, Concetti, Tipi di testo, Tipi di risorsa, Altre entità (tipi di animale, tipi di oggetto, tipi di evento) per il caso strumentale.

Il caso o ruolo causativo o di causa è quello relativo all'entità (inanimata) o attività che provoca involontariamente il compimento di un processo; a differenza dello strumento, non può essere combinato con un agente; il caso reciproco è il caso decausativo (l'attività coinvolta dalla causa, che ne costituisce l'effetto). Un esempio di relazione sintagmatica 
causativa/decausativa è "[decausativo:] Cancro - [causativo:] Fumo", ovvero "Cancro da fumo". Come abbiamo accennato più sopra (vedi 3.3.6.4.2.2.3), la relazione sintagmatica causativa/decausativa è una relazione causa-effetto, ma diversa da quelle che sono rappresentate, generalmente come relazioni associative, dalle lingue documentarie: mentre quelle esprimono generalmente la relazione tra una causa e il suo effetto tipico (a priori) o viceversa, la relazione sintagmatica causativa/decausativa della nostra semantica sintagmatica prescinde da valutazioni sul carattere tipico o meno che lega l'elemento lessicale che svolge il caso causativo e quello che svolge il caso decausativo.

Il caso o ruolo benefattivo o di beneficiario è quello relativo al beneficiario di un'azione (o entità, vedi sotto), e riguarda invariabilmente entità (collettive e inviduali) animate (anche Luoghi, se usati nell'accezione della comunità relativa a un Luogo); suo caso reciproco è il caso debenefattivo; a differenza di quanto previsto dal sistema dei casi profondi di Fillmore, nel nostro il sistema il caso debenefattivo può essere non soltanto un'attività, ma qualsiasi entità rivolta al beneficiario; quindi, esempi di relazione sintagmatica benefattiva/debenefattiva sono non soltanto relazioni del tipo "[debenefattivo:] Sport - [benefattivo:] Bambini", ovvero "Sport per bambini", ma anche relazioni del tipo "[debenefattivo:] Ospedali - [benefattivo:] Bambini", ovvero "Ospedali per bambini" (cioè ospedali pediatrici). Se ci riferiamo alle entità del modello concettuale, la relazione sintagmatica benefattiva/debenefattiva può riguardare: per quanto riguarda le relazioni tra un medesimo tipo di entità: Tipi di persona e Tipi di ente; per quanto riguarda relazioni tra tipi differenti di entità: Tipi di testo (debenefattivo) e Tipi di persona (benefattivo); Tipi di testo (debenefattivo) e Tipi di ente (benefattivo); Tipi di risorsa (debenefattivo) e Tipi di persona (benefattivo); Tipi di risorsa (debenefattivo) e Tipi di ente (benefattivo); Altre entità (tipi di evento, tipi di animale) e Tipi di persona; Altre entità (tipi di evento, tipi di animale) e Tipi di ente.

Il caso o ruolo funzionale o di funzione è quello relativo a un'entità o attività che rappresenti la destinazione di un'entità (collettiva, sia animata che inanimata); suo caso reciproco è il caso defunzionale (l'entità che ha una certa destinazione). Si tratta di un caso profondo concettualmente simile al beneficiario, ma rivolto a tipologie lessicali diverse da entità animate. Nel nostro sistema di casi profondi, il caso funzionale è un po' anomalo, perché è l'unico in cui il caso reciproco (defunzionale) non possa essere svolto da un'attività, ma solo da un'entità. Esempi di relazioni sintagmatiche funzionali/defunzionali sono "[defunzionale: Canne - [funzionale:] Pesca" ovvero "Canne da pesca", ma anche "[defunzionale:] Strutture - [funzionale:] Edifici civili" ovvero "Strutture per edifici civili". 355 Il caso o ruolo locativo o di stato in luogo è quello relativo alla collocazione spaziale dell'azione (o di un'entità, vedi sotto), e indica uno stato in luogo; suo caso reciproco è il caso delocativo. A differenza di quanto previsto dal sistema dei casi profondi di Fillmore, e come già anticipato, nel nostro sistema di casi profondi il caso locativo può riguardare anche la collocazione spaziale di un'entità. In questa prospettiva, sono esempi di relazioni sintagmatiche locative/delocative "[delocativo:] Educazione - [locativo:] Italia" ovvero

355 ASSOCIAZIONE ITALIANA BIBLIOTECHE. GRUPPO DI RICERCA SULL'INDICIZZAZIONE

PER SOGGETTO, Guida all'indicizzazione per soggetto, cit., p. 43, considera sintagmi come "impianti sportivi per le scuole" e "strutture in acciaio per edifici civili" come relazioni sintagmatiche partitive (e non strumentali o benefattive), sulla base del criterio che: 1) l'intero e la parte appartengono allo stesso tipo di elemento lessicale; 2) la testa del sintagma può essere effettivamente considerata una parte dell'elemento che funge da modificare; relazioni introdotte da "per" che però non soddisfino le due suddette condizioni rientrerebbero nella relazione sintagmatica strumentale. 
"Educazione in Italia", ma anche "[delocativo:] Biblioteche - [locativo:] Italia", ovvero "Biblioteche in Italia" o "Biblioteche italiane". Riferendoci al modello concettuale, la relazione sintagmatica locativa/delocativa può riguardare: Luoghi, per quanto riguarda il caso locativo, e Tipi di testo, Tipi di risorsa, Enti, Tipi di persona, Tipi di ente, Lingue, Concetti, Altre entità (tipi di oggetto, tipi di animale, tipi di evento, etc.), ed Eventi, per quanto riguarda il caso delocativo.

Inoltre, nel nostro sistema sintagmatico, e in linea con quanto detto nella precedente sezione (vedi 3.2.7), il caso locativo può essere svolto non soltanto una località geografica, ma anche una collocazione spaziale in senso astratto, un ambiente (che può essere un'attività o un'entità) in cui ha luogo qualche fenomeno. Parleremo in queste situazioni di caso "locativo concettuale" o relazione sintagmatica locativa concettuale: esempi ne sono "[delocativo:] Sessismo - [locativo:] Politica", ovvero "Sessismo in politica" o "Sessismo nella politica", o ancora "[delocativo:] Donne - [locativo:] Politica", ovvero "Donne in politica". Se ci riferiamo alle entità del modello concettuale, la relazione sintagmatica locativa/delocativa di questo tipo può riguardare: Concetti, Tipi di persona, Tipi di ente e Altre entità (tipi di evento) per quanto riguarda il caso delocativo; Tipi di testo, Tipi di risorsa, Tipi di persona, Tipi di ente, Concetti, Altre entità (tipi di evento, tipi di oggetto, tipi di animale) per quanto riguarda il caso locativo.

Abbiamo già detto della possibile sovrapposizione tra relazioni sintagmatiche locative e oggettive per alcuni tipi di evento o processo. La relazione sintagmatica locativa/delocativa può inoltre sovrapporsi alla relazione sintagmatica partitiva, in alcuni casi in cui entrambi i termini della relazione siano entità (non attività). La problematicità dei confini tra relazione sintagmatica locativa e partitiva è notata anche dal GRIS. Per esempio, i "Fiumi in Sicilia" (relazione locativa) sono anche in "Fiumi della Sicilia" (relazione sintagmatica partitiva, vedi sotto); gli "Edifici a Roma" sono anche gli "Edifici di Roma". Come nel caso della sovrapposizione tra relazioni sintagmatiche oggettive e locative siamo al cospetto di una situazione in cui la scelta tra una relazione o l'altra è teoricamente problematica; e come in quel caso le soluzioni sono dello stesso tipo. In questo caso riteniamo opportuno che la relazione sintagmatica partitiva sia da riservare soltanto a elementi lessicali relativi a entità individuali (p.e. "[meronimico:] Centro storico - [olonimico:] Palermo", ovvero "Centro storico di Palermo"), e che in tutti gli altri casi sia da preferire la scelta della relazione sintagmatica locativa (p.e. "[delocativo:] Centri storici - [locativo:] Sicilia" ovvero "Centri storici della Sicilia" o "Centri storici in Sicilia"); tale criterio coincide in sostanza con uno dei due suggeriti dal GRIS per risolvere la problematicità dei confini tra relazione sintagmatica locativa e partitiva, l'altro essendo quello della natura dell'entità: GRIS suggerisce di trattare come relazioni sintagmatiche locative tutte le relazioni del tipo sopra menzionato che riguardino entità di tipo diverso. ${ }^{356}$

Il caso o ruolo originario o di origine o di fonte è quello relativo alla provenienza spaziale dell'attività (o dell'entità, nel nostro sistema), e indicativo di moto da luogo; il caso reciproco è il caso deoriginario (attività o entità con una certa provenienza spaziale). Un esempio di relazione sintagmatica originaria/deoriginaria è "[deoriginario:] Deportati [originario:] Italia", ovvero "Deportati dall'Italia".

Il caso o ruolo direttivo o di direzione o meta è il caso relativo alla direzione spaziale spaziale dell'attività (o dell'entità, nel nostro sistema), e indicativo di moto a luogo; il caso re-

356 ASSOCIAZIONE ITALIANA BIBLIOTECHE. GRUPPO DI RICERCA SULL'INDICIZZAZIONE PER SOGGETTO, Guida all'indicizzazione per soggetto, cit., p. 50. 
ciproco è il caso dedirettivo (attività o entità con una certa direzione spaziale). Un esempio di relazione sintagmatica direttiva/dedirettiva è: "[dedirettivo:] Deportati - [direttivo:] Germania", ovvero "Deportati in Germania".

Il caso o ruolo temporale è quello che indica la circostanza temporale di un'azione (o di un'entità, nel nostro sistema); il caso reciproco è il caso detemporale (attività o entità che ha luogo in una determinata circostanza temporale). Un esempio di relazione sintagmatica temporale/detemporale è "[detemporale] Arte - [temporale:] Ottocento" ovvero "Arte dell'Ottocento" o "Arte ottecentesca". Riferendoci alle entità del modello concettuale, la relazione sintagmatica temporale/detemporale può riguardare un Tempo (caso

temporale) in relazione con: Tipi di testo, Tipi di risorsa, Enti, Famiglie, Tipi di persona, Tipi di ente, Luoghi, Lingue, Eventi, Concetti, Altre entità (caso detemporale).

Con la discussione del caso temporale si chiude la discussione dei casi profondi della nostra semantica sintagmatica e si passa alle relazioni sintagmatiche grammaticali di tipo diverso - anche se, solo per comodità, continueremo a parlare di casi.

Il caso o ruolo possessivo o di proprietà o possesso è un caso di natura residuale relativo a un oggetto o a un'entità collettiva dipendente da un'altra entità o attività; il caso reciproco è il caso depossessivo (possessore di un'entità, può essere un oggetto o un'entità collettiva). Un esempio di relazione sintagmatica possessiva/depossessiva è "[depossessivo:] Durezza - [possessivo:] Metalli”, ovvero "Durezza dei metalli”, oppure "[depossessivo:] Beni [possessivo:] Chiesa", ovvero "Beni della Chiesa". Il caso depossessivo non può reggere altri casi profondi o d'altro tipo. Riferendoci alle entità del modello concettuale, la relazione sintagmatica possessiva/depossessiva può riguardare Concetti, Oggetti e Altre entità (tipi di oggetto) che svolgano un caso depossessivo e: Testi, Risorse, Tipi di Testo, Tipi di risorsa, Persone, Enti, Famiglie, Tipi di persona, Tipi di ente, Luoghi, Tempi, Lingue, Eventi, Concetti e Altre entità che svoltano un caso possessivo.

Infine, anche nel caso della relazione sintagmatica possessiva/depossessiva è possibile una sovrapposizione con la relazione sintagmatica partitiva, così come segnalato anche da GRIS. Esempi di casi di sovrapposizione tra relazioni sintagmatiche partitive e possessive sono sintagmi come "Popolazione dell'Italia" o quello degli enti in forma gerarchica come "Biblioteca dell'Università di Firenze". Anche in questo caso valgono problemi e possibili soluzioni proposte più sopra, ma con una preferenza per le relazioni sintagmatiche partitive rispetto alle relazioni sintagmatiche possessive: GRIS raccomanda di preferire la relazione sintagmatica partitiva alla relazione sintagmatica possessiva almeno in tutti quei casi in cui non sia possibile predicare un'autentica relazione di possesso, per esempio nel caso sopra menzionato, in cui l'Italia non possiede autenticamente la popolazione; ${ }^{357}$ anche per quanto riguarda il caso degli enti gerarchici riteniamo che anch'essi vadano considerati come elementi lessicali legati da relazioni sintagmatiche partitive e non possessive.

Passiamo a discutere la relazione sintagmatica partitiva, o di partonomia o partonomica. Essa non differisce nella sostanza dalla omologa relazione paradigmatica: il caso olonimico indica l'intero rispetto alle sue parti; il caso reciproco (meronimico) indica la parte; all'interno del nostro approccio, sia il caso olonimico sia quello meronimico possono riguardare soltanto entità. Esempio di relazioni sintagmatiche olonimiche/meronimiche sono "[meronimico:] Motori - [olonimico:] Automobili" ovvero "Motori di automobili", e "[meronimico:] Personale - [olonimico:] Biblioteche" ovvero "Personale delle biblioteche".

357 ASSOCIAZIONE ITALIANA BIBLIOTECHE. GRUPPO DI RICERCA SULL'INDICIZZAZIONE PER SOGGETTO, Guida all'indicizzazione per soggetto, cit., p. 51. 
Relazioni sintagmatiche partitive sono anche quelle che riguardino elementi lessicali in forma composta relativi a entità individuali (forme parzialmente collegate) come "[meronimico:] Biblioteca - [olonimico:] CGIL” ovvero "Biblioteca della CGIL”. Relazioni sintagmatiche partitive e relazioni (paradigmatiche) partitive pure sono mutualmente esclusive; ribadiamo anche che le relazioni sintagmatiche partitive sono invece correlate alle relazioni eponimiche e semiche/sememiche partitive. Ripetiamo che in diversi casi possono esservi sovrapposizioni tra relazioni sintagmatiche partitive e d'altro tipo (locative, possessive, etc.), da risolvere secondo le indicazioni già fornite.

Un'altra relazione sintagmatica grammaticale importante della sottolingua è la relazione sintagmatica eponimica (iperonimica o iponimica) atipica, o semplicemente relazione sintagmatica gerarchica atipica, chiamata "relazione di classe quasi generica" da PRECIS e GRIS. Essa ha una diretta corrispondenza con la sua omologa relazione (eponimica e se$\mathrm{mica} / \mathrm{sememica}$ ) paradigmatica: indica la relazione tra un elemento e una prospettiva secondo cui può essere visto ovvero un insieme o classe in cui può essere compreso, e che però non siano quelli tipici in un dato contesto. Parleremo rispettivamente di caso iponimico atipico per l'individuo e di caso iperonimico atipico per la classe; sia il caso iponimico atipico sia il caso iperonimico atipico possono coinvolgere tanto entità quanto attività. Esempio di relazione sintagmatica eponimica atipica è "[iponimico atipico:] Acqua - [iperonimico atipico:] Combustibile" ovvero "Acqua come combustibile". La relazione sintagmatica eponimica atipica è l'unico tipo di relazione eponimica che può comparire sul piano sintagmatico. Come abbiamo già accennato, gli elementi lessicali che presentano una relazione sintagmatica eponimica atipica hanno soltanto forme composte, e soltanto non collegate o totalmente collegate; esse sono concettualmente incompatibili con forme lessicali semplici. Le forme composte non collegate hanno una forma inevitabilmente artificiosa ("Acqua come combustibile", "Granchi in quanto cibo", etc.). Riferendoci alle entità del modello concettuale, la relazione sintagmatica eponimica atipica può riguardare: 1) per quanto riguarda relazioni tra un medesimo tipo di entità: Tipi di persona, Altre entità (tipi di oggetto, tipi di animale, tipi di evento) e Concetti; 2) per quanto riguarda relazioni tra tipi diversi di entità: Testi (caso iponimico atipico) e Concetti, Altre entità o Tipi di testo (caso iperonimico atipico), come in "Bibbia come fonte storica" o "Bibbia come letteratura"; o ancora qualsiasi tipo di entità (caso iponimico atipico) e Concetti o Altre entità (caso iperonimico atipico). Così come per altre relazioni sintagmatiche, sia il caso iponimico atipico che il caso iperonimico atipico possono essere composti da più elementi lessicali tra loro in relazione. Un caso tipico di relazione sintagmatica eponimica atipica con il caso iperonimico atipico costituito da una combinazione di elementi lessicali (tra loro in relazione sintagmatica possessiva/depossessiva) è quello dei temi letterari o artistici, per esempio "[iponimico atipico:] Donne - [iperonimico atipico: depossessivo:] Tema [iperonimico atipico: possessivo:] Letteratura", cioè "Donne come tema letterario" ovvero "Donne nella letteratura".

\subsection{Relazioni sintagmatiche logiche}

Con la discussione della relazione sintagmatica eponimica atipica si chiude la nostra discussione delle relazioni sintagmatiche grammaticali e si passa alla discussione delle relazioni sintagmatiche logiche. Di esse abbiamo già parlato nella sezione precedente (vedi 
3.2.8): si tratta di relazioni di natura fondamentalmente sintattica, perché esclusivamente funzionali alla costruzione di sintagmi e che non hanno alcuna corrispondenza a livello paradigmatico; anch'esse non sono tuttavia esenti dai vincoli posti dalla semantica paradigmatica. Come abbiamo anticipato, il sistema di organizzazione sintagmatico logico non è alternativo ma piuttosto si sovrappone a quello sintagmatico grammaticale: in alcuni sintagmi sono presenti esclusivamente relazioni sintagmatiche grammaticali; in altri solo relazioni sintagmatiche logiche; in altri ancora una loro combinazione. Oltre alla relazione di coordinazione, la semantica sintagmatica della sottolingua prevede un'ulteriore tipologia di relazione logica sintagmatica, la relazione di interazione.

La relazione sintagmatica di coordinazione, prevista anche da PRECIS e GRIS, ${ }^{358}$ è quella relazione sintagmatica logica che lega due o più elementi che svolgono lo stesso caso grammaticale (caso profondo o d'altro tipo); è una relazione non orientata, ovvero non gerarchizzata: non vi è subordinazione o sovraordinazione tra i termini di una relazione di coordinazione, ma pariteticità. Inoltre, per poter essere legati da una relazione di coordinazione, due elementi lessicali devono appartenere alla medesima tipologia lessicale. Infine, ricordiamo che nella sottolingua proposta è prevista la possibilità di una relazione di coordinazione (all'interno dello stesso elemento lessicale in forma composta) di co-iponimi, co-meronimi e co-sememi. Esempi di relazione di coordinazione sono "[delocativo:] Educazione - [locativo: coordinato] Italia - [locativo: coordinato] Francia" ovvero "Educazione in Italia e in Francia", e "[delocativo: coordinato] Didattica - [delocativo: coordinato] Ricerca - [locativo:] Italia" ovvero "Didattica e ricerca in Italia".

Concludiamo la nostra trattazione della semantica sintagmatica della sottolingua con la relazione (sintagmatica) di interazione. La relazione di interazione è una relazione semantica caratteristica, sotto differenti forme e denominazioni, di diverse lingue documentarie. ${ }^{359}$ Essa non è distinta in quanto tale dalla linguistica, ma è una relazione che ritroviamo già nel linguaggio naturale e che esprime l'interazione o azione reciproca tra due o più elementi lessicali componenti il sintagma. Esempi di relazione di interazione nel linguaggio naturale sono "Stato e Chiesa", "Fede e ragione", ma anche "Relazioni diplomatiche tra Italia e Francia", "Confini dell'Italia e dell'Austria", etc. A rigor di termini non si tratterebbe di una relazione sintagmatica logica, ma piuttosto di una relazione sintagmatica grammaticale, tuttavia nel nostro modello di sottolingua la consideremo come una relazione sintagmatica logica. Questo tipo di relazione è non solo esterna, ma anche anomala rispetto al sistema dei casi profondi di Fillmore: in essa, infatti, gli elementi lessicali coinvolti assumono al contempo il caso agentivo e oggettivo, in modo reciproco, tramite un terzo elemento lessicale che funge da caso interattivo e che connette i due elementi lessicali componenti tra loro in relazione di interazione. Per esempio: "[deagentivo + deoggettivo:] Commercio - [agentivo: interattivo: oggettivo] Italia - [oggettivo: interattivo: agentivo:] Francia", ovvero "Commercio tra Italia e Francia" o "Commercio tra Francia e Italia" o ancora "Commercio dell'Italia con la Francia" o "Commercio della Francia con l'Italia". Nel caso in cui l'elemento che ha funzione di mettere in relazione i termini della relazione di interazione sia di natura assolutamente generica, esso può anche rimanere implicito

358 ASSOCIAZIONE ITALIANA BIBLIOTECHE. GRUPPO DI RICERCA SULL'INDICIZZAZIONE PER SOGGETTO, Guida all'indicizzazione per soggetto, cit., p. 56-57.

359 Anche GRIS riconosce, come "categoria particolare di azioni" (attivitità) l'interazione, e specifica che è una azione reciproca "tra concetti che svolgono contemporaneamente le funzioni di agente e di oggetto" (ASSOCIAZIONE ITALIANA BIBLIOTECHE. GRUPPO DI RICERCA SULL'INDICIZZAZIONE PER SOGGETTO, Guida all'indicizzazione per soggetto, cit., p. 30). 
Tesi di dottorato di Giuliano Genetasio, discussa presso l'Università degli Studi di Udine

nelle forme non collegate dell'elemento lessicale, oppure essere esplicitato. Per esempio: "[deagentivo + deoggettivo:] Relazioni - [agentivo: interattivo: oggettivo] Stato - [oggettivo: interattivo: agentivo:] Chiesa", ovvero "Stato e Chiesa" o "Chiesa e Stato", "Relazioni tra Stato e Chiesa”, etc. 


\title{
Lista riassuntiva delle relazioni semantiche della sottolingua
}

\author{
Relazioni paradigmatiche \\ Relazioni gerarchiche \\ Relazioni eponimiche pure \\ Relazioni partonomiche pure \\ Relazioni eponimiche, semiche e sememiche specificate \\ Relazione iperonimica deagentiva, relazione iponimica agentiva \\ Relazione iperonimica deoggettiva, relazione iponimica oggettiva \\ Relazione iperonimica destrumentale, relazione iponimica strumentale \\ Relazione iperonimica debenefattiva, relazione iponimica benefattiva \\ Relazione iperonimica decausativa, relazione iponimica causativa \\ Relazione iperonimica defunzionale, relazione iponimica funzionale \\ Relazione iperonimica delocativa, relazione iponimica locativa \\ Relazione iperonimica deoriginaria, relazione iponimica originaria \\ Relazione iperonimica dedirettiva, relazione iponimica direttiva \\ Relazione iperonimica detemporale, relazione iponimica temporale \\ Relazione iperonimica depossessiva, relazione iponimica possessiva \\ Relazione iperonimica meronimica, relazione iponimica olonimica \\ Relazione iperonimica atipica, relazione iponimica atipica* \\ Relazione semica agentiva, relazione sememica deagentiva \\ Relazione semica oggettiva, relazione sememica deoggettiva \\ Relazione semica strumentale, relazione sememica destrumentale \\ Relazione semica benefattiva, relazione sememica debenefattiva \\ Relazione semica causativa, relazione sememica decausativa \\ Relazione semica funzionale, relazione sememica defunzionale \\ Relazione semica locativa, relazione sememica delocativa \\ Relazione semica originaria, relazione sememica deoriginaria \\ Relazione semica direttiva, relazione sememica dedirettiva \\ Relazione semica temporale, relazione sememica detemporale \\ Relazione semica possessiva, relazione sememica dedepossessiva \\ Relazione semica olonimica, relazione sememica meronimica \\ Relazione semica atipica, relazione sememica atipica.
}

Relazioni co-iponimiche, co-meronimiche e co-sememiche*

Relazioni tra nominalizzazioni

Relazioni di antonimia

scalare $^{* *}$

polare

complementare

Relazioni di equivalenza***

non controllate 
Tesi di dottorato di Giuliano Genetasio, discussa presso l'Università degli Studi di Udine

monocontrollate

bicontrollate

Relazioni di omonimia, polisemia e omotassia***

Relazioni ad hoc

Relazioni sintagmatiche

grammaticali

Casi profondi

Relazione sintagmatica agentiva, relazione sintagmatica deagentiva

Relazione sintagmatica strumentale, relazione sintagmatica destrumentale

Relazione sintagmatica causativa, relazione sintagmatica decausativa

Relazione sintagmatica benefattiva, relazione sintagmatica debenefattiva

Relazione sintagmatica funzionale, relazione sintagmatica defunzionale

Relazione sintagmatica locativa, relazione sintagmatica delocativa

Relazione sintagmatica originaria, relazione sintagmatica deoriginaria

Relazione sintagmatica direttiva, relazione sintagmatica dedirettiva

Relazione sintagmatica temporale, relazione sintagmatica detemporale

Relazione sintagmatica possessiva, relazione sintagmatica depossessiva

Relazione sintagmatica olonimica, relazione sintagmatica meronimica

Relazione sintagmatica iperonimica atipica, relazione sintagmatica iponimica atipica logiche

Relazione di coordinazione

Relazione di interazione

* Trattate implicitamente nel nostro modello di lingua documentaria, con l'eccezione delle relazioni tra nominalizzazioni

** Opzionalmente, la relazione di antonimia scalare può essere trattata come relazione di equivalenza

*** Opzionalmente, è possibile differenziare le relazioni di equivalenza sulla base della tipologia di sinonimia o equivalenza semantica

**** Trattate implicitamente nel nostro modello di lingua documentaria 
Tesi di dottorato di Giuliano Genetasio, discussa presso l'Università degli Studi di Udine

\section{Figure}

Fig. A

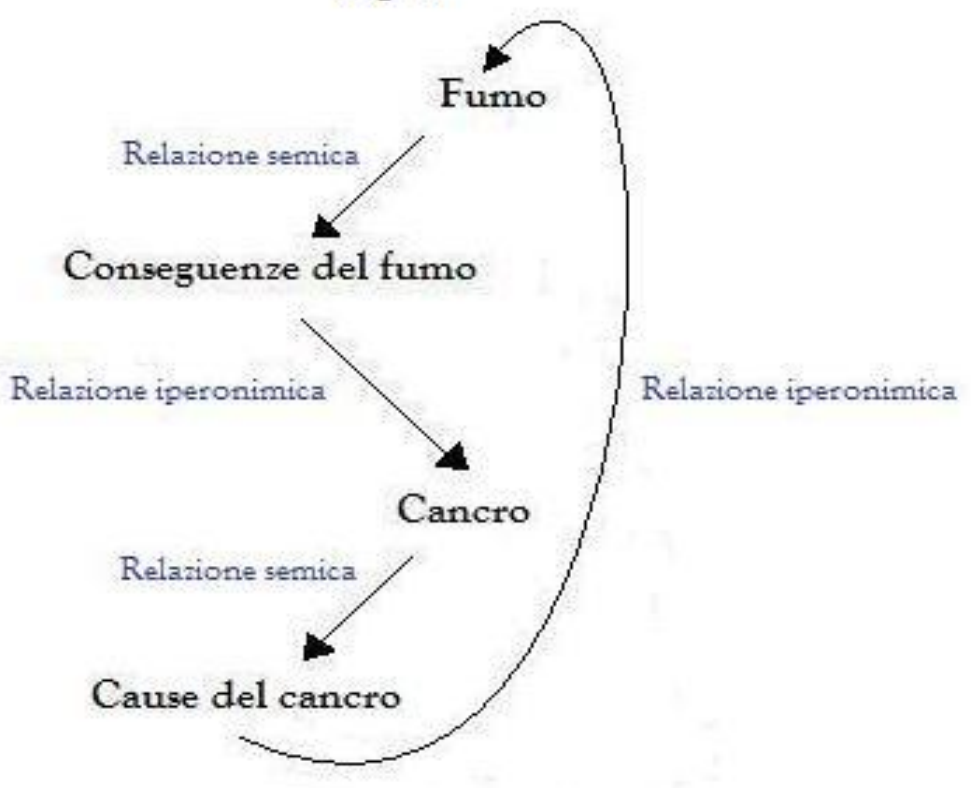


Tesi di dottorato di Giuliano Genetasio, discussa presso l'Università degli Studi di Udine

Fig. $B 1$

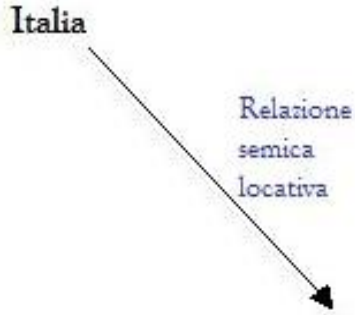

Coltivazione in Italia

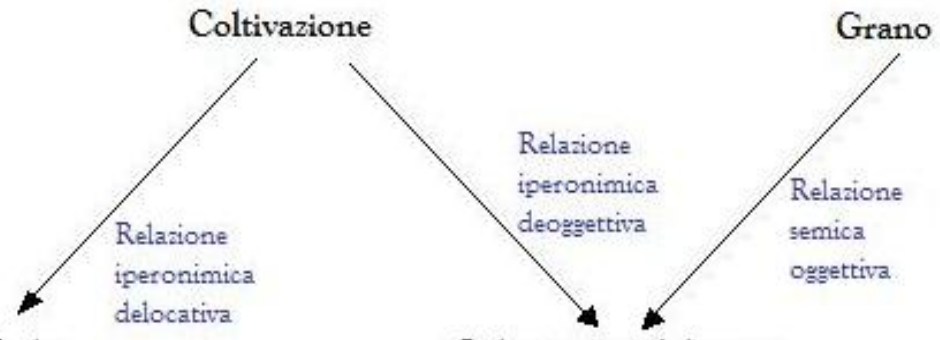

Coltivazione del grano

Fig. B2

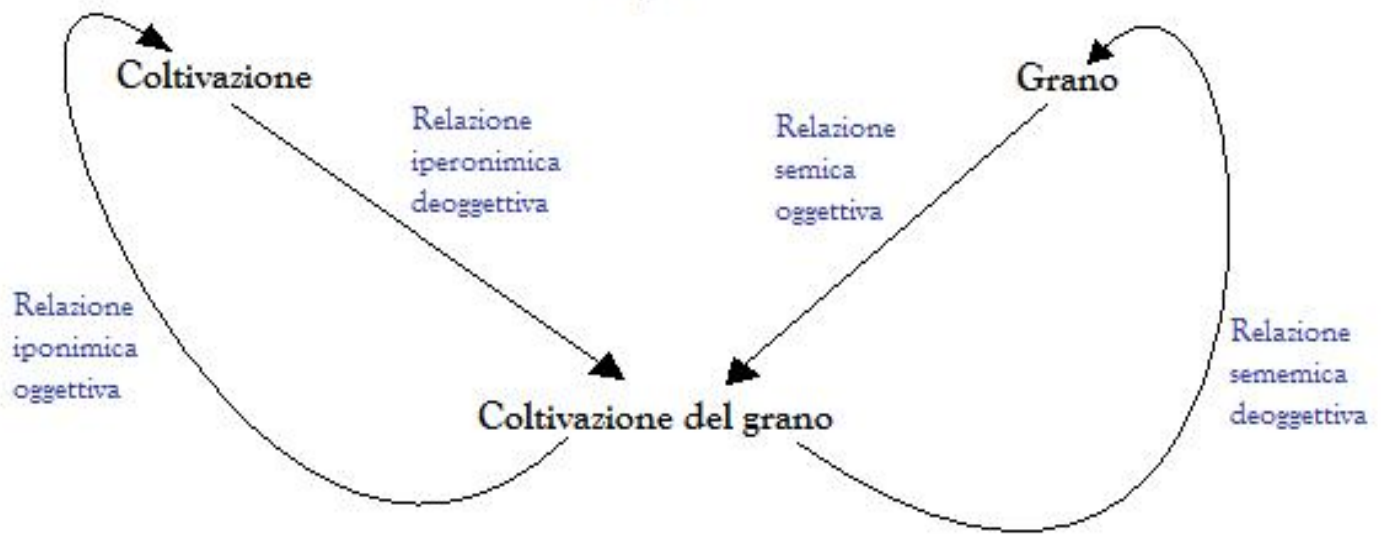


Tesi di dottorato di Giuliano Genetasio, discussa presso l'Università degli Studi di Udine

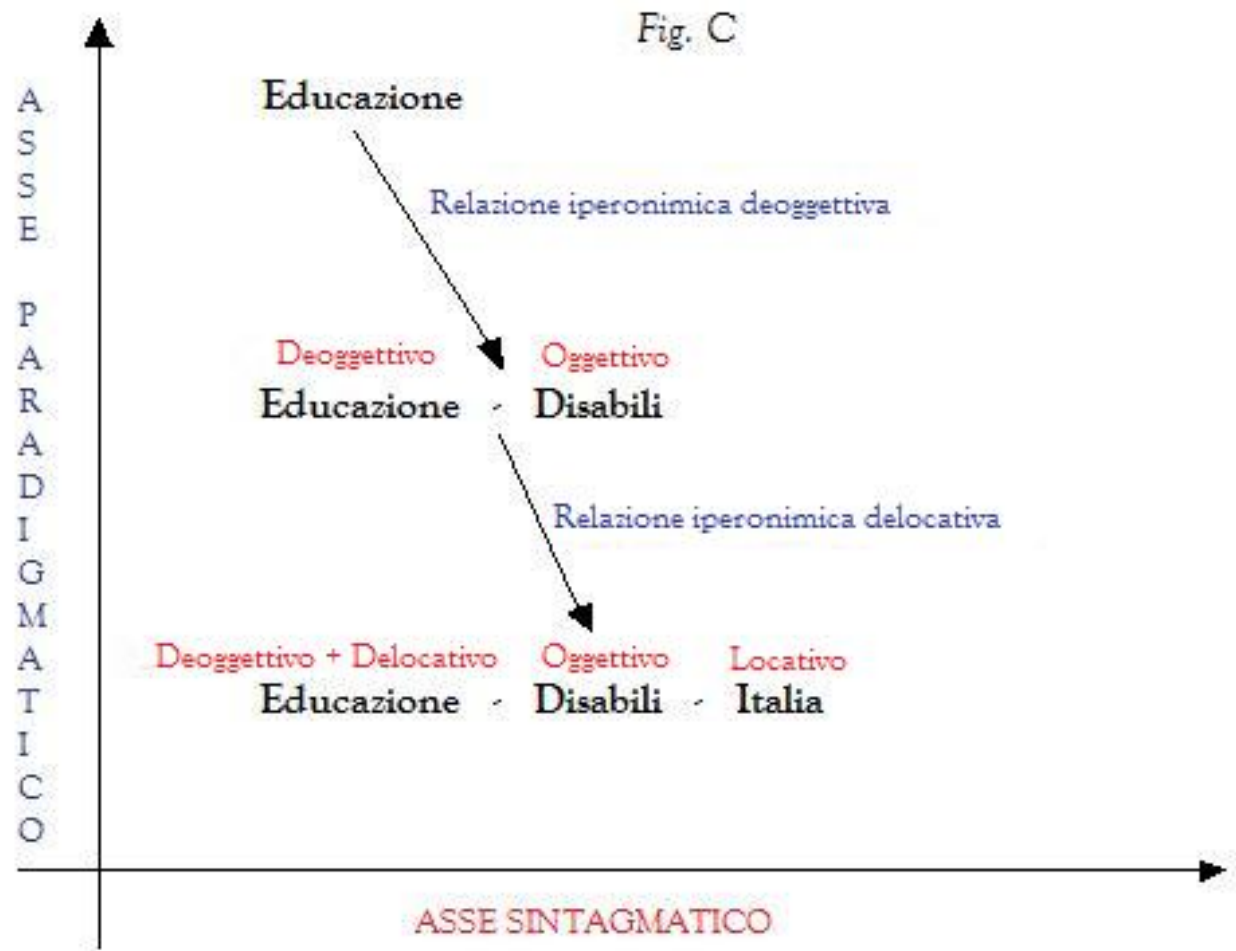

Fig. D

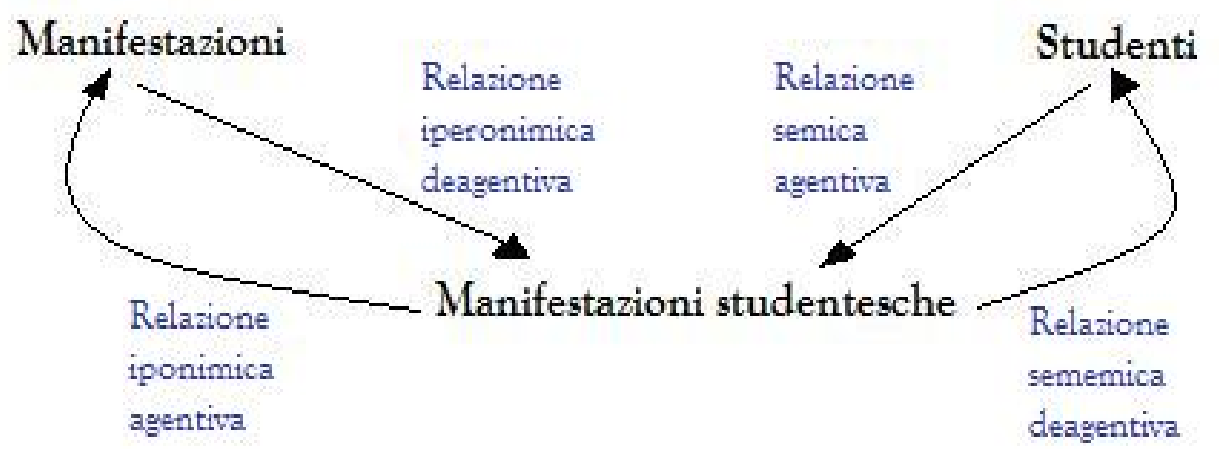


Tesi di dottorato di Giuliano Genetasio, discussa presso l'Università degli Studi di Udine

Fig. E1

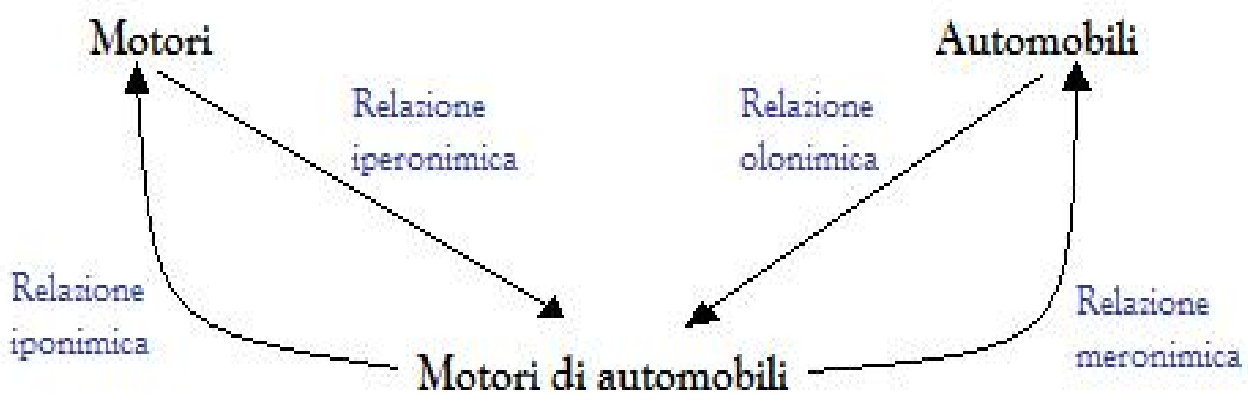

Fig. E2

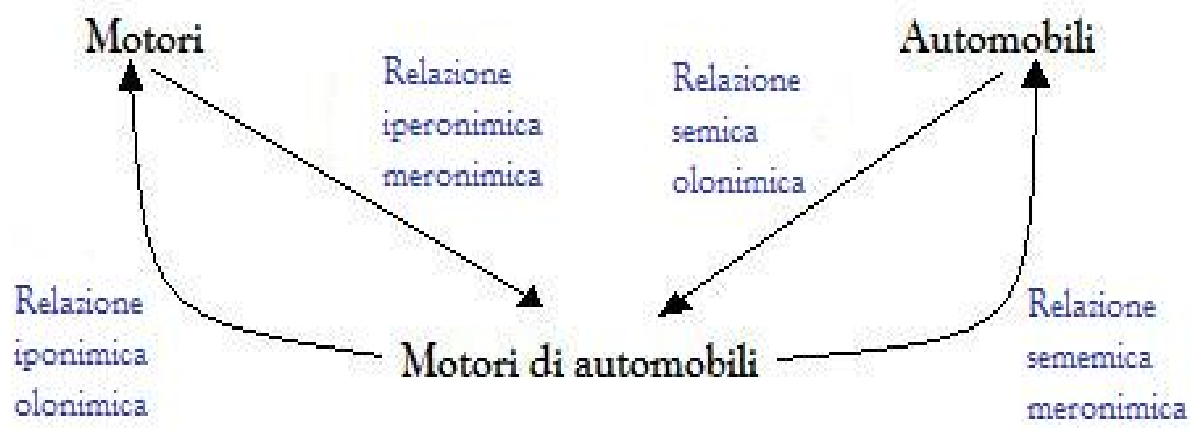


Tesi di dottorato di Giuliano Genetasio, discussa presso l'Università degli Studi di Udine

\section{Fig. F1}

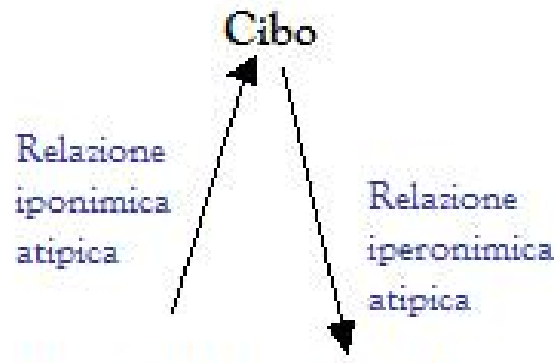

Granchi in quanto cibo
Fig. F2

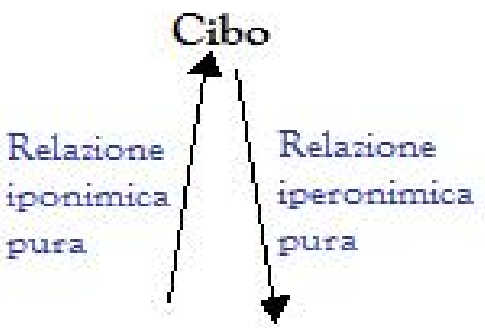

Granchi

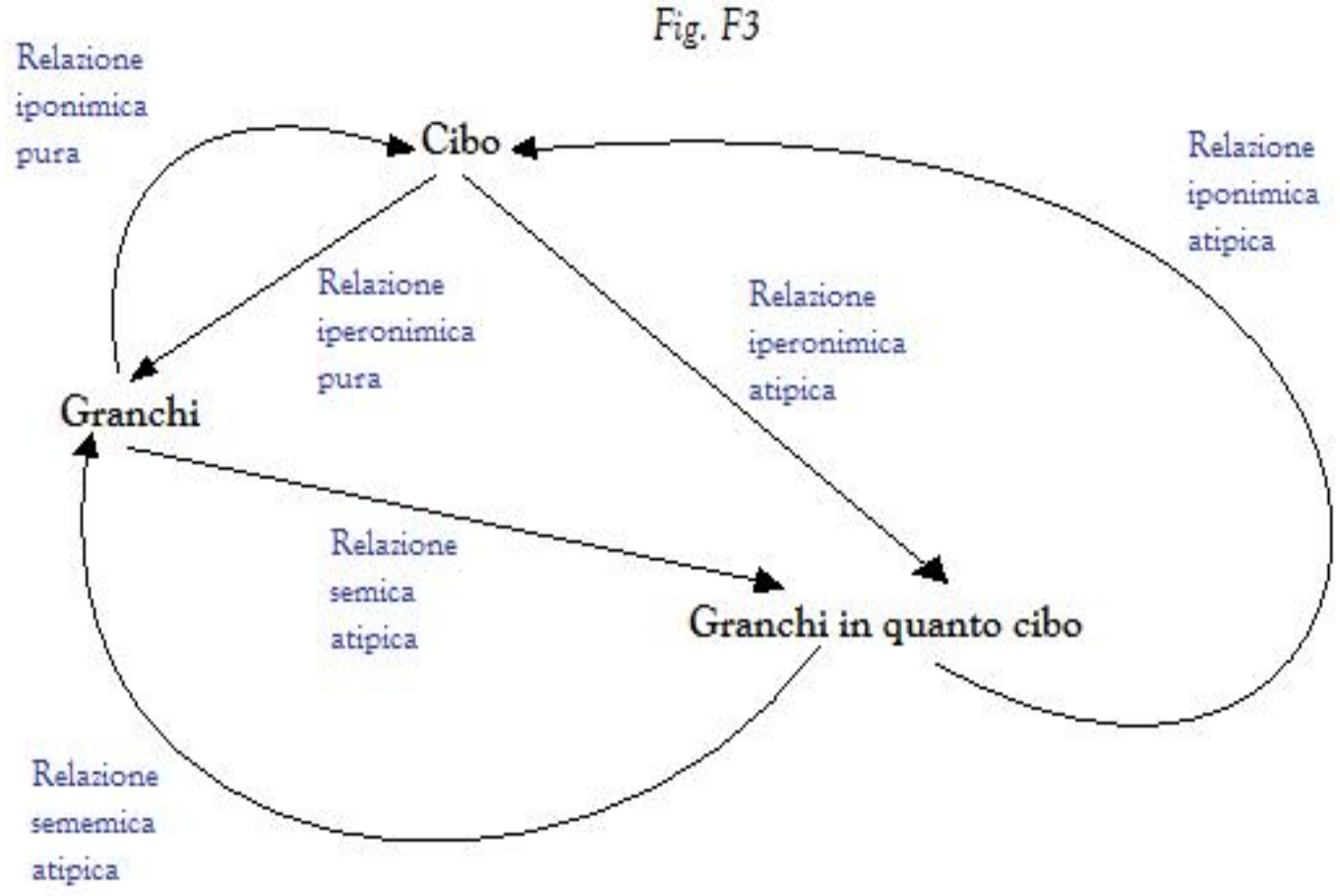


Tesi di dottorato di Giuliano Genetasio, discussa presso l'Università degli Studi di Udine

\section{Glossario}

Il Glossario include una selezione di termini utilizzati nel testo, appartenenti a una delle seguenti categorie: 1) termini di nostra elaborazione; 2) termini di biblioteconomia impiegati secondo una particolare accezione all'interno del testo; 3) termini di linguistica; 4) altri termini utilizzati spesso nelle definizioni. Non sempre vi è simmetria nella scelta dei termini e delle forme dei termini da includere, perché il Glossario include esclusivamente quei termini e quelle forme dei termini presenti nel testo. La scelta della forma preferita del termine risponde generalmente all'uso del testo; all'interno delle definizioni, vengono utilizzate sia forme preferite sia, quando opportuno, forme non preferite dei termini.

Accesso bibliografico Processo di interazione con le registrazioni bibliografiche da parte di indicizzatori e utenti finali, comprensivo di indicizzazione e reperimento dell'informazione.

\section{Agente vedi Caso agentivo}

Allotassia Parafrasi tra elementi lessicali in forma composta (sintagmi) i cui elementi lessicali componenti hanno sequenze diverse.

Altre entità Super-entità che raccoglie tutti gli elementi dell'universo bibliografico che non sono stati distinti come entità a sé stanti: animali, personaggi fittizi e leggendari, tipi di animale, tipi di evento, tipi di oggetto, tipi di luogo, etc.

Analisi componenziale Analisi linguistica volta a individuare gli elementi lessicali (semi) concettualmente componenti un altro elemento lessicale (semema).

Antonimia vedi Relazione di antonimia

Antonimia complementare vedi Relazione di antonimia complementare

Antonimia polare vedi Relazione di antonimia polare

Antonimia scalare vedi Relazione di antonimia scalare

Antonimo Elemento lessicale di significato opposto rispetto a un altro elemento lessicale.

Antonimo complementare Antonimo il cui significato si definisce in rapporto all'altro antonimo. 
Tesi di dottorato di Giuliano Genetasio, discussa presso l'Università degli Studi di Udine

Antonimo scalare Antonimo che si situa all'estremo di un continuum o scala.

Approccio concettuale all'indicizzazione Approccio di studio all'indicizzazione che si rifà ai modelli concettuali entità-relazioni tipici del database design, in cui un dato dominio di interesse viene rappresentato schematicamente in oggetti (entità), caratteristiche degli oggetti (attributi) e relazioni tra questi oggetti.

Approccio linguistico all'indicizzazione Approccio di studio all'indicizzazione che analizza i rapporti tra linguaggio e indicizzazione, ovvero che affronta lo studio degli strumenti di indicizzazione in quanto lingue documentarie.

Argomento vedi Tema

Aspetto linguistico Aspetto fondamentale di una lingua, concernente l'insieme delle regole e dei rapporti di un certo tipo, e generalmente corrispondente a una determinata branca della linguistica; gli aspetti linguistici da noi individuati sono: semantica, morfosintassi e pragmatica.

Asse paradigmatico del linguaggio Dimensione linguistica relativa alle relazioni paradigmatiche e alle tipologie lessicali.

Asse sintagmatico del linguaggio Dimensione linguistica relativa alla combinazione degli elementi lessicali in un sintagma; l'asse sintagmatico è composto da una struttura di superficie e da una struttura profonda.

Asse sintagmatico di superficie vedi Struttura di superficie del linguaggio

Asse sintagmatico profondo vedi Struttura profonda del linguaggio

Attività Azione o processo.

Attributo Caratteristica o elemento informativo di un'entità.

Attributo esterno Attributo (nominale) di un'entità che fa riferimento ad altre entità.

Attributo interno Attributo di un'entità che non fa riferimento ad altre entità.

Attributo nominale Nome, titolo, termine o altro appellativo di un'entità.

Attributo nominale complesso Attributo nominale di un'entità che esplicita relazioni con ulteriori entità, caratterizzato da relazioni sintagmatiche tra le entità i cui nomi compongono il nome complesso.

Attributo nominale semplice Attributo nominale di un'entità che non esplicita relazioni con ulteriori entità. 
Tesi di dottorato di Giuliano Genetasio, discussa presso l'Università degli Studi di Udine

Attributo non nominale Attributo diverso da un nome.

\section{Beneficiario vedi Caso benefattivo}

Biplanarità del lessico Condizione tipica del linguaggio naturale per cui ogni elemento lessicale esiste secondo due piani distinti: significato e significante.

Caso agentivo Caso profondo relativo all'entità che compie volontariamente un'azione.

Caso benefattivo Caso profondo relativo al beneficiario di un'azione.

Caso causativo Caso profondo relativo all'entità o attività che provoca involontariamente il compimento di un processo.

Caso deagentivo Caso profondo relativo all'attività coinvolta dall'agente.

Caso debenefattivo Caso profondo relativo all'attività o entità rivolta al beneficiario.

Caso decausativo Caso profondo relativo all'attività coinvolta dalla causa, ovvero all'effetto.

Caso dedirettivo Caso profondo relativo all'attività o entità con una certa direzione spaziale.

Caso defunzionale Caso profondo relativo all'entità che ha una certa destinazione.

Caso delocativo Caso profondo relativo all'attività coinvolta dall'agente.

Caso deoggettivo Caso profondo relativo all'attività che coinvolge l'oggetto.

Caso deoriginario Caso profondo relativo all'attività o entità con una certa provenienza spaziale.

Caso depossessivo Caso residuale relativo al possessore (entità o attività) di un'entità.

Caso destrumentale Caso profondo relativo all'attività coinvolta dallo strumento.

Caso detemporale Caso profondo relativo all'attività o entità che ha luogo in una determinata circostanza temporale.

Caso direttivo Caso profondo relativo alla direzione spaziale dell'attività o entità, indicante moto a luogo.

Caso funzionale Caso profondo relativo a un'entità o attività che rappresenta la destinazione di un'entità. 
Tesi di dottorato di Giuliano Genetasio, discussa presso l'Università degli Studi di Udine

Caso interattivo Caso sintagmatico logico relativo a un elemento lessicale (entità o attività) che connette due o più elementi lessicali coinvolti in una relazione di interazione.

Caso iperonimico atipico Caso residuale relativo a una classe o iperonimo atipico.

Caso iponimico atipico Caso residuale relativo a un individuo o iponimo atipico.

Caso locativo Caso profondo relativo alla collocazione spaziale dell'azione, indicante stato in luogo.

Caso meronimico Caso residuale relativo a un'entità che costituisce la parte rispetto all'intero.

Caso oggettivo Caso profondo relativo all'oggetto del compimento di un'azione.

Caso olonimico Caso residuale relativo a un'entità che costituisce l'intero rispetto alle parti.

Caso originario Caso profondo relativo alla provenienza spaziale dell'attività o entità, indicante moto da luogo.

Caso possessivo Caso residuale che individua una dipendenza di un'entità rispetto a un'altra entità $\mathrm{O}$ attività.

Caso profondo Argomento grammaticale o ruolo assunto da un elemento lessicale componente all'interno di un sintagma a livello di struttura profonda.

Caso strumentale Caso profondo relativo all'entità che serve involontariamente al compimento di un'azione.

Caso temporale Caso profondo relativo alla circostanza temporale di un'azione.

\section{Causa vedi Caso causativo}

Circalità estensionale Insieme degli argomenti trattati esplicitamente da un Testo.

Circalità intensionale Insieme degli argomenti implicati da un Testo.

Circolo gerarchico Catena circolare di relazioni gerarchiche di tipo inverso.

Co-iponimia vedi Relazione di co-iponimia

Collegamento Struttura catalografica fisica che registra una o più relazioni tra entità.

Co-meronimia vedi Relazione co-meronimica 
Tesi di dottorato di Giuliano Genetasio, discussa presso l'Università degli Studi di Udine

\section{Commento vedi Rema}

Complementarietà vedi Relazione di antonimia complementare

Componente semantica vedi Sema

Contenuto editoriale Particolare aggregazione di Opere (e relative Espressioni) che può trovare realizzazione in una o più Manifestazioni, come atti di convegno, miscellanee in onore, etc.

Controllo dell'universo bibliografico vedi Controllo linguistico

Controllo formale vedi Controllo linguistico

Controllo linguistico Controllo degli aspetti linguistici esercitato dalle lingue documentarie sulla base delle esigenze e caratteristiche dell'accesso bibliografico, realizzato tramite specifiche strutture catalografiche (registrazioni, indici e collegamenti).

Coordinazione vedi Relazione di coordinazione

Co-sememia vedi Relazione co-sememica

Effetto vedi Caso decausativo

Elemento componente vedi Elemento lessicale componente

Elemento descrittivo Indice funzionale a Identificare e Selezionare.

Elemento in forma metalessicale vedi Elemento metalessicale

Elemento indicale vedi Indice

Elemento inventariale Indice funzionale a Ottenere.

Elemento lessicale Parola o locuzione dotata di significato; ogni elemento lessicale esiste al contempo come significato (semema) e significante.

Elemento lessicale componente Elemento lessicale che fa parte di una forma composta di un altro elemento lessicale e contribuisce a definirne il significato complessivo.

Elemento lessicale composto in forma collegata vedi Elemento lessicale in forma collegata

Elemento lessicale composto in forma non collegata vedi Elemento lessicale in forma non collegata 
Tesi di dottorato di Giuliano Genetasio, discussa presso l'Università degli Studi di Udine

Elemento lessicale composto in forma parzialmente collegata vedi Elemento lessicale in forma parzialmente collegata

Elemento lessicale composto in forma totalmente collegata vedi Elemento lessicale in forma totalmente collegata

Elemento lessicale in forma collegata Elemento lessicale in forma composta che fa riferimento a ulteriori elementi lessicali significativi ed è espresso in una forma artificiale, che presenta gli elementi componenti significativi in forma sostantivale e con le reciproche relazioni sintagmatiche espresse in forma metalessicale; ovvero, secondo la terminologia dell'approccio concettuale all'indicizzazione, indice controllato relativo a un nome complesso, che registra esplicitamente le relazioni sintagmatiche tra le entità i cui nomi compongono il nome complesso.

Elemento lessicale in forma composta Elemento lessicale formato da una combinazione di più parole tra loro in relazione.

Elemento lessicale in forma non collegata Elemento lessicale in forma composta che fa riferimento a ulteriori elementi lessicali significativi ed è espresso in linguaggio naturale, cioè in forma discorsiva e non controllata formalmente, con le relazioni sintagmatiche espresse lessicalmente; ovvero, secondo la terminologia dell'approccio concettuale all'indicizzazione, indice relativo a un nome complesso, che non registra esplicitamente le relazioni sintagmatiche tra le entità i cui nomi compongono il nome complesso.

Elemento lessicale in forma paradigmatica vedi Elemento lessicale in forma semplice

Elemento lessicale in forma parzialmente collegata Elemento lessicale in forma collegata in cui l'elemento lessicale componente che rappresenta la classe non è un elemento lessicale significativo, ovvero elemento lessicale in cui non tutti gli elementi lessicali componenti sono significativi; secondo la terminologia dell'approccio concettuale all'indicizzazione, indice controllato relativo a un nome complesso, in cui però almeno un elemento (a esclusione di preposizioni, articoli, etc.) non fa riferimento a ulteriori entità e non sia dunque registrato autonomamente.

Elemento lessicale in forma semplice Elemento lessicale formato da un'unica parola.

\section{Elemento lessicale in forma sintagmatica vedi Elemento lessicale in forma composta}

Elemento lessicale in forma totalmente collegata Elemento lessicale in forma collegata in cui l'elemento lessicale componente che rappresenta la classe è un elemento lessicale significativo, ovvero elemento lessicale in cui tutti gli elementi lessicali componenti sono significativi secondo la terminologia dell'approccio concettuale all'indicizzazione, indice controllato relativo a un nome complesso, in cui la totalità degli elementi (a esclusione di preposizioni, articoli, etc.) fa riferimento a ulteriori entità e sia registrato autonomamente. 
Tesi di dottorato di Giuliano Genetasio, discussa presso l'Università degli Studi di Udine

Elemento lessicale non significativo Elemento lessicale componente che non fa riferimento a un'entità concettuale, ovvero che non è registrato autonomamente.

Elemento lessicale significativo Elemento lessicale che fa riferimento a un'entità concettuale, ovvero che è registrato autonomamente.

\section{Elemento metaindicale vedi Metaindice}

Elemento metalessicale Elemento lessicale esterno al lessico vero e proprio della lingua documentaria, che fornisce informazioni su un elemento lessicale della lingua documentaria.

Elemento non ricercabile Indice non funzionale a Trovare a Navigare; comprende elementi descrittivi e inventariali.

Entità Cosa o oggetto; termine che può riferirsi a seconda dei casi o alle entità concettuali o alle entità linguistiche.

Entità animata Entità di tipo linguistico vivente.

Entità bibliografica Aspetto dell'interesse dell'utente nei prodotti di un'attività artistica o intellettuale; le entità del Gruppo 1 comprendono Testi e Risorse.

Entità collettiva Entità di tipo linguistico la cui estensione include ulteriori entità.

Entità concettuale Aspetto bibliografico fondamentale dell'interesse dell'utente, ovvero dell'oggetto della catalogazione.

\section{Entità del Gruppo 1 di FRBR vedi Entità bibliografica}

Entità del modello concettuale vedi Entità concettuale

Entità di tipo linguistico vedi Tipologia lessicale

Entità inanimata Entità di tipo linguistico non vivente.

Entità individuale Entità di tipo linguistico unica, la cui estensione non include ulteriori entità.

Entità linguistica vedi Tipologia lessicale

Equivalenza semantica vedi Relazione di equivalenza

Esigenza dell'utente vedi Funzione dell'utente

Estensione Insieme dei sememi inclusi da un semema. 
Famiglia semantica vedi Relazione di famiglia semantica

Forma collegata vedi Elemento lessicale in forma collegata

Forma lessicale collegata vedi Elemento lessicale in forma collegata

Forma lessicale composta collegata vedi Elemento lessicale in forma collegata

Forma lessicale composta non collegata vedi Elemento lessicale in forma non collegata

Forma lessicale composta parzialmente collegata vedi Elemento lessicale in forma parzialmente collegata

Forma lessicale composta totalmente collegata vedi Elemento lessicale in forma totalmente collegata

Forma lessicale non collegata vedi Elemento lessicale in forma non collegata

Forma lessicale parzialmente collegata vedi Elemento lessicale in forma parzialmente collegata

Forma lessicale totalmente collegata vedi Elemento lessicale in forma totalmente collegata

Forma non collegata vedi Elemento lessicale in forma non collegata

Forma parzialmente collegata vedi Elemento lessicale in forma parzialmente collegata

Forma sintagmatica collegata vedi Elemento lessicale in forma collegata

Forma sintagmatica non collegata vedi Elemento lessicale in forma non collegata

Forma sintagmatica parzialmente collegata vedi Elemento lessicale in forma parzialmente collegata

Forma sintagmatica totalmente collegata vedi Elemento lessicale in forma totalmente collegata

Forma totalmente collegata vedi Elemento lessicale in forma totalmente collegata

Fonte vedi Caso originario

Funzione Obiettivo o azione; termine che può riferirsi a seconda dei casi o alle funzioni dell'utente o alle funzioni linguistiche o ancora al caso funzionale. 
Tesi di dottorato di Giuliano Genetasio, discussa presso l'Università degli Studi di Udine

Funzione catalografica vedi Funzione dell'utente

Funzione cognitiva del linguaggio vedi Funzione referenziale del linguaggio

Funzione conativa del linguaggio Azione o funzione del linguaggio volta a promuovere o evitare un'azione da parte del destinatario.

Funzione del catalogo vedi Funzione dell'utente

Funzione del linguaggio vedi Funzione linguistica

Funzione dell'utente Obiettivo informativo fondamentale che un utente finale può esprimere nei confronti degli oggetti di interesse, ovvero che il catalogo è teso a soddisfare; ogni funzione dell'utente corrisponde a una precisa operazione compiuta dall'utente finale.

Funzione denotativa del linguaggio vedi Funzione referenziale del linguaggio

Funzione descrittiva del linguaggio vedi Funzione referenziale del linguaggio

Funzione di contatto del linguaggio vedi Funzione fatica del linguaggio

Funzione di navigazione vedi Navigare

Funzione emotiva del linguaggio vedi Funzione espressiva del linguaggio

Funzione espressiva del linguaggio Azione o funzione del linguaggio volta a esprimere determinate emozioni, sentimenti o opinioni del parlante.

Funzione estetica del linguaggio vedi Funzione poetica del linguaggio

Funzione fatica del linguaggio Azione o funzione del linguaggio volta a stabilire, prolungare o chiudere la comunicazione stessa.

Funzione linguistica Azione o funzione svolta dal linguaggio.

Funzione metalinguistica del linguaggio Azione o funzione del linguaggio volta a fornire informazioni e spiegazioni sulla comunicazione stessa.

Funzione persuasiva del linguaggio vedi Funzione conativa del linguaggio

Funzione poetica del linguaggio Azione o funzione del linguaggio volta a produrre messaggi esteticamente efficaci o divertenti.

Funzione referenziale del linguaggio Azione o funzione del linguaggio volta a produrre descrizioni, spiegazioni o informazioni sulla realtà extralinguistica. 
Tesi di dottorato di Giuliano Genetasio, discussa presso l'Università degli Studi di Udine

\section{Grammatica vedi Morfosintassi}

Indice Struttura catalografica fisica che registra uno o più attributi di un'entità.

Indice assegnato Indice relativo a un attributo di un'entità bibliografica o di carattere non segnico, o di carattere segnico ma rielaborato dall'indicizzatore, o ancora escogitato interamente dall'indicizzatore sulla base di un'analisi dell'entità bibliografica.

Indice assegnato di circalità estensionale Indice assegnato che registra la circalità estensionale di un Testo.

Indice collegato vedi Elemento lessicale in forma collegata

Indice controllato Indice relativo a un attributo nominale, che registra esplicitamente relazioni con ulteriori entità o con ulteriori attributi di un'entità.

Indice derivato Indice relativo a un attributo di un'entità bibliografica, derivato direttamente da essa o da entità bibliografiche associate.

Indice derivato di circalità estensionale Indice derivato che registra la circalità estensionale di un Testo.

Indice di circalità estensionale Indice che registra la circalità estensionale di un testo.

Indice di circalità intensionale Indice che registra la circalità intensionale di un Testo.

Indice di punto di vista Indice che registra i punti di vista di un Testo.

Indice di rema Indice assegnato che registra il rema di un Testo.

Indice di tema estensionale Indice assegnato che registra un tema di un Testo.

Indice di valutazione vedi Indice valutativo

Indice gerarchico generico Indice che registra una relazione gerarchica generica o eponimica.

Indice gerarchico iperonimico Indice che registra un iponimo ovvero una relazione di iperonimia.

Indice gerarchico iponimico Indice che registra un iperonimo ovvero una relazione di iperonimia.

Indice gerarchico meronimico Indice che registra un olonimo ovvero una relazione di meronimia. 
Indice gerarchico olonimico Indice che registra un meronimo ovvero una relazione di olonimia.

Indice gerarchico sememico Indice che registra un sema (differente da genere o classe) ovvero una relazione di sememia.

Indice gerarchico semico Indice che registra un semema ovvero una relazione di semia.

Indice esterno Indice che registra un attributo esterno.

Indice interno Indice che registra un attributo interno.

Indice nominale Indice che registra un attributo nominale.

Indice non collegato vedi Elemento lessicale in forma non collegata

Indice non controllato Indice relativo a un attributo nominale, che non registra esplicitamente relazioni con ulteriori entità.

Indice non nominale Indice che registra un attributo non nominale.

Indice parzialmente collegato vedi Elemento lessicale in forma parzialmente collegata

Indice totalmente collegato vedi Elemento lessicale in forma totalmente collegata

Indice valutativo Indice che registra la valutazione di un'entità o di un attributo di un'entità.

Intensione Insieme delle proprietà che caratterizzano un semema, ovvero degli elementi semantici (semi) costituenti un semema.

\section{Interazione vedi Relazione di interazione}

Interesse dell'utente Insieme delle funzioni dell'utente, degli oggetti di interesse e degli indici dell'approccio concettuale, in quanto subordinati all'utente finale.

Iperonimia vedi Relazione di iperonimia

Iperonimo Elemento lessicale che funge da genere o classe rispetto ad altri elementi lessicali, ovvero elemento lessicale la cui estensione include altri elementi lessicali.

Iponimia vedi Relazione di iponimia

Iponimo Elemento lessicale che funge da specie o individuo rispetto ad altri elementi lessicali, ovvero elemento lessicale la cui intensione include altri elementi lessicali. 
Tesi di dottorato di Giuliano Genetasio, discussa presso l'Università degli Studi di Udine

\section{Lessema vedi Elemento lessicale}

Lessicalizzazione degli aspetti linguistici Processo conseguente dal controllo linguistico esercitato dalle lingue documentarie, a causa del quale gli aspetti linguistici devono essere espressi necessariamente tramite ulteriori elementi lessicali con funzione metalinguistica.

Lingua Sistema linguistico; è una delle entità caratteristiche del modello concettuale proposto.

\section{Lingua bibliografica vedi Lingua documentaria}

Lingua documentaria Strumento di indicizzazione, soprattutto in quanto considerato dal punto di vista linguistico.

\section{Lingua documentaria interna vedi Sottolingua}

\section{Locativo vedi Caso locativo}

Marca semantica vedi Sema

\section{Meronimia vedi Relazione di meronimia}

Meronimo Elemento lessicale che costituisce la parte rispetto all'intero.

\section{Meta vedi Caso direttivo}

Metaindice Struttura catalografica fisica che fornisce informazioni su un indice.

\section{Metalingua vedi Lingua documentaria}

Metasemantica delle relazioni semantiche Insieme delle relazioni semantiche tra le diverse tipologie di relazioni semantiche di una lingua; l'espressione è utilizzata prevalentemtente in riferimento alla sottolingua.

Metasintagma Sintagma implicito formato dalla combinazione di un elemento lessicale e del relativo elemento metalessicale.

Monogerarchia Architettura delle relazioni gerarchiche ad albero, caratterizzata da relazioni di tipo uno-a-molti, secondo cui un determinato elemento lessicale può avere più elementi lessicali subordinati ma soltanto un elemento lessicale sovraordinato. Cfr. 3.3.6.4.2.2.2.1

Morfologia Studio delle forme grammaticali degli elementi lessicali.

Morfosintassi Studio dei significanti degli elementi lessicali; comprende lo studio della 
Tesi di dottorato di Giuliano Genetasio, discussa presso l'Università degli Studi di Udine

morfologia (forme grammaticali degli elementi lessicali) e della sintassi (regole di combinazione grammaticale tra gli elementi lessicali).

Navigare Funzione dell'utente relativa all'acquisizione di informazioni su e alla ricerca di entità correlate a quelle trovate.

Nome vedi Attributo nominale

Nome complesso vedi Attributo nominale complesso

Nome semplice vedi Attributo nominale semplice

Nominalizzazione Elemento lessicale relativo a un'entità che racchiude in sé il concetto di un'attività, e che sul piano sintagmatico si comporta almeno parzialmente come un'attività; a livello morfosintattico, le nominalizzazioni sono sostantivi derivati da verbi.

Poligerarchia Architettura delle relazioni gerarchiche di tipo reticolare, caratterizzata da rapporti molti-a-molti, secondo cui un elemento lessicale può avere più elementi lessicali subordinati ma anche più elementi lessicali sovraordinati.

Obiettivo dell'utente vedi Funzione dell'utente

\section{Oggetto vedi Caso oggettivo}

Oggetto di interesse Aspetto bibliografico dell'interesse dell'utente: un'entità, un attributo o una relazione.

Oggettivismo del soggetto Approccio indicale in cui il soggetto è visto come l'argomento trattato da un Testo e l'indicizzazione per soggetto è vista come una rappresentazione oggettiva e sintetica del contenuto di un tTesto ottenibile a partire dai suoi contrassegni formali.

Olonimia vedi Relazione di olonimia

Olonimo Elemento lessicale che costituisce l'intero rispetto alle parti.

Omotassia vedi Relazione di omotassia

Operazione dell'utente vedi Funzione dell'utente

Origine vedi Caso originario

Paradigmaticità vedi Asse paradigmatico del linguaggio

Parafrasi Equivalenza semantica tra elementi lessicali in forma semplice o composta da un lato ed elementi lessicali in forma composta dall'altro. 
Tesi di dottorato di Giuliano Genetasio, discussa presso l'Università degli Studi di Udine

Piano formale vedi Piano linguistico formale

Piano linguistico formale Piano relativo alla suddivisione di una lingua in aspetti linguistici distinti (semantica, morfosintassi, etc.), e all'esistenza di determinate tipologie lessicali e di relazioni semantiche o sintattiche.

Piano linguistico sostanziale Piano relativo al lessico di una lingua e alla sua specifica semantica lessicale.

Piano sostanziale vedi Piano linguistico sostanziale

Polarità vedi Relazione di antonimia polare

Possesso vedi Caso possessivo

Pragmatica Studio del rapporto tra linguaggio e contesto (linguistico ed extralinguistico), ovvero studio del linguaggio in quanto azione; comprende lo studio delle funzioni linguistiche.

Pragmatismo del soggetto Approccio indicale in cui il soggetto è visto come significato (di carattere soggettivo, storico e legato al contesto d'uso) che il Testo assume per l'utente, e l'indicizzazione per soggetto è vista come creazione di strumenti utili per l'utente.

Primo grado di controllo linguistico vedi Semantica delle entità concettuali e delle registrazioni

\section{Proprietà vedi Caso possessivo}

Punto di accesso Indice funzionale a Trovare e Navigare, oltre che a Identificare e Selezionare.

Punto di vista Ideologia assunta o metodologia utilizzata per lo studio di un argomento.

Referente Oggetto a cui si riferisce il segno, secondo la semiotica di Peirce.

Relazione Rapporto tra entità o tra attributi di un'entità, ovvero tra elementi lessicali o forme di elementi lessicali

Relazione a posteriori Relazione semantica che non è comunemente accettata o tipica all'interno di un dato contesto.

Relazione a priori Relazione semantica comunemente accettata o tipica in un dato contesto.

Relazione ad hoc Relazione tipica dell'accesso bibliografico, normalmente non trattata in 
Tesi di dottorato di Giuliano Genetasio, discussa presso l'Università degli Studi di Udine

linguistica, in quanto considerata come parte della semantica delle lingue documentarie.

Relazione analitica vedi Relazione a priori

Relazione antonimica vedi Relazione di antonimia

Relazione causa-effetto vedi Relazione sintagmatica causativa

Relazione co-iponimica vedi Relazione di co-iponimia

Relazione co-meronimica Relazione tra parti di un medesimo intero.

Relazione co-sememica Relazione tra sememi del medesimo sema (non generico).

Relazione co-sememica tra nominalizzazioni vedi Relazione tra nominalizzazioni

Relazione di allotassia vedi Allotassia

Relazione di antonimia Relazione tra elementi lessicali di significato opposto.

Relazione di antonimia complementare Relazione tra antonimi che si definiscono reciprocamente.

Relazione di antonimia polare Relazione tra antonimi che non prevedono gradi intermedi.

Relazione di antonimia scalare Relazione tra antonimi che si situano agli estremi di un unico continuum.

Relazione di co-iponimia Relazione tra specie o individui del medesimo genere o classe.

\section{Relazione di co-meronimia vedi Relazione co-meronimica}

Relazione di complementarietà vedi Relazione di antonimia complementare

Relazione di coordinazione Relazione sintagmatica logica tra due o più elementi che svolgono lo stesso caso (profondo o d'altro tipo).

Relazione di co-sememia vedi Relazione co-sememica

Relazione di eponimia Relazione gerarchica tra una classe e i suoi individui o viceversa, ovvero relazione tra un primo semema e un secondo semema incluso nell'estensione del primo semema o viceversa.

Relazione di eponimia specificata Relazione di eponimia specificata secondo un particolare criterio; il criterio di specificazione adottato è basato sulle relazioni 
Tesi di dottorato di Giuliano Genetasio, discussa presso l'Università degli Studi di Udine

sintagmatiche grammaticali della sottolingua.

Relazione di equivalenza Relazione tra elementi lessicali (in forma semplice o composta) morfosintatticamente differenti ma semanticamente identici o quasi, ovvero - in virtù dell'eliminazione della biplanarità del lessico tipica delle lingue documentarie - relazione tra le diverse forme di un elemento lessicale.

Relazione di equivalenza bicontrollata Relazione di equivalenza tra forme collegate di un elemento lessicale.

Relazione di equivalenza monocontrollata Relazione di equivalenza tra una forma collegata e una forma non collegata di un elemento lessicale.

Relazione di equivalenza non controllata Relazione di equivalenza tra forme non collegate di un elemento lessicale.

Relazione di equivalenza semantica vedi Relazione di equivalenza

Relazione di famiglia semantica Relazione che lega tutti i lessemi che condividono uno stesso sema e anche parte del significante.

Relazione di interazione Relazione sintagmatica logica che esprime l'interazione o azione reciproca tra due o più elementi lessicali componenti il sintagma.

Relazione di iperonimia Relazione tra un genere o classe e le sue specie o individui, ovvero relazione tra un primo semema e un secondo semema incluso nell'estensione del primo semema.

Relazione di iponimia Relazione tra una specie o individuo e i suoi generi o classi, ovvero relazione tra un primo semema e un secondo semema incluso nell'intensione del primo semema.

Relazione di meronimia Relazione tra una parte e il suo intero.

Relazione di olonimia Relazione tra l'intero e le sue parti.

Relazione di omotassia Relazione tra sintagmi dai significanti identici ma dai significati differenti, e perciò causa di ambiguità semantica.

Relazione di parafrasi vedi Parafrasi

Relazione di partonomia vedi Relazione partitiva

Relazione di polarità vedi Relazione di antonimia polare

Relazione di scalarità vedi Relazione di antonimia scalare 
Relazione di sememia vedi Relazione sememica

Relazione di sememia specificata vedi Relazione sememica specificata

Relazione di semia vedi Relazione semica

Relazione di semia specificata vedi Relazione semica specificata

Relazione di sinonimia vedi Sinonimia

Relazione eponimica vedi Relazione di eponimia

Relazione eponimica a priori vedi Relazione eponimica pura

Relazione eponimica a posteriori vedi Relazione eponimica atipica

Relazione eponimica atipica Relazione eponimica tra una classe e un individuo non tipico della classe, o viceversa; la relazione eponimica atipica considera un elemento lessicale secondo uno specifica prospettiva, funzione o punto di vista atipici in un determinato contesto culturale.

Relazione eponimica non specificata vedi Relazione eponimica pura

Relazione eponimica partitiva Relazione di eponimia tra un iperonimo che costituisce la parte e un iponimo che costituisce l'intero.

Relazione eponimica pura Relazione di eponimia comunemente accettata in un dato contesto, il cui criterio di specificazione non è stato distinto; all'interno della sottolingua, le relazioni eponimiche pure sono tutte quelle in cui l'iponimo specifica l'iperonimo tramite differenze che non individuano ulteriori elementi lessicali significativi.

Relazione eponimica specificata vedi Relazione di eponimia specificata

Relazione eponimica tipica vedi Relazione eponimica pura

Relazione generica vedi Relazione di eponimia

Relazione gerarchica Relazione in cui è presente un concetto di gerarchia tra i termini; sono relazioni gerarchiche le relazioni eponimiche, semiche/sememiche e partitive.

Relazione gerarchica di eponimia specificata vedi Relazione di eponimia specificata

Relazione gerarchica di sememia vedi Relazione sememica

Relazione gerarchica di sememia specificata vedi Relazione sememica specificata 
Tesi di dottorato di Giuliano Genetasio, discussa presso l'Università degli Studi di Udine

Relazione gerarchica di semia vedi Relazione semica

Relazione gerarchica di semia specificata vedi Relazione semica specificata

Relazione gerarchica eponimica vedi Relazione di eponimia

Relazione gerarchica eponimica specificata vedi Relazione di eponimia specificata

Relazione gerarchica generica vedi Relazione di eponimia

Relazione gerarchica generica specificata vedi Relazione di eponimia specificata

Relazione gerarchica partitiva vedi Relazione partitiva

Relazione gerarchica sememica vedi Relazione sememica

Relazione gerarchica sememica specificata vedi Relazione sememica specificata

Relazione gerarchica semica vedi Relazione semica

Relazione gerarchica semica specificata vedi Relazione semica specificata

Relazione gerarchica specificata Relazione gerarchica specificata secondo un particolare criterio; il criterio di specificazione adottato è basato sulle relazioni sintagmatiche grammaticali della sottolingua; comprende le relazioni eponimiche specificate e semiche/ sememiche specificate.

Relazione in forma lessicale Relazione (sintagmatica) espressa tramite un elemento lessicale.

Relazione in forma metalessicale Relazione espressa tramite un elemento metalessicale.

Relazione iperonimica vedi Relazione di iperonimia

Relazione iperonimica atipica Relazione di iperonimia tra una classe e un individuo non tipico della classe.

Relazione iperonimica deagentiva Relazione di iperonimia in cui l'iperonimo esprime l'attività svolta dall'agente dell'iponimo.

Relazione iperonimica debenefattiva Relazione di iperonimia in cui l'iperonimo esprime l'attività o entità rivolta al beneficiario dell'iponimo.

Relazione iperonimica decausativa Relazione di iperonimia in cui l'iperonimo esprime l'attività coinvolta dalla causa dell'iponimo. 
Relazione iperonimica dedirettiva Relazione di iperonimia in cui l'iperonimo esprime l'attività o entità con una certa direzione spaziale.

Relazione iperonimica defunzionale Relazione di iperonimia in cui l'iperonimo esprime l'entità che ha una certa destinazione dell'iponimo.

Relazione iperonimica delocativa Relazione di iperonimia in cui l'iperonimo esprime l'entità o attività con una certa collocazione spaziale dell'iponimo.

Relazione iperonimica deoggettiva Relazione di iperonimia in cui l'iperonimo esprime l'attività che coinvolge l'oggetto dell'iponimo.

Relazione iperonimica deoriginaria Relazione di iperonimia in cui l'iperonimo esprime l'attività o entità con una certa provenienza spaziale dell'iponimo.

Relazione iperonimica depossessiva Relazione di iperonimia in cui l'iperonimo esprime il possessore (entità o attività) di un'entità.

Relazione iperonimica destrumentale Relazione di iperonimia in cui l'iperonimo esprime l'attività coinvolta dallo strumento dell'iponimo.

Relazione iperonimica detemporale Relazione di iperonimia in cui l'iperonimo esprime l'attività o entità che ha luogo in una determinata circostanza temporale.

Relazione iperonimica meronimica Relazione di iperonimia in cui l'iperonimo esprime un'entità che costituisce la parte rispetto all'intero.

Relazione iponimica vedi Relazione di iponimia

Relazione iponimica agentiva Relazione di iponimia in cui l'iponimo specifica l'iperonimo tramite l'aggiunta di un agente.

Relazione iponimica atipica Relazione di iponimia tra un individuo e una classe non tipica dell'individuo.

Relazione iponimica benefattiva Relazione di iponimia in cui l'iponimo specifica l'iperonimo tramite l'aggiunta di un beneficiario.

Relazione iponimica causativa Relazione di iponimia in cui l'iponimo specifica l'iperonimo tramite l'aggiunta di una causa.

Relazione iponimica direttiva Relazione di iponimia in cui l'iponimo specifica l'iperonimo tramite l'aggiunta di una direzione spaziale.

Relazione iponimica funzionale Relazione di iponimia in cui l'iponimo specifica 
Tesi di dottorato di Giuliano Genetasio, discussa presso l'Università degli Studi di Udine

l'iperonimo tramite l'aggiunta di una destinazione.

Relazione iponimica locativa Relazione di iponimia in cui l'iponimo specifica l'iperonimo tramite l'aggiunta di una collocazione spaziale.

Relazione iponimica oggettiva Relazione di iponimia in cui l'iponimo specifica l'iperonimo tramite l'aggiunta di un oggetto.

Relazione iponimica olonimica Relazione di iponimia in cui l'iponimo specifica l'iperonimo tramite l'aggiunta di un'entità che rappresenta l'intero rispetto alle parti.

Relazione iponimica originaria Relazione di iponimia in cui l'iponimo specifica l'iperonimo tramite l'aggiunta di una provenienza spaziale.

Relazione iponimica possessiva Relazione di iponimia in cui l'iponimo specifica l'iperonimo tramite l'aggiunta di un'entità dipendente.

Relazione iponimica strumentale Relazione di iponimia in cui l'iponimo specifica l'iperonimo tramite l'aggiunta di uno strumento.

Relazione iponimica temporale Relazione di iponimia in cui l'iponimo specifica l'iperonimo tramite l'aggiunta di una circostanza temporale.

Relazione meronimica vedi Relazione di meronimia

Relazione olonimica vedi Relazione di olonimia

Relazione paradigmatica Relazione esistente a prescindere da specifici discorsi (sintagmi).

Relazione partitiva Relazione gerarchica tra l'intero e le sue parti o viceversa.

Relazione partitiva pura Relazione partitiva che riguarda elementi lessicali che presentano forme semplici o forme composte che non individuano ulteriori elementi lessicali significativi.

Relazione partonomica vedi Relazione partitiva

Relazione partonomica non specificata vedi Relazione partitiva pura

Relazione partonomica pura vedi Relazione partitiva pura

Relazione semantica Rapporto di natura semantica tra entità o tra attributi di un'entità.

Relazione semantica ad hoc vedi Relazione ad hoc

Relazione semantica in forma lessicale vedi Relazione in forma lessicale 
Tesi di dottorato di Giuliano Genetasio, discussa presso l'Università degli Studi di Udine

Relazione semantica in forma metalessicale vedi Relazione in forma metalessicale

Relazione semantica paradigmatica vedi Relazione paradigmatica

Relazione semantica sintagmatica vedi Relazione sintagmatica

Relazione sememica Relazione tra un semema e i suoi semi (non generici).

Relazione sememica atipica Relazione sememica tra un elemento lessicale considerato in quanto individuo di una classe non tipica da un lato e lo stesso elemento lessicale in sé dall'altro; nella relazione sememica atipica il semema si differenzia dal sema per l'aggiunta di un caso iperonimico atipico.

Relazione sememica deagentiva Relazione sememica in cui il semema si differenzia dal sema tramite l'aggiunta di un'attività svolta dall'agente.

Relazione sememica debenefattiva Relazione sememica in cui il semema si differenzia dal sema tramite l'aggiunta di un'attività o entità rivolta al beneficiario.

Relazione sememica decausativa Relazione sememica in cui il semema si differenzia dal sema tramite l'aggiunta di un'attività provocata dalla causa.

Relazione sememica dedirettiva Relazione sememica in cui il semema si differenzia dal sema tramite l'aggiunta di un'entità o attività relativa a una direzione spaziale.

Relazione sememica defunzionale Relazione sememica in cui il semema si differenzia dal sema tramite l'aggiunta di un'entità relativa a una destinazione.

Relazione sememica delocativa Relazione sememica in cui il semema si differenzia dal sema tramite l'aggiunta di un'entità o attività relativa a una collocazione spaziale.

Relazione sememica deoggettiva Relazione sememica in cui il semema si differenzia dal sema tramite l'aggiunta di un'attività che coinvolge l'oggetto.

Relazione sememica deoriginaria Relazione sememica in cui il semema si differenzia dal sema tramite l'aggiunta di un'entità o attività relativa a una provenienza spaziale.

Relazione sememica depossessiva Relazione sememica in cui il semema si differenzia dal sema tramite l'aggiunta di un possessore (entità o attività).

Relazione sememica destrumentale Relazione sememica in cui il semema si differenzia dal sema tramite l'aggiunta di un'attività servita involontariamente dallo strumento.

Relazione sememica detemporale Relazione sememica in cui il semema si differenzia dal sema tramite l'aggiunta di un'entità o attività relativa a una circostanza temporale. 
Tesi di dottorato di Giuliano Genetasio, discussa presso l'Università degli Studi di Udine

Relazione sememica meronimica Relazione sememica in cui il semema si differenzia dal sema tramite l'aggiunta di un'entità che costituisce la parte rispetto all'intero.

Relazione sememica specificata Relazione sememica specificata secondo un particolare criterio; il criterio di specificazione adottato è basato sulle relazioni sintagmatiche grammaticali della sottolingua.

Relazione semica Relazione tra un sema (non generico) e i suoi sememi.

Relazione semica agentiva Relazione semica in cui il sema esprime l'agente del semema.

Relazione semica atipica Relazione semica tra un elemento lessicale da un lato e lo stesso elemento lessicale considerato in quanto individuo di una classe non tipica dall'altro; nella relazione semica atipica il sema (non generico) esprime il caso iponimico atipico del semema.

Relazione semica benefattiva Relazione semica in cui il sema esprime il beneficiario del semema.

Relazione semica causativa Relazione semica in cui il sema esprime la causa del semema.

Relazione semica direttiva Relazione semica in cui il sema esprime la direzione spaziale del semema.

Relazione semica funzionale Relazione semica in cui il sema esprime la destinazione del semema.

Relazione semica locativa Relazione semica in cui il sema esprime la collocazione spaziale del semema.

Relazione semica oggettiva Relazione semica in cui il sema esprime l'oggetto del semema.

Relazione semica olonimica Relazione semica in cui il sema esprime l'entità che costituisce l'intero rispetto alle parti.

Relazione semica originaria Relazione semica in cui il sema esprime la provenienza spaziale del semema.

Relazione semica possessiva Relazione semica in cui il sema esprime l'entità dipendente del semema.

Relazione semica specificata Relazione semica specificata secondo un particolare criterio; il criterio di specificazione adottato è basato sulle relazioni sintagmatiche grammaticali della sottolingua. 
Tesi di dottorato di Giuliano Genetasio, discussa presso l'Università degli Studi di Udine

Relazione semica strumentale Relazione semica in cui il sema esprime lo strumento del semema.

Relazione semica temporale Relazione semica in cui il sema esprime la circostanza temporale del semema.

Relazione sintagmatica Relazione posta all'interno di uno specifico discorso (sintagma).

Relazione sintagmatica agentiva Relazione sintagmatica tra caso agentivo e caso deagentivo.

Relazione sintagmatica benefattiva Relazione sintagmatica tra caso benefattivo e caso debenefattivo.

Relazione sintagmatica causativa Relazione sintagmatica tra caso causativo e caso decausativo.

Relazione sintagmatica deagentiva Relazione sintagmatica tra caso deagentivo e caso agentivo.

Relazione sintagmatica debenefattiva Relazione sintagmatica tra caso debenefattivo e caso benefattivo.

Relazione sintagmatica decausativa Relazione sintagmatica tra caso decausativo e caso causativo.

Relazione sintagmatica dedirettiva Relazione sintagmatica tra caso dedirettivo e caso direttivo.

Relazione sintagmatica defunzionale Relazione sintagmatica tra caso defunzionale e caso funzionale.

Relazione sintagmatica delocativa Relazione sintagmatica tra caso delocativo e caso locativo.

Relazione sintagmatica deoggettiva Relazione sintagmatica tra caso deoggettivo e caso oggettivo.

Relazione sintagmatica deoriginaria Relazione sintagmatica tra caso deoriginario e caso originario.

Relazione sintagmatica depossessiva Relazione sintagmatica tra caso depossessivo e caso possessivo.

Relazione sintagmatica destrumentale Relazione sintagmatica tra caso destrumentale e caso strumentale. 
Relazione sintagmatica detemporale Relazione sintagmatica tra caso detemporale e caso temporale.

Relazione sintagmatica di coordinazione vedi Relazione di coordinazione

Relazione sintagmatica di interazione vedi Relazione di interazione

Relazione sintagmatica di partonomia vedi Relazione sintagmatica partitiva

Relazione sintagmatica direttiva Relazione sintagmatica tra caso direttivo e caso dedirettivo.

Relazione sintagmatica eponimica atipica Relazione sintagmatica tra caso iperonimico atipico e caso iponimico atipico o viceversa.

Relazione sintagmatica funzionale Relazione sintagmatica tra caso funzionale e caso defunzionale.

Relazione sintagmatica gerarchica atipica vedi Relazione sintagmatica eponimica atipica

Relazione sintagmatica grammaticale Relazione sintagmatica che ha un corrispettivo a livello di relazioni paradigmatiche; le relazioni sintagmatiche grammaticali includono casi profondi e relazioni sintagmatiche grammaticali d'altro tipo.

Relazione sintagmatica iperonimica atipica Relazione sintagmatica tra caso iperonimico atipico e caso iponimico atipico.

Relazione sintagmatica iponimica atipica Relazione sintagmatica tra caso iponimico atipico e caso iperonimico atipico.

Relazione sintagmatica locativa Relazione sintagmatica tra caso locativo e caso delocativo.

Relazione sintagmatica logica Relazione sintagmatica che non ha un corrispettivo a livello di relazioni paradigmatiche e che è esclusivamente funzionale alla costruzione di sintagmi.

Relazione sintagmatica meronimica Relazione sintagmatica tra caso meronimico e caso olonimico.

Relazione sintagmatica oggettiva Relazione sintagmatica tra caso oggettivo e caso deoggettivo.

Relazione sintagmatica olonimica Relazione sintagmatica tra caso olonimico e caso meronimico. 
Tesi di dottorato di Giuliano Genetasio, discussa presso l'Università degli Studi di Udine

Relazione sintagmatica originaria Relazione sintagmatica tra caso originario e caso deoriginario.

Relazione sintagmatica partitiva Relazione sintagmatica tra intero (caso olonimico) e parte (caso meronimico), e viceversa.

Relazione sintagmatica partonomica vedi Relazione sintagmatica partitiva

Relazione sintagmatica possessiva Relazione sintagmatica tra caso possessivo e caso depossessivo.

Relazione sintagmatica strumentale Relazione sintagmatica tra caso strumentale e caso destrumentale.

Relazione sintagmatica temporale Relazione sintagmatica tra caso temporale e caso detemporale.

Relazione sintetica vedi Relazione a posteriori

Relazione tra nominalizzazioni Relazione tra co-sememi che siano nominalizzazioni di uno stesso verbo e che appartengono però a tipologie lessicali differenti.

Rema Insieme delle affermazioni dell'autore relative agli argomenti di un Testo.

Rete sindetica vedi Semantica delle relazioni della sottolingua

Risorsa Entità del Gruppo 1 di natura materiale; comprende Manifestazioni e Item.

Ruolo vedi Caso profondo

Ruolo agentivo vedi Caso agentivo

Ruolo benefattivo vedi Caso benefattivo

Ruolo causativo vedi Caso causativo

Ruolo direttivo vedi Caso direttivo

Ruolo funzionale vedi Caso funzionale

Ruolo interattivo vedi Caso interattivo

Ruolo locativo vedi Caso locativo

Ruolo oggettivo vedi Caso oggettivo 
Tesi di dottorato di Giuliano Genetasio, discussa presso l'Università degli Studi di Udine

Ruolo originario vedi Caso originario

Ruolo possessivo vedi Caso possessivo

Ruolo semantico vedi Caso profondo

Ruolo strumentale vedi Caso strumentale

Ruolo tematico vedi Caso profondo

Ruolo temporale vedi Caso temporale

Scalarità vedi Relazione di antonimia scalare

Scopo vedi Caso funzionale

Secondo grado di controllo linguistico vedi Semantica degli attributi e degli indici

Segno sinolo di significato e significante; oggetto di studio della semiotica.

Segno linguistico vedi Elemento lessicale

Sema Elemento lessicale componente il significato di un altro elemento lessicale (semema).

Semantica Studio del significato (semi e sememi) degli elementi lessicali.

Semantica degli attributi e degli indici Secondo grado di controllo linguistico delle lingue documentarie con cui un certo numero di elementi di significato del linguaggio naturale relativi alle caratteristiche delle entità viene selezionato per la sua rilevanza rispetto alle esigenze degli utenti finali e controllato esplicitamente tramite indici; corrisponde all'insieme degli attributi e degli indici delineati nell'approccio concettuale all'indicizzazione.

Semantica delle entità concettuali e delle registrazioni Primo grado di controllo linguistico delle lingue documentarie, con cui con cui un certo numero di elementi di significato del linguaggio naturale viene selezionato per la sua rilevanza rispetto alle esigenze degli utenti finali e controllato esplicitamente tramite registrazioni bibliografiche; corrisponde all'insieme delle entità concettuali delineate nell'approccio concettuale all'indicizzazione.

Semantica delle entità linguistiche vedi Semantica delle tipologie lessicali

Semantica delle relazioni della sottolingua Insieme delle relazioni semantiche (paradigmatiche e sintagmatiche) della sottolingua. 
Semantica delle relazioni e dei collegamenti Terzo grado di controllo linguistico delle lingue documentarie, con cui un certo numero di relazioni di significato del linguaggio naturale viene selezionato per la sua rilevanza rispetto alle esigenze degli utenti finali e controllato esplicitamente tramite indici e collegamenti; corrisponde all'insieme delle relazioni, degli indici controllati e dei collegamenti delineati nell'approccio concettuale all'indicizzazione.

Semantica delle tipologie lessicali Insieme delle tipologie semantiche fondamentali degli elementi lessicali di una lingua.

Semantica paradigmatica vedi Asse paradigmatico del linguaggio

Semantica paradigmatica dei sintagmi vedi Semantica paradigmatica vigente nei sintag$\mathrm{mi}$

Semantica paradigmatica delle entità linguistiche vedi Semantica delle tipologie lessicali

Semantica paradigmatica delle relazioni Insieme delle relazioni paradigmatiche di una lingua; l'espressione è utilizzata prevalentemente in riferimento alla sottolingua.

Semantica paradigmatica delle tipologie lessicali vedi Semantica delle tipologie lessicali

Semantica paradigmatica vigente nei sintagmi Semantica paradigmatica in quanto condizionante la combinazione degli elementi lessicali e dunque la formazione di sintagmi.

Semantica sintagmatica Insieme delle relazioni sintagmatiche, ovvero semantica relativa ai significati che gli elementi lessicali assumono all'interno di un sintagma.

Semema Significato di un elemento lessicale in quanto composto da un insieme di semi.

Sememia vedi Relazione sememica

Semia vedi Relazione semica

Significante Espressione fonica o grafica di un segno; oggetto di studio della morfosintassi.

Significato Contenuto concettuale che scaturisce dalla combinazione dei significanti; oggetto di studio della semantica.

Sinonimia Relazione tra elementi lessicali morfosintatticamente differenti ma semanticamente identici o quasi; la sinonimia vera e propria non riguarda Persone, Enti, Famiglie ed entità bibliografiche.

Sinonimia sintagmatica vedi Allotassia 
Sinonimo Elemento lessicale morfosintatticamente differente ma semanticamente identico o molto simile a un altro elemento lessicale, ovvero elemento lessicale che condivide tutti o quasi tutti i semi di un altro elemento lessicale.

Sintagma vedi Elemento lessicale in forma composta

Sintagmaticità vedi Asse sintagmatico del linguaggio

Sintassi Studio delle regole di combinazione grammaticale tra gli elementi lessicali.

Sintassi della struttura profonda vedi Semantica sintagmatica

Sintassi profonda vedi Semantica sintagmatica

Soggetto Insieme degli argomenti per cui un Testo è potenzialmente utile perché in esso trattati o implicati.

Sostantivo deverbale vedi Nominalizzazione

Sottolingua Ulteriore lingua documentaria interna a una lingua documentaria, che scaturisce dal terzo grado di controllo linguistico e che è dotata di lessico e aspetti linguistici propri.

Sottolingua documentaria vedi Sottolingua

Strumento vedi Caso strumentale

Struttura di superficie dell'asse sintagmatico vedi Struttura di superficie del linguaggio

Struttura di superficie del linguaggio Livello fenomenico e superficiale della morfosintassi, diverso per ogni lingua e corrispondente alle sue manifestazioni fonetiche e grafiche.

Struttura poligerarchica vedi Poligerarchia

Struttura profonda del linguaggio Livello profondo della morfosintassi, presente come tale in tutte le lingue, e composto dalla combinazione della semantica sintagmatica (o sintassi profonda) con la semantica paradigmatica vigente nei sintagmi.

Struttura profonda dell'asse sintagmatico vedi Struttura profonda del linguaggio

Struttura superficiale del linguaggio vedi Struttura di superficie del linguaggio

Tema Argomento trattato esplicitamente da un Testo.

Tema estensionale vedi Tema 
Tesi di dottorato di Giuliano Genetasio, discussa presso l'Università degli Studi di Udine

Tempo Data o arco temporale; è una delle entità caratteristiche del modello concettuale proposto.

Termine generico vedi Iperonimo

Termine specifico vedi Iponimo

Terzo grado di controllo linguistico vedi Sottolingua

Testo Entità del Gruppo 1 di natura artistica o intellettuale; comprende Opere, Espressioni e Contenuti editoriali.

Tipo di ente Categoria di appartenenza di un Ente; è una delle entità caratteristiche del modello concettuale proposto.

Tipo di persona Categoria di appartenenza di una Persona; è una delle entità caratteristiche del modello concettuale proposto.

Tipo di risorsa Tipologia di supporto su cui sono registrate e codificate le Risorse, sia di carattere generale che specifico; è una delle entità caratteristiche del modello concettuale proposto.

Tipo di testo Tipologia formale o concettuale di Testo; è una delle entità caratteristiche del modello concettuale proposto.

Tipologia lessicale Tipologia semantica fondamentale di un elemento lessicale.

Universale linguistico Elemento o caratteristica presente in tutte le lingue.

Universale linguistico formale Universale linguistico relativo al piano formale del linguaggio.

Universale linguistico sostanziale Universale linguistico relativo al piano sostanziale del linguaggio. 
Tesi di dottorato di Giuliano Genetasio, discussa presso l'Università degli Studi di Udine

\section{Bibliografia}

La bibliografia comprende tutti i testi consultati e ritenuti pertinenti, anche se non citati in testo. URL controllati per l'ultima volta il 19-01-2012.

AMERICAN LIBRARY ASSOCIATION, List of subject headings for use in dictionary catalogs, Boston, Library Bureau, 1895, disponibile all'indirizzo

<http://ia600304.us.archive.org/4/items/ed2listofsubject00ameruoft/ed2listofsubject00 ameruoft.pdf>

JAMES D. ANDERSON - JOSÉ PÉREZ-CARBALLO, The nature of indexing. How humans and machines analyze messages and texts for retrieval. Part I, Research, and the nature of human indexing, "Information processing \& management", 37, 2 (2001), p. 231-254, disponibile all'indirizzo

<http://www.mariapinto.es/ciberabstracts/Articulos/artAnderson2001a.pdf>

JAMES D. ANDERSON - JOSÉ PÉREZ-CARBALLO, The nature of indexing. How humans and machines analyze messages and texts for retrieval. Part II, Machine indexing, and the allocation of human versus machine effort, "Information processing \& management", 37, 2 (2001), p. 255-277

RICHARD S. ANGELL, Library of Congress subject headings. Review and forecast, in Subject retrieval in the seventies. New directions. Proceedings of an international symposyium held at the Center for Adult Education, University of Maryland, College Park, May 14 to 15, 1971, edited by Hans (Hanan) Wellisch and Thomas D. Wilson, Westport, Greenwood, 1972, p. $143-162$

SUSAN ARTANDI, Machine indexing. Linguistic and semiotic implications, "Journal of the American Society for Information Science”, 27, 4 (1976), p. 235-239

BENEDETTO ASCHERO, Teoria e tecnica dellindicizzazione per soggetto, nuova ed. riveduta e ampliata, Milano, Editrice Bibliografica, 1993

ASSOCIAZIONE ITALIANA BIBLIOTECHE. GRUPPO DI RICERCA

SULL'INDICIZZAZIONE PER SOGGETTO, Guida all'indicizzazione per soggetto, Roma, Associazione italiana biblioteche, 2001

DEREK AUSTIN, Basic concept classes and primitive relations, in Universal classification I. Subject analysis and ordering systems. Proceedings. 4th Internatl. Study Conference on Classification Research, 6th Annual Conference of Gesellschaft für Klassification e.V., Augsburg, 
Tesi di dottorato di Giuliano Genetasio, discussa presso l'Università degli Studi di Udine

28 June-2 July 1982. Vol. 1, editor Ingetraut Dahlberg, Frankfurt, Indeks, 1982, p. 86-94

DEREK AUSTIN, PRECIS. A manual of concept analysis and subject indexing, London, Council of the British National Bibliography, 1974

F. H. AYRES, Time for change. A new approach to cataloguing concepts, "Cataloging \& classification quarterly", 28, 2/3 (2000), p. 3-16

MARIA BRUNA BALDACCI, Rappresentazione e ricerca delle informazioni. Come comunicare attraverso $i$ sistemi informativi automatizzati, Roma, NIS, 1988

MARIO GUIDO BARITE, The notion of 'category'. Its implications in subject analysis and in the construction and evaluation of indexing languages, "Knowledge organization", 27, 1/2 (2000), p. 4-10

MARCIA J. BATES, Factors affecting subject catalog search success, "Journal of the American Society for Information Science”, 28, 3 (1977), p. 161-169

MARCIA J. BATES, Rethinking subject cataloging in the online environment, "Library resources and technical services", 33, 4 (1989), p. 400-412

MARCIA J. BATES, Subject access in online catalogs. A design model, "Journal of the American Society for Information Science”, 37, 6 (1986), p. 357-376

DAVID BATTY, Reflections on active and passive voices in indexing, "Journal of library and information science", 10, 1 (1984), p. 13-21

CLARE BEGHTOL, Bibliographic classification theory and text linguistics. Aboutness analysis, intertextuality and the cognitive act of classifying documents, "Journal of documentation", 42, 2 (1986), p. 84-113

BARBARA L. BERMAN, Form headings in subject cataloging, "Library resources and technical services", 33, 2 (1989), p. 134-139

SANFORD BERMAN, Proposal for reforms to improve subject searching, "American libraries", 15, 4 (1984), p. 254

GAETANO BERRUTO, Nozioni di linguistica generale, 3. ed., Napoli, Liguori, 2004

SUZANNE BERTRAND-GASTALDY - DIANE LANTEIGNE - LUC GIROUX CLAIRE DAVID, Convergent theories. Using a multidisciplinary approach to explain indexing results, in ASIS '95. Proceedings of the 58th ASIS Annual Meeting, Chicago, Illinois, October 9-12. Forging new partnerships in information, 1995, proceedings editor Tom Kinney, Medford, N.J., Information today, 1995, p. 56-60 
Tesi di dottorato di Giuliano Genetasio, discussa presso l'Università degli Studi di Udine

K. BHATTACHARYYA, The effectiveness of natural language in science indexing and retrieval, "Journal of documentation", 30, 3 (1974), p. 235-254

CARLO BIANCHINI, Riflessioni sull'universo bibliografico. Funzioni, oggetti e modelli della catalogazione per autore e titolo, Milano, Sylvestre Bonnard, 2005

BIBLIOTECA NAZIONALE CENTRALE DI FIRENZE, Nuovo soggettario, disponibile all'indirizzo <http://thes.bncf.firenze.sbn.it/>

DAVID C. BLAIR, Language and representation in information retrieval, Amsterdam, New York, Elsevier Science Publishers, 1990

DAVID BODOFF - AJIT KAMBIL, Partial coordination. 1, The best of pre-coordination and post-coordination, "Journal of the American Society for Information Science", 49, 14 (1998), p. 1254-1269

BERT R. BOYCE, Beyond topicality. A two stage view of relevance and the retrieval process, 18 , 3 (1982), p. 105-109

DIANE BROOKING, Comments for Working Group, in Testimony submitted to the Library of Congress Working Group on the Future of Bibliographic Control. Part II, 2007, p. 14-17

VANDA BROUGHTON, The need for a faceted classification as the basis of all methods of information retrieval, "Aslib proceedings", 58, 1/2, 2006, p. $49-72$

JOHN M. BUDD, The complexity of information retrieval. An hypothetical example, "Journal of academic librarianship", 22, 2 (1996), p. 111-117

JOHN M. BUDD, Discourse analysis and the study of communication in LIS, "Library trends", 55, 1 (2006), p. 65-82

JOHN M. BUDD, Phenomenology and information studies, "Journal of documentation", 61, 1 (2005), p. 44-59

JOHN M. BUDD, Relevance. Language, semantics, philosophy, "Library trends", 52, 3 (2004), p. $447-462$

PINO BUIZZA, 025.4 Analisi e controllo per soggetto, in Biblioteconomia. Guida classificata, diretta da Mauro Guerrini, condirettore Gianfranco Crupi, a cura di Stefano Gambari, collaborazione di Vincenzo Fugaldi, presentazione di Luigi Crocetti, Milano, Editrice Bibliografica, 2007, p. 564-584

PINO BUIZZA, Indicizzazione per soggetto e FRBR, "Bibliotime", 6, 1 (2003), disponibile all'indirizzo <http://didattica.spbo.unibo.it/bibliotime/num-vi-1/buizza.htm>

PINO BUIZZA - MAURO GUERRINI, Un modello concettuale per il Nuovo soggettario. 
Tesi di dottorato di Giuliano Genetasio, discussa presso l'Università degli Studi di Udine

L'indicizzazione per soggetto alla luce di FRBR, "Bollettino AIB", 41, 3 (2001), p. 327-336

VANNEVAR BUSH, As we may think, "The Atlantic monthly", 176, 1 (1945), p. 101-108

KAREN CALHOUN, The changing nature of the catalog and its integration with other discovery tools. Final report. March 17, 2006, prepared for the Library of Congress, 2006, disponibile all'indirizzo <http://www.loc.gov/catdir/calhoun-report-final.pdf>

KAREN CALHOUN - JOANNE CANTRELL - PEGGY GALLAGHER - JANET

HAWK, Online catalogs. What users and librarians want. An OCLC report, Dublin, Ohio, OCLC, 2009, disponibile all'indirizzo

<http://www.oclc.org/reports/onlinecatalogs/fullreport.pdf>

RAFAEL CAPURRO, Hermeneutics facing the 'information framing', "Ubiquity", 9, 8 (2008), disponibile all'indirizzo <http://ubiquity.acm.org/article.cfm?id=1361368>

and the phenomenon of information, in Metaphysics, epistemology and technology, edited by Carl Mitcham, Amsterdam, Jai, 2000, p. 79-85

BIANCA CASTAÑÓN MORENO, Análisis temático documental, "Investigación bibliotecológica”, 6,12 (1992), p. 4-10

LOIS MAI CHAN, Exploiting LCSH, LCC and DDC to retrieve networked resources. Issues and challenges, in Proceedings of the Bicentennial Conference on Bibliographic Control for the New Millennium: Confronting the Challenges of Networked Resources and the Web, Washington, D.C., November 15-17, 2000, edited by Ann M. Sandberg-Fox, Washington, D.C., Library of Congress, Cataloging Distribution Service, 2001, p. 159-78, disponibile all'indirizzo <http://www.loc.gov/catdir/bibcontrol/chan_paper.html>

LOIS MAI CHAN, The future of LCSH. A response, "Cataloging \& classification quarterly", 46, 4 (2008), p. 433-436

LOIS MAI CHAN, Library of Congress subject headings. Principles and application, 3rd ed., Englewood,Colo., Libraries Unlimited,1995

LOIS MAI CHAN, Still robust at 100. A century of LC subject headings, "Library of Congress Information Bulletin”, 57, 8 (1998), p. 200-201, disponibile all'indirizzo <http://www.loc.gov/loc/lcib/9808/lcsh-100.html>

LOIS MAI CHAN, Subject analysis tools online. The challenge ahead, "Information technology and libraries”, 9, 2 (1990), p. 258-262

LOIS M. CHAN, Thoughts on LCSH, in Library of Congress subject headings. Pre-vs. Postcoordination and related issues, 2007, disponibile all'indirizzo <http://www.loc.gov/catdir/cpso/pre_vs_post.pdf>, p. 14-20 
Tesi di dottorato di Giuliano Genetasio, discussa presso l'Università degli Studi di Udine

LOIS MAI CHAN - ERIC CHILDRESS - REBECCA J. DEAN - EDWARD T.

O'NEILL - DIANE VIZINE-GOETZ, A faceted approach to subject data in the Dublin Core metadata record, "Journal of internet cataloging", 4, 1/2 (2001), p. 35-47

LOIS MAI CHAN - T. HODGES, Entering the new millennium. A new century for LCSH, "Cataloging \& classification quarterly", 29, 1/2 (2000), p. 232-233

ALBERTO CHETI, Le categorie nell'indicizzazione, "Biblioteche oggi", 8, 1 (1990), p. 29-49

ALBERTO CHETI, Il punto di vista del GRIS sulla 'relazione di soggetto' in FRBR, in Principi di catalogazione internazionali. Una piattaforma europea? Considerazioni sull'IME ICC di Francoforte e Buenos Aires. Atti del convegno internazionale, Roma, Bibliocom, 51. Congresso AIB, 27 ottobre 2004, a cura di Mauro Guerrini, Roma, Associazione italiana biblioteche, 2008, p. $91-100$

ALBERTO CHETI, Il negozio di ferramenta e la ricerca per soggetto dei documenti, "Bollettino AIB”, 41, 1 (2001), p. 69-76

NOAM CHOMSKY, La grammatica trasformazionale. Scritti espositivi, Torino, Boringhieri, 1975, traduzione di Logical structures in language, A transformational approach to syntax, On the notion 'Rule of grammar', The formal nature of language

JUNE CHRESSANTHIS, An introduction to form subdivisions in subject headings,

"Mississippi libraries", 64, 1 (2000), p. 17-18

CLARA M. CHU - ANN O'BRIEN, Subject analysis. The critical first stage in indexing, "Journal of information science", 19, 6 (1993), p. 439-454

GUGLIELMO CINQUE, Introduzione del curatore, in La semantica generativa, a cura di Guglielmo Cinque, Torino, Boringhieri, 1979

CLASSIFICATION RESEARCH GROUP, The need for a faceted classification as the basis of all methods of information retrieval, "Library Association record", 57, 7 (1955), p. 262-268, poi pubblicato in Theory of subject analysis. A sourcebook, edited by Lois Mai Chan, Phyllis A. Richmond, Elaine Svenonius, Littleton, Colo., Libraries unlimited, 1985

ERIC J. COATES, Progress in documentation. Some properties of relationships in the structure of indexing languages, "Journal of documentation", 29, 4 (1973), p. 390-404

ERIC J. COATES, Subject catalogues. Headings and structure, London, Library Association, 1988

PAULINE ATHERTON COCHRANE, The future of LCSH. Comments on Lois Chan's Response, "Cataloging \& classification quarterly", 46, 4 (2008), p. 436-438 
Tesi di dottorato di Giuliano Genetasio, discussa presso l'Università degli Studi di Udine

PAULINE ATHERTHON COCHRANE, Improving LCSH for use in online catalogs. Exercises for self-help with a selection of background readings, Littleton, Colo., Libraries Unlimited, 1986

PAULINE ATHERTON COCHRANE, Improving LCSH for use in online catalogs revisited. What progress has been made? What issues still remain?, "Cataloging \& classification quarterly", 29, 1/2 (2000), p. 73-89

LOURDES Y. COLLANTES, Degree of agreement in naming objects and concepts for information retrieval, "Journal of the American Society for Information Science", 46, 2 (1995), p. 116-132

JUD H. COPELAND, RDA and FRBR. A brave new world in cataloging. A guide to understanding the fundamental changes in cataloging, "Arkansas libraries", 67, 2 (2010), p. $14-19$

WALTER A. COOK, A case grammar matrix, "Languages and linguistics", 6 (1972), p. $15-47$

WILLIAM S. COOPER, Is interindexer consistency a hobgoblin?, "American documentation”, 20, 3 (1969), p. 268-278

YVES GILBERT COURRIER, Document analysis, verbs and case grammar, Pittsburg, University of Pittsburg, 1976

MAURICE COYAUD, Introduction a l'ètude des langages documentaires. Thèse de doctorat $d u$ 3e cycle présentée à la Faculté des lettres et sciences humaines de Paris, Paris, Klincksieck, 1966

MAURICE COYAUD, Linguistique et documentation. Les articulations logiques du discours, Paris, Larousse, 1972

KAREN COYLE, Machine indexing, "Journal of academic librarianship", 34, 6 (2008), p. $530-531$

ANA L. CRISTÁN, SACO and subject gateways, "Cataloging \& classification quarterly" $39,1 / 2$

(2004), p. 309-322

R. C. CROS - JEAN-CLAUDE GARDIN - F. LÉVY, L'automatisation des recherches documentaires. Un modèle général 'Le SYNTOL', 2e édition revue et augmentée, Paris, Gauthier-Villars, 1968

GIANFRANCO CRUPI, 010.72. Strumenti e strategie di ricerca bibliografica, in Biblioteconomia. Guida classificata, diretta da Mauro Guerrini, condirettore Gianfranco Crupi, a cura di Stefano Gambari, collaborazione di Vincenzo Fugaldi, presentazione di Luigi Crocetti, Milano, Editrice Bibliografica, 2007, p. 107-134 
Tesi di dottorato di Giuliano Genetasio, discussa presso l'Università degli Studi di Udine

DAVID CRYSTAL, Linguistics and indexing, "The indexer", 14, 1 (1984), p. 3-7

DAVID CRYSTAL, Reverse indexing, "The indexer", 26, 1 (2008), p. 14-17

DAVID CRYSTAL, Semantic targeting. Past, present and future, "Aslib proceedings", 62, 4/5 (2010), p. 355-365

CHARLES A. CUTTER, Rules for a dictionary catalog, 4th edition rewritten, Washington, D.C., Government Printing Office, 1904

INGETRAUT DAHLBERG, A referent-oriented, analytical concept theory of INTERCONCEPT, "International classification", 5, 3 (1978), p. 142-151

IONE T. DAMASCO, FAST. Faceted application of subject terminology. A new spin on an old standard, "TechKnow", 12, 2 (2006), p. 1-8

JAY ELWOOD DAILY, The grammar of subject headings. A formulation of rules for subject headings based on a syntactical and morphological analysis of the Library of Congress list, Ann Arbor, Mich., University Microfilms, 1957

ARMANDO DE PALMA, Presentazione, in NOAM CHOMSKY, La grammatica trasformazionale. Scritti espositivi, Torino, Boringhieri, 1975, traduzione di Logical structures in language, A transformational approach to syntax, On the notion 'Rule of grammar', The formal nature of language, p. 9-15

REBECCA J. DEAN, FAST. Development of simplified headings for metadata, in Authority control in organizing and accessing information. Definition and international experience, Arlene G. Taylor, Barbara B. Tillett editors, with the assistance of Mauro Guerrini, Murtha Baca, New York, Haworth, 2004, p. 331-352, disponibile all'indirizzo <http://www.unifi.it/universita/biblioteche/ac/relazioni/dean_eng.pdf >

TOM DELSEY, Modeling subject access. Extending the FRBR and FRANAR conceptual models, "Cataloging \& classification quarterly", 39, 3/4 (2005), p. 49-61

RICHARD L. DERR, The concept of information in ordinary discourse, "Information processing \& management", 21, 6 (1985), p. 489-499

BRENDA DERVIN, Useful theory of librarianship. Communication, not information, "Drexel library quarterly", 13, 3 (1977), p. 16-32

STELLA G. DEXTRE CLARKE, Evolution towards ISO 25964. An international standard with guidelines for thesauri and other type of controlled vocabulary, "Information Wissenschaft \& Praxis", 58, 8 (2007), p. 441-444 
Tesi di dottorato di Giuliano Genetasio, discussa presso l'Università degli Studi di Udine

FRANCIS JAWAHAR DEVADASON, Computer generation of different types of subject index entries based on deep structure of subject indexing languages. Deep structure indexing system, in ASIS '85. Proceedings of the 48th ASIS Annual Meeting, Las Vegas, Nevada, October 20-24, 1985, edited by Carol A. Parkhurst, Knowledge Industry Publications, White Plains, New York, 1985, p. 88-96

ROSSELLA DINI, Principi e standard, in Il futuro della descrizione bibliografica. Atti della giornata di studio, Firenze, 13 novembre 1987, a cura di Mauro Guerrini, Roma, Associazione italiana biblioteche, 1988, p. 9-15

S. DUTTA - P. K. SINHA, Pragmatic approach to subject indexing. A new concept, "Journal of the American Society for Information Science”, 35, 6 (1984), p. 325-331

UMBERTO ECO, I limiti dell'interpretazione, 2. ed., Milano, Bompiani, 1995

UMBERTO ECO, La ricerca della lingua perfetta nella cultura europea, 5. ed., Roma, Laterza, 2006

UMBERTO ECO, Trattato di semiotica generale, 17. ed., Milano, Bompiani, 1999 Eco1999

SANDA ERDELEZ, Information encountering. A conceptual framework for accidental information discovery, in Information seeking in context. Proceedings of an international conference on research and information needs, seeking and use in different contexts, 14-16 August, 1996, Tampere, Finland, ed. by Perrti Vakkari, Reijo Savolainen and Brenda Dervin, London, Taylor Graham, 1997, p. 412-421

BERNHARD EVERSBERG, On the theory of library catalogs and search engines. Supplementing a presentation on 'Principles and goals of cataloging', 2007, disponibile all'indirizzo <http://www.allegro-c.de/formate/tlcse.htm>

ROBERT A. FAIRTHORNE, Content analysis, specification, and control, "Annual review of information science and technology”, 4 (1969), p. 73-109

JASON E. L. FARRADANE, Analysis and organization of knowledge for retrieval, "Aslib proceedings", 22, 12 (1970), p. 607-616

JASON E. L. FARRADANE, Relational indexing. Part II, "Journal of information science principles and practice”, 1, 6 (1980), p. 313-324

JOHN F. FARROW, All in the mind. Concept analysis in indexing, "The indexer", 19, 4 (1995), p. 243-247

JOHN F. FARROW, A cognitive process model of document indexing, "Journal of documentation”, 47, 2 (1991), p. 149-166 
Tesi di dottorato di Giuliano Genetasio, discussa presso l'Università degli Studi di Udine

JOHN F. FARROW, Propositional analysis and macrorules in indexing, "Library review", 45, 1 (1996), p. 6-15

EMILY GALLUP FAYEN, Guidelines for the construction, format, and management of monolingual controlled vocabularies. A revision of ANSI/NISO Z39.19 for the 21st century, "Information Wissenschaft \& Praxis", 58, 8, 2007, p. 445-448

RAYA FIDEL, User-centered indexing, "Journal of the American Society of Information Science”, 45, 8 (1994), p. 572-576

CHARLES J. FILLMORE, Il caso del caso, traduzione di G. R. Cardona, in Gli universali nella teoria linguistica, Torino, Boringhieri, 1978, p. 27-131, traduzione di The case for case, p. $1-88$

CHARLES J. FILLMORE, Pragmatics and the description of discourse, in Radical pragmatics, edited by Peter Cole, New York, Academic Press, 1981, p. 143-167

CHARLES J. FILLMORE, Si riapre il caso del caso, traduzione di G. R. Cardona, in Gli universali nella teoria linguistica, Torino, Boringhieri, 1978, p. 271-300, traduzione di The case for case reopened

KAREN S. FISCHER, Critical views of LCSH, 1990-2001. The third bibliographic essay, “Cataloging \& classification quarterly", 41, 1 (2005), p. 63-109

ANTONY CHARLES FOSKETT, The subject approach to information, 2nd ed. revised and enlarged, London, Bingley, 1971

INA FOURIE, Book indexing. A reflection on the contrasting complexities and ease of conceptualization and how we can deepen our understanding, "Mousaion", 26, 1 (2008), p. $111-125$

ELENA FRANCHINI, 025.47. Tesauri, in Biblioteconomia. Guida classificata, diretta da Mauro Guerrini, condirettore Gianfranco Crupi, a cura di Stefano Gambari, collaborazione di Vincenzo Fugaldi, presentazione di Luigi Crocetti, Milano, Editrice Bibliografica, 2007, p. 645-649

VICTORIA FRÂNCU, Subjects in FRBR and poly-hierarchical thesauri as possible knowledge organizing tools, in Knowledge organization for a global learning society. Proceedings of the ninth International ISKO Conference, 4-7 July 2006, Vienna, Austria, edited by Gerhard Budin, Christian Swertz, Konstantin Mitgutsch, Würzburg, Ergon, 2006, p. 375-382

THOMAS J. FROEHLICH, Relevance reconsidered. Towards an agenda for the 21st century. Introduction to special topic issue on relevance research, "Journal of the American Society for Information Science”, 45, 3, p. 124-133, disponibile all'indirizzo <http://www.cs.oswego.edu/ ${ }^{\sim}$ kyoon/course/ISC220/readings/froelich94.pdf> 
Tesi di dottorato di Giuliano Genetasio, discussa presso l'Università degli Studi di Udine

BERND FROHMANN, Discourse analysis as a research method in library and information science, "Library and information science research", 16, 2 (1994), p. 119-138

BERND FROHMANN, An investigation of the semantic bases of some theoretical principles of classification proposed by Austin and the CRG, "Cataloging \& classification quarterly", 4, 1 (1983), p. 11-27

BERND FROHMANN, Rules of indexing. A critique of mentalism in information retrieval theory, "Journal of documentation", 46, 2 (1990), p. 81-101

ROBERT FUGMANN, The complementarity of natural and index language in the field of information supply. An overview of their specific capabilities and limitations, "Knowledge organization”, 29, 3 (2002), p. 217-230

ROBERT FUGMANN, The Five-axiom theory of indexing and information supply, "Journal of the American Society for Information Science”, 36, 2 (1985), p. 116-129

ROBERT FUGMANN, On the practice of indexing and its theoretical foundations, "International classification", 7, 1 (1980), p. 13-20

ROBERT FUGMANN, Representational predictability. Key to the resolution of several pending issues in indexing and information supply, in Knowledge organization and quality management. Proceedings of the third International ISKO Conference, 20-24 June 1994, Copenhagen, Denmark, organized by the Royal School of Librarianship, Copenhagen, Denmark in cooperation with the International Society for Knowledge Organization, Germany, with support from Dan Fink's Foundation, Denmark, edited by Hanne Albrechtsen and Susanne Oernager, Frankfurt/Main, Indeks, 1994, p. 414-422

ROBERT FUGMANN, Toward a theory of information supply and indexing, "International classification”, 6, 1 (1979), p. 3-15

JONATHAN FURNER, Information studies without information, "Library trends", 52, 3 (2004), p. 427-446

The future of subdivisions in the Library of Congress subject headings system. Report from the Subject Subdivisions Conference sponsored by the Library of Congress May 9-12, 1991, edited by Martha O'Hara Conway, Washington, D.C., Library of Congress, Cataloging Distribution Service, 1992

JEAN-CLAUDE GARDIN, Document analysis and linguistic theory, "Journal of documentation", 29, 2 (1973), p. 137-168

JEAN-CLAUDE GARDIN, Semantic analysis procedures in the sciences of man, "Social science information”, 8, 1 (1969), p. 17-42 
Tesi di dottorato di Giuliano Genetasio, discussa presso l'Università degli Studi di Udine

EUGENE GARFIELD, The relationship between mechanical indexing, structural linguistics and information retrieval, "Journal of information science", 18, 5 (1992), p. 343-354

TED GEMBERLING, Thema and FRBR's third group, "Cataloging \& classification quarterly", 48, 5 (2010), p. 445-449

CLAUDIO GNOLI, Animals belonging to the emperor. Enabling viewpoint warrant in classification, in Subject access. Preparing for the future, edited by Patrice Landry, Leda Bultrini, Edward T. O’Neill and Sandra K. Roe, Berlin, De Gruyter, 2011, p. 91-100

CLAUDIO GNOLI, Naturalism vs pragmatism in knowledge organization, in Knowledge organization and the global information society. Proceedings of the eighth International ISKO Conference, 13-16 July 2004, London, UK, organized by ISKO and School of Library, Archive \& Information Studies, University College London, edited by Ian C. McIlwaine, Würzburg, Ergon, 2004, p. 263-268

GIOVANNI GOBBER - MORENO MORANI, Linguistica generale, Milano, McGrawHill, 2010

MICHAEL GORMAN, An administrator's view. Factors influencing organized subject access, “Technicalities", 15, 10 (1995), p. 11-14

MICHAEL GORMAN, Cataloging and the new technologies, in The nature and future of the catalog. Proceedings of the ALA's Information Science and Automation Division's 1975 and 1977 Institutes on the Catalog, edited by Maurice J. Freedman and S. Michael Malinconico, Phoenix, Oryx, 1979, p. 127-152

M. A. GOPINATH, Descriptors and their role in information retrieval, "Library science with a slant to documentation and information studies”, 30, 3 (1993), p. 118-123

REBECCA GREEN, Syntagmatic relationships in index languages. A reassessment, "Library quarterly”, 65, 4 (1995), p. 365-385

REBECCA GREEN, Topical relevance relationships. I, Why topic matching fails, "Journal of the American Society for Information Science”, 46, 9 (1995), p. 646-653

REBECCA GREEN - C. BEAN, Topical relevance relationships. II, An exploratory study and preliminary tipology, "Journal of the American Society for Information Science", 46, 9 (1995), p. 654-662

ALAN M. GREENBERG, Scope notes in Library of Congress subject headings, "Cataloging \& classification quarterly", 1, 2/3 (1982), p. 95-104

ALGIRDAS JULIEN GREIMAS, Semantica strutturale, Roma, Meltemi, 2000, traduzione di Sémantique structurale. Recherche de méthode, Paris, Larousse, 1966 
Tesi di dottorato di Giuliano Genetasio, discussa presso l'Università degli Studi di Udine

H. PAUL GRICE, Meaning, "The Philosophical Review”, 66, 3 (1957), p. 377-88

TERESA GRIMALDI, 025.47. Catalogazione per soggetto (Teoria dell'indicizzazione semantica), in Biblioteconomia. Guida classificata, diretta da Mauro Guerrini, condirettore Gianfranco Crupi, a cura di Stefano Gambari, collaborazione di Vincenzo Fugaldi, presentazione di Luigi Crocetti, Milano, Editrice Bibliografica, 2007, p. 625-628

TERESA GRIMALDI, L'indicizzazione dal punto di vista cognitivo, "Il bibliotecario", 12, 1 (1995), p. 277-301

TERESA GRIMALDI, L'indicizzazione dal punto di vista cognitivo (II), "Il bibliotecario", 13, 2 (1996), p. 259-293

ÉRIC DE GROLIER, A study of general categories applicable to classification and coding in documentation, translated from the French text by the National Science Foundation, revised by Anthony Thompson, Paris, UNESCO, 1962

ANDRZEJ GRZEGORCZYK, The pragmatic foundations of semantics, "Synthese", 8, 6/7 (1950-1951), p. 300-324

MAURO GUERRINI, I Principi internazionali di catalogazione (ICP). Universo bibliografico e teoria catalografica all'inizio del XXI secolo, con Giuliano Genetasio, postfazione di Attilio Mauro Caproni, Milano, Editrice Bibliografica, 2012

JOACIM HANSSON, Hermeneutics as a bridge between the modern and the postmodern in library and information science, "Journal of documentation", 61, 1 (2005), p. 102-113

COREY A. HARPER - BARBARA B. TILLETT, Library of Congress controlled vocabularies and their application to the semantic web, "Cataloging \& classification quarterly", 43, 3/4 (2007), p. 47-68, disponibile all'indirizzo <https://scholarsbank.uoregon.edu/jspui/bitstream/1794/3269/1/ccq_sem_web.pdf>

DAVID JUDSON HAYKIN, Subject headings. A practical guide, New York, Gordon, 1978

MAGDA HEINER-FREILING, Survey on subject heading languages used in national libraries and bibliographies, "Cataloging \& classification quarterly", 29, 1/2 (2000), p. $189-198$

HARRIETTE HEMMASI - DAVID P. MILLER - MARY CHARLES LASATER, Access to form data in online catalogs, 1999, disponibile all'indirizzo

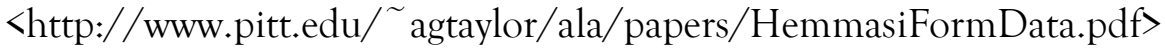

DORALYN J. HICKEY, Subject analysis. An interpretive survey, "Library trends", 25, 1 (1976), p. 273-291 
Tesi di dottorato di Giuliano Genetasio, discussa presso l'Università degli Studi di Udine

CHARLES. R. HILDRETH, Online catalog design models. Are we moving in the right direction? Report prepared for the Council on Library Resources, 1995, disponibile all'indirizzo <http://myweb.cwpost.liu.edu/childret/clr-opac.html>

SAMANTHA SCHMEHL HINES, Librarians at the bounds of rationality. How bounded rationality can help us help others, "Behavioral \& social sciences librarian", 28, 3 (2009), p. $80-86$

LOUIS T. HJELMSLEV, I fondamenti della teoria del linguaggio, introduzione e traduzione di Giulio C. Lepschy, Torino, Einaudi, 1987, traduzione di Omkring Sprogteoriens Grundlæggelse

BIRGER HJØRLAND, The concept of 'subject' in information science, "Journal of documentation", 48, 2 (1992), p. 172-200

BIRGER HJØRLAND, Theory of knowledge organization and the feasibility of universal solutions. Presented at the Eighth International ISKO Conference, London, July 13-16, 2004, 2004, disponibile all'indirizzo

<http://arizona.openrepository.com/arizona/bitstream/10150/105303/1/hjorlandisko8 .pdf>

BIRGER HJØRLAND, Information retrieval, text composition, and semantics, "Knowledge organization", 25, 1/2 (1998), p. 16-31

BIRGER HJØRLAND, Information seeking and subject representation. An activity-theoretical approach to information science, Wetsport, Conn., London, Greenwood, 1997

BIRGER HJØRLAND, Nine principles of knowledge organization, in Knowledge organization and quality management. Proceedings of the third International ISKO Conference, 20-24 June 1994, Copenhagen, Denmark, organized by the Royal School of Librarianship, Copenhagen, Denmark in cooperation with the International Society for Knowledge Organization, Germany, with support from Dan Fink's Foundation, Denmark, edited by Hanne Albrechtsen and Susanne Oernager, Frankfurt/Main, Indeks, 1994, p. 91-100

BIRGER HJØRLAND, Theory and metatheory of information science. A new interpretation, "Journal of documentation", 54, 5 (1998), p. 606-621

BIRGER HJØRLAND, Towards a theory of aboutness, subject, topicality, theme, domain, field, content...and relevance, "Journal of the American Society for Information Science and Technology”, 52, 9 (2001), p. 774-778

BIRGER HJØRLAND - LYKKE KYLLESBECH NIELSEN, Subject access points in electronic retrieval, "Annual review of information science and technology", 35 (2001), p. 249-298 
Tesi di dottorato di Giuliano Genetasio, discussa presso l'Università degli Studi di Udine

BIRGER HJØRLAND - KARSTEN NISSEN PEDERSEN, A substantive theory of classification for information retrieval, "Journal of documentation", 61, 5 (2005), p. 582-597

HEIDI LEE HOERMAN - KEVIN A. FURNISS, Turning practice into principles. A comparison of the IFLA Principles underlying subject heading languages (SHLs) and the principles underlying the Library of Congress subject headings system, Cataloging \& classification quarterly", 29, 1/2 (2000), p. 31-52, disponibile all'indirizzo <http://polaris.gseis.ucla.edu/gleazer/462_readings/Hoerman.pdf>

HERBERT H. HOFFMAN, Making sense of serials. Subject access to works in online catalogs, "Technicalities", 21, 5 (2001), p. 9-13

DARA LEE HOWARD, Pertinence as reflected in personal constructs, "Journal of the American Society for Information Science”, 45, 3 (1994), p. 172-185

W. JOHN HUTCHINS, The concept of 'aboutness' in subject indexing, "Aslib proceedings", 30,5 , p. $172-181$

W. JOHN HUTCHINS, Languages of indexing and classification. A linguistic study of structures and functions, Stevenage, Peregrinus, 1975

W. JOHN HUTCHINS, Linguistic processes in the indexing and retrieval of documents, "Linguistics", 61 (1970), p. 29-64

IFLA WORKING GROUP ON THE FUNCTIONAL REQUIREMENTS FOR SUBJECT AUTHORITY RECORDS, Functional requirements for subject authority data (FRSAD). A conceptual model, editors: Marcia Lei Zeng, Maja Žumer, Athena Salaba, approved by the Standing Committee

of the IFLA Section on Classification and Indexing, June 2010, <http://www.ifla.org/files/classification-and-indexing/functional-requirements-forsubject-authority-data/frsad-finalreport.pdf $>$

\section{IFLA STUDY GROUP ON THE FUNCTIONAL REQUIREMENTS FOR}

BIBLIOGRAPHIC RECORDS, Functional requirements for bibliographic records. Final report, approved by the Standing Committee of the IFLA Section on Cataloguing, München, Saur, 1998, disponibile all'indirizzo <http://www.ifla.org/files/cataloguing/frbr/frbr.pdf>

IFLA UBCIM WORKING GROUP ON FUNCTIONAL REQUIREMENTS AND NUMBERING OF AUTHORITY RECORDS (FRANAR), Functional requirements for authority records. A conceptual model, draft 2005-06-15, 2005, <http://www.cidoc crm.org/docs/frbr_oo/frbr_docs/FRANARConceptual-M-Draft-e.pdf>

PETER INGWERSEN - IRENE WORMELL, Means to improved subject access and representation in modern information retrieval, "Libri", 38, 2 (1988), p. 94-119

INTERNATIONAL CONFERENCE ON CATALOGUING PRINCIPLES, International 
Tesi di dottorato di Giuliano Genetasio, discussa presso l'Università degli Studi di Udine

Conference on Cataloguing Principles. Paris, 9th-18th October, 1961. Report, edited by A. H. Chaplin and Dorothy Anderson, London, Organizing Committee of the International Conference on Cataloguing Principles, 1963, disponibile all'indirizzo <http://www.dnb.de/standardisierung/pdf/paris_principles_1961.pdf>

\section{INTERNATIONAL FEDERATION OF LIBRARY ASSOCIATIONS AND}

INSTITUTIONS, Dichiarazione di principi internazionali di catalogazione, traduzione a cura di Carlo Bianchini, Maria De Panicis, Mauro Guerrini, Cristina Magliano, Paola Manoni, in INTERNATIONAL FEDERATION OF LIBRARY ASSOCIATIONS AND

INSTITUTIONS, IFLA cataloguing principles. The Statement

of international cataloguing principles (ICP) and its Glossary. In 20 languages, edited by Barbara

B. Tillett and Ana Lupe Cristán, München, Saur, 2009, p. 126-139, disponibile all'indirizzo

〈http://www.ifla.org/files/cataloguing/icp/icp_2009-it.pdf>.

\section{INTERNATIONAL FEDERATION OF LIBRARY ASSOCIATIONS AND}

INSTITUTIONS, International standard bibliographic description (ISBD). Preliminary consolidated edition, recommended by the ISBD Review Group, approved by the Standing Committee of the IFLA Cataloguing

Section, München, Saur, 2007

INTERNATIONAL ORGANIZATION FOR STANDARDIZATION, International standard ISO 5963. Documentation. Methods for examining documents, determining their subjects, and selecting indexing terms, Géneve, ISO, 1985

INTERNATIONAL SOCIETY FOR KNOWLEDGE ORGANIZATION. SEZIONE ITALIA. INTEGRATIVE LEVELS CLASSIFICATION, León manifesto, 2007, disponibile all'indirizzo <http://www.iskoi.org/ilc/leon.php>

SHEILA S. INTNER, ASCR. The American subject cataloging rules. Part 1, "Technicalities", 8,7 (1988), p. 5-7

JOSEPHINE I. IWE, Linguistics and information processing. Provision of syntactic and semantic consistency in the language of Library of Congress subject headings (LCSH) pertaining to literature and librarianship. A comparative analysis, "Cataloging \& classification quarterly", 32, 2 (2001), p. 107-126

HEMALATA IYER, Natural language representation. Transformational rules, "International classification” 17, 1 (1990), p. 8-13

ROMAN JAKOBSON, Saggi di linguistica generale, cura e introduzione di Luigi Heilmann, traduzione di Luigi Heilmann e Letizia Grassi, 3a ed., Milano, Feltrinelli, 2008, traduzione di Essais de linguistique générale

QIANG JIN, Is FAST the right direction for a new system of subject cataloging and metadata?, "Cataloging \& classification quarterly", 45, 3 (2008), p. 91-110 
Tesi di dottorato di Giuliano Genetasio, discussa presso l'Università degli Studi di Udine

THOMAS JOHANSEN, Elements of the non-linguistic approach to subject-relationships, "International classification" 14, 1 (1987), p. 11-18

JOINT STEERING COMMITTEE FOR REVISION OF AACR, Regole di catalogazione angloamericane. Seconda edizione. Revisione del 1988, redatte sotto la direzione del Joint Steering Committee for Revision of AACR, a cura di Michael Gorman e Paul W. Winkler, edizione italiana a cura di Rossella Dini e Luigi Crocetti, Milano, Editrice Bibliografica, 1997, traduzione di Anglo-American cataloguing rules

JOINT STEERING COMMITTEE FOR DEVELOPMENT OF RDA, Resource description $\mathscr{F}$ access. RDA, developed in a collaborative process led by the Joint Steering Committee for Development of RDA (JSC), Chicago, American Library Association, 2010-

KEVIN P. JONES, Towards a theory of indexing, "Journal of documentation", 32, 2 (1976), p. $118-123$

DANIEL N. JOUDREY - ARLENE G. TAYLOR, RE: LCSH Strings - some thoughts, in Library of Congress subject headings. Pre-vs. Post-coordination and related issues, 2007, disponibile all'indirizzo <http://www.loc.gov/catdir/cpso/pre_vs_post.pdf>, p. 21-24

JULIUS OTTO KAISER, Systematic indexing, London, Pitman, 1911

MURAT KARAMUFTUOGLU, Need for a systemic theory of classification in information science, "Journal of the American Society for Information Science and Technology", 58, 13 (2007), p. 1977-1987

JERROLD J. KATZ, Semantic theory, New York, Harper and Row, 1972

JERROLD J. KATZ - JERRY ALAN FODOR, The structure of a semantic theory, "Language", 39, 2 (1963), p. 170-210

CRISTOPHER S. G. KHOO - JIN-CHEON NA, Semantic relations in information science, "Annual review of information science and technology", 40 (2006), p. 157-228

SAM A. KHOSH-KUI, Effects of subject specificity. Part I, Specificity of LC subject headings and depth of subject analysis in monographic records, "Technical services quarterly", 4, 2 (1986), p. 59-67

GIYEONG KIM, Relationship between index term specificity and relevance judgment, "Information processing \& management", 42, 5 (2006), p. 1218-1229

WALTER KINTSCH - TEUN A. VAN DIJK, Toward a model of text comprehension and production, "Psychological review", 85, 5 (1978), p. 363-394 
Tesi di dottorato di Giuliano Genetasio, discussa presso l'Università degli Studi di Udine

MONIKA KIRTLAND - PAULINE COCHRANE, Critical views of LCSH - Library of Congres subject headings. A bibliographic and bibliometric essay, Washington, D.C., distributed by ERIC Clearinghouse, 1981

OLEG KREYMER, 'The mysteries of memory...' (The analytical language of T. Mann), 2007, disponibile all'indirizzo <http://oknyc.blogspot.com/2007/06/all-mysteries-of-memoryanalytical.html>

SYDNEY M. LAMB, The sememic approach to structural semantics, "American anthropologist", 66, 3 (1964), p. 57-78

FREDERICK WILFRID LANCASTER, Vocabulary control for information retrieval, 2nd ed., Arlington, Va., Information Resources Press, 1986

BERTRAND C. LANDRY - JAMES E. RUSH, Towards a theory of indexing. II, "Journal of the American Society for Information Science" 21, 1970, p. 358-367

DEREK W. LANGRIDGE, Subject analysis. Principles and procedures, London, BowkerSaur, 1989

RAY R. LARSON, Classification clustering, probabilistic information retrieval, and the online catalog, "Library quarterly", 61, 2 (1991), p. 133-173

RAY R. LARSON, The decline of subject searching. Long-term trends and patterns of index use in an online catalog, "Journal of the American Society for Information Science", 42, 3 (1991), p. 197-215, disponibile all'indirizzo <http://polaris.gseis.ucla.edu/gleazer/462_readings/ Larson_1991.pdf>

PATRICK LE BCEUF, Brave new FRBR world, in IFLA MEETING OF EXPERTS ON AN INTERNATIONAL CATALOGUING CODE, 1, IFLA cataloguing principles. Steps towards an international cataloguing code. Report from the 1st IFLA Meeting of Experts on an International Cataloguing Code, Frankfurt, 2003, edited by Barbara B. Tillett, Renate Gömpel and Susanne Oehlschläger,München, Saur, 2004, p. 40-53, disponibile all'indirizzo <http://www.d-nb.de/standardisierung/pdf/papers_leboeuf.pdf>

CHINGKWEI ADRIENNE LEE - JOHN N. OLSGAARD, Linguistics and information science, in Principles and applications of information science for library professionals, John N. Olsgaard editor, Chicago, American Library Association, 1989, p. 27-36

LIBRARY OF CONGRESS. CATALOGING POLICY AND SUPPORT OFFICE, Library of Congress subject headings. Pre- vs. Post-coordination and related issues, 2007, disponibile all'indirizzo <http://www.loc.gov/catdir/cpso/pre_vs_post.pdf>

LIBRARY OF CONGRESS. CATALOGING POLICY AND SUPPORT OFFICE, Subject Headings Manual, Washington, D.C., Library of Congress, Cataloging Policy and Support Office, 2008- 
Tesi di dottorato di Giuliano Genetasio, discussa presso l'Università degli Studi di Udine

LIBRARY OF CONGRESS. POLICY AND STANDARDS DIVISION, Library of Congress subject headings, 32nd ed., Washington, D.C., Library of Congress, Cataloging Distribution Service, 2010

LIBRARY OF CONGRESS. WORKING GROUP ON THE FUTURE OF BIBLIOGRAPHIC CONTROL, On the Record. Report of the Library of Congress Working Group on the Future of Bibliographic Control, Washington, D.C., Library of Congress, 2008, disponibile all'indirizzo <http://www.loc.gov/bibliographic-future/news/lcwgontherecord-jan08-final.pdf>

B. LONG, Linguistique et indexation, "Documentaliste”, 17, 3 (1980), p. 99-106

CAIMEI LU - JUNG-RAN PARK - XIAOHUA HU, User tags versus expert-assigned subject terms. A comparison of LibraryThing tags and Library of Congress subject headings, "Journal of Information Science”, 36, 6 (2010), p. 763-779, disponibile all'indirizzo <http://jis.sagepub.com/content/36/6/763.full.pdf>

HOWARD MACLAY, Overview, in Semantics. An interdisciplinary reader in philosophy, linguistics and psychology. Part II. Linguistics, edited by Danny D. Steinberg \& Leon A. Jakobovits, Cambridge, Cambridge University Press, 1971, p. 157-182

R. K. MAHAPATRA, Information needs. A conceptual investigation, "SRELS journal of information management", 39, 4 (2002), 371-382

JENS-ERIK MAI, Semiotics and indexing. An analysis of the subject indexing process, "Journal of documentation", 57, 5 (2001), p. 591-622

S. MICHAEL MALINCONICO, The library catalog in a computerized environment, in The nature and future of the catalog. Proceedings of the ALA's Information Science and Automation Division's 1975 and 1977 Institutes on the Catalog, edited by Maurice J. Freedman and S. Michael Malinconico, Phoenix, Oryx, 1979, p. 46-71

DIEGO MALTESE, La biblioteca come linguaggio e come sistema, Milano, Bibliografica, 1985

DIEGO MALTESE, Elementi di indicizzazione per soggetto. L'analisi dei documenti e l'indicizzazione a catena, Milano, Bibliografica, 1982

DIEGO MALTESE, Principi di catalogazione e regole italiane, Firenze, Olschki, 1965

DIEGO MALTESE - ALBERTO PETRUCCIANI, Un'esperienza di indicizzazione per soggetto. Materiali perla versione italiana del PRECIS, Roma, Associazione italiana biblioteche, 1990 
Tesi di dottorato di Giuliano Genetasio, discussa presso l'Università degli Studi di Udine

JACQUES MANIEZ, Actualité des langages documentaires. Les fondements théoriques de la recherche d'information, Paris, ADBS, 2002

JACQUES MANIEZ, Les langages documentaires et classificatoires. Conception, construction et utilisation dans les systèmes documentaires, Paris, Editions d'organisation, 1987

THOMAS MANN, 'Cataloging must change!' and indexer consistency studies. Misreading the evidence at our peril, "Cataloging \& classification quarterly", 23, 3/4 (1997), p. 3-45

THOMAS MANN, The changing nature of the catalog and its integration with other discovery tools. Final report. March 17, 2006. Prepared for the Library of Congress by Karen Calhoun. A critical review, 2006, disponibile all'indirizzo

<http://www.guild2910.org/AFSCMECalhounReviewREV.pdf>

THOMAS MANN, Will Google's keyword searching eliminate the need of LC cataloging and classification?, 2005, disponibile all'indirizzo <http://www.guild2910.org/searching.htm>

MARC 21 format for authority data. Including guidelines for content designation, prepared by Network Development and MARC Standards Office, Library of Congress in cooperation with Standards and Support, Library and Archives Canada, Bibliographic Standards and Systems, British Library, Washington, Library of Congress, Cataloging Distribution Service, 1999.

MARC 21 format for bibliographic data. Including guidelines for content designation, prepared by Network Development and MARC Standards Office, Library of Congress in cooperation with Standards and Support, Library and Archives Canada, Bibliographic Standards and Systems, British Library, Washington, Library of Congress, Cataloging Distribution Service, 1999.

DEANNA B. MARCUM, Response to On the record. Report of the Library of Congress Working Group on the Future of Bibliographic Control, Washington, D.C., Library of Congress, 2008, disponibile all'indirizzo <http://www.loc.gov/bibliographic-future/news/ LCWGRptResponse_DM_053008.pdf>

KAREN MARKEY, Integrating the machine-readable LCSH into online catalogs, 1988

KAREN MARKEY, Interpreting the findings of 'A study of library users and their understanding of subject headings', "Technicalities”, 19, 4 (1999), p. 1, 13-15

KAREN MARKEY - B. A. ROEBER DEDE - M. LEAVITT, The change of meaning in subdivided subject headings, 38, 3 (1999), p. 19-43

KAREN MARKEY - SCHELLE SIMCOX - EILEEN G. FENTON, Do patrons understand Library of Congress subject headings?, "Technicalities”, 19, 1 (1999), p. 7-11 
Tesi di dottorato di Giuliano Genetasio, discussa presso l'Università degli Studi di Udine

KAREN MARKEY - SCHELLE SIMCOX - EILEEN G. FENTON, End-user

understanding of subject headings in library catalogs, "Library resources and technical services", 43, 3 (1999), p. 140-60

KAREN MARKEY - SCHELLE SIMCOX - MARIE WILLIAMS, Do librarians understand the subject headings?, "Reference and user services quarterly", 38, 4 (1999), p. 369-87

KAREN MARKEY - DIANE VIZINE-GOETZ, Using subject headings for online retrieval. Theory, practice, and potential, San Diego, Academic Press, 1994

KAREN MARKEY - MARJORIE S. WELLER, Testing a new design for subject searching in online catalogs, "Library hi tech", 12, 1 (1994), p. 67-86

A. M. MARQUES CINTRA, Elementos de lingüística para estudos de indexaçao, "Ciência da informação", 12, 1 (1983), p. 5-22

M. E. MARON, On indexing, retrieval and the meaning of about, "Journal of the American Society for Information Science”, 28, 1 (1977), p. 38-43

LINNEA MARSHALL, Specific and generic subject headings. Increasing subject access to library materials, "Cataloging \& classification quarterly", 36, 2 (2003), p. 59-87

JESSICA S. MELTON, A use for the techniques of structural linguistics in documentation research, in Classification research. Proceedings of the second International Study Conference held at Hotel Prins Hamlet, Elsinoore, Denmark, 14th to 18th September, 1964, edited by Pauline Atherton, Copenhagen, Munksgaard, 1965

EVA MÉNDEZ RODRÍGUEZ - JOSÉ A. MOREIRO GONZÁLEZ, Lenguaje natural e indización automatizada, "Ciencias de la información", 30 , 3 (1999), p.11-24

TANJA MERČUN - MAJA ŽUMER, New generation of catalogues for the new generation of users. A comparison of six library catalogues, "Program", 42, 3 (2008), p. 243-261, disponibile all'indirizzo <http://www.ff.uni-

lj.si/oddelki/biblio/oddelek/osebje/dokumenti/MercunZumer08_New_generation_of_c atalogues_for_the_new_generation_of_users.pdf $>$

JOHN WALLACE METCALFE, Tentative code of rules for alphabetico-specific entry, in Subject classifying and indexing, by John Wallace Metcalfe, New York, Scarecrow, 1959, p. 263-292

JOHN WALLACE METCALFE, Information indexing and subject cataloging. Alphabetical. Classified. Coordinate. Mechanical, New York, Scarecrow, 1957

FABIO METITIERI, L'OPAC collaborativo, tra folksonomia e socialità, "Biblioteche oggi", 
Tesi di dottorato di Giuliano Genetasio, discussa presso l'Università degli Studi di Udine

27, 2 (2009), p. 7-12, disponibile all'indirizzo

<http://www.bibliotecheoggi.it/2009/20090200701.pdf>

DOUGLAS P. METZLER - TERRY NOREAULT - BRIAN HEIDORN, Syntactic parsing for information retrieval, in Productivity in the information age. Proceedings of the 46th ASIS Annual Meeting, Washington, D.C.,October 2-6, 1983, edited by Raymond F. Vondran, Anne Caputo, Carol Wasserman, Richard A. V. Diener, White Plains, New York, Knowledge Industry Publications, 1983, p. 269-273

JIA MI - CATHY WENG, Revitalizing the library OPAC. Interface, searching, and display challenges, "Information technology and libraries", 27, 1 (2008), p. 5-22

FRANCIS L. MIKSA, Information organization and the mysterious information user, "Libraries $\&$ the cultural record", 44, 3 (2009), p. 343-370

DAVID P. MILLER, Out from under. Form/genre access in LCSH, "Cataloging \& classification quarterly", 29, 1/2 (2000), p.169-188

DAVID P. MILLER, The SACO Program in the lives of our institutions, "Cataloging \& classification quarterly", 48, 2/3 (2010), p. 194-220

JACK MILLS, Faceted classification and logical division in information retrieval, "Library trends", 52, 3 (2004), p. 541-570

JESSICA L. MILSTEAD, Natural versus inverted word order in subject headings, "Library resources and technical services”, 24, 2 (1980), p. 174-178

WILLIAM MISCHO, Library of Congress subject headings. A review of the problems, and prospects for improved subject access, "Cataloging \& classification quarterly", 1, 2/3 (1982), p. $105-124$

CHARLES W. MORRIS, Lineamenti di una teoria dei segni, nella traduzione di Ferruccio Rossi-Landi, a cura di Susan Petrilli, nuova ed. rivista e aggiornata, Lecce, Pensa multimedia, 2009, traduzione di Foundations of the theory of signs

JANE MORRIS, Individual differences in the interpretation of text. Implications for information science, "Journal of the American Society for Information Science and Technology", 61, 1 (2010), p. 141-149

MAGDELEINE MOUREAU, Les aspects linguistiques des stratégies d'interrogation dans la rcherche bibliographique sur ordinateur, "Documentaliste”, 13, 5/6 (1976), p. 190-195

WIDAD MUSTAFA-ELHADI, The contribution of terminology to the theoretical conception of classificatory languages and document indexing, in Tools for knowledge organization and the human interface. Proceedings. 1st International ISKO-Conference, Darmstadt, 14-17 August 
Tesi di dottorato di Giuliano Genetasio, discussa presso l'Università degli Studi di Udine

1990, organized by the International Society for Knowledge Organization (ISKO), edited by R. Fugmann, Frankfurt/Main, Indeks, 1990, p. 98-106

YUKIO NAKAMURA, A language for knowledge representation, in Knowledge organization and quality management. Proceedings of the third International ISKO Conference, 20-24 June 1994, Copenhagen, Denmark, organized by the Royal School of Librarianship, Copenhagen, Denmark in cooperation with the International Society for Knowledge Organization, Germany, edited by Hanne Albrechtsen and Susanne Oernager, Frankfurt am Main, Indeks, 1994, p. 127-133

NATIONAL INFORMATION STANDARDS ORGANIZATION, Guidelines for the construction, format, and management of monolingual controlled thesauri. ANSI/NISO Z39.19-2005, Bethesda (Md.), NISO Press, 2005

SAMUEL D. NEILL, The dilemma of the subjective in information organisation and retrieval, "Journal of documentation", 43, 3 (1987), p. 193-211

ROBERT T. ODDY - ELIZABETH DUROSS LIDDY - BHASKARAN BALAKRISHNAN - ANN BISHOP - JOSEPH ELEWONONI - EILEEN MARTIN, Towards the use of situational information in information retrieval, "Journal of documentation”, 48, 2 (1992), p. 123-171

NORMAN ODER, The end of LC subject headings?, "Library journal", 13, 9 (2006), p. $14-15$

HOPE A. OLSON, Universal models. A history of the organization of knowledge, in Knowledge organization and quality management. Proceedings of the third International ISKO Conference, 20-24 June 1994, Copenhagen, Denmark, organized by the Royal School of Librarianship, Copenhagen, Denmark in cooperation with the International Society for Knowledge Organization, Germany, edited by Hanne Albrechtsen and Susanne Oernager, Frankfurt am Main, Indeks, 1994, p. 72-80

HOPE A. OLSON - LISA M. GIVEN, Indexing and the 'organized' researcher, "The indexer", 23, 3 (2003), p. 129-133

EDWARD T. O'NEILL - LOIS MAI CHAN, FAST (Faceted application of subject terminology). A simplified vocabulary based on Library of Congress subject headings, "IFLA Journal”, 29, 4 (2003), p. 336-342

EDWARD T. O'NEILL - ERIC CHILDRESS - REBECCA J. DEAN - KERRE KAMMERER - DIANE VIZINE-GOETZ - LOIS MAI CHAN - LYNN EL-HOSHY, FAST. Faceted application of subject terminology, in Subject retrieval in a networked environment. Proceedings of the IFLA satellite meeting held in Dublin, OH, 14-16 August 2001 and sponsored by the IFLA Classification and Indexing Section, the IFLA Information Technology Section and OCLC, edited by I. C. McIlwaine, Saur, München, 2003, p. 140-146 
Tesi di dottorato di Giuliano Genetasio, discussa presso l'Università degli Studi di Udine

EDWARD T. O'NEILL - DIANE VIZINE-GOETZ, Bibliographic relationships. Implications for the function of the catalog, in The conceptual foundations of descriptive cataloging, edited by Elaine Svenonius, San Diego, Academic Press, 1989, p. 167-179

C. C. OSIGWE, The effect of semantic and syntactic relationships in the choice of descriptor elements for precision in document indexing and information retrieval, "Quarterly bulletin of the International Association of Agricultural Librarians and Documentalists”, 37, 4 (1992), p. 205-208

JOSEPH W. PALMER, Subject authority control and syndetic structure - myth and realities. An inquiry into certain subject heading practices and some questions about their implications, "Cataloging \& classification quarterly", 7, 2 (1986), p. 71-95

IOANNIS PAPADAKIS - KONSTANTINOS KYPRIANOS - ROSA MAVROPODI MICHALIS STEFANIDAKIS, Subject-based information retrieval within digital libraries employing LCSHs, "D-lib magazine", 15, 9/10 (2009), disponibile all'indirizzo <http://www.dlib.org/dlib/september09/papadakis/09papadakis.html>

IOANNIS PAPADAKIS - MICHALIS STEFANIDAKIS - AIKATERINI TZALI, Semantic navigating an OPAC by subject headings meta-information, "Electronic library", 27, 5 (2008), p. 779-791

JUNG-RAN PARK, Evolution of concept networks and implications for knowledge representation, "Journal of documentation", 63, 6 (2007), p. 963-983

CHARLES SANDERS PEIRCE, Semiotica, testi scelti e introdotti da Massimo A. Bonfantini, Letizia Grassi, Roberto Grazia, Torino, Einaudi, 1980

CHARLES SANDERS PEIRCE, Collected papers of Charles Sanders Peirce. Volume 1, Principles of philosophy, and Volume II, Elements of logic, edited by Charles Hartshorne and Paul Weiss, Belknap Press of Harvard University Press, Cambdridge, Massachusetts, 1960

JEAN M. PERREAULT, Categories and relators. A new schema, "Knowledge organization", 21, 4 (1994), p. 189-198

ELAINE PETERSON, Parallel systems. The coexistence of subject cataloging and folksonomy, "Library philosophy nad practice", 2008, disponibile all'indirizzo <http://unllib.unl.edu/ LPP/e-peterson3.htm>

ELAINE PETERSON, Patron preferences for folksonomy tags. Research findings when both hierarchical subject headings and folksonomy tags are used, "Evidence based library and information practice”, 4, 1 (2009), p. 53-56

ALBERTO PETRUCCIANI, I fondamenti semantici della catalogazione per soggetto, "Accademie e biblioteche d'Italia" 50, 3 (1982), p. 219-234 
Tesi di dottorato di Giuliano Genetasio, discussa presso l'Università degli Studi di Udine

ALBERTO PETRUCCIANI, Funzione e struttura del catalogo per autore, Firenze, Giunta regionale toscana, La nuova Italia, 1984

JULIA PETTEE, The development of authorship entry and the formulation of authorship rules as found in the Anglo-American code, in Foundations of cataloging. A sourcebook, edited by Michael Carpenter and Elaine Svenonius, Littleton, Colo., Libraries Unlimited, 1985, p. 79-80

JULIA PETTEE, Subject headings. The history and theory of the alphabetical subject approach to books, New York, H. M. Wilson, 1946

AUGUSTO PONZIO, Il linguaggio e le lingue. Introduzione alla linguistica generale, Bari, B.A. graphis, 2004

A. R. D. PRASAD - NABONITA GUHA, Concept naming vs concept categorisation. A faceted approach to semantic annotation, "Online information review", 32, 4 (2008), p. 500-510

EMANUELE QUINTARELLI, Folksonomies. Power to the people, paper presented at the ISKO ITALY-UNIMB Meeting, Milan, June 24, 2005, disponibile all'indirizzo <http://www.iskoi.org/doc/folksonomies.htm>

ANDREW RADFORD, La sintassi trasformazionale. Introduzione alla Teoria Standard Estesa di Chomsky, Bologna, Il mulino, 1983, traduzione di Transformational syntax. A student's guide to Chomsky's extended standard theory

GARY P. RADFORD, Flaubert, Foucault, and the bibliotheque fantastique. Toward a postmodern epistemology for library science, "Library trends", 46, 4 (1998), p. 616-634

GARY P. RADFORD - MARIE L. RADFORD, Structuralism, post-structuralism and the library. De Saussure and Foucault, "Journal of documentation", 61, 1 (2005), p. 60-78

SHIYALI RAMAMRITA RANGANATHAN, Prolegomena to library classification, assisted by M. A. Gopinath, 3rd ed., New York, Asia, 1967, disponibile all'indirizzo <http://arizona.openrepository.com/arizona/handle/10150/106370>

Regole italiane di catalogazione. REICAT, a cura della Commissione permanente per la revisione delle regole italiane di catalogazione, Roma, ICCU, 2009

SALLY JO REYNOLDS, In theory there is no solution, The impediments to a subject cataloging code, "Library quarterly", 59, 3 (1989), p. 223-238

PHYLLIS ALLEN RICHMOND, Contribution toward a new generalized theory of classification, in Classification research. Proceedings of the second International Study Conference held at Hotel Prins Hamlet, Elsinoore, Denmark, 14th to 18th September, 1964, edited by Pauline Atherton, Copenhagen, Munksgaard, 1965, p. 39-54 
Tesi di dottorato di Giuliano Genetasio, discussa presso l'Università degli Studi di Udine

ALEXIS RIVIER, Construction des langages d'indexation. Aspects théoretiques, "Documentaliste", 27, 6 (1990), p. 263-274

STEPHEN E. ROBERTSON, Between aboutness and meaning, in Informatics 5. The analysis of meaning. Proceedings of a conference held by the ASLIB Informatics Group and the BCS Information Retrieval Specialist Group, 26-28 march 1979 Oxford, edited by Maxime McCafferty and Kathleen Gray, London, Aslib, 1979, p. 202-205

MASSIMO ROLLE, Per un nuovo modello di linguaggio documentario, "Bollettino AIB", 41, 3 (2001), p. 315-326

JAN ROSS, A new way of information retrieval. 3-D indexing and concept mapping, "Learned publishing", 13, 2 (2000), p. 119-123

ATHENA SALABA, End-user understanding of indexing language information, "Cataloging \& classification quarterly", 47, 1 (2009), p. 23-51

ALENKA ŠAUPERL, Catalogers' common ground and shared knowledge, "“Journal of the American Society for Information Science and Technology”, 55, 1 (2004), p. 55-63

ALENKA ŠAUPERL, Precoordination or not? A new view of the old question, "Journal of documentation”, 65, 5 (2009), p. 817-833

SAUSSURE, FERDINAND DE, Corso di linguistica generale, traduzione e commento di Tullio De Mauro, 22. ed., Roma, GLF Laterza, 2009, traduzione di Cours de linguistique générale

ADAM L. SCHIFF, The future of LCSH, 2008, disponibile all'indirizzo <http://depts.washington.edu/aluw/activities/0708/The_Future_of_LCSH.ppt>

ADAM L. SCHIFF, Special libraries and the Program for Cooperative Cataloging, "Information outlook", 3, 10 (1999), p. 14

ALFREDO SERRAI, Critica dei Functional requirements for bibliographic records (FRBR), "Bibliotheca", 1, 2 (2002), p. 207-215

ALFREDO SERRAI, Del catalogo alfabetico per soggetti. Semantica del rapporto indicale, Roma, Bulzoni, 1979

ALFREDO SERRAI, Indici logica e linguaggio. Problemi di catalogazione semantica, Roma, Consiglio nazionale delle ricerche, Laboratorio di studi sulla ricerca e sulla documentazione scientifica, 1974

ALFREDO SERRAI, Problemi linguistici e implicazioni epistemologiche della catalogazione semantica, "Accademie e biblioteche d'Italia", 40, 2 (1972), p. 109-116 
Tesi di dottorato di Giuliano Genetasio, discussa presso l'Università degli Studi di Udine

JOHN ANTHONY ARTHUR SILLINCE, Literature searching with unclear objectives. A new approach using argumentation, "Online information review", 16, 6 (1992), p. 391-410

HERBERT A. SIMON, Il comportamento amministrativo, Bologna, Il mulino, 2001, traduzione di Administrative behavior. A study of decision-making processes in administrative organization

RICHARD P. SMIRAGLIA, The progress of theory in knowledge organization, "Library trends", 50, 3 (2002), p. 330-349

DAGOBERT SOERGEL, A general model for indexing languages. The basis for campatibility and integration, in Subject retrieval in the seventies. New directions. Proceedings of an international symposyium held at the Center for Adult Education, University of Maryland, College Park, May 14 to 15, 1971, edited by Hans (Hanan) Wellisch and Thomas D. Wilson, Westport, Greenwood, 1972, p. 36-61

DAGOBERT SOERGEL, Organizing information. Principles of data base and retrieval systems, Orlando, Academic Press, 1985

JUTTA SØRENSEN - DEREK AUSTIN, PRECIS in a multilingual context. Part 2, A linguistic and logical explanation of the syntax, "Libri", 26, 3 (1976), p. 108-139

KAREN SPARCK-JONES - MARTIN KAY, Linguistics and information science, New York, Academic Press, 1973

SIMON SPERO, LCSH is to thesaurus as doorbell is to mammal. Visualizing structural problems in the Library of Congress subject headings, in Proceedings of the 2008 International Conference on Dublin Core and Metadata Applications, 2008, disponibile all'indirizzo <http://edoc.hu-berlin.de/conferences/dc-2008/spero-simon-203/PDF/spero.pdf>

LOUISE SPITERI, A simplified model for facet analysis, "Canadian journal of information and library science”, 23, 1/2 (1998), p. 1-30

GARY STEELE, The wisdom of the cataloguers. Using collective intelligence to assign Library of Congress subject headings, 2010, disponibile all'indirizzo <http://www.iskouk.org/conf2011/papers/steele.pdf>

TOM STEELE, The new cooperative cataloging, "Library hi tech", 27, 1 (2009), p. 68 - 77

WOLFGANG G. STOCK, Concepts and semantic relations in information science, "Journal of the American Society for Information Science and Technology”, 61, 10 (2010), p. 1951-1969

N. A. STOKOLOVA, Syntactic tools and semantic power of information languages. (Pt. II of 
Tesi di dottorato di Giuliano Genetasio, discussa presso l'Università degli Studi di Udine

'Elements of sa semantic theory of information retrieval'), "International classification" 3, 2 (1976), p. 75-81

ALVA T. STONE, The LCSH century. A brief history of the Library of Congress subject headings, and Introduction to the centennial essays, "Cataloging \& classification quarterly", 29, 1 (2000), p. $1-15$

ALVA T. STONE, That elusive concept of 'aboutness'. Ten year's work in subject analysis, 1992, "Library resources and technical services", 37, 3 (1993), p. 277-298

WILLIAM E. STUDWELL, Application astray keeps retrieval away. Five basic principles for application of LC subject headings, "Technicalities", 10, 6 (1990), p. 8-9

WILLIAM E. STUDWELL, Cutterism, codism, and consumerism. The past, present and future of LC subject access, "Technical services quarterly", 9, 1 (1991), p. 19-25

WILLIAM E. STUDWELL, Gathering levels in LC subject headings. Revisiting an excellent aid to subject retrieval, "Technicalities", 11, 5 (1991), p. 8-9

WILLIAM E. STUDWELL, LC's head in the sand, or, Why the Subject cataloging manual is not enough, "Technical services quarterly", 10, 3 (1993), p. 45-50

WILLIAM E. STUDWELL, Library of Congress subject headings. Philosophy, practice, and prospects, New York, Haworth, 1990

WILLIAM E. STUDWELL, Of eggs and baskets. Getting more access out of LC subject headings in an online environment, "Cataloging \& classification quarterly", 13, 3/4 (1992), p. $91-102$

WILLIAM E. STUDWELL, USE, the universal subject environment. A new subject approach in the time of the internet, "Journal of internet cataloging", 2, 3/4 (2000), p. 197-209

OLOF SUNDIN - JENNY JOHANNISSON, Pragmatism, neo-pragmatism and sociocultural theory. Communicative participation as a perspective in LIS, "Journal of documentation", 61, 1 (2005), p. 23-43

ELAINE SVENONIUS, Directions for research in indexing, classification, and cataloging, "Library resources and technical services", 25, 1 (1981), p. 88-103

ELAINE SVENONIUS, The epistemological foundations of knowledge representations, "Library trends", 52, 3 (2004), p. 571-587

ELAINE SVENONIUS, Facet definition. A case study, "International classification" 5, 3 (1978), p. 134-141 
Tesi di dottorato di Giuliano Genetasio, discussa presso l'Università degli Studi di Udine

ELAINE SVENONIUS, The intellectual foundation of information organization, Cambridge, Mass., MIT Press, 2000

ELAINE SVENONIUS, Indexical contexts, in Universal classification I. Subject analysis and ordering systems. Proceedings. 4th Internatl. Study Conference on Classification Research, 6th Annual Conference of Gesellschaft für Klassification e.V., Augsburg, 28 June-2 July 1982. Vol. 1, editor Ingetraut Dahlberg, Frankfurt, Indeks, 1982, p. 125-138

ELAINE SVENONIUS, LCSH. Semantics, syntax and specificity, "Cataloging \& classification quarterly", 29, 1/2 (2000), p. 17-30

ELAINE SVENONIUS - HELEN F. SCHMIERER, Current issues in the subject control of information, "Library quarterly", 47, 3 (1977), p. 326-346

D. F. SWIFT - V. WINN - D. BRAMER, 'Aboutness' as a strategy for retrieval in the social sciences, "Aslib proceedings", 30, 5 1978, p. 182-187

D. F. SWIFT - V. WINN - D. BRAMER, Information systems for subject specialist. A multimodal approach to indexing and classification, Washington, D.C., distributed by ERIC Clearinghouse, 1977, disponibile all'indirizzo <http://www.eric.ed.gov/contentdelivery/servlet/ERICServlet?accno=ED139396>

V. D. SYDORCHENKO, Typological classification of thesaurus semantic structures, "Automatic documentation and mathematical linguistics", 9, 2 (1975), p. 16-23

SANNA TALJA - KIMMO TUOMINEN - REIJO SAVOLAINEN, 'Isms' in information science. Constructivism, collettivism and constructionism, "Journal of documentation", 61, 1 (2005), p. 79-101

SHOICHI TANIGUCHI, Design of cataloging rules using conceptual modeling of cataloging process, "Journal of the American Society for Information Science and Technology", 55, 6 (2004), p. 498-512

STEFANO TARTAGLIA, La sintassi delle stringhe di soggetto. Metodo e principi, "Bollettino AIB”, 37, 3 (1997), p. 329-333

ARLENE G. TAYLOR, On the subject of subjects, "Journal of academic librarianship", 21, 6 (1995), p. 484-491

ROY TENNANT, MARC must die, "Library journal”, 127, 17 (2002), p. 26-28, disponibile all'indirizzo <http://www.libraryjournal.com/article/CA250046.html>

MARLIESE THOMAS - DANA M. CAUDLE - CECILIA M. SCHMITZ, To tag or not to tag?, "Library hi tech", 27, 3 (2009), p. 411-434 
Tesi di dottorato di Giuliano Genetasio, discussa presso l'Università degli Studi di Udine

CLARE THORNLEY, Dilemmas in information science (IS) and information retrieval (IR).

Recurring challenges or new solutions?, "Aslib proceedings", 61, 3 (2009), p. 323-330

CLARE THORNLEY - FORBES GIBB, A dialectical approach to information retrieval, "Journal of documentation", 63, 5 (2007), p. 755-764

CLARE THORNLEY - FORBES GIBB, Meaning in philosophy and meaning in information retrieval (IR), "Journal of documentation", 65, 1 (2009), p. 133-150

BARBARA B. TILLETT, AACR and authority control, in The future of the descriptive cataloging rules. Papers from the ALCTS Preconference. AACR2000, American Library Association Annual Conference, Chicago, June 22, 1995, edited by Brian E. C. Schottlaender, Chicago, American Library Association, 1998, p. 30-39

BARBARA B. TILLETT, Bibliographic relationships. Toward a conceptual structure of bibliographic information used in cataloging, Ann Arbor, Mi., University Microfilms International, 1987

ROSS J. TODD, Academic indexing. What's it all about?, "The indexer”, 18, 2 (1992), p. $101-104$

JOHN TOFANELLI, Written testimony for Working Group, in Testimony submitted to the Library of Congress Working Group on the Future of Bibliographic Control. Part II, 2007, p. 97-98

YASAR TONTA, LCSH and PRECIS in library and information science. A comparative study, Board of Trustees of The University of Illinois, 1992, disponibile all'indirizzo <http://yunus.hacettepe.edu.tr/ ${ }^{\sim}$ tonta/yayinlar/tonta-lcsh-precis-gslis.pdf>

UNIVERSITY OF CALIFORNIA LIBRARIES. BIBLIOGRAPHIC SERVICES TASK FORCE, Rethinking how we provide bibliographic services for the University of California. Final report, 2005, disponibile all'indirizzo

<http://libraries.universityofcalifornia.edu/sopag/BSTF/Final.pdf>

TEUN A. VAN DIJK, Relevance assignment in discourse comprehension, "Discourse processes", 2 , 2 (1979), p. 113-126

SHERRY L. VELLUCCI, Bibliographic relationships in music catalogs, with a foreword by D. W. Krummel, Lanham, Md., Scarecrow, 1997

ROBERTO VENTURA, La biblioteca tra linguaggio e rappresentazione. Per un'indagine filosofica della biblioteconomia, "JLIS.it", 1, 1 (2010), p. 41-61

VIAF. Virtual international authority file, disponibile all'indirizzo <http://www.viaf.org/> 
Tesi di dottorato di Giuliano Genetasio, discussa presso l'Università degli Studi di Udine

BRIAN C. VICKERY, Knowledge representation. A brief review, "Journal of documentation", 42, 3 (1986), p. 145-159

DIANE VIZINE-GOETZ, FAST headings as tags for WorldCat, 2009, in Subject access. Preparing for the future, edited by Patrice Landry, Leda Bultrini, Edward T. O'Neill and Sandra K. Roe, Berlin, De Gruyter, 2011, p. 181-188

AMY J. WARNER, Quantitative and qualitative assessments of the impact of linguistic theory on informations science, "Journal of the American Society for Information Science", 42, 1 (1991), p. 64-71

AMY J. WARNER, A reaction to the findings of 'A study of library users and their understanding of subject headings', "Technicalities”, 19, 4 (1999), p. 4-5

AMY J. WARNER - PATRICK H. WENZEL, A linguistic analysis and categorization of nominal expressions, in Proceedings of the 54th ASIS Annual Meeting, Washington DC, October 27-31, 1991, editor and contributed papers chair José-Marie Griffiths, Medford, New Jersey, Learned Information, 1991, p. 186-195

JULIAN WARNER, Analogies between linguistics and information theory, "Journal of the American Society for Information Science and Technology", 58, 3 (2007), p. 309-321

JULIAN WARNER, Linguistics and information theory. Analytic advantages, "Journal of the American Society for Information Science and Technology", 58, 2 (2007) p. 275-285

JULIAN WARNER, Semiotics, information science, documents and computers, "Journal of documentation”, 46, 1 (1990), p. 16-32

PAUL GABRIELE WESTON, Il catalogo elettronico. Dalla biblioteca cartacea alla biblioteca digitale, postfazione di Giovanni Solimine, Roma, Carocci, 2005

PAUL GABRIELE WESTON, FRBR e utente. Considerazioni sulla ricerca, in SEMINARIO FRBR, Seminario FRBR. Functional requirements for bibliographic records, Requisiti funzionali per record bibliografici, Firenze, 27-28 gennaio 2000, atti a cura di Mauro Guerrini, Roma, Associazione italiana biblioteche, 2000, p. 118-127, disponibile all'indirizzo <http://www.aib.it/aib/sezioni/toscana/conf/frbr/weston.htm>

MARGARET WILLETTS, An investigation of the nature of the relation between terms in thesauri, "Journal of documentation", 31, 3 (1975), p. 158-184

NANCY J. WILLIAMSON, Standards and rules for subject access, "Cataloging \& classification quarterly", 21, 3/4 (1996), p. 155-176

NANCY J. WILLIAMSON, Standards and standardization in subject analysis systems. Current status and future directions, in Subject indexing. Principles and practices in the 90's. Proceedings of 
Tesi di dottorato di Giuliano Genetasio, discussa presso l'Università degli Studi di Udine

the IFLA Satellite Meeting, Lisbon, Portugal, 17-18 August, 1993, edited by Robert P. Holley, Dorothy McGarry, Donna Duncan, Elaine Svenonius, München, Saur, 1995, p. 278-291

NANCY J. WILLIAMSON, Subject cataloguing and LCSH, in Standards for the international exchange of bibliographic information. Papers presented at a course held at the School of Library, Archive and Information Studies, University College London, 3-18 August 1990, edited by I. C. McIlwaine, London, Library Association, 1991, p. 137-145

MARY DABNEY WILSON, Specificity, syndetic structure, and subject access to works about individual corporate bodies, "Library resources and technical services", 42, 4 (1998), p. 272-81

PATRICK WILSON, Two kinds of power. An essay on bibliographic control, Berkeley, University of California Press, 1978

THOMAS D. WILSON, On user studies and information needs, "Journal of documentation" 37, 1 (1981), p. 3-15

LUDWIG WITTGENSTEIN, Ricerche filosofiche, edizione italiana a cura di Mario Trinchero, Torino, Einaudi, 2009, traduzione di Philosophische Untersuchungen

GREGORY WOOL, Filing and precoordination. How subject headings are displayed in online catalogs and why it matters, "Cataloging \& classification quarterly", 29, 1/2 (2000), p. 91-106

IRENE WORMELL, Subject access redefined. How new technology changes the conception of subject representation, in Knowledge organization and quality management. Proceedings of the third International ISKO Conference, 20-24 June 1994, Copenhagen, Denmark, organized by the Royal School of Librarianship, Copenhagen, Denmark in cooperation with the International Society for Knowledge Organization, Germany, with support from Dan Fink's Foundation, Denmark, edited by Hanne Albrechtsen and Susanne Oernager, Frankfurt/Main, Indeks, 1994, p. 431-439

KWAN YI - LOIS MAI CHAN, Linking folksonomy to Library of Congress subject headings. An exploratory study, "Journal of documentation", 65, 6 (2009), p. 872-900

KWAN YI - LOIS MAI CHAN, Revisiting the syntactical and structural analysis of Library of Congress subject headings for the digital environment, "Journal of the American Society for Information Science and Technology", 46, 4 (2010), p. 677-687

MARCIA LEI ZENG - MAJA ŽUMER, Introducing FRSAD and mapping it with SKOS and other models, "International cataloguing and bibliographic control", 39, 3 (2010), p. 53-56

MARCIA LEI ZENG, Knowledge organization systems (KOS), 2008, "Knowledge organization”, 35, 2/3 (2008), p. 160-182 
Tesi di dottorato di Giuliano Genetasio, discussa presso l'Università degli Studi di Udine

PAUL ZIFF, On H. P. Grice's account of meaning, "Analysis”, 28, 1 (1967), p. 1-8

MAJA ŽUMER, FRBR. The end of the road or a new beginning?, "Bulletin of the American Society for Information Science and Technology”, 33, 6 (2007), p. 27-29

MAJA ŽUMER - GERHARD J. A. RIESTHUIS, Consequences of implementing FRBR. Are we ready to open Pandora's box?, "Knowledge organization", 29, 2 (2002), p. 78-86 
Tesi di dottorato di Giuliano Genetasio, discussa presso l'Università degli Studi di Udine

\section{Indice}

$\begin{array}{ll}\text { Abstract } & 2\end{array}$

1. Introduzione 3

1.1. L'attuale contesto dell'accesso bibliografico e il dibattito suscitato dal Rapporto Calhoun

1.2. Verità del dibattito e ragioni della ricerca: carenze teoriche e inadeguatezza tecnologica dell'indicizzazione

1.3. Contenuti e obiettivi della ricerca: approccio concettuale e linguistico all'indicizzazione

1.4. Limiti della ricerca

1.4.1. Ambito di analisi

1.4.2. Approccio scelto

1.4.3. Letteratura analizzata

2. Un approccio concettuale all'indicizzazione 10

2.1. L'approccio concettuale all'indicizzazione $\quad 10$

2.2. L'accesso bibliografico 12

2.3. L'interesse dell'utente 13

2.4. Le funzioni dell'utente 15

2.5. Gli oggetti di interesse 19

2.6. Le entità 19

2.7. Entità del Gruppo 1 di FRBR: Testi e Risorse 20

2.8. Entità del Gruppo 2 di FRBR 22

2.9. Entità del Gruppo 3 di FRBR 23

2.10. Entità nuove rispetto a FRBR 24

2.11. Entità concettuali e tipologie lessicali 26

$\begin{array}{ll}2.12 \text { Attributi e relazioni } & 26\end{array}$

2.13. Attributi nominali 28

2.14. Attributi nominali specifici 30

2.15. Attributi non nominali 36

2.16. Il soggetto 37

2.16.1. Introduzione $\quad 37$

2.16.2. Le diverse concezioni del soggetto 37

2.16.3. Antinomie del soggetto: pragmatismo e oggettivismo del soggetto 41

2.16.4. Verso una visione pragmatista e complessa del soggetto: circalità
estensionale e intensionale

2.16.5. Gli elementi della circalità estensionale $\quad 50$

2.16.6. Punti di vista 51

2.16.7. Una definizione di soggetto e di indicizzazione per soggetto 52

2.16.8. L'oggetto dell'indicizzazione per soggetto 53 
Tesi di dottorato di Giuliano Genetasio, discussa presso l'Università degli Studi di Udine

2.17. Dal modello concettuale alla sua realizzazione fisica 54

2.18. Indici e collegamenti: premessa: l'impatto dei cambiamenti tecnologici 55

2.18.1. Introduzione $\quad 55$

2.18.2. Ordine lineare e uniformità 56

2.18.3. Oltre la separazione tra indicizzazione per autore e per soggetto, verso una visione unitaria dell'accesso bibliografico: prima parte $\quad 57$

2.18.4. Oltre la precoordinazione? 58

2.18.5. Dal catalogo locale al catalogo in rete: ancora sull'uniformità 59

2.19. Indici e collegamenti: introduzione $\quad 60$

2.20. Indici nominali interni $\quad 65$

2.21. Indici nominali esterni 66

2.22. Indici non nominali corrispondenti ad attributi 67

$\begin{array}{ll}\text { 2.23. Indici valutativi } & 68\end{array}$

2.24. Metaindici 69

2.25. Indici di soggetto $\quad 70$

2.26. Indicizzazione alfabetica e sistematica per soggetto 72

2.27. Oltre la separazione tra indicizzazione per autore e per soggetto, verso una visione unitaria dell'accesso bibliografico: seconda parte 75

$\begin{array}{ll}\text { Lista riassuntiva delle entità } & 78\end{array}$

$\begin{array}{ll}\text { Lista riassuntiva degli attributi e delle relazioni } & 79\end{array}$

Lista riassuntiva degli indici e dei collegamenti $\quad 81$

3. Un approccio linguistico all'indicizzazione 83

3.1. Introduzione $\quad 83$

3.1.1. Opportunità di un approccio linguistico 83

3.1.2. L'applicazione della linguistica all'indicizzazione: letteratura favorevole 84

3.1.3. L'applicazione della linguistica all'indicizzazione: discipline correlate $\begin{array}{ll}\text { e letteratura contraria } & 87\end{array}$

3.2. Nozioni di linguistica generale $\quad 88$

3.2.1. Nozioni base della semiotica 88

3.2.2. Il presupposto fondamentale della linguistica generale: gli universali linguistici 90

3.2.3. Langue e parole, competenza ed esecuzione, sistema e processo, asse paradigmatico e sintagmatico 92

3.2.4. Funzioni linguistiche 96

3.2.5. Aspetti e componenti del linguaggio 97

$\begin{array}{ll}\text { 3.2.5.1. Introduzione } & 97\end{array}$

3.2.5.2. Lessico 97

3.2.5.3. Aspetti linguistici e branche della linguistica 98

$\begin{array}{ll}3.2 .5 .3 .1 . & 98\end{array}$

3.2.5.3.2. Morfosintassi $\quad 99$

$\begin{array}{ll}3.2 .5 .3 .3 . \text { Semantica } & 100\end{array}$

3.2.5.3.4. Rapporti tra sintassi, semantica e pragmatica 109 
Tesi di dottorato di Giuliano Genetasio, discussa presso l'Università degli Studi di Udine

3.2.6. Ancora su asse paradigmatico e sintagmatico, semantica e sintassi 112

3.2.7. Asse sintagmatico: semantica sintagmatica o sintassi profonda 114

3.2.8. Asse sintagmatico: relazioni sintagmatiche logiche 116

3.3. L'uso del linguaggio per l'indicizzazione 117

3.3.1. Indicizzazione e linguaggio 117

3.3.2. Universali linguistici e lingua documentaria ideale 119

$\begin{array}{ll}\text { 3.3.3. Funzioni linguistiche dell'indicizzazione } & 121\end{array}$

3.3.4. I diversi gradi di controllo linguistico 122

3.3.4.1. Introduzione 122

3.3.4.2. Semantica delle entità concettuali e delle registrazioni 123

$\begin{array}{ll}\text { 3.3.4.3. Semantica degli attributi e degli indici } & 123\end{array}$

3.3.4.4. Modalità di realizzazione del controllo linguistico: linguaggio naturale e lingue documentarie $\quad 124$

$\begin{array}{ll}\text { 3.3.4.5. Semantica delle relazioni e dei collegamenti } & 124\end{array}$

3.3.4.6. Unitarietà e semanticità dell'indicizzazione 125

3.3.4.7. Implicazioni per gli aspetti linguistici: lessicalizzazione e metasintagmi 125

$\begin{array}{ll}\text { 3.3.5. Asse paradigmatico e sintagmatico: prima parte } & 126\end{array}$

$\begin{array}{ll}\text { 3.3.6. La sottolingua documentaria } & 128\end{array}$

3.3.6.1. Asse paradigmatico e sintagmatico: seconda parte 129

3.3.6.2. Lessico 130

3.3.6.2.1. Controllo della biplanarità del lessico della sottolingua e suoi limiti 130

3.3.6.2.2. Natura parzialmente chiusa del lessico della sottolingua 132

3.3.6.2.3. Elementi lessicali e metalessicali 133

3.3.6.2.4. Elementi lessicali in forma semplice e composta 133

3.3.6.2.5. Elementi lessicali in forma composta non collegata, parzialmente collegata e totalmente collegata 133

3.3.6.2.6. Uso degli elementi lessicali e semantica paradigmatica dei sintagmi 136

$\begin{array}{ll}\text { 3.3.6.3. Morfosintassi di superficie } & 137\end{array}$

$\begin{array}{ll}\text { 3.3.6.4. Semantica } & 140\end{array}$

$\begin{array}{ll}3.3 .6 .4 .1 \text {. Introduzione } & 140\end{array}$

$\begin{array}{ll}3.3 .6 .4 .2 \text {. Semantica paradigmatica } & 142\end{array}$

3.3.6.4.2.1. Introduzione 142

3.3.6.4.2.2. Semantica paradigmatica delle relazioni 143

3.3.6.4.2.2.1. Introduzione 143

3.3.6.4.2.2.2. Relazioni gerarchiche $\quad 144$

3.3.6.4.2.2.2.1. Introduzione 144

3.3.6.4.2.2.2.2. Relazioni eponimiche pure 146

3.3.6.4.2.2.2.3. Relazioni partonomiche pure 147

3.3.6.4.2.2.2.4. Relazioni eponimiche, semiche e sememiche
specificate

3.3.6.4.2.2.2.5. Relazioni co-iponimiche, co-meronimiche e
co-sememiche

3.3.6.4.2.2.2.6. Relazioni tra nominalizzazioni 154

3.3.6.4.2.2.3. Relazioni associative $\quad 155$ 
Tesi di dottorato di Giuliano Genetasio, discussa presso l'Università degli Studi di Udine

3.3.6.4.2.2.4. Relazioni di antonimia 156

3.3.6.4.2.2.5. Relazioni di equivalenza 157

3.3.6.4.2.2.6. Relazioni di omonimia, polisemia e omotassia 163

3.3.6.4.2.2.7. Relazioni ad hoc 165

3.3.6.4.2.3. Semantica paradigmatica delle tipologie lessicali 166

3.3.6.4.3. Semantica sintagmatica o sintassi profonda 167

$\begin{array}{ll}3.3 .6 .4 .3 .1 . & 167\end{array}$

$\begin{array}{ll}\text { 3.3.6.4.3.2. Relazioni sintagmatiche } & 170\end{array}$

$\begin{array}{ll}3.3 .6 .4 .3 .3 \text {. Relazioni sintagmatiche grammaticali } & 175\end{array}$

3.3.6.4.3.4. Relazioni sintagmatiche logiche 180

Lista riassuntiva delle relazioni semantiche della sottolingua 183

$\begin{array}{ll}\text { Figure } & 185\end{array}$

$\begin{array}{ll}\text { Glossario } & 190\end{array}$

$\begin{array}{ll}\text { Bibliografia } & 219\end{array}$ 